January 2022

\title{
Basics of Thermal Field Theory
}

\author{
A Tutorial on Perturbative Computations ${ }^{1}$ \\ Mikko Laine $^{\mathrm{a}}$ and Aleksi Vuorinen ${ }^{\mathrm{b}}$ \\ ${ }^{a} A E C$, Institute for Theoretical Physics, University of Bern, \\ Sidlerstrasse 5, CH-3012 Bern, Switzerland \\ ${ }^{\mathrm{b}}$ Department of Physics, University of Helsinki, \\ P.O. Box 64, FI-00014 University of Helsinki, Finland
}

\begin{abstract}
These lecture notes, suitable for a two-semester introductory course or self-study, offer an elementary and self-contained exposition of the basic tools and concepts that are encountered in practical computations in perturbative thermal field theory. Selected applications to heavy ion collision physics and cosmology are outlined in the last chapter.
\end{abstract}

\footnotetext{
${ }^{1}$ An earlier version of these notes is available as an ebook (Springer Lecture Notes in Physics 925) at dx.doi.org/10.1007/978-3-319-31933-9; an eprint can be found at arxiv.org/abs/1701.01554; the very latest version is kept up to date at www.laine.itp.unibe.ch/basics.pdf.
} 



\section{Contents}

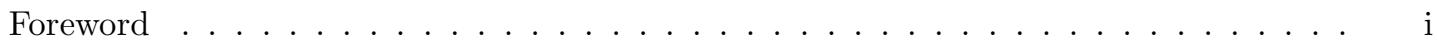

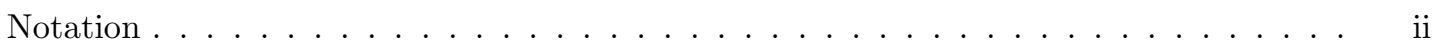

General outline ............................... iii

1 Quantum mechanics . . . . . . . . . . . . . . . . . . 1

1.1 Path integral representation of the partition function . . . . . . . . . 1

1.2 Evaluation of the path integral for the harmonic oscillator . . . . . . . 6

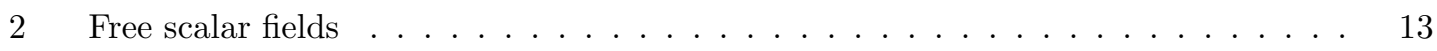

2.1 Path integral for the partition function . . . . . . . . . . . . 13

$2.2 \quad$ Evaluation of thermal sums and their low-temperature limit . . . . . . . 16

$2.3 \quad$ High-temperature expansion . . . . . . . . . . . . . . . . . . . . 23

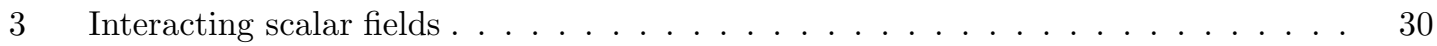

$3.1 \quad$ Principles of the weak-coupling expansion . . . . . . . . . . . . 30

3.2 Problems of the naive weak-coupling expansion $\ldots \ldots \ldots \ldots$

3.3 Proper free energy density to $\mathcal{O}(\lambda)$ : ultraviolet renormalization . . . . . . . 40

$3.4 \quad$ Proper free energy density to $\mathcal{O}\left(\lambda^{\frac{3}{2}}\right)$ : infrared resummation . . . . . . . . 44

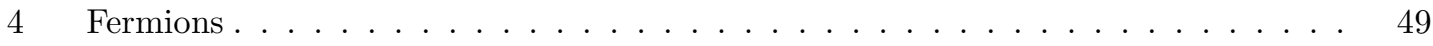

4.1 Path integral for the partition function of a fermionic oscillator . . . . . . 49

4.2 The Dirac field at finite temperature . . . . . . . . . . . . . 53

$5 \quad$ Gauge fields . . . . . . . . . . . . . . . . . . . . . 61

$5.1 \quad$ Path integral for the partition function . . . . . . . . . . . . 61

5.2 Weak-coupling expansion $\ldots \ldots \ldots \ldots \ldots$. . . . . . . . 67

5.3 Thermal gluon mass . . . . . . . . . . . . . . . . . . . . 73 
$5.4 \quad$ Free energy density to $\mathcal{O}\left(g^{3}\right) \ldots \ldots \ldots \ldots \ldots$

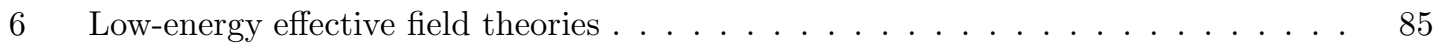

6.1 The infrared problem of thermal field theory $\ldots \ldots \ldots \ldots$. . . . . . 85

6.2 Dimensionally reduced effective field theory for hot QCD . . . . . . . . 91

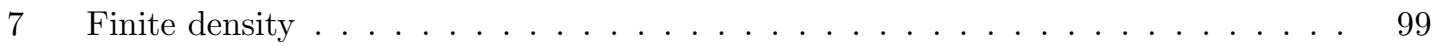

$7.1 \quad$ Complex scalar field and effective potential . . . . . . . . . . . . . 99

7.2 Dirac fermion with a finite chemical potential . . . . . . . . . . . . 105

8 Real-time observables . . . . . . . . . . . . . . . . . . . . . . . . 114

8.1 Different Green's functions . . . . . . . . . . . . . . . . . . . . . . . 114

8.2 From a Euclidean correlator to a spectral function . . . . . . . . . . . . 126

8.3 Real-time formalism . . . . . . . . . . . . . . . . . . . . . . 132

$8.4 \quad$ Hard Thermal Loops . . . . . . . . . . . . . . . . . . . . . . . 137

$9 \quad$ Applications . . . . . . . . . . . . . . . . . . . . . 155

9.1 Thermal phase transitions . . . . . . . . . . . . . . . . . . 155

9.2 Bubble nucleation rate . . . . . . . . . . . . . . . . . . . 164

$9.3 \quad$ Particle production rate . . . . . . . . . . . . . . . . . . 174

$9.4 \quad$ Embedding rates in cosmology . . . . . . . . . . . . . . . 183

9.5 Evolution of a long-wavelength field in a thermal environment . . . . . . . . 192

9.6 Linear response theory and transport coefficients . . . . . . . . . . . . . 197

$9.7 \quad$ Equilibration rates / damping coefficients . . . . . . . . . . . . . . 205

9.8 Resonances in medium . . . . . . . . . . . . . . . . . . . . . . 213

Appendix: Extended Standard Model in Euclidean spacetime . . . . . . . . . . . . . . 223

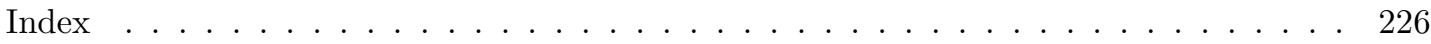




\section{Foreword}

These notes are based on lectures delivered at the Universities of Bielefeld and Helsinki, between 2004 and 2015, as well as at a number of summer and winter schools, between 1996 and 2018. The early sections were strongly influenced by lectures by Keijo Kajantie at the University of Helsinki, in the early 1990s. Obviously, the lectures additionally owe an enormous gratitude to existing text books and literature, particularly the classic monograph by Joseph Kapusta.

There are several good text books on finite-temperature field theory, and no attempt is made here to join that group. Rather, the goal is to offer an elementary exposition of the basics of the field, in an explicit "hands-on" style which can hopefully more or less directly be transported to the classroom. The presentation is meant to be self-contained and display also intermediate steps. The idea is, roughly, that each numbered section could constitute a single lecture. Referencing is sparse; on more advanced topics, as well as on historically accurate references, the reader is advised to consult the text books and review articles in refs. [0.1]-[0.17].

These notes could not have been put together without the helpful influence of many people, varying from students with persistent requests for clarification; colleagues who have used parts of an early version of these notes in their own lectures and shared their experiences with us; colleagues whose interest in specific topics has inspired us to add corresponding material to these notes; alert readers who have informed us about typographic errors and suggested improvements; and collaborators from whom we have learned parts of the material presented here. Let us gratefully acknowledge in particular Gert Aarts, Chris Korthals Altes, Dietrich Bödeker, Yannis Burnier, Stefano Capitani, Simon Caron-Huot, Jacopo Ghiglieri, Ioan Ghisoiu, Kimmo Kainulainen, Keijo Kajantie, Aleksi Kurkela, Harvey Meyer, Guy Moore, Paul Romatschke, Kari Rummukainen, York Schröder, Mikhail Shaposhnikov, Markus Thoma, Tanmay Vachaspati, and Mikko Vepsäläinen.

Mikko Laine and Aleksi Vuorinen 


\section{Notation}

In thermal field theory, both Euclidean and Minkowskian spacetimes play a role.

In the Euclidean case, we write

$$
X \equiv\left(\tau, x^{i}\right), \quad x \equiv|\mathbf{x}|, \quad S_{E}=\int_{X} L_{E}
$$

where $i=1, \ldots, d$,

$$
\int_{X} \equiv \int_{0}^{\beta} \mathrm{d} \tau \int_{\mathbf{x}}, \quad \int_{\mathbf{x}} \equiv \int^{d} \mathrm{~d}^{d} \mathbf{x}, \quad \beta \equiv \frac{1}{T},
$$

and $d$ is the space dimensionality. Fourier analysis is carried out in the Matsubara formalism via

$$
K \equiv\left(k_{n}, k_{i}\right), \quad k \equiv|\mathbf{k}|, \quad \phi(X)=\sum_{K} \tilde{\phi}(K) e^{i K \cdot X}
$$

where

$$
\mathcal{F}_{K} \equiv T \sum_{k_{n}} \int_{\mathbf{k}}, \quad \int_{\mathbf{k}} \equiv \int \frac{\mathrm{d}^{d} \mathbf{k}}{(2 \pi)^{d}} .
$$

Here, $k_{n}$ stands for discrete Matsubara frequencies, which at times are also denoted by $\omega_{n}$. In the case of antiperiodic functions, the summation is written as $T \sum_{\left\{k_{n}\right\}}$. The squares of four-vectors read $K^{2}=k_{n}^{2}+k^{2}$ and $X^{2}=\tau^{2}+x^{2}$, but the Euclidean scalar product between $K$ and $X$ is defined as

$$
K \cdot X=k_{n} \tau+\sum_{i=1}^{d} k_{i} x^{i}=k_{n} \tau-\mathbf{k} \cdot \mathbf{x},
$$

where the vector notation is reserved for contravariant Minkowskian vectors: $\mathbf{x}=\left(x^{i}\right), \mathbf{k}=\left(k^{i}\right)$. If a chemical potential is also present, we denote $\tilde{k}_{n} \equiv k_{n}+i \mu$.

In the Minkowskian case, we have

$$
\mathcal{X} \equiv(t, \mathbf{x}), \quad x \equiv|\mathbf{x}|, \quad \mathcal{S}_{M}=\int_{\mathcal{X}} \mathcal{L}_{M}
$$

where $\int_{\mathcal{X}} \equiv \int \mathrm{d} x^{0} \int_{\mathbf{x}}$. Fourier analysis proceeds via

$$
\mathcal{K} \equiv\left(k^{0}, \mathbf{k}\right), \quad k \equiv|\mathbf{k}|, \quad \phi(\mathcal{X})=\int_{\mathcal{K}} \tilde{\phi}(\mathcal{K}) e^{i \mathcal{K} \cdot \mathcal{X}},
$$

where $\int_{\mathcal{K}}=\int \frac{\mathrm{d} k^{0}}{2 \pi} \int_{\mathbf{k}}$, and the metric is chosen to be of the "mostly minus" form,

$$
\mathcal{K} \cdot \mathcal{X}=k^{0} x^{0}-\mathbf{k} \cdot \mathbf{x} .
$$

No special notation is introduced for the case where a Minkowskian four-vector is on-shell, i.e. when $\mathcal{K}=\left(\epsilon_{k}, \mathbf{k}\right)$; this is to be understood from the context.

The argument of a field $\phi$ is taken to indicate whether the configuration space is Euclidean or Minkowskian. If not specified otherwise, momentum integrations are regulated by defining the spatial measure in $d=3-2 \epsilon$ dimensions, whereas the spacetime dimensionality is denoted by $D=4-2 \epsilon$. A Greek index takes values in the set $\{0, \ldots, d\}$, and a Latin one in $\{1, \ldots, d\}$.

Finally, we note that we work consistently in units where the speed of light $c$ and the Boltzmann constant $k_{\mathrm{B}}$ have been set to unity. The reduced Planck constant $\hbar$ also equals unity in most places, excluding the first chapter (on quantum mechanics) as well as some later discussions where we want to emphasize the distinction between quantum and classical descriptions. 


\section{General outline}

\section{Physics context}

From the physics point of view, there are two important contexts in which relativistic thermal field theory is being widely applied: cosmology and the theoretical description of heavy ion collision experiments.

In cosmology, the temperatures considered vary hugely, ranging from $T \simeq 10^{15} \mathrm{GeV}$ to $T \simeq$ $10^{-3} \mathrm{eV}$. Contemporary challenges in the field include figuring out explanations for the existence of dark matter, the observed antisymmetry in the amounts of matter and antimatter, and the formation of large-scale structures from small initial density perturbations. (The origin of initial density perturbations itself is generally considered to be a non-thermal problem, associated with an early period of inflation.) An important further issue is that of equilibration, i.e. details of the processes through which the inflationary state turned into a thermal plasma, and in particular what the highest temperature reached during this epoch was. It is notable that most of these topics are assumed to be associated with weak or even superweak interactions, whereas strong interactions (QCD) only play a background role. A notable exception to this is light element nucleosynthesis, but this well-studied topic is not in the center of our current focus.

In heavy ion collisions, in contrast, strong interactions do play a major role. The lifetime of the thermal fireball created in such a collision is $\sim 10 \mathrm{fm} / \mathrm{c}$ and the maximal temperature reached is in the range of a few hundred $\mathrm{MeV}$. Weak interactions are too slow to take place within the lifetime of the system. Prominent observables are the yields of different particle species, the quenching of energetic jets, and the hydrodynamic properties of the plasma that can be deduced from the observed particle yields. An important issue is again how fast an initial quantum-mechanical state turns into an essentially incoherent thermal plasma.

Despite many differences in the physics questions posed and in the microscopic forces underlying cosmology and heavy ion collision phenomena, there are also similarities. Most importantly, gauge interactions (whether weak or strong) are essential in both contexts. Because of asymptotic freedom, the strong interactions of QCD also become "weak" at sufficiently high temperatures. It is for this reason that many techniques, such as the resummations that are needed for developing a formally consistent weak-coupling expansion, can be applied in both contexts. The topics covered in the present notes have been chosen with both fields of application in mind.

\section{Organization of these notes}

The notes start with the definition and computation of basic "static" thermodynamic quantities, such as the partition function and free energy density, in various settings. Considered are in turn quantum mechanics (sec. 1), free and interacting scalar field theories (secs. 2 and 3, respectively), fermionic systems (sec. 4), and gauge fields (sec. 5). The main points of these sections include the introduction of the so-called imaginary-time formalism; the functioning of renormalization at finite temperature; and the issue of infrared problems that complicates almost every computation in relativistic thermal field theory. The last of these issues leads us to introduce the concept of effective field theories (sec. 6), after which we consider the changes caused by the introduction of a finite density or chemical potential (sec. 7). After these topics, we move on to a new set of observ- 
ables, so-called real-time quantities, which play an essential role in many modern phenomenological applications of thermal field theory (sec. 8). In the final chapter of the book, a number of concrete applications of the techniques introduced are discussed (sec. 9).

We note that secs. 1-7 are presented on an elementary and self-contained level and require no background knowledge beyond statistical physics, quantum mechanics, and rudiments of quantum field theory. They could constitute the contents of a one-semester basic introduction to perturbative thermal field theory. In sec. 8, the level increases gradually, and parts of the discussion in sec. 9 are already close to the research level, requiring more background knowledge. Conceivably the topics of secs. 8 and 9 could be covered in an advanced course on perturbative thermal field theory, or in a graduate student seminar. In addition the whole book is suitable for self-study, and is then advised to be read in the order in which the material has been presented.

\section{Recommended literature}

A pedagogical presentation of thermal field theory, concentrating mostly on Euclidean observables and the imaginary-time formalism, can be found in ref. [0.1]. The current notes borrow significantly from this classic treatise.

In thermal field theory, the community is somewhat divided between those who find the imaginarytime formalism more practicable, and those who prefer to use the so-called real-time formalism from the beginning. Particularly for the latter community, the standard reference is ref. [0.2], which also contains an introduction to particle production rate computations.

A modern textbook, partly an update of ref. [0.1] but including also a full account of real-time observables, as well as reviews on many recent developments, is provided by ref. [0.3].

Lecture notes on transport coefficients, infrared resummations, and non-equilibrium phenomena such as thermalization, can be found in ref. [0.4]. Reviews with varying foci are offered by refs. [0.5]$[0.16]$.

Finally, an extensive review of efforts to approach a non-perturbative understanding of real-time thermal field theory has been presented in ref. [0.17]. 


\section{Literature}

[0.1] J.I. Kapusta, Finite-temperature Field Theory (Cambridge University Press, Cambridge, 1989).

[0.2] M. Le Bellac, Thermal Field Theory (Cambridge University Press, Cambridge, 2000).

[0.3] J.I. Kapusta and C. Gale, Finite-Temperature Field Theory: Principles and Applications (Cambridge University Press, Cambridge, 2006).

[0.4] P. Arnold, Quark-Gluon Plasma and Thermalization, Int. J. Mod. Phys. E 16 (2007) 2555 [0708.0812].

[0.5] V.A. Rubakov and M.E. Shaposhnikov, Electroweak baryon number non-conservation in the early Universe and in high-energy collisions, Usp. Fiz. Nauk 166 (1996) 493 [Phys. Usp. 39 (1996) 461] [hep-ph/9603208].

[0.6] L.S. Brown and R.F. Sawyer, Nuclear reaction rates in a plasma, Rev. Mod. Phys. 69 (1997) 411 [astro-ph/9610256].

[0.7] J.P. Blaizot and E. Iancu, The quark-gluon plasma: collective dynamics and hard thermal loops, Phys. Rept. 359 (2002) 355 [hep-ph/0101103].

[0.8] D.H. Rischke, The quark-gluon plasma in equilibrium, Prog. Part. Nucl. Phys. 52 (2004) 197 [nucl-th/0305030].

[0.9] U. Kraemmer and A. Rebhan, Advances in perturbative thermal field theory, Rept. Prog. Phys. 67 (2004) 351 [hep-ph/0310337].

[0.10] S. Davidson, E. Nardi and Y. Nir, Leptogenesis, Phys. Rept. 466 (2008) 105 [0802.2962].

[0.11] P. Kovtun, Lectures on hydrodynamic fluctuations in relativistic theories, J. Phys. A 45 (2012) 473001 [1205.5040].

[0.12] D.E. Morrissey and M.J. Ramsey-Musolf, Electroweak baryogenesis, New J. Phys. 14 (2012) 125003 [1206.2942].

[0.13] J. Ghiglieri and D. Teaney, Parton energy loss and momentum broadening at NLO in high temperature QCD plasmas, Int. J. Mod. Phys. E 24 (2015) 1530013 [1502.03730].

[0.14] J. Ghiglieri, A. Kurkela, M. Strickland and A. Vuorinen, Perturbative thermal QCD: Formalism and applications, Phys. Rept. 880 (2020) 1 [2002.10188].

[0.15] M.B. Hindmarsh, M. Lüben, J. Lumma and M. Pauly, Phase transitions in the early universe, SciPost Phys. Lect. Notes 24 (2021) 1 [2008.09136].

[0.16] D. Bödeker and W. Buchmüller, Baryogenesis from the weak scale to the grand unification scale, Rev. Mod. Phys. 93 (2021) 035004 [2009.07294].

[0.17] H.B. Meyer, Transport properties of the quark-gluon plasma: A lattice QCD perspective, Eur. Phys. J. A 47 (2011) 86 [1104.3708]. 


\section{Quantum mechanics}

Abstract: After recalling some basic concepts of statistical physics and quantum mechanics, the partition function of a harmonic oscillator is defined and evaluated in the standard canonical formalism. An imaginary-time path integral representation is subsequently developed for the partition function, the path integral is evaluated in momentum space, and the earlier result is reproduced upon a careful treatment of the zero-mode contribution. Finally, the concept of 2-point functions (propagators) is introduced, and some of their key properties are derived in imaginary time.

Keywords: Partition function, Euclidean path integral, imaginary-time formalism, Matsubara modes, 2-point function.

\subsection{Path integral representation of the partition function}

\section{Basic structure}

The properties of a quantum-mechanical system are defined by its Hamiltonian, which for nonrelativistic spin-0 particles in one dimension takes the form

$$
\hat{H}=\frac{\hat{p}^{2}}{2 m}+V(\hat{x})
$$

where $m$ is the particle mass. The dynamics of the states $|\psi\rangle$ is governed by the Schrödinger equation,

$$
i \hbar \frac{\partial}{\partial t}|\psi\rangle=\hat{H}|\psi\rangle
$$

which can formally be solved in terms of a time-evolution operator $\hat{U}\left(t ; t_{0}\right)$. This operator satisfies the relation

$$
|\psi(t)\rangle=\hat{U}\left(t ; t_{0}\right)\left|\psi\left(t_{0}\right)\right\rangle,
$$

and for a time-independent Hamiltonian takes the explicit form

$$
\hat{U}\left(t ; t_{0}\right)=e^{-\frac{i}{\hbar} \hat{H}\left(t-t_{0}\right)} .
$$

It is useful to note that in the classical limit, the system of eq. (1.1) can be described by the Lagrangian

$$
\mathcal{L}=\mathcal{L}_{M}=\frac{1}{2} m \dot{x}^{2}-V(x),
$$

which is related to the classical version of the Hamiltonian via a simple Legendre transform:

$$
p \equiv \frac{\partial \mathcal{L}_{M}}{\partial \dot{x}}, \quad H=\dot{x} p-\mathcal{L}_{M}=\frac{p^{2}}{2 m}+V(x) .
$$

Returning to the quantum-mechanical setting, various bases can be chosen for the state vectors. The so-called $|x\rangle$-basis satisfies the relations

$$
\left\langle x|\hat{x}| x^{\prime}\right\rangle=x\left\langle x \mid x^{\prime}\right\rangle=x \delta\left(x-x^{\prime}\right), \quad\left\langle x|\hat{p}| x^{\prime}\right\rangle=-i \hbar \partial_{x}\left\langle x \mid x^{\prime}\right\rangle=-i \hbar \partial_{x} \delta\left(x-x^{\prime}\right),
$$

whereas in the energy basis we simply have

$$
\hat{H}|n\rangle=\epsilon_{n}|n\rangle .
$$


An important concrete realization of a quantum-mechanical system is provided by the harmonic oscillator, defined by the potential

$$
V(\hat{x}) \equiv \frac{1}{2} m \omega^{2} \hat{x}^{2} .
$$

In this case the energy eigenstates $|n\rangle$ can be found explicitly, with the corresponding eigenvalues equalling

$$
\epsilon_{n}=\hbar \omega\left(n+\frac{1}{2}\right), \quad n=0,1,2, \ldots .
$$

All the states are non-degenerate.

It turns out to be useful to view (quantum) mechanics formally as $(1+0)$-dimensional (quantum) field theory: the operator $\hat{x}$ can be viewed as a field operator $\hat{\phi}$ at a certain point, implying the correspondence

$$
\hat{x} \leftrightarrow \hat{\phi}(\mathbf{0}) .
$$

In quantum field theory operators are usually represented in the Heisenberg picture; correspondingly, we then have

$$
\hat{x}_{H}(t) \leftrightarrow \hat{\phi}_{H}(t, \mathbf{0}) .
$$

In the following we adopt an implicit notation whereby showing the time coordinate $t$ as an argument of a field automatically implies the use of the Heisenberg picture, and the corresponding subscript is left out.

\section{Canonical partition function}

Taking our quantum-mechanical system to a finite temperature $T$, the fundamental quantity of interest is the partition function, $\mathcal{Z}$. We employ the canonical ensemble, whereby $\mathcal{Z}$ is a function of $T$; introducing units in which $k_{\mathrm{B}}=1$ (i.e., $T_{\text {here }} \equiv k_{\mathrm{B}} T_{\mathrm{SI}-\text { units }}$ ), the partition function is defined by

$$
\mathcal{Z}(T) \equiv \operatorname{Tr}\left[e^{-\beta \hat{H}}\right], \quad \beta \equiv \frac{1}{T},
$$

where the trace is taken over the full Hilbert space. From this quantity, other observables, such as the free energy $F$, entropy $S$, and average energy $E$ can be obtained via standard relations:

$$
\begin{aligned}
F & =-T \ln \mathcal{Z}, \\
S & =-\frac{\partial F}{\partial T}=\ln \mathcal{Z}+\frac{1}{T \mathcal{Z}} \operatorname{Tr}\left[\hat{H} e^{-\beta \hat{H}}\right]=-\frac{F}{T}+\frac{E}{T}, \\
E & =\frac{1}{\mathcal{Z}} \operatorname{Tr}\left[\hat{H} e^{-\beta \hat{H}}\right] .
\end{aligned}
$$

Let us now explicitly compute these quantities for the harmonic oscillator. This becomes a trivial exercise in the energy basis, given that we can immediately write

$$
\mathcal{Z}=\sum_{n=0}^{\infty}\left\langle n\left|e^{-\beta \hat{H}}\right| n\right\rangle=\sum_{n=0}^{\infty} e^{-\beta \hbar \omega\left(\frac{1}{2}+n\right)}=\frac{e^{-\beta \hbar \omega / 2}}{1-e^{-\beta \hbar \omega}}=\frac{1}{2 \sinh \left(\frac{\hbar \omega}{2 T}\right)} .
$$

Consequently,

$$
F=T \ln \left(e^{\frac{\hbar \omega}{2 T}}-e^{-\frac{\hbar \omega}{2 T}}\right)=\frac{\hbar \omega}{2}+T \ln \left(1-e^{-\beta \hbar \omega}\right)
$$




$$
\begin{aligned}
& \approx \begin{cases}\frac{\hbar \omega}{2}, & T \ll \hbar \omega \\
-T \ln \left(\frac{T}{\hbar \omega}\right), & T \gg \hbar \omega\end{cases} \\
S & =-\ln \left(1-e^{-\beta \hbar \omega}\right)+\frac{\hbar \omega}{T} \frac{1}{e^{\beta \hbar \omega}-1} \\
& \approx \begin{cases}\frac{\hbar \omega}{T} e^{-\frac{\hbar \omega}{T}}, & T \ll \hbar \omega \\
1+\ln \frac{T}{\hbar \omega}, & T \gg \hbar \omega\end{cases} \\
E & =F+T S=\hbar \omega\left(\frac{1}{2}+\frac{1}{e^{\beta \hbar \omega}-1}\right) \\
& \approx \begin{cases}\frac{\hbar \omega}{2}, & T \ll \hbar \omega \\
T, & T \gg \hbar \omega\end{cases}
\end{aligned}
$$

Note how in most cases one can separate the contribution of the ground state, dominating at low temperatures $T \ll \hbar \omega$, from that of the thermally excited states, characterized by the appearance of the Bose distribution $n_{\mathrm{B}}(\hbar \omega) \equiv 1 /[\exp (\beta \hbar \omega)-1]$. Note also that $E$ rises linearly with $T$ at high temperatures; the coefficient is said to count the number of degrees of freedom of the system.

\section{Path integral for the partition function}

In the case of the harmonic oscillator, the energy eigenvalues are known in an analytic form, and $\mathcal{Z}$ could be easily evaluated. In many other cases the $\epsilon_{n}$ are, however, difficult to compute. A more useful representation of $\mathcal{Z}$ is obtained by writing it as a path integral.

In order to get started, let us recall some basic relations. First of all, it follows from the form of the momentum operator in the $|x\rangle$-basis that

$$
\langle x|\hat{p}| p\rangle=p\langle x \mid p\rangle=-i \hbar \partial_{x}\langle x \mid p\rangle \Rightarrow\langle x \mid p\rangle=A e^{\frac{i p x}{\hbar}},
$$

where $A$ is some constant. Second, we need completeness relations in both $|x\rangle$ and $|p\rangle$-bases, which take the respective forms

$$
\int \mathrm{d} x|x\rangle\left\langle x\left|=\hat{\mathbb{1}}, \quad \int \frac{\mathrm{d} p}{B}\right| p\right\rangle\langle p|=\hat{\mathbb{1}},
$$

where $B$ is another constant. The choices of $A$ and $B$ are not independent; indeed,

$$
\begin{aligned}
\hat{\mathbb{1}} & =\int \mathrm{d} x \int \frac{\mathrm{d} p}{B} \int \frac{\mathrm{d} p^{\prime}}{B}|p\rangle\langle p \mid x\rangle\left\langle x \mid p^{\prime}\right\rangle\left\langle p^{\prime}\left|=\int \mathrm{d} x \int \frac{\mathrm{d} p}{B} \int \frac{\mathrm{d} p^{\prime}}{B}\right| p\right\rangle|A|^{2} e^{\frac{i\left(p^{\prime}-p\right) x}{\hbar}}\left\langle p^{\prime}\right| \\
& =\int \frac{\mathrm{d} p}{B} \int \frac{\mathrm{d} p^{\prime}}{B}|p\rangle|A|^{2} 2 \pi \hbar \delta\left(p^{\prime}-p\right)\left\langle p^{\prime}\left|=\frac{2 \pi \hbar|A|^{2}}{B} \int \frac{\mathrm{d} p}{B}\right| p\right\rangle\langle p|=\frac{2 \pi \hbar|A|^{2}}{B} \hat{\mathbb{1}},
\end{aligned}
$$

implying that $B=2 \pi \hbar|A|^{2}$. We choose $A \equiv 1$ in the following, so that $B=2 \pi \hbar$.

Next, we move on to evaluate the partition function, which we do in the $x$-basis, so that our starting point becomes

$$
\mathcal{Z}=\operatorname{Tr}\left[e^{-\beta \hat{H}}\right]=\int \mathrm{d} x\left\langle x\left|e^{-\beta \hat{H}}\right| x\right\rangle=\int \mathrm{d} x\left\langle x\left|e^{-\frac{\epsilon \hat{H}}{\hbar}} \cdots e^{-\frac{\epsilon \hat{H}}{\hbar}}\right| x\right\rangle .
$$

Here we have split $e^{-\beta \hat{H}}$ into a product of $N \gg 1$ different pieces, defining $\epsilon \equiv \beta \hbar / N$. 
A crucial trick at this point is to insert

$$
\hat{\mathbb{1}}=\int \frac{\mathrm{d} p_{i}}{2 \pi \hbar}\left|p_{i}\right\rangle\left\langle p_{i}\right|, \quad i=1, \ldots, N,
$$

on the left side of each exponential, with $i$ increasing from right to left; and

$$
\hat{\mathbb{1}}=\int \mathrm{d} x_{i}\left|x_{i}\right\rangle\left\langle x_{i}\right|, \quad i=1, \ldots, N,
$$

on the right side of each exponential, with again $i$ increasing from right to left. Thereby we are left to consider matrix elements of the type

$$
\begin{aligned}
\left\langle x_{i+1} \mid p_{i}\right\rangle\left\langle p_{i}\left|e^{-\frac{\epsilon}{\hbar} \hat{H}(\hat{p}, \hat{x})}\right| x_{i}\right\rangle & =e^{\frac{i p_{i} x_{i+1}}{\hbar}}\left\langle p_{i}\left|e^{-\frac{\epsilon}{\hbar} H\left(p_{i}, x_{i}\right)+\mathcal{O}\left(\epsilon^{2}\right)}\right| x_{i}\right\rangle \\
& =\exp \left\{-\frac{\epsilon}{\hbar}\left[\frac{p_{i}^{2}}{2 m}-i p_{i} \frac{x_{i+1}-x_{i}}{\epsilon}+V\left(x_{i}\right)+\mathcal{O}(\epsilon)\right]\right\} .
\end{aligned}
$$

Moreover, we note that at the very right, we have

$$
\left\langle x_{1} \mid x\right\rangle=\delta\left(x_{1}-x\right)
$$

which allows us to carry out the integral over $x$. Similarly, at the very left, the role of $\left\langle x_{i+1}\right|$ is played by the state $\langle x|=\left\langle x_{1}\right|$. Finally, we remark that the $\mathcal{O}(\epsilon)$ correction in eq. (1.30) can be eliminated by sending $N \rightarrow \infty$.

In total, we can thus write the partition function in the form

$$
\mathcal{Z}=\left.\lim _{N \rightarrow \infty} \int\left[\prod_{i=1}^{N} \frac{\mathrm{d} x_{i} \mathrm{~d} p_{i}}{2 \pi \hbar}\right] \exp \left\{-\frac{1}{\hbar} \sum_{j=1}^{N} \epsilon\left[\frac{p_{j}^{2}}{2 m}-i p_{j} \frac{x_{j+1}-x_{j}}{\epsilon}+V\left(x_{j}\right)\right]\right\}\right|_{x_{N+1} \equiv x_{1}, \epsilon \equiv \beta \hbar / N},
$$

which is often symbolically expressed as a "continuum" path integral

$$
\mathcal{Z}=\int_{x(\beta \hbar)=x(0)} \mathcal{D} x \mathcal{D}\left(\frac{p}{2 \pi \hbar}\right) \exp \left\{-\frac{1}{\hbar} \int_{0}^{\beta \hbar} \mathrm{d} \tau\left[\frac{[p(\tau)]^{2}}{2 m}-i p(\tau) \dot{x}(\tau)+V(x(\tau))\right]\right\} .
$$

The integration measure here is understood as the limit indicated in eq. (1.32); the discrete $x_{i}$ 's have been collected into a function $x(\tau)$; and the maximal value of the $\tau$-coordinate has been obtained from $\epsilon N=\beta \hbar$.

Returning to the discrete form of the path integral, we note that the integral over the momenta $p_{i}$ is Gaussian, and can thereby be carried out explicitly:

$$
\int_{-\infty}^{\infty} \frac{\mathrm{d} p_{i}}{2 \pi \hbar} \exp \left\{-\frac{\epsilon}{\hbar}\left[\frac{p_{i}^{2}}{2 m}-i p_{i} \frac{x_{i+1}-x_{i}}{\epsilon}\right]\right\}=\sqrt{\frac{m}{2 \pi \hbar \epsilon}} \exp \left[-\frac{m\left(x_{i+1}-x_{i}\right)^{2}}{2 \hbar \epsilon}\right] .
$$

Using this, eq. (1.32) becomes

$$
\mathcal{Z}=\left.\lim _{N \rightarrow \infty} \int\left[\prod_{i=1}^{N} \frac{\mathrm{d} x_{i}}{\sqrt{2 \pi \hbar \epsilon / m}}\right] \exp \left\{-\frac{1}{\hbar} \sum_{j=1}^{N} \epsilon\left[\frac{m}{2}\left(\frac{x_{j+1}-x_{j}}{\epsilon}\right)^{2}+V\left(x_{j}\right)\right]\right\}\right|_{x_{N+1} \equiv x_{1}, \epsilon \equiv \beta \hbar / N}
$$

which may also be written in a continuum form. Of course the measure then contains a factor which appears quite divergent at large $N$,

$$
C \equiv\left(\frac{m}{2 \pi \hbar \epsilon}\right)^{N / 2}=\exp \left[\frac{N}{2} \ln \left(\frac{m N}{2 \pi \hbar^{2} \beta}\right)\right] .
$$


This factor is, however, independent of the properties of the potential $V\left(x_{j}\right)$ and thereby contains no dynamical information, so that we do not need to worry too much about the apparent divergence. For the moment, then, we can simply write down a continuum "functional integral",

$$
\mathcal{Z}=C \int_{x(\beta \hbar)=x(0)} \mathcal{D} x \exp \left\{-\frac{1}{\hbar} \int_{0}^{\beta \hbar} \mathrm{d} \tau\left[\frac{m}{2}\left(\frac{\mathrm{d} x(\tau)}{\mathrm{d} \tau}\right)^{2}+V(x(\tau))\right]\right\} .
$$

Let us end by giving an "interpretation" to the result in eq. (1.37). We recall that the usual quantum-mechanical path integral at zero temperature contains the exponential

$$
\exp \left(\frac{i}{\hbar} \int \mathrm{d} t \mathcal{L}_{M}\right), \quad \mathcal{L}_{M}=\frac{m}{2}\left(\frac{\mathrm{d} x}{\mathrm{~d} t}\right)^{2}-V(x) .
$$

We note that eq. (1.37) can be obtained from its zero-temperature counterpart with the following recipe [1.1]:

(i) Carry out a Wick rotation, denoting $\tau \equiv i t$.

(ii) Introduce

$$
L_{E} \equiv-\mathcal{L}_{M}(\tau=i t)=\frac{m}{2}\left(\frac{\mathrm{d} x}{\mathrm{~d} \tau}\right)^{2}+V(x) .
$$

(iii) Restrict $\tau$ to the interval $(0, \beta \hbar)$.

(iv) Require periodicity of $x(\tau)$, i.e. $x(\beta \hbar)=x(0)$.

With these steps (and noting that $i \mathrm{~d} t=\mathrm{d} \tau$ ), the exponential becomes

$$
\exp \left(\frac{i}{\hbar} \int \mathrm{d} t \mathcal{L}_{M}\right) \stackrel{(\mathrm{i})-(\text { iv) }}{\longrightarrow} \exp \left(-\frac{1}{\hbar} S_{E}\right) \equiv \exp \left(-\frac{1}{\hbar} \int_{0}^{\beta \hbar} \mathrm{d} \tau L_{E}\right)
$$

where the subscript $E$ stands for "Euclidean". Because of step (i), the path integral in eq. (1.40) is also known as the imaginary-time formalism. It turns out that this recipe works, with few modifications, also in quantum field theory, and even for spin- $1 / 2$ and spin- 1 particles, although the derivation of the path integral itself looks quite different in those cases. We return to these issues in later chapters of the book. 


\subsection{Evaluation of the path integral for the harmonic oscillator}

As an independent crosscheck of the results of sec. 1.1, we now explicitly evaluate the path integral of eq. (1.37) in the case of a harmonic oscillator, and compare the result with eq. (1.17). To make the exercise more interesting, we carry out the evaluation in Fourier space with respect to the time coordinate $\tau$. Moreover we would like to deduce the information contained in the divergent constant $C$ without making use of its actual value, given in eq. (1.36).

Let us start by representing an arbitrary function $x(\tau), 0<\tau<\beta \hbar$, with the property $x\left((\beta \hbar)^{-}\right)=$ $x\left(0^{+}\right)$(referred to as "periodicity") as a Fourier sum

$$
x(\tau) \equiv T \sum_{n=-\infty}^{\infty} x_{n} e^{i \omega_{n} \tau},
$$

where the factor $T$ is a convention. Imposing periodicity requires that

$$
e^{i \omega_{n} \beta \hbar}=1, \quad \text { i.e. } \omega_{n} \beta \hbar=2 \pi n, \quad n \in \mathbb{Z},
$$

where the values $\omega_{n}=2 \pi T n / \hbar$ are called Matsubara frequencies. The corresponding amplitudes $x_{n}$ are called Matsubara modes.

Apart from periodicity, we also impose reality on $x(\tau)$ :

$$
x(\tau) \in \mathbb{R} \Rightarrow x^{*}(\tau)=x(\tau) \Rightarrow x_{n}^{*}=x_{-n} .
$$

If we write $x_{n}=a_{n}+i b_{n}$, it then follows that

$$
x_{n}^{*}=a_{n}-i b_{n}=x_{-n}=a_{-n}+i b_{-n} \Rightarrow\left\{\begin{array}{c}
a_{n}=a_{-n} \\
b_{n}=-b_{-n}
\end{array},\right.
$$

and moreover that $b_{0}=0$ and $x_{-n} x_{n}=a_{n}^{2}+b_{n}^{2}$. Thereby we now have the representation

$$
x(\tau)=T\left\{a_{0}+\sum_{n=1}^{\infty}\left[\left(a_{n}+i b_{n}\right) e^{i \omega_{n} \tau}+\left(a_{n}-i b_{n}\right) e^{-i \omega_{n} \tau}\right]\right\},
$$

where $a_{0}$ is called (the amplitude of) the Matsubara zero mode.

With the representation of eq. (1.41), general quadratic structures can be expressed as

$$
\begin{aligned}
\frac{1}{\hbar} \int_{0}^{\beta \hbar} \mathrm{d} \tau x(\tau) y(\tau) & =T^{2} \sum_{m, n} x_{n} y_{m} \frac{1}{\hbar} \int_{0}^{\beta \hbar} \mathrm{d} \tau e^{i\left(\omega_{n}+\omega_{m}\right) \tau} \\
& =T^{2} \sum_{m, n} x_{n} y_{m} \frac{1}{T} \delta_{n,-m}=T \sum_{n} x_{n} y_{-n}
\end{aligned}
$$

In particular, the argument of the exponential in eq. (1.37) becomes

$$
\begin{aligned}
-\frac{1}{\hbar} \int_{0}^{\beta \hbar} \mathrm{d} \tau & \frac{m}{2}\left[\frac{\mathrm{d} x(\tau)}{\mathrm{d} \tau} \frac{\mathrm{d} x(\tau)}{\mathrm{d} \tau}+\omega^{2} x(\tau) x(\tau)\right] \\
\stackrel{(1.46)}{=} & -\frac{m T}{2} \sum_{n=-\infty}^{\infty} x_{n}\left[i \omega_{n} i \omega_{-n}+\omega^{2}\right] x_{-n} \\
\stackrel{\omega_{-n}=-\omega_{n}}{=} & -\frac{m T}{2} \sum_{n=-\infty}^{\infty}\left(\omega_{n}^{2}+\omega^{2}\right)\left(a_{n}^{2}+b_{n}^{2}\right) \\
\stackrel{(1.44)}{=} & -\frac{m T}{2} \omega^{2} a_{0}^{2}-m T \sum_{n=1}^{\infty}\left(\omega_{n}^{2}+\omega^{2}\right)\left(a_{n}^{2}+b_{n}^{2}\right) .
\end{aligned}
$$


Next, we need to consider the integration measure. To this end, let us make a change of variables from $x(\tau), \tau \in(0, \beta \hbar)$, to the Fourier components $a_{n}, b_{n}$. As we have seen, the independent variables are $a_{0}$ and $\left\{a_{n}, b_{n}\right\}, n \geq 1$, whereby the measure becomes

$$
\mathcal{D} x(\tau)=\left|\operatorname{det}\left[\frac{\delta x(\tau)}{\delta x_{n}}\right]\right| \mathrm{d} a_{0}\left[\prod_{n \geq 1} \mathrm{~d} a_{n} \mathrm{~d} b_{n}\right] .
$$

The change of bases is purely kinematical and independent of the potential $V(x)$, implying that we can define

$$
C^{\prime} \equiv C\left|\operatorname{det}\left[\frac{\delta x(\tau)}{\delta x_{n}}\right]\right|
$$

and regard now $C^{\prime}$ as an unknown coefficient.

Making use of the Gaussian integral $\int_{-\infty}^{\infty} \mathrm{d} x \exp \left(-c x^{2}\right)=\sqrt{\pi / c}, c>0$, as well as the above integration measure, the expression in eq. (1.37) becomes

$$
\begin{aligned}
\mathcal{Z} & =C^{\prime} \int_{-\infty}^{\infty} \mathrm{d} a_{0} \int_{-\infty}^{\infty}\left[\prod_{n \geq 1} \mathrm{~d} a_{n} \mathrm{~d} b_{n}\right] \exp \left[-\frac{1}{2} m T \omega^{2} a_{0}^{2}-m T \sum_{n \geq 1}\left(\omega_{n}^{2}+\omega^{2}\right)\left(a_{n}^{2}+b_{n}^{2}\right)\right] \\
& =C^{\prime} \sqrt{\frac{2 \pi}{m T \omega^{2}}} \prod_{n=1}^{\infty} \frac{\pi}{m T\left(\omega_{n}^{2}+\omega^{2}\right)}, \quad \omega_{n}=\frac{2 \pi T n}{\hbar} .
\end{aligned}
$$

The remaining task is to determine $C^{\prime}$. This can be achieved via the following observations:

- Since $C^{\prime}$ is independent of $\omega$ (which only appears in $V(x)$ ), we can determine it in the limit $\omega=0$, whereby the system simplifies.

- The integral over the zero mode $a_{0}$ in eq. (1.50) is, however, divergent for $\omega \rightarrow 0$. We may call such a divergence an infrared divergence: the zero mode is the lowest-energy mode.

- We can still take the $\omega \rightarrow 0$ limit, if we momentarily regulate the integration over the zero mode in some way. Noting from eq. (1.45) that

$$
\frac{1}{\beta \hbar} \int_{0}^{\beta \hbar} \mathrm{d} \tau x(\tau)=T a_{0}
$$

we see that $T a_{0}$ represents the average value of $x(\tau)$ over the $\tau$-interval. We may thus regulate the system by "putting it in a periodic box", i.e. by restricting the (average) value of $x(\tau)$ to some (large but finite) interval $\Delta x$.

With this setup, we can now proceed to find $C^{\prime}$ via matching.

"Effective theory computation": In the $\omega \rightarrow 0$ limit but in the presence of the regulator, eq. (1.50) becomes

$$
\begin{aligned}
\lim _{\omega \rightarrow 0} \mathcal{Z}_{\text {regulated }} & =C^{\prime} \int_{\Delta x / T} \mathrm{~d} a_{0} \int_{-\infty}^{\infty}\left[\prod_{n \geq 1} \mathrm{~d} a_{n} \mathrm{~d} b_{n}\right] \exp \left[-m T \sum_{n \geq 1} \omega_{n}^{2}\left(a_{n}^{2}+b_{n}^{2}\right)\right] \\
& =C^{\prime} \frac{\Delta x}{T} \prod_{n=1}^{\infty} \frac{\pi}{m T \omega_{n}^{2}}, \quad \omega_{n}=\frac{2 \pi T n}{\hbar} .
\end{aligned}
$$


"Full theory computation": In the presence of the regulator, and in the absence of $V(x)$ (implied by the $\omega \rightarrow 0$ limit), eq. (1.27) can be computed in a very simple way:

$$
\begin{aligned}
\lim _{\omega \rightarrow 0} \mathcal{Z}_{\text {regulated }} & =\int_{\Delta x} \mathrm{~d} x\left\langle x\left|e^{-\frac{\hat{p}^{2}}{2 m T}}\right| x\right\rangle \\
& =\int_{\Delta x} \mathrm{~d} x \int_{-\infty}^{\infty} \frac{\mathrm{d} p}{2 \pi \hbar}\left\langle x\left|e^{-\frac{\hat{p}^{2}}{2 m T}}\right| p\right\rangle\langle p \mid x\rangle \\
& =\int_{\Delta x} \mathrm{~d} x \int_{-\infty}^{\infty} \frac{\mathrm{d} p}{2 \pi \hbar} e^{-\frac{p^{2}}{2 m T}} \underbrace{\langle x \mid p\rangle\langle p \mid x\rangle}_{1} \\
& =\frac{\Delta x}{2 \pi \hbar} \sqrt{2 \pi m T} .
\end{aligned}
$$

Matching the two sides: Equating eqs. (1.53) and (1.54), we find the formal expression

$$
C^{\prime}=\frac{T}{2 \pi \hbar} \sqrt{2 \pi m T} \prod_{n=1}^{\infty} \frac{m T \omega_{n}^{2}}{\pi}
$$

Since the regulator $\Delta x$ has dropped out, we may call $C^{\prime}$ an "ultraviolet" matching coefficient.

With $C^{\prime}$ determined, we can now continue with eq. (1.51), obtaining the finite expression

$$
\begin{aligned}
\mathcal{Z} & =\frac{T}{\hbar \omega} \prod_{n=1}^{\infty} \frac{\omega_{n}^{2}}{\omega_{n}^{2}+\omega^{2}} \\
& =\frac{T}{\hbar \omega} \frac{1}{\prod_{n=1}^{\infty}\left[1+\frac{(\hbar \omega / 2 \pi T)^{2}}{n^{2}}\right]} .
\end{aligned}
$$

Making use of the identity

$$
\frac{\sinh \pi x}{\pi x}=\prod_{n=1}^{\infty}\left(1+\frac{x^{2}}{n^{2}}\right)
$$

we directly reproduce our earlier result for the partition function, eq. (1.17). Thus, we have managed to correctly evaluate the path integral without ever making recourse to eq. (1.36) or, for that matter, to the discretization that was present in eqs. (1.32) and (1.35).

Let us end with a few remarks:

- In quantum mechanics, the partition function $\mathcal{Z}$ as well as all other observables are finite functions of the parameters $T, m$, and $\omega$, if computed properly. We saw that with path integrals this is not obvious at every intermediate step, but at the end it did work out. In quantum field theory, on the contrary, "ultraviolet" (UV) divergences may remain in the results even if we compute everything correctly. These are then taken care of by renormalization. However, as our quantum-mechanical example demonstrated, the "ambiguity" of the functional integration measure (through $C^{\prime}$ ) is not in itself a source of UV divergences.

- It is appropriate to stress that in many physically relevant observables, the coefficient $C^{\prime}$ drops out completely, and the above procedure is thereby even simpler. An example of such a quantity is given in eq. (1.60) below.

- Finally, some of the concepts and techniques that were introduced with this simple example - zero modes, infrared divergences, their regularization, matching computations, etc - also play a role in non-trivial quantum field theoretic examples that we encounter later on. 


\section{Appendix A: 2-point function}

Defining a Heisenberg-like operator (with it $\rightarrow \tau$ )

$$
\hat{x}(\tau) \equiv e^{\frac{\hat{H} \tau}{\hbar}} \hat{x} e^{-\frac{\hat{H} \tau}{\hbar}}, \quad 0<\tau<\beta \hbar,
$$

we define a "2-point Green's function" or a "propagator" through

$$
G(\tau) \equiv \frac{1}{\mathcal{Z}} \operatorname{Tr}\left[e^{-\beta \hat{H}} \hat{x}(\tau) \hat{x}(0)\right] .
$$

The corresponding path integral can be shown to read

$$
G(\tau)=\frac{\int_{x(\beta \hbar)=x(0)} \mathcal{D} x x(\tau) x(0) \exp \left[-S_{E} / \hbar\right]}{\int_{x(\beta \hbar)=x(0)} \mathcal{D} x \exp \left[-S_{E} / \hbar\right]},
$$

whereby the normalization of $\mathcal{D} x$ plays no role. In the following, we compute $G(\tau)$ explicitly for the harmonic oscillator, by making use of

(a) the canonical formalism, i.e. expressing $\hat{H}$ and $\hat{x}$ in terms of the annihilation and creation operators $\hat{a}$ and $\hat{a}^{\dagger}$,

(b) the path integral formalism, working in Fourier space.

Starting with the canonical formalism, we write all quantities in terms of $\hat{a}$ and $\hat{a}^{\dagger}$ :

$$
\hat{H}=\hbar \omega\left(\hat{a}^{\dagger} \hat{a}+\frac{1}{2}\right), \quad \hat{x}=\sqrt{\frac{\hbar}{2 m \omega}}\left(\hat{a}+\hat{a}^{\dagger}\right), \quad\left[\hat{a}, \hat{a}^{\dagger}\right]=1 .
$$

In order to construct $\hat{x}(\tau)$, we make use of the expansion

$$
e^{\hat{A}} \hat{B} e^{-\hat{A}}=\hat{B}+[\hat{A}, \hat{B}]+\frac{1}{2 !}[\hat{A},[\hat{A}, \hat{B}]]+\frac{1}{3 !}[\hat{A},[\hat{A},[\hat{A}, \hat{B}]]]+\ldots
$$

Noting that

$$
\begin{aligned}
{[\hat{H}, \hat{a}] } & =\hbar \omega\left[\hat{a}^{\dagger} \hat{a}, \hat{a}\right]=-\hbar \omega \hat{a}, \\
{[\hat{H},[\hat{H}, \hat{a}]] } & =(-\hbar \omega)^{2} \hat{a}, \\
{\left[\hat{H}, \hat{a}^{\dagger}\right] } & =\hbar \omega\left[\hat{a}^{\dagger} \hat{a}, \hat{a}^{\dagger}\right]=\hbar \omega \hat{a}^{\dagger}, \\
{\left[\hat{H},\left[\hat{H}, \hat{a}^{\dagger}\right]\right] } & =(\hbar \omega)^{2} \hat{a}^{\dagger},
\end{aligned}
$$

and so forth, we can write

$$
\begin{aligned}
e^{\frac{\hat{H} \tau}{\hbar}} \hat{x} e^{-\frac{\hat{H} \tau}{\hbar}} & =\sqrt{\frac{\hbar}{2 m \omega}}\left\{\hat{a}\left[1-\omega \tau+\frac{1}{2 !}(-\omega \tau)^{2}+\ldots\right]+\hat{a}^{\dagger}\left[1+\omega \tau+\frac{1}{2 !}(\omega \tau)^{2}+\ldots\right]\right\} \\
& =\sqrt{\frac{\hbar}{2 m \omega}}\left(\hat{a} e^{-\omega \tau}+\hat{a}^{\dagger} e^{\omega \tau}\right) .
\end{aligned}
$$

Inserting now $\mathcal{Z}$ from eq. (1.17), eq. (1.60) becomes

$$
G(\tau)=2 \sinh \left(\frac{\beta \hbar \omega}{2}\right) \sum_{n=0}^{\infty}\left\langle n\left|e^{-\beta \hbar \omega\left(n+\frac{1}{2}\right)} \frac{\hbar}{2 m \omega}\left(\hat{a} e^{-\omega \tau}+\hat{a}^{\dagger} e^{\omega \tau}\right)\left(\hat{a}+\hat{a}^{\dagger}\right)\right| n\right\rangle .
$$


With the relations $\hat{a}^{\dagger}|n\rangle=\sqrt{n+1}|n+1\rangle$ and $\hat{a}|n\rangle=\sqrt{n}|n-1\rangle$ we can identify the non-zero matrix elements,

$$
\left\langle n\left|\hat{a} \hat{a}^{\dagger}\right| n\right\rangle=n+1, \quad\left\langle n\left|\hat{a}^{\dagger} \hat{a}\right| n\right\rangle=n .
$$

Thereby we obtain

$$
G(\tau)=\frac{\hbar}{m \omega} \sinh \left(\frac{\beta \hbar \omega}{2}\right) \exp \left(-\frac{\beta \hbar \omega}{2}\right) \sum_{n=0}^{\infty} e^{-\beta \hbar \omega n}\left[e^{-\omega \tau}+n\left(e^{-\omega \tau}+e^{\omega \tau}\right)\right],
$$

where the terms are quickly evaluated as geometric sums,

$$
\begin{aligned}
\sum_{n=0}^{\infty} e^{-\beta \hbar \omega n} & =\frac{1}{1-e^{-\beta \hbar \omega}}, \\
\sum_{n=0}^{\infty} n e^{-\beta \hbar \omega n} & =-\frac{1}{\beta \hbar} \frac{\mathrm{d}}{\mathrm{d} \omega} \frac{1}{1-e^{-\beta \hbar \omega}}=\frac{e^{-\beta \hbar \omega}}{\left(1-e^{-\beta \hbar \omega}\right)^{2}} .
\end{aligned}
$$

In total, we then have

$$
\begin{aligned}
G(\tau) & =\frac{\hbar}{2 m \omega}\left(1-e^{-\beta \hbar \omega}\right)\left[\frac{e^{-\omega \tau}}{1-e^{-\beta \hbar \omega}}+\left(e^{-\omega \tau}+e^{\omega \tau}\right) \frac{e^{-\beta \hbar \omega}}{\left(1-e^{-\beta \hbar \omega}\right)^{2}}\right] \\
& =\frac{\hbar}{2 m \omega} \frac{1}{1-e^{-\beta \hbar \omega}}\left[e^{-\omega \tau}+e^{\omega(\tau-\beta \hbar)}\right] \\
& =\frac{\hbar}{2 m \omega} \frac{e^{\omega \tau}+e^{\omega(\beta \hbar-\tau)}}{e^{\beta \hbar \omega}-1} \\
& =\frac{\hbar}{2 m \omega} \frac{\cosh \left[\left(\frac{\beta \hbar}{2}-\tau\right) \omega\right]}{\sinh \left[\frac{\beta \hbar \omega}{2}\right]} .
\end{aligned}
$$

As far as the path integral treatment goes, we employ the same representation as in eq. (1.50), noting that $C^{\prime}$ drops out in the ratio of eq. (1.61). Recalling the Fourier representation of eq. (1.45),

$$
\begin{aligned}
& x(\tau)=T\left\{a_{0}+\sum_{k=1}^{\infty}\left[\left(a_{k}+i b_{k}\right) e^{i \omega_{k} \tau}+\left(a_{k}-i b_{k}\right) e^{-i \omega_{k} \tau}\right]\right\}, \\
& x(0)=T\left\{a_{0}+\sum_{l=1}^{\infty} 2 a_{l}\right\},
\end{aligned}
$$

the observable of our interest becomes

$$
G(\tau)=\langle x(\tau) x(0)\rangle \equiv \frac{\int \mathrm{d} a_{0} \int \prod_{n \geq 1} \mathrm{~d} a_{n} \mathrm{~d} b_{n} x(\tau) x(0) \exp \left[-S_{E} / \hbar\right]}{\int \mathrm{d} a_{0} \int \prod_{n \geq 1} \mathrm{~d} a_{n} \mathrm{~d} b_{n} \exp \left[-S_{E} / \hbar\right]} .
$$

At this point, we employ the fact that the exponential is quadratic in $a_{0}, a_{n}, b_{n} \in \mathbb{R}$, which immediately implies

$$
\left\langle a_{0} a_{k}\right\rangle=\left\langle a_{0} b_{k}\right\rangle=\left\langle a_{k} b_{l}\right\rangle=0, \quad\left\langle a_{k} a_{l}\right\rangle=\left\langle b_{k} b_{l}\right\rangle \propto \delta_{k l},
$$

with the expectation values defined in the sense of eq. (1.73). Thereby we obtain

$$
G(\tau)=T^{2}\left\langle a_{0}^{2}+\sum_{k=1}^{\infty} 2 a_{k}^{2}\left(e^{i \omega_{k} \tau}+e^{-i \omega_{k} \tau}\right)\right\rangle
$$


where

$$
\begin{aligned}
\left\langle a_{0}^{2}\right\rangle & =\frac{\int \mathrm{d} a_{0} a_{0}^{2} \exp \left(-\frac{1}{2} m T \omega^{2} a_{0}^{2}\right)}{\int \mathrm{d} a_{0} \exp \left(-\frac{1}{2} m T \omega^{2} a_{0}^{2}\right)} \\
& =-\frac{2}{m \omega^{2}} \frac{\mathrm{d}}{\mathrm{d} T}\left[\ln \int \mathrm{d} a_{0} \exp \left(-\frac{1}{2} m T \omega^{2} a_{0}^{2}\right)\right]=-\frac{2}{m \omega^{2}} \frac{\mathrm{d}}{\mathrm{d} T}\left[\ln \sqrt{\frac{2 \pi}{m \omega^{2} T}}\right] \\
& =\frac{1}{m \omega^{2} T} \\
\left\langle a_{k}^{2}\right\rangle & =\frac{\int \mathrm{d} a_{k} a_{k}^{2} \exp \left[-m T\left(\omega_{k}^{2}+\omega^{2}\right) a_{k}^{2}\right]}{\int \mathrm{d} a_{k} \exp \left[-m T\left(\omega_{k}^{2}+\omega^{2}\right) a_{k}^{2}\right]} \\
& =\frac{1}{2 m\left(\omega_{k}^{2}+\omega^{2}\right) T} .
\end{aligned}
$$

Inserting these into eq. (1.75) we get

$$
G(\tau)=\frac{T}{m}\left(\frac{1}{\omega^{2}}+\sum_{k=1}^{\infty} \frac{e^{i \omega_{k} \tau}+e^{-i \omega_{k} \tau}}{\omega_{k}^{2}+\omega^{2}}\right)=\frac{T}{m} \sum_{k=-\infty}^{\infty} \frac{e^{i \omega_{k} \tau}}{\omega_{k}^{2}+\omega^{2}}, \quad \omega_{k}=\frac{2 \pi T k}{\hbar} .
$$

There are various ways to evaluate the sum in eq. (1.78). We encounter a generic method in sec. 2.2 , so let us present a different approach here. We start by noting that

$$
\left(-\frac{\mathrm{d}^{2}}{\mathrm{~d} \tau^{2}}+\omega^{2}\right) G(\tau)=\frac{T}{m} \sum_{k=-\infty}^{\infty} e^{i \omega_{k} \tau}=\frac{\hbar}{m} \delta(\tau \bmod \beta \hbar),
$$

where we made use of the standard summation formula $\sum_{k=-\infty}^{\infty} e^{i \omega_{k} \tau}=\beta \hbar \delta(\tau \bmod \beta \hbar){ }^{2}$

Next, we solve eq. (1.79) for $0<\tau<\beta \hbar$, obtaining

$$
\left(-\frac{\mathrm{d}^{2}}{\mathrm{~d} \tau^{2}}+\omega^{2}\right) G(\tau)=0 \quad \Rightarrow \quad G(\tau)=A e^{\omega \tau}+B e^{-\omega \tau}
$$

where $A, B$ are unknown constants. The solution can be further restricted by noting that the definition of $G(\tau)$, eq. (1.78), indicates that $G(\beta \hbar-\tau)=G(\tau)$. Using this condition to obtain $B$, we then get

$$
G(\tau)=A\left[e^{\omega \tau}+e^{\omega(\beta \hbar-\tau)}\right] .
$$

The remaining unknown $A$ can be obtained by integrating eq. (1.79) over the source at $\tau=0$ and making use of the periodicity of $G(\tau), G(\tau+\beta \hbar)=G(\tau)$. This finally produces

$$
G^{\prime}\left((\beta \hbar)^{-}\right)-G^{\prime}\left(0^{+}\right)=\frac{\hbar}{m} \quad \Rightarrow \quad 2 \omega A\left(e^{\omega \beta \hbar}-1\right)=\frac{\hbar}{m},
$$

which together with eq. (1.81) yields our earlier result, eq. (1.70).

The agreement of the two different computations, eqs. (1.60) and (1.61), once again demonstrates the equivalence of the canonical and path integral approaches to solving thermodynamic quantities in a quantum-mechanical setting.

\footnotetext{
2 "Proof": $\sum_{k=-\infty}^{\infty} e^{i \omega_{k} \tau}=1+\lim _{\epsilon \rightarrow 0} \sum_{k=1}^{\infty}\left[\left(e^{i \frac{2 \pi \tau}{\beta \hbar}-\epsilon}\right)^{k}+\left(e^{-i \frac{2 \pi \tau}{\beta \hbar}-\epsilon}\right)^{k}\right]=\lim _{\epsilon \rightarrow 0}\left[\frac{1}{1-e^{i \frac{2 \pi \tau}{\beta \hbar}-\epsilon}}-\frac{1}{1-e^{i \frac{2 \pi \tau}{\beta \hbar}+\epsilon}}\right]$. If $\tau \neq 0 \bmod \beta \hbar$, then the limit $\epsilon \rightarrow 0$ can be taken, and the two terms cancel against each other. But if $\frac{2 \pi \tau}{\beta \hbar} \approx 0$, we can expand to leading order in a Taylor series, obtaining $\lim _{\epsilon \rightarrow 0}\left[\frac{i}{\frac{2 \pi \tau}{\beta \hbar}+i \epsilon}-\frac{i}{\frac{2 \pi \tau}{\beta \hbar}-i \epsilon}\right]=2 \pi \delta\left(\frac{2 \pi \tau}{\beta \hbar}\right)=\beta \hbar \delta(\tau)$.
} 


\section{Literature}

[1.1] R.P. Feynman and A.R. Hibbs, Quantum Mechanics and Path Integrals (McGraw-Hill, New York, 1965). 


\section{Free scalar fields}

Abstract: The concepts of sec. 1 are generalized to the case of a free massive scalar field living in a $d+1$ dimensional spacetime. This can be viewed as a system of infinitely many coupled harmonic oscillators. The resulting imaginary-time path integral for the partition function is expressed in Fourier representation. Matsubara sums are evaluated both in a low-temperature and a hightemperature expansion. The numerical convergence of these expansions, as well as some of their general properties, are discussed.

Keywords: Field theory, Matsubara sum, low-temperature expansion, high-temperature expansion, dimensional regularization, chemical potential, Euler gamma function, Riemann zeta function.

\subsection{Path integral for the partition function}

A path integral representation for the partition function of a scalar field theory can be derived from the result obtained for the quantum-mechanical harmonic oscillator (HO) in sec. 1.2.

In quantum field theory, the form of the theory is most economically defined in terms of the corresponding classical (Minkowskian) Lagrangian $\mathcal{L}_{M}$, rather than the Hamiltonian $\hat{H}$; for instance, Lorentz symmetry is explicit only in $\mathcal{L}_{M}$. Let us therefore start from eq. (1.5) for the quantum harmonic oscillator, and re-interpret $x$ as an "internal" degree of freedom $\phi$, situated at the origin $\mathbf{0}$ of $d$-dimensional space, like in eq. (1.11):

$$
\begin{aligned}
\mathcal{S}_{M}^{\mathrm{HO}} & =\int \mathrm{d} t \mathcal{L}_{M}^{\mathrm{HO}}, \\
\mathcal{L}_{M}^{\mathrm{HO}} & =\frac{m}{2}\left(\frac{\partial \phi(t, \mathbf{0})}{\partial t}\right)^{2}-V(\phi(t, \mathbf{0})) .
\end{aligned}
$$

We may compare this with the usual action of a scalar field theory (SFT) in $d$-dimensional space,

$$
\begin{aligned}
\mathcal{S}_{M}^{\mathrm{SFT}} & =\int \mathrm{d} t \int_{\mathbf{x}} \mathcal{L}_{M}^{\mathrm{SFT}}, \\
\mathcal{L}_{M}^{\mathrm{SFT}} & =\frac{1}{2} \partial^{\mu} \phi \partial_{\mu} \phi-V(\phi)=\frac{1}{2}\left(\partial_{t} \phi\right)^{2}-\frac{1}{2}\left(\partial_{i} \phi\right)\left(\partial_{i} \phi\right)-V(\phi),
\end{aligned}
$$

where we assume that repeated indices are summed over (irrespective of whether they are up and down), and the metric is $(+---)$.

Comparing eq. (2.2) with eq. (2.4), we see that scalar field theory is formally nothing but a collection of almost independent harmonic oscillators with $m=1$, one at every $\mathbf{x}$. These oscillators interact via the derivative term $\left(\partial_{i} \phi\right)\left(\partial_{i} \phi\right)$ which, in the language of statistical physics, couples nearest neighbours through

$$
\partial_{i} \phi \approx \frac{\phi\left(t, \mathbf{x}+a \mathbf{e}_{i}\right)-\phi(t, \mathbf{x})}{a},
$$

where $\mathbf{e}_{i}$ is a unit vector in the direction $i$ and $a$ is the lattice spacing.

Next, we note that a coupling of the above type does not change the derivation of the path integral in sec. 1.1 in any essential way: it was only important that the Hamiltonian was quadratic in the canonical momenta, $p=m \dot{x} \leftrightarrow \partial_{t} \phi$. In other words, the derivation of the path integral is 
only concerned with objects having to do with time dependence, and these appear in eqs. (2.2) and (2.4) in identical manners. Therefore, we can directly take over the result of eqs. (1.37)-(1.40):

$$
\begin{aligned}
\mathcal{Z}^{\mathrm{SFT}}(T) & =\int_{\phi(\beta \hbar, \mathbf{x})=\phi(0, \mathbf{x})} \prod_{\mathbf{x}}[C \mathcal{D} \phi(\tau, \mathbf{x})] \exp \left[-\frac{1}{\hbar} \int_{0}^{\beta \hbar} \mathrm{d} \tau \int_{\mathbf{x}} L_{E}^{\mathrm{SFT}}\right], \\
L_{E}^{\mathrm{SFT}} & =-\mathcal{L}_{M}^{\mathrm{SFT}}(t \rightarrow-i \tau)=\frac{1}{2}\left(\frac{\partial \phi}{\partial \tau}\right)^{2}+\sum_{i=1}^{d} \frac{1}{2}\left(\frac{\partial \phi}{\partial x^{i}}\right)^{2}+V(\phi) .
\end{aligned}
$$

For brevity, we drop out the superscript SFT in the following, and write $L_{E}=\frac{1}{2} \partial_{\mu} \phi \partial_{\mu} \phi+V(\phi)$.

\section{Fourier representation}

We now parallel the strategy of sec. 1.2 and rewrite the path integral in a Fourier representation. In order to simplify the notation, we measure time in units where $\hbar=1.05 \times 10^{-34} \mathrm{Js}=1$. Then the dependence of the scalar field on $\tau$ can be expressed as

$$
\phi(\tau, \mathbf{x})=T \sum_{n=-\infty}^{\infty} \tilde{\phi}\left(\omega_{n}, \mathbf{x}\right) e^{i \omega_{n} \tau}, \quad \omega_{n}=2 \pi T n, \quad n \in \mathbb{Z} .
$$

With the spatial coordinates, it is useful to make each direction finite for a moment, denoting the corresponding extents by $L_{i}$, and to impose periodic boundary conditions in each direction. Then the dependence of the field $\phi$ on a given $x^{i}$ can be represented in the form

$$
f\left(x^{i}\right)=\frac{1}{L_{i}} \sum_{n_{i}=-\infty}^{\infty} \tilde{f}\left(n_{i}\right) e^{i k_{i} x^{i}}, \quad k_{i}=\frac{2 \pi n_{i}}{L_{i}}, \quad n_{i} \in \mathbb{Z},
$$

where $1 / L_{i}$ plays the same role as $T$ in the time direction. In the infinite volume limit, the sum in eq. (2.9) goes over to the usual Fourier integral,

$$
\frac{1}{L_{i}} \sum_{n_{i}}=\frac{1}{2 \pi} \sum_{n_{i}} \Delta k_{i} \stackrel{L_{i} \rightarrow \infty}{\longrightarrow} \int \frac{\mathrm{d} k_{i}}{2 \pi}
$$

where $\Delta k_{i}=2 \pi / L_{i}$ is the width of the unit shell. The entire function in eq. (2.8) then reads

$$
\phi(\tau, \mathbf{x})=T \sum_{\omega_{n}} \frac{1}{V} \sum_{\mathbf{k}} \tilde{\phi}\left(\omega_{n}, \mathbf{k}\right) e^{i \omega_{n} \tau-i \mathbf{k} \cdot \mathbf{x}}, \quad V \equiv L_{1} L_{2} \ldots L_{d}
$$

where the sign conventions correspond to those in eq. (0.5).

Like in sec. 1.2 , the reality of $\phi(\tau, \mathbf{x})$ implies that the Fourier modes satisfy

$$
\left[\tilde{\phi}\left(\omega_{n}, \mathbf{k}\right)\right]^{*}=\tilde{\phi}\left(-\omega_{n},-\mathbf{k}\right) .
$$

Thereby only half of the Fourier modes are independent. We can choose, for instance,

$$
\tilde{\phi}\left(\omega_{n}, \mathbf{k}\right), \quad n \geq 1 ; \quad \tilde{\phi}(0, \mathbf{k}), \quad k_{1}>0 ; \quad \tilde{\phi}\left(0,0, k_{2}, \ldots\right), \quad k_{2}>0 ; \ldots ; \quad \text { and } \tilde{\phi}(0, \mathbf{0})
$$

as the integration variables. Note again the presence of a zero mode.

With the above conventions, quadratic forms can be written in the form

$$
\int_{0}^{\beta} \mathrm{d} \tau \int_{\mathbf{x}} \phi_{1}(\tau, \mathbf{x}) \phi_{2}(\tau, \mathbf{x})=T \sum_{\omega_{n}} \frac{1}{V} \sum_{\mathbf{k}} \tilde{\phi}_{1}\left(-\omega_{n},-\mathbf{k}\right) \tilde{\phi}_{2}\left(\omega_{n}, \mathbf{k}\right),
$$


implying that in the free case, i.e. for $V(\phi) \equiv \frac{1}{2} m^{2} \phi^{2}$, the exponent in eq. (2.6) becomes

$$
\begin{aligned}
\exp \left(-S_{E}\right) & =\exp \left(-\int_{0}^{\beta} \mathrm{d} \tau \int_{\mathbf{x}} L_{E}\right) \\
& =\exp \left[-\frac{1}{2} T \sum_{\omega_{n}} \frac{1}{V} \sum_{\mathbf{k}}\left(\omega_{n}^{2}+\mathbf{k}^{2}+m^{2}\right)\left|\tilde{\phi}\left(\omega_{n}, \mathbf{k}\right)\right|^{2}\right] \\
& =\prod_{\mathbf{k}}\left\{\exp \left[-\frac{T}{2 V} \sum_{\omega_{n}}\left(\omega_{n}^{2}+\mathbf{k}^{2}+m^{2}\right)\left|\tilde{\phi}\left(\omega_{n}, \mathbf{k}\right)\right|^{2}\right]\right\} .
\end{aligned}
$$

The exponential here is precisely of the same form as in eq. (1.50), with the replacements

$$
m^{(\mathrm{HO})} \rightarrow \frac{1}{V}, \quad\left(\omega^{(\mathrm{HO})}\right)^{2} \rightarrow \mathbf{k}^{2}+m^{2}, \quad\left|x_{n}^{(\mathrm{HO})}\right|^{2} \rightarrow\left|\tilde{\phi}\left(\omega_{n}, \mathbf{k}\right)\right|^{2} .
$$

Thus, we see that the result for the partition function factorizes into a product of harmonic oscillator partition functions, for which we know the answer already.

In order to take advantage of the above observation, we rewrite eqs. (1.50), (1.56) and (1.18) for the case $\hbar=1$. This allows us to represent the harmonic oscillator partition function in the form

$$
\begin{aligned}
\mathcal{Z}^{\mathrm{HO}} & =C^{\prime} \int\left[\prod_{n \geq 0} \mathrm{~d} x_{n}\right] \exp \left[-\frac{m T}{2} \sum_{n=-\infty}^{\infty}\left(\omega_{n}^{2}+\omega^{2}\right)\left|x_{n}\right|^{2}\right] \\
& =\frac{T}{\omega} \prod_{n=1}^{\infty} \frac{\omega_{n}^{2}}{\omega^{2}+\omega_{n}^{2}} \\
& =T \prod_{n=-\infty}^{\infty}\left(\omega_{n}^{2}+\omega^{2}\right)^{-\frac{1}{2}} \prod_{n^{\prime}=-\infty}^{\infty}\left(\omega_{n}^{2}\right)^{\frac{1}{2}} \\
& =\exp \left\{-\frac{1}{T}\left[\frac{\omega}{2}+T \ln \left(1-e^{-\beta \omega}\right)\right]\right\},
\end{aligned}
$$

where $n^{\prime}$ means that the zero mode $n=0$ is omitted.

Combining now eq. (2.15) with eqs. (2.17)-(2.20), we obtain two useful representations for $\mathcal{Z}^{\mathrm{SFT}}$. First of all, denoting

$$
\epsilon_{k} \equiv \sqrt{\mathbf{k}^{2}+m^{2}}
$$

eq. (2.19) yields

$$
\begin{aligned}
\mathcal{Z}^{\mathrm{SFT}}=\exp \left(-\frac{F^{\mathrm{SFT}}}{T}\right) & =\prod_{\mathbf{k}}\left\{T \prod_{n}\left(\omega_{n}^{2}+\epsilon_{k}^{2}\right)^{-\frac{1}{2}} \prod_{n^{\prime}}\left(\omega_{n}^{2}\right)^{\frac{1}{2}}\right\} \\
& =\exp \left\{\sum_{\mathbf{k}}\left[\ln T+\frac{1}{2} \sum_{n^{\prime}} \ln \omega_{n}^{2}-\frac{1}{2} \sum_{n} \ln \left(\omega_{n}^{2}+\epsilon_{k}^{2}\right)\right]\right\} .
\end{aligned}
$$

Taking the infinite-volume limit, the free-energy density, $F / V$, can thus be written as

$$
\lim _{V \rightarrow \infty} \frac{F^{\mathrm{SFT}}}{V}=\int \frac{\mathrm{d}^{d} \mathbf{k}}{(2 \pi)^{d}}\left[T \sum_{\omega_{n}} \frac{1}{2} \ln \left(\omega_{n}^{2}+\epsilon_{k}^{2}\right)-T \sum_{\omega_{n}^{\prime}} \frac{1}{2} \ln \left(\omega_{n}^{2}\right)-\frac{T}{2} \ln \left(T^{2}\right)\right] .
$$

Second, making directly use of eq. (2.20), we get the alternative representation

$$
\begin{aligned}
\mathcal{Z}^{\mathrm{SFT}}=\exp \left(-\frac{F^{\mathrm{SFT}}}{T}\right) & =\prod_{\mathbf{k}}\left\{\exp \left[-\frac{1}{T}\left(\frac{\epsilon_{k}}{2}+T \ln \left(1-e^{-\beta \epsilon_{k}}\right)\right)\right]\right\}, \\
\lim _{V \rightarrow \infty} \frac{F^{\mathrm{SFT}}}{V} & =\int \frac{\mathrm{d}^{d} \mathbf{k}}{(2 \pi)^{d}}\left[\frac{\epsilon_{k}}{2}+T \ln \left(1-e^{-\beta \epsilon_{k}}\right)\right] .
\end{aligned}
$$

We return to the momentum integrations in eqs. (2.24) and (2.26) in secs. 2.2 and 2.3. 


\subsection{Evaluation of thermal sums and their low-temperature limit}

Thanks to the previously established equality between eqs. (2.19) and (2.20), we have arrived at two different representations for the free energy density of a free scalar field theory, namely eqs. (2.24) and (2.26). The purpose of this section is to take the step from eq. (2.24) to (2.26) directly, and learn to carry out thermal sums such as those in eq. (2.24) also in more general cases.

As a first observation, we note that the sum in eq. (2.24) contains two physically very different structures. The first term depends on the energy (and thus on the mass of the field), and can be classified as a "physical" contribution. At the same time, the second and third terms represent "unphysical" subtractions, which are independent of the energy, but are needed in order to make the entire sum convergent. It is evident that only the contribution of the energy-dependent term survives in eq. (2.26).

In order not to lose the focus of our discussion on the subtraction terms, we mostly concentrate on another, convergent sum in the following:

$$
i\left(\epsilon_{k}\right) \equiv \frac{1}{\epsilon_{k}} \frac{\mathrm{d} j\left(\epsilon_{k}\right)}{\mathrm{d} \epsilon_{k}}=T \sum_{\omega_{n}} \frac{1}{\omega_{n}^{2}+\epsilon_{k}^{2}} .
$$

The term appearing in eq. (2.24),

$$
j\left(\epsilon_{k}\right) \equiv T \sum_{\omega_{n}} \frac{1}{2} \ln \left(\omega_{n}^{2}+\epsilon_{k}^{2}\right)-T \sum_{\omega_{n}^{\prime}} \frac{1}{2} \ln \left(\omega_{n}^{2}\right)-\frac{T}{2} \ln \left(T^{2}\right), \quad \omega_{n}=2 \pi T n,
$$

can be obtained from $i\left(\epsilon_{k}\right)$ through integration, apart from an $\epsilon_{k}$-independent integration constant.

Let now $f(p)$ be a generic function, analytic in the complex plane (apart from isolated singularities), and in particular regular on the real axis. We may then consider the sum

$$
\sigma \equiv T \sum_{\omega_{n}} f\left(\omega_{n}\right)
$$

where the $\omega_{n}$ are the Matsubara frequencies defined above (e.g. in eq. (2.28)). It turns out to be useful to define the auxiliary function

$$
i n_{\mathrm{B}}(i p) \equiv \frac{i}{\exp (i \beta p)-1},
$$

where $n_{\mathrm{B}}$ is the Bose distribution. Eq. (2.30) can be seen to have poles exactly at $\beta p=2 \pi n, n \in \mathbb{Z}$, i.e. at $p=\omega_{n}$. Expanding this function in a Laurent series around any of the poles, we get

$$
i n_{\mathrm{B}}\left(i\left[\omega_{n}+z\right]\right)=\frac{i}{\exp \left(i \beta\left[\omega_{n}+z\right]\right)-1}=\frac{i}{\exp (i \beta z)-1} \approx \frac{T}{z}+\mathcal{O}(1),
$$

which implies that the residue at each pole is $T$. This means that we can replace the sum in eq. (2.29) by the complex integral

$$
\sigma=\oint \frac{\mathrm{d} p}{2 \pi i} f(p) i n_{\mathrm{B}}(i p) \equiv \int_{-\infty-i 0^{+}}^{+\infty-i 0^{+}} \frac{\mathrm{d} p}{2 \pi} f(p) n_{\mathrm{B}}(i p)+\int_{+\infty+i 0^{+}}^{-\infty+i 0^{+}} \frac{\mathrm{d} p}{2 \pi} f(p) n_{\mathrm{B}}(i p),
$$

where the integration contour runs anti-clockwise around the real axis of the complex $p$-plane.

The above result can be further simplified by substituing $p \rightarrow-p$ in the latter term of eq. (2.32), and noting that

$$
n_{\mathrm{B}}(-i p)=\frac{1}{\exp (-i \beta p)-1}=\frac{\exp (i \beta p)-1+1}{1-\exp (i \beta p)}=-1-n_{\mathrm{B}}(i p) .
$$


This leads to the formula

$$
\begin{aligned}
\sigma & =\int_{-\infty-i 0^{+}}^{+\infty-i 0^{+}} \frac{\mathrm{d} p}{2 \pi}\left\{f(-p)+[f(p)+f(-p)] n_{\mathrm{B}}(i p)\right\} \\
& =\int_{-\infty}^{+\infty} \frac{\mathrm{d} p}{2 \pi} f(p)+\int_{-\infty-i 0^{+}}^{+\infty-i 0^{+}} \frac{\mathrm{d} p}{2 \pi}[f(p)+f(-p)] n_{\mathrm{B}}(i p),
\end{aligned}
$$

where we returned to the real axis in the first term, made possible by the lack of singularities there. All in all, we have thus converted the sum of eq. (2.29) into a rather convenient complex integral.

Inspecting the integral in eq. (2.34), we note that its first term is temperature-independent: it gives the zero-temperature, or "vacuum", contribution to $\sigma$. The latter term determines how thermal effects change the result. Let us note, furthermore, that in the lower half-plane we have

$$
\left|n_{\mathrm{B}}(i p)\right| \stackrel{p=x-i y}{=}\left|\frac{1}{e^{i \beta x} e^{\beta y}-1}\right| \stackrel{y \gg T}{\approx} e^{-\beta y} \stackrel{y \gg x}{\approx} e^{-\beta|p|} .
$$

Therefore, it looks likely that if the function $f(p)$ grows slower than $e^{\beta|p|}$ at large $|p|$ (in particular, polynomially), the integration contour for the finite- $T$ term of eq. (2.34) can be closed in the lower half-plane, whereby the result is determined by the poles and residues of the function $f(p)+f(-p)$. Physically, we say that the thermal contribution to $\sigma$ is related to "on-shell" particles.

Let us now apply the general formula in eq. (2.34) to the particular example of eq. (2.27). In fact, without any additional cost, we can consider a slight generalization,

$$
i\left(\epsilon_{k} ; c\right) \equiv T \sum_{\omega_{n}} \frac{1}{\left(\omega_{n}+c\right)^{2}+\epsilon_{k}^{2}}, \quad c \in \mathbb{C},
$$

so that in the notation of eq. (2.29) we have

$$
\begin{aligned}
f(p) & =\frac{1}{(p+c)^{2}+\epsilon_{k}^{2}}=\frac{i}{2 \epsilon_{k}}\left[\frac{1}{p+c+i \epsilon_{k}}-\frac{1}{p+c-i \epsilon_{k}}\right], \\
f(p)+f(-p) & =\frac{i}{2 \epsilon_{k}}\left[\frac{1}{p+c+i \epsilon_{k}}+\frac{1}{p-c+i \epsilon_{k}}-\frac{1}{p+c-i \epsilon_{k}}-\frac{1}{p-c-i \epsilon_{k}}\right] .
\end{aligned}
$$

For eq. (2.34), we need the poles of these functions in the lower half-plane, which for $|\operatorname{Im} c|<\epsilon_{k}$ are located at $p= \pm c-i \epsilon_{k}$. According to eqs. (2.37) and (2.38), the residue at each lower half-plane pole is $i / 2 \epsilon_{k}$. Thus the vacuum term in eq. (2.34) produces

$$
\frac{1}{2 \pi}(-2 \pi i) \frac{i}{2 \epsilon_{k}}=\frac{1}{2 \epsilon_{k}}
$$

whereas the thermal part yields

$$
\frac{1}{2 \pi}(-2 \pi i) \frac{i}{2 \epsilon_{k}}\left[\frac{1}{e^{\beta\left(\epsilon_{k}-i c\right)}-1}+\frac{1}{e^{\beta\left(\epsilon_{k}+i c\right)}-1}\right]
$$

In total, we obtain

$$
i\left(\epsilon_{k} ; c\right)=\frac{1}{2 \epsilon_{k}}\left[1+n_{\mathrm{B}}\left(\epsilon_{k}-i c\right)+n_{\mathrm{B}}\left(\epsilon_{k}+i c\right)\right],
$$

which is clearly periodic in $c \rightarrow c+2 \pi T n, n \in \mathbb{Z}$, as it must be according to eq. (2.36). We also note that the appearance of $i c$ resembles that of a chemical potential. Indeed, as shown around eqs. (2.45) and (2.46), setting $i c \rightarrow-\mu$ corresponds to a situation where we have averaged over a particle (chemical potential $\mu$ ) and an antiparticle (chemical potential $-\mu) .^{3}$

\footnotetext{
${ }^{3}$ Apart from a chemical potential, the parameter $c$ can also appear in a system with "shifted boundary conditions" over a compact direction, cf. e.g. ref. [2.1].
} 
To conclude the discussion, we integrate eq. (2.41) with respect to $\epsilon_{k}$ in order to obtain the function in eq. (2.28) (generalized to include $c$ ),

$$
j\left(\epsilon_{k} ; c\right) \equiv T \sum_{\omega_{n}} \frac{1}{2} \ln \left[\left(\omega_{n}+c\right)^{2}+\epsilon_{k}^{2}\right]-\left(\epsilon_{k} \text {-independent terms }\right) .
$$

Eq. (2.27) clearly continues to hold in the presence of $c$, so noting that

$$
\frac{1}{e^{x}-1}=\frac{e^{-x}}{1-e^{-x}}=\frac{\mathrm{d} \ln \left(1-e^{-x}\right)}{\mathrm{d} x}
$$

eq. (2.41) immediately yields

$$
j\left(\epsilon_{k} ; c\right)=\text { const. }+\frac{\epsilon_{k}}{2}+\frac{T}{2}\left\{\ln \left[1-e^{-\beta\left(\epsilon_{k}-i c\right)}\right]+\ln \left[1-e^{-\beta\left(\epsilon_{k}+i c\right)}\right]\right\} .
$$

The constant term in this result can depend both on $T$ and $c$, but not on $\epsilon_{k}$.

For $c=0$, a comparison of eq. (2.44) with eq. (2.26) shows that the role of the extra terms in eq. (2.24) is to eliminate the integration constant in eq. (2.44). This implies that the full physical result for $j\left(\epsilon_{k} ; 0\right)$ can be deduced directly from $i\left(\epsilon_{k} ; 0\right)$. The same is true even for $\mu \equiv-i c \neq 0$, if we interpret $j\left(\epsilon_{k} ; c\right)$ as a free energy density averaged over a particle and an antiparticle, as we next show.

\section{Extension to a chemical potential}

Considering a harmonic oscillator in the presence of a chemical potential, our task becomes to compute the partition function

$$
e^{-\beta F(T, \mu)} \equiv \mathcal{Z}(T, \mu) \equiv \operatorname{Tr}\left[e^{-\beta(\hat{H}-\mu \hat{N})}\right],
$$

where $\hat{N} \equiv \hat{a}^{\dagger} \hat{a}$. We show that the expression

$$
\frac{1}{2}[F(T, i c)+F(T,-i c)]
$$

agrees with the $\epsilon_{k}$-dependent part of eq. (2.44).

To start with, we observe that

$$
\langle n|(\hat{H}-\mu \hat{N})| n\rangle=\hbar \omega\left(n+\frac{1}{2}\right)-\mu n=(\hbar \omega-\mu) n+\frac{\hbar \omega}{2},
$$

so that evaluating the partition function in the energy basis yields

$$
\mathcal{Z}^{\text {HО }}=\sum_{n=0}^{\infty} \exp \left(-\frac{\hbar \omega}{2 T}-\frac{\hbar \omega-\mu}{T} n\right)=\frac{\exp \left(-\frac{\hbar \omega}{2 T}\right)}{1-\exp \left(-\frac{\hbar \omega-\mu}{T}\right)} .
$$

Setting now $\hbar \rightarrow 1, \omega \rightarrow \epsilon_{k}, \mu \rightarrow-i c$, we can rewrite the result as

$$
\mathcal{Z}^{\mathrm{HO}}=\exp \left\{-\frac{1}{T}\left[\frac{\epsilon_{k}}{2}+T \ln \left(1-e^{-\frac{\epsilon_{k}+i c}{T}}\right)\right]\right\} .
$$

Reading from here $F(T, \mu)$ according to eq. (2.45), and computing $\frac{1}{2}[F(T, i c)+F(T,-i c)]$, clearly yields exactly the $\epsilon_{k}$-dependent part of eq. (2.44). 


\section{Low-temperature expansion}

Our next goal is to carry out the momentum integration in eq. (2.24) and/or (2.26). To this end, we denote

$$
\begin{aligned}
J(m, T) & \equiv \int \frac{\mathrm{d}^{d} \mathbf{k}}{(2 \pi)^{d}}\left[\frac{\epsilon_{k}}{2}+T \ln \left(1-e^{-\beta \epsilon_{k}}\right)\right] \\
& =T \sum_{\omega_{n}} \int \frac{\mathrm{d}^{d} \mathbf{k}}{(2 \pi)^{d}}\left[\frac{1}{2} \ln \left(\omega_{n}^{2}+\epsilon_{k}^{2}\right)-\text { const. }\right], \\
I(m, T) & \equiv \frac{1}{m} \frac{\mathrm{d}}{\mathrm{d} m} J(m, T) \\
& =\int \frac{\mathrm{d}^{d} \mathbf{k}}{(2 \pi)^{d}} \frac{1}{2 \epsilon_{k}}\left[1+2 n_{\mathrm{B}}\left(\epsilon_{k}\right)\right] \\
& =T \sum_{\omega_{n}} \int \frac{\mathrm{d}^{d} \mathbf{k}}{(2 \pi)^{d}} \frac{1}{\omega_{n}^{2}+\epsilon_{k}^{2}},
\end{aligned}
$$

where $d \equiv 3-2 \epsilon$ is the space dimensionality, $\epsilon_{k} \equiv \sqrt{k^{2}+m^{2}}$, and we made use of the fact that inside the integral $m^{-1} \partial_{m}=\epsilon_{k}^{-1} \partial_{\epsilon_{k}}$. In order to simplify the notation, we further denote

$$
\oiint_{K} \equiv T \sum_{\omega_{n}} \int \frac{\mathrm{d}^{d} \mathbf{k}}{(2 \pi)^{d}}, \quad \oint_{K}^{\prime} \equiv T \sum_{\omega_{n}^{\prime}} \int \frac{\mathrm{d}^{d} \mathbf{k}}{(2 \pi)^{d}}, \quad \int_{\mathbf{k}} \equiv \int \frac{\mathrm{d}^{d} \mathbf{k}}{(2 \pi)^{d}},
$$

where $K \equiv\left(\omega_{n}, \mathbf{k}\right)$, and a prime denotes that the zero mode $\left(\omega_{n}=0\right)$ is omitted.

At low temperatures, $T \ll m$, we may expect the results to resemble those of the zero-temperature theory. To this end, we write

$$
J(m, T)=J_{0}(m)+J_{T}(m), \quad I(m, T)=I_{0}(m)+I_{T}(m),
$$

where $J_{0}$ is the temperature-independent vacuum energy density,

$$
J_{0}(m) \equiv \int_{\mathbf{k}} \frac{\epsilon_{k}}{2}
$$

and $J_{T}$ the thermal part of the free energy density,

$$
J_{T}(m) \equiv \int_{\mathbf{k}} T \ln \left(1-e^{-\beta \epsilon_{k}}\right) .
$$

The sum-integral $I(m, T)$ is divided in a similar way. It is clear that $J_{0}$ is ultraviolet divergent, and can only be evaluated in the presence of a regulator; our choice is typically dimensional regularization, as indicated in eq. (2.55). In contrast, the integrand in $J_{T}$ is exponentially small for $k \gg T$, and therefore the integral is convergent.

Let us start from the evaluation of $J_{0}(m)$. Writing out the mass dependence explicitly, the task becomes to compute

$$
J_{0}(m)=\int_{\mathbf{k}} \frac{1}{2}\left(k^{2}+m^{2}\right)^{\frac{1}{2}}
$$

For generality and future reference, we first consider a somewhat more generic integral,

$$
\Phi(m, d, A) \equiv \int \frac{\mathrm{d}^{d} \mathbf{k}}{(2 \pi)^{d}} \frac{1}{\left(k^{2}+m^{2}\right)^{A}},
$$


and obtain then $J_{0}$ as $J_{0}(m)=\frac{1}{2} \Phi\left(m, d,-\frac{1}{2}\right)$.

Owing to the fact that our integrand only depends on $k$, all angular integrations can be carried out at once, and the integration measure obtains the well-known form ${ }^{4}$

$$
\mathrm{d}^{d} \mathbf{k}=\frac{\pi^{\frac{d}{2}}}{\Gamma\left(\frac{d}{2}\right)}\left(k^{2}\right)^{\frac{d-2}{2}} \mathrm{~d}\left(k^{2}\right),
$$

where $\Gamma(s)$ is the Euler gamma function, discussed in further detail in sec. 2.3. Substituting now $k^{2} \rightarrow z \rightarrow m^{2} t$ in eq. (2.60), we get

$$
\begin{aligned}
\Phi(m, d, A) & =\frac{\pi^{\frac{d}{2}}}{\Gamma\left(\frac{d}{2}\right)} \frac{1}{(2 \pi)^{d}} \int_{0}^{\infty} \mathrm{d} z z^{\frac{d-2}{2}}\left(z+m^{2}\right)^{-A} \\
& =\frac{m^{d-2 A}}{(4 \pi)^{\frac{d}{2}} \Gamma\left(\frac{d}{2}\right)} \int_{0}^{\infty} \mathrm{d} t t^{\frac{d}{2}-1}(1+t)^{-A},
\end{aligned}
$$

from which the further substitution $t \rightarrow 1 / s-1, \mathrm{~d} t \rightarrow-\mathrm{d} s / s^{2}$ yields

$$
\Phi(m, d, A)=\frac{m^{d-2 A}}{(4 \pi)^{\frac{d}{2}} \Gamma\left(\frac{d}{2}\right)} \int_{0}^{1} \mathrm{~d} s s^{A-\frac{d}{2}-1}(1-s)^{\frac{d}{2}-1} .
$$

Here we recognize a standard integral that can be expressed in terms of the Euler $\Gamma$-function, producing finally

$$
\Phi(m, d, A)=\int \frac{\mathrm{d}^{d} \mathbf{k}}{(2 \pi)^{d}} \frac{1}{\left(\mathbf{k}^{2}+m^{2}\right)^{A}}=\frac{1}{(4 \pi)^{\frac{d}{2}}} \frac{\Gamma\left(A-\frac{d}{2}\right)}{\Gamma(A)} \frac{1}{\left(m^{2}\right)^{A-\frac{d}{2}}} .
$$

Let us now return to $J_{0}(m)$ in eq. (2.59), setting $A=-\frac{1}{2}$ and $d=3-2 \epsilon$ in eq. (2.64) and multiplying the result by $\frac{1}{2}$. The basic property $\Gamma(s)=s^{-1} \Gamma(s+1)$ allows us to transport the arguments of the $\Gamma$-functions to the vicinity of $1 / 2$ or 1 , where Taylor expansions are readily carried out, yielding (some helpful formulae are listed in eqs. (2.96)-(2.102)):

$$
\begin{aligned}
\Gamma(-2+\epsilon) & =\frac{\Gamma(1+\epsilon)}{(-2+\epsilon)(-1+\epsilon) \epsilon} \\
& =\frac{1}{2 \epsilon}\left(1+\frac{\epsilon}{2}\right)(1+\epsilon)\left(1-\gamma_{\mathrm{E}} \epsilon\right)+\mathcal{O}(\epsilon), \\
\Gamma\left(-\frac{1}{2}\right) & =-2 \Gamma\left(\frac{1}{2}\right)=-2 \sqrt{\pi} .
\end{aligned}
$$

The other parts of eq. (2.64) can be written as

$$
\begin{aligned}
(4 \pi)^{-\frac{3}{2}+\epsilon} & =\frac{2 \sqrt{\pi}}{(4 \pi)^{2}}[1+\epsilon \ln (4 \pi)]+\mathcal{O}\left(\epsilon^{2}\right), \\
\left(m^{2}\right)^{2-\epsilon} & =m^{4} \mu^{-2 \epsilon}\left(\frac{\mu^{2}}{m^{2}}\right)^{\epsilon}=m^{4} \mu^{-2 \epsilon}\left(1+\epsilon \ln \frac{\mu^{2}}{m^{2}}\right)+\mathcal{O}\left(\epsilon^{2}\right),
\end{aligned}
$$

where $\mu$ is an arbitrary (renormalization) scale parameter, introduced through $1=\mu^{-2 \epsilon} \mu^{2 \epsilon} .{ }^{5}$

\footnotetext{
${ }^{4}$ A quick derivation: On one hand, $\int \mathrm{d}^{d} \mathbf{k} e^{-t k^{2}}=\left[\int_{-\infty}^{\infty} \mathrm{d} k_{1} e^{-t k_{1}^{2}}\right]^{d}=(\pi / t)^{\frac{d}{2}}$. On the other hand, $\int \mathrm{d}^{d} \mathbf{k} e^{-t k^{2}}=$ $c(d) \int_{0}^{\infty} \mathrm{d} k k^{d-1} e^{-t k^{2}}=c(d) t^{-\frac{d}{2}} \int_{0}^{\infty} \mathrm{d} x x^{d-1} e^{-x^{2}}=c(d) \Gamma\left(\frac{d}{2}\right) / 2 t^{\frac{d}{2}}$. Thereby $c(d)=2 \pi^{\frac{d}{2}} / \Gamma\left(\frac{d}{2}\right)$.

${ }^{5}$ When systems with a finite chemical potential are considered, cf. eq. (2.45), one has to abandon the standard convention of denoting the scale parameter by $\mu$; frequently the notation $\Lambda$ is used instead, cf. eqs. (7.64) and (7.65).
} 
Collecting everything together, we obtain from above

$$
J_{0}(m)=-\frac{m^{4} \mu^{-2 \epsilon}}{64 \pi^{2}}\left[\frac{1}{\epsilon}+\ln \frac{\mu^{2}}{m^{2}}+\ln (4 \pi)-\gamma_{\mathrm{E}}+\frac{3}{2}+\mathcal{O}(\epsilon)\right]
$$

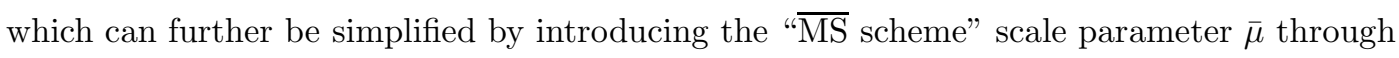

$$
\ln \bar{\mu}^{2} \equiv \ln \mu^{2}+\ln (4 \pi)-\gamma_{\mathrm{E}} .
$$

This leads us to

$$
J_{0}(m)=-\frac{m^{4} \mu^{-2 \epsilon}}{64 \pi^{2}}\left[\frac{1}{\epsilon}+\ln \frac{\bar{\mu}^{2}}{m^{2}}+\frac{3}{2}+\mathcal{O}(\epsilon)\right],
$$

from which a differentiation with respect to the mass parameter produces

$$
I_{0}(m)=\frac{1}{m} \frac{\mathrm{d}}{\mathrm{d} m} J_{0}(m)=\int_{\mathbf{k}} \frac{1}{2 \epsilon_{k}}=-\frac{m^{2} \mu^{-2 \epsilon}}{16 \pi^{2}}\left[\frac{1}{\epsilon}+\ln \frac{\bar{\mu}^{2}}{m^{2}}+1+\mathcal{O}(\epsilon)\right] .
$$

Interestingly, we note that $\int_{-\infty}^{\infty} \frac{\mathrm{d} k_{0}}{2 \pi} \frac{1}{k_{0}^{2}+\epsilon_{k}^{2}}=\frac{1}{2 \epsilon_{k}}$, so that $I_{0}(m)$ can also be written as

$$
I_{0}(m)=\int \frac{\mathrm{d}^{d+1} \mathbf{k}}{(2 \pi)^{d+1}} \frac{1}{k^{2}+m^{2}} .
$$

This is a very natural result, considering that the quantity we are determining is the $T=0$ limit of the sum-integral

$$
I(m, T)=\sum_{K} \frac{1}{K^{2}+m^{2}},
$$

with $\lim _{T \rightarrow 0} T \sum_{k_{n}}=\int \frac{\mathrm{d} k_{0}}{2 \pi}$, cf. eq. (2.10).

Next, we consider the finite-temperature integrals $J_{T}(m)$ and $I_{T}(m)$ which, as already mentioned, are both finite. Therefore we can normally set $d=3$ within them, even though it is good to recall that in multiloop computations these functions sometimes get multiplied by a divergent term, in which case contributions of $\mathcal{O}(\epsilon)$ (or higher) are needed as well. ${ }^{6}$ Neglecting this subtlety for now and substituting $k \rightarrow T x$ in eqs. (2.58) and (2.53), we find

$$
\begin{aligned}
& J_{T}(m)=\frac{T^{4}}{2 \pi^{2}} \int_{0}^{\infty} \mathrm{d} x x^{2} \ln \left(1-e^{-\sqrt{x^{2}+y^{2}}}\right)_{y \equiv \frac{m}{T}}, \\
& I_{T}(m)=\left.\frac{T^{2}}{2 \pi^{2}} \int_{0}^{\infty} \frac{\mathrm{d} x x^{2}}{\sqrt{x^{2}+y^{2}}} \frac{1}{e^{\sqrt{x^{2}+y^{2}}}-1}\right|_{y \equiv \frac{m}{T}} .
\end{aligned}
$$

These integrals cannot be expressed in terms of elementary functions, ${ }^{7}$ but their numerical evaluation is rather straightforward.

Even though eq. (2.76) cannot be evaluated exactly, we can still find approximate expressions valid in various limits. In this section we are interested in low temperatures, i.e. $y=m / T \gg 1$. We thus evaluate the leading term of eq. (2.76) in an expansion in $\exp (-y)$ and $1 / y$, which produces

$$
\int_{0}^{\infty} \mathrm{d} x x^{2} \ln \left(1-e^{-\sqrt{x^{2}+y^{2}}}\right) \quad=\quad-\int_{0}^{\infty} \mathrm{d} x x^{2} e^{-\sqrt{x^{2}+y^{2}}}+\mathcal{O}\left(e^{-2 y}\right)
$$

\footnotetext{
${ }^{6}$ The $\mathcal{O}(\epsilon)$ terms could be obtained by noting from eq. (2.61) that for $d=3-2 \epsilon, \mu^{2 \epsilon} \mathrm{d}^{d} \mathbf{k} /(2 \pi)^{d}=\mathrm{d}^{3} \mathbf{k} /(2 \pi)^{3}\{1+$ $\left.\epsilon\left[\ln \left(\bar{\mu}^{2} / 4 k^{2}\right)+2\right]+\mathcal{O}\left(\epsilon^{2}\right)\right\}$.

${ }^{7}$ However the following convergent sum representations apply: $J_{T}(m)=-\frac{m^{2} T^{2}}{2 \pi^{2}} \sum_{n=1}^{\infty} \frac{1}{n^{2}} K_{2}\left(\frac{n m}{T}\right), I_{T}(m)=$ $\frac{m T}{2 \pi^{2}} \sum_{n=1}^{\infty} \frac{1}{n} K_{1}\left(\frac{n m}{T}\right)$, with $K_{n}$ a modified Bessel function.
} 


$$
\begin{aligned}
& w \equiv \sqrt{=\sqrt{x^{2}+y^{2}}}-\int_{y}^{\infty} \mathrm{d} w w \sqrt{w^{2}-y^{2}} e^{-w}+\mathcal{O}\left(e^{-2 y}\right) \\
& v \equiv \underline{\underline{w}-y}-e^{-y} \int_{0}^{\infty} \mathrm{d} v(v+y) \sqrt{2 v y+v^{2}} e^{-v}+\mathcal{O}\left(e^{-2 y}\right) \\
& =-\sqrt{2} y^{\frac{3}{2}} e^{-y} \int_{0}^{\infty} \mathrm{d} v v^{\frac{1}{2}}\left(1+\frac{v}{y}\right)\left(1+\frac{v}{2 y}\right)^{\frac{1}{2}} e^{-v}+\mathcal{O}\left(e^{-2 y}\right) \\
& =-\sqrt{2} \Gamma\left(\frac{3}{2}\right) y^{\frac{3}{2}} e^{-y}\left[1+\mathcal{O}\left(\frac{1}{y}\right)+\mathcal{O}\left(e^{-y}\right)\right],
\end{aligned}
$$

where $\Gamma\left(\frac{3}{2}\right)=\sqrt{\pi} / 2$. It may be noted that the power-suppressed terms amount to an asymptotic (non-convergent) series, but can be accounted for through the leading term of a convergent expansion in terms of modified Bessel functions given in footnote $7,-y^{2} K_{2}(y)\left[1+\mathcal{O}\left(e^{-y}\right)\right]$.

Inserting the above expression into eq. (2.76), we have obtained

$$
J_{T}(m)=-T^{4}\left(\frac{m}{2 \pi T}\right)^{\frac{3}{2}} e^{-\frac{m}{T}}\left[1+\mathcal{O}\left(\frac{T}{m}\right)+\mathcal{O}\left(e^{-\frac{m}{T}}\right)\right],
$$

whereas the derivative in eq. (2.52) yields

$$
I_{T}(m)=\frac{T^{3}}{m}\left(\frac{m}{2 \pi T}\right)^{\frac{3}{2}} e^{-\frac{m}{T}}\left[1+\mathcal{O}\left(\frac{T}{m}\right)+\mathcal{O}\left(e^{-\frac{m}{T}}\right)\right] .
$$

Thereby we have arrived at the main conclusion of this section: at low temperatures, $T \ll m$, finite-temperature effects in a free theory with a mass gap are exponentially suppressed by the Boltzmann factor, $\exp (-m / T)$, like in non-relativistic statistical mechanics. Consequently, the functions $J(m, T)$ and $I(m, T)$ can be well approximated by their respective zero-temperature limits $J_{0}(m)$ and $I_{0}(m)$, which are given in eqs. (2.72) and (2.73). 


\subsection{High-temperature expansion}

Next, we move on to consider a limit opposite to that of the previous section, i.e. $T \gg m$ or, in terms of eq. (2.76), $y=m / T \ll 1$. It may appear that the procedure should then be a simple Taylor expansion of the integrand in eq. (2.76) around $y^{2}=0$. The zeroth order term indeed yields

$$
J_{T}(0)=\frac{T^{4}}{2 \pi^{2}} \int_{0}^{\infty} \mathrm{d} x x^{2} \ln \left(1-e^{-\sqrt{x^{2}}}\right)=-\frac{\pi^{2} T^{4}}{90},
$$

which is nothing but the free-energy density (minus the pressure) of black-body radiation with one massless degree of freedom. A correction term of order $\mathcal{O}\left(y^{2}\right)$ can also be worked out exactly.

However, $\mathcal{O}\left(y^{2}\right)$ is as far as it goes: trying to proceed to the next order, $\mathcal{O}\left(y^{4}\right)$, one finds that the integral for the coefficient of $y^{4}$ is power-divergent at small $x \equiv k / T$. In other words, the function $J_{T}(m)$ is non-analytic in the variable $m^{2}$ around the point $m^{2}=0$. A generalized high-temperature expansion nevertheless exists, and turns out to take the form

$$
J_{T}(m)=-\frac{\pi^{2} T^{4}}{90}+\frac{m^{2} T^{2}}{24}-\frac{m^{3} T}{12 \pi}-\frac{m^{4}}{2(4 \pi)^{2}}\left[\ln \left(\frac{m e^{\gamma_{\mathrm{E}}}}{4 \pi T}\right)-\frac{3}{4}\right]+\frac{m^{6} \zeta(3)}{3(4 \pi)^{4} T^{2}}+\mathcal{O}\left(\frac{m^{8}}{T^{4}}\right)+\mathcal{O}(\epsilon),
$$

where $m \equiv\left(m^{2}\right)^{1 / 2}$. It is the cubic term in eq. (2.82) that first indicates that $J_{T}(m)$ is non-analytic in $m^{2}$ - after all, the function $z^{3 / 2}$ contains a branch cut. This term plays a very important role in certain physics contexts, as will be seen in sec. 9.1.

Our goal in this section is to derive eq. (2.82). A classic derivation, starting directly from the definition in eq. (2.76), was presented by Dolan and Jackiw [2.2]. It is, however, easier, and ultimately more useful, to tackle the task in a slightly different way: we start from eq. (2.51) rather than eq. (2.50), and carry out first the integration $\int_{\mathbf{k}}$, and only then the sum $\sum_{\omega_{n}}$ (cf. e.g. ref. [2.3]). A slight drawback in this strategy is that eq. (2.51) contains inconvenient constant terms. Fortunately, we already know the mass-independent value $J(0, T)$ : it is given by eq. $(2.81)$. Therefore it is enough to study $I(m, T)$, in which case the starting point is eq. (2.54), which we may subsequently integrate as

$$
J(m, T)=\int_{0}^{m} \mathrm{~d} m^{\prime} m^{\prime} I\left(m^{\prime}, T\right)+J(0, T) .
$$

Proceeding now with $I(m, T)$ from eq. (2.54), the essential insight is to split the Matsubara sum into the contribution of the zero mode, $\omega_{n}=0$, and that of the non-zero modes, $\omega_{n} \neq 0$. Using the notation of eq. (2.55), we thus write

$$
\oiint_{K}=\oiint_{K}^{\prime}+T \int_{\mathbf{k}}
$$

and first consider the contribution of the last term, which is denoted by $I^{(n=0)}$.

To start with, we return to the infrared divergences alluded to above. Trying naively a simple Taylor expansion of the integrand of $I^{(n=0)}$ in powers of $m^{2}$, we would get

$$
I^{(n=0)}=T \int_{\mathbf{k}} \frac{1}{k^{2}+m^{2}} \stackrel{?}{=} T \int \frac{\mathrm{d}^{d} \mathbf{k}}{(2 \pi)^{d}}\left[\frac{1}{k^{2}}-\frac{m^{2}}{k^{4}}+\frac{m^{4}}{k^{6}}+\ldots\right] .
$$

For $d=3-2 \epsilon$, the first term is "ultraviolet divergent", i.e. grows at large $k$, whereas the second and subsequent terms are "infrared divergent", i.e. grow at small $k$ too fast to be integrable. Of 
course, in dimensional regularization, every expanded term in eq. (2.85) appears to be zero; the total result is, however, non-zero, cf. eq. (2.86) below. The bottom line is that the Taylor expansion in eq. (2.85) is not justified.

Next, we compute the integral in eq. (2.85) properly. The result can be read from eq. (2.64), by just setting $d=3-2 \epsilon, A=1$ :

$$
I^{(n=0)}=T \Phi(m, 3-2 \epsilon, 1)=\frac{T}{(4 \pi)^{3 / 2-\epsilon}} \frac{\Gamma\left(-\frac{1}{2}+\epsilon\right)}{\Gamma(1)} \frac{1}{\left(m^{2}\right)^{-1 / 2+\epsilon}} \stackrel{\Gamma\left(-\frac{1}{2}\right)=-2 \sqrt{\pi}}{=}-\frac{T m}{4 \pi}+\mathcal{O}(\epsilon) .
$$

We thus see that a linearly divergent integral over a manifestly positive function is finite and negative in dimensional regularization! According to eq. (2.52), the corresponding term in $J^{(n=0)}$ reads

$$
J^{(n=0)}=-\frac{T m^{3}}{12 \pi}+\mathcal{O}(\epsilon) .
$$

Given the importance of the result and its somewhat counter-intuitive appearance, it is worthwhile to demonstrate that eq. (2.86) is not an artifact of dimensional regularization. Indeed, let us compute the integral with cutoff regularization, by restricting $k$ to be smaller than an explicit upper bound $\Lambda$ :

$$
\begin{aligned}
I^{(n=0)} & =T \frac{4 \pi}{(2 \pi)^{3}} \int_{0}^{\Lambda} \frac{\mathrm{d} k k^{2}}{k^{2}+m^{2}}=\frac{T}{2 \pi^{2}}\left[\Lambda-m^{2} \int_{0}^{\Lambda} \frac{\mathrm{d} k}{k^{2}+m^{2}}\right] \\
& =\frac{T}{2 \pi^{2}}\left[\Lambda-m \arctan \left(\frac{\Lambda}{m}\right)\right] \stackrel{m \leqq \Lambda}{=} T\left[\frac{\Lambda}{2 \pi^{2}}-\frac{m}{4 \pi}+\mathcal{O}\left(\frac{m^{2}}{\Lambda}\right)\right] .
\end{aligned}
$$

We observe that, due to the first term, eq. (2.88) is positive. This term is unphysical, however: it must cancel against similar terms emerging from the non-zero Matsubara modes, since the temperature-dependent part of eq. (2.53) is manifestly finite. Owing to the fact that it represents a power divergence, it does not appear in dimensional regularization at all. The second term in eq. (2.88) is the physical one, and it agrees with eq. (2.86). The remaining terms in eq. (2.88) vanish when the cutoff is taken to infinity, and are analogous to the $\mathcal{O}(\epsilon)$-terms of eq. (2.86).

Next, we turn to the non-zero Matsubara modes, whose contribution to the integral is denoted by $I^{\prime}(m, T)$ (the prime is not to be confused with a derivative). It is important to realize that in this case, a Taylor expansion in $m^{2}$ can formally be carried out (we do not worry about the radius of convergence here): the integrals are of the type

$$
\int_{\mathbf{k}} \frac{\left(m^{2}\right)^{n}}{\left(\omega_{n}^{2}+k^{2}\right)^{n+1}}, \quad \omega_{n} \neq 0,
$$

and thus the integrand remains finite for small $k$, i.e., there are no infrared divergences. For the small- $n$ terms, ultraviolet divergences may on the other hand remain, but these are taken care of by the regularization.

More explicitly, we obtain

$$
\begin{aligned}
I^{\prime}(m, T) & = \\
& \stackrel{\text { Taylor }}{=} 2 T \sum_{\omega_{n}^{\prime}} \int \frac{\mathrm{d}^{d} \mathbf{k}}{(2 \pi)^{d}} \frac{1}{\omega_{n}^{2}+k^{2}+m^{2}} \\
& \stackrel{\mathrm{d}^{d} \mathbf{k}}{(2 \pi)^{d}} \sum_{l=0}^{\infty}(-1)^{l} \frac{m^{2 l}}{\left[(2 \pi n T)^{2}+k^{2}\right]^{l+1}} \\
\stackrel{(2.64)}{=} & 2 T \sum_{n=1}^{\infty} \sum_{l=0}^{\infty}(-1)^{l} m^{2 l} \frac{1}{(4 \pi)^{\frac{d}{2}}} \frac{\Gamma\left(l+1-\frac{d}{2}\right)}{\Gamma(l+1)} \frac{1}{(2 \pi n T)^{2 l+2-d}}
\end{aligned}
$$




$$
=\frac{2 T}{(4 \pi)^{\frac{d}{2}}(2 \pi T)^{2-d}} \sum_{l=0}^{\infty}\left[\frac{-m^{2}}{(2 \pi T)^{2}}\right]^{l} \frac{\Gamma\left(l+1-\frac{d}{2}\right)}{\Gamma(l+1)} \zeta(2 l+2-d),
$$

where in the last step we interchanged the orders of the two summations, and identified the sum over $n$ as a Riemann zeta function, $\zeta(s) \equiv \sum_{n=1}^{\infty} n^{-s}$. Some properties of $\zeta(s)$ are summarized in appendix A below.

For the sake of illustration, let us work out the terms $l=0,1,2$ of the above sum explicitly. For $d=3-2 \epsilon$, the order $l=0$ requires evaluating $\Gamma\left(-\frac{1}{2}+\epsilon\right)$ and $\zeta(-1+2 \epsilon) ; l=1$ requires evaluating $\Gamma\left(\frac{1}{2}+\epsilon\right)$ and $\zeta(1+2 \epsilon)$; and $l=2$ requires evaluating $\Gamma\left(\frac{3}{2}+\epsilon\right)$ and $\zeta(3+2 \epsilon)$. Applying results listed in appendix A of this section, a straightforward computation (cf. appendix B for intermediate steps) yields

$$
I^{\prime}(m, T)=\frac{T^{2}}{12}-\frac{2 m^{2} \mu^{-2 \epsilon}}{(4 \pi)^{2}}\left[\frac{1}{2 \epsilon}+\ln \left(\frac{\bar{\mu} e^{\gamma_{\mathrm{E}}}}{4 \pi T}\right)\right]+\frac{2 m^{4} \zeta(3)}{(4 \pi)^{4} T^{2}}+\mathcal{O}\left(\frac{m^{6}}{T^{4}}\right)+\mathcal{O}(\epsilon) .
$$

Adding to this the zero-mode contribution from eq. (2.86), we get

$$
I(m, T)=\frac{T^{2}}{12}-\frac{m T}{4 \pi}-\frac{2 m^{2} \mu^{-2 \epsilon}}{(4 \pi)^{2}}\left[\frac{1}{2 \epsilon}+\ln \left(\frac{\bar{\mu} e^{\gamma_{\mathrm{E}}}}{4 \pi T}\right)\right]+\frac{2 m^{4} \zeta(3)}{(4 \pi)^{4} T^{2}}+\mathcal{O}\left(\frac{m^{6}}{T^{4}}\right)+\mathcal{O}(\epsilon) .
$$

Subtracting eq. (2.73) to isolate the $T$-dependent part finally yields

$$
I_{T}(m)=\frac{T^{2}}{12}-\frac{m T}{4 \pi}-\frac{2 m^{2}}{(4 \pi)^{2}}\left[\ln \left(\frac{m e^{\gamma_{\mathrm{E}}}}{4 \pi T}\right)-\frac{1}{2}\right]+\frac{2 m^{4} \zeta(3)}{(4 \pi)^{4} T^{2}}+\mathcal{O}\left(\frac{m^{6}}{T^{4}}\right)+\mathcal{O}(\epsilon) .
$$

Note how the divergences and $\bar{\mu}$ have cancelled in our result for $I_{T}(m)$, as must be the case.

To transport the above results to various versions of the function $J$, we make use of eqs. (2.81) and (2.83). From eq. (2.91), we first get

$$
J^{\prime}(m, T)=-\frac{\pi^{2} T^{4}}{90}+\frac{m^{2} T^{2}}{24}-\frac{m^{4} \mu^{-2 \epsilon}}{2(4 \pi)^{2}}\left[\frac{1}{2 \epsilon}+\ln \left(\frac{\bar{\mu} e^{\gamma_{E}}}{4 \pi T}\right)\right]+\frac{m^{6} \zeta(3)}{3(4 \pi)^{4} T^{2}}+\mathcal{O}\left(\frac{m^{8}}{T^{4}}\right)+\mathcal{O}(\epsilon) .
$$

Adding the zero-mode contribution from eq. (2.87) then leads to

$J(m, T)=-\frac{\pi^{2} T^{4}}{90}+\frac{m^{2} T^{2}}{24}-\frac{m^{3} T}{12 \pi}-\frac{m^{4} \mu^{-2 \epsilon}}{2(4 \pi)^{2}}\left[\frac{1}{2 \epsilon}+\ln \left(\frac{\bar{\mu} e^{\gamma_{\mathrm{E}}}}{4 \pi T}\right)\right]+\frac{m^{6} \zeta(3)}{3(4 \pi)^{4} T^{2}}+\mathcal{O}\left(\frac{m^{8}}{T^{4}}\right)+\mathcal{O}(\epsilon)$.

Subtracting the zero-temperature part, $J_{0}(m)$, of eq. (2.72) leads to the expansion for $J_{T}(m)$ that was given in eq. (2.82). We may again note the cancellation of $1 / \epsilon$ and $\bar{\mu}$ in $J_{T}(m)$. The numerical convergence of the high-temperature expansion is illustrated in fig. 1 on p. 28.

\section{Appendix A: Properties of the Euler $\Gamma$ and Riemann $\zeta$ functions}

$\Gamma(s)$

The function $\Gamma(s)$ is to be viewed as a complex-valued function of a complex variable $s$. For $\operatorname{Re}(s)>0$, it can be defined as

$$
\Gamma(s) \equiv \int_{0}^{\infty} \mathrm{d} x x^{s-1} e^{-x},
$$

whereas for $\operatorname{Re}(s) \leq 0$, the values can be obtained through the iterative use of the relation

$$
\Gamma(s)=\frac{\Gamma(s+1)}{s} .
$$


On the real axis, $\Gamma(s)$ is regular at $s=1$; as a consequence of eq. (2.97), it then has first-order poles at $s=0,-1,-2, \ldots$. A useful relation, reflecting the pole structure, reads $\Gamma(s) \Gamma(1-s)=\frac{\pi}{\sin (\pi s)}$.

In practical applications, the argument $s$ is typically close to an integer or a half-integer. In the former case, we can use eq. (2.97) to relate the desired value to the behavior of $\Gamma(s)$ and its derivatives around $s=1$, which can in turn be worked out from the convergent integral representation in eq. (2.96). In particular,

$$
\Gamma(1)=1, \quad \Gamma^{\prime}(1)=-\gamma_{\mathrm{E}},
$$

where $\gamma_{\mathrm{E}}$ is the Euler constant, $\gamma_{\mathrm{E}}=0.577215664901 \ldots$. In the latter case, we can similarly use eq. (2.97) to relate the desired value to $\Gamma(s)$ and its derivatives around $s=\frac{1}{2}$, which can again be worked out from the integral representation in eq. (2.96), producing

$$
\Gamma\left(\frac{1}{2}\right)=\sqrt{\pi}, \quad \Gamma^{\prime}\left(\frac{1}{2}\right)=\sqrt{\pi}\left(-\gamma_{\mathrm{E}}-2 \ln 2\right) .
$$

The values required for eq. (2.91) thus become

$$
\begin{aligned}
\Gamma\left(-\frac{1}{2}+\epsilon\right) & =-2 \sqrt{\pi}+\mathcal{O}(\epsilon), \\
\Gamma\left(\frac{1}{2}+\epsilon\right) & =\sqrt{\pi}\left[1-\epsilon\left(\gamma_{\mathrm{E}}+2 \ln 2\right)+\mathcal{O}\left(\epsilon^{2}\right)\right], \\
\Gamma\left(\frac{3}{2}+\epsilon\right) & =\frac{\sqrt{\pi}}{2}+\mathcal{O}(\epsilon) .
\end{aligned}
$$

We have gone one order higher in the middle expansion, because this function is multiplied by $1 / \epsilon$ in the result (cf. eq. (2.112)).

$$
\zeta(s)
$$

The function $\zeta(s)$ is also to be viewed as a complex-valued function of a complex argument $s$. For $\operatorname{Re}(s)>1$, it can be defined as

$$
\zeta(s)=\sum_{n=1}^{\infty} n^{-s}=\frac{1}{\Gamma(s)} \int_{0}^{\infty} \frac{\mathrm{d} x x^{s-1}}{e^{x}-1},
$$

where the equivalence of the two forms can be seen by writing $1 /\left(e^{x}-1\right)=e^{-x} /\left(1-e^{-x}\right)=$ $\sum_{n=1}^{\infty} e^{-n x}$, and using the definition of the $\Gamma$-function in eq. (2.96). Some remarkable properties of $\zeta(s)$ follow from the fact that by writing

$$
\frac{1}{e^{x}-1}=\frac{1}{\left(e^{x / 2}-1\right)\left(e^{x / 2}+1\right)}=\frac{1}{2}\left[\frac{1}{e^{x / 2}-1}-\frac{1}{e^{x / 2}+1}\right]
$$

and then substituting integration variables through $x \rightarrow 2 x$, we can find an alternative integral representation,

$$
\zeta(s)=\frac{1}{\left(1-2^{1-s}\right) \Gamma(s)} \int_{0}^{\infty} \frac{\mathrm{d} x x^{s-1}}{e^{x}+1},
$$

defined for $\operatorname{Re}(s)>0, s \neq 1$. Even though the integral here clearly diverges at $s \rightarrow 0$, the function $\Gamma(s)$ also diverges at the same point, making $\zeta(s)$ regular around origin:

$$
\begin{aligned}
\zeta(0) & =-\frac{1}{2}, \\
\zeta^{\prime}(0) & =-\frac{1}{2} \ln (2 \pi) .
\end{aligned}
$$

Finally, for $\operatorname{Re}(s) \leq 0$, an analytic continuation is obtained through the relation

$$
\zeta(s)=\underbrace{\frac{(2 \pi)^{s}}{\Gamma\left(\frac{s}{2}\right) \Gamma\left(1-\frac{s}{2}\right)}}_{2^{s} \pi^{s-1} \sin \left(\frac{\pi s}{2}\right)} \Gamma(1-s) \zeta(1-s) .
$$


On the real axis, $\zeta(s)$ has a pole only at $s=1$. Its values at even arguments are "easy"; in fact, at even negative integers, eq. (2.108) implies that

$$
\zeta(-2 n)=0, \quad n=1,2,3, \ldots,
$$

whereas at positive even integers the values can be related to the Bernoulli numbers,

$$
\zeta(2)=\frac{\pi^{2}}{6}, \quad \zeta(4)=\frac{\pi^{4}}{90}, \ldots
$$

Negative odd integers can be related to positive even ones through eq. (2.108), which also allows us to determine the behaviour of the function around the pole at $s=1$. In contrast, odd positive integers larger than unity, i.e. $s=3,5, \ldots$, yield new transcendental numbers.

The values required in eq. (2.91) become

$$
\begin{aligned}
\zeta(-1+2 \epsilon) & =-\frac{1}{2 \pi^{2}} \Gamma(2) \zeta(2)+\mathcal{O}(\epsilon)=-\frac{1}{12}+\mathcal{O}(\epsilon) \\
\zeta(1+2 \epsilon) & =2^{1+2 \epsilon} \pi^{2 \epsilon}\left[\sin \left(\frac{\pi}{2}\right)+\pi \epsilon \cos \left(\frac{\pi}{2}\right)\right]\left(-\frac{1}{2 \epsilon}\right) \Gamma(1-2 \epsilon) \zeta(-2 \epsilon) \\
& =2(1+2 \epsilon \ln 2)(1+2 \epsilon \ln \pi)\left(-\frac{1}{2 \epsilon}\right)\left(1+2 \epsilon \gamma_{\mathrm{E}}\right)\left(-\frac{1}{2}\right)(1-2 \epsilon \ln 2 \pi)+\mathcal{O}(\epsilon) \\
& =\frac{1}{2 \epsilon}+\gamma_{\mathrm{E}}+\mathcal{O}(\epsilon) \\
\zeta(3+2 \epsilon) & =\zeta(3)+\mathcal{O}(\epsilon) \approx 1.2020569031 \ldots+\mathcal{O}(\epsilon)
\end{aligned}
$$

where in the first two cases we made use of eq. (2.108), and in the second also of eqs. (2.106) and (2.107).

\section{Appendix B: Numerical convergence}

We complete here the derivation of eq. (2.91), and sketch the regimes where the low and hightemperature expansions are numerically accurate by inspecting $J_{T}(m)$ from eq. (2.82).

First of all, for the term $l=0$ in eq. (2.90), we make use of the results of eqs. (2.100), (2.111):

$$
\left.I^{\prime}(m, T)\right|_{l=0}=\frac{2 T}{(4 \pi)^{3 / 2}}(2 \pi T) \frac{-2 \sqrt{\pi}}{1}\left(-\frac{1}{12}\right)+\mathcal{O}(\epsilon)=\frac{T^{2}}{12}+\mathcal{O}(\epsilon) .
$$

For the term $l=1$, we on the other hand insert the values of eqs. (2.101) and (2.112):

$$
\begin{aligned}
\left.I^{\prime}(m, T)\right|_{l=1} & =2 T \frac{(4 \pi)^{\epsilon}}{(4 \pi)^{3 / 2}}(2 \pi T)^{1-2 \epsilon}\left[\frac{-m^{2}}{(2 \pi T)^{2}}\right] \sqrt{\pi}\left[1-\epsilon\left(\gamma_{\mathrm{E}}+2 \ln 2\right)\right] \frac{1}{2 \epsilon}\left(1+2 \epsilon \gamma_{\mathrm{E}}\right)+\mathcal{O}(\epsilon) \\
1= & \stackrel{\mu^{-2 \epsilon}}{=} \mu^{2 \epsilon}-\frac{m^{2} \mu^{-2 \epsilon}}{(4 \pi)^{2}}\left\{\frac{1}{\epsilon}+\ln \frac{\mu^{2}}{T^{2}}+\ln (4 \pi)-\gamma_{\mathrm{E}}+2\left[\gamma_{\mathrm{E}}-\ln (4 \pi)\right]\right\}+\mathcal{O}(\epsilon) \\
& \stackrel{(2.71)}{=}-\frac{m^{2} \mu^{-2 \epsilon}}{(4 \pi)^{2}}\left\{\frac{1}{\epsilon}+\ln \frac{\bar{\mu}^{2}}{T^{2}}+2 \ln \left(\frac{e^{\gamma_{\mathrm{E}}}}{4 \pi}\right)\right\}+\mathcal{O}(\epsilon) .
\end{aligned}
$$

Finally, for the term $l=2$, we make use of eqs. (2.102) and (2.113), giving

$$
\left.I^{\prime}(m, T)\right|_{l=2}=\frac{2 T}{(4 \pi)^{3 / 2}}(2 \pi T) \frac{m^{4}}{(2 \pi T)^{4}} \frac{\frac{1}{2} \sqrt{\pi}}{2} \zeta(3)+\mathcal{O}(\epsilon)=\frac{2 m^{4} \zeta(3)}{(4 \pi)^{4} T^{2}}+\mathcal{O}(\epsilon) .
$$




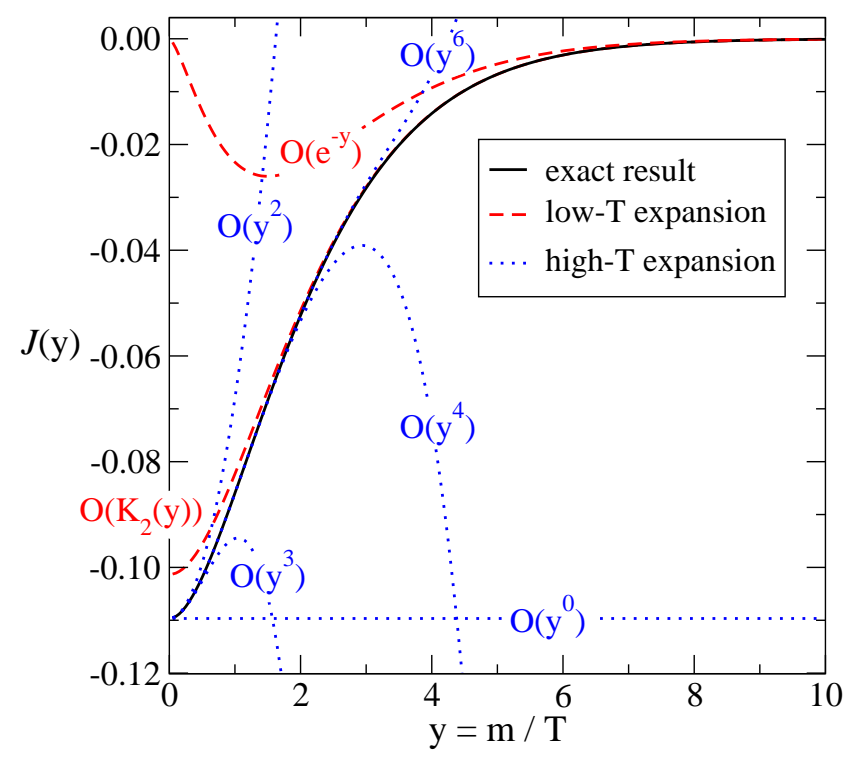

Figure 1: The behavior of $\mathcal{J}(y)$ and its various approximations. Shown are the exact numerical result from eq. (2.117), the two low-temperature approximations from eq. (2.118) (with exponential and powerlike corrections, respectively), as well as the high-temperature expansion from eq. (2.119).

For the numerical evaluation of $J_{T}(m)$, we again denote $y \equiv m / T$ and inspect the function

$$
\mathcal{J}(y) \equiv \frac{J_{T}(m)}{T^{4}}=\frac{1}{2 \pi^{2}} \int_{0}^{\infty} \mathrm{d} x x^{2} \ln \left(1-e^{-\sqrt{x^{2}+y^{2}}}\right) .
$$

We contrast this with the low-temperature results from footnote 7 and from eq. (2.79),

$$
\mathcal{J}(y) \stackrel{y \gtrsim 1}{\approx}-\frac{y^{2} K_{2}(y)}{2 \pi^{2}} \stackrel{y \gg 1}{\approx}-\left(\frac{y}{2 \pi}\right)^{\frac{3}{2}} e^{-y}
$$

as well as with the high-temperature expansion from eq. (2.82),

$$
\mathcal{J}(y) \stackrel{y \ll 1}{\approx}=-\frac{\pi^{2}}{90}+\frac{y^{2}}{24}-\frac{y^{3}}{12 \pi}-\frac{y^{4}}{2(4 \pi)^{2}}\left[\ln \left(\frac{y e^{\gamma_{\mathrm{E}}}}{4 \pi}\right)-\frac{3}{4}\right]+\frac{y^{6} \zeta(3)}{3(4 \pi)^{4}}
$$

The result of the comparison is shown in fig. 1. We observe that if we keep terms up to $y^{6}$ in the high-temperature expansion, its numerical convergence is good for $y \lesssim 3$. On the other hand, the low-temperature expansion with power corrections converges reasonably well for $y \gtrsim 6$. In between, either a numerical evaluation or the low-temperature expansion in terms of Bessel functions is necessary. It should be stressed that these statements are to be understood in a pragmatic sense, rather than as mathematically defined convergence radii. 


\section{Literature}

[2.1] L. Giusti and H.B. Meyer, Thermodynamic potentials from shifted boundary conditions: the scalar-field theory case, JHEP 11 (2011) 087 [1110.3136].

[2.2] L. Dolan and R. Jackiw, Symmetry behavior at finite temperature, Phys. Rev. D 9 (1974) 3320.

[2.3] P. Arnold and C. Zhai, Three-loop free energy for pure gauge QCD, Phys. Rev. D 50 (1994) 7603 [hep-ph/9408276]. 


\section{Interacting scalar fields}

Abstract: The key concepts of a perturbative or weak-coupling expansion are introduced in the context of evaluating the imaginary-time path integral representation for the partition function of an interacting scalar field. The issues of ultraviolet and infrared divergences are brought up. These problems are cured through renormalization and resummation, respectively.

Keywords: Weak-coupling expansion, Wick's theorem, propagator, contraction, ultraviolet and infrared divergences, renormalization, resummation, ring diagrams.

\subsection{Principles of the weak-coupling expansion}

In order to move from a free to an interacting theory, we now include a quartic term in the potential in eq. (2.4),

$$
V(\phi) \equiv \frac{1}{2} m^{2} \phi^{2}+\frac{1}{4} \lambda \phi^{4},
$$

where $\lambda>0$ is a dimensionless coupling constant. Thereby the Minkowskian and Euclidean Lagrangians become

$$
\begin{aligned}
\mathcal{L}_{M} & =\frac{1}{2} \partial^{\mu} \phi \partial_{\mu} \phi-\frac{1}{2} m^{2} \phi^{2}-\frac{1}{4} \lambda \phi^{4}, \\
L_{E} & =\frac{1}{2} \partial_{\mu} \phi \partial_{\mu} \phi+\frac{1}{2} m^{2} \phi^{2}+\frac{1}{4} \lambda \phi^{4},
\end{aligned}
$$

where repeated indices are summed over, irrespective of whether they are up and down or all down. The case with all indices down implies the use of Euclidean metric like in eq. (2.7).

In the presence of $\lambda>0$, it is no longer possible to determine the partition function of the system exactly, neither in the canonical formalism nor through a path integral approach. We therefore need to develop approximation schemes, which could in principle be either analytic or numerical. In the following we restrict our attention to the simplest analytic procedure which, as we will see, already teaches us a lot about the nature of the system.

In a weak-coupling expansion, the theory is solved by formally assuming that $\lambda \ll 1$, and by expressing the result for the observable in question as a (generalized) Taylor series in $\lambda$. The physical observable that we are interested in is the partition function defined according to eq. (2.6). Denoting the free and interacting parts of the Euclidean action by

$$
\begin{aligned}
S_{0} & \equiv \int_{0}^{\beta} \mathrm{d} \tau \int_{\mathbf{x}}\left[\frac{1}{2} \partial_{\mu} \phi \partial_{\mu} \phi+\frac{1}{2} m^{2} \phi^{2}\right], \\
S_{I} & \equiv \lambda \int_{0}^{\beta} \mathrm{d} \tau \int_{\mathbf{x}}\left[\frac{1}{4} \phi^{4}\right],
\end{aligned}
$$

the partition function can be written in the form

$$
\begin{aligned}
\mathcal{Z}^{\mathrm{SFT}}(T) & =C \int \mathcal{D} \phi \exp \left(-S_{0}-S_{I}\right) \\
& =C \int \mathcal{D} \phi e^{-S_{0}}\left[1-S_{I}+\frac{1}{2} S_{I}^{2}-\frac{1}{6} S_{I}^{3}+\ldots\right] \\
& =\mathcal{Z}_{(0)}^{\mathrm{SFT}}\left[1-\left\langle S_{I}\right\rangle_{0}+\frac{1}{2}\left\langle S_{I}^{2}\right\rangle_{0}-\frac{1}{6}\left\langle S_{I}^{3}\right\rangle_{0}+\ldots\right] .
\end{aligned}
$$


Here,

$$
\mathcal{Z}_{(0)}^{\mathrm{SFT}} \equiv C \int \mathcal{D} \phi e^{-S_{0}}
$$

is the free partition function determined in sec. 2 , and the expectation value $\langle\cdots\rangle_{0}$ is defined as

$$
\langle\cdots\rangle_{0} \equiv \frac{\int \mathcal{D} \phi[\cdots] \exp \left(-S_{0}\right)}{\int \mathcal{D} \phi \exp \left(-S_{0}\right)}
$$

With this result, the free energy density reads

$$
\begin{aligned}
& \frac{F^{\mathrm{SFT}}(T, V)}{V}=-\frac{T}{V} \ln \mathcal{Z}^{\mathrm{SFT}} \\
& =\frac{F_{(0)}^{\mathrm{SFT}}}{V}-\frac{T}{V} \ln \left(1-\left\langle S_{I}\right\rangle_{0}+\frac{1}{2}\left\langle S_{I}^{2}\right\rangle_{0}-\frac{1}{6}\left\langle S_{I}^{3}\right\rangle_{0}+\ldots\right) \\
& =\frac{F_{(0)}^{\mathrm{SFT}}}{V}-\frac{T}{V}\left\{-\left\langle S_{I}\right\rangle_{0}+\frac{1}{2}\left[\left\langle S_{I}^{2}\right\rangle_{0}-\left\langle S_{I}\right\rangle_{0}^{2}\right]\right. \\
& \left.-\frac{1}{6}\left[\left\langle S_{I}^{3}\right\rangle_{0}-3\left\langle S_{I}\right\rangle_{0}\left\langle S_{I}^{2}\right\rangle_{0}+2\left\langle S_{I}\right\rangle_{0}^{3}\right]+\ldots\right\},
\end{aligned}
$$

where we have Taylor-expanded the logarithm, $\ln (1-x)=-x-x^{2} / 2-x^{3} / 3+\ldots$. The first term, $F_{(0)}^{\mathrm{SFT}} / V$, is given in eq. (2.26), whereas the subsequent terms correspond to corrections of orders $\mathcal{O}(\lambda), \mathcal{O}\left(\lambda^{2}\right)$, and $\mathcal{O}\left(\lambda^{3}\right)$, respectively. As we will see, the combinations that appear within the square brackets in eq. (3.10) have a specific significance: eq. (3.10) is simpler than eq. (3.6)!

For future reference, let us denote

$$
f(T) \equiv \lim _{V \rightarrow \infty} \frac{F(T, V)}{V},
$$

where we have dropped the superscript "SFT" for simplicity. With this definition eq. (3.10) can be compactly represented by the formula $f=f_{(0)}+f_{(\geq 1)}$, where

$$
\begin{aligned}
f_{(\geq 1)}(T) & =-\frac{T}{V}\left\langle\exp \left(-S_{I}\right)-1\right\rangle_{0, \mathrm{c}} \\
& =\left\langle S_{I}-\frac{1}{2} S_{I}^{2}+\ldots\right\rangle_{0, \mathrm{c}, \text { drop overall } \int_{X}},
\end{aligned}
$$

where the subscript $(\ldots)_{c}$ refers to "connected" contractions, the precise meaning of which is discussed momentarily, and an "overall $\int_{X}$ " is dropped because it cancels against the prefactor $T / V$.

Inserting eq. (3.5) into the various terms of eq. (3.10), we are led to evaluate expectation values of the type

$$
\left\langle\phi\left(X_{1}\right) \phi\left(X_{2}\right) \ldots \phi\left(X_{n}\right)\right\rangle_{0} .
$$

These can be reduced to products of free 2-point correlators, $\left\langle\phi\left(X_{k}\right) \phi\left(X_{l}\right)\right\rangle_{0}$, through the Wick's theorem, as we now discuss.

\section{Wick's theorem}

Wick's theorem states that free (Gaussian) expectation values of any number of integration variables can be reduced to products of 2-point correlators, according to

$$
\left\langle\phi\left(X_{1}\right) \phi\left(X_{2}\right) \ldots \phi\left(X_{n-1}\right) \phi\left(X_{n}\right)\right\rangle_{0}=\sum_{\text {all combinations }}\left\langle\phi\left(X_{1}\right) \phi\left(X_{2}\right)\right\rangle_{0} \cdots\left\langle\phi\left(X_{n-1}\right) \phi\left(X_{n}\right)\right\rangle_{0} .
$$


Before applying this to the terms of eq. (3.10), we briefly recall how the theorem can be derived with (path) integration techniques.

Let us assume that we can discretise spacetime such that the coordinates $X$ only take a finite number of values, which in particular requires the volume to be finite. Then we can collect the values $\phi(X), \forall X$, into a single vector $v$, and subsequently write the free action in the form $S_{0}=\frac{1}{2} v^{T} A v$, where $A$ is a matrix. Here, we assume that $A^{-1}$ exists and that $A$ is symmetric, i.e. $A^{T}=A$; then it also follows that $\left(A^{-1}\right)^{T}=A^{-1}$.

The trick allowing us to evaluate integrals weighted by $\exp \left(-S_{0}\right)$ is to introduce a source vector $b$, and to take derivatives with respect to its components. Specifically, we define

$$
\begin{array}{rll}
\exp [W(b)] & \equiv & \int \mathrm{d} v \exp \left[-\frac{1}{2} v_{i} A_{i j} v_{j}+b_{i} v_{i}\right] \\
& \stackrel{v_{i} \rightarrow v_{i}+A_{i j}^{-1} b_{j}}{=} & \exp \left[\frac{1}{2} b_{i} A_{i j}^{-1} b_{j}\right] \int \mathrm{d} v \exp \left[-\frac{1}{2} v_{i} A_{i j} v_{j}\right],
\end{array}
$$

where we made a substitution of integration variables at the second equality. We then obtain

$$
\begin{aligned}
\left\langle v_{k} v_{l} \ldots v_{n}\right\rangle_{0} & =\frac{\int \mathrm{d} v\left(v_{k} v_{l} \ldots v_{n}\right) \exp \left[-\frac{1}{2} v_{i} A_{i j} v_{j}\right]}{\int \mathrm{d} v \exp \left[-\frac{1}{2} v_{i} A_{i j} v_{j}\right]} \\
& =\frac{\left\{\frac{\mathrm{d}}{\mathrm{d} b_{k}} \frac{\mathrm{d}}{\mathrm{d} b_{l}} \cdots \frac{\mathrm{d}}{\mathrm{d} b_{n}} \exp [W(b)]\right\}_{b=0}}{\exp [W(0)]} \\
& =\left\{\frac{\mathrm{d}}{\mathrm{d} b_{k}} \frac{\mathrm{d}}{\mathrm{d} b_{l}} \ldots \frac{\mathrm{d}}{\mathrm{d} b_{n}} \exp \left[\frac{1}{2} b_{i} A_{i j}^{-1} b_{j}\right]\right\}_{b=0} \\
& =\left\{\frac{\mathrm{d}}{\mathrm{d} b_{k}} \frac{\mathrm{d}}{\mathrm{d} b_{l}} \ldots \frac{\mathrm{d}}{\mathrm{d} b_{n}}\left[1+\frac{1}{2} b_{i} A_{i j}^{-1} b_{j}+\frac{1}{2}\left(\frac{1}{2}\right)^{2} b_{i} A_{i j}^{-1} b_{j} b_{r} A_{r s}^{-1} b_{s}+\ldots\right]\right\}_{b=0} .
\end{aligned}
$$

Taking the derivatives in eq. (3.16), we observe that:

- $\langle 1\rangle_{0}=1$.

- If there is an odd number of components of $v$ in the expectation value, the result is zero.

- $\left\langle v_{k} v_{l}\right\rangle_{0}=A_{k l}^{-1}$.

- $\left\langle v_{k} v_{l} v_{m} v_{n}\right\rangle_{0}=A_{k l}^{-1} A_{m n}^{-1}+A_{k m}^{-1} A_{l n}^{-1}+A_{k n}^{-1} A_{l m}^{-1}$

$$
=\left\langle v_{k} v_{l}\right\rangle_{0}\left\langle v_{m} v_{n}\right\rangle_{0}+\left\langle v_{k} v_{m}\right\rangle_{0}\left\langle v_{l} v_{n}\right\rangle_{0}+\left\langle v_{k} v_{n}\right\rangle_{0}\left\langle v_{l} v_{m}\right\rangle_{0} .
$$

- At higher orders, we obtain a discretized version of eq. (3.14).

- Since all the operations were purely combinatorial, removing the discretization does not modify the result, so that eq. (3.14) holds also in the infinite volume and continuum limits.

Let us now use eq. (3.14) in connection with eq. (3.10). From eqs. (2.26), (2.50) and (3.10), we read off the familiar leading-order result,

$$
f_{(0)}(T)=J(m, T) .
$$

At the first order, linear in $\lambda$, we on the other hand get

$$
f_{(1)}(T)=\lim _{V \rightarrow \infty} \frac{T}{V}\left\langle S_{I}\right\rangle_{0}=\lim _{V \rightarrow \infty} \frac{T}{V} \int_{0}^{\beta} \mathrm{d} \tau \int_{\mathbf{x}} \frac{\lambda}{4}\langle\phi(X) \phi(X) \phi(X) \phi(X)\rangle_{0},
$$


where we can now use Wick's theorem. Due to translational invariance, $\langle\phi(X) \phi(Y)\rangle_{0}$ can only depend on $X-Y$, so the spacetime integral becomes trivial, and we obtain

$$
f_{(1)}(T)=\frac{3}{4} \lambda\langle\phi(0) \phi(0)\rangle_{0}\langle\phi(0) \phi(0)\rangle_{0} .
$$

Finally, at the second order, we get

$$
\begin{aligned}
f_{(2)}(T)=\lim _{V \rightarrow \infty} & \left\{-\frac{T}{2 V}\left[\left\langle S_{I}^{2}\right\rangle_{0}-\left\langle S_{I}\right\rangle_{0}^{2}\right]\right\} \\
=\lim _{V \rightarrow \infty} & \left\{-\frac{T}{2 V}\left[\int_{X, Y}\left(\frac{\lambda}{4}\right)^{2}\langle\phi(X) \phi(X) \phi(X) \phi(X) \phi(Y) \phi(Y) \phi(Y) \phi(Y)\rangle_{0}\right.\right. \\
& \left.\left.-\int_{X} \frac{\lambda}{4}\langle\phi(X) \phi(X) \phi(X) \phi(X)\rangle_{0} \int_{Y} \frac{\lambda}{4}\langle\phi(Y) \phi(Y) \phi(Y) \phi(Y)\rangle_{0}\right]\right\},
\end{aligned}
$$

where we have again denoted (cf. eq. (0.2))

$$
\int_{X} \equiv \int_{0}^{\beta} \mathrm{d} \tau \int_{V} \mathrm{~d}^{d} \mathbf{x}
$$

Upon carrying out the contractions in eq. (3.20) according to Wick's theorem, the role of the "subtraction term", i.e. the second one in eq. (3.20), becomes clear: it cancels all disconnected contractions where all fields at point $X$ are contracted with other fields at the same point. In other words, the combination in eq. (3.20) amounts to taking into account only the connected contractions; this is the meaning of the subscript c in eq. (3.12). This combinatorial effect is caused by the logarithm in eq. (3.10), i.e., by going from the partition function to the free energy.

As far as the connected contractions go, we obtain through a (repeated) use of Wick's theorem:

$$
\begin{aligned}
\langle\phi(X) \phi & (X) \phi(X) \phi(X) \phi(Y) \phi(Y) \phi(Y) \phi(Y)\rangle_{0, \mathrm{c}} \\
= & 4\langle\phi(X) \phi(Y)\rangle_{0}\langle\phi(X) \phi(X) \phi(X) \phi(Y) \phi(Y) \phi(Y)\rangle_{0, \mathrm{c}} \\
& +3\langle\phi(X) \phi(X)\rangle_{0}\langle\phi(X) \phi(X) \phi(Y) \phi(Y) \phi(Y) \phi(Y)\rangle_{0, \mathrm{c}} \\
= & 4 \times 3\langle\phi(X) \phi(Y)\rangle_{0}\langle\phi(X) \phi(Y)\rangle_{0}\langle\phi(X) \phi(X) \phi(Y) \phi(Y)\rangle_{0, \mathrm{c}} \\
& +4 \times 2\langle\phi(X) \phi(Y)\rangle_{0}\langle\phi(X) \phi(X)\rangle_{0}\langle\phi(X) \phi(Y) \phi(Y) \phi(Y)\rangle_{0, \mathrm{c}} \\
& +3 \times 4\langle\phi(X) \phi(X)\rangle_{0}\langle\phi(X) \phi(Y)\rangle_{0}\langle\phi(X) \phi(Y) \phi(Y) \phi(Y)\rangle_{0, \mathrm{c}} \\
=\quad & 4 \times 3 \times 2\langle\phi(X) \phi(Y)\rangle_{0}\langle\phi(X) \phi(Y)\rangle_{0}\langle\phi(X) \phi(Y)\rangle_{0}\langle\phi(X) \phi(Y)\rangle_{0} \\
& +(4 \times 3+4 \times 2 \times 3+3 \times 4 \times 3)\langle\phi(X) \phi(X)\rangle_{0}\langle\phi(X) \phi(Y)\rangle_{0}\langle\phi(X) \phi(Y)\rangle_{0}\langle\phi(Y) \phi(Y)\rangle_{0} .
\end{aligned}
$$

Inspecting the 2-point correlators in this result, we note that they either depend on $X-Y$, or on neither $X$ nor $Y$, the latter case corresponding to the contraction of fields at the same point. Thereby one of the spacetime integrals is trivial (just substitute $X \rightarrow X+Y$, and note that $\left.\langle\phi(X+Y) \phi(Y)\rangle_{0}=\langle\phi(X) \phi(0)\rangle_{0}\right)$, and cancels against the factor $T / V=1 /(\beta V)$ in eq. (3.20). In total, we then have

$$
f_{(2)}(T)=-\left(\frac{\lambda}{4}\right)^{2}\left[12 \int_{X}\left\{\langle\phi(X) \phi(0)\rangle_{0}\right\}^{4}+36\left\{\langle\phi(0) \phi(0)\rangle_{0}\right\}^{2} \int_{X}\left\{\langle\phi(X) \phi(0)\rangle_{0}\right\}^{2}\right] .
$$

Graphically this can be represented as

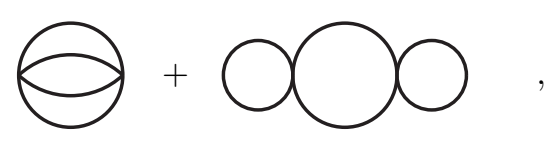


where solid lines denote propagators, and the vertices at which they cross denote spacetime points, in this case $X$ and 0 .

We could in principle go on with the third-order terms in eq. (3.10). Again, it could be verified that the "subtraction terms" cancel all disconnected contractions, so that only the connected ones contribute to $f(T)$, and that one spacetime integral cancels against the explicit factor $T / V$. These features are of general nature, and hold at any order in the weak-coupling expansion.

In summary, Wick's theorem has allowed us to convert the terms in eq. (3.10) to various structures made of the 2-point correlator $\langle\phi(X) \phi(0)\rangle_{0}$. We now turn to the properties of this function.

\section{Propagator}

The 2-point correlator $\langle\phi(X) \phi(Y)\rangle_{0}$ is usually called the free propagator. Denoting

$$
\delta(P+Q) \equiv \int_{X} e^{i(P+Q) \cdot X}=\beta \delta_{p_{n}+q_{n}, 0}(2 \pi)^{d} \delta^{(d)}(\mathbf{p}+\mathbf{q}),
$$

where $P \equiv\left(p_{n}, \mathbf{p}\right)$ and $p_{n}$ are bosonic Matsubara frequencies, and employing the representation

$$
\phi(X) \equiv \sum_{P} \tilde{\phi}(P) e^{i P \cdot X},
$$

we recall from basic quantum field theory that the (Euclidean) propagator can be written as

$$
\begin{aligned}
\langle\tilde{\phi}(P) \tilde{\phi}(Q)\rangle_{0} & =\delta(P+Q) \frac{1}{P^{2}+m^{2}}, \\
\langle\phi(X) \phi(Y)\rangle_{0} & =\xi_{P} e^{i P \cdot(X-Y)} \frac{1}{P^{2}+m^{2}} .
\end{aligned}
$$

Before inserting these expressions into eqs. (3.19) and (3.23), we briefly review their derivation, working in a finite volume $V$ and proceeding like in sec. 2.1.

First, we insert eq. (3.26) into the definition of the propagator,

$$
\langle\phi(X) \phi(Y)\rangle_{0}=\sum_{P, Q} e^{i P \cdot X+i Q \cdot Y}\langle\tilde{\phi}(P) \tilde{\phi}(Q)\rangle_{0},
$$

as well as to the free action, $S_{0}$,

$$
S_{0}=\frac{1}{2} \oiint_{P} \tilde{\phi}(-P)\left(P^{2}+m^{2}\right) \tilde{\phi}(P)=\frac{1}{2} \oiint_{P}\left(P^{2}+m^{2}\right)|\tilde{\phi}(P)|^{2} .
$$

Here, we may further write $\tilde{\phi}(P)=a(P)+i b(P)$, with $a(-P)=a(P), b(-P)=-b(P)$, and subsequently note that only half of the Fourier components are independent. We may choose these according to eq. (2.13).

Restricting the sum to the independent components, and making use of the symmetry properties of $a(P)$ and $b(P)$, eq. (3.30) becomes

$$
S_{0}=\frac{T}{V} \sum_{P_{\text {indep }}}\left(P^{2}+m^{2}\right)\left[a^{2}(P)+b^{2}(P)\right] .
$$


The Gaussian integral,

$$
\frac{\int \mathrm{d} x x^{2} \exp \left(-c x^{2}\right)}{\int \mathrm{d} x \exp \left(-c x^{2}\right)}=\frac{1}{2 c},
$$

and the symmetries of $a(P)$ and $b(P)$ then imply the results

$$
\begin{aligned}
\langle a(P) b(Q)\rangle_{0} & =0 \\
\langle a(P) a(Q)\rangle_{0} & =\left(\delta_{P, Q}+\delta_{P,-Q}\right) \frac{V}{2 T} \frac{1}{P^{2}+m^{2}}, \\
\langle b(P) b(Q)\rangle_{0} & =\left(\delta_{P, Q}-\delta_{P,-Q}\right) \frac{V}{2 T} \frac{1}{P^{2}+m^{2}},
\end{aligned}
$$

where the $\delta$-functions are of the Kronecker-type. Using these, the momentum-space propagator becomes

$$
\begin{aligned}
\langle\tilde{\phi}(P) \tilde{\phi}(Q)\rangle_{0} & =\langle a(P) a(Q)+i a(P) b(Q)+i b(P) a(Q)-b(P) b(Q)\rangle_{0} \\
& =\delta_{P,-Q} \frac{V}{T} \frac{1}{P^{2}+m^{2}}=\beta \delta_{p_{n}+q_{n}, 0} V \delta_{\mathbf{p}+\mathbf{q}, \mathbf{0}} \frac{1}{P^{2}+m^{2}},
\end{aligned}
$$

which in the infinite-volume limit (cf. eq. (2.10)), viz.

$$
\frac{1}{V} \sum_{\mathbf{p}} \longrightarrow \int \frac{\mathrm{d}^{d} \mathbf{p}}{(2 \pi)^{d}}, \quad V \delta_{\mathbf{p}, \mathbf{0}} \longrightarrow(2 \pi)^{d} \delta^{(d)}(\mathbf{p})
$$

becomes exactly eq. (3.27). Inserting this into eq. (3.29) we also recover eq. (3.28).

It is useful to study the behaviour of the propagator $\langle\phi(X) \phi(Y)\rangle_{0}$ at small and large separations $X-Y$. For this we may use the result of eq. (1.70),

$$
T \sum_{p_{n}} \frac{e^{i p_{n} \tau}}{p_{n}^{2}+\epsilon^{2}}=\frac{1}{2 \epsilon} \frac{\cosh \left[\left(\frac{\beta}{2}-\tau\right) \epsilon\right]}{\sinh \left[\frac{\beta \epsilon}{2}\right]}, \quad \beta=\frac{1}{T}, \quad 0 \leq \tau \leq \beta .
$$

Even though this equation was derived for $0 \leq \tau \leq \beta$, it is clear from the left-hand side that we can extend its validity to $-\beta \leq \tau \leq \beta$ by replacing $\tau$ by $|\tau|$. Thereby, the propagator in eq. (3.28) becomes

$$
G_{0}(X-Y) \equiv\langle\phi(X) \phi(Y)\rangle_{0}=\left.\int \frac{\mathrm{d}^{d} \mathbf{p}}{(2 \pi)^{d}} e^{i \mathbf{p} \cdot(\mathbf{y}-\mathbf{x})} \frac{1}{2 \epsilon_{p}} \frac{\cosh \left[\left(\frac{\beta}{2}-\left|x_{0}-y_{0}\right|\right) \epsilon_{p}\right]}{\sinh \left[\frac{\beta \epsilon_{p}}{2}\right]}\right|_{\epsilon_{p} \equiv \sqrt{p^{2}+m^{2}}}, \quad
$$

where we may set $Y=0$ with no loss of generality.

Consider first short distances, $|\mathbf{x}|,\left|x_{0}\right| \ll \frac{1}{T}, \frac{1}{m}$. We may expect the dominant contribution in the Fourier transform of eq. (3.39) to come from the regime $|\mathbf{p}||\mathbf{x}| \sim 1$, so we assume $|\mathbf{p}| \gg T, m$. Then $\epsilon_{p} \approx p$ and $\beta \epsilon_{p} \approx p / T \gg 1$, and consequently,

$$
\frac{\cosh \left[\left(\frac{\beta}{2}-\left|x_{0}\right|\right) \epsilon_{p}\right]}{\sinh \left[\frac{\beta \epsilon_{p}}{2}\right]} \approx \frac{\exp \left[\left(\frac{\beta}{2}-\left|x_{0}\right|\right) \epsilon_{p}\right]}{\exp \left[\frac{\beta \epsilon_{p}}{2}\right]} \approx e^{-\left|x_{0}\right| p} .
$$

Noting that

$$
\frac{1}{2 p} e^{-\left|x_{0}\right| p}=\int_{-\infty}^{\infty} \frac{\mathrm{d} p_{0}}{2 \pi} \frac{e^{i p_{0} x_{0}}}{p_{0}^{2}+\mathbf{p}^{2}}
$$


this implies

$$
G_{0}(X) \approx \int \frac{\mathrm{d}^{d+1} P}{(2 \pi)^{d+1}} \frac{e^{i P \cdot X}}{P^{2}},
$$

with $P \equiv\left(p_{0}, \mathbf{p}\right)$. We recognize this as the coordinate space propagator of a massless scalar field at zero temperature.

At this point we make use of the $d+1$-dimensional rotational symmetry of Euclidean spacetime, and choose $X=\left(x_{0}, \mathbf{x}\right)$ to point in the direction of the component $p_{0}$. Then,

$$
\begin{aligned}
\int \frac{\mathrm{d}^{d+1} P}{(2 \pi)^{d+1}} \frac{e^{i P \cdot X}}{P^{2}} & =\int \frac{\mathrm{d}^{d} \mathbf{p}}{(2 \pi)^{d}} \int_{-\infty}^{\infty} \frac{\mathrm{d} p_{0}}{2 \pi} \frac{e^{i p_{0}|X|}}{p_{0}^{2}+\mathbf{p}^{2}} \\
& =\int \frac{\mathrm{d}^{d} \mathbf{p}}{(2 \pi)^{d}} \frac{e^{-p|X|}}{2 p} \\
\stackrel{(2.61)}{=} \frac{1}{(2 \pi)^{d}} \frac{\pi^{\frac{d}{2}}}{\Gamma\left(\frac{d}{2}\right)} \int_{0}^{\infty} \mathrm{d} p p^{d-2} e^{-p|X|} & \frac{\Gamma(d-1)}{(4 \pi)^{\frac{d}{2}} \Gamma\left(\frac{d}{2}\right)|X|^{d-1}},
\end{aligned}
$$

from which, inserting $d=3$ and $\Gamma\left(\frac{3}{2}\right)=\sqrt{\pi} / 2$, we find

$$
G_{0}(X) \approx \frac{1}{4 \pi^{2}|X|^{2}}, \quad|X| \ll \frac{1}{T}, \frac{1}{m} .
$$

The result is independent of $T$ and $m$, signifying that at short distances (in the "ultraviolet" regime), temperature and masses do not play a role. We may further note that the propagator rapidly diverges in this regime.

Next, we consider the opposite limit of large distances, $x=|\mathbf{x}| \gg 1 / T$, noting that the periodic temporal coordinate $x_{0}$ is always "small", i.e. at most $1 / T$. We expect that the Fourier transform of eq. (3.39) is now dominated by small momenta, $p \ll T$. If we simplify the situation further by assuming that we are also at very high temperatures, $m \ll T$, then $\beta \epsilon_{p} \ll 1$, and we can expand the hyperbolic functions in Taylor series, approximating $\cosh (\epsilon) \approx 1, \sinh (\epsilon) \approx \epsilon$. We then obtain from eq. (3.39)

$$
G_{0}(X) \approx T \int \frac{\mathrm{d}^{d} \mathbf{p}}{(2 \pi)^{d}} \frac{e^{-i \mathbf{p} \cdot \mathbf{x}}}{p^{2}+m^{2}}, \quad x \gg \frac{1}{T} .
$$

Note that the integrand here is also the $p_{n}=0$ contribution from the left-hand side of eq. (3.38). Setting $d=3,{ }^{8}$ and denoting $z \equiv \mathbf{p} \cdot \mathbf{x} /(p x)$, the remaining integral can be worked out as

$$
\begin{aligned}
G_{0}(X) & \approx \frac{T}{(2 \pi)^{2}} \int_{-1}^{+1} \mathrm{~d} z \int_{0}^{\infty} \mathrm{d} p p^{2} \frac{e^{-i p x z}}{p^{2}+m^{2}} \\
& =\frac{T}{(2 \pi)^{2}} \int_{0}^{\infty} \frac{\mathrm{d} p p^{2}}{p^{2}+m^{2}} \frac{e^{i p x}-e^{-i p x}}{i p x} \\
& =\frac{T}{(2 \pi)^{2} i x} \int_{-\infty}^{\infty} \frac{\mathrm{d} p p e^{i p x}}{p^{2}+m^{2}} \\
& =\frac{T e^{-m x}}{4 \pi x}, \quad x \gg \frac{1}{T} .
\end{aligned}
$$

In the last step the integration contour was closed in the upper half-plane (recalling that $x>0$ ).

\footnotetext{
${ }^{8}$ For a general $d, \int \frac{\mathrm{d}^{d} \mathbf{p}}{(2 \pi)^{d}} \frac{e^{-i \mathbf{p} \cdot \mathbf{x}}}{p^{2}+m^{2}}=(2 \pi)^{-\frac{d}{2}}\left(\frac{m}{x}\right)^{\frac{d}{2}-1} K_{\frac{d}{2}-1}(m x)$, where $K$ is a modified Bessel function.
} 
We note from eq. (3.46) that at large distances (in the "infrared" regime), thermal effects modify the behaviour of the propagator in an essential way. In particular, if we were to set the mass to zero, then eq. (3.44) would be the exact behaviour at zero temperature, both at small and at large distances, whereas eq. (3.46) shows that a finite temperature would "slow down" the long-distance decay to $T /(4 \pi|\mathbf{x}|)$. In other words, we can say that at finite temperature the theory is more sensitive to infrared physics than at zero temperature.

The considerations just discussed play a physical role in plasma physics, i.e. the study of electrodynamics in a statistical environment. We defer an illustration to appendix B of sec. 5.2, by which time gauge fields will have been introduced. 


\subsection{Problems of the naive weak-coupling expansion}

\section{$\mathcal{O}(\lambda):$ ultraviolet divergences}

We now proceed with the evaluation of the weak-coupling expansion for the free energy density in a scalar field theory, the first three orders of which are given by eqs. (3.17), (3.19) and (3.23). Noting from eqs. (2.54) and (3.28) that $G_{0}(0)=I(m, T)$, we obtain

$$
f(T)=J(m, T)+\frac{3}{4} \lambda[I(m, T)]^{2}+\mathcal{O}\left(\lambda^{2}\right) .
$$

According to eqs. (2.72) and (2.73), we have

$$
\begin{aligned}
& J(m, T)=-\frac{m^{4} \mu^{-2 \epsilon}}{64 \pi^{2}}\left[\frac{1}{\epsilon}+\ln \frac{\bar{\mu}^{2}}{m^{2}}+\frac{3}{2}+\mathcal{O}(\epsilon)\right]+J_{T}(m), \\
& I(m, T)=-\frac{m^{2} \mu^{-2 \epsilon}}{16 \pi^{2}}\left[\frac{1}{\epsilon}+\ln \frac{\bar{\mu}^{2}}{m^{2}}+1+\mathcal{O}(\epsilon)\right]+I_{T}(m),
\end{aligned}
$$

where the finite functions $J_{T}(m)$ and $I_{T}(m)$ were evaluated in various limits in eqs. (2.79), (2.80), (2.82) and (2.93).

Inserting eqs. (3.48) and (3.49) into eq. (3.47), we note that the result is, in general, ultraviolet divergent. For instance, restricting for simplicity to very high temperatures, $T \gg m$, and making use of eq. (2.93),

$$
I_{T}(m) \approx \frac{T^{2}}{12}-\frac{m T}{4 \pi}+\mathcal{O}\left(m^{2}\right)
$$

the dominant term at $\epsilon \rightarrow 0$ reads

$$
f(T) \approx-\frac{\mu^{-2 \epsilon}}{64 \pi^{2} \epsilon}\left\{m^{4}+\lambda\left[\frac{1}{2} T^{2} m^{2}-\frac{3}{2 \pi} T m^{3}+\mathcal{O}\left(m^{4}\right)\right]+\mathcal{O}\left(\lambda^{2}\right)\right\}+\mathcal{O}(1) .
$$

This result is clearly non-sensical; in particular the divergences depend on the temperature, i.e. cannot be removed by subtracting a $T$-independent "vacuum" contribution. To properly handle this issue requires renormalization, to which we return in sec. 3.3 .

\section{$\mathcal{O}\left(\lambda^{2}\right)$ : infrared divergences}

Let us next consider the $\mathcal{O}\left(\lambda^{2}\right)$ correction to eq. (3.47), given by eq. (3.23). With the notation of eq. (3.39), it can be written as

$$
f_{(2)}(T)=-\frac{3}{4} \lambda^{2} \int_{X}\left[G_{0}(X)\right]^{4}-\frac{9}{4} \lambda^{2}[I(m, T)]^{2} \int_{X}\left[G_{0}(X)\right]^{2} .
$$

It is particularly interesting to inspect what happens if we take the particle mass $m$ to be very small in units of the temperature, $m \ll T$.

As eqs. (2.82), (3.47) and (3.50) show, at $\mathcal{O}(\lambda)$ the small-mass limit is perfectly well-defined. At the next order, we on the other hand must analyze the two terms of eq. (3.52). Starting with the first one, we know from eq. (3.44) that the behaviour of $G_{0}$ is independent of $m$ at small $x$, and thus nothing particular happens for $x \ll T^{-1}$. On the other hand, for large $x, G_{0}$ is given by eq. (3.46), and we may thus estimate the contribution of this region as

$$
\int_{x \gtrsim \beta}\left[G_{0}(X)\right]^{4} \sim \int_{0}^{\beta} \mathrm{d} \tau \int_{x \gtrsim \beta} \mathrm{d}^{3} \mathbf{x}\left(\frac{T e^{-m x}}{4 \pi x}\right)^{4} .
$$


This integral is convergent even for $m \rightarrow 0$.

Consider then the second term of eq. (3.52). Repeating the previous argument, we see that the long-distance contribution to the free energy density is proportional to the integral

$$
\int_{x \gtrsim \beta}\left[G_{0}(X)\right]^{2} \sim \int_{0}^{\beta} \mathrm{d} \tau \int_{x \gtrsim \beta} \mathrm{d}^{3} \mathbf{x}\left(\frac{T e^{-m x}}{4 \pi x}\right)^{2} .
$$

If we now attempt to set $m \rightarrow 0$, we run into a linearly divergent integral. Because this problem emerges from large distances, we call this an infrared divergence.

In fact, it is easy to be more precise about the form of the divergence. We can namely write

$$
\begin{aligned}
\int_{X}\left[G_{0}(X)\right]^{2} & =\int_{X} \oiint_{P} \frac{e^{i P \cdot X}}{P^{2}+m^{2}} \sum_{Q} \frac{e^{i Q \cdot X}}{Q^{2}+m^{2}} \\
& =\oiint_{P Q} \delta(P+Q) \frac{1}{\left(P^{2}+m^{2}\right)\left(Q^{2}+m^{2}\right)} \\
& =\sum_{P} \frac{1}{\left[P^{2}+m^{2}\right]^{2}} \\
& =-\frac{\mathrm{d}}{\mathrm{d} m^{2}} I(m, T) .
\end{aligned}
$$

Inserting eq. (3.50), we get

$$
\int_{X}\left[G_{0}(X)\right]^{2}=-\frac{1}{2 m} \frac{\mathrm{d}}{\mathrm{d} m} I(m, T)=\frac{T}{8 \pi m}+\mathcal{O}(1),
$$

so that for $m \ll T$, eq. (3.52) evaluates to

$$
f_{(2)}(T)=-\frac{9}{4} \lambda^{2} \frac{T^{4}}{144} \frac{T}{8 \pi m}+\mathcal{O}\left(m^{0}\right) .
$$

This indeed diverges for $m \rightarrow 0$.

It is clear that like the ultraviolet divergence in eq. (3.51), the infrared divergence in eq. (3.57) must be an artifact of some sort: the pressure and other thermodynamic properties of a plasma of weakly interacting massless scalar particles should be finite, as we know to be the case for a plasma of massless photons. We return to the resolution of this "paradox" in sec. 3.4. 


\subsection{Proper free energy density to $\mathcal{O}(\lambda)$ : ultraviolet renormalization}

In sec. 3.2 we attempted to compute the free energy density $f(T)$ of a scalar field theory up to $\mathcal{O}(\lambda)$, but found a result which appeared to be ultraviolet (UV) divergent. Let us now show that, as must be the case in a renormalizable theory, the divergences disappear order-by-order in perturbation theory, if we re-express $f(T)$ in terms of renormalized parameters. Furthermore the renormalization procedure is identical to that at zero temperature.

In order to proceed, we need to change the notation somewhat. The zero-temperature parameters we employed before, i.e. $m^{2}, \lambda$, are now re-interpreted to be bare parameters, $m_{\mathrm{B}}^{2}, \lambda_{\mathrm{B}}{ }^{9}{ }^{9}$ The expansion in eq. (3.47) can then be written in the schematic form

$$
f(T)=\phi^{(0)}\left(m_{\mathrm{B}}^{2}, T\right)+\lambda_{\mathrm{B}} \phi^{(1)}\left(m_{\mathrm{B}}^{2}, T\right)+\mathcal{O}\left(\lambda_{\mathrm{B}}^{2}\right) .
$$

As a second step, we introduce the renormalized parameters $m_{\mathrm{R}}^{2}, \lambda_{\mathrm{R}}$. These could either be directly physical quantities (say, the mass of the scalar particle, and the scattering amplitude with particular kinematics), or quantities which are not directly physical, but are related to physical quantities by finite equations (say, so-called $\overline{\mathrm{MS}}$ scheme parameters). In any case, it is natural to choose the renormalized parameters such that in the limit of an extremely weak interaction, $\lambda_{\mathrm{R}} \ll 1$, they formally agree with the bare parameters. In other words, we may write

$$
\begin{aligned}
m_{\mathrm{B}}^{2} & =m_{\mathrm{R}}^{2}+\lambda_{\mathrm{R}} f^{(1)}\left(m_{\mathrm{R}}^{2}\right)+\mathcal{O}\left(\lambda_{\mathrm{R}}^{2}\right), \\
\lambda_{\mathrm{B}} & =\lambda_{\mathrm{R}}+\lambda_{\mathrm{R}}^{2} g^{(1)}\left(m_{\mathrm{R}}^{2}\right)+\mathcal{O}\left(\lambda_{\mathrm{R}}^{3}\right),
\end{aligned}
$$

where it is important to note that the renormalized parameters are defined at zero temperature (no $T$ appears in these relations). The functions $f^{(i)}$ and $g^{(i)}$ are in general divergent in the limit that the regularization is removed; for instance, in dimensional regularization, they are expected to contain poles, such as $1 / \epsilon$ or higher.

The idea now is to convert the expansion in eq. (3.58) into an expansion in $\lambda_{\mathrm{R}}$ by inserting in it the expressions from eqs. (3.59) and (3.60) and Taylor-expanding the result in $\lambda_{R}$. This produces

$$
f(T)=\phi^{(0)}\left(m_{\mathrm{R}}^{2}, T\right)+\lambda_{\mathrm{R}}\left[\phi^{(1)}\left(m_{\mathrm{R}}^{2}, T\right)+\frac{\partial \phi^{(0)}\left(m_{\mathrm{R}}^{2}, T\right)}{\partial m_{\mathrm{R}}^{2}} f^{(1)}\left(m_{\mathrm{R}}^{2}\right)\right]+\mathcal{O}\left(\lambda_{\mathrm{R}}^{2}\right),
$$

where we note that to $\mathcal{O}\left(\lambda_{\mathrm{R}}^{2}\right)$ only the mass parameter needs to be renormalized.

To carry out renormalization in practice, we need to choose a scheme. We adopt here the so-called pole mass scheme, where $m_{\mathrm{R}}^{2}$ is taken to be the physical mass squared of the $\phi$-particle, denoted by $m_{\text {phys }}^{2}$. In Minkowskian spacetime, this quantity appears as an exponential time evolution,

$$
e^{-i \epsilon_{0} t} \equiv e^{-i m_{\text {phys }} t}
$$

in the propagator of a particle at rest, $\mathbf{p}=\mathbf{0}$. In Euclidean spacetime, it on the other hand corresponds to an exponential fall-off, $\exp \left(-m_{\mathrm{phys}} \tau\right)$, in the imaginary-time propagator. Therefore, in order to determine $m_{\text {phys }}^{2}$ to $\mathcal{O}\left(\lambda_{\mathrm{R}}\right)$, we need to compute the full propagator, $G(X)$, to $\mathcal{O}\left(\lambda_{\mathrm{R}}\right)$ at zero temperature.

The full propagator can be defined as the generalization of eq. (3.39) to the interacting case:

$$
G(X) \equiv \frac{\left\langle\phi(X) \phi(0) \exp \left(-S_{I}\right)\right\rangle_{0}}{\left\langle\exp \left(-S_{I}\right)\right\rangle_{0}}
$$

\footnotetext{
${ }^{9}$ The temperature, in contrast, is a physical property of the system, and is not subject to any modification.
} 


$$
\begin{aligned}
& =\frac{\langle\phi(X) \phi(0)\rangle_{0}-\left\langle\phi(X) \phi(0) S_{I}\right\rangle_{0}+\mathcal{O}\left(\lambda_{\mathrm{B}}^{2}\right)}{1-\left\langle S_{I}\right\rangle_{0}+\mathcal{O}\left(\lambda_{\mathrm{B}}^{2}\right)} \\
& =\langle\phi(X) \phi(0)\rangle_{0}-\left[\left\langle\phi(X) \phi(0) S_{I}\right\rangle_{0}-\langle\phi(X) \phi(0)\rangle_{0}\left\langle S_{I}\right\rangle_{0}\right]+\mathcal{O}\left(\lambda_{\mathrm{B}}^{2}\right) .
\end{aligned}
$$

We note that just like the subtractions in eq. (3.10), the second term inside the square brackets serves to cancel disconnected contractions. Therefore, like in eq. (3.12), we can drop the second term, if we replace the expectation value in the first one by $\langle\ldots\rangle_{0, c}$.

Let us now inspect the leading (zeroth order) term in eq. (3.63), in order to learn how $m_{\text {phys }}$ could most conveniently be extracted from the propagator. Introducing the notation

$$
\int_{P} \equiv \lim _{T \rightarrow 0} \mathcal{F}_{P}=\int \frac{\mathrm{d}^{d+1} P}{(2 \pi)^{d+1}},
$$

and working in the $T=0$ limit for the time being, the free propagator reads (cf. eq. (3.28))

$$
G_{0}(X)=\langle\phi(X) \phi(0)\rangle_{0}=\int_{P} \frac{e^{i P \cdot X}}{P^{2}+m^{2}} .
$$

For eq. (3.62), we need to project to zero spatial momentum, $\mathbf{p}=\mathbf{0}$; evidently this can be achieved by taking a spatial average of $G_{0}(X)$ via

$$
\int_{\mathbf{x}}\langle\phi(\tau, \mathbf{x}) \phi(0)\rangle_{0}=\int \frac{\mathrm{d} p_{0}}{2 \pi} \frac{e^{i p_{0} \tau}}{p_{0}^{2}+m^{2}}
$$

We see that we get an integral which can be evaluated with the help of the Cauchy theorem and, in particular, that the exponential fall-off of the correlation function is determined by the pole position of the momentum-space propagator:

$$
\int_{\mathbf{x}}\langle\phi(\tau, \mathbf{x}) \phi(0)\rangle_{0}=\frac{1}{2 \pi} 2 \pi i \frac{e^{-m \tau}}{2 i m}, \quad \tau \geq 0 .
$$

Hence,

$$
\left.m_{\text {phys }}^{2}\right|_{\lambda=0}=m^{2} .
$$

More generally, the physical mass can be extracted by determining the pole position of the full propagator in momentum space, for $\mathbf{p}=\mathbf{0}$.

We then proceed to the second term in eq. (3.63), keeping still $T=0$ :

$$
\begin{aligned}
-\left\langle\phi(X) \phi(0) S_{I}\right\rangle_{0, \mathrm{c}} & =-\frac{\lambda_{\mathrm{B}}}{4} \int_{Y}\langle\phi(X) \phi(0) \phi(Y) \phi(Y) \phi(Y) \phi(Y)\rangle_{0, \mathrm{c}} \\
& =-\frac{\lambda_{\mathrm{B}}}{4} \int_{Y} 4 \times 3\langle\phi(X) \phi(Y)\rangle_{0}\langle\phi(Y) \phi(0)\rangle_{0}\langle\phi(Y) \phi(Y)\rangle_{0} \\
& =-3 \lambda_{\mathrm{B}} G_{0}(0) \int_{Y} G_{0}(Y) G_{0}(X-Y) \\
& =-3 \lambda_{\mathrm{B}} \int_{P} \frac{1}{P^{2}+m_{\mathrm{B}}^{2}} \int_{Y} \int_{Q, R} e^{i Q \cdot Y} e^{i R \cdot(X-Y)} \frac{1}{Q^{2}+m_{\mathrm{B}}^{2}} \frac{1}{R^{2}+m_{\mathrm{B}}^{2}} \\
& =-3 \lambda_{\mathrm{B}} I_{0}\left(m_{\mathrm{B}}\right) \int_{R} \frac{e^{i R \cdot X}}{\left(R^{2}+m_{\mathrm{B}}^{2}\right)^{2}} .
\end{aligned}
$$

Summing this expression together with eq. (3.65), the full propagator reads

$$
\begin{aligned}
G(X) & =\int_{P} e^{i P \cdot X}\left[\frac{1}{P^{2}+m_{\mathrm{B}}^{2}}-\frac{3 \lambda_{\mathrm{B}} I_{0}\left(m_{\mathrm{B}}\right)}{\left(P^{2}+m_{\mathrm{B}}^{2}\right)^{2}}+\mathcal{O}\left(\lambda_{\mathrm{B}}^{2}\right)\right] \\
& =\int_{P} \frac{e^{i P \cdot X}}{P^{2}+m_{\mathrm{B}}^{2}+3 \lambda_{\mathrm{B}} I_{0}\left(m_{\mathrm{B}}\right)}+\mathcal{O}\left(\lambda_{\mathrm{B}}^{2}\right)
\end{aligned}
$$


where we have resummed a series of higher-order corrections in a way that is correct to the indicated order of the weak-coupling expansion.

The same steps that led us from eq. (3.66) to (3.68) now produce

$$
m_{\text {phys }}^{2}=m_{\mathrm{B}}^{2}+3 \lambda_{\mathrm{B}} I_{0}\left(m_{\mathrm{B}}\right)+\mathcal{O}\left(\lambda_{\mathrm{B}}^{2}\right) .
$$

Recalling from eq. (3.60) that $m_{\mathrm{B}}^{2}=m_{\mathrm{R}}^{2}+\mathcal{O}\left(\lambda_{\mathrm{R}}\right), \lambda_{\mathrm{B}}=\lambda_{\mathrm{R}}+\mathcal{O}\left(\lambda_{\mathrm{R}}^{2}\right)$, this relation can be inverted to give

$$
m_{\mathrm{B}}^{2}=m_{\text {phys }}^{2}-3 \lambda_{\mathrm{R}} I_{0}\left(m_{\text {phys }}\right)+\mathcal{O}\left(\lambda_{\mathrm{R}}^{2}\right),
$$

which corresponds to eq. (3.59). The function $I_{0}$, given in eq. (2.73), furthermore diverges in the limit $\epsilon \rightarrow 0$,

$$
I_{0}\left(m_{\mathrm{phys}}\right)=-\frac{m_{\mathrm{phys}}^{2} \mu^{-2 \epsilon}}{16 \pi^{2}}\left[\frac{1}{\epsilon}+\ln \frac{\bar{\mu}^{2}}{m_{\mathrm{phys}}^{2}}+1+\mathcal{O}(\epsilon)\right],
$$

and we may hope that this divergence cancels those we found in $f(T)$.

Indeed, let us repeat the steps from eq. (3.58) to eq. (3.61) employing the explicit expression for the free energy density from eq. (3.47),

$$
f(T)=J\left(m_{\mathrm{B}}, T\right)+\frac{3}{4} \lambda_{\mathrm{B}}\left[I\left(m_{\mathrm{B}}, T\right)\right]^{2}+\mathcal{O}\left(\lambda_{\mathrm{B}}^{2}\right) .
$$

Recalling from eq. (2.52) that

$$
I(m, T)=\frac{1}{m} \frac{\mathrm{d}}{\mathrm{d} m} J(m, T)=2 \frac{\mathrm{d}}{\mathrm{d} m^{2}} J(m, T),
$$

we can expand the two terms in eq. (3.74) as a Taylor series around $m_{\text {phys }}^{2}$, obtaining

$$
\begin{aligned}
J\left(m_{\mathrm{B}}, T\right) & =J\left(m_{\text {phys }}, T\right)+\left(m_{\mathrm{B}}^{2}-m_{\text {phys }}^{2}\right) \frac{\partial J\left(m_{\text {phys }}, T\right)}{\partial m_{\text {phys }}^{2}}+\mathcal{O}\left(\lambda_{\mathrm{R}}^{2}\right) \\
& =J\left(m_{\text {phys }}, T\right)-\frac{3}{2} \lambda_{\mathrm{R}} I_{0}\left(m_{\text {phys }}\right) I\left(m_{\text {phys }}, T\right)+\mathcal{O}\left(\lambda_{\mathrm{R}}^{2}\right), \\
\lambda_{\mathrm{B}}\left[I\left(m_{\mathrm{B}}, T\right)\right]^{2} & =\lambda_{\mathrm{R}}\left[I\left(m_{\text {phys }}, T\right)\right]^{2}+\mathcal{O}\left(\lambda_{\mathrm{R}}^{2}\right),
\end{aligned}
$$

where in eq. (3.76) we inserted eq. (3.72). With this input, eq. (3.74) becomes

$$
\begin{aligned}
f(T) & =J\left(m_{\text {phys }}, T\right)+\frac{3}{4} \lambda_{\mathrm{R}}\left[I^{2}\left(m_{\text {phys }}, T\right)-2 I_{0}\left(m_{\text {phys }}\right) I\left(m_{\text {phys }}, T\right)\right]+\mathcal{O}\left(\lambda_{\mathrm{R}}^{2}\right) \\
& =\underbrace{\left\{J_{0}\left(m_{\text {phys }}\right)-\frac{3}{4} \lambda_{\mathrm{R}} I_{0}^{2}\left(m_{\text {phys }}\right)\right\}}_{T=0 \text { part }}+\underbrace{\left\{J_{T}\left(m_{\text {phys }}\right)+\frac{3}{4} \lambda_{\mathrm{R}} I_{T}^{2}\left(m_{\text {phys }}\right)\right\}}_{T \neq 0 \text { part }}+\mathcal{O}\left(\lambda_{\mathrm{R}}^{2}\right),
\end{aligned}
$$

where we inserted the definitions $J(m, T)=J_{0}(m)+J_{T}(m)$ and $I(m, T)=I_{0}(m)+I_{T}(m)$.

Recalling eqs. (2.72) and (2.73), we observe that the first term in eq. (3.78), the " $T=0$ part", is still divergent. However, this term is independent of the temperature, and thus plays no role in thermodynamics. Rather, it corresponds to a vacuum energy density that only matters in connection with gravity. If we included gravity, however, we should also include a bare cosmological constant, $\Lambda_{\mathrm{B}}$, in the bare Lagrangian; this would contribute additively to eq. (3.78), and we could simply identify the physical cosmological constant as

$$
\Lambda_{\text {phys }} \equiv \Lambda_{\mathrm{B}}+J_{0}\left(m_{\text {phys }}\right)-\frac{3}{4} \lambda_{\mathrm{R}} I_{0}^{2}\left(m_{\text {phys }}\right)+\mathcal{O}\left(\lambda_{\mathrm{R}}^{2}\right) .
$$


The divergences would now be cancelled by $\Lambda_{\mathrm{B}}$, and $\Lambda_{\text {phys }}$ would be finite.

In contrast, the second term in eq. (3.78), the " $T \neq 0$ part", is finite: it contains the functions $J_{T}, I_{T}$ for which we have analytically determined various limiting values in eqs. (2.79), (2.80), (2.82) and (2.93), as well as general integral representations in eqs. (2.76) and (2.77). Therefore all thermodynamic quantities obtained from derivatives of $f(T)$, such as the entropy density or specific heat, are manifestly finite. In other words, the temperature-dependent ultraviolet divergences that we found in sec. 3.2 have disappeared through zero-temperature renormalization. 


\subsection{Proper free energy density to $\mathcal{O}\left(\lambda^{\frac{3}{2}}\right)$ : infrared resummation}

We now move on to a topic which is in a sense maximally different from the UV issues discussed in the previous section, and consider the limit where the physical mass of the scalar field, $m_{\text {phys }}$, tends to zero. With a few technical modifications, this would be the case (in perturbation theory) for, say, gluons in QCD. According to eq. (3.72), this limit corresponds to $m_{\mathrm{B}} \rightarrow 0$, since $I_{0}(0)=0$; then we are faced with the infrared problem discussed in sec. 3.2.

In the limit of a small mass, we can employ high-temperature expansions for the functions $J(m, T)$ and $I(m, T)$, given in eqs. (2.92) and (2.95). Employing eqs. (3.47) and (3.57), we write the leading terms in the small- $m_{\mathrm{B}}$ expansion as

$$
\begin{aligned}
& \mathcal{O}\left(\lambda_{\mathrm{B}}^{0}\right): \quad f_{(0)}(T)=J\left(m_{\mathrm{B}}, T\right)=-\frac{\pi^{2} T^{4}}{90}+\frac{m_{\mathrm{B}}^{2} T^{2}}{24}-\frac{m_{\mathrm{B}}^{3} T}{12 \pi}+\mathcal{O}\left(m_{\mathrm{B}}^{4}\right), \\
& \mathcal{O}\left(\lambda_{\mathrm{B}}^{1}\right): \quad f_{(1)}(T)=\frac{3}{4} \lambda_{\mathrm{B}}\left[I\left(m_{\mathrm{B}}, T\right)\right]^{2} \\
& =\frac{3}{4} \lambda_{\mathrm{B}}\left[\frac{T^{2}}{12}-\frac{m_{\mathrm{B}} T}{4 \pi}+\mathcal{O}\left(m_{\mathrm{B}}^{2}\right)\right]^{2} \\
& =\frac{3}{4} \lambda_{\mathrm{B}}\left[\frac{T^{4}}{144}-\frac{m_{\mathrm{B}} T^{3}}{24 \pi}+\mathcal{O}\left(m_{\mathrm{B}}^{2} T^{2}\right)\right] \text {, } \\
& \mathcal{O}\left(\lambda_{\mathrm{B}}^{2}\right): \quad f_{(2)}(T)=-\frac{9}{4} \lambda_{\mathrm{B}}^{2} \frac{T^{4}}{144} \frac{T}{8 \pi m_{\mathrm{B}}}+\mathcal{O}\left(m_{\mathrm{B}}^{0}\right) .
\end{aligned}
$$

Let us inspect, in particular, odd powers of $m_{\mathrm{B}}$, which according to eqs. (3.80)-(3.82) are becoming increasingly important as we go further in the expansion. We remember from sec. 2.3 that odd powers of $m_{\mathrm{B}}$ are necessarily associated with contributions from the Matsubara zero mode. In fact, the odd power in eq. (3.80) is directly the zero-mode contribution to eq. (2.87),

$$
\delta_{\text {odd }} f_{(0)}=J^{(n=0)}=-\frac{m_{\mathrm{B}}^{3} T}{12 \pi} .
$$

The odd power in eq. (3.81) on the other hand originates from a cross-term between the zero-mode contribution and the leading non-zero mode contribution to $I(0, T)$ :

$$
\delta_{\text {odd }} f_{(1)}=\frac{3}{2} \lambda_{\mathrm{B}} \times I^{\prime}(0, T) \times I^{(n=0)}=-\frac{\lambda_{\mathrm{B}} m_{\mathrm{B}} T^{3}}{32 \pi} .
$$

Finally, the small- $m_{\mathrm{B}}$ divergence in eq. (3.82) comes from a product of two non-zero mode contributions and a particularly infrared sensitive zero-mode contribution:

$$
\delta_{\text {odd }} f_{(2)}=\frac{9}{4} \lambda_{\mathrm{B}}^{2} \times\left[I^{\prime}(0, T)\right]^{2} \times \frac{\mathrm{d} I^{(n=0)}}{\mathrm{d} m_{\mathrm{B}}^{2}}=-\frac{\lambda_{\mathrm{B}}^{2} T^{5}}{8^{3} \pi m_{\mathrm{B}}} .
$$

Comparing these structures, we see that the "expansion parameter" related to odd powers is

$$
\frac{\delta_{\text {odd }} f_{(1)}}{\delta_{\text {odd }} f_{(0)}} \sim \frac{\delta_{\text {odd }} f_{(2)}}{\delta_{\text {odd }} f_{(1)}} \sim \frac{\lambda_{\mathrm{B}} T^{2}}{8 m_{\mathrm{B}}^{2}} .
$$

Thus, if we try to set $m_{\mathrm{B}}^{2} \rightarrow 0$ (or even just $m_{\mathrm{B}}^{2} \ll \lambda_{\mathrm{B}} T^{2} / 8$ ), the loop expansion shows no convergence.

In order to cure the problem with the infrared (IR) sensitivity of the loop expansion, our goal now becomes to identify and sum the divergent terms to all orders. We may then expect that 
the complete sum obtains a form where we can set $m_{\mathrm{B}}^{2} \rightarrow 0$ without meeting divergences. This procedure is often referred to as resummation.

Fortunately, it is indeed possible to identify the problematic terms. Eqs. (3.83)-(3.85) already suggest that at order $N$ in $\lambda_{\mathrm{B}}$, they are associated with terms containing $N$ non-zero mode contributions $I^{\prime}(0, T)$, and one zero-mode contribution. Graphically, this corresponds to a single loop formed by a zero-mode propagator, dressed with $N$ non-zero mode "bubbles". Such graphs are usually called "ring" or "daisy" diagrams, and can be illustrated as follows (the dashed line is a zero-mode propagator, solid lines are non-zero mode propagators):

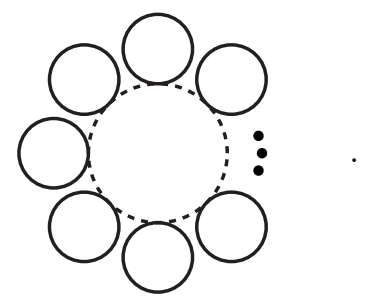

To be more quantitative, we consider eq. (3.12) at order $\lambda_{\mathrm{B}}^{N}$. A straightforward combinatorial analysis then gives

$$
\begin{aligned}
& f(T)=\left\langle S_{I}-\frac{1}{2} S_{I}^{2}+\ldots+\frac{(-1)^{N+1}}{N !} S_{I}^{N}\right\rangle_{0, \mathrm{c}, \text { drop overall } \int_{X}}
\end{aligned}
$$

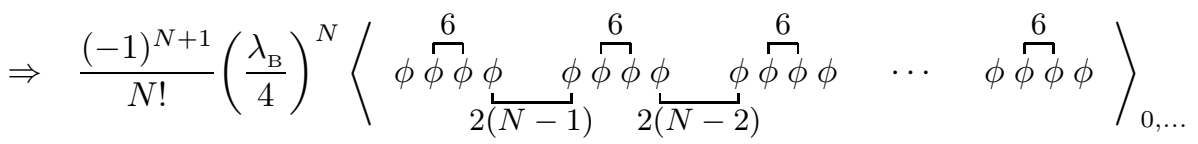

$$
\begin{aligned}
& =\frac{(-1)^{N+1}}{N !}\left(\frac{\lambda_{\mathrm{B}}}{4}\right)^{N} 6^{N} \underbrace{[2(N-1)][2(N-2)] \ldots[2]}[\underbrace{\frac{T^{2}}{12}}]^{N} \underbrace{T \int \frac{\mathrm{d}^{d} \mathbf{p}}{(2 \pi)^{d}}\left(\frac{1}{p^{2}+m_{\mathrm{B}}^{2}}\right)^{N}}, \\
& 2^{N-1}(N-1) ! \quad I^{\prime}(0, T) \quad \text { zero-mode part }
\end{aligned}
$$

where we have indicated the contractions from which the various factors originate. Let us compute the zero-mode part for the first few orders, omitting for simplicity terms of $\mathcal{O}(\epsilon)$ :

$$
\begin{array}{cc}
N=1: & \int_{\mathbf{p}} \frac{1}{p^{2}+m_{\mathrm{B}}^{2}}=-\frac{m_{\mathrm{B}}}{4 \pi}=\frac{\mathrm{d}}{\mathrm{d} m_{\mathrm{B}}^{2}}\left(-\frac{m_{\mathrm{B}}^{3}}{6 \pi}\right), \\
N=2: & \int_{\mathbf{p}} \frac{1}{\left(p^{2}+m_{\mathrm{B}}^{2}\right)^{2}}=-\frac{\mathrm{d}}{\mathrm{d} m_{\mathrm{B}}^{2}}\left(-\frac{m_{\mathrm{B}}}{4 \pi}\right)=-\frac{\mathrm{d}}{\mathrm{d} m_{\mathrm{B}}^{2}} \frac{\mathrm{d}}{\mathrm{d} m_{\mathrm{B}}^{2}}\left(-\frac{m_{\mathrm{B}}^{3}}{6 \pi}\right), \\
\text { generally : } & \int_{\mathbf{p}} \frac{1}{\left(p^{2}+m_{\mathrm{B}}^{2}\right)^{N}}=-\frac{1}{N-1} \frac{\mathrm{d}}{\mathrm{d} m_{\mathrm{B}}^{2}} \int_{\mathbf{p}} \frac{1}{\left(p^{2}+m_{\mathrm{B}}^{2}\right)^{N-1}} \\
=\left(\frac{-1}{N-1}\right)\left(\frac{-1}{N-2}\right) \cdots\left(\frac{-1}{1}\right)\left(\frac{\mathrm{d}}{\mathrm{d} m_{\mathrm{B}}^{2}}\right)^{N-1} \int_{\mathbf{p}} \frac{1}{p^{2}+m_{\mathrm{B}}^{2}} \\
=\frac{(-1)^{N}}{(N-1) !}\left(\frac{\mathrm{d}}{\mathrm{d} m_{\mathrm{B}}^{2}}\right)^{N}\left(\frac{m_{\mathrm{B}}^{3}}{6 \pi}\right) .
\end{array}
$$

Combining eqs. (3.88) and (3.89), we get

$$
\begin{aligned}
\delta_{\text {odd }} f_{(N)} & =\frac{(-1)^{N+1}}{N !}\left(\frac{3 \lambda_{\mathrm{B}}}{2}\right)^{N} 2^{N-1}(N-1) !\left(\frac{T^{2}}{12}\right)^{N} T \frac{(-1)^{N}}{(N-1) !}\left(\frac{\mathrm{d}}{\mathrm{d} m_{\mathrm{B}}^{2}}\right)^{N}\left(\frac{m_{\mathrm{B}}^{3}}{6 \pi}\right) \\
& =-\frac{T}{2} \frac{1}{N !}\left(\frac{\lambda_{\mathrm{B}} T^{2}}{4}\right)^{N}\left(\frac{\mathrm{d}}{\mathrm{d} m_{\mathrm{B}}^{2}}\right)^{N}\left(\frac{m_{\mathrm{B}}^{3}}{6 \pi}\right) .
\end{aligned}
$$


As a crosscheck, it can be verified that this expression reproduces eqs. (3.83)-(3.85).

Now, owing to the fact that eq. (3.90) has precisely the right structure to correspond to a Taylor expansion, we can sum the contributions in eq. (3.90) to all orders, obtaining

$$
\sum_{N=0}^{\infty} \frac{1}{N !}\left(\frac{\lambda_{\mathrm{B}} T^{2}}{4}\right)^{N}\left(\frac{\mathrm{d}}{\mathrm{d} m_{\mathrm{B}}^{2}}\right)^{N}\left(-\frac{m_{\mathrm{B}}^{3} T}{12 \pi}\right)=-\frac{T}{12 \pi}\left(m_{\mathrm{B}}^{2}+\frac{\lambda_{\mathrm{B}} T^{2}}{4}\right)^{\frac{3}{2}} .
$$

We observe that a "miracle" has happened: in eq. (3.91) the limit $m_{\mathrm{B}}^{2} \rightarrow 0$ can be taken without divergences. But there is a surprise: setting the mass parameter to zero, we arrive at a contribution

of $\mathcal{O}\left(\lambda_{\mathrm{B}}^{3 / 2}\right)$, rather than $\mathcal{O}\left(\lambda_{\mathrm{B}}^{2}\right)$ as naively expected in sec. 3.2. In other words, infrared divergences modify qualitatively the structure of the weak-coupling expansion.

Setting finally $m_{\mathrm{B}}^{2} \rightarrow 0$ everywhere, and collecting all finite terms from eqs. (3.80), (3.81) and (3.91), we find the correct expansion of $f(T)$ in the massless limit,

$$
\begin{aligned}
f(T) & =-\frac{\pi^{2} T^{4}}{90}+\frac{\lambda_{\mathrm{B}} T^{4}}{4 \times 48}-\frac{T}{12 \pi}\left(\frac{\lambda_{\mathrm{B}} T^{2}}{4}\right)^{3 / 2}+\mathcal{O}\left(\lambda_{\mathrm{B}}^{2} T^{4}\right) \\
& =-\frac{\pi^{2} T^{4}}{90}\left[1-\frac{15}{32} \frac{\lambda_{\mathrm{R}}}{\pi^{2}}+\frac{15}{16}\left(\frac{\lambda_{\mathrm{R}}}{\pi^{2}}\right)^{\frac{3}{2}}+\mathcal{O}\left(\lambda_{\mathrm{R}}^{2}\right)\right]
\end{aligned}
$$

where at the last stage we inserted $\lambda_{\mathrm{B}}=\lambda_{\mathrm{R}}+\mathcal{O}\left(\lambda_{\mathrm{R}}^{2}\right)$.

It is appropriate to add that despite the complications we have found, higher-order corrections can be computed to eq. (3.93). In fact, as of today, the coefficients of the seven subsequent terms, of orders $\mathcal{O}\left(\lambda_{\mathrm{R}}^{2}\right), \mathcal{O}\left(\lambda_{\mathrm{R}}^{5 / 2} \ln \lambda_{\mathrm{R}}\right), \mathcal{O}\left(\lambda_{\mathrm{R}}^{5 / 2}\right), \mathcal{O}\left(\lambda_{\mathrm{R}}^{3} \ln \lambda_{\mathrm{R}}\right), \mathcal{O}\left(\lambda_{\mathrm{R}}^{3}\right), \mathcal{O}\left(\lambda_{\mathrm{R}}^{7 / 2}\right)$, and $\mathcal{O}\left(\lambda_{\mathrm{R}}^{8} \ln \lambda_{\mathrm{R}}\right)$, are known $[3.1,3.2]$. This progress is possible due to the fact that the resummation of higher-order contributions that we carried out explicitly in this section can be implemented more elegantly and systematically with so-called effective field theory methods. We return to this general procedure in sec. 6 , but some flavour can be obtained by organizing the above computation in yet another way, outlined in the appendix below.

\section{Appendix A: An alternative method for resummation}

In this appendix we show that the previous resummation can also be implemented through the following steps:

(i) Following the computation of $m_{\text {phys }}^{2}$ in eq. (3.71) but working now at finite temperature, we determine a specific $T$-dependent pole mass in the $m_{\mathrm{B}} \rightarrow 0$ limit. The result can be called an effective thermal mass, $m_{\text {eff }}^{2}$.

(ii) We argue that in the weak-coupling limit $\left(\lambda_{\mathrm{R}} \ll 1\right)$, the thermal mass is important only for the Matsubara zero mode [3.3].

(iii) Writing the Lagrangian (for $m_{\mathrm{B}}^{2}=0$ ) in the form

$$
L_{E}=\underbrace{\frac{1}{2} \partial_{\mu} \phi \partial_{\mu} \phi+\frac{1}{2} m_{\mathrm{eff}}^{2} \phi_{n=0}^{2}}_{L_{0}}+\underbrace{\frac{1}{4} \lambda_{\mathrm{B}} \phi^{4}-\frac{1}{2} m_{\mathrm{eff}}^{2} \phi_{n=0}^{2}}_{L_{I}},
$$


we treat $L_{0}$ as the free theory and $L_{I}$ as an interaction of order $\lambda_{\mathrm{R}}$. With this reorganization of the theory, we write down the contributions $f_{(0)}$ and $f_{(1)}$ to the free energy density, and check that we obtain a well-behaved perturbative expansion that reproduces eq. (3.93).

Starting with the effective mass parameter, the computation proceeds precisely like the one 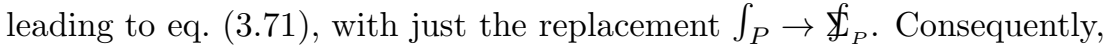

$$
m_{\text {eff }}^{2}=\lim _{m_{\mathrm{B}}^{2} \rightarrow 0}\left[m_{\mathrm{B}}^{2}+3 \lambda_{\mathrm{B}} I\left(m_{\mathrm{B}}, T\right)\right]=3 \lambda_{\mathrm{B}} I(0, T)=\frac{\lambda_{\mathrm{R}} T^{2}}{4}+\mathcal{O}\left(\lambda_{\mathrm{R}}^{2}\right) .
$$

We note that for the non-zero Matsubara modes, with $\omega_{n} \neq 0$, we have $m_{\text {eff }}^{2} \ll \omega_{n}^{2}$ in the weakcoupling limit $\lambda_{\mathrm{R}} \ll(4 \pi)^{2}$, so that the thermal mass plays a subdominant role in the propagator. In contrast, for the Matsubara zero mode, $m_{\text {eff }}^{2}$ modifies the propagator significantly for $p^{2} \ll m_{\text {eff }}^{2}$, removing any infrared divergences. This observation justifies the fact that the thermal mass was only introduced for the $n=0$ mode in eq. (3.94).

With our new reorganization, the free propagators become different for the Matsubara zero $\left(\tilde{\phi}_{n=0}\right)$ and non-zero $\left(\tilde{\phi}^{\prime}\right)$ modes:

$$
\begin{aligned}
\left\langle\tilde{\phi}^{\prime}(P) \tilde{\phi}^{\prime}(Q)\right\rangle_{0} & =\delta(P+Q) \frac{1}{\omega_{n}^{2}+p^{2}}, \\
\left\langle\tilde{\phi}_{n=0}(P) \tilde{\phi}_{n=0}(Q)\right\rangle_{0} & =\delta(P+Q) \frac{1}{p^{2}+m_{\mathrm{eff}}^{2}} .
\end{aligned}
$$

Consequently, eq. (3.17) gets replaced with

$$
\begin{aligned}
f_{(0)}(T) & =\oint_{P}^{\prime} \frac{1}{2} \ln \left(P^{2}\right)+T \int_{\mathbf{p}} \frac{1}{2} \ln \left(p^{2}+m_{\mathrm{eff}}^{2}\right)-\text { const. } \\
& =J^{\prime}(0, T)+J^{(n=0)}\left(m_{\mathrm{eff}}, T\right) \\
& =-\frac{\pi^{2} T^{4}}{90}-\frac{m_{\mathrm{eff}}^{3} T}{12 \pi} .
\end{aligned}
$$

In the massless first term, the omission of the zero mode made no difference.

With $f_{(1)}$ now coming from $L_{I}$ in eq. (3.94), eq. (3.19) is modified into

$$
\begin{aligned}
f_{(1)}(T) & =\frac{3}{4} \lambda_{\mathrm{B}}\langle\phi(0) \phi(0)\rangle_{0}\langle\phi(0) \phi(0)\rangle_{0}-\frac{1}{2} m_{\mathrm{eff}}^{2}\left\langle\phi_{n=0}(0) \phi_{n=0}(0)\right\rangle_{0} \\
& =\frac{3}{4} \lambda_{\mathrm{B}}\left[I^{\prime}(0, T)+I^{(n=0)}\left(m_{\mathrm{eff}}, T\right)\right]^{2}-\frac{1}{2} m_{\mathrm{eff}}^{2} I^{(n=0)}\left(m_{\mathrm{eff}}, T\right) \\
& =\frac{3}{4} \lambda_{\mathrm{B}}\left[\frac{T^{4}}{144}-\frac{m_{\mathrm{eff}} T^{3}}{24 \pi}+\frac{m_{\mathrm{eff}}^{2} T^{2}}{16 \pi^{2}}\right]+\frac{1}{2} m_{\mathrm{eff}}^{2} \frac{m_{\mathrm{eff}} T}{4 \pi} .
\end{aligned}
$$

Inserting eq. (3.95) into the last term of eq. (3.99), we see that this contribution precisely cancels against the linear term within the square brackets. As we recall from eq. (3.84), the linear term was part of the problematic series that needed to be resummed. Combining eqs. (3.98) and (3.99), we instead get

$$
f(T)=-\frac{\pi^{2} T^{4}}{90}+\frac{3 \lambda_{\mathrm{R}}}{4} \frac{T^{4}}{144}-\frac{m_{\mathrm{eff}}^{3} T}{12 \pi}+\mathcal{O}\left(\lambda_{\mathrm{R}}^{2}\right),
$$

which agrees with eq. (3.93).

The cancellation that took place in eq. (3.99) can also be verified at higher orders. In particular, proceeding to $\mathcal{O}\left(\lambda_{\mathrm{R}}^{2}\right)$, it can be seen that the structure in eq. (3.85) gets cancelled as well. Indeed, the resummation of infrared divergences that we carried out explicitly in eq. (3.91) can be fully captured by the reorganization in eq. (3.94). 


\section{Literature}

[3.1] A. Gynther, M. Laine, Y. Schröder, C. Torrero and A. Vuorinen, Four-loop pressure of massless $O(N)$ scalar field theory, JHEP 04 (2007) 094 [hep-ph/0703307].

[3.2] J.O. Andersen, L. Kyllingstad and L.E. Leganger, Pressure to order $g^{8} \log g$ of massless $\phi^{4}$ theory at weak coupling, JHEP 08 (2009) 066 [0903.4596].

[3.3] P.B. Arnold and O. Espinosa, Effective potential and first-order phase transitions: Beyond leading order, Phys. Rev. D 47 (1993) 3546; ibid. 50 (1994) 6662 (E) [hep-ph/9212235]. 


\section{Fermions}

Abstract: A fermionic (spin-1/2) field is considered at finite temperature. Starting with a fermionic analogue of the harmonic oscillator and proceeding to the case of a field satisfying a Dirac equation, an imaginary-time path integral representation is derived for the partition function. This leads to the concept of Grassmann variables satisfying antiperiodic boundary conditions. The corresponding Matsubara frequencies are introduced, the partition function is evaluated in the low and high-temperature expansions, and the structures of these expansions are compared with those of a scalar field theory.

Keywords: Fermionic oscillator, Grassmann variables, antiperiodic boundary conditions, Dirac field, Dirac matrices, low and high-temperature expansions for fermions.

\subsection{Path integral for the partition function of a fermionic oscillator}

Just like in the bosonic case, the structure of the path integral for the partition function of a fermionic field [4.1] can be derived most easily by first considering a non-interacting field living in a zero-dimensional space $(d=0)$. We refer to this system as a fermionic oscillator.

In order to introduce the fermionic oscillator, let us start by recapitulating the main formulae of the bosonic case. In the operator description, the commutation relations, the Hamiltonian, the energy eigenstates, as well as the completeness relations can be expressed as

$$
\begin{aligned}
& {[\hat{a}, \hat{a}]=0, \quad\left[\hat{a}^{\dagger}, \hat{a}^{\dagger}\right]=0, \quad\left[\hat{a}, \hat{a}^{\dagger}\right]=1 ;} \\
& \hat{H}=\hbar \omega\left(\hat{a}^{\dagger} \hat{a}+\frac{1}{2}\right)=\frac{\hbar \omega}{2}\left(\hat{a}^{\dagger} \hat{a}+\hat{a} \hat{a}^{\dagger}\right) ; \\
& \hat{a}^{\dagger}|n\rangle=\sqrt{n+1}|n+1\rangle, \quad \hat{a}|n\rangle=\sqrt{n}|n-1\rangle, \quad n=0,1,2, \ldots ; \\
& \mathbb{1}=\sum_{n}|n\rangle\left\langle n\left|=\int \mathrm{d} x\right| x\right\rangle\left\langle x\left|=\int \frac{\mathrm{d} p}{2 \pi \hbar}\right| p\right\rangle\langle p|,
\end{aligned}
$$

where we have momentarily reinstated $\hbar$. The observable we are interested in is $\mathcal{Z}=\operatorname{Tr}[\exp (-\beta \hat{H})]$, and the various path integral representations we obtained for it read (cf. eqs. (1.33), (1.37), (1.40))

$$
\begin{aligned}
\mathcal{Z} & =\int_{x(\beta \hbar)=x(0)} \mathcal{D} x \mathcal{D}\left(\frac{p}{2 \pi \hbar}\right) \exp \left\{-\frac{1}{\hbar} \int_{0}^{\beta \hbar} \mathrm{d} \tau\left[\frac{p^{2}(\tau)}{2 m}-i p(\tau) \dot{x}(\tau)+V(x(\tau))\right]\right\} \\
& =C \int_{x(\beta \hbar)=x(0)} \mathcal{D} x \exp \left\{-\frac{1}{\hbar} \int_{0}^{\beta \hbar} \mathrm{d} \tau\left[\frac{m}{2}\left(\frac{\mathrm{d} x(\tau)}{\mathrm{d} \tau}\right)^{2}+V(x(\tau))\right]\right\} \\
& =C \int_{x(\beta \hbar)=x(0)} \mathcal{D} x \exp \left(-\frac{1}{\hbar} \int_{0}^{\beta \hbar} \mathrm{d} \tau L_{E}\right), \quad L_{E}=-\mathcal{L}_{M}(t \rightarrow-i \tau),
\end{aligned}
$$

where $C$ is a constant, independent of the potential $V(x)=\frac{1}{2} m \omega^{2} x^{2}$.

In the fermionic case, we replace the algebra of eq. (4.1) by

$$
\{\hat{a}, \hat{a}\}=0, \quad\left\{\hat{a}^{\dagger}, \hat{a}^{\dagger}\right\}=0, \quad\left\{\hat{a}, \hat{a}^{\dagger}\right\}=1 .
$$

Considering the Hilbert space, i.e. the analogue of eq. (4.3), we define the vacuum state $|0\rangle$ by

$$
\hat{a}|0\rangle \equiv 0
$$


and subsequently the one-particle state $|1\rangle$ by

$$
|1\rangle \equiv \hat{a}^{\dagger}|0\rangle
$$

It is easy to see that the Hilbert space contains no other states: operating on $|1\rangle$ with $\hat{a}$ or $\hat{a}^{\dagger}$ gives either the already known state $|0\rangle$, or nothing:

$$
\begin{aligned}
& \hat{a}|1\rangle=\hat{a} \hat{a}^{\dagger}|0\rangle=\left[1-\hat{a}^{\dagger} \hat{a}\right]|0\rangle=|0\rangle, \\
& \hat{a}^{\dagger}|1\rangle=\hat{a}^{\dagger} \hat{a}^{\dagger}|0\rangle=0 .
\end{aligned}
$$

Here 0 stands for a null vector; $|0\rangle$ is a non-trivial vector representing the vacuum state.

The Hamiltonian, i.e. the analogue of eq. (4.2), is an operator acting in this vector space, and can be defined through

$$
\hat{H} \equiv \frac{\hbar \omega}{2}\left(\hat{a}^{\dagger} \hat{a}-\hat{a} \hat{a}^{\dagger}\right)=\hbar \omega\left(\hat{a}^{\dagger} \hat{a}-\frac{1}{2}\right)
$$

The observable of our interest is the partition function,

$$
\mathcal{Z}=\operatorname{Tr}\left[e^{-\beta \hat{H}}\right]=\left\langle 0\left|e^{-\beta \hat{H}}\right| 0\right\rangle+\left\langle 1\left|e^{-\beta \hat{H}}\right| 1\right\rangle
$$

which this time can be evaluated almost trivially due to the simplicity of the Hilbert space:

$$
\begin{aligned}
\mathcal{Z} & =[\langle 0 \mid 0\rangle+\sum_{n=0}^{\infty} \frac{(-\beta \hbar \omega)^{n}}{n !} \underbrace{\left\langle 1\left|\left(\hat{a}^{\dagger} \hat{a}\right)^{n}\right| 1\right\rangle}_{1}] e^{\frac{\beta \hbar \omega}{2}} \\
& =\left[1+e^{-\beta \hbar \omega}\right] e^{\frac{\beta \hbar \omega}{2}}=2 \cosh \left(\frac{\hbar \omega}{2 T}\right) .
\end{aligned}
$$

This can be compared with eq. (1.17). The corresponding free energy reads

$$
F=-T \ln \mathcal{Z}=-T \ln \left(e^{\frac{\hbar \omega}{2 T}}+e^{-\frac{\hbar \omega}{2 T}}\right)=-\frac{\hbar \omega}{2}-T \ln \left(1+e^{-\beta \hbar \omega}\right)
$$

which can be compared with eq. (1.18). However, like in the bosonic case, it is ultimately more useful to write a path integral representation for the partition function, and this is indeed our goal.

An essential ingredient in the derivation of the bosonic path integral was a repeated use of the completeness relations of eq. (4.4) (cf. sec. 1.1). We now need to find some analogues of these relations for the fermionic system. This can be achieved with the help of Grassmann variables. In short, the answer is that whereas in the bosonic case the system of eqs. (4.1) leads to commuting classical fields, $x(\tau), p(\tau)$, in the fermionic case the system of eqs. (4.8) leads to anti-commuting Grassmann fields, $c(\tau), c^{*}(\tau)$. Furthermore, whereas $x(\tau)$ is periodic, and there is no constraint on $p(\tau)$, the fields $c(\tau), c^{*}(\tau)$ are both anti-periodic over the compact $\tau$-interval.

We now define the Grassmann variables $c, c^{*}$ (more generally, the Grassmann fields $c(\tau), c^{*}(\tau)$ ) through the following axioms:

- $c, c^{*}$ are treated as independent variables, like $x, p$.

- $c^{2}=\left(c^{*}\right)^{2} \equiv 0, c c^{*}=-c^{*} c$.

- Integration proceeds through $\int \mathrm{d} c=\int \mathrm{d} c^{*} \equiv 0, \int \mathrm{d} c c=\int \mathrm{d} c^{*} c^{*} \equiv 1$. 
- The integration is also Grassmann-like, in the sense that $\{c, \mathrm{~d} c\}=\left\{c, \mathrm{~d} c^{*}\right\}=\left\{c^{*}, \mathrm{~d} c\right\}=$ $\left\{c^{*}, \mathrm{~d} c^{*}\right\}=0$, and similarly $\{\mathrm{d} c, \mathrm{~d} c\}=\left\{\mathrm{d} c, \mathrm{~d} c^{*}\right\}=\left\{\mathrm{d} c^{*}, \mathrm{~d} c^{*}\right\}=0$.

- By convention, we write the integration measure in the order $\int \mathrm{d} c^{*} \mathrm{~d} c$.

- A field $c(\tau)$ is a collection of independent Grassmann variables, one at each point $\tau \in(0, \beta \hbar)$.

- $c, c^{*}$ are defined to anticommute with $\hat{a}, \hat{a}^{\dagger}$ as well, so that products like $c \hat{a}^{\dagger}$ act as regular bosonic operators, e.g. $\left[c \hat{a}^{\dagger}, c^{*}\right]=0$.

We now define a ket-state, $|c\rangle$, and a bra-state, $\langle c|$, which are eigenstates of $\hat{a}$ ("from the left") and $\hat{a}^{\dagger}$ ("from the right"), respectively,

$$
\begin{array}{ll}
|c\rangle \equiv e^{-c \hat{a}^{\dagger}}|0\rangle=\left(1-c \hat{a}^{\dagger}\right)|0\rangle ; \quad \hat{a}|c\rangle=c|0\rangle=c|c\rangle, \\
\langle c| \equiv\langle 0| e^{-\hat{a} c^{*}}=\langle 0|\left(1-\hat{a} c^{*}\right) ; \quad\langle c| \hat{a}^{\dagger}=\langle 0| c^{*}=\langle c| c^{*},
\end{array}
$$

where we used $\langle 0| \hat{a}^{\dagger}=0$, corresponding to $\hat{a}|0\rangle=0$. Such states possess the transition amplitude

$$
\left\langle c^{\prime} \mid c\right\rangle=\left\langle 0\left|\left(1-\hat{a} c^{\prime *}\right)\left(1-c \hat{a}^{\dagger}\right)\right| 0\right\rangle=1+\left\langle 0\left|\hat{a} c^{\prime *} c \hat{a}^{\dagger}\right| 0\right\rangle=1+c^{\prime *} c=e^{c^{\prime *} c} .
$$

With these states, we can define the objects needed,

$$
\begin{aligned}
\int \mathrm{d} c^{*} \mathrm{~d} c e^{-c^{*} c}|c\rangle\langle c| & =\int \mathrm{d} c^{*} \mathrm{~d} c\left(1-c^{*} c\right)\left(1-c \hat{a}^{\dagger}\right)|0\rangle\langle 0|\left(1-\hat{a} c^{*}\right) \\
& =|0\rangle\left\langle 0\left|+\int \mathrm{d} c^{*} \mathrm{~d} c c \hat{a}^{\dagger}\right| 0\right\rangle\langle 0| \hat{a} c^{*} \\
& =|0\rangle\langle 0|+| 1\rangle\langle 1|=\mathbb{1}, \\
\int \mathrm{d} c^{*} \mathrm{~d} c e^{-c^{*} c}\langle-c|\hat{A}| c\rangle & =\int \mathrm{d} c^{*} \mathrm{~d} c\left(1-c^{*} c\right)\left\langle 0\left|\left(1+\hat{a} c^{*}\right) \hat{A}\left(1-c \hat{a}^{\dagger}\right)\right| 0\right\rangle \\
& =\langle 0|\hat{A}| 0\rangle-\int \mathrm{d} c^{*} \mathrm{~d} c\left\langle 0\left|\hat{a} c^{*} \hat{A} c \hat{a}^{\dagger}\right| 0\right\rangle \\
& =\langle 0|\hat{A}| 0\rangle-\left\langle 1\left|\int \mathrm{d} c^{*} \mathrm{~d} c c^{*} c \hat{A}\right| 1\right\rangle \\
& =\langle 0|\hat{A}| 0\rangle+\langle 1|\hat{A}| 1\rangle=\operatorname{Tr}[\hat{A}],
\end{aligned}
$$

where we assumed $\hat{A}$ to be a "bosonic" operator, for instance the Hamiltonian. The minus sign in the bra-state on the left-hand side of eq. (4.21) originates essentially from interchanging the order of the state vectors on the left-hand side of eq. (4.20).

Representing now the trace in eq. (4.14) as in eq. (4.21), and splitting the exponential into a product of $N$ small terms like in eq. (1.27), we can write

$$
\mathcal{Z}=\int \mathrm{d} c^{*} \mathrm{~d} c e^{-c^{*} c}\left\langle-c\left|e^{-\frac{\epsilon \hat{H}}{\hbar}} \cdots e^{-\frac{\epsilon \hat{H}}{\hbar}}\right| c\right\rangle, \quad \epsilon \equiv \frac{\beta \hbar}{N} .
$$

Then we insert eq. (4.20) in between the exponentials, as $\mathbb{1}=\int \mathrm{d} c_{i}^{*} \mathrm{~d} c_{i} e^{-c_{i}^{*} c_{i}}\left|c_{i}\right\rangle\left\langle c_{i}\right|$, whereby we are faced with objects like

$$
\begin{array}{rlrl}
e^{-c_{i+1}^{*} c_{i+1}}\left\langle c_{i+1}\left|e^{-\frac{\epsilon}{\hbar} \hat{H}\left(\hat{a}^{\dagger}, \hat{a}\right)}\right| c_{i}\right\rangle & \stackrel{(4.17),}{=}(4.18) & \exp \left(-c_{i+1}^{*} c_{i+1}\right)\left\langle c_{i+1} \mid c_{i}\right\rangle \exp \left[-\frac{\epsilon}{\hbar} H\left(c_{i+1}^{*}, c_{i}\right)\right] \\
& \stackrel{(4.19)}{=} & \exp \left[-c_{i+1}^{*} c_{i+1}+c_{i+1}^{*} c_{i}-\frac{\epsilon}{\hbar} H\left(c_{i+1}^{*}, c_{i}\right)\right] \\
= & \exp \left\{-\frac{\epsilon}{\hbar}\left[\hbar c_{i+1}^{*} \frac{c_{i+1}-c_{i}}{\epsilon}+H\left(c_{i+1}^{*}, c_{i}\right)\right]\right\} .
\end{array}
$$


Finally, attention needs to be paid to the right-most and left-most exponentials in eq. (4.22). We may define $c_{1} \equiv c$, which clarifies the fate of the right-most exponential, but the left-most one needs to be inspected in detail:

$$
\begin{aligned}
& \int \mathrm{d} c_{1}^{*} \mathrm{~d} c_{1} e^{-c_{1}^{*} c_{1}}\left\langle-c_{1}\left|e^{-\frac{\epsilon}{\hbar} \hat{H}\left(\hat{a}^{\dagger}, \hat{a}\right)}\right| \int \mathrm{d} c_{N}^{*} \mathrm{~d} c_{N} \mid c_{N}\right\rangle \\
= & \int \mathrm{d} c_{1}^{*} \mathrm{~d} c_{1} \int \mathrm{d} c_{N}^{*} \mathrm{~d} c_{N} \exp \left[-c_{1}^{*} c_{1}-c_{1}^{*} c_{N}-\frac{\epsilon}{\hbar} H\left(-c_{1}^{*}, c_{N}\right)\right] \\
= & \int \mathrm{d} c_{1}^{*} \mathrm{~d} c_{1} \int \mathrm{d} c_{N}^{*} \mathrm{~d} c_{N} \exp \left\{-\frac{\epsilon}{\hbar}\left[\hbar c_{1}^{*} \frac{c_{1}+c_{N}}{\epsilon}+H\left(-c_{1}^{*}, c_{N}\right)\right]\right\} \\
= & \int \mathrm{d} c_{1}^{*} \mathrm{~d} c_{1} \int \mathrm{d} c_{N}^{*} \mathrm{~d} c_{N} \exp \left\{-\frac{\epsilon}{\hbar}\left[-\hbar c_{1}^{*} \frac{-c_{1}-c_{N}}{\epsilon}+H\left(-c_{1}^{*}, c_{N}\right)\right]\right\} .
\end{aligned}
$$

Thereby, we obtain in total

$$
\begin{aligned}
\mathcal{Z} & =\int \mathrm{d} c_{N}^{*} \mathrm{~d} c_{N} \cdots \int \mathrm{d} c_{1}^{*} \mathrm{~d} c_{1} \exp \left(-\frac{S_{E}}{\hbar}\right), \\
S_{E} & =\left.\epsilon \sum_{i=1}^{N}\left[\hbar c_{i+1}^{*} \frac{c_{i+1}-c_{i}}{\epsilon}+H\left(c_{i+1}^{*}, c_{i}\right)\right]\right|_{c_{N+1} \equiv-c_{1}, c_{N+1}^{*} \equiv-c_{1}^{*}} .
\end{aligned}
$$

Finally, taking the formal limit $N \rightarrow \infty, \epsilon \rightarrow 0$, with $\beta \hbar=\epsilon N$ kept fixed, we arrive at

$$
\mathcal{Z}=\int_{\substack{c(\beta \hbar)=-c(0), c^{*}(\beta \hbar)=-c^{*}(0)}} \mathcal{D} c^{*}(\tau) \mathcal{D} c(\tau) \exp \left\{-\frac{1}{\hbar} \int_{0}^{\beta \hbar} \mathrm{d} \tau\left[\hbar c^{*}(\tau) \frac{\mathrm{d} c(\tau)}{\mathrm{d} \tau}+H\left(c^{*}(\tau), c(\tau)\right)\right]\right\} .
$$

In other words, the fermionic path integral resembles the bosonic one in eq. (4.5), but the Grassmann fields obey antiperiodic boundary conditions over the Euclidean time interval. In fact, the analogy between bosonic and fermionic path integrals can be pushed even further, but for that we need to specify precisely the form of the fermionic Hamiltonian. For this, we turn to Dirac fields. 


\subsection{The Dirac field at finite temperature}

In order to make use of the results of the previous section, we need to construct the Hamiltonian of the Dirac field and identify the objects that play the roles of the operators $\hat{a}$ and $\hat{a}^{\dagger}$. Our starting point is the "classical" Minkowskian Lagrangian,

$$
\mathcal{L}_{M}=\bar{\psi}\left(i \gamma^{\mu} \partial_{\mu}-m\right) \psi
$$

where $\bar{\psi} \equiv \psi^{\dagger} \gamma^{0}$ and $m \equiv m \cdot \mathbb{1}_{4 \times 4}$. The Dirac $\gamma$-matrices obey the relations

$$
\left\{\gamma^{\mu}, \gamma^{\nu}\right\} \equiv 2 \eta^{\mu \nu}, \quad\left(\gamma^{\mu}\right)^{\dagger}=\gamma^{0} \gamma^{\mu} \gamma^{0}
$$

The conjugate momentum is defined by

$$
\pi=\frac{\partial \mathcal{L}_{M}}{\partial\left(\partial_{0} \psi\right)}=\bar{\psi} i \gamma^{0}=i \psi^{\dagger}
$$

and the Hamiltonian density subsequently becomes

$$
\mathcal{H}=\pi \partial_{0} \psi-\mathcal{L}_{M}=\bar{\psi}\left[-i \gamma^{k} \partial_{k}+m\right] \psi .
$$

If we now switch to operator language and recall the canonical (anti)commutation relations,

$$
\begin{aligned}
& \left\{\hat{\psi}_{\alpha}\left(x^{0}, \mathbf{x}\right), \hat{\psi}_{\beta}\left(x^{0}, \mathbf{y}\right)\right\}=\left\{\hat{\psi}_{\alpha}^{\dagger}\left(x^{0}, \mathbf{x}\right), \hat{\psi}_{\beta}^{\dagger}\left(x^{0}, \mathbf{y}\right)\right\}=0, \\
& \left\{\hat{\psi}_{\alpha}\left(x^{0}, \mathbf{x}\right), \hat{\pi}_{\beta}\left(x^{0}, \mathbf{y}\right)\right\}=\left\{\hat{\psi}_{\alpha}\left(x^{0}, \mathbf{x}\right), i \hat{\psi}_{\beta}^{\dagger}\left(x^{0}, \mathbf{y}\right)\right\}=i \delta^{(d)}(\mathbf{x}-\mathbf{y}) \delta_{\alpha \beta},
\end{aligned}
$$

where the subscripts refer to the Dirac indices, $\alpha, \beta \in\{1, \ldots, 4\}$, we note that $\hat{\psi}_{\alpha}, \hat{\psi}_{\beta}^{\dagger}$ play precisely the same roles as $\hat{a}, \hat{a}^{\dagger}$ in eq. (4.8). Furthermore, from the operator point of view, the Hamiltonian has indeed the structure of eq. (4.13) (apart from the constant term),

$$
\hat{H}=\int_{\mathbf{x}} \hat{\psi}^{\dagger}\left(x^{0}, \mathbf{x}\right)\left[-i \gamma^{0} \gamma^{k} \partial_{k}+m \gamma^{0}\right] \hat{\psi}\left(x^{0}, \mathbf{x}\right) .
$$

Rephrasing eq. (4.27) by denoting $c^{*} \rightarrow \psi^{\dagger}, c \rightarrow \psi$, and setting again $\hbar=1$, the object within the square brackets, which we define to be the Euclidean Lagrangian, then reads

$$
L_{E} \equiv \psi^{\dagger} \partial_{\tau} \psi+\psi^{\dagger}\left[-i \gamma^{0} \gamma^{k} \partial_{k}+m \gamma^{0}\right] \psi=\bar{\psi}\left[\gamma^{0} \partial_{\tau}-i \gamma^{k} \partial_{k}+m\right] \psi .
$$

Most remarkably, a comparison of eqs. (4.28) and (4.35) shows that our recipe from eq. (1.39), $L_{E}=-\mathcal{L}_{M}(\tau=i t)$, apparently again works. (Note that $i \partial_{0}=i \partial_{t} \rightarrow-\partial_{\tau}$.)

It is conventional and convenient to simplify the appearance of eq. (4.35) by introducing so-called Euclidean Dirac matrices, through

$$
\tilde{\gamma}_{0} \equiv \gamma^{0}, \quad \tilde{\gamma}_{k} \equiv-i \gamma^{k}, \quad k=1, \ldots, d,
$$

which according to eq. (4.29) satisfy the algebra

$$
\left\{\tilde{\gamma}_{\mu}, \tilde{\gamma}_{\nu}\right\}=2 \delta_{\mu \nu}, \quad \tilde{\gamma}_{\mu}^{\dagger}=\tilde{\gamma}_{\mu} .
$$

We also denote

$$
\partial_{0} \equiv \partial_{\tau}
$$


from now on, understanding that repeated lower indices imply the use of the Euclidean metric, and furthermore drop tildes from $\tilde{\gamma}_{\mu}$ 's. Thereby eq. (4.35) can be written in the simple form

$$
L_{E}=\bar{\psi}\left[\gamma_{\mu} \partial_{\mu}+m\right] \psi
$$

and the partition function becomes (in continuum)

$$
\mathcal{Z}=\int_{\substack{\psi(\beta, \mathbf{x})=-\psi(0, \mathbf{x}), \bar{\psi}(\beta, \mathbf{x})=-\bar{\psi}(0, \mathbf{x})}} \mathcal{D} \bar{\psi}(\tau, \mathbf{x}) \mathcal{D} \psi(\tau, \mathbf{x}) \exp \left\{-\int_{0}^{\beta} \mathrm{d} \tau \int_{\mathbf{x}} L_{E}\right\},
$$

where we substituted the integration variables from $\psi^{\dagger}$ to $\bar{\psi}$. Note that in the path integral formulation, $\psi$ and $\bar{\psi}$ are to be regarded as independent integration variables.

In order to evaluate $\mathcal{Z}$, it is useful to go to Fourier space; to this end, we write

$$
\psi(X) \equiv \oiint_{\{P\}} e^{i P \cdot X} \tilde{\psi}(P), \quad \bar{\psi}(X) \equiv \sum_{\{P\}} e^{-i P \cdot X} \tilde{\bar{\psi}}(P),
$$

where the curly brackets remind us of the fermionic nature of thermal sums. The anti-periodicity in eq. (4.40) requires that $P$ be of the form

$$
P=\left(\omega_{n}^{\mathrm{f}}, \mathbf{p}\right), \quad e^{i \omega_{n}^{\mathrm{f}} \beta}=-1,
$$

whereby the fermionic Matsubara frequencies become

$$
\omega_{n}^{\mathrm{f}}=2 \pi T\left(n+\frac{1}{2}\right), \quad n \in \mathbb{Z},
$$

i.e. $\omega_{n}^{\mathrm{f}}= \pm \pi T, \pm 3 \pi T, \ldots$. Note in particular that anti-periodicity removes the Matsubara zero mode from the spectrum, implying (recalling the discussion of sec. 3.4) that there are no infrared problems associated with fermions, at least when it comes to "static" observables like the partition function. In the following, we drop the superscript from $\omega_{n}^{\mathrm{f}}$ and indicate the fermionic nature of the Matsubara frequency with curly brackets like in eq. (4.41).

In the Fourier representation, the exponent in eq. (4.40) becomes

$$
\begin{aligned}
S_{E} & \equiv \int_{0}^{\beta} \mathrm{d} \tau \int_{\mathbf{x}} \bar{\psi}(X)\left[\gamma_{\mu} \partial_{\mu}+m\right] \psi(X) \\
& =\int_{X} \sum_{\{P\}} \sum_{\{Q\}} e^{i(P-Q) \cdot X} \tilde{\bar{\psi}}(Q)\left[i \gamma_{\mu} P_{\mu}+m\right] \tilde{\psi}(P) \\
& =\sum_{\{P\}} \tilde{\bar{\psi}}(P)[i \not P+m] \tilde{\psi}(P),
\end{aligned}
$$

where we made use of eq. (3.25), and defined $\not P \equiv \gamma_{\mu} P_{\mu}$. In contrast to real scalar fields, all Fourier modes are independent in the fermionic case. Up to an overall constant, we can then change the integration variables in eq. (4.40) to be the Fourier modes. Another useful result is the generalization of the simple identities

$$
\begin{aligned}
& \int \mathrm{d} c^{*} \mathrm{~d} c e^{-c^{*} a c}=\int \mathrm{d} c^{*} \mathrm{~d} c\left[-c^{*} a c\right]=a, \\
& \frac{\int \mathrm{d} c^{*} \mathrm{~d} c c c^{*} e^{-c^{*} a c}}{\int \mathrm{d} c^{*} \mathrm{~d} c e^{-c^{*} a c}}=\frac{\int \mathrm{d} c^{*} \mathrm{~d} c c c^{*}}{\int \mathrm{d} c^{*} \mathrm{~d} c\left[-c^{*} a c\right]}=\frac{1}{a},
\end{aligned}
$$


to a multicomponent case:

$$
\begin{aligned}
& \int\left\{\prod_{i} \mathrm{~d} c_{i}^{*} \mathrm{~d} c_{i}\right\} \exp \left(-c_{i}^{*} M_{i j} c_{j}\right)=\operatorname{det}(M) \\
& \frac{\int\left\{\prod_{i} \mathrm{~d} c_{i}^{*} \mathrm{~d} c_{i}\right\} c_{k} c_{l}^{*} \exp \left(-c_{i}^{*} M_{i j} c_{j}\right)}{\int\left\{\prod_{i} \mathrm{~d} c_{i}^{*} \mathrm{~d} c_{i}\right\} \exp \left(-c_{i}^{*} M_{i j} c_{j}\right)}=\left(M^{-1}\right)_{k l}
\end{aligned}
$$

Armoured with this knowledge, we can derive explicit results for the partition function $\mathcal{Z}$ as well as for the fermion propagator, needed for computing perturbative corrections to the partition function. From eqs. (4.40), (4.44) and (4.47), we first obtain

$$
\begin{aligned}
\mathcal{Z} & =\tilde{C} \prod_{\{P\}} \operatorname{det}[i \not P+m] \\
& =\tilde{C}\left(\prod_{\{P\}} \operatorname{det}[i \not P+m] \prod_{\{P\}} \operatorname{det}[-i \not P+m]\right)^{\frac{1}{2}}
\end{aligned}
$$

where $\tilde{C}$ is some constant, and have we "replicated" the determinant and compensated for that by taking the square root of the result. The reason for the replication is that we may now write

$$
[i \not P+m][-i \not P+m]=\not P \not P+m^{2}=\left(P^{2}+m^{2}\right) \mathbb{1}_{4 \times 4}
$$

where we applied eq. (4.37). Thereby we get

$$
\mathcal{Z}=\tilde{C}\left(\prod_{\{P\}} \operatorname{det}\left[\left(P^{2}+m^{2}\right) \mathbb{1}_{4 \times 4}\right]\right)^{\frac{1}{2}}=\tilde{C} \prod_{\{P\}}\left(P^{2}+m^{2}\right)^{2}
$$

and the free energy density $f(T)$ becomes

$$
\begin{aligned}
f(T) & =\lim _{V \rightarrow \infty} \frac{F}{V}=\lim _{V \rightarrow \infty}\left(-\frac{T}{V} \ln \mathcal{Z}\right) \\
& =-\lim _{V \rightarrow \infty} \frac{T}{V} \times 2 \sum_{\{P\}} \ln \left(P^{2}+m^{2}\right)+\text { const. } \\
& =-4 \sum_{\{P\}} \frac{1}{2} \ln \left(P^{2}+m^{2}\right)+\text { const. }
\end{aligned}
$$

where we identified the sum-integration measure from eq. (2.11).

The following remarks are in order:

- The sum-integral appearing in eq. (4.52) is similar to the bosonic one in eq. (2.51), but is preceded by a minus sign, and contains fermionic Matsubara frequencies. These are the characteristic properties of fermions.

- The factor 4 in eq. (4.52) corresponds to the four spin degrees of freedom of a Dirac spinor.

- Like for scalar field theory in eq. (2.51) or (2.24), there is a constant part in $f(T)$, independent of the particle mass. We do not specify this term explicitly here; rather, there will be an implicit specification below (cf. eq. (4.55)), where we relate generic fermionic thermal sums to the known bosonic ones. Alternatively, the result can be extracted from eq. (4.16). 
Finally, from eqs. (4.44) and (4.48), we find the propagator

$$
\left\langle\tilde{\psi}_{\alpha}(P) \tilde{\bar{\psi}}_{\beta}(Q)\right\rangle_{0}=\delta(P-Q)[i \not P+m \mathbb{1}]_{\alpha \beta}^{-1}=\delta(P-Q) \frac{[-i \not P+m \mathbb{1}]_{\alpha \beta}}{P^{2}+m^{2}},
$$

where the argument of the $\delta$-function is $P-Q$ (instead of $P+Q$ ) due to the form of eq. (4.44). Once interactions are added, their effects can again be reduced to sum-integrals over products of propagators through the application of Wick's theorem, cf. sec. 3.1. However, the Grassmann nature of the Dirac fields produces a minus sign in every commutation.

\section{Fermionic thermal sums}

Let us now consider the same problem as in sec. 2.2, but with fermionic Matsubara frequencies. That is, we need to perform sums of the type

$$
\sigma_{\mathrm{f}} \equiv T \sum_{\left\{\omega_{n}\right\}} f\left(\omega_{n}\right)
$$

Denoting for clarity the corresponding sum in eq. (2.29) by $\sigma_{\mathrm{b}}$, we can write:

$$
\begin{aligned}
\sigma_{\mathrm{f}}(T)= & T[\ldots+f(-3 \pi T)+f(-\pi T)+f(\pi T)+\ldots] \\
= & T[\ldots+f(-3 \pi T)+f(-2 \pi T)+f(-\pi T)+f(0)+f(\pi T)+f(2 \pi T)+\ldots] \\
& -T[\ldots+f(-2 \pi T)+f(0)+f(2 \pi T)+\ldots] \\
= & 2 \times \frac{T}{2}\left[\ldots+f\left(-6 \pi \frac{T}{2}\right)+f\left(-4 \pi \frac{T}{2}\right)+f\left(-2 \pi \frac{T}{2}\right)+f(0)+f\left(2 \pi \frac{T}{2}\right)+f\left(4 \pi \frac{T}{2}\right)+\ldots\right] \\
& -T[\ldots+f(-2 \pi T)+f(0)+f(2 \pi T)+\ldots] \\
= & 2 \sigma_{\mathrm{b}}\left(\frac{T}{2}\right)-\sigma_{\mathrm{b}}(T) .
\end{aligned}
$$

Thereby all fermionic sums follow from the known bosonic ones, while the converse is not true.

To give a concrete example, consider eq. (2.34),

$$
\sigma_{\mathrm{b}}(T)=\int_{-\infty}^{+\infty} \frac{\mathrm{d} p}{2 \pi} f(p)+\int_{-\infty-i 0^{+}}^{+\infty-i 0^{+}} \frac{\mathrm{d} p}{2 \pi}[f(p)+f(-p)] n_{\mathrm{B}}(i p) .
$$

Eq. (4.55) implies

$$
\sigma_{\mathrm{f}}(T)=\int_{-\infty}^{+\infty} \frac{\mathrm{d} p}{2 \pi} f(p)+\int_{-\infty-i 0^{+}}^{+\infty-i 0^{+}} \frac{\mathrm{d} p}{2 \pi}[f(p)+f(-p)]\left[2 n_{\mathrm{B}}^{\left(\frac{T}{2}\right)}(i p)-n_{\mathrm{B}}^{(T)}(i p)\right],
$$

so that the finite-temperature part has the new weight

$$
\begin{aligned}
2 n_{\mathrm{B}}^{\left(\frac{T}{2}\right)}(i p)-n_{\mathrm{B}}^{(T)}(i p) & =\frac{2}{\exp (2 i p \beta)-1}-\frac{1}{\exp (i p \beta)-1} \\
& =\frac{1}{\exp (i p \beta)-1}\left[\frac{2}{\exp (i p \beta)+1}-1\right]=\frac{1-\exp (i p \beta)}{[\exp (i p \beta)-1][\exp (i p \beta)+1]} \\
& =-n_{\mathrm{F}}^{(T)}(i p),
\end{aligned}
$$

where $n_{\mathrm{F}}(p) \equiv 1 /[\exp (\beta p)+1]$ is the Fermi distribution. In total, then, fermionic sums can be converted to integrals according to

$$
\sigma_{\mathrm{f}}(T)=\int_{-\infty}^{+\infty} \frac{\mathrm{d} p}{2 \pi} f(p)-\int_{-\infty-i 0^{+}}^{+\infty-i 0^{+}} \frac{\mathrm{d} p}{2 \pi}[f(p)+f(-p)] n_{\mathrm{F}}(i p) .
$$




\section{Appendix A: Low and high-temperature expansions for fermions}

Defining the fermionic sum-integrals

$$
\begin{aligned}
\tilde{J}(m, T) & \equiv \frac{1}{2} \mathcal{F}_{\{P\}}\left[\ln \left(P^{2}+m^{2}\right)-\text { const. }\right], \\
\tilde{I}(m, T) & \equiv \sum_{\{P\}} \frac{1}{P^{2}+m^{2}},
\end{aligned}
$$

we first divide them into zero and finite-temperature parts via

$$
\tilde{J}(m, T)=J_{0}(m)+\tilde{J}_{T}(m), \quad \tilde{I}(m, T)=I_{0}(m)+\tilde{I}_{T}(m),
$$

where we have used the fact that at $T=0$, the integrals reduce to their bosonic counterparts, i.e. $J_{0}(m)$ and $I_{0}(m)$ from eqs. (2.70) and (2.73), respectively. In the following, our goal is to find general expressions for the functions $\tilde{J}_{T}(m)$ and $\tilde{I}_{T}(m)$. In addition we work out their low and high-temperature expansions, noting in particular the absence of odd powers of $m$ in the high-temperature limits. Finally, we also determine the fermionic version of eq. (1.78),

$$
\tilde{G}(\tau) \equiv T \sum_{\left\{\omega_{n}\right\}} \frac{e^{i \omega_{n} \tau}}{\omega_{n}^{2}+\omega^{2}}, \quad 0 \leq \tau \leq \beta .
$$

Let us proceed according to eq. (4.55). From eq. (2.50), i.e.

$$
J_{T}(m)=\int_{\mathbf{k}} T \ln \left(1-e^{-\beta \epsilon_{k}}\right),
$$

we immediately obtain

$$
\begin{aligned}
\tilde{J}_{T}(m) & =\int_{\mathbf{k}} T\left[\ln \left(1-e^{-2 \beta \epsilon_{k}}\right)-\ln \left(1-e^{-\beta \epsilon_{k}}\right)\right] \\
& =\int_{\mathbf{k}} T \ln \left(1+e^{-\beta \epsilon_{k}}\right),
\end{aligned}
$$

whereas from eq. (2.53), i.e.

$$
I_{T}(m)=\int_{\mathbf{k}} \frac{n_{\mathrm{B}}\left(\epsilon_{k}\right)}{\epsilon_{k}},
$$

the same steps as in eq. (4.58) lead us to

$$
\tilde{I}_{T}(m)=-\int_{\mathbf{k}} \frac{n_{\mathrm{F}}\left(\epsilon_{k}\right)}{\epsilon_{k}} .
$$

Unfortunately these integrals cannot be expressed in terms of known elementary functions. ${ }^{10}$

Concerning the expansions, we know from eq. (2.79) that the low-temperature limit of $J_{T}$ reads

$$
J_{T}(m) \approx-T^{4}\left(\frac{m}{2 \pi T}\right)^{\frac{3}{2}} e^{-\beta m} .
$$

In eq. (4.55), the first term is exponentially suppressed, and thus we obtain in the fermionic case

$$
\tilde{J}_{T}(m) \approx T^{4}\left(\frac{m}{2 \pi T}\right)^{\frac{3}{2}} e^{-\beta m}
$$

\footnotetext{
${ }^{10}$ Rapidly convergent sum representations in terms of the modified Bessel function can, however, be obtained: $\tilde{J}_{T}(m)=-\frac{m^{2} T^{2}}{2 \pi^{2}} \sum_{n=1}^{\infty} \frac{(-1)^{n}}{n^{2}} K_{2}\left(\frac{n m}{T}\right), \tilde{I}_{T}(m)=\frac{m T}{2 \pi^{2}} \sum_{n=1}^{\infty} \frac{(-1)^{n}}{n} K_{1}\left(\frac{n m}{T}\right)$.
} 
From eq. (2.80), the low-temperature expansion for $I_{T}$ reads

$$
I_{T}(m) \approx \frac{T^{3}}{m}\left(\frac{m}{2 \pi T}\right)^{\frac{3}{2}} e^{-\beta m}
$$

Again, the first term in eq. (4.55) is exponentially suppressed, so that we get

$$
\tilde{I}_{T}(m) \approx-\frac{T^{3}}{m}\left(\frac{m}{2 \pi T}\right)^{\frac{3}{2}} e^{-\beta m}
$$

Moving on to the high- $T$ limit, the high-temperature expansion of $J_{T}$ reads from eq. (2.82)

$$
J_{T}(m)=-\frac{\pi^{2} T^{4}}{90}+\frac{m^{2} T^{2}}{24}-\frac{m^{3} T}{12 \pi}-\frac{m^{4}}{2(4 \pi)^{2}}\left[\ln \left(\frac{m e^{\gamma_{\mathrm{E}}}}{4 \pi T}\right)-\frac{3}{4}\right]+\frac{m^{6} \zeta(3)}{3(4 \pi)^{4} T^{2}}+\ldots
$$

According to eq. (4.55), we then get

$$
\begin{aligned}
\tilde{J}_{T}(m) & =-\frac{1}{8} \frac{\pi^{2} T^{4}}{90}+\frac{1}{2} \frac{m^{2} T^{2}}{24}-\frac{m^{3} T}{12 \pi}-2 \frac{m^{4}}{2(4 \pi)^{2}}\left[\ln \left(\frac{m e^{\gamma_{\mathrm{E}}}}{4 \pi T}\right)-\frac{3}{4}+\ln 2\right]+\frac{8 m^{6} \zeta(3)}{3(4 \pi)^{4} T^{2}} \\
& +\frac{\pi^{2} T^{4}}{90}-\frac{m^{2} T^{2}}{24}+\frac{m^{3} T}{12 \pi}+\frac{m^{4}}{2(4 \pi)^{2}}\left[\ln \left(\frac{m e^{\gamma_{\mathrm{E}}}}{4 \pi T}\right)-\frac{3}{4}\right]-\frac{m^{6} \zeta(3)}{3(4 \pi)^{4} T^{2}}-\ldots \\
& =\frac{7}{8} \frac{\pi^{2} T^{4}}{90}-\frac{m^{2} T^{2}}{48}-\frac{m^{4}}{2(4 \pi)^{2}}\left[\ln \left(\frac{m e^{\gamma_{\mathrm{E}}}}{\pi T}\right)-\frac{3}{4}\right]+\frac{7 m^{6} \zeta(3)}{3(4 \pi)^{4} T^{2}}+\ldots,
\end{aligned}
$$

where we note in particular the disappearance of the term cubic in $m$. Finally, from eq. (2.93), we may read off the high-temperature expansion of $I_{T}$,

$$
I_{T}(m)=\frac{T^{2}}{12}-\frac{m T}{4 \pi}-\frac{2 m^{2}}{(4 \pi)^{2}}\left[\ln \left(\frac{m e^{\gamma_{\mathrm{E}}}}{4 \pi T}\right)-\frac{1}{2}\right]+\frac{2 m^{4} \zeta(3)}{(4 \pi)^{4} T^{2}}+\ldots
$$

which together with eq. (4.55) yields

$$
\begin{aligned}
\tilde{I}_{T}(m) & =\frac{1}{2} \frac{T^{2}}{12}-\frac{m T}{4 \pi}-\frac{4 m^{2}}{(4 \pi)^{2}}\left[\ln \left(\frac{m e^{\gamma_{\mathrm{E}}}}{4 \pi T}\right)-\frac{1}{2}+\ln 2\right]+\frac{16 m^{4} \zeta(3)}{(4 \pi)^{4} T^{2}} \\
& -\frac{T^{2}}{12}+\frac{m T}{4 \pi}+\frac{2 m^{2}}{(4 \pi)^{2}}\left[\ln \left(\frac{m e^{\gamma_{\mathrm{E}}}}{4 \pi T}\right)-\frac{1}{2}\right]-\frac{2 m^{4} \zeta(3)}{(4 \pi)^{4} T^{2}}+\ldots \\
& =-\frac{T^{2}}{24}-\frac{2 m^{2}}{(4 \pi)^{2}}\left[\ln \left(\frac{m e^{\gamma_{\mathrm{E}}}}{\pi T}\right)-\frac{1}{2}\right]+\frac{14 m^{4} \zeta(3)}{(4 \pi)^{4} T^{2}}+\ldots
\end{aligned}
$$

Again, the term odd in $m$ has disappeared. Note also that the term $-2 m^{2} \ln (m) /(4 \pi)^{2}$ is $T$ independent and cancels against a corresponding logarithm in $I_{0}(m)$ if we consider $\tilde{I}(m, T)$, cf. eq. (2.73), so that $\tilde{I}(m, T)$ is formally a genuine power series in $m^{2}$.

Finally, we know from eq. (1.70) that the bosonic imaginary-time propagator reads

$$
\begin{aligned}
G(\tau) & =T \sum_{\omega_{n}} \frac{e^{i \omega_{n} \tau}}{\omega_{n}^{2}+\omega^{2}} \\
& =\frac{1}{2 \omega} \frac{e^{(\beta-\tau) \omega}+e^{\tau \omega}}{e^{\beta \omega}-1}=\frac{n_{\mathrm{B}}(\omega)}{2 \omega}\left[e^{(\beta-\tau) \omega}+e^{\tau \omega}\right], \quad 0 \leq \tau \leq \beta .
\end{aligned}
$$

Employing eq. (4.55), we obtain from here

$$
\tilde{G}(\tau)=\frac{1}{2 \omega}\left\{\frac{2}{e^{2 \beta \omega}-1}\left[e^{(2 \beta-\tau) \omega}+e^{\tau \omega}\right]-\frac{1}{e^{\beta \omega}-1}\left[e^{(\beta-\tau) \omega}+e^{\tau \omega}\right]\right\}
$$




$$
\begin{aligned}
& =\frac{1}{2 \omega} \frac{1}{\left(e^{\beta \omega}-1\right)\left(e^{\beta \omega}+1\right)} \underbrace{\left\{2 e^{(2 \beta-\tau) \omega}+2 e^{\tau \omega}-\left(e^{\beta \omega}+1\right)\left[e^{(\beta-\tau) \omega}+e^{\tau \omega}\right]\right\}}_{\left(e^{\beta \omega}-1\right)\left[e^{(\beta-\tau) \omega}-e^{\tau \omega}\right]} \\
& =\frac{n_{\mathrm{F}}(\omega)}{2 \omega}\left[e^{(\beta-\tau) \omega}-e^{\tau \omega}\right], \quad 0 \leq \tau \leq \beta .
\end{aligned}
$$

For good measure, we end the section by rederiving eq. (4.77) more directly, similarly to the procedure applied around eq. (2.30). Consider the auxiliary function

$$
n_{\mathrm{F}}(i p) \equiv \frac{1}{e^{i \beta p}+1}
$$

which has poles at $\exp (i \beta p)=-1$, i.e. $p=\omega_{n}^{\mathrm{f}}$. The residue around each of these poles is

$$
n_{\mathrm{F}}\left(i\left[\omega_{n}^{\mathrm{f}}+z\right]\right)=\frac{1}{-e^{i \beta z}+1} \approx \frac{i T}{z}+\mathcal{O}(1) .
$$

Therefore, letting $f(p)$ be a generic function regular along the real axis, we can write

$$
T \sum_{\left\{\omega_{n}\right\}} f\left(\omega_{n}\right)=\frac{-i}{2 \pi i} \oint \mathrm{d} p f(p) n_{\mathrm{F}}(i p),
$$

where the integration contour runs anti-clockwise around the real axis of the complex $p$-plane. Choosing then

$$
f(p) \equiv \frac{e^{i p \tau}}{p^{2}+\omega^{2}},
$$

we note that for $0<\tau<\beta$ both half-planes are "safe", i.e. $e^{i p \tau} n_{\mathrm{F}}(i p)$ vanishes fast for $p \rightarrow \pm i \infty$. (The values for $\tau=0$ and $\tau=\beta$ can be obtained in the end from continuity.) Therefore we can close the two parts of the integration contour in a clockwise manner in the upper and lower half-planes, respectively. Picking up the poles of $f(p)$ from $\pm i \omega$, this yields

$$
T \sum_{\left\{\omega_{n}\right\}} \frac{e^{i \omega_{n} \tau}}{\omega_{n}^{2}+\omega^{2}}=\frac{-i}{2 \pi i}(-2 \pi i)\left[\frac{e^{-\omega \tau}}{2 i \omega} n_{\mathrm{F}}(-\omega)+\frac{e^{\omega \tau}}{-2 i \omega} n_{\mathrm{F}}(\omega)\right],
$$

and noting that

$$
n_{\mathrm{F}}(-\omega)=\frac{1}{e^{-\beta \omega}+1}=\frac{e^{\beta \omega}}{e^{\beta \omega}+1}=e^{\beta \omega} n_{\mathrm{F}}(\omega),
$$

we directly obtain eq. (4.77). 


\section{Literature}

[4.1] F.A. Berezin, The method of second quantization, Pure Appl. Phys. 24 (1966) 1-228. 


\section{Gauge fields}

Abstract: After introducing the concept of non-Abelian gauge invariance, associated with the existence of spin-1 gauge fields, the main elements of the canonical quantization of gauge fields are recalled. Subsequently, an imaginary-time path integral expression is motivated for the partition function of such fields. The rules for carrying out a weak-coupling expansion of this quantity are formulated, and the corresponding Feynman rules are derived. This machinery is employed for defining and computing a thermal gluon mass, also known as a Debye mass. Finally, the free energy density of non-Abelian black-body radiation is determined up to third order in the coupling constant, revealing a highly non-trivial structure in this asymptotic series.

Keywords: Yang-Mills theory, gauge invariance, covariant derivative, Gauss law, gauge fixing, ghosts, black-body radiation, Stefan-Boltzmann law, Debye mass, screening, QED, QCD.

\subsection{Path integral for the partition function}

Like with fermions in sec. 4.2, our starting point with new fields is their classical Lagrangian in Minkowskian spacetime. For non-Abelian gauge fields, this has the familiar Yang-Mills form

$$
\mathcal{L}_{M}=-\frac{1}{4} F^{a \mu \nu} F_{\mu \nu}^{a} \quad F_{\mu \nu}^{a}=\partial_{\mu} A_{\nu}^{a}-\partial_{\nu} A_{\mu}^{a}+g f^{a b c} A_{\mu}^{b} A_{\nu}^{c}
$$

where $g$ is the (bare) gauge coupling and $f^{a b c}$ are the structure constants of the gauge group, typically taken to be $\mathrm{SU}\left(N_{\mathrm{c}}\right)$ with some $N_{\mathrm{c}}$. Introducing a covariant derivative in the adjoint representation,

$$
\mathcal{D}_{\mu}^{a c} \equiv \partial_{\mu} \delta^{a c}+g f^{a b c} A_{\mu}^{b}
$$

we note for later reference that $F_{\mu \nu}^{a}$ can be expressed in the equivalent forms

$$
F_{\mu \nu}^{a}=\partial_{\mu} A_{\nu}^{a}-\mathcal{D}_{\nu}^{a c} A_{\mu}^{c}=\mathcal{D}_{\mu}^{a c} A_{\nu}^{c}-\partial_{\nu} A_{\mu}^{a} .
$$

We can also supplement eq. (5.1) with matter fields: for instance, letting $\psi$ be a fermion in the fundamental representation, $\phi$ a scalar in the fundamental representation, and $\Phi$ a scalar in the adjoint representation, we could add the terms

$$
\delta \mathcal{L}_{M}=\bar{\psi}\left(i \gamma^{\mu} D_{\mu}-m\right) \psi+\left(D^{\mu} \phi\right)^{\dagger} D_{\mu} \phi+\frac{1}{2} \mathcal{D}^{a c \mu} \Phi^{c} \mathcal{D}_{\mu}^{a d} \Phi^{d}-V\left(\phi^{\dagger} \phi, \Phi^{a} \Phi^{a}\right),
$$

where $D_{\mu}=\partial_{\mu}-i g A_{\mu}^{a} T^{a}$ is a covariant derivative in the fundamental representation. The $N_{\mathrm{c}} \times N_{\mathrm{c}}$ matrices $T^{a}$ are the Hermitean generators of $\mathrm{SU}\left(N_{\mathrm{c}}\right)$, satisfying the algebra $\left[T^{a}, T^{b}\right]=i f^{a b c} T^{c}$, and conventionally normalized as $\operatorname{Tr}\left[T^{a} T^{b}\right]=\delta^{a b} / 2$.

The construction principle behind eqs. (5.1) and (5.4) is that of local gauge invariance. With $U \equiv \exp \left[i g \theta^{a}(x) T^{a}\right]$, the Lagrangian is invariant in the transformations $A_{\mu} \rightarrow A_{\mu}^{\prime}, \psi \rightarrow \psi^{\prime}, \phi \rightarrow \phi^{\prime}$, $\Phi \rightarrow \Phi^{\prime}$, with

$$
\begin{aligned}
A_{\mu}^{\prime} \equiv A_{\mu}^{\prime a} T^{a} & =U A_{\mu} U^{-1}+\frac{i}{g} U \partial_{\mu} U^{-1}=A_{\mu}+i g \theta^{a}\left[T^{a}, A_{\mu}\right]+T^{a} \partial^{\mu} \theta^{a}+\mathcal{O}\left(\theta^{2}\right) \\
\Leftrightarrow A_{\mu}^{\prime a} & =A_{\mu}^{a}+\mathcal{D}_{\mu}^{a c} \theta^{c}+\mathcal{O}\left(\theta^{2}\right), \\
\psi^{\prime} & =U \psi=\left(\mathbb{1}+i g \theta^{a} T^{a}\right) \psi+\mathcal{O}\left(\theta^{2}\right),
\end{aligned}
$$




$$
\begin{aligned}
\phi^{\prime} & =U \phi=\left(\mathbb{1}+i g \theta^{a} T^{a}\right) \phi+\mathcal{O}\left(\theta^{2}\right), \\
\Phi^{\prime} \equiv \Phi^{\prime a} T^{a} & =U \Phi U^{-1}=\Phi+i g \theta^{a}\left[T^{a}, \Phi\right]+\mathcal{O}\left(\theta^{2}\right) \\
\Leftrightarrow \Phi^{\prime a} & =\Phi^{a}+g f^{a b c} \Phi^{b} \theta^{c}+\mathcal{O}\left(\theta^{2}\right) .
\end{aligned}
$$

We would now like to quantize the theory of eqs. (5.1) and (5.4), and in particular derive a path integral representation for its partition function. At this point, the role of gauge invariance becomes conceptually slightly convoluted. It will namely turn out that:

- The classical theory is constructed by insisting on gauge invariance.

- Canonical quantization and the derivation of the Euclidean path integral necessitate an explicit breaking of gauge invariance.

- The final Euclidean path integral again displays gauge invariance.

- Formulating perturbation theory within the Euclidean path integral necessitates yet again an explicit breaking of gauge invariance.

- Nevertheless, only gauge invariant observables are considered physical.

A proper discussion of these issues goes beyond the scope of this book but we note in passing that a deeper reason for why the breaking of gauge invariance by gauge fixing is not considered to be a serious issue is that the theory nevertheless maintains a certain global symmetry, called the BRST symmetry, which is sufficient for guaranteeing many basic properties of the theory, such as the existence of Slavnov-Taylor identities, or physical ("gauge-invariant") states in its Hilbert space.

As far as canonical quantization and the derivation of the Euclidean path integral are concerned, there are (at least) two procedures followed in the literature. The idea of the perhaps most common one is to carry out a complete gauge fixing (going to the axial gauge $A_{3}^{a}=0$ ), identifying physical degrees of freedom, ${ }^{11}$ and then following the quantization procedure of scalar field theory.

We take here a different approach, where the idea is to do as little gauge fixing as possible; the price to pay is that one then has to be careful about the states over which the physical Hilbert space is constructed. ${ }^{12}$ The advantage of this approach is that the role of gauge invariance remains less compromised during quantization. If the evaluation of the resulting Euclidean path integral were also to be carried out non-perturbatively (within lattice regularization, for instance), then it would become rather transparent why only gauge invariant observables are physical.

\section{Canonical quantization}

For simplicity, let us restrict to eq. (5.1) in the following, omitting the matter fields for the time being. For canonical quantization, the first step is to construct the Hamiltonian. We do this after setting

$$
A_{0}^{a} \equiv 0,
$$

\footnotetext{
${ }^{11}$ These are $A_{1}^{a}, A_{2}^{a}$ and the corresponding canonical momenta; $A_{0}^{a}$ is expressed in terms of these by imposing a further constraint, the Gauss law, which reads $\mathcal{D}_{i}^{a b} F_{0 i}^{b}=0$ if no matter fields are present.

${ }^{12}$ This approach dates back to ref. [5.1], and can be given a precise meaning within lattice gauge theory [5.2,5.3].
} 
which, however, fixes the gauge only partially; according to eq. (5.6), time-independent gauge transformations are still allowed, given that $A_{0}^{a}$ remains zero in them. In some sense, our philosophy is to break gauge invariance only to the same "soft" degree that Lorentz invariance is necessarily broken in the canonical formulation through the special role that is given to the time coordinate.

The spatial components $A_{i}^{a}$ are now treated as the canonical fields (coordinates). According to eq. (5.3), $F_{0 i}^{a}=\partial_{0} A_{i}^{a}$, and eq. (5.1) thus becomes

$$
\mathcal{L}_{M}=\frac{1}{2} \partial_{0} A_{i}^{a} \partial_{0} A_{i}^{a}-\frac{1}{4} F_{i j}^{a} F_{i j}^{a} .
$$

The canonical momenta corresponding to $A_{i}^{a}$, denoted by $E_{i}^{a}$, take the form

$$
E_{i}^{a} \equiv \frac{\partial \mathcal{L}_{M}}{\partial\left(\partial_{0} A_{i}^{a}\right)}=\partial_{0} A_{i}^{a},
$$

and the Hamiltonian density subsequently reads

$$
\mathcal{H}=E_{i}^{a} \partial_{0} A_{i}^{a}-\mathcal{L}_{M}=\frac{1}{2} E_{i}^{a} E_{i}^{a}+\frac{1}{4} F_{i j}^{a} F_{i j}^{a} .
$$

We also note that the "multiplier" of $A_{0}^{a}$ in the action (before gauge fixing) reads, according to eq. (5.3),

$$
\frac{\delta \mathcal{S}_{M}}{\delta A_{0}^{a}}=\frac{\delta}{\delta A_{0}^{a}} \int_{\mathcal{X}}\left[\frac{1}{2}\left(\partial_{0} A_{i}^{b}-\mathcal{D}_{i}^{b c} A_{0}^{c}\right) F_{0 i}^{b}\right]=\mathcal{D}_{i}^{a b} F_{0 i}^{b},
$$

where we made use of the identity

$$
\int_{\mathcal{X}} f^{a}(\mathcal{X}) \mathcal{D}_{\mu}^{a b} g^{b}(\mathcal{X})=-\int_{\mathcal{X}} g^{a}(\mathcal{X}) \mathcal{D}_{\mu}^{a b} f^{b}(\mathcal{X}) .
$$

The object in eq. (5.15) is identified as the left-hand side of the non-Abelian Gauss law.

The theory can now be canonically quantized by promoting $A_{i}^{a}$ and $E_{i}^{a}$ into operators, and by imposing standard bosonic equal-time commutation relations between them,

$$
\left[\hat{A}_{i}^{a}(t, \mathbf{x}), \hat{E}_{j}^{b}(t, \mathbf{y})\right]=i \delta^{a b} \delta_{i j} \delta(\mathbf{x}-\mathbf{y}) .
$$

According to eq. (5.14), the Hamiltonian then becomes

$$
\hat{H}=\int_{\mathbf{x}}\left(\frac{1}{2} \hat{E}_{i}^{a} \hat{E}_{i}^{a}+\frac{1}{4} \hat{F}_{i j}^{a} \hat{F}_{i j}^{a}\right)
$$

A very important role in the quantization is played by the so-called Gauss law operators, cf. eq. (5.15). Combining this expression with $F_{0 i}^{b}=\partial_{0} A_{i}^{b}=E_{i}^{b}$, we write them in the form

$$
\hat{G}^{a}=\hat{\mathcal{D}}_{i}^{a b} \hat{E}_{i}^{b}, \quad a=1, \ldots, N_{\mathrm{c}}^{2}-1,
$$

and furthermore define an operator parametrized by time-independent gauge transformations,

$$
\hat{U} \equiv \exp \left\{-i \int_{\mathbf{x}} \theta^{a}(\mathbf{x}) \hat{G}^{a}(\mathbf{x})\right\} .
$$

We now claim that $\hat{U}$ generates time-independent gauge transformations. Let us prove this to the leading non-trivial order in $\theta^{a}$. First of all,

$$
\hat{U} \hat{A}_{j}^{b}(\mathbf{y}) \hat{U}^{-1}=\hat{A}_{j}^{b}(\mathbf{y})-i \int_{\mathbf{x}} \theta^{a}(\mathbf{x})\left[\hat{G}^{a}(\mathbf{x}), \hat{A}_{j}^{b}(\mathbf{y})\right]+\mathcal{O}\left(\theta^{2}\right)
$$




$$
\begin{aligned}
& =\hat{A}_{j}^{b}(\mathbf{y})-i \int_{\mathbf{x}} \theta^{a}(\mathbf{x})\left\{\partial_{i}^{\mathbf{x}}\left[\hat{E}_{i}^{a}(\mathbf{x}), \hat{A}_{j}^{b}(\mathbf{y})\right]+g f^{a c d} \hat{A}_{i}^{c}(\mathbf{x})\left[\hat{E}_{i}^{d}(\mathbf{x}), \hat{A}_{j}^{b}(\mathbf{y})\right]\right\}+\mathcal{O}\left(\theta^{2}\right) \\
& =\hat{A}_{j}^{b}(\mathbf{y})-\int_{\mathbf{x}} \theta^{a}(\mathbf{x})\left\{\partial_{i}^{\mathbf{x}} \delta^{a b} \delta_{i j} \delta(\mathbf{x}-\mathbf{y})+g f^{a c d} \hat{A}_{i}^{c}(\mathbf{x}) \delta^{d b} \delta_{i j} \delta(\mathbf{x}-\mathbf{y})\right\}+\mathcal{O}\left(\theta^{2}\right) \\
& =\hat{A}_{j}^{b}(\mathbf{y})+\partial_{j} \theta^{b}(\mathbf{y})+g f^{b c a} \hat{A}_{i}^{c}(\mathbf{y}) \theta^{a}(\mathbf{y})+\mathcal{O}\left(\theta^{2}\right) \\
& =\hat{A}_{j}^{b}(\mathbf{y})+\hat{\mathcal{D}}_{j}^{b a} \theta^{a}(\mathbf{y})+\mathcal{O}\left(\theta^{2}\right) \\
& =\hat{A}_{j}^{b}(\mathbf{y})+\mathcal{O}\left(\theta^{2}\right)
\end{aligned}
$$

where we used the antisymmetry of the structure constants as well as eq. (5.6). Similarly,

$$
\begin{aligned}
\hat{U} \hat{E}_{j}^{b}(\mathbf{y}) \hat{U}^{-1} & =\hat{E}_{j}^{b}(\mathbf{y})-i \int_{\mathbf{x}} \theta^{a}(\mathbf{x})\left[\hat{G}^{a}(\mathbf{x}), \hat{E}_{j}^{b}(\mathbf{y})\right]+\mathcal{O}\left(\theta^{2}\right) \\
& =\hat{E}_{j}^{b}(\mathbf{y})-i \int_{\mathbf{x}} \theta^{a}(\mathbf{x})\left\{+g f^{a c d}\left[\hat{A}_{i}^{c}(\mathbf{x}), \hat{E}_{j}^{b}(\mathbf{y})\right] \hat{E}_{i}^{d}(\mathbf{x})\right\}+\mathcal{O}\left(\theta^{2}\right) \\
& =\hat{E}_{j}^{b}(\mathbf{y})+\int_{\mathbf{x}} \theta^{a}(\mathbf{x}) g f^{a c d} \delta^{c b} \delta_{i j} \delta(\mathbf{x}-\mathbf{y}) \hat{E}_{i}^{d}(\mathbf{x})+\mathcal{O}\left(\theta^{2}\right) \\
& =\hat{E}_{j}^{b}(\mathbf{y})+g f^{b d a} \hat{E}_{j}^{d}(\mathbf{y}) \theta^{a}(\mathbf{y})+\mathcal{O}\left(\theta^{2}\right) \\
& =\hat{E}_{j}^{\prime b}(\mathbf{y})+\mathcal{O}\left(\theta^{2}\right)
\end{aligned}
$$

where the result corresponds to the transformation law of an adjoint scalar, cf. eq. (5.10).

One important consequence of eqs. (5.21) and (5.22) is that the operators $\hat{G}^{a}$ commute with the Hamiltonian $\hat{H}$. This follows from the fact that the Hamiltonian of eq. (5.18) is gauge-invariant in time-independent gauge transformations, as long as $\hat{E}_{i}^{a}$ transforms as an adjoint scalar. This leads to

$$
\hat{U} \hat{H} \hat{U}^{-1}=\hat{H} \quad \Rightarrow \quad\left[\hat{G}^{a}(\mathbf{x}), \hat{H}\right]=0 \quad \forall \mathbf{x} .
$$

Another implication of these results is that $\hat{U}$ transforms eigenstates as well: if $\hat{A}_{i}^{a}\left|A_{i}^{a}\right\rangle=A_{i}^{a}\left|A_{i}^{a}\right\rangle$, then

$$
\begin{aligned}
\hat{A}_{i}^{a} \hat{U}^{-1}\left|A_{i}^{a}\right\rangle & =\hat{U}^{-1} \hat{A}_{i}^{\prime a}\left|A_{i}^{a}\right\rangle=\hat{U}^{-1}\left[\hat{A}_{i}^{a}+\hat{\mathcal{D}}_{j}^{a b} \theta^{b}+\mathcal{O}\left(\theta^{2}\right)\right]\left|A_{i}^{a}\right\rangle \\
& =\hat{U}^{-1}\left[A_{i}^{a}+\mathcal{D}_{j}^{a b} \theta^{b}+\mathcal{O}\left(\theta^{2}\right)\right]\left|A_{i}^{a}\right\rangle=\hat{U}^{-1} A_{i}^{\prime a}\left|A_{i}^{a}\right\rangle \\
& =A_{i}^{\prime a} \hat{U}^{-1}\left|A_{i}^{a}\right\rangle,
\end{aligned}
$$

where we made use of eq. (5.21). Consequently, we can identify

$$
\hat{U}^{-1}\left|A_{i}^{a}\right\rangle=\left|A_{i}^{\prime a}\right\rangle
$$

Let us now define a physical state, "|phys $\rangle$ ", to be one which is gauge-invariant: $\hat{U}^{-1} \mid$ phys $\rangle=$ $\mid$ phys $\rangle$. Expanding to first order in $\theta^{a}$, we see that these states must satisfy

$$
\left.\hat{G}^{a}(\mathbf{x}) \mid \text { phys }\right\rangle=0 \quad \forall \mathbf{x},
$$

which is an operator manifestation of the statement that physical states must obey the Gauss law. Moreover, given that the Hamiltonian commutes with $\hat{G}^{a}$, we can choose the basis vectors of the Hilbert space to be simultaneous eigenstates of $\hat{H}$ and $\hat{G}^{a}$. Among all of these states, only the ones with zero eigenvalue of $\hat{G}^{a}$ are physical; it is then only these states which are to be used in the evaluation of $\mathcal{Z}=\operatorname{Tr}[\exp (-\beta \hat{H})]$. 
After these preparations, we are finally in a position to derive a path integral expression for $\mathcal{Z}$. In terms of quantum mechanics, we have a system with a Hamiltonian $\hat{H}$ and a commuting operator, $\hat{Q}$, whose role is played by $\hat{G}^{a}$. One could in principle consider the grand canonical partition function, $\mathcal{Z}(T, \mu)=\operatorname{Tr}\{\exp [-\beta(\hat{H}-\mu \hat{Q})]\}$, but according to the discussion above we are only interested in the contribution to $\mathcal{Z}$ from the states with zero "charge", $\hat{Q} \mid$ phys $\rangle=0$. To this end, it is more natural to remain in the canonical picture, and we thus label the states with the eigenvalues $\epsilon_{q}, q$, so that $\hat{H}\left|\epsilon_{q}, q\right\rangle=\epsilon_{q}\left|\epsilon_{q}, q\right\rangle, \hat{Q}\left|\epsilon_{q}, q\right\rangle=q\left|\epsilon_{q}, q\right\rangle$. Assuming for concreteness that the eigenvalues $q$ of $\hat{Q}$ are integers, we can write the relevant partition function by taking a trace over all states, but inserting a Kronecker- $\delta$ inside the trace,

$$
\mathcal{Z}_{\text {phys }} \equiv \sum_{\epsilon_{0}}\left\langle\epsilon_{0}, 0\left|e^{-\beta \epsilon_{0}}\right| \epsilon_{0}, 0\right\rangle=\sum_{\epsilon_{q}, q}\left\langle\epsilon_{q}, q\left|\delta_{q, 0} e^{-\beta \epsilon_{q}}\right| \epsilon_{q}, q\right\rangle=\operatorname{Tr}\left[\delta_{\hat{Q}, \hat{0}} e^{-\beta \hat{H}}\right],
$$

where $\delta_{\hat{Q}, \hat{0}}\left|\epsilon_{q}, q\right\rangle \equiv \delta_{q, 0}\left|\epsilon_{q}, q\right\rangle$.

Given that $\delta_{\hat{Q}, \hat{0}}=\delta_{\hat{Q}, \hat{0}} \delta_{\hat{Q}, \hat{0}}$ and $[\hat{H}, \hat{Q}]=0$, we can write

$$
\mathcal{Z}_{\text {phys }}=\operatorname{Tr}[\underbrace{\delta_{\hat{Q}, \hat{0}} e^{-\epsilon \hat{H}} \delta_{\hat{Q}, \hat{0}} e^{-\epsilon \hat{H}} \ldots \delta_{\hat{Q}, \hat{0}} e^{-\epsilon \hat{H}}}_{N \text { parts }}],
$$

where $\epsilon=\beta / N$ and $N \rightarrow \infty$ as before. Here, we may further represent

$$
\delta_{\hat{Q}, \hat{0}}=\int_{-\pi}^{\pi} \frac{\mathrm{d} \theta_{i}}{2 \pi} e^{i \theta_{i} \hat{Q}}=\int_{-\pi / \epsilon}^{\pi / \epsilon} \frac{\mathrm{d} y_{i}}{2 \pi \epsilon^{-1}} e^{i \epsilon y_{i} \hat{Q}},
$$

and insert unit operators as in eq. (1.30), but placing now the momentum state representation between $\delta_{\hat{Q}, \hat{0}}$ and $\exp (-\epsilon \hat{H})$. The typical building block of the discretised path integral then reads

$$
\begin{aligned}
& \left\langle x_{i+1}\left|e^{i \epsilon y_{i} \hat{Q}(\hat{x}, \hat{p})}\right| p_{i}\right\rangle\left\langle p_{i}\left|e^{-\epsilon \hat{H}(\hat{p}, \hat{x})}\right| x_{i}\right\rangle \\
& \quad=\exp \left\{-\epsilon\left[-i y_{i} Q\left(x_{i+1}, p_{i}\right)+\frac{p_{i}^{2}}{2 m}-i p_{i} \frac{x_{i+1}-x_{i}}{\epsilon}+V\left(x_{i}\right)+\mathcal{O}(\epsilon)\right]\right\} .
\end{aligned}
$$

It remains to take the limit $\epsilon \rightarrow 0$, whereby $x_{i}, p_{i}, y_{i}$ become functions, $x(\tau), p(\tau), y(\tau)$, and to replace $x(\tau) \rightarrow A_{i}^{a}, p(\tau) \rightarrow E_{i}^{a}, y(\tau) \rightarrow \tilde{A}_{0}^{a}, Q \rightarrow \mathcal{D}_{i}^{a b} E_{i}^{b}, m \rightarrow 1$. Then the integral over the square brackets in eq. (5.30) becomes

$$
\begin{aligned}
\int_{X}\left[-i \tilde{A}_{0}^{a} \mathcal{D}_{i}^{a b} E_{i}^{b}+\frac{1}{2} E_{i}^{a} E_{i}^{a}-i E_{i}^{a} \partial_{\tau} A_{i}^{a}+\frac{1}{4} F_{i j}^{a} F_{i j}^{a}\right] \\
=\int_{X}\left[\frac{1}{2} E_{i}^{a} E_{i}^{a}-i E_{i}^{a}\left(\partial_{\tau} A_{i}^{a}-\mathcal{D}_{i}^{a b} \tilde{A}_{0}^{b}\right)+\frac{1}{4} F_{i j}^{a} F_{i j}^{a}\right],
\end{aligned}
$$

where we made use of eq. (5.16).

At this point, we make a curious observation: inside the round brackets in eq. (5.31) there is an expression of the form we encountered in eq. (5.3). Of course, the field $\tilde{A}_{0}^{a}$ is not the original $A_{0}^{a}$-field, which was set to zero, but rather a new field, which we are however free to rename as $A_{0}^{a}$. Indeed, in the following we leave out the tilde from $\tilde{A}_{0}^{a}$, and redefine a Euclidean field strength tensor according to

$$
F_{0 i}^{a} \equiv \partial_{\tau} A_{i}^{a}-\mathcal{D}_{i}^{a b} A_{0}^{b}
$$

Noting furthermore that

$$
\frac{1}{2} E_{i}^{a} E_{i}^{a}-i E_{i}^{a} F_{0 i}^{a}=\frac{1}{2}\left(E_{i}^{a}-i F_{0 i}^{a}\right)^{2}+\frac{1}{2} F_{0 i}^{a} F_{0 i}^{a},
$$


we can carry out the Gaussian integral over $E_{i}^{a}$, and end up with the desired path integral expression for the partition function of the theory:

$$
\mathcal{Z}_{\text {phys }}=C \int \mathcal{D} A_{0}^{a} \int_{A_{i}^{a}(\beta, \mathbf{x})=A_{i}^{a}(0, \mathbf{x})} \mathcal{D} A_{i}^{a} \exp \left\{-\int_{0}^{\beta} \mathrm{d} \tau \int_{\mathbf{x}} L_{E}\right\}, \quad L_{E}=\frac{1}{4} F_{\mu \nu}^{a} F_{\mu \nu}^{a} .
$$

In the next section, we address the evaluation of this quantity using a weak-coupling expansion, which requires us to return to the question of gauge fixing.

Two final remarks are in order:

- The field $A_{0}^{a}$ was introduced in order to impose the Gauss law at every $\tau$, and therefore the integrations at each $\tau$ are independent of each other. In other words, it is not obvious from the derivation of the path integral whether the field $A_{0}^{a}$ should satisfy periodic boundary conditions like the spatial components $A_{i}^{a}$ do.

It may be noted, however, that the fields to which the $A_{0}^{a}$ couple in eq. (5.34) do obey periodic boundary conditions. This suggests that we can consider them to live on a circle, and therefore make the same choice for $A_{0}^{a}$ itself. Further evidence comes from a perturbative computation around eq. (5.61), showing that only a periodic $A_{0}^{a}(\tau)$ leads to physical results in a simple way. We make this choice in the following. It is perhaps also appropriate to remark that in lattice gauge theory, the fields $A_{0}^{a}$ live on timelike "links", rather than "sites", and the question of periodicity does not directly concern them.

- For scalar field theory and fermions, eqs. (2.7) and (4.35), we found after a careful derivation of the Euclidean path integral that the result could be interpreted in terms of a simple recipe: $L_{E}=-\mathcal{L}_{M}(t \rightarrow-i \tau)$. We may now ask whether the same is true for gauge fields.

A comparison of eqs. (5.1) and (5.34) shows that, indeed, the recipe again works. The only complication is that the Minkowskian $A_{0}^{a}$ needs to be replaced with $i \tilde{A}_{0}^{a}$ (of which we have normally left out the tilde), just like $\partial_{t}$ gets replaced with $i \partial_{\tau}$. This reflects the structure of gauge invariance, implying that covariant derivatives change as $D_{t} \rightarrow i D_{\tau}$. 


\subsection{Weak-coupling expansion}

\section{Gauge fixing and ghosts}

The path integral representation in eq. (5.34) is manifestly gauge invariant and could in principle (after a suitable regularization) be evaluated as such. As before we restrict our treatment here to perturbation theory; in this case, it turns out that gauge invariance needs to be broken once again, because the quadratic part of $L_{E}$ otherwise contains a non-invertible matrix, so that no propagators can be defined. For completeness, let us recall the main steps of this procedure.

Let $G^{a}$ now be some function of the path integration variables in eq. (5.34), for instance $G^{a}(X)=$ $A_{3}^{a}(X)$ or $G^{a}(X)=-\partial_{\mu} A_{\mu}^{a}(X)$ (note that our notation has changed here, and this function has no relation to the Gauss law). Ideally the function should be so chosen that the equation $G^{a}=0$ has a unique solution for $A_{\mu}^{a}$; otherwise we are faced with the so-called Gribov ambiguity. The idea is then to insert the object

$$
\prod_{X, Y, a, b} \delta\left(G^{a}\right) \operatorname{det}\left[\frac{\delta G^{a}(X)}{\delta \theta^{b}(Y)}\right]
$$

as a multiplier in front of the exponential in eq. (5.34), in order to remove the (infinite) redundancy related to integrating over physically equivalent gauge configurations, the "gauge orbits". Indeed, it appears that this insertion does not change the value of gauge invariant expectation values, but merely induces an overall constant in $\mathcal{Z}$, analogous to $C$. First of all, since $L_{E}$ is gauge invariant, its value within each gauge orbit does not depend on the particular form of the constraint $G^{a}=0$. Second, inspecting the integration measure, we can imagine dividing the integration into one over gauge non-equivalent fields, $\bar{A}_{\mu}$, and another over gauge transformations thereof, parametrized by $\theta$. Then

$$
\begin{aligned}
\int & \mathcal{D} A_{\mu} \delta\left(G^{a}\right) \operatorname{det}\left[\frac{\delta G^{a}}{\delta \theta^{b}}\right] \exp \left\{-\int_{X} L_{E}\left(A_{\mu}\right)\right\} \\
& =\int \mathcal{D} \bar{A}_{\mu} \int \mathcal{D} \theta^{b} \delta\left(G^{a}\right) \operatorname{det}\left[\frac{\delta G^{a}}{\delta \theta^{b}}\right] \exp \left\{-\int_{X} L_{E}\left(\bar{A}_{\mu}\right)\right\} \\
& =\int \mathcal{D} \bar{A}_{\mu} \int \mathcal{D} G^{a} \delta\left(G^{a}\right) \exp \left\{-\int_{X} L_{E}\left(\bar{A}_{\mu}\right)\right\} \\
& =\int \mathcal{D} \bar{A}_{\mu} \exp \left\{-\int_{X} L_{E}\left(\bar{A}_{\mu}\right)\right\} .
\end{aligned}
$$

In other words, the result seems to exhibit no dependence on the particular choice of $G^{a} .{ }^{13}$

Given that the outcome is independent of $G^{a}$, it is conventional and convenient to replace $\delta\left(G^{a}\right)$ by $\delta\left(G^{a}-f^{a}\right)$, where $f^{a}$ is some $A_{\mu}^{a}$-independent function, and then to average over the $f^{a}$ 's with a Gaussian weight. This implies writing

$$
\begin{aligned}
\delta\left(G^{a}\right) & \rightarrow \int \mathcal{D} f^{a} \delta\left(G^{a}-f^{a}\right) \exp \left(-\frac{1}{2 \xi} \int_{X} f^{a} f^{a}\right) \\
& =\exp \left(-\frac{1}{2 \xi} \int_{X} G^{a} G^{a}\right),
\end{aligned}
$$

where an arbitrary parameter, $\xi$, has been introduced. Its presence at intermediate stages of a perturbative calculation permits for a very efficient and non-trivial crosscheck, since all dependence on it must vanish in the final results for physical (gauge invariant) quantities.

\footnotetext{
${ }^{13}$ The arguments presented are heuristic in nature. The manipulations can be given a precise meaning in lattice regularization, where the integration measure is well defined as the gauge invariant Haar measure on $\mathrm{SU}\left(N_{\mathrm{c}}\right)$.
} 
Finally, the other structure in eq. (5.35), namely the determinant, can be written in terms of Faddeev-Popov ghosts [5.4], making use of eq. (4.47),

$$
\operatorname{det}(M)=\int \mathcal{D} \bar{c} \mathcal{D} c \exp (-\bar{c} M c)
$$

Given that the "matrix" $\delta G^{a} / \delta \theta^{b}$ is purely bosonic, ghost fields should obey the same boundary conditions as gauge fields, i.e. be periodic in spite of their Grassmann nature.

In total, then, we can write the gauge-fixed version of eq. (5.34), adding now also Dirac fermions to complete the theory into $\mathrm{QCD}$. The result reads

$$
\begin{aligned}
\mathcal{Z}_{\text {phys }} & =C \int_{\text {periodic }} \mathcal{D} A_{0}^{a} \mathcal{D} A_{k}^{a} \int_{\text {periodic }} \mathcal{D} \bar{c}^{a} \mathcal{D} c^{a} \int_{\text {anti-periodic }} \mathcal{D} \bar{\psi} \mathcal{D} \psi \\
& \times \exp \left\{-\int_{0}^{\beta} \mathrm{d} \tau \int_{\mathbf{x}}\left[\frac{1}{4} F_{\mu \nu}^{a} F_{\mu \nu}^{a}+\frac{1}{2 \xi} G^{a} G^{a}+\bar{c}^{a}\left(\frac{\delta G^{a}}{\delta \theta^{b}}\right) c^{b}+\bar{\psi}\left(\gamma_{\mu} D_{\mu}+m\right) \psi\right]\right\}
\end{aligned}
$$

where we have on purpose simplified the quark mass term by assuming the existence of one flavourdegenerate mass $m .{ }^{14}$ We remark again, although do not prove here, that the argument of the exponent in eq. (5.39) is invariant under BRST symmetry. It will turn out to be convenient to make a particular choice for the functions $G^{a}$ by selecting covariant gauges, defined by

$$
\begin{aligned}
G^{a} & \equiv-\partial_{\mu} A_{\mu}^{a}, \\
\frac{1}{2 \xi} G^{a} G^{a} & =\frac{1}{2 \xi} \partial_{\mu} A_{\mu}^{a} \partial_{\nu} A_{\nu}^{a}, \\
\frac{\delta G^{a}}{\delta \theta^{b}} & =+\overleftarrow{\partial}_{\mu} \frac{\delta A_{\mu}^{a}}{\delta \theta^{b}}=\overleftarrow{\partial}_{\mu}\left[\vec{\partial}_{\mu} \delta^{a b}+g f^{a c b} A_{\mu}^{c}\right], \\
\bar{c}^{a}\left(\frac{\delta G^{a}}{\delta \theta^{b}}\right) c^{b} & =\partial_{\mu} \bar{c}^{a} \partial_{\mu} c^{a}+g f^{a b c} \partial_{\mu} \bar{c}^{a} A_{\mu}^{b} c^{c} .
\end{aligned}
$$

Here we made use of eqs. (5.2) and (5.6).

\section{Feynman rules for Euclidean continuum QCD}

For completeness, we now collect together the Feynman rules that apply to computations within the theory defined by eq. (5.39), when the gauge is fixed according to eq. (5.40).

Consider first the free (quadratic) part of the Euclidean action. Expressing everything in the Fourier representation, this becomes

$$
\begin{aligned}
S_{E, 0} & =\oint_{P Q} \delta(P+Q)\left\{\frac{1}{2} i P_{\mu} \tilde{A}_{\nu}^{a}(P)\left[i Q_{\mu} \tilde{A}_{\nu}^{a}(Q)-i Q_{\nu} \tilde{A}_{\mu}^{a}(Q)\right]+\frac{1}{2 \xi} i P_{\mu} \tilde{A}_{\mu}^{a}(P) i Q_{\nu} \tilde{A}_{\nu}^{a}(Q)\right\} \\
& +\oint_{P Q} \delta(-P+Q)\left[-i P_{\mu} \tilde{\bar{c}}^{a}(P) i Q_{\mu} \tilde{c}^{a}(Q)\right]+\oint_{\{P Q\}} \delta(-P+Q) \tilde{\bar{\psi}}_{A}(P)\left[i \gamma_{\mu} Q_{\mu}+m\right] \tilde{\psi}_{A}(Q) \\
& =\sum_{P Q} \delta(P+Q)\left\{\frac{1}{2} \tilde{A}_{\mu}^{a}(P) \tilde{A}_{\nu}^{a}(Q)\left[P^{2} \delta_{\mu \nu}-\left(1-\frac{1}{\xi}\right) P_{\mu} P_{\nu}\right]\right\} \\
& +\oint_{P Q} \delta(-P+Q)\left[\tilde{\bar{c}}^{a}(P) \tilde{c}^{a}(Q) P^{2}\right]+\oint_{\{P Q\}} \delta(-P+Q) \tilde{\bar{\psi}}_{A}(P)[i \not P+m] \tilde{\psi}_{A}(Q),
\end{aligned}
$$

\footnotetext{
${ }^{14}$ A more general Euclidean Lagrangian, incorporating all fields of the Standard Model, is given on p. 223.
} 
where the index $A$ for the quarks is assumed to comprise both colour and flavour indices, whereas in the Dirac space $\bar{\psi}$ and $\psi$ are treated as vectors. The propagators are obtained by inverting the matrices in this expression:

$$
\begin{aligned}
\left\langle\tilde{A}_{\mu}^{a}(P) \tilde{A}_{\nu}^{b}(Q)\right\rangle_{0} & =\delta^{a b} \delta(P+Q)\left[\frac{\delta_{\mu \nu}-\frac{P_{\mu} P_{\nu}}{P^{2}}}{P^{2}}+\frac{\frac{\xi P_{\mu} P_{\nu}}{P^{2}}}{P^{2}}\right], \\
\left\langle\tilde{c}^{a}(P) \tilde{\bar{c}}^{b}(Q)\right\rangle_{0} & =\delta^{a b} \delta(P-Q) \frac{1}{P^{2}} \\
\left\langle\tilde{\psi}_{A}(P) \tilde{\bar{\psi}}_{B}(Q)\right\rangle_{0} & =\delta_{A B} \delta(P-Q) \frac{-i \not P+m}{P^{2}+m^{2}} .
\end{aligned}
$$

Finally, we list the interactions, which are most conveniently written in a maximally symmetric form, obtained through changes of integration and summation variables. Thereby the three-gluon vertex becomes

$$
\begin{aligned}
S_{I}^{(A A A)}= & \int_{X} \frac{1}{2}\left(\partial_{\mu} A_{\nu}^{a}-\partial_{\nu} A_{\mu}^{a}\right) g f^{a b c} A_{\mu}^{b} A_{\nu}^{c} \\
= & \sum_{P Q R} \frac{1}{3 !} \tilde{A}_{\mu}^{a}(P) \tilde{A}_{\nu}^{b}(Q) \tilde{A}_{\rho}^{c}(R) \delta(P+Q+R) \\
& \quad \times i g f^{a b c}\left[\delta_{\mu \rho}\left(P_{\nu}-R_{\nu}\right)+\delta_{\rho \nu}\left(R_{\mu}-Q_{\mu}\right)+\delta_{\nu \mu}\left(Q_{\rho}-P_{\rho}\right)\right],
\end{aligned}
$$

the four-gluon vertex

$$
\begin{aligned}
& S_{I}^{(A A A A)}=\int_{X} \frac{1}{4} g^{2} f^{a b c} f^{a d e} A_{\mu}^{b} A_{\nu}^{c} A_{\mu}^{d} A_{\nu}^{e} \\
& \quad=\sum_{P Q R S} \frac{1}{4 !} \tilde{A}_{\mu}^{a}(P) \tilde{A}_{\nu}^{b}(Q) \tilde{A}_{\rho}^{c}(R) \tilde{A}_{\sigma}^{d}(S) \delta(P+Q+R+S) \\
& \quad \times g^{2}\left[f^{e a b} f^{e c d}\left(\delta_{\mu \rho} \delta_{\nu \sigma}-\delta_{\mu \sigma} \delta_{\nu \rho}\right)+f^{e a c} f^{e b d}\left(\delta_{\mu \nu} \delta_{\rho \sigma}-\delta_{\mu \sigma} \delta_{\nu \rho}\right)+f^{e a d} f^{e b c}\left(\delta_{\mu \nu} \delta_{\rho \sigma}-\delta_{\mu \rho} \delta_{\nu \sigma}\right)\right],
\end{aligned}
$$

the ghost interaction

$$
\begin{aligned}
S_{I}^{(\bar{c} A c)} & =\int_{X} \partial_{\mu} \bar{c}^{a} g f^{a b c} A_{\mu}^{b} c^{c} \\
& =\oint_{P Q R} \tilde{\tilde{c}}^{a}(P) \tilde{A}_{\mu}^{b}(Q) \tilde{c}^{c}(R) \delta(-P+Q+R)\left(-i g f^{a b c} P_{\mu}\right),
\end{aligned}
$$

and finally the fermion interaction

$$
\begin{aligned}
S_{I}^{(\bar{\psi} A \psi)} & =\int_{X} \bar{\psi}_{A} \gamma_{\mu}\left(-i g T_{A B}^{a}\right) A_{\mu}^{a} \psi_{B} \\
& =\sum_{Q\{P R\}} \tilde{\bar{\psi}}_{A}(P) \gamma_{\mu} \tilde{A}_{\mu}^{a}(Q) \tilde{\psi}_{B}(R) \delta(-P+Q+R)\left(-i g T_{A B}^{a}\right) .
\end{aligned}
$$

\section{Appendix A: Non-Abelian black-body radiation in the free limit}

In this appendix, we compute the free energy density $f(T)$ for $N_{\mathrm{c}}$ colours of free gluons and $N_{\mathrm{f}}$ flavours of massless quarks, starting from eq. (5.44), and use the outcome to deduce a result for the usual electromagnetic blackbody radiation. This is an interesting exercise because, inspite of us being in the free limit, ghosts turn out to play a role at finite temperature. 
To start with, we recall from eqs. (2.51), (2.81), (4.60) and (4.73) that

$$
\begin{aligned}
& J(0, T)=\frac{1}{2} \oiint_{P}\left[\ln \left(P^{2}\right)-\text { const. }\right]=-\frac{\pi^{2} T^{4}}{90}, \\
& \tilde{J}(0, T)=\frac{1}{2} \oiint_{\{P\}}\left[\ln \left(P^{2}\right)-\text { const. }\right]=\frac{7}{8} \frac{\pi^{2} T^{4}}{90} .
\end{aligned}
$$

Our task is to figure out the prefactors of these terms, corresponding to the contributions of gluons, ghosts and quarks.

In the gluonic case, we are faced with the matrix

$$
M_{\mu \nu}=P^{2} \delta_{\mu \nu}-\left(1-\frac{1}{\xi}\right) P_{\mu} P_{\nu}
$$

which is conveniently handled by introducing two further matrices,

$$
\mathbb{P}_{\mu \nu}^{\mathrm{T}} \equiv \delta_{\mu \nu}-\frac{P_{\mu} P_{\nu}}{P^{2}}, \quad \mathbb{P}_{\mu \nu}^{\mathrm{L}} \equiv \frac{P_{\mu} P_{\nu}}{P^{2}} .
$$

As matrices, these satisfy $\mathbb{P}^{\mathrm{T}} \mathbb{P}^{\mathrm{T}}=\mathbb{P}^{\mathrm{T}}, \mathbb{P}^{\mathrm{L}} \mathbb{P}^{\mathrm{L}}=\mathbb{P}^{\mathrm{L}}, \mathbb{P}^{\mathrm{T}} \mathbb{P}^{\mathrm{L}}=0, \mathbb{P}^{\mathrm{T}}+\mathbb{P}^{\mathrm{L}}=\mathbb{1}$, making them projection operators and implying that their eigenvalues are either zero or unity. The numbers of the unit eigenvalues can furthermore be found by taking the appropriate traces: $\operatorname{Tr}\left[\mathbb{P}^{\mathrm{T}}\right]=$ $\delta_{\mu \mu}-1=d, \operatorname{Tr}\left[\mathbb{P}^{\mathrm{L}}\right]=1$.

We can clearly write

$$
M_{\mu \nu}=P^{2} \mathbb{P}_{\mu \nu}^{\mathrm{T}}+\frac{1}{\xi} P^{2} \mathbb{P}_{\mu \nu}^{\mathrm{L}},
$$

from which we see that $M$ has $d$ eigenvalues of $P^{2}$ and one $P^{2} / \xi$. Also, there are $a=1, \ldots, N_{\mathrm{c}}^{2}-1$ copies of this structure, so that in total

$$
\begin{aligned}
\left.f(T)\right|_{\text {gluons }} & =\left(N_{\mathrm{c}}^{2}-1\right)\left\{d \times \frac{1}{2} \sum_{P}\left[\ln \left(P^{2}\right)-\text { const. }\right]+\frac{1}{2} \sum_{P}\left[\ln \left(\frac{1}{\xi} P^{2}\right)-\text { const. }\right]\right\} \\
& =\left(N_{\mathrm{c}}^{2}-1\right)\left\{-\frac{1}{2} \sum_{P} \ln (\xi)+(d+1) J(0, T)\right\} .
\end{aligned}
$$

The first term vanishes in dimensional regularization, because it contains no scales.

For the ghosts, the Gaussian integral yields (cf. eq. (4.47))

$$
\int \prod_{a} \mathrm{~d} \tilde{\bar{c}}^{a} \mathrm{~d} \tilde{c}^{a} \exp \left(-\tilde{\bar{c}}^{a} P^{2} \tilde{c}^{a}\right)=\prod_{a} P^{2}=\exp \left\{-\left[-2\left(N_{\mathrm{c}}^{2}-1\right) \frac{1}{2} \ln \left(P^{2}\right)\right]\right\} .
$$

Recalling that ghosts obey periodic boundary conditions, we obtain from here

$$
\left.f(T)\right|_{\text {ghosts }}=-2\left(N_{\mathrm{c}}^{2}-1\right) J(0, T) .
$$

Finally, quarks function as in eq. (4.52), except that they now come in $N_{\mathrm{c}}$ colours and $N_{\mathrm{f}}$ flavours, giving

$$
\left.f(T)\right|_{\text {quarks }}=-4 N_{\mathrm{c}} N_{\mathrm{f}} \tilde{J}(0, T) .
$$

Summing together eqs. (5.57), (5.59) and (5.60), inserting the values of $J$ and $\tilde{J}$ from eqs. (5.52) and (5.53), and setting $d=3$, we get

$$
\left.f(T)\right|_{\mathrm{QCD}}=-\frac{\pi^{2} T^{4}}{90}\left[2\left(N_{\mathrm{c}}^{2}-1\right)+\frac{7}{2} N_{\mathrm{f}} N_{\mathrm{c}}\right] .
$$


This result is often referred to as (the QCD-version of) the Stefan-Boltzmann law.

It is important to realize that the contribution from the ghosts was essential above: according to eq. (5.59), it cancels half of the result in eq. (5.57), thereby yielding the correct number of physical degrees of freedom in a massless gauge field as the multiplier in eq. (5.61). In addition, the assumption that $A_{0}^{a}$ is periodic has played a role: had it also had an antiperiodic part, eq. (5.61) would have received a further unphysical term.

To finish the section, we finally note that the case of QED can be obtained by setting $N_{\mathrm{c}} \rightarrow 1$ and $N_{\mathrm{c}}^{2}-1 \rightarrow 1$, recalling that the gauge group of QED is $\mathrm{U}(1)$. This produces

$$
\left.f(T)\right|_{\mathrm{QED}}=-\frac{\pi^{2} T^{4}}{90}\left(2+\frac{7}{2} N_{\mathrm{f}}\right)
$$

where the factor 2 inside the square brackets corresponds to the two photon polarizations, and the factor 4 multiplying $\frac{7}{8} N_{\mathrm{f}}$ to the degrees of freedom of a spin- $\frac{1}{2}$ particle and a spin- $\frac{1}{2}$ antiparticle. If left-handed neutrinos were to be included here, they would contribute an additional term of $2 \times \frac{7}{8} N_{\mathrm{f}}=\frac{7}{4} N_{\mathrm{f}}$. Eq. (5.62) together with the contribution of the neutrinos gives the free energy density determining the expansion rate of the universe for temperatures in the $\mathrm{MeV}$ range.

\section{Appendix B: 2-point correlator of magnetic field fluctuations}

As a second illustration of perturbative computations with thermal gauge fields, we consider the correlation function of magnetic fields in QED. This discussion bears a resemblance to that for scalar fields at the end of sec. 3.1, with the difference that the magnetic field involves derivatives. There is a clear physics motivation now, in that correlators of magnetic fields play an important role in astrophysics, where they are measureable to an extent (cf., e.g., ref. [5.5]).

In analogy with eq. (3.39), let us consider the "equal-time" $\left(x_{0}=y_{0}\right)$ correlator

$$
G_{i j}(\mathbf{x}-\mathbf{y}) \equiv\left\langle\mathcal{B}_{i}(\mathbf{x}) \mathcal{B}_{j}(\mathbf{y})\right\rangle_{0},
$$

where the magnetic field is defined as $\mathcal{B}_{i} \equiv \frac{1}{2} \epsilon_{i j k} F_{j k}=\epsilon_{i j k} \partial_{j} A_{k}$, with $A_{k}$ denoting an Abelian gauge field. Going to momentum space and inserting the propagator from eq. (5.45), eq. (5.63) becomes

$$
\begin{aligned}
G_{i j}(\mathbf{x}-\mathbf{y}) & =\oiint_{P, Q} \epsilon_{i k l} \epsilon_{j m n}\left(-i p_{k}\right)\left(-i q_{m}\right) e^{-i(\mathbf{p} \cdot \mathbf{x}+\mathbf{q} \cdot \mathbf{y})}\left\langle\tilde{A}_{l}(P) \tilde{A}_{n}(Q)\right\rangle_{0} \\
& =\oiint_{P} \epsilon_{i k l} \epsilon_{j m n} p_{k} p_{m} e^{i \mathbf{p} \cdot(\mathbf{y}-\mathbf{x})}\left[\frac{\delta_{l n}}{P^{2}}+\frac{(\xi-1) p_{l} p_{n}}{\left(P^{2}\right)^{2}}\right] .
\end{aligned}
$$

The antisymmetry of the Levi-Civita symbol implies that the longitudinal part, proportional to $p_{l} p_{n}$, drops out, and simultaneously with it any dependence on the gauge parameter $\xi$. Employing $\epsilon_{i k l} \epsilon_{j m n} \delta_{l n}=\delta_{i j} \delta_{k m}-\delta_{i m} \delta_{k j}$, and pulling spatial momenta in front of the sum-integration, we get

$$
G_{i j}(\mathbf{x}-\mathbf{y})=\left(\partial_{i} \partial_{j}-\delta_{i j} \nabla^{2}\right) \sum_{P} \frac{e^{i \mathbf{p} \cdot(\mathbf{y}-\mathbf{x})}}{p_{n}^{2}+p^{2}},
$$

where the derivatives operate on $\mathbf{x}$. The Matsubara sum can be carried out, with the help of eq. (2.41), however it is actually helpful to first carry out the integral, according to eq. (3.46), and the Matsubara sum only afterwards (it is typical of thermal computations that, depending on the 
problem, one or the other ordering may offer for a nice simplification). Showing the first steps in both directions, we get

$$
\begin{aligned}
& G_{i j}(\mathbf{x}-\mathbf{y}) \stackrel{\stackrel{(2.41)}{=}}{=}\left(\partial_{i} \partial_{j}-\delta_{i j} \nabla^{2}\right) \int_{\mathbf{p}} e^{i \mathbf{p} \cdot(\mathbf{y}-\mathbf{x})} \frac{1+2 n_{\mathrm{B}}(p)}{2 p} \\
& \stackrel{(3.46)}{=} \quad\left(\partial_{i} \partial_{j}-\delta_{i j} \nabla^{2}\right) T \sum_{p_{n}} \frac{e^{-\left|p_{n}\right||\mathbf{x}-\mathbf{y}|}}{4 \pi|\mathbf{x}-\mathbf{y}|} \\
&=\left(\partial_{i} \partial_{j}-\delta_{i j} \nabla^{2}\right) \frac{T}{4 \pi|\mathbf{x}-\mathbf{y}|}\left[-1+\frac{2}{1-e^{-2 \pi T|\mathbf{x}-\mathbf{y}|}}\right] \\
&=\left(\partial_{i} \partial_{j}-\delta_{i j} \nabla^{2}\right) \frac{T \operatorname{coth}(\pi T|\mathbf{x}-\mathbf{y}|)}{4 \pi|\mathbf{x}-\mathbf{y}|} .
\end{aligned}
$$

The function here only depends on $r \equiv|\mathbf{x}-\mathbf{y}|$, so the partial derivatives can be expressed as

$$
\begin{aligned}
& \partial_{i} \partial_{j}=\frac{r_{i} r_{j} \partial_{r}^{2}}{r^{2}}+\left(\delta_{i j}-\frac{r_{i} r_{j}}{r^{2}}\right) \frac{\partial_{r}}{r}, \quad \nabla^{2}=\partial_{r}^{2}+\frac{2 \partial_{r}}{r} \\
\Longrightarrow & \partial_{i} \partial_{j}-\delta_{i j} \nabla^{2}=\frac{r_{i} r_{j}}{r^{2}}\left(\partial_{r}^{2}-\frac{\partial_{r}}{r}\right)-\delta_{i j}\left(\partial_{r}^{2}+\frac{\partial_{r}}{r}\right) .
\end{aligned}
$$

The radial derivatives could be taken, however that does not make the physics more transparent.

There are a few lessons to be learned from eq. (5.66). First, if we consider the limit of short distances, then $\operatorname{coth}(\pi T|\mathbf{x}-\mathbf{y}|) \rightarrow 1 /(\pi T|\mathbf{x}-\mathbf{y}|)$, and $\frac{T \operatorname{coth}(\pi T|\mathbf{x}-\mathbf{y}|)}{4 \pi|\mathbf{x}-\mathbf{y}|} \rightarrow 1 /\left(4 \pi^{2}|\mathbf{x}-\mathbf{y}|^{2}\right)$. This is just the vacuum behaviour from eq. (3.44). So, once again, thermal effects are not important at short distances. At large distances, in turn, $\operatorname{coth}(\pi T|\mathbf{x}-\mathbf{y}|) \rightarrow 1$, and $\frac{T \operatorname{coth}(\pi T|\mathbf{x}-\mathbf{y}|)}{4 \pi|\mathbf{x}-\mathbf{y}|} \rightarrow T /(4 \pi|\mathbf{x}-\mathbf{y}|)$. This represents the same physics as discussed below eq. (3.46). Incidentally, in terms of the Matsubara sum in eq. (5.66), the long-distance behaviour originates from the Matsubara zero mode, $p_{n}=0$. This anticipates its important role, as will be discussed in sec. 6.1. 


\subsection{Thermal gluon mass}

We consider next the gauge field propagator, in particular the Matsubara zero-mode sector thereof. We wish to see whether an effective thermal mass $m_{\text {eff }}$ is generated for this field mode, as was the case for a scalar field (cf. eq. (3.95)). The observable to consider is the full propagator, i.e. the analogue of eq. (3.63). Note that we do not consider non-zero Matsubara modes since, like in eq. (3.94), the thermal mass corrections are parametrically subdominant if we assume the coupling to be weak, $g^{2} T^{2} \ll(2 \pi T)^{2}$. For the same reason, we do not need to consider thermal mass corrections for fermions at the present order.

In order to simplify the task somewhat, we choose to carry out the computation in the so-called Feynman gauge, $\xi \equiv 1$, whereby the free propagator of eq. (5.45) becomes

$$
\left\langle\tilde{A}_{\mu}^{a}(K) \tilde{A}_{\nu}^{b}(Q)\right\rangle_{0}=\delta^{a b} \delta(K+Q)\left[\frac{\delta_{\mu \nu}-\frac{K_{\mu} K_{\nu}}{K^{2}}}{K^{2}}+\frac{\frac{\xi K_{\mu} K_{\nu}}{K^{2}}}{K^{2}}\right] \stackrel{\xi=1}{=} \quad \delta^{a b} \delta(K+Q) \frac{\delta_{\mu \nu}}{K^{2}} .
$$

Specifically, our goal is to compute the 1-loop gluon self-energy $\Pi_{\mu \nu}$, defined via

$$
\frac{\left\langle\tilde{A}_{\mu}^{a}(K) \tilde{A}_{\nu}^{b}(Q) e^{-S_{\mathrm{I}}}\right\rangle_{0}}{\left\langle e^{\left.-S_{\mathrm{I}}\right\rangle_{0}}\right.}=\delta^{a b} \delta(K+Q)\left[\frac{\delta_{\mu \nu}}{K^{2}}-\frac{\Pi_{\mu \nu}(K)}{K^{4}}+\mathcal{O}\left(g^{4}\right)\right],
$$

where the role of the denominator of the left-hand side is to cancel the disconnected contributions.

At 1-loop level, there are several distinct contributions to $\Pi_{\mu \nu}$ : two of these involve gauge loops (via a quartic vertex from $-S_{I}$ and two cubic vertices from $+S_{I}^{2} / 2$, respectively), one involves a ghost loop (via two cubic vertices from $+S_{I}^{2} / 2$ ), and one a fermion loop (via two cubic vertices from $+S_{I}^{2} / 2$ ). If the theory were to contain additional scalar fields, then two additional graphs similar to the gauge loops would be generated. For future purposes we treat the external momentum $K$ of the graphs as a general Euclidean four-momentum, even though for the Matsubara zero modes (that we are ultimately interested in) only the spatial part is non-zero.

Let us begin by considering the gauge loop originating from a quartic vertex. Denoting the structure in eq. (5.49) by

$$
C_{\mu \nu \rho \sigma}^{a b c d} \equiv f^{e a b} f^{e c d}\left(\delta_{\mu \rho} \delta_{\nu \sigma}-\delta_{\mu \sigma} \delta_{\nu \rho}\right)+f^{e a c} f^{e b d}\left(\delta_{\mu \nu} \delta_{\rho \sigma}-\delta_{\mu \sigma} \delta_{\nu \rho}\right)+f^{e a d} f^{e b c}\left(\delta_{\mu \nu} \delta_{\rho \sigma}-\delta_{\mu \rho} \delta_{\nu \sigma}\right),
$$

we get

$$
\begin{aligned}
& \left\langle\tilde{A}_{\mu}^{a}(K) \tilde{A}_{\nu}^{b}(Q)\left(-S_{I}\right)\right\rangle_{0, \mathrm{c}}=\xi_{m^{2}}^{m^{m}} \xi \\
& =-\frac{g^{2}}{24}\left\langle\tilde{A}_{\mu}^{a}(K) \tilde{A}_{\nu}^{b}(Q) \sum_{R P T U} \tilde{A}_{\alpha}^{c}(R) \tilde{A}_{\beta}^{d}(P) \tilde{A}_{\rho}^{e}(T) \tilde{A}_{\sigma}^{f}(U) \delta(R+P+T+U) C_{\alpha \beta \rho \sigma}^{c d e f}\right\rangle_{0, \mathrm{c}} \\
& =-\frac{g^{2}}{2} \sum_{R P T U} \delta(R+P+T+U)\left\langle\tilde{A}_{\mu}^{a}(K) \tilde{A}_{\alpha}^{c}(R)\right\rangle_{0}\left\langle\tilde{A}_{\nu}^{b}(Q) \tilde{A}_{\beta}^{d}(P)\right\rangle_{0}\left\langle\tilde{A}_{\rho}^{e}(T) \tilde{A}_{\sigma}^{f}(U)\right\rangle_{0} C_{\alpha \beta \rho \sigma}^{c d e f},
\end{aligned}
$$

where we made use of the complete symmetry of $C_{\alpha \beta \rho \sigma}^{c d e f}$. Inserting here eq. (5.68), this becomes

$$
\begin{aligned}
\left\langle\tilde{A}_{\mu}^{a}(K) \tilde{A}_{\nu}^{b}(Q)\left(-S_{I}\right)\right\rangle_{0, \mathrm{c}}= & -\frac{g^{2}}{2} \oint_{R P T U} \delta(R+P+T+U) \delta(K+R) \delta(Q+P) \delta(T+U) \\
& \times \frac{1}{K^{2} Q^{2} T^{2}} \delta^{a c} \delta^{b d} \delta^{e f} \delta_{\mu \alpha} \delta_{\nu \beta} \delta_{\rho \sigma} C_{\alpha \beta \rho \sigma}^{c d e f} .
\end{aligned}
$$


The sum-integrals over $R, P, T$ in the above expression are trivially carried out, and amount to $R \rightarrow-K, P \rightarrow-Q, T \rightarrow-U$. The remaining constraint becomes $\delta(R+P+T+U) \rightarrow \delta(K+Q)$, whereby $Q \rightarrow-K$. Moreover, we note that

$$
\begin{aligned}
\delta^{a c} \delta^{b d} \delta^{e f} \delta_{\mu \alpha} \delta_{\nu \beta} \delta_{\rho \sigma} C_{\alpha \beta \rho \sigma}^{c d e f} & =\delta^{e f} \delta_{\rho \sigma}\left[f^{g a e} f^{g b f}\left(\delta_{\mu \nu} \delta_{\rho \sigma}-\delta_{\mu \sigma} \delta_{\nu \rho}\right)+f^{g a f} f^{g b e}\left(\delta_{\mu \nu} \delta_{\rho \sigma}-\delta_{\mu \rho} \delta_{\nu \sigma}\right)\right] \\
& =2 d f^{a g e} f^{b g e} \delta_{\mu \nu},
\end{aligned}
$$

where we made use of the antisymmetry of the structure constants, as well as of the fact that $\delta_{\sigma \sigma}=d+1=4-2 \epsilon$. Noting that the structure constants furthermore satisfy $f^{a g e} f^{b g e}=N_{\mathrm{c}} \delta^{a b}$, we get in total

$$
\left\langle\tilde{A}_{\mu}^{a}(K) \tilde{A}_{\nu}^{b}(Q)\left(-S_{I}\right)\right\rangle_{0, \mathrm{c}}=-g^{2} N_{\mathrm{c}} d I_{T}(0) \delta^{a b} \delta(K+Q) \frac{\delta_{\mu \nu}}{\left(K^{2}\right)^{2}}
$$

where $I_{T}(0)=\mathscr{\&}_{U} \frac{1}{U^{2}}$, cf. eqs. (2.54) and (2.56). Note that the $\delta$-functions as well as the colour and spacetime indices appear here just like in eq. (5.69), allowing us to straightforwardly read off the contribution of this graph to $\Pi_{\mu \nu}(K)$.

Next, we move on to the gluon loop originating from two cubic interaction vertices. Denoting the combination of $\delta$-functions and momenta in eq. (5.48) by

$$
D_{\mu \nu \rho}(P, Q, R) \equiv \delta_{\mu \rho}\left(P_{\nu}-R_{\nu}\right)+\delta_{\rho \nu}\left(R_{\mu}-Q_{\mu}\right)+\delta_{\nu \mu}\left(Q_{\rho}-P_{\rho}\right),
$$

we get for this contribution

$$
\begin{aligned}
\left\langle\tilde{A}_{\mu}^{a}(K) \tilde{A}_{\nu}^{b}(Q)\right. & \left.\left(\frac{1}{2} S_{I}^{2}\right)\right\rangle_{0, c}^{(1)}=\tilde{\xi}_{=-\frac{g^{2}}{72}}\left\langle\tilde{A}_{\mu}^{a}(K) \tilde{A}_{\nu}^{b}(Q) \sum_{R P T} \tilde{A}_{\alpha}^{c}(R) \tilde{A}_{\beta}^{d}(P) \tilde{A}_{\gamma}^{e}(T) \sum_{U V X} \tilde{A}_{\zeta}^{g}(U) \tilde{A}_{\eta}^{h}(V) \tilde{A}_{\rho}^{i}(X)\right\rangle_{0, c} \\
& \times f^{c d e} f^{g h i} \delta(R+P+T) \delta(U+V+X) D_{\alpha \beta \gamma}(R, P, T) D_{\zeta \eta \rho}(U, V, X) \\
=- & \frac{g^{2}}{2} \sum_{R P T U V X}\left\langle\tilde{A}_{\mu}^{a}(K) \tilde{A}_{\alpha}^{c}(R)\right\rangle_{0}\left\langle\tilde{A}_{\nu}^{b}(Q) \tilde{A}_{\zeta}^{g}(U)\right\rangle_{0}\left\langle\tilde{A}_{\beta}^{d}(P) \tilde{A}_{\eta}^{h}(V)\right\rangle_{0}\left\langle\tilde{A}_{\gamma}^{e}(T) \tilde{A}_{\rho}^{i}(X)\right\rangle_{0} \\
& \times f^{c d e} f^{g h i} \delta(R+P+T) \delta(U+V+X) D_{\alpha \beta \gamma}(R, P, T) D_{\zeta \eta \rho}(U, V, X),
\end{aligned}
$$

where we made use of the complete symmetry of $f^{c d e} D_{\alpha \beta \gamma}(R, P, T)$ in simultaneous interchanges of all indices labelling a particular gauge field (for instance $c, \alpha, R \leftrightarrow d, \beta, P$ ).

Inserting eq. (5.68) into the above expression, let us inspect in turn the colour indices, spacetime indices, and momenta. The colour contractions are easily carried out, and result in the overall factor

$$
\delta^{a c} \delta^{b g} \delta^{d h} \delta^{e i} f^{c d e} f^{g h i}=f^{a d e} f^{b d e}=N_{\mathrm{c}} \delta^{a b} .
$$

The spacetime contractions can all be transported to the $D$-functions, noting that the effect can be summarized with the substitution rules $\alpha \rightarrow \mu, \zeta \rightarrow \nu, \eta \rightarrow \beta, \rho \rightarrow \gamma$. The momenta get fixed as $R \rightarrow-K, U \rightarrow-Q, V \rightarrow-P, X \rightarrow-T$, whereby

$$
\delta(R+P+T) \delta(U+V+X) \rightarrow \delta(-K+P+T) \delta(-Q-P-T)=\delta(-K+P+T) \delta(K+Q) .
$$

Then $T \rightarrow K-P$, and

$$
D_{\mu \beta \gamma}(R, P, T) D_{\nu \beta \gamma}(U, V, X) \rightarrow D_{\mu \beta \gamma}(-K, P, K-P) D_{\nu \beta \gamma}(K,-P, P-K) .
$$


Only the sum-integral over $P$ is left over, giving us

$$
\begin{aligned}
& \left\langle\tilde{A}_{\mu}^{a}(K) \tilde{A}_{\nu}^{b}(Q)\left(\frac{1}{2} S_{I}^{2}\right)\right\rangle_{0, \mathrm{c}}^{(1)} \\
& =-\frac{g^{2} N_{\mathrm{c}}}{2} \frac{\delta^{a b} \delta(K+Q)}{\left(K^{2}\right)^{2}} \oint_{P} \frac{1}{P^{2}(K-P)^{2}} D_{\mu \beta \gamma}(-K, P, K-P) D_{\nu \beta \gamma}(K,-P,-K+P) .
\end{aligned}
$$

Finally, we are faced with the tedious task of inserting eq. (5.75) into the above expression and carrying out all contractions - a task most conveniently handled using programming languages intended for carrying out symbolic manipulations, such as FORM [5.6]. Here we perform the contractions by hand, obtaining first

$$
\begin{aligned}
D_{\mu \beta \gamma}( & -K, P, K-P) D_{\nu \beta \gamma}(K,-P,-K+P) \\
= & -\left[\delta_{\mu \gamma}\left(-2 K_{\beta}+P_{\beta}\right)+\delta_{\gamma \beta}\left(K_{\mu}-2 P_{\mu}\right)+\delta_{\beta \mu}\left(K_{\gamma}+P_{\gamma}\right)\right] \\
& \times\left[\delta_{\nu \gamma}\left(-2 K_{\beta}+P_{\beta}\right)+\delta_{\gamma \beta}\left(K_{\nu}-2 P_{\nu}\right)+\delta_{\beta \nu}\left(K_{\gamma}+P_{\gamma}\right)\right] \\
= & -\delta_{\mu \nu}\left(4 K^{2}-4 K \cdot P+P^{2}+K^{2}+2 K \cdot P+P^{2}\right) \\
& -(d+1)\left(K_{\mu} K_{\nu}-2 K_{\mu} P_{\nu}-2 K_{\nu} P_{\mu}+4 P_{\mu} P_{\nu}\right) \\
& -\left[\left(-2 K_{\mu}+P_{\mu}\right)\left(K_{\nu}-2 P_{\nu}\right)+\left(-2 K_{\mu}+P_{\mu}\right)\left(K_{\nu}+P_{\nu}\right)+\left(K_{\mu}-2 P_{\mu}\right)\left(K_{\nu}+P_{\nu}\right)+(\mu \leftrightarrow \nu)\right] \\
= & -\delta_{\mu \nu}\left[4 K^{2}+(K-P)^{2}+P^{2}\right]-(d-5) K_{\mu} K_{\nu}+(2 d-1)\left(K_{\mu} P_{\nu}+K_{\nu} P_{\mu}\right)-(4 d-2) P_{\mu} P_{\nu} .
\end{aligned}
$$

Because the propagators in eq. (5.79) are identical, we can furthermore simplify the structure $K_{\mu} P_{\nu}+K_{\nu} P_{\mu}$ by renaming one of the integration variables as $P \rightarrow K-P$ in "one half of this term", i.e. by writing

$$
\begin{aligned}
K_{\mu} P_{\nu}+K_{\nu} P_{\mu} & \rightarrow \frac{1}{2}\left[K_{\mu} P_{\nu}+K_{\nu} P_{\mu}+K_{\mu}\left(K_{\nu}-P_{\nu}\right)+K_{\nu}\left(K_{\mu}-P_{\mu}\right)\right] \\
& =K_{\mu} K_{\nu} .
\end{aligned}
$$

Therefore a representation equivalent to eq. (5.80) is

$$
\begin{aligned}
& D_{\mu \beta \gamma}(-K, P, K-P) D_{\nu \beta \gamma}(K,-P,-K+P) \\
& \quad \rightarrow \quad-\delta_{\mu \nu}\left[4 K^{2}+(K-P)^{2}+P^{2}\right]+(d+4) K_{\mu} K_{\nu}-(4 d-2) P_{\mu} P_{\nu} .
\end{aligned}
$$

Inserting now eq. (5.82) into eq. (5.79), we observe that the result depends in a non-trivial way on the "external" momentum $K$. This is an important fact that plays a role later on. For the moment, we however note that since the tree-level gluon propagator of eq. (5.68) is massless, the leading order pole position lies at $K^{2}=0$. This may get shifted by the loop corrections that we are currently investigating, like in the case of a scalar field theory (cf. eq. (3.95)). Since this correction is suppressed by a factor of $\mathcal{O}\left(g^{2}\right)$, in our perturbative calculation we may insert $K=0$ in eq. (5.82), making only an error of $\mathcal{O}\left(g^{4}\right)$. Proceeding this way, we get

$$
\left\langle\tilde{A}_{\mu}^{a}(K) \tilde{A}_{\nu}^{b}(Q)\left(\frac{1}{2} S_{I}^{2}\right)\right\rangle_{0, \mathrm{c}}^{(1)} \approx \frac{g^{2} N_{\mathrm{c}}}{2} \frac{\delta^{a b} \delta(K+Q)}{\left(K^{2}\right)^{2}} \oint_{P} \frac{1}{\left(P^{2}\right)^{2}}\left[2 P^{2} \delta_{\mu \nu}+(4 d-2) P_{\mu} P_{\nu}\right] .
$$

Now, symmetries tell us that the integral in eq. (5.83) can only depend on two second rank tensors, $\delta_{\mu \nu}$ and $\delta_{\mu 0} \delta_{\nu 0}$, of which the latter originates from the breaking of Lorentz symmetry by 
the rest frame of the heat bath. Denoting $P=\left(p_{n}, \mathbf{p}\right)$, this allows us to split the latter term into two parts according to (note that $\delta_{\mu i} \delta_{\nu i}=\delta_{\mu \nu}-\delta_{\mu 0} \delta_{\nu 0}$ )

$$
\begin{aligned}
\oiint_{P} \frac{P_{\mu} P_{\nu}}{\left(P^{2}\right)^{2}} & =\delta_{\mu 0} \delta_{\nu 0} \oiint_{P} \frac{P_{0}^{2}}{\left(P^{2}\right)^{2}}+\delta_{\mu i} \delta_{\nu i} \oint_{P} \frac{P_{i}^{2}}{\left(P^{2}\right)^{2}} \\
& =\delta_{\mu 0} \delta_{\nu 0} \oiint_{P} \frac{p_{n}^{2}}{\left(P^{2}\right)^{2}}+\delta_{\mu i} \delta_{\nu i} \frac{1}{d} \oiint_{P} \frac{p^{2}}{\left(P^{2}\right)^{2}} \\
& =\delta_{\mu 0} \delta_{\nu 0} \oiint_{P} \frac{p_{n}^{2}}{\left(P^{2}\right)^{2}}+\delta_{\mu i} \delta_{\nu i} \frac{1}{d} \oiint_{P} \frac{P^{2}-p_{n}^{2}}{\left(P^{2}\right)^{2}} .
\end{aligned}
$$

At this point, let us inspect the familiar sum-integral (cf. eq. (2.92))

$$
I_{T}(0)=\sum_{P} \frac{1}{P^{2}}=T \sum_{n=-\infty}^{\infty} \int_{\mathbf{p}} \frac{1}{(2 \pi n T)^{2}+p^{2}}=\frac{T^{2}}{12}+\mathcal{O}(\epsilon) .
$$

Taking the derivative $T^{2} \frac{\mathrm{d}}{\mathrm{d} T^{2}}=\frac{T}{2} \frac{\mathrm{d}}{\mathrm{d} T}$ on both sides, we find

$$
\frac{T}{2} \sum_{n=-\infty}^{\infty} \int_{\mathbf{p}} \frac{1}{(2 \pi n T)^{2}+p^{2}}-T \sum_{n=-\infty}^{\infty} \int_{\mathbf{p}} \frac{(2 \pi n T)^{2}}{\left[(2 \pi n T)^{2}+\mathbf{p}^{2}\right]^{2}}=\frac{T^{2}}{12}+\mathcal{O}(\epsilon)
$$

which can be used in order to solve for the only unknown sum-integral in eq. (5.84),

$$
\oiint_{P} \frac{p_{n}^{2}}{\left(P^{2}\right)^{2}}=-\frac{I_{T}(0)}{2}+\mathcal{O}(\epsilon) .
$$

Inserting this result into eq. (5.84), we thereby obtain in $d=3-2 \epsilon$ dimensions

$$
\oint_{P} \frac{P_{\mu} P_{\nu}}{\left(P^{2}\right)^{2}}=\frac{1}{2}\left(-\delta_{\mu 0} \delta_{\nu 0}+\delta_{\mu i} \delta_{\nu i}\right) \frac{T^{2}}{12}+\mathcal{O}(\epsilon)
$$

which turns eq. (5.83) finally into

$$
\begin{aligned}
\left\langle\tilde{A}_{\mu}^{a}(K) \tilde{A}_{\nu}^{b}(Q)\left(\frac{1}{2} S_{I}^{2}\right)\right\rangle_{0, \mathrm{c}}^{(1)} \approx \frac{g^{2} N_{\mathrm{c}}}{2} \frac{\delta^{a b} \delta(K+Q)}{\left(K^{2}\right)^{2}}\left\{\delta_{\mu 0} \delta_{\nu 0}\left[2-\frac{1}{2}(4 d-2)\right]\right. \\
\left.+\delta_{\mu i} \delta_{\nu i}\left[2+\frac{1}{2}(4 d-2)\right]\right\} I_{T}(0)+\mathcal{O}(\epsilon) \\
\stackrel{d=3-2 \epsilon}{=} \frac{g^{2} N_{\mathrm{c}}}{2} \frac{\delta^{a b} \delta(K+Q)}{\left(K^{2}\right)^{2}}\left\{-3 \delta_{\mu 0} \delta_{\nu 0}+7 \delta_{\mu i} \delta_{\nu i}\right\} \frac{T^{2}}{12}+\mathcal{O}(\epsilon) .
\end{aligned}
$$

Moving on to the ghost loop, we apply the vertex of eq. (5.50) but otherwise proceed as in eq. (5.76). This produces

$$
\begin{aligned}
& \left\langle\tilde{A}_{\mu}^{a}(K) \tilde{A}_{\nu}^{b}(Q)\left(\frac{1}{2} S_{I}^{2}\right)\right\rangle_{0, \mathrm{c}}^{(2)}= \\
& =-\frac{g^{2}}{2}\left\langle\tilde{A}_{\mu}^{a}(K) \tilde{A}_{\nu}^{b}(Q) \sum_{R P T} \tilde{\tilde{c}}^{c}(R) \tilde{A}_{\alpha}^{d}(P) \tilde{c}^{e}(T) \oiint_{U V X} \tilde{\tilde{c}}^{g}(U) \tilde{A}_{\beta}^{h}(V) \tilde{c}^{i}(X)\right\rangle_{0, \mathrm{c}} \\
& \quad \times f^{c d e} f^{g h i} \delta(-R+P+T) \delta(-U+V+X) R_{\alpha} U_{\beta} \\
& =g^{2} \sum_{R P T U V X}\left\langle\tilde{A}_{\mu}^{a}(K) \tilde{A}_{\alpha}^{d}(P)\right\rangle_{0}\left\langle\tilde{A}_{\nu}^{b}(Q) \tilde{A}_{\beta}^{h}(V)\right\rangle_{0}\left\langle\tilde{c}^{e}(T) \tilde{\bar{c}}^{g}(U)\right\rangle_{0}\left\langle\tilde{c}^{i}(X) \tilde{\bar{c}}^{c}(R)\right\rangle_{0} \\
& \times f^{c d e} f^{g h i} \delta(-R+P+T) \delta(-U+V+X) R_{\alpha} U_{\beta},
\end{aligned}
$$


where the Grassmann nature of the ghosts induced a minus sign at the second equality sign.

Inserting now the gluon propagator from eq. (5.68) and the ghost propagator from eq. (5.46), we inspect in turn the colour indices, spacetime indices, and momenta. The colour contractions result in the familiar factor

$$
\delta^{a d} \delta^{b h} \delta^{e g} \delta^{i c} f^{c d e} f^{g h i}=f^{c a e} f^{e b c}=-N_{\mathrm{c}} \delta^{a b},
$$

whereas the spacetime indices can be directly transported to the momenta: $\delta_{\mu \alpha} \delta_{\nu \beta} R_{\alpha} U_{\beta}=R_{\mu} U_{\nu}$. The momenta get fixed as $P \rightarrow-K, V \rightarrow-Q, U \rightarrow T, R \rightarrow X$, whereby

$\delta(-R+P+T) \delta(-U+V+X) \rightarrow \delta(-X-K+T) \delta(-T-Q+X)=\delta(-X-K+T) \delta(K+Q)$.

This implies that $R_{\mu} U_{\nu} \rightarrow X_{\mu} T_{\nu} \rightarrow\left(T_{\mu}-K_{\mu}\right) T_{\nu}$. Renaming finally $T \rightarrow P$, we obtain

$$
\left\langle\tilde{A}_{\mu}^{a}(K) \tilde{A}_{\nu}^{b}(Q)\left(\frac{1}{2} S_{I}^{2}\right)\right\rangle_{0, \mathrm{c}}^{(2)}=-g^{2} N_{\mathrm{c}} \frac{\delta^{a b} \delta(K+Q)}{\left(K^{2}\right)^{2}} \sum_{P} \frac{1}{P^{2}(K-P)^{2}}\left(P_{\mu}-K_{\mu}\right) P_{\nu} .
$$

Repeating the trick of eq. (5.81), this can be turned into

$$
\left\langle\tilde{A}_{\mu}^{a}(K) \tilde{A}_{\nu}^{b}(Q)\left(\frac{1}{2} S_{I}^{2}\right)\right\rangle_{0, \mathrm{c}}^{(2)}=-\frac{g^{2} N_{\mathrm{c}}}{2} \frac{\delta^{a b} \delta(K+Q)}{\left(K^{2}\right)^{2}} \oiint_{P} \frac{1}{P^{2}(K-P)^{2}}\left(2 P_{\mu} P_{\nu}-K_{\mu} K_{\nu}\right),
$$

which in the $K \rightarrow 0$ limit produces, upon setting $d \rightarrow 3$ and using eq. (5.88),

$$
\left\langle\tilde{A}_{\mu}^{a}(K) \tilde{A}_{\nu}^{b}(Q)\left(\frac{1}{2} S_{I}^{2}\right)\right\rangle_{0, \mathrm{c}}^{(2)} \approx-\frac{g^{2} N_{\mathrm{c}}}{2} \frac{\delta^{a b} \delta(K+Q)}{\left(K^{2}\right)^{2}}\left(-\delta_{\mu 0} \delta_{\nu 0}+\delta_{\mu i} \delta_{\nu i}\right) \frac{T^{2}}{12}+\mathcal{O}(\epsilon) .
$$

Finally, we consider the fermion loop, originating from the vertex of eq. (5.51). Proceeding as above, we obtain

$$
\begin{aligned}
& \left\langle\tilde{A}_{\mu}^{a}(K) \tilde{A}_{\nu}^{b}(Q)\left(\frac{1}{2} S_{I}^{2}\right)\right\rangle_{0, \mathrm{c}}^{(3)}= \\
& =-\frac{g^{2}}{2}\left\langle\tilde{A}_{\mu}^{a}(K) \tilde{A}_{\nu}^{b}(Q) \sum_{P\{R T\}} \tilde{\bar{\psi}}_{A}(R) \gamma_{\alpha} \tilde{A}_{\alpha}^{c}(P) \tilde{\psi}_{B}(T) \sum_{V\{U X\}} \tilde{\bar{\psi}}_{C}(U) \gamma_{\beta} \tilde{A}_{\beta}^{d}(V) \tilde{\psi}_{D}(X)\right\rangle_{0, \mathrm{c}} \\
& \quad \times \delta(-R+P+T) \delta(-U+V+X) T_{A B}^{c} T_{C D}^{d} \\
& =g^{2} \oint_{P V\{R T U X\}}\left\langle\tilde{A}_{\mu}^{a}(K) \tilde{A}_{\alpha}^{c}(P)\right\rangle_{0}\left\langle\tilde{A}_{\nu}^{b}(Q) \tilde{A}_{\beta}^{d}(V)\right\rangle_{0} \operatorname{Tr}\left[\left\langle\tilde{\psi}_{D}(X) \tilde{\bar{\psi}}_{A}(R)\right\rangle_{0} \gamma_{\alpha}\left\langle\tilde{\psi}_{B}(T) \tilde{\bar{\psi}}_{C}(U)\right\rangle_{0} \gamma_{\beta}\right] \\
& \quad \times \delta(-R+P+T) \delta(-U+V+X) T_{A B}^{c} T_{C D}^{d},
\end{aligned}
$$

where the Grassmann nature of the fermions induced a minus sign. As noted earlier, the capital indices originating from the quark spinors stand both for colour and flavour quantum numbers.

Inserting next the gluon propagator from eq. (5.68) and the fermion propagator from eq. (5.47), let us once more inspect in turn the colour and flavour indices, Lorentz indices, and momenta. The colour and flavour contractions result this time in the factor

$$
\delta^{a c} \delta^{b d} \delta_{D A} \delta_{B C} T_{A B}^{c} T_{C D}^{d}=\operatorname{Tr}\left[T^{a} T^{b}\right]=\frac{N_{\mathrm{f}}}{2},
$$

where we assumed the flavours to be degenerate in mass and in addition took advantage of the assumed normalization of the fundamental representation generators $T^{a}$. The spacetime indices yield on the other hand

$$
\begin{aligned}
\delta_{\mu \alpha} \delta_{\nu \beta} \operatorname{Tr}\left[(-i \not R+m) \gamma_{\alpha}(-i \Psi+m) \gamma_{\beta}\right] & =4\left[-R_{\sigma} U_{\rho}\left(\delta_{\sigma \mu} \delta_{\rho \nu}-\delta_{\sigma \rho} \delta_{\mu \nu}+\delta_{\sigma \nu} \delta_{\rho \mu}\right)+m^{2} \delta_{\mu \nu}\right] \\
& =4\left[\delta_{\mu \nu}\left(R \cdot U+m^{2}\right)-R_{\mu} U_{\nu}-R_{\nu} U_{\mu}\right]
\end{aligned}
$$


where we used standard results for the traces of Euclidean $\gamma$-matrices. Momenta get fixed as $P \rightarrow-K, V \rightarrow-Q, R \rightarrow X, U \rightarrow T$. Thereby

$\delta(-R+P+T) \delta(-U+V+X) \rightarrow \delta(-X-K+T) \delta(-T-Q+X)=\delta(-X-K+T) \delta(K+Q)$,

which implies that we can substitute $f(R, U) \rightarrow f(X, T) \rightarrow f(T-K, T)$ in eq. (5.98). Renaming finally $T \rightarrow P$, the contribution of the quark loop diagram to the self-energy becomes

$$
\begin{aligned}
& \left\langle\tilde{A}_{\mu}^{a}(K) \tilde{A}_{\nu}^{b}(Q)\left(\frac{1}{2} S_{I}^{2}\right)\right\rangle_{0, c}^{(3)} \\
& \quad=2 g^{2} N_{\mathrm{f}} \frac{\delta^{a b} \delta(K+Q)}{\left(K^{2}\right)^{2}} \oint_{\{P\}} \frac{\delta_{\mu \nu}\left(P^{2}-K \cdot P+m^{2}\right)-2 P_{\mu} P_{\nu}+K_{\mu} P_{\nu}+K_{\nu} P_{\mu}}{\left[P^{2}+m^{2}\right]\left[(K-P)^{2}+m^{2}\right]} .
\end{aligned}
$$

For vanishing chemical potential, a shift like in eq. (5.81) works also with fermionic four-momenta, so that this expression further simplifies to

$$
\begin{aligned}
& \left\langle\tilde{A}_{\mu}^{a}(K) \tilde{A}_{\nu}^{b}(Q)\left(\frac{1}{2} S_{I}^{2}\right)\right\rangle_{0, \mathrm{c}}^{(3)} \\
& =g^{2} N_{\mathrm{f}} \frac{\delta^{a b} \delta(K+Q)}{\left(K^{2}\right)^{2}} \sum_{\{P\}} \frac{\delta_{\mu \nu}\left(2 P^{2}-K^{2}+2 m^{2}\right)-4 P_{\mu} P_{\nu}+2 K_{\mu} K_{\nu}}{\left[P^{2}+m^{2}\right]\left[(K-P)^{2}+m^{2}\right]} .
\end{aligned}
$$

The structure in the numerator of eq. (5.101) is similar to that in eq. (5.82), except that the Matsubara frequencies are fermionic. In particular, if we again set the external momentum to zero, and for simplicity also consider the limit $T \gg m$, so that quark masses can be ignored, the entire term becomes proportional to

$$
\begin{aligned}
& \mathcal{F}_{\{P\}} \frac{\delta_{\mu \nu} P^{2}-2 P_{\mu} P_{\nu}}{\left(P^{2}\right)^{2}}=\delta_{\mu 0} \delta_{\nu 0} \oint_{\{P\}} \frac{P^{2}-2 P_{0}^{2}}{\left(P^{2}\right)^{2}}+\delta_{\mu i} \delta_{\nu i} \oint_{\{P\}} \frac{P^{2}-2 P_{i}^{2}}{\left(P^{2}\right)^{2}} \\
& =\delta_{\mu 0} \delta_{\nu 0} \oiint_{\{P\}} \frac{P^{2}-2 p_{n}^{2}}{\left(P^{2}\right)^{2}}+\delta_{\mu i} \delta_{\nu i} \mathcal{F}_{\{P\}} \frac{P^{2}-\left(\frac{2}{d}\right) p^{2}}{\left(P^{2}\right)^{2}} \\
& =\delta_{\mu 0} \delta_{\nu 0} \mathcal{F}_{\{P\}} \frac{P^{2}-2 p_{n}^{2}}{\left(P^{2}\right)^{2}}+\delta_{\mu i} \delta_{\nu i} \oint_{\{P\}} \frac{\left(1-\frac{2}{d}\right) P^{2}+\left(\frac{2}{d}\right) p_{n}^{2}}{\left(P^{2}\right)^{2}} .
\end{aligned}
$$

The relation in eq. (5.87) continues to hold in the fermionic case, so setting $d \rightarrow 3$, we get

$$
\sum_{\{P\}} \frac{\delta_{\mu \nu} P^{2}-2 P_{\mu} P_{\nu}}{\left(P^{2}\right)^{2}}=\delta_{\mu 0} \delta_{\nu 0} \times 2 \tilde{I}_{T}(0)+\delta_{\mu i} \delta_{\nu i} \times\left(\frac{1}{3}-\frac{1}{3}\right) \tilde{I}_{T}(0)+\mathcal{O}(\epsilon) .
$$

Inserting here finally $\tilde{I}_{T}(0)=-T^{2} / 24$ from eq. (4.75), we arrive at the final result for the fermionic contribution,

$$
\left\langle\tilde{A}_{\mu}^{a}(K) \tilde{A}_{\nu}^{b}(Q)\left(\frac{1}{2} S_{I}^{2}\right)\right\rangle_{0, \mathrm{c}}^{(3)} \approx-g^{2} N_{\mathrm{f}} \frac{\delta^{a b} \delta(K+Q)}{\left(K^{2}\right)^{2}}\left\{\delta_{\mu 0} \delta_{\nu 0}+0 \times \delta_{\mu i} \delta_{\nu i}\right\} \frac{T^{2}}{6}+\mathcal{O}(\epsilon) .
$$

Summing together eqs. (5.74), (5.89), (5.95) and (5.104) and omitting the terms of $\mathcal{O}(\epsilon)$, we find the surprisingly compact expression

$$
\begin{aligned}
& \left\langle\tilde{A}_{\mu}^{a}(K) \tilde{A}_{\nu}^{b}(Q)\left(-S_{I}+\frac{1}{2} S_{I}^{2}\right)\right\rangle_{0, \mathrm{c}} \\
& \approx-g^{2} \frac{\delta^{a b} \delta(K+Q)}{\left(K^{2}\right)^{2}}\left\{\left[\left(3+\frac{3}{2}-\frac{1}{2}\right) N_{\mathrm{c}}+2 N_{\mathrm{f}}\right] \delta_{\mu 0} \delta_{\nu 0}+\left[\left(3-\frac{7}{2}+\frac{1}{2}\right) N_{\mathrm{c}}\right] \delta_{\mu i} \delta_{\nu i}\right\} \frac{T^{2}}{12} \\
& =-\frac{\delta^{a b} \delta_{\mu 0} \delta_{\nu 0} \delta(K+Q)}{\left(K^{2}\right)^{2}} \times g^{2} T^{2}\left(\frac{N_{\mathrm{c}}}{3}+\frac{N_{\mathrm{f}}}{6}\right) .
\end{aligned}
$$


It is important to note that all corrections have cancelled from the spatial part. ${ }^{15}$ Due to WardTakahashi identities (or more properly their non-Abelian generalizations, Slavnov-Taylor identities), the gauge field self-energy must be transverse with respect to the external four-momentum, which in the case of the Matsubara zero mode takes the form $K=(0, \mathbf{k})$. Since we computed the self-energy with $\mathbf{k}=\mathbf{0}$, the transverse structure $\delta_{i j} k^{2}-k_{i} k_{j}$ cannot appear, and the spatial part must vanish altogether.

The result obtained above has a direct physical meaning. Indeed, we recall from the discussion of scalar field theory, eq. (3.70), that eqs. (5.69) and (5.105) can be interpreted as a (resummed) full propagator of the form

$$
\left\langle\tilde{A}_{\mu}^{a}(K) \tilde{A}_{\nu}^{b}(Q)\right\rangle \quad \stackrel{K \approx 0}{\approx} \frac{\delta^{a b} \delta_{\mu \nu} \delta(K+Q)}{K^{2}+\delta_{\mu 0} \delta_{\nu 0} m_{\mathrm{E}}^{2}},
$$

where

$$
m_{\mathrm{E}}^{2} \equiv g^{2} T^{2}\left(\frac{N_{\mathrm{c}}}{3}+\frac{N_{\mathrm{f}}}{6}\right)
$$

is called the Debye mass parameter. Its existence corresponds to the fact the colour-electric field $A_{0}$ gets exponentially screened in a thermal plasma, like the scalar field propagator in eq. (3.46). In contrast, the colour-magnetic field $A_{i}$ does not get screened, at least at this order.

We conclude with two remarks:

- If we consider the full Standard Model rather than QCD (the corresponding Euclidean Lagrangian is given on p. 223), then there is a separate thermal mass for the temporal components of all three gauge fields, and for the Matsubara zero mode of the Higgs field. These can be found in ref. [5.7].

- The definition of a Debye mass becomes ambiguous at higher orders. One possibility is to define it as a "matching coefficient" in a certain "effective theory"; this is discussed in more detail in sec. 6.2, cf. eq. (6.37). In that case higher-order corrections to the expression in eq. (5.107) can be computed [5.8]. On the other hand, if we want to define the Debye mass as a physical quantity, the result becomes non-perturbative already at the next-to-leading order [5.9], and a proper definition and extraction requires a lattice approach [5.10].

\footnotetext{
${ }^{15}$ We checked this for $d=3$ but with some more effort it is possible to verify that the same is true for general $d$. The generalization of the coefficient to general $d$ is given in eq. (8.148).
} 


\subsection{Free energy density to $\mathcal{O}\left(g^{3}\right)$}

As an application of the results of the previous section, we now compute the free energy density of QCD up to $\mathcal{O}\left(g^{3}\right)$, parallelling the method introduced for scalar field theory around eq. (3.94). We recall that the essential insight in this treatment was to supplement the quadratic part of the Lagrangian for the Matsubara zero modes by an effective thermal mass computed from the full propagator, and to treat minus the same term as part of the interaction Lagrangian. The "noninteracting" free energy density computed with the corrected propagator then yields the result for the ring sum, whereas the bilinear interaction term cancels the corresponding, infrared (IR) divergent contributions order by order in a loop expansion. ${ }^{16}$ In the present case, given the result of eq. (5.106), we see that only the temporal components of the gauge fields need to be corrected with a mass term. This is in accordance with the gauge transformation properties of static colourelectric and colour-magnetic fields, which forbid the spatial components from having a mass; we return to this in sec. 6.2 .

With the above considerations in mind, the correction of $\mathcal{O}\left(g^{3}\right)$ [5.11] to the tree-level result in eq. (5.61) can immediately be written down, if we employ eq. (2.87) and take into account that there are $N_{\mathrm{c}}^{2}-1$ copies of the gauge field. This produces

$$
\begin{aligned}
\left.f_{\left(\frac{3}{2}\right)}(T)\right|_{\mathrm{QCD}} & =\left(N_{\mathrm{c}}^{2}-1\right)\left(-\frac{T m_{\mathrm{E}}^{3}}{12 \pi}\right) \\
& =\left(N_{\mathrm{c}}^{2}-1\right) T^{4} g^{3}\left(-\frac{1}{12 \pi}\right)\left(\frac{N_{\mathrm{c}}}{3}+\frac{N_{\mathrm{f}}}{6}\right)^{\frac{3}{2}} \\
& =-\frac{\pi^{2} T^{4}}{3} 2\left(N_{\mathrm{c}}^{2}-1\right)\left(\frac{g^{2}}{4 \pi^{2}}\right)^{\frac{3}{2}}\left(\frac{N_{\mathrm{c}}}{3}+\frac{N_{\mathrm{f}}}{6}\right)^{\frac{3}{2}},
\end{aligned}
$$

where the effective mass $m_{\mathrm{E}}$ was taken from eq. (5.107).

Next, we consider the contributions of $\mathcal{O}\left(g^{2}\right)$. In analogy with eq. (3.99), these terms $[5.12,5.13]$ come from the non-zero mode contributions to the 2-loop "vacuum"-type graphs in (cf. eq. (3.12))

$$
\left.f_{(1)}(T)\right|_{\mathrm{QCD}}=\left\langle S_{I}-\frac{1}{2} S_{I}^{2}+\ldots\right\rangle_{0, \mathrm{c}, \text { drop overall } \int_{X}} .
$$

It is useful to compare this expression with the computation of the full propagator in the previous section, eq. (5.105). We note that, apart from an overall minus sign, the two computations are quite similar at the present order. In fact, we claim that we only need to "close" the gluon line in the results of the previous section and simultaneously divide the graphs by $-1 / 2 n$, where $n$ is the number of gluon lines in the vacuum graph in question. Let us prove this by direct inspection.

Consider first the $\mathcal{O}\left(g^{2}\right)$ contribution from the 4-gluon vertex. In vacuum graphs, this leads to the combinatorial factor

$$
\langle\tilde{A} \tilde{A} \tilde{A} \tilde{A}\rangle_{0, \mathrm{c}}=3\langle\tilde{A} \tilde{A}\rangle_{0}\langle\tilde{A} \tilde{A}\rangle_{0},
$$

whereas in the propagator calculation we arrived at

$$
-\langle\tilde{A} \tilde{A} \tilde{A} \tilde{A} \tilde{A} \tilde{A}\rangle_{0, \mathrm{c}}=-4 \times 3\langle\tilde{A} \tilde{A}\rangle_{0}\langle\tilde{A} \tilde{A}\rangle_{0}\langle\tilde{A} \tilde{A}\rangle_{0} .
$$

\footnotetext{
${ }^{16}$ This cancellation is the only role that the subtraction plays at the order that we are considering, cf. the discussion below eq. (3.99). In the following we simplify the procedure by computing the contribution of $\mathcal{O}\left(g^{2}\right)$ with massless propagators, whereby no odd powers of thermal masses are generated and the subtraction can be omitted as well.
} 
The difference is $-4=-2 \times n$, with $n=2$ being the number of contractions in eq. (5.110). Similarly, with the contribution from two 3 -gluon vertices, the vacuum graphs lead to the combinatorial factor

$$
-\langle\tilde{A} \tilde{A} \tilde{A} \tilde{A} \tilde{A} \tilde{A}\rangle_{0, \mathrm{c}}=-3 \times 2\langle\tilde{A} \tilde{A}\rangle_{0}\langle\tilde{A} \tilde{A}\rangle_{0}\langle\tilde{A} \tilde{A}\rangle_{0},
$$

whereas when considering the propagator we got

$$
\langle\tilde{A} \tilde{A} \tilde{A} \tilde{A} \tilde{A} \tilde{A} \tilde{A} \tilde{A}\rangle_{0, \mathrm{c}}=6 \times 3 \times 2\langle\tilde{A} \tilde{A}\rangle_{0}\langle\tilde{A} \tilde{A}\rangle_{0}\langle\tilde{A} \tilde{A}\rangle_{0}\langle\tilde{A} \tilde{A}\rangle_{0} .
$$

There is evidently a difference of $-6=-2 \times n$, with $n=3$ the number of contractions in eq. (5.112). Finally, the ghost and fermion contributions to the vacuum graphs lead to the combinatorial factor

$$
-\langle\tilde{\bar{c}} \tilde{A} \tilde{c} \tilde{\bar{c}} \tilde{A} \tilde{c}\rangle_{0, c}=\langle\tilde{A} \tilde{A}\rangle_{0}\langle\tilde{c} \tilde{\bar{c}}\rangle_{0}\langle\tilde{c} \tilde{\bar{c}}\rangle_{0},
$$

whereas in the propagator computation we obtained

$$
\langle\tilde{A} \tilde{A} \tilde{\bar{c}} \tilde{A} \tilde{c} \tilde{\bar{c}} \tilde{A} \tilde{c}\rangle_{0, \mathrm{c}}=-2\langle\tilde{A} \tilde{A}\rangle_{0}\langle\tilde{A} \tilde{A}\rangle_{0}\langle\tilde{c} \tilde{\bar{c}}\rangle_{0}\langle\tilde{c} \tilde{\bar{c}}\rangle_{0} .
$$

So once more a difference of $-2 \times n$, with $n=1$ the number of gluon contractions in eq. (5.114).

With the insights gained, the contribution of the 4-gluon vertex to the free energy density of QCD can be extracted directly from eq. (5.74):

$$
\begin{aligned}
\sum_{\text {man }} & =-\frac{1}{4}\left\{-g^{2} N_{\mathrm{c}} d I_{T}(0) \sum_{K} \frac{\delta^{a a} \delta_{\mu \mu}}{K^{2}}\right\} \\
& =\frac{g^{2}}{4} N_{\mathrm{c}}\left(N_{\mathrm{c}}^{2}-1\right) d(d+1)\left[I_{T}(0)\right]^{2} .
\end{aligned}
$$

The contribution of the 3 -gluon vertices is similarly obtained from eq. (5.79): noting from eq. (5.80) that

$$
\begin{aligned}
\delta_{\mu \nu} & D_{\mu \beta \gamma}(-K, P, K-P) D_{\nu \beta \gamma}(K,-P,-K+P) \\
& =-(d+1)\left[4 K^{2}+(K-P)^{2}+P^{2}\right]-(d-5) K^{2}+2(2 d-1) K \cdot P-(4 d-2) P^{2} \\
& =-\left\{K^{2}[5 d+5+d-5]+K \cdot P[-2 d-2-4 d+2]+P^{2}[2 d+2+4 d-2]\right\} \\
& =-3 d\left\{K^{2}+(K-P)^{2}+P^{2}\right\},
\end{aligned}
$$

we get from eq. (5.79)

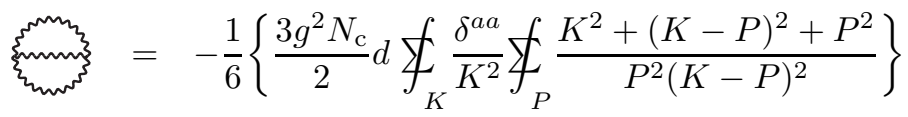

$$
\begin{aligned}
& =-\frac{g^{2}}{4} N_{\mathrm{c}}\left(N_{\mathrm{c}}^{2}-1\right) d \times 3\left[I_{T}(0)\right]^{2} .
\end{aligned}
$$

Note that unlike in eq. (5.83), for the present calculation it was crucial to keep the full $K$ dependence in the two-point function, because all values of $K$ are now integrated over.

Similarly, the contribution of the ghost loop can be extracted from eq. (5.93), producing

$$
\begin{aligned}
& =-\frac{1}{2}\left\{-g^{2} N_{\mathrm{c}} \sum_{K} \frac{\delta^{a a}}{K^{2}} \sum_{P} \frac{P^{2}-K \cdot P}{P^{2}(K-P)^{2}}\right\} \\
& =\frac{g^{2}}{4} N_{\mathrm{c}}\left(N_{\mathrm{c}}^{2}-1\right) \oint_{K P} \frac{P^{2}+(K-P)^{2}-K^{2}}{K^{2} P^{2}(K-P)^{2}} \\
& =\frac{g^{2}}{4} N_{\mathrm{c}}\left(N_{\mathrm{c}}^{2}-1\right)\left[I_{T}(0)\right]^{2},
\end{aligned}
$$


whereas the contribution of the fermion loop is obtained from eq. (5.100):

$$
7 m=-\frac{1}{2}\left\{2 g^{2} N_{\mathrm{f}} \sum_{K} \frac{\delta^{a a}}{K^{2}} \mathcal{F}_{\{P\}} \frac{(d+1)\left(P^{2}-K \cdot P+m^{2}\right)-2 P^{2}+2 K \cdot P}{\left[P^{2}+m^{2}\right]\left[(K-P)^{2}+m^{2}\right]}\right\} .
$$

Simplifying the last expression by setting $m / T \rightarrow 0$, we get

$$
\begin{aligned}
\text { mun } & =-g^{2} N_{\mathrm{f}}\left(N_{\mathrm{c}}^{2}-1\right) \oint_{K\{P\}} \frac{(d-1)\left(P^{2}-K \cdot P\right)}{K^{2} P^{2}(K-P)^{2}} \\
& =-g^{2} N_{\mathrm{f}}\left(N_{\mathrm{c}}^{2}-1\right) \frac{d-1}{2} \oint_{K\{P\}} \frac{P^{2}+(K-P)^{2}-K^{2}}{K^{2} P^{2}(K-P)^{2}} \\
& =-\frac{g^{2}}{2} N_{\mathrm{f}}\left(N_{\mathrm{c}}^{2}-1\right)(d-1)\left\{2 I_{T}(0) \tilde{I}_{T}(0)-\left[\tilde{I}_{T}(0)\right]^{2}\right\} .
\end{aligned}
$$

Here careful attention needed to be paid to the nature of the Matsubara frequencies appearing in the propagators.

Adding together the terms from eqs. (5.116), (5.118), (5.119) and (5.121), setting $d=3$ (note the absence of divergences), and using $I_{T}(0)=T^{2} / 12, \tilde{I}_{T}(0)=-T^{2} / 24$, we get as the full $\mathcal{O}\left(g^{2}\right)$ contribution to the free energy density

$$
\begin{aligned}
\left.f_{(1)}(T)\right|_{\mathrm{QCD}} & =g^{2}\left(N_{\mathrm{c}}^{2}-1\right) \frac{T^{4}}{144}\left[\left(3-\frac{9}{4}+\frac{1}{4}\right) N_{\mathrm{c}}-\left(-2 \times \frac{1}{2}-\frac{1}{4}\right) N_{\mathrm{f}}\right] \\
& =g^{2}\left(N_{\mathrm{c}}^{2}-1\right) \frac{T^{4}}{144}\left(N_{\mathrm{c}}+\frac{5}{4} N_{\mathrm{f}}\right) \\
& =-\frac{\pi^{2} T^{4}}{90}\left(N_{\mathrm{c}}^{2}-1\right)\left(-\frac{5}{2} \frac{g^{2}}{4 \pi^{2}}\right)\left(N_{\mathrm{c}}+\frac{5}{4} N_{\mathrm{f}}\right) .
\end{aligned}
$$

Adding to this the effects of eqs. (5.61) and (5.108), the final result reads

$$
\begin{aligned}
\left.f(T)\right|_{\mathrm{QCD}}=-\frac{\pi^{2} T^{4}}{45}\left(N_{\mathrm{c}}^{2}-1\right)\{1 & +\frac{7}{4} \frac{N_{\mathrm{f}} N_{\mathrm{c}}}{N_{\mathrm{c}}^{2}-1}-\frac{5}{4}\left(N_{\mathrm{c}}+\frac{5}{4} N_{\mathrm{f}}\right) \frac{\alpha_{s}}{\pi} \\
& \left.+30\left(\frac{N_{\mathrm{c}}}{3}+\frac{N_{\mathrm{f}}}{6}\right)^{\frac{3}{2}}\left(\frac{\alpha_{s}}{\pi}\right)^{\frac{3}{2}}+\mathcal{O}\left(\alpha_{s}^{2}\right)\right\},
\end{aligned}
$$

were we have denoted $\alpha_{s} \equiv g^{2} / 4 \pi$.

A few remarks are in order:

- The result in eq. (5.123) can be compared with that for a scalar field theory in eq. (3.93). The general structure is identical, and in particular the first relative correction is negative in both cases. This means that the interactions between the particles in a plasma tend to decrease the pressure that the plasma exerts.

- The second correction to the pressure turns out to be positive. Such an alternating structure indicates that it may be difficult to quantitatively estimate the magnitude of radiative corrections to the non-interacting result. We may recall, however, that $1-\frac{1}{2}+\frac{1}{3}-\frac{1}{4} \ldots=\ln 2=$ $0.693 \ldots$, whereas $1-\frac{1}{2}-\frac{1}{3}-\frac{1}{4} \ldots=-\infty$; in principle an alternating structure is beneficial as far as (asymptotic) convergence goes.

- The coefficients of the four subsequent terms, of orders $\mathcal{O}\left(\alpha_{s}^{2} \ln \alpha_{s}\right), \mathcal{O}\left(\alpha_{s}^{2}\right), \mathcal{O}\left(\alpha_{s}^{5 / 2}\right)$, and $\mathcal{O}\left(\alpha_{s}^{3} \ln \alpha_{s}\right)$, are also known [5.14]-[5.18]. Like for scalar field theory, this progress is possible thanks to the use of effective field theory methods that we discuss in the next chapter. 


\section{Appendix A: Do ghosts develop a thermal mass?}

In the computation of the present section, we have assumed that only the Matsubara zero modes of the fields $A_{0}^{a}$ need to be resummed, i.e. get an effective thermal mass. The fact that fermions do not need to be resummed is clear, but the case of ghosts is less obvious. To this end, let us finish the section by demonstrating that ghosts do not get any thermal mass, and thus behave like the spatial components of the gauge fields.

The tree-level ghost propagator is given in eq. (5.46), and we now consider corrections to this expression. The relevant vertex is the one in eq. (5.50), yielding for the only correction of $\mathcal{O}\left(g^{2}\right)$

$$
\begin{aligned}
& \left\langle\tilde{c}^{a}(K) \tilde{\bar{c}}^{b}(Q)\left(\frac{1}{2} S_{I}^{2}\right)\right\rangle_{0, \mathrm{c}}= \\
& =-\frac{g^{2}}{2}\left\langle\tilde{c}^{a}(K) \tilde{\bar{c}}^{b}(Q) \sum_{R P T} \tilde{\tilde{c}}^{c}(R) \tilde{A}_{\alpha}^{d}(P) \tilde{c}^{e}(T) \sum_{U V X} \tilde{\tilde{c}}^{g}(U) \tilde{A}_{\beta}^{h}(V) \tilde{c}^{i}(X)\right\rangle_{0, \mathrm{c}} \\
& \quad \times f^{c d e} f^{g h i} \delta(-R+P+T) \delta(-U+V+X) R_{\alpha} U_{\beta} \\
& =-g^{2} \sum_{R P T U V X}\left\langle\tilde{c}^{a}(K) \tilde{\bar{c}}^{c}(R)\right\rangle_{0}\left\langle\tilde{c}^{e}(T) \tilde{\bar{c}}^{g}(U)\right\rangle_{0}\left\langle\tilde{c}^{i}(X) \tilde{\bar{c}}^{b}(Q)\right\rangle_{0}\left\langle\tilde{A}_{\alpha}^{d}(P) \tilde{A}_{\beta}^{h}(V)\right\rangle_{0} \\
& \times f^{c d e} f^{g h i} \delta(-R+P+T) \delta(-U+V+X) R_{\alpha} U_{\beta},
\end{aligned}
$$

where an even number of minus signs originated from the commutations of Grassmann fields. Inserting here the gluon propagator from eq. (5.68) as well as the free ghost propagator from eq. (5.46), we again end up inspecting colour indices, Lorentz indices, and momenta in the resulting expression. The colour contractions are seen to result in the factor

$$
\delta^{a c} \delta^{e g} \delta^{i b} \delta^{d h} f^{c d e} f^{g h i}=f^{a d e} f^{e d b}=-N_{\mathrm{c}} \delta^{a b},
$$

whereas the spacetime indices yield simply $\delta_{\alpha \beta}$. Momenta get fixed as $R \rightarrow K, U \rightarrow T, X \rightarrow Q$, $V \rightarrow-P$. Thereby

$$
\delta(-R+P+T) \delta(-U+V+X) \rightarrow \delta(-K+P+T) \delta(-T-P+Q)=\delta(-K+P+T) \delta(-K+Q),
$$

implying that $T=K-P$ and $R \cdot U \rightarrow K \cdot T \rightarrow K \cdot(K-P)$. Inserting all this, eq. (5.124) turns into

$$
\left\langle\tilde{c}^{a}(K) \tilde{\bar{c}}^{b}(Q)\left(\frac{1}{2} S_{I}^{2}\right)\right\rangle_{0, \mathrm{c}}=g^{2} N_{\mathrm{c}} \frac{\delta^{a b} \delta(K-Q)}{\left(K^{2}\right)^{2}} \oint_{P} \frac{K \cdot(K-P)}{P^{2}(K-P)^{2}} .
$$

The expression in eq. (5.127) is proportional to the external momentum $K$. Therefore, it does not represent an effective mass correction, but is rather a "wave function (re)normalization" contribution, as can be made explicit through a shift like in eq. (5.81). 


\section{Literature}

[5.1] C.W. Bernard, Feynman rules for gauge theories at finite temperature, Phys. Rev. D 9 (1974) 3312 .

[5.2] J.B. Kogut and L. Susskind, Hamiltonian formulation of Wilson's lattice gauge theories, Phys. Rev. D 11 (1975) 395.

[5.3] M. Lüscher, Construction of a selfadjoint, strictly positive transfer matrix for Euclidean lattice gauge theories, Commun. Math. Phys. 54 (1977) 283.

[5.4] L.D. Faddeev and V.N. Popov, Feynman diagrams for the Yang-Mills field, Phys. Lett. B 25 (1967) 29.

[5.5] T. Vachaspati, Progress on cosmological magnetic fields, Rept. Prog. Phys. 84 (2021) 074901 [2010.10525].

[5.6] J. Kuipers, T. Ueda, J.A.M. Vermaseren and J. Vollinga, FORM version 4.0, Comput. Phys. Commun. 184 (2013) 1453 [1203.6543].

[5.7] M.E. Carrington, Effective potential at finite temperature in the Standard Model, Phys. Rev. D 45 (1992) 2933.

[5.8] I. Ghisoiu, J. Möller and Y. Schröder, Debye screening mass of hot Yang-Mills theory to three-loop order, JHEP 11 (2015) 121 [1509.08727].

[5.9] A.K. Rebhan, Non-Abelian Debye mass at next-to-leading order, Phys. Rev. D 48 (1993) 3967 [hep-ph/9308232].

[5.10] P.B. Arnold and L.G. Yaffe, Non-Abelian Debye screening length beyond leading order, Phys. Rev. D 52 (1995) 7208 [hep-ph/9508280].

[5.11] J.I. Kapusta, Quantum Chromodynamics at high temperature, Nucl. Phys. B 148 (1979) 461.

[5.12] E.V. Shuryak, Theory of hadronic plasma, Sov. Phys. JETP 47 (1978) 212.

[5.13] S.A. Chin, Transition to hot quark matter in relativistic heavy-ion collision, Phys. Lett. B 78 (1978) 552.

[5.14] T. Toimela, The next term in the thermodynamic potential of QCD, Phys. Lett. B 124 (1983) 407.

[5.15] P. Arnold and C. Zhai, Three-loop free energy for pure gauge QCD, Phys. Rev. D 50 (1994) 7603 [hep-ph/9408276].

[5.16] C. Zhai and B. Kastening, Free energy of hot gauge theories with fermions through $g^{5}$, Phys. Rev. D 52 (1995) 7232 [hep-ph/9507380].

[5.17] E. Braaten and A. Nieto, Free energy of QCD at high temperature, Phys. Rev. D 53 (1996) 3421 [hep-ph/9510408].

[5.18] K. Kajantie, M. Laine, K. Rummukainen and Y. Schröder, Pressure of hot QCD up to $g^{6} \ln (1 / g)$, Phys. Rev. D 67 (2003) 105008 [hep-ph/0211321]. 


\section{Low-energy effective field theories}

Abstract: The existence of a so-called infrared (IR) problem in relativistic thermal field theory is pointed out, both from a physical and a formal (imaginary-time) point of view. The notion of effective field theories is introduced, and the main issues related to their construction and use are illustrated with the help of a simple example. Subsequently this methodology is applied to the imaginary-time path integral represention for the partition function of non-Abelian gauge field theory. This leads to the construction of a dimensionally reduced effective field theory for capturing certain (so-called "static", i.e. time-independent) properties of QCD (or more generally Standard Model) thermodynamics in the high-temperature limit.

Keywords: Infrared divergences, power counting, Matsubara zero mode, Bose enhancement, Linde problem, hard and soft modes, effective theories, Electrostatic QCD, Magnetostatic QCD, symmetries, matching, truncation.

\subsection{The infrared problem of thermal field theory}

Let us start by considering the types of integrals that appear in thermal perturbation theory. According to eqs. (2.34) and (4.59), each new loop order (corresponding to an additional loop momentum) produces one of

$$
\begin{aligned}
& \mathcal{F}_{P} f\left(\omega_{n}, \mathbf{p}\right)=\int_{\mathbf{p}}\left\{\frac{1}{2} \int_{-\infty-i 0^{+}}^{+\infty-i 0^{+}} \frac{\mathrm{d} \omega}{2 \pi}[f(\omega, \mathbf{p})+f(-\omega, \mathbf{p})]\left[1+2 n_{\mathrm{B}}(i \omega)\right]\right\}, \\
& \mathcal{F}_{\{P\}} f\left(\omega_{n}, \mathbf{p}\right)=\int_{\mathbf{p}}\left\{\frac{1}{2} \int_{-\infty-i 0^{+}}^{+\infty-i 0^{+}} \frac{\mathrm{d} \omega}{2 \pi}[f(\omega, \mathbf{p})+f(-\omega, \mathbf{p})]\left[1-2 n_{\mathrm{F}}(i \omega)\right]\right\},
\end{aligned}
$$

depending on whether the new line is bosonic or fermionic. The functions $f$ here contain propagators and additional structures emerging from vertices; in the simplest case, $f(\omega, \mathbf{p}) \sim 1 /\left(\omega^{2}+\epsilon_{p}^{2}\right)$, where we denote $\epsilon_{p} \equiv \sqrt{p^{2}+m^{2}}$.

Now, the structures which are the most important, or yield the largest contributions, are those where the functions $f$ are largest. Let us inspect this question in terms of the left and right-hand sides of eqs. (6.1) and (6.2).

For bosons, the largest contribution on the left-hand side of eq. (6.1) is clearly associated with the Matsubara zero mode, $\omega_{n}=0$; in the case $f(\omega, \mathbf{p}) \sim 1 /\left(\omega^{2}+\epsilon_{p}^{2}\right)$, this gives simply

$$
\left.\left\{T \sum_{\omega_{n}} f\right\}\right|_{\omega_{n}=0} \sim \frac{T}{\epsilon_{p}^{2}} .
$$

On the right-hand side, we on the other hand close the contour in the lower half-plane, whereby the largest contribution is associated with Bose enhancement around the pole $\omega=-i \epsilon_{p}$ :

$$
\begin{aligned}
\{\ldots\} & \sim \frac{1}{2} \frac{-2 \pi i}{2 \pi} \frac{2}{-2 i \epsilon_{p}}\left[1+2 n_{\mathrm{B}}\left(\epsilon_{p}\right)\right]=\frac{1}{\epsilon_{p}}\left(\frac{1}{2}+\frac{1}{e^{\epsilon_{p} / T}-1}\right) \\
& \approx \frac{1}{\epsilon_{p}}\left(\frac{1}{2}+\frac{1}{\epsilon_{p} / T+\epsilon_{p}^{2} / 2 T^{2}}+\ldots\right)=\frac{T}{\epsilon_{p}^{2}}+\mathcal{O}\left(\frac{1}{T}\right) .
\end{aligned}
$$


On the second row, we performed an expansion in powers of $\epsilon_{p} / T$, which is valid for small energies at high temperatures.

For fermions, there is no Matsubara zero mode on the left-hand side of eq. (6.2), so that the largest terms have at most (i.e. for $\epsilon_{p} \ll \pi T$ ) the magnitude

$$
\left.\left\{T \sum_{\left\{\omega_{n}\right\}} f\right\}\right|_{\omega_{n}= \pm \pi T} \sim \frac{T}{(\pi T)^{2}} \sim \frac{1}{\pi^{2} T} .
$$

Similarly, in terms of the right-hand side of eq. (6.2), we can estimate

$$
\begin{aligned}
\{\ldots\} & \sim \frac{1}{2} \frac{-2 \pi i}{2 \pi} \frac{2}{-2 i \epsilon_{p}}\left[1-2 n_{\mathrm{F}}\left(\epsilon_{p}\right)\right]=\frac{1}{\epsilon_{p}}\left(\frac{1}{2}-\frac{1}{e^{\epsilon_{p} / T}+1}\right) \\
& \approx \frac{1}{\epsilon_{p}}\left(\frac{1}{2}-\frac{1}{2+\epsilon_{p} / T}+\ldots\right)=\mathcal{O}\left(\frac{1}{T}\right) .
\end{aligned}
$$

Given the estimates above, let us construct a dimensionless expansion parameter associated with the loop expansion. Apart from an additional propagator, each loop order also brings in an additional vertex or vertices; we denote the corresponding coupling by $g^{2}$, as would be the case in gauge theory. Moreover, the Matsubara summation involves a factor $T$, so we can assume that the expansion parameter contains the combination $g^{2} T$. We now have to use the other scales in the problem to transform this into a dimensionless number. For the Matsubara zero modes, eq. (6.3) tells us that we are allowed to use inverse powers of $\epsilon_{p}$ or, after integration over the spatial momenta, inverse powers of $m$. Therefore, we can assume that for large temperatures, $\pi T \gg m$, the largest possible expansion parameter is

$$
\epsilon_{\mathrm{b}} \sim \frac{g^{2} T}{\pi m} .
$$

For fermions, in contrast, eq. (6.5) suggests that inverse powers of $\epsilon_{p}$ or, after integration over spatial momenta, $m$, cannot appear in the denominator, even if $m \ll \pi T$; we are thus led to the estimate

$$
\epsilon_{\mathrm{f}} \sim \frac{g^{2} T}{\pi^{2} T} \sim \frac{g^{2}}{\pi^{2}}
$$

In these estimates most numerical factors have been omitted for simplicity.

Assuming that we work in the weak-coupling limit, $g^{2} \ll \pi^{2}$, we can thus conclude the following:

- Fermions appear to be purely perturbative in computations concerning "static" observables, with the corresponding weak-coupling expansion proceeding in powers of $g^{2} / \pi^{2}$.

- Bosonic Matsubara zero modes appear to suffer from bad convergence in the limit $m \rightarrow 0$.

- The resummations that we saw around eq. (3.94) for scalar field theory and in sec. 5.3 for QCD produce an effective thermal mass, $m_{\text {eff }}^{2} \sim g^{2} T^{2}$. Thus, we may expect the expansion parameter in eq. (6.7) to become $\sim g^{2} T /(\pi g T)=g / \pi$. In other words, a small expansion parameter exists in principle if $g \ll \pi$, but the structure of the weak-coupling series is peculiar, with odd powers of $g$ appearing.

- As we found in eq. (5.106), colour-magnetic fields do not develop a thermal mass squared at $\mathcal{O}\left(g^{2} T^{2}\right)$. This might still happen at higher orders, so we can state that $m_{\text {eff }} \lesssim g^{2} T / \pi$ for 
these modes. Thereby the expansion parameter in eq. (6.7) reads $\epsilon_{\mathrm{b}} \gtrsim g^{2} T / g^{2} T=1$. In other words, colour-magnetic fields cannot be treated perturbatively; this is known as the infrared problem (or "Linde problem") of thermal gauge theory [6.1].

The situation that we have encountered, namely that infrared problems exist but that they are related to particular degrees of freedom, is common in (quantum) field theory. Correspondingly, there is also a generic tool, called the effective field theory approach, which allows us to isolate the infrared problems into a simple Lagrangian, and treat them in this setting. The concept of effective field theories is not restricted to finite-temperature physics, but applies also at zero temperature, if the system possesses a scale hierarchy. In fact, the high-temperature case can be considered a special case of this, with the corresponding hierarchy often expressed as $g^{2} T / \pi \ll g T \ll \pi T$, where the first scale refers to the non-perturbative one associated with colour-magnetic fields. Given the generic nature of effective field theories, we first discuss the basic idea in a zero-temperature setting, before moving on to finite-temperature physics.

\section{A simple example of an effective field theory}

Let us consider a Lagrangian containing two different scalar fields, $\phi$ and $H$, with masses $m$ and $M$, respectively: ${ }^{17}$

$$
L_{\text {full }} \equiv \frac{1}{2} \partial_{\mu} \phi \partial_{\mu} \phi+\frac{1}{2} m^{2} \phi^{2}+\frac{1}{2} \partial_{\mu} H \partial_{\mu} H+\frac{1}{2} M^{2} H^{2}+g^{2} \phi^{2} H^{2}+\frac{1}{4} \lambda \phi^{4}+\frac{1}{4} \kappa H^{4} .
$$

We assume that there exists a hierarchy $m \ll M$ or, to be more precise, $m_{\mathrm{R}} \ll M_{\mathrm{R}}$, though we leave out the subscripts in the following. Our goal is to study to what extent the physics described by this theory can be captured by a simpler effective theory of the form

$$
L_{\text {eff }}=\frac{1}{2} \partial_{\mu} \bar{\phi} \partial_{\mu} \bar{\phi}+\frac{1}{2} \bar{m}^{2} \bar{\phi}^{2}+\frac{1}{4} \bar{\lambda} \bar{\phi}^{4}+\ldots
$$

where infinitely many higher-dimensional operators have been dropped. ${ }^{18}$

The main statement concerning the effective description goes as follows. Let us assume that $m \lesssim g M$ and that all couplings are parametrically of similar magnitude, $\lambda \sim \kappa \sim g^{2}$, and proceed to consider external momenta $P \lesssim g M$. Then the one-particle-irreducible Green's functions $\bar{\Gamma}_{n}$, computed within the effective theory, reproduce those of the full theory, $\Gamma_{n}$, with a relative error

$$
\frac{\delta \bar{\Gamma}_{n}}{\bar{\Gamma}_{n}} \equiv \frac{\left|\bar{\Gamma}_{n}-\Gamma_{n}\right|}{\bar{\Gamma}_{n}} \lesssim \mathcal{O}\left(g^{k}\right), \quad k>0
$$

if the parameters $\bar{m}^{2}$ and $\bar{\lambda}$ of eq. (6.10) are tuned suitably. The number $k$ may depend on the dimensionality of spacetime as well as on $n$, although a universal lower bound should exist. This lower bound can furthermore be increased by adding suitable higher-dimensional operators to $L_{\text {eff }}$; in the limit of infinitely many such operators the effective description should become exact.

A weaker form of the effective theory statement, although already sufficiently strong for practical purposes, is that Green's functions are matched only "on-shell", rather than for arbitrary external momenta. This form of the statement is implemented, for instance, in the so-called nonperturbative Symanzik improvement program of lattice QCD [6.3] (for a nice review, see ref. [6.4]).

\footnotetext{
${ }^{17}$ The discussion follows closely that in ref. [6.2].

${ }^{18}$ If we also wanted to describe gravity with these theories, we could add a "fundamental" cosmological constant $\Lambda$ in $L_{\text {full }}$, and an "effective" cosmological constant $\bar{\Lambda}$ in $L_{\text {eff }}$.
} 
It has been fittingly said that the effective theory assertion is almost trivial yet very difficult to prove. We will not attempt a formal proof here, but rather try to get an impression on how it arises, by inspecting with some care the 2-point Green's function of the light field $\phi$. In the full theory, at 1-loop level, the inverse of this ("amputated") quantity reads

$$
\begin{aligned}
G^{-1} & =\cdots+\bigcap^{\prime} \\
& =P^{2}+m^{2}+\Pi_{l}^{(1)}\left(0 ; m^{2}\right)+\Pi_{h}^{(1)}\left(0 ; M^{2}\right),
\end{aligned}
$$

where the dashed line represents the light field and the solid one the heavy field, while the subscripts $l, h$ stand for light and heavy, respectively. The first argument of the functions $\Pi_{l}^{(1)}, \Pi_{h}^{(1)}$ is the external momentum; as the notation indicates, closed bubbles contain no dependence on it.

Within the effective theory, the same computation yields

$$
\begin{aligned}
\bar{G}^{-1} & =\cdots+\cdots+\cdots \\
& =P^{2}+\bar{m}^{2}+\bar{\Pi}_{l}^{(1)}\left(0 ; \bar{m}^{2}\right) .
\end{aligned}
$$

The equivalence of all Green's functions at the on-shell point should imply the equivalence of pole masses, i.e. the locations of the on-shell points. By matching eqs. (6.12) and (6.13), we see that this can indeed be achieved provided that

$$
\bar{m}^{2}=m^{2}+\Pi_{h}^{(1)}\left(0 ; M^{2}\right)+\mathcal{O}\left(g^{4}\right) .
$$

Note that within perturbation theory the matching is carried out "order-by-order": $\bar{\Pi}_{l}^{(1)}\left(0 ; \bar{m}^{2}\right)$ is already of 1-loop order, so inside it $\bar{\lambda}$ and $\bar{m}^{2}$ can be replaced by $\lambda$ and $m^{2}$, respectively, given that the difference between $\bar{\lambda}$ and $\lambda$ as well as $\bar{m}^{2}$ and $m^{2}$ is itself of 1-loop order.

The situation becomes considerably more complicated once we go to the 2-loop level. To this end, let us analyze various types of graphs that exist in the full theory, and try to understand how they could be matched onto the simpler contributions within the effective theory.

First of all, there are graphs involving only light fields,

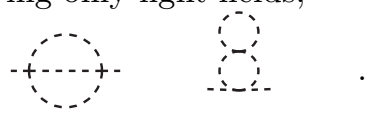

These can directly be matched with the corresponding graphs within the effective theory; as above, the fact that different parameters appear in the propagators (and vertices) is a higher-order effect.

Second, there are graphs which account for the "insignificant higher-order effects" that we omitted in the 1-loop matching, but that would play a role once we go to the 2-loop level:

$$
\begin{aligned}
& \Leftrightarrow \quad \Leftrightarrow \quad\left(\bar{m}^{2}-m^{2}\right) \frac{\partial \Pi_{l}^{(1)}\left(0 ; m^{2}\right)}{\partial m^{2}}, \\
& \Leftrightarrow(\bar{\lambda}-\lambda) \frac{\partial \Pi_{l}^{(1)}\left(0 ; m^{2}\right)}{\partial \lambda} \text {. }
\end{aligned}
$$

As indicated here, these two combine to reproduce (a part of) the 1-loop effective theory expression $\bar{\Pi}_{l}^{(1)}\left(0 ; \bar{m}^{2}\right)$ with 2-loop full theory accuracy.

Third, there are graphs only involving heavy fields in the loops:

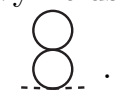


Obviously we can account for their effects by a 2-loop correction to $\bar{m}^{2}$.

Finally, there remain the most complicated graphs: structures involving both heavy and light fields, in a way that the momenta flowing through the two sets of lines do not get factorized:

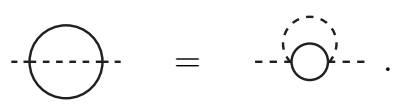

Naively, the representation on the right-hand side might suggest that this graph is simply part of the correction $(\bar{\lambda}-\lambda) \partial \Pi_{l}^{(1)}\left(0 ; m^{2}\right) / \partial \lambda$, just like the graph in eq. (6.17). This, however, is not the case, because the substructure appearing,

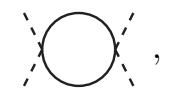

is momentum-dependent, unlike the effective vertex $\bar{\lambda}$.

Nevertheless, it should be possible to split eq. (6.19) into two parts, pictorially represented by

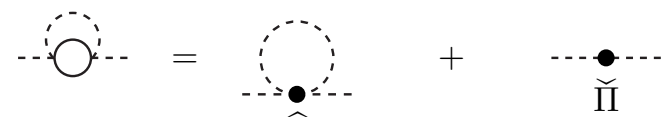

$$
\begin{aligned}
& \widehat{\Pi} \\
& \Leftrightarrow \quad \Pi_{\text {mixed }}^{(2)}\left(P^{2} ; m^{2}, M^{2}\right)=\widehat{\Pi}_{\text {mixed }}^{(2)}\left(P^{2} ; m^{2}, M^{2}\right)+\breve{\Pi}_{\text {mixed }}^{(2)}\left(P^{2} ; m^{2}, M^{2}\right) .
\end{aligned}
$$

The first part $\widehat{\Pi}^{(2)}$ is, by definition, characterized by the fact that it depends non-analytically on the mass parameter $m^{2}$ of the light field; therefore the internal $\phi$ field is soft in this part, i.e. gets a contribution from momenta $Q \sim m$. In this situation, the momentum dependence of eq. (6.20) is of subleading importance. In other words, this part of the graph does contribute simply to $(\bar{\lambda}-\lambda) \partial \Pi_{l}^{(1)}\left(0 ; m^{2}\right) / \partial \lambda$, as we naively expected.

The second part $\check{\Pi}^{(2)}$ is, by definition, analytic in the mass parameter $m^{2}$. We associate this with a situation where the internal $\phi$ is hard: even though its mass is small, it can have a large internal momentum $Q \sim M$, transmitted to it through interactions with the heavy modes. In this situation, the momentum dependence of eq. (6.20) plays an essential role. At the same time, the fact that all internal momenta are hard, permits for a Taylor expansion in the small external momentum:

$$
\begin{aligned}
\breve{\Pi}_{\text {mixed }}^{(2)}\left(P^{2} ; m^{2}, M^{2}\right)= & \breve{\Pi}_{\text {mixed }}^{(2)}\left(0 ; m^{2}, M^{2}\right)+P^{2} \frac{\partial}{\partial P^{2}} \breve{\Pi}_{\text {mixed }}^{(2)}\left(0 ; m^{2}, M^{2}\right) \\
& +\frac{1}{2}\left(P^{2}\right)^{2} \frac{\partial^{2}}{\partial\left(P^{2}\right)^{2}} \breve{\Pi}_{\text {mixed }}^{(2)}\left(0 ; m^{2}, M^{2}\right)+\ldots .
\end{aligned}
$$

The first term here represents a 2-loop correction to $\bar{m}^{2}$, just like the graph in eq. (6.18), whereas the second term can be compensated for by a change of the normalization of the field $\bar{\phi}$. Finally, the further terms have the appearance of higher-order (derivative) operators, truncated from the structure shown explicitly in eq. (6.10). Comparing with the leading kinetic term, the magnitude of the third term is very small,

$$
\frac{g^{4} \frac{\left(P^{2}\right)^{2}}{M^{2}}}{P^{2}} \lesssim g^{6}
$$

for $P \lesssim g M$, justifying the truncation of the effective action up to a certain relative accuracy. The structures in eq. (6.23) are collectively denoted by the 2-point "blob" in eq. (6.21).

To summarize, we see that the explicit construction of an effective field theory becomes subtle at higher loop orders. Another illuminating example of the difficulties met with "mixed graphs" 
is given around eq. (6.45) below. Nevertheless, we may formulate the following practical recipe for the effective field theory description of a Euclidean theory with a scale hierarchy:

(1) Identify the "light" or "soft" degrees of freedom, i.e. the ones that are IR-sensitive.

(2) Write down the most general Lagrangian for them, respecting all the symmetries of the system, and including local operators of arbitrary order.

(3) The parameters of this Lagrangian can be determined by matching:

- Compute the same observable in the full and effective theories, applying the same UVregularization and IR-cutoff.

- Subtract the results.

- The IR-cutoff should now disappear, and the result of the subtraction be analytic in $P^{2}$. This allows for a matching of the parameters and field normalizations of the effective theory.

- If the IR-cutoff does not disappear, the degrees of freedom, or the form of the effective theory, have not been correctly identified.

(4) Truncate the effective theory by dropping higher-dimensional operators suppressed by $1 / M^{k}$, which can only give a relative contribution of order

$$
\sim\left(\frac{m}{M}\right)^{k} \sim g^{k}
$$

where the dimensionless coefficient $g$ parametrizes the scale hierarchy. 


\subsection{Dimensionally reduced effective field theory for hot QCD}

We now apply the effective theory recipe to the problem outlined at the beginning of sec. 6.1, i.e. accounting for the soft contributions to the free energy density of thermal QCD. In this process, we follow the numbering introduced at the end of sec. 6.1.

(1) Identification of the soft degrees of freedom. As discussed earlier, the soft degrees of freedom in perturbative Euclidean thermal field theory are the bosonic Matsubara zero modes. Since they do not depend on the coordinate $\tau$, they live in $d=3-2 \epsilon$ spatial dimensions; for this reason, the construction of the effective theory is in this context called high-temperature dimensional reduction $[6.5,6.6]$. For simplicity, we concentrate on the dimensional reduction of QCD in the present section, but within perturbation theory the same procedure can also be (and indeed has been) applied to the full Standard Model [6.7], as well as many extensions thereof.

(2) Symmetries. Since the heat bath breaks Lorentz invariance, the time direction and the space directions are not interchangeable. Therefore, the spacetime symmetries of the effective theory are merely invariances in spatial rotations and translations.

In addition, the full theory possesses a number of discrete symmetries: QCD is invariant in $\mathrm{C}$, $\mathrm{P}$ and $\mathrm{T}$ separately. The effective theory inherits these symmetries, and it turns out that $L_{\text {eff }}$ is symmetric in $\bar{A}_{0} \rightarrow-\bar{A}_{0}$, where the low-energy fields are denoted by $\bar{A}_{\mu}$ (the symmetry $\bar{A}_{0} \rightarrow-\bar{A}_{0}$ is absent if the $\mathrm{C}$ symmetry of QCD is broken by coupling the quarks to a chemical potential).

Finally, consider the gauge symmetry from eq. (5.5):

$$
A_{\mu}^{\prime}=U A_{\mu} U^{-1}+\frac{i}{g} U \partial_{\mu} U^{-1}
$$

Since we now restrict to static (i.e. $\tau$-independent) fields, $U$ should not depend on $\tau$, either, and the effective theory should be invariant under

$$
\begin{aligned}
\bar{A}_{i}^{\prime} & =U \bar{A}_{i} U^{-1}+\frac{i}{g} U \partial_{i} U^{-1}, \\
\bar{A}_{0}^{\prime} & =U \bar{A}_{0} U^{-1} .
\end{aligned}
$$

In other words, the spatial components $\bar{A}_{i}$ remain gauge fields, whereas the temporal component $\bar{A}_{0}$ has turned into a scalar field in the adjoint representation (cf. eq. (5.9)).

With these ingredients, we can postulate the general form of the effective Lagrangian. It is illuminating to start by simply writing down the contribution of the soft degrees of freedom to the full Yang-Mills Lagrangian, eq. (5.34). Noting from eq. (5.32), viz.

$$
F_{0 i}^{a} \equiv \partial_{\tau} A_{i}^{a}-\mathcal{D}_{i}^{a b} A_{0}^{b},
$$

that in the static case $F_{i 0}^{a}=\mathcal{D}_{i}^{a b} A_{0}^{b}$, we end up with

$$
L_{E}=\frac{1}{4} F_{i j}^{a} F_{i j}^{a}+\frac{1}{2}\left(\mathcal{D}_{i}^{a b} A_{0}^{b}\right)\left(\mathcal{D}_{i}^{a c} A_{0}^{c}\right) .
$$

At this point, it is convenient to note that

$$
T^{a} \mathcal{D}_{i}^{a b} A_{0}^{b}=\partial_{i} A_{0}+g f^{a c b} T^{a} A_{i}^{c} A_{0}^{b}=\partial_{i} A_{0}-i g\left[A_{i}, A_{0}\right]=\left[D_{i}, A_{0}\right],
$$


where $D_{i}=\partial_{i}-i g A_{i}$ is the covariant derivative in the fundamental representation. Thereby we obtain as the "tree-level" terms of our effective theory

$$
L_{\text {eff }}^{(0)}=\frac{1}{4} \bar{F}_{i j}^{a} \bar{F}_{i j}^{a}+\operatorname{Tr}\left\{\left[\bar{D}_{i}, \bar{A}_{0}\right]\left[\bar{D}_{i}, \bar{A}_{0}\right]\right\},
$$

where we have now replaced $A_{\mu} \rightarrow \bar{A}_{\mu}$.

Next, we complete the tree-level structure by adding all mass and interaction terms allowed by symmetries. In this process, it is useful to proceed in order of increasing dimensionality, whereby we obtain at the three lowest orders:

$$
\begin{array}{lll}
\operatorname{dim}=2: & \operatorname{Tr}\left[\bar{A}_{0}^{2}\right] ; \\
\operatorname{dim}=4: & \operatorname{Tr}\left[\bar{A}_{0}^{4}\right], \quad\left(\operatorname{Tr}\left[\bar{A}_{0}^{2}\right]\right)^{2} ; \\
\operatorname{dim}=6: & \operatorname{Tr}\left\{\left[\bar{D}_{i}, \bar{F}_{i j}\right]\left[\bar{D}_{k}, \bar{F}_{k j}\right]\right\}, \ldots .
\end{array}
$$

In the last case, we have only shown one example operator, while many others are listed in ref. [6.8]. Note also that for $N_{\mathrm{c}}=2$ and 3 , there exists a linear relation between the two operators of dimensionality 4 , but from $N_{\mathrm{c}}=4$ onwards they are fully independent.

Combining eqs. (6.32)-(6.34), we can write the effective action in the form

$$
S_{\text {eff }}=\frac{1}{T} \int_{\mathbf{x}}\left\{\frac{1}{4} \bar{F}_{i j}^{a} \bar{F}_{i j}^{a}+\operatorname{Tr}\left(\left[\bar{D}_{i}, \bar{A}_{0}\right]\left[\bar{D}_{i}, \bar{A}_{0}\right]\right)+\bar{m}^{2} \operatorname{Tr}\left[\bar{A}_{0}^{2}\right]+\bar{\lambda}^{(1)}\left(\operatorname{Tr}\left[\bar{A}_{0}^{2}\right]\right)^{2}+\bar{\lambda}^{(2)} \operatorname{Tr}\left[\bar{A}_{0}^{4}\right]+\ldots\right\} .
$$

The prefactor $1 / T$, appearing like in classical statistical physics, comes from the integration $\int_{0}^{\beta} \mathrm{d} \tau$, since none of the soft fields depend on $\tau$. This theory is referred to as EQCD, for "Electrostatic QCD". Note that in the presence of a finite chemical potential, cf. sec. 7, charge conjugation symmetry is broken and the additional operator $i \bar{\gamma} \operatorname{Tr}\left[\bar{A}_{0}^{3}\right]$ appears in the effective action [6.9].

(3) Matching. If we restrict to 1-loop order, then the matching of the parameters in eq. (6.36) is rather simple, as explained around eq. (6.14): we just need to compute Green's functions for the soft fields with vanishing external momenta, with the heavy modes appearing in the internal propagators. For the parameter $\bar{m}^{2}$, this is furthermore precisely the computation that we carried out in sec. 5.3, so the result can be directly read off from eq. (5.107):

$$
\bar{m}^{2}=g^{2} T^{2}\left(\frac{N_{\mathrm{c}}}{3}+\frac{N_{\mathrm{f}}}{6}\right)+\mathcal{O}\left(g^{4} T^{2}\right) .
$$

The parameters $\bar{\lambda}^{(1)}, \bar{\lambda}^{(2)}$ can, in turn, be obtained by considering 4-point functions with soft modes of $A_{0}$ on the external legs, and non-zero Matsubara modes in the loop:

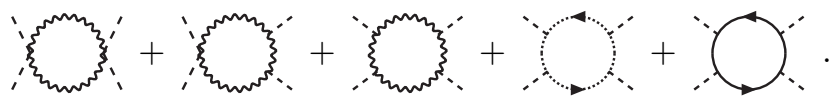

These graphs are clearly of $\mathcal{O}\left(g^{4}\right)$, and the actual values of the two parameters read $[6.10,6.11]$

$$
\bar{\lambda}^{(1)}=\frac{g^{4}}{4 \pi^{2}}+\mathcal{O}\left(g^{6}\right), \quad \bar{\lambda}^{(2)}=\frac{g^{4}}{12 \pi^{2}}\left(N_{\mathrm{c}}-N_{\mathrm{f}}\right)+\mathcal{O}\left(g^{6}\right) .
$$

The gauge coupling $\bar{g}$ appearing in $\bar{D}_{i}$ and $\bar{F}_{i j}^{a}$ is of the form $\bar{g}^{2}=g^{2}+\mathcal{O}\left(g^{4}\right)$ and needs to be matched as well $[6.12,6.13]$. If there are non-zero chemical potentials $\mu_{i}$ in the problem, the same is true for $\bar{\gamma}=\sum_{i=1}^{N_{\mathrm{f}}} \mu_{i} g^{3} /\left(3 \pi^{2}\right)+\mathcal{O}\left(g^{5}\right)[6.9]$. 
(4) Truncation of higher-dimensional operators. The most non-trivial part of any effective theory construction is the quantitative analysis of the error made, when operators beyond a given dimensionality are dropped. In other words, the challenge is to determine the constant $k$ in eq. (6.11). We illustrate this by considering the error made when dropping the operator in eq. (6.35).

First of all, we need to know the parametric magnitude of the coefficient with which the neglected operator would enter $L_{\text {eff }}$, if it were kept. The operator of eq. (6.35) could be generated through the momentum dependence of graphs like

$$
\text { , ismons. }
$$

where the dashed lines now stand for the spatial components of the gauge field, $\bar{A}_{i}$. If we drop this term, the corresponding Green's function will not be computed correctly; however, it still has some value, namely that which would be obtained within the effective theory via the graph

$$
\text { , }
$$

Here, we have noted that to account for the momentum dependence of the graph, represented by the derivative $\partial_{i}$ in front of $\bar{F}_{i j}^{a}$, one needs to Taylor-expand the integral to the first non-trivial order in external momentum, explaining why the propagator is raised to power three in eq. (6.41). An explicit computation further shows that the coefficient in eq. (6.41) comes with a negative sign, but this has no significance for our general discussion.

Next, we note that the value of the Green's function within the (truncated) effective theory, eq. (6.41), is in fact larger than what the contribution of the omitted operator would have been, cf. eq. (6.40). Therefore, the error made through the omission of eq. (6.40) is small:

$$
\frac{\delta \bar{\Gamma}}{\bar{\Gamma}} \sim \frac{g^{2}}{T^{2}} \frac{\bar{m}^{3}}{g^{2} T} \sim\left(\frac{\bar{m}}{T}\right)^{3} \sim g^{3} .
$$

In other words, for the Green's function considered and the dimensionally reduced effective theory of hot QCD truncated beyond dimension 4, we can expect the relative accuracy exponent of eq. (6.11) to take the value $k=3[6.14]$.

Having now completed the construction of the effective theory of eq. (6.36), we can take a further step: the field $\bar{A}_{0}$ is massive, and can thus be integrated out, should we wish to study distance scales longer than $1 / \bar{m}$. Thereby we arrive at an even simpler effective theory,

$$
S_{\text {eff }}^{\prime}=\frac{1}{T} \int_{\mathbf{x}}\left\{\frac{1}{4} \overline{\bar{F}}_{i j}^{a} \overline{\bar{F}}_{i j}^{a}+\ldots\right\}
$$

referred to as MQCD, for "Magnetostatic QCD". It is important to realize that this theory, i.e. three-dimensional Yang-Mills theory (up to higher-order operators such as the one in eq. (6.35)), only has one parameter, the gauge coupling. Furthermore, if the fields $\overline{\bar{A}}_{i}^{a}$ are rescaled by an appropriate power of $T^{1 / 2}, \overline{\bar{A}}_{i}^{a} \rightarrow \overline{\bar{A}}_{i}^{a} T^{1 / 2}$, then the coefficient $1 / T$ in eq. (6.43) disappears. The coupling constant squared that appears afterwards is $\overline{\bar{g}}^{2} T$, and this is the only scale in the system. Therefore all dimensionful quantities (correlation lengths, string tension, free energy density, ...) must be proportional to an appropriate power of $\bar{g}^{2} T$, with a non-perturbative coefficient. This is 
the essence of the non-perturbative physics pointed out by Linde [6.1]. ${ }^{19}$

The implication of the above setup for the properties of the weak-coupling expansion is the following. Consider a generic observable $\mathcal{O}$, with an expectation value of the form

$$
\langle\mathcal{O}\rangle \sim g^{m} T^{n}\left[1+\alpha g^{r}+\ldots\right] .
$$

There are now four distinct possibilities:

(i) $r$ is even, and $\alpha$ is determined by the heavy scale $\sim \pi T$ and is purely perturbative. This is the case for instance for the leading correction to the free energy density $f(T)$, cf. eq. (5.123).

(ii) $r$ is odd, and $\alpha$ is determined by the intermediate scale $\sim g T$, being still purely perturbative. This is the case for the next-to-leading order corrections to many real-time quantities in thermal QCD, for instance to the heavy quark diffusion coefficient [6.18].

(iii) $m+r$ is even, and $\alpha$ is non-perturbatively determined by the soft scale $\sim g^{2} T / \pi$. This is the case e.g. for the next-to-leading order correction to the physical Debye screening length [5.9, $5.10]$ and for one of the subleading corrections to $f(T)$ in a non-Abelian plasma [6.1,6.19].

(iv) $r>k$, and $\alpha$ can only be determined correctly by adding higher-dimensional operators to the effective theory.

A few final remarks are in order:

- We have seen that the omission of higher-order operators in the construction of an effective theory usually leads to a small error, since the same Green's function is produced with a larger coefficient within it. It could happen, however, that there is some approximate symmetry in the full theory, which becomes exact within the effective theory, if we truncate its derivation to a given order. For instance, many Grand Unified Theories violate baryon minus lepton number $(B-L)$, whereas in the classic Standard Model this is an exact symmetry, to be broken only by some higher-dimensional operator [6.20,6.21]. Therefore, if such a Grand Unified Theory represented a true description of Nature and we considered $B-L$ violation within the classic Standard Model, we would make an infinitely large relative error.

- There are several reasons why effective theories constitute a useful framework. First of all, they allow us to justify and extend resummations such as those discussed in sec. 3.4 systematically to higher orders in the weak-coupling expansion. As mentioned below eqs. (3.93) and (5.123), this has led to the determination of many subsequent terms in the weak-coupling series. Second, effective theories permit for a simple non-perturbative study of the infrared sector affected by the Linde problem; examples are provided by refs. [6.9,6.19,6.22,6.23], and further ones will be encountered below.

- When proceeding to higher orders in the matching computations, they are often most conveniently formulated in the so-called background field gauge [6.24], rather than in the covariant gauge of eq. (5.40), cf. e.g. ref. [6.25].

\footnotetext{
${ }^{19}$ In contrast, topological configurations such as instantons, which play an important role for certain nonperturbative phenomena in vacuum, only play a minor role at finite temperatures $[6.15,6.16]$, save for special observables where the anomalous $\mathrm{U}_{\mathrm{A}}(1)$ breaking dominates the signal (cf. ref. [6.17] and references therein). The reason is that the Euclidean topological susceptibility (measuring topological "activity") vanishes to all orders in perturbation theory, and is numerically small.
} 


\section{Appendix A: Subtleties related to the low-energy expansion}

Let us consider the full theory

$$
L_{\text {full }} \equiv \frac{1}{2} \partial_{\mu} \phi \partial_{\mu} \phi+\frac{1}{2} m^{2} \phi^{2}+\frac{1}{2} \partial_{\mu} H \partial_{\mu} H+\frac{1}{2} M^{2} H^{2}+\frac{1}{6} \gamma H \phi^{3} .
$$

For simplicity (more precisely, in order to avoid ultraviolet divergences), we assume that the dimensionality of spacetime is 3, i.e. $d=2-2 \epsilon$ in our standard notation, and moreover we work at zero temperature, like in sec. 6.1. We then take the following steps:

(i) Integrating out $H$ in order to construct an effective theory, we compute the graph

$$
\begin{array}{c:c}
\vdots & \vdots \\
- & \vdots
\end{array}
$$

After Taylor-expanding the result in external momenta, we write down all the corresponding operators.

(ii) We focus on the 4-point function of the $\bar{\phi}$ field at vanishing external momenta, and determine the contributions of the operators computed in step (i) to this Green's function.

(iii) Finally we consider directly the full theory graph

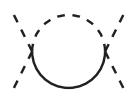

at vanishing external momenta. Comparing with the Taylor-expanded result obtained from step (ii), we demonstrate how a "careless" Taylor expansion can lead to wrong results.

The construction of the effective theory proceeds essentially as in eq. (3.12), except that only the $H$-field is now integrated out. We get from here

$$
\begin{aligned}
S_{\mathrm{eff}} & \approx\left\langle-\frac{1}{2} S_{I}^{2}\right\rangle_{H, \mathrm{c}} \\
& =-\frac{\gamma^{2}}{72} \int_{X, Y} \phi^{3}(X) \phi^{3}(Y)\langle H(X) H(Y)\rangle_{0} \\
& =-\frac{\gamma^{2}}{72} \int_{X, Y} \phi^{3}(X) \phi^{3}(Y) \int_{P} \frac{e^{i P \cdot(X-Y)}}{P^{2}+M^{2}} \\
& =-\frac{\gamma^{2}}{72} \int_{X, Y} \phi^{3}(X) \phi^{3}(Y) \int_{P} e^{i P \cdot(X-Y)}\left[\sum_{n=0}^{\infty} \frac{(-1)^{n}\left(P^{2}\right)^{n}}{\left(M^{2}\right)^{n+1}}\right] \\
& =-\frac{\gamma^{2}}{72} \int_{X, Y} \phi^{3}(X) \phi^{3}(Y)\left[\sum_{n=0}^{\infty} \frac{\left(\nabla_{X}^{2}\right)^{n}}{\left(M^{2}\right)^{n+1}}\right] \delta(X-Y) \\
& =-\frac{\gamma^{2}}{72} \int_{X} \sum_{n=0}^{\infty} \phi^{3}(X) \frac{\left(\nabla_{X}^{2}\right)^{n}}{\left(M^{2}\right)^{n+1}} \phi^{3}(X),
\end{aligned}
$$

where an expansion was carried out assuming $P^{2} \ll M^{2}$, and partial integrations were performed at the last step.

Using eq. (6.48), we can extract the corresponding contribution to the 4-point function at vanishing momenta:

$$
\left\langle\tilde{\phi}(0) \tilde{\phi}(0) \tilde{\phi}(0) \tilde{\phi}(0) e^{-S_{\text {eff }}}\right\rangle
$$




$$
\begin{aligned}
\Rightarrow & \frac{\gamma^{2}}{72}\left\langle\tilde{\phi}(0) \tilde{\phi}(0) \tilde{\phi}(0) \tilde{\phi}(0) \int_{P_{1}, \ldots, P_{6}} \delta\left(\Sigma_{i} P_{i}\right) \tilde{\phi}\left(P_{1}\right) \ldots \tilde{\phi}\left(P_{6}\right)\right\rangle \sum_{n=0}^{\infty} \frac{\left[-\left(P_{4}+P_{5}+P_{6}\right)^{2}\right]^{n}}{\left(M^{2}\right)^{n+1}} \\
= & \frac{\gamma^{2}}{72} \times 6 \times(2 \times 3 \times 2+3 \times 4 \times 2) \int_{P_{1}, \ldots, P_{6}} \delta\left(\Sigma_{i} P_{i}\right) \sum_{n=0}^{\infty} \frac{\left[-\left(P_{4}+P_{5}+P_{6}\right)^{2}\right]^{n}}{\left(M^{2}\right)^{n+1}} \\
& \times\left\langle\tilde{\phi}(0) \tilde{\phi}\left(P_{1}\right)\right\rangle_{0}\left\langle\tilde{\phi}(0) \tilde{\phi}\left(P_{2}\right)\right\rangle_{0}\left\langle\tilde{\phi}(0) \tilde{\phi}\left(P_{5}\right)\right\rangle_{0}\left\langle\tilde{\phi}(0) \tilde{\phi}\left(P_{6}\right)\right\rangle_{0}\left\langle\tilde{\phi}\left(P_{3}\right) \tilde{\phi}\left(P_{4}\right)\right\rangle_{0} \\
= & 3 \gamma^{2} \frac{\delta(0)}{\left(\bar{m}^{2}\right)^{4}} \int_{P_{3}} \frac{1}{P_{3}^{2}+\bar{m}^{2}} \sum_{n=0}^{\infty} \frac{\left(-P_{3}^{2}\right)^{n}}{\left(M^{2}\right)^{n+1}},
\end{aligned}
$$

where we denoted by $\bar{m}$ the mass of the effective-theory field $\bar{\phi}$ and by $\tilde{\phi}$ its Fourier representation. Furthermore we noted that the result vanishes unless the fields $\tilde{\phi}\left(P_{i}\right)$ are contracted so that one of the momenta $P_{4}, P_{5}$ and $P_{6}$ remains an integration variable. The integrals appearing in the result can be carried out in dimensional regularization; for instance, the two leading terms read

$$
\begin{aligned}
& n=0: \quad \frac{1}{M^{2}} \int_{P_{3}} \frac{1}{P_{3}^{2}+\bar{m}^{2}}=\frac{1}{M^{2}}\left(-\frac{\bar{m}}{4 \pi}\right), \\
& n=1: \quad-\frac{1}{M^{4}} \int_{P_{3}} \frac{P_{3}^{2}}{P_{3}^{2}+\bar{m}^{2}}=\frac{\bar{m}^{2}}{M^{4}} \int_{P_{3}} \frac{1}{P_{3}^{2}+\bar{m}^{2}}=-\frac{1}{M^{4}} \frac{\bar{m}^{3}}{4 \pi},
\end{aligned}
$$

where we made use of eq. (2.86) and of the vanishing of scale-free integrals in dimensional regularization. We note that the terms get smaller with increasing $n$, apparently justifying a posteriori the Taylor expansion we carried out above.

Let us finally carry out the integral corresponding to eq. (6.47) exactly. The contractions remain as above, and we simply need to replace the integral in eq. (6.49) by

$$
\begin{aligned}
\int_{P_{3}} \frac{1}{P_{3}^{2}+\bar{m}^{2}} \frac{1}{P_{3}^{2}+M^{2}} & =\int_{P_{3}} \frac{1}{M^{2}-\bar{m}^{2}}\left[\frac{1}{P_{3}^{2}+\bar{m}^{2}}-\frac{1}{P_{3}^{2}+M^{2}}\right] \\
& =\frac{1}{M^{2}-\bar{m}^{2}}\left(\frac{-1}{4 \pi}\right)(\bar{m}-M) \\
& =\frac{1}{4 \pi(M+\bar{m})} \\
& =\frac{1}{4 \pi M}\left(1-\frac{\bar{m}}{M}+\frac{\bar{m}^{2}}{M^{2}}-\frac{\bar{m}^{3}}{M^{3}}+\ldots\right) .
\end{aligned}
$$

Comparing eqs. (6.50) and (6.51) with eq. (6.52), we note that by carrying out the Taylor expansion, i.e. the naive matching of the effective theory parameters, we missed the leading contribution in eq. (6.52). The largest term we found, eq. (6.50), is only next-to-leading in eq. (6.52). It furthermore appears that we missed all even powers of $\bar{m}$ in the sum of eq. (6.52).

The reason for the problem encountered is the same as in eq. (6.21): it again has to be taken into account that the light fields $\phi$ can also carry large momenta $P_{3} \sim M$, in which case a Taylor expansion of $1 /\left(P_{3}^{2}+M^{2}\right)$ is not justified. Rather, we have to view eq. (6.47) in analogy with eq. (6.21),

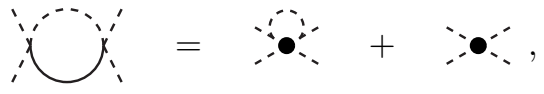

where the first term corresponds to a naive replacement of eq. (6.46) by a momentum-independent 6 -point vertex, and the second term to a contribution from hard $\phi$-modes to an effective 4-point vertex. In accordance with our discussion around eq. (6.21), we see that the result of eq. (6.50) (and more generally eq. (6.49)) is indeed non-analytic in the parameter $\bar{m}^{2}$, whereas the supplementary terms in eq. (6.52) that the naive Taylor expansion missed are analytic in it. 


\section{Literature}

[6.1] A.D. Linde, Infrared problem in thermodynamics of the Yang-Mills gas, Phys. Lett. B 96 (1980) 289.

[6.2] J.C. Collins, Renormalization (Cambridge University Press, 1984).

[6.3] K. Jansen et al., Non-perturbative renormalization of lattice QCD at all scales, Phys. Lett. B 372 (1996) 275 [hep-lat/9512009].

[6.4] P. Weisz, Renormalization and lattice artifacts, arXiv:1004.3462.

[6.5] P. Ginsparg, First and second order phase transitions in gauge theories at finite temperature, Nucl. Phys. B 170 (1980) 388.

[6.6] T. Appelquist and R.D. Pisarski, High-temperature Yang-Mills theories and threedimensional Quantum Chromodynamics, Phys. Rev. D 23 (1981) 2305.

[6.7] K. Kajantie, M. Laine, K. Rummukainen and M.E. Shaposhnikov, Generic rules for high temperature dimensional reduction and their application to the Standard Model, Nucl. Phys. B 458 (1996) 90 [hep-ph/9508379].

[6.8] S. Chapman, New dimensionally reduced effective action for QCD at high temperature, Phys. Rev. D 50 (1994) 5308 [hep-ph/9407313].

[6.9] A. Hart, M. Laine and O. Philipsen, Static correlation lengths in QCD at high temperatures and finite densities, Nucl. Phys. B 586 (2000) 443 [hep-ph/0004060].

[6.10] S. Nadkarni, Dimensional reduction in finite-temperature Quantum Chromodynamics. II, Phys. Rev. D 38 (1988) 3287.

[6.11] N.P. Landsman, Limitations to dimensional reduction at high temperature, Nucl. Phys. B $322(1989) 498$.

[6.12] S. Huang and M. Lissia, The relevant scale parameter in the high temperature phase of QCD, Nucl. Phys. B 438 (1995) 54 [hep-ph/9411293].

[6.13] M. Laine and Y. Schröder, Two-loop QCD gauge coupling at high temperatures, JHEP 03 (2005) 067 [hep-ph/0503061].

[6.14] K. Kajantie, M. Laine, K. Rummukainen and M.E. Shaposhnikov, High temperature dimensional reduction and parity violation, Phys. Lett. B 423 (1998) 137 [hep-ph/9710538].

[6.15] D.J. Gross, R.D. Pisarski and L.G. Yaffe, $Q C D$ and instantons at finite temperature, Rev. Mod. Phys. 53 (1981) 43.

[6.16] A. Boccaletti and D. Nogradi, The semi-classical approximation at high temperature revisited, JHEP 03 (2020) 045 [2001.03383].

[6.17] T. Kanazawa and N. Yamamoto, U(1) axial symmetry and Dirac spectra in QCD at high temperature, JHEP 01 (2016) 141 [1508.02416].

[6.18] S. Caron-Huot and G.D. Moore, Heavy quark diffusion in perturbative QCD at next-toleading order, Phys. Rev. Lett. 100 (2008) 052301 [0708.4232]. 
[6.19] F. Di Renzo, M. Laine, V. Miccio, Y. Schröder and C. Torrero, The leading non-perturbative coefficient in the weak-coupling expansion of hot QCD pressure, JHEP 07 (2006) 026 [hep$\mathrm{ph} / 0605042]$.

[6.20] S. Weinberg, Baryon- and Lepton-Nonconserving Processes, Phys. Rev. Lett. 43 (1979) 1566.

[6.21] F. Wilczek and A. Zee, Operator Analysis of Nucleon Decay, Phys. Rev. Lett. 43 (1979) 1571.

[6.22] P.B. Arnold and L.G. Yaffe, Non-Abelian Debye screening length beyond leading order, Phys. Rev. D 52 (1995) 7208 [hep-ph/9508280].

[6.23] M. D'Onofrio, K. Rummukainen and A. Tranberg, Sphaleron Rate in the Minimal Standard Model, Phys. Rev. Lett. 113 (2014) 141602 [1404.3565].

[6.24] L.F. Abbott, The background field method beyond one loop, Nucl. Phys. B 185 (1981) 189.

[6.25] I. Ghisoiu, J. Möller and Y. Schröder, Debye screening mass of hot Yang-Mills theory to three-loop order, JHEP 11 (2015) 121 [1509.08727]. 


\title{
7. Finite density
}

\begin{abstract}
The concept of a system at a finite density or, equivalently, at a finite chemical potential, is introduced. Considering first a complex scalar field, an imaginary-time path integral representation is derived for the partition function. The evaluation of the partition function reveals infrared problems, which are this time related to the phenomenon of Bose-Einstein condensation. A generic tool applicable to any scalar field theory, called the effective potential, is introduced in order to handle this situation. Subsequently the case of a Dirac fermion at a finite chemical potential is discussed. The concept of a susceptibility is introduced. The quark number susceptibility in QCD is evaluated up to second order in the gauge coupling.
\end{abstract}

Keywords: Noether's theorem, global symmetry, Bose-Einstein condensation, condensate, constrained effective potential, susceptibility.

\subsection{Complex scalar field and effective potential}

Let us consider a system which possesses some conserved global charge, $Q$. We assume the conserved charge to be additive, i.e. the charge can in principle have any (integer) value. Physical examples of possible $Q$ 's include:

- The baryon number $B$ and the lepton number $L$. (In fact, within the classic Standard Model, the combination $B+L$ is not conserved because of an anomaly [7.1], so that strictly speaking only the linear combination $B-L$ is conserved; however, the rate of $B+L$ violation is exponentially small at $T<160 \mathrm{GeV}$ [7.2], so in this regime we can treat both $B$ and $L$ as separate conserved quantities.)

- If weak interactions are switched off (i.e., if we inspect phenomena at temperatures well below $50 \mathrm{GeV}$, time scales well shorter than $10^{-10} \mathrm{~s}$, or distances well below $1 \mathrm{~cm}$ within the collision region of a particle experiment), then flavour quantum numbers such as the strangeness $S$ are conserved. One prominent example of this is QCD thermodynamics, where one typically considers the chemical potentials of all quark flavors to be independent parameters.

- In non-relativistic field theories, the particle number $N$ is conserved.

- In some supersymmetric theories, there is a quantity called the $R$-charge which is conserved. (However this is normally a multiplicative rather than an additive charge. As discussed below, this leads to a qualitatively different behaviour.)

The case of a conserved $Q$ turns out to be analogous to the case of gauge fields, treated in sec. 5; indeed the introduction of a chemical potential, $\mu$, as a conjugate variable to $Q$, is closely related to the introduction of the gauge field $A_{0}$ that was needed for imposing the Gauss law, " $Q=0$ ". However, in contrast to that situation, we work in a grand canonical ensemble in the following, so that the quantum mechanical partition function is of the type

$$
\mathcal{Z}(T, \mu) \equiv \operatorname{Tr}\left[e^{-\beta(\hat{H}-\mu \hat{Q})}\right] .
$$


In sec. 5 the projection operator $\delta_{\hat{Q}, \hat{0}}$ was effectively imposed as

$$
\delta_{\hat{Q}, \hat{0}}=\int_{-\pi}^{\pi} \frac{\mathrm{d} \theta}{2 \pi} e^{i \theta \hat{Q}}
$$

where $\theta \propto A_{0}$ and we assumed the eigenvalues of $\hat{Q}$ to be integers. Comparing eqs. (7.1) and (7.2), a chemical potential is seen to correspond, roughly speaking, to a constant purely imaginary Euclidean gauge field $A_{0}$.

Now, let us go back to classical field theory for a moment, and recall that if the system possesses a global U(1) symmetry, then there exists, according to Noether's theorem, a conserved current, $\mathcal{J}_{\mu}$. The integral of the zeroth component of the current, i.e. the charge density, over the spatial volume, defines the conserved charge,

$$
Q \equiv \int_{\mathbf{x}} \mathcal{J}_{0}(t, \mathbf{x})
$$

Conversely, one can expect that a system which does have a conserved global charge should also display a global U(1) symmetry in its field-theoretic description. Usually this is indeed the case, and we restrict to these situations in the following. (One notable exception is free field theory where, due to a lack of interactions, particle number is conserved even without a global symmetry. Another is that a discrete symmetry, $\phi \rightarrow-\phi$, may also lead to the concept of a generalized "parity", which acts as a multiplicative quantum number, with possible values \pm 1 ; however, in this case no non-trivial charge density $\rho=\langle\hat{Q}\rangle / V$ can be defined in the thermodynamic limit.)

As the simplest example of a system with an additive conserved charge and a global U(1) symmetry, consider a complex scalar field. The classical Lagrangian of a complex scalar field reads

$$
\mathcal{L}_{M}=\partial^{\mu} \phi^{*} \partial_{\mu} \phi-V(\phi)
$$

where the potential has the form

$$
V(\phi) \equiv m^{2} \phi^{*} \phi+\lambda\left(\phi^{*} \phi\right)^{2}
$$

The system is invariant in the (position-independent) phase transformation

$$
\phi \rightarrow e^{-i \alpha} \phi, \quad \phi^{*} \rightarrow e^{i \alpha} \phi^{*}
$$

where $\alpha \in \mathbb{R}$. The corresponding Noether current can be defined as

$$
\begin{aligned}
\mathcal{J}_{\mu} & \equiv \frac{\partial \mathcal{L}_{M}}{\partial\left(\partial^{\mu} \phi\right)} \frac{\delta \phi}{\delta \alpha}+\frac{\partial \mathcal{L}_{M}}{\partial\left(\partial^{\mu} \phi^{*}\right)} \frac{\delta \phi^{*}}{\delta \alpha} \\
& =-\partial_{\mu} \phi^{*} i \phi+\partial_{\mu} \phi i \phi^{*} \\
& =-i\left[\left(\partial_{\mu} \phi^{*}\right) \phi-\phi^{*} \partial_{\mu} \phi\right]=-2 \operatorname{Im}\left[\phi^{*} \partial_{\mu} \phi\right] .
\end{aligned}
$$

The overall sign (i.e., what we call particles and antiparticles) is a matter of convention; we could equally well have defined the global symmetry through $\phi \rightarrow e^{i \alpha} \phi, \phi^{*} \rightarrow e^{-i \alpha} \phi^{*}$, and then $\mathcal{J}_{\mu}$ would have the opposite sign.

The first task, as always, is to write down a path integral expression for the partition function, eq. (7.1). Subsequently, we may try to evaluate the partition function, in order to see what kind of phenomena take place in this system. 
In order to write down the path integral, we start from the known expression of $\mathcal{Z}$ of a real scalar field $\phi_{1}$ without a chemical potential, i.e. the generalization to field theory of eq. (1.33):

$$
\mathcal{Z} \propto \int_{\text {periodic }} \mathcal{D} \phi_{1} \int \mathcal{D} \pi_{1} \exp \left\{-\int_{0}^{\beta} \mathrm{d} \tau \int_{\mathbf{x}}\left[\frac{1}{2} \pi_{1}^{2}-i \pi_{1} \partial_{\tau} \phi_{1}+\frac{1}{2}\left(\partial_{i} \phi_{1}\right)^{2}+V\left(\phi_{1}\right)\right]\right\},
$$

where $\pi_{1}=\partial \phi_{1} / \partial t$ (cf. the discussion in sec. 2.1). Here the combination $\frac{1}{2} \pi_{1}^{2}+\frac{1}{2}\left(\partial_{i} \phi_{1}\right)^{2}+V\left(\phi_{1}\right)$ is nothing but the classical Hamiltonian density, $\mathcal{H}\left(\pi_{1}, \phi_{1}\right)$.

In order to make use of eq. (7.8), let us rewrite the complex scalar field $\phi$ as $\phi=\left(\phi_{1}+i \phi_{2}\right) / \sqrt{2}$, $\phi_{i} \in \mathbb{R}$. Then

$$
\partial^{\mu} \phi^{*} \partial_{\mu} \phi=\frac{1}{2} \partial^{\mu} \phi_{1} \partial_{\mu} \phi_{1}+\frac{1}{2} \partial^{\mu} \phi_{2} \partial_{\mu} \phi_{2}, \quad \phi^{*} \phi=\frac{1}{2}\left(\phi_{1}^{2}+\phi_{2}^{2}\right),
$$

and the classical Hamiltonian density reads

$$
\mathcal{H}=\frac{1}{2}\left[\pi_{1}^{2}+\pi_{2}^{2}+\left(\partial_{i} \phi_{1}\right)^{2}+\left(\partial_{i} \phi_{2}\right)^{2}+m^{2} \phi_{1}^{2}+m^{2} \phi_{2}^{2}\right]+\frac{1}{4} \lambda\left(\phi_{1}^{2}+\phi_{2}^{2}\right)^{2} .
$$

For the grand canonical ensemble, we need to add from eqs. (7.3) and (7.7) the classical version of $-\mu \hat{Q}$ to the Hamiltonian, cf. eq. (7.1):

$$
\begin{aligned}
-\mu Q & =\mu \int_{\mathbf{x}} \operatorname{Im}\left[\left(\phi_{1}-i \phi_{2}\right)\left(\partial_{t} \phi_{1}+i \partial_{t} \phi_{2}\right)\right] \\
& =\int_{\mathbf{x}} \mu\left(\pi_{2} \phi_{1}-\pi_{1} \phi_{2}\right) .
\end{aligned}
$$

Since the charge can be expressed in terms of the canonical variables, nothing changes in the derivation of the path integral, and we can simply replace the Hamiltonian of eq. (7.8) by the sum of eqs. (7.10) and (7.11).

Finally, we carry out the Gaussian integrals over $\pi_{1}, \pi_{2}$ :

$$
\begin{aligned}
& \int \mathrm{d} \pi_{1} \exp \left\{-\mathrm{d}^{D} X\left[\frac{1}{2} \pi_{1}^{2}+\pi_{1}\left(-i \frac{\partial \phi_{1}}{\partial \tau}-\mu \phi_{2}\right)\right]\right\}=C \exp \left\{-\frac{1}{2} \mathrm{~d}^{D} X\left(\frac{\partial \phi_{1}}{\partial \tau}-i \mu \phi_{2}\right)^{2}\right\} \\
& \int \mathrm{d} \pi_{2} \exp \left\{-\mathrm{d}^{D} X\left[\frac{1}{2} \pi_{2}^{2}+\pi_{2}\left(-i \frac{\partial \phi_{2}}{\partial \tau}+\mu \phi_{1}\right)\right]\right\}=C \exp \left\{-\frac{1}{2} \mathrm{~d}^{D} X\left(\frac{\partial \phi_{2}}{\partial \tau}+i \mu \phi_{1}\right)^{2}\right\} .
\end{aligned}
$$

Afterwards we go back to the complex notation, writing

$$
\begin{aligned}
& \frac{1}{2}\left(\frac{\partial \phi_{1}}{\partial \tau}-i \mu \phi_{2}\right)^{2}+\frac{1}{2}\left(\frac{\partial \phi_{2}}{\partial \tau}+i \mu \phi_{1}\right)^{2} \\
= & \frac{1}{2}\left[\left(\frac{\partial \phi_{1}}{\partial \tau}\right)^{2}+\left(\frac{\partial \phi_{2}}{\partial \tau}\right)^{2}\right]-\mu \times \underbrace{\left.i \phi_{2} \frac{\partial \phi_{1}}{\partial \tau}-\phi_{1} \frac{\partial \phi_{2}}{\partial \tau}\right]}_{\phi \partial_{\tau} \phi^{*}-\phi^{*} \partial_{\tau} \phi}-\frac{1}{2} \mu^{2}\left(\phi_{1}^{2}+\phi_{2}^{2}\right) \\
= & {\left[\left(\partial_{\tau}+\mu\right) \phi^{*}\right]\left[\left(\partial_{\tau}-\mu\right) \phi\right] . }
\end{aligned}
$$

In total, then, the path integral representation for the grand canonical partition function of a complex scalar field reads

$$
\mathcal{Z}(T, \mu)=C \int_{\text {periodic }} \mathcal{D} \phi \exp \left\{-\int_{0}^{\beta} \mathrm{d} \tau \int_{\mathbf{x}}\left[\left(\partial_{\tau}+\mu\right) \phi^{*}\left(\partial_{\tau}-\mu\right) \phi+\partial_{i} \phi^{*} \partial_{i} \phi+m^{2} \phi^{*} \phi+\lambda\left(\phi^{*} \phi\right)^{2}\right]\right\} .
$$


As anticipated, $\mu$ appears in a way reminiscent of an imaginary gauge field $A_{0}$.

Let us work out the properties of the free theory in the presence of $\mu$. Going to momentum space with $P=\left(\omega_{n}, \mathbf{p}\right)$, the quadratic part of the Euclidean action becomes

$$
\begin{aligned}
S_{E}^{(0)} & =\sum_{P} \tilde{\phi}^{*}(P)\left[\left(-i \omega_{n}+\mu\right)\left(i \omega_{n}-\mu\right)+p^{2}+m^{2}\right] \tilde{\phi}(P) \\
& =\sum_{P} \tilde{\phi}^{*}(P) \tilde{\phi}(P)\left[\left(\omega_{n}+i \mu\right)^{2}+p^{2}+m^{2}\right] .
\end{aligned}
$$

We observe that the chemical potential induces a shift of the Matsubara frequencies by a constant imaginary term (this was the reason for considering the corresponding sum in eq. (2.36)). In particular, the propagator reads

$$
\left\langle\tilde{\phi}(P) \tilde{\phi}^{*}(Q)\right\rangle_{0}=\delta(P-Q) \frac{1}{\left(\omega_{n}+i \mu\right)^{2}+p^{2}+m^{2}},
$$

whereas the grand canonical free energy density (sometimes referred to as the grand potential in the literature) is obtained from eqs. (2.44) and (2.49). We just need to replace $c \rightarrow i \mu$ and note that for a complex scalar field, all Fourier modes are independent, whereby the structures in eqs. (2.44) and (2.49) are to be multiplied by a factor 2 :

$$
f(T, \mu)=\left.\int_{\mathbf{p}}\left\{\epsilon_{p}+T\left[\ln \left(1-e^{-\beta\left(\epsilon_{p}-\mu\right)}\right)+\ln \left(1-e^{-\beta\left(\epsilon_{p}+\mu\right)}\right)\right]\right\}\right|_{\epsilon_{p}=\sqrt{p^{2}+m^{2}}} .
$$

We may wonder how the existence of $\mu \neq 0$ affects the infrared problem of finite-temperature field theory, discussed in sec. 6.1. In sec. 2.3 we found that the high-temperature expansion $(T \gg m)$ of eq. (7.18) at $\mu=0$ has a peculiar structure, because of a branch cut starting at $m^{2}=0$. From the second term in eq. (7.18), we note that this problem has become worse in the presence of $\mu>0$ : the integrand is complex-valued if $\mu>m$, because then $\exp \left(-\beta\left(\epsilon_{p}-\mu\right)\right)>1$ at small $p$. In an

interacting theory, thermal corrections generate an effective mass $m_{\text {eff }}^{2} \sim \lambda T^{2}$ (cf. eq. (3.95)), which postpones the problem to a larger $\mu$. Nevertheless, for large enough $\mu$ it still exists.

It turns out that there is a physics consequence from this infrared problem: the existence of Bose-Einstein condensation, to which we now turn.

\section{Bose-Einstein condensation}

In order to properly treat complex scalar field theory with a chemical potential, two things need to be realized:

(i) In contrast to gauge field theory, the infrared problem exists even in the non-interacting limit. Therefore it cannot be cured by a perturbatively or non-perturbatively generated effective mass. Rather, it corresponds to a strong dependence of the properties of the system on the volume, so we should keep the volume finite to start with.

(ii) The chemical potential $\mu$ is a most useful quantity in theoretical computations, but it is somewhat "abstract" from a practical point of view; the physical properties of the system are typically best characterized not by $\mu$ but by the intensive variable conjugate to $\mu$, i.e. the number density of the conserved charge. Therefore, rather than trying to give $\mu$ some specific value, we should fix the number density. 
Motivated by point (i), let us put the system in a periodic box, $V=L_{1} L_{2} L_{3}$. The spatial momenta get discretized like in eq. (2.9),

$$
\mathbf{p}=2 \pi\left(\frac{n_{1}}{L_{1}}, \frac{n_{2}}{L_{2}}, \frac{n_{3}}{L_{3}}\right),
$$

with $n_{i} \in \mathbb{Z}$. The mode with $\omega_{n}=0, \mathbf{p}=\mathbf{0}$ will be called the condensate, and denoted by $\bar{\phi}$. Note that the condensate is a Matsubara zero mode but in addition a spatial zero mode.

We now rewrite the partition function of eq. (7.15) as

$$
\begin{aligned}
\mathcal{Z}(T, \mu) & =\int_{-\infty}^{\infty} \mathrm{d} \bar{\phi}\left\{\int_{\text {periodic, } P \neq 0} \mathcal{D} \phi^{\prime} e^{-S_{E}\left[\phi=\bar{\phi}+\phi^{\prime}\right]}\right\} \\
& \equiv \int_{-\infty}^{\infty} \mathrm{d} \bar{\phi} \exp \left[-\frac{V}{T} V_{\text {eff }}\left(\bar{\phi}^{*} \bar{\phi}\right)\right]
\end{aligned}
$$

Here $\phi^{\prime}$ contains all modes with $P \neq 0$, and $V_{\text {eff }}$ is called the (constrained) effective potential. The factor $V / T$ is the trivial spacetime integral, $\int_{0}^{\beta} \mathrm{d} \tau \int_{V} \mathrm{~d}^{d} \mathbf{x}$.

Let us write down the effective potential explicitly for very weak interactions, $\lambda \approx 0$. It turns out that the limit $\lambda \rightarrow 0$ is subtle, so for the moment we keep $\lambda$ non-zero in the zero-mode part. From eqs. (7.15) and (7.16) we get

$$
\begin{aligned}
S_{E}\left[\phi=\bar{\phi}+\phi^{\prime}\right]=\frac{V}{T} & {\left[\left(m^{2}-\mu^{2}\right) \bar{\phi}^{*} \bar{\phi}+\lambda\left(\bar{\phi}^{*} \bar{\phi}\right)^{2}\right] } \\
& +\sum_{P \neq 0}\left\{\tilde{\phi}^{\prime *}(P) \tilde{\phi}^{\prime}(P)\left[\left(\omega_{n}+i \mu\right)^{2}+p^{2}+m^{2}\right]+\mathcal{O}(\lambda)\right\},
\end{aligned}
$$

where we made use of the fact that the crossterm between $\bar{\phi}$ and $\phi^{\prime}$ vanishes, given that by definition $\phi^{\prime}$ has no zero-momentum mode:

$$
\int_{0}^{\beta} \mathrm{d} \tau \int_{V} \mathrm{~d}^{d} \mathbf{x} \phi^{\prime}=0
$$

The path integral over the latter term in eq. (7.21) yields then eq. (7.18); in the limit of a large volume, the omission of a single mode does not matter (its effect is $\propto(T / V) \ln \left(m^{2}-\mu^{2}\right)$ ). Thereby the effective potential reads

$$
V_{\mathrm{eff}}\left(\bar{\phi}^{*} \bar{\phi}\right)=\left(m^{2}-\mu^{2}\right) \bar{\phi}^{*} \bar{\phi}+\lambda\left(\bar{\phi}^{*} \bar{\phi}\right)^{2}+f(T, \mu)+\mathcal{O}\left(\frac{1}{V}, \lambda\right) .
$$

Physically, the first two terms correspond to the contribution of the particles that have formed a condensate, whereas the third term represents propagating particle modes in the plasma.

Now, if we go to the limit of very small temperatures, $T \ll m$, and assume furthermore that $|\mu| \leq m$, which is required in order for eq. (7.18) to be defined, then the thermal part of eq. (7.18) vanishes. (It has a "non-relativistic" limiting value for $\mu \rightarrow m^{-}$, which scales as $-T^{4}\left(\frac{m}{2 \pi T}\right)^{\frac{3}{2}}$.) The vacuum contribution to eq. (7.18) is on the other hand independent of $T$ and $\mu$, and can be omitted. Therefore,

$$
V_{\mathrm{eff}}\left(\bar{\phi}^{*} \bar{\phi}\right) \approx\left(m^{2}-\mu^{2}\right) \bar{\phi}^{*} \bar{\phi}+\lambda\left(\bar{\phi}^{*} \bar{\phi}\right)^{2}
$$

The remaining task is to carry out the integral over $\bar{\phi}$ in eq. (7.20). At this point we need to make contact with the particle number density. From $\mathcal{Z}=\operatorname{Tr}[\exp (-\beta \hat{H}+\beta \mu \hat{Q})]$ and the definition 
of $V_{\text {eff }}$ in eq. (7.20), we obtain

$$
\begin{aligned}
\rho & \equiv \frac{\langle\hat{Q}\rangle}{V}=\frac{T}{V} \frac{\partial \ln \mathcal{Z}}{\partial \mu} \\
& =\frac{\int \mathrm{d} \bar{\phi} 2 \mu \bar{\phi}^{*} \bar{\phi} \exp \left[-\frac{V}{T} V_{\text {eff }}\left(\bar{\phi}^{*} \bar{\phi}\right)\right]}{\int \mathrm{d} \bar{\phi} \exp \left[-\frac{V}{T} V_{\text {eff }}\left(\bar{\phi}^{*} \bar{\phi}\right)\right]} \equiv 2 \mu\left\langle\bar{\phi}^{*} \bar{\phi}\right\rangle,
\end{aligned}
$$

where $\left\langle\bar{\phi}^{*} \bar{\phi}\right\rangle$ is the expectation value of $\bar{\phi}^{*} \bar{\phi}$.

Let us consider a situation where we decrease the temperature, $T \ll m$, and attempt simultaneously to keep the particle number density, the left-hand side of eq. (7.26), fixed. How should we choose $\mu$ in this situation? There are three possibilities:

(i) If $|\mu|<m$, the integrals in eq. (7.26) can be carried out even for $\lambda \rightarrow 0^{+}$. In fact the result corresponds to the "propagator" of $\bar{\phi}$ :

$$
\lim _{\lambda \rightarrow 0^{+}} \rho=\frac{2 \mu T}{V\left(m^{2}-\mu^{2}\right)} .
$$

We note that if $T \rightarrow 0$, then $\rho \rightarrow 0$. This conflicts with our assumption that the number density stays constant; therefore this range of $\mu$ is not physically relevant for our situation.

(ii) If $|\mu|>m$, the integrals in eq. (7.26) are defined only for $\lambda>0$. For $V \rightarrow \infty$ they can be determined by the saddle point approximation:

$$
V_{\mathrm{eff}}^{\prime}\left(\bar{\phi}^{*} \bar{\phi}\right)=0 \Rightarrow \bar{\phi}^{*} \bar{\phi}=\frac{\mu^{2}-m^{2}}{2 \lambda} \quad \Rightarrow \quad \rho=\frac{\mu\left(\mu^{2}-m^{2}\right)}{\lambda} .
$$

We see that for $\lambda \rightarrow 0^{+}$, we need to send $\mu \rightarrow m^{+}$, in order to keep $\rho$ finite.

(iii) According to the preceding points, the only possible choice at $\lambda=0^{+}$is $|\mu|=m$. For $\rho>0$ we need to choose $\mu=m$. In this limit eq. (7.26) can be expressed as

$$
\rho=2 m\left\langle\bar{\phi}^{*} \bar{\phi}\right\rangle,
$$

which should be thought of as a condition for the field $\bar{\phi}$.

Eq. (7.29) manifests the phenomenon of Bose-Einstein condensation (at zero temperature in the free limit): the conserved particle number is converted into a non-zero scalar condensate.

It is straightforward to include the effects of a finite temperature in these considerations, by starting from eq. (7.23) so that $-\partial_{\mu} f(T, \mu)$ gives another contribution to the charge density, and the effects of interactions, by keeping $\lambda>0$. These very interesting developments go beyond the scope of the present lectures (cf. e.g. refs. [7.3]-[7.5]). On the other hand, the concepts of a condensate and an effective potential will be met again in later chapters. 


\subsection{Dirac fermion with a finite chemical potential}

The Lagrangian of a Dirac fermion,

$$
\mathcal{L}_{M}=\bar{\psi}_{A}\left(i \not D_{A B}-m \delta_{A B}\right) \psi_{B}, \quad \not D_{A B}=\gamma^{\mu}\left(\delta_{A B} \partial_{\mu}-i g A_{\mu}^{a} T_{A B}^{a}\right),
$$

possesses a global symmetry,

$$
\psi_{A} \rightarrow e^{-i \alpha} \psi_{A}, \quad \bar{\psi}_{A} \rightarrow e^{i \alpha} \bar{\psi}_{A},
$$

in addition to the usual non-Abelian (local) gauge symmetry. Therefore there is a conserved quantity, and we can consider the behaviour of the system in the presence of a chemical potential.

The conserved Noether current reads

$$
\begin{aligned}
\mathcal{J}_{\mu} & =\frac{\partial \mathcal{L}_{M}}{\partial\left(\partial^{\mu} \psi_{A}\right)} \frac{\delta \psi_{A}}{\delta \alpha} \\
& =-\bar{\psi}_{A} i \gamma_{\mu} i \psi_{A}=\bar{\psi}_{A} \gamma_{\mu} \psi_{A} .
\end{aligned}
$$

The corresponding charge is $Q=\int_{\mathbf{x}} \mathcal{J}_{0}$, and as an operator it commutes with the Hamiltonian, $[\hat{H}, \hat{Q}]=0$. Therefore, like with scalar field theory, we can treat the combination $\hat{H}-\mu \hat{Q}$ as an "effective" Hamiltonian, and directly write down the corresponding path integral, by adding

$$
-\mu Q=-\mu \int_{\mathbf{x}} \bar{\psi}_{A} \gamma_{0} \psi_{A}
$$

to the Euclidean action. The path integral thereby reads

$$
\mathcal{Z}(T, \mu)=\int_{\text {antiperiodic }} \mathcal{D} \overline{\mathcal{D}} \psi \exp \left\{-\int_{0}^{\beta} \mathrm{d} \tau \int_{\mathbf{x}} \bar{\psi}\left[\gamma_{\mu} D_{\mu}-\gamma_{0} \mu+m\right] \psi\right\}
$$

For perturbation theory, let us consider the quadratic part of the Euclidean action. Going to momentum space with $P=\left(\omega_{n}, \mathbf{p}\right)$, we get

$$
S_{E}^{(0)}=\oint_{\{P\}} \tilde{\bar{\psi}}(P)\left[i \gamma_{0} \omega_{n}+i \gamma_{i} p_{i}-\gamma_{0} \mu+m\right] \tilde{\psi}(P) .
$$

Therefore, just like in sec. 7.1, the existence of a chemical potential corresponds to a shift $\omega_{n} \rightarrow$ $\omega_{n}+i \mu$ of the Matsubara frequencies.

Let us write down the free energy density of a single free Dirac fermion. Compared with a complex scalar field, there is an overall factor -2 (rather than -4 like in eq. (4.52), where we compared with a real scalar field). Otherwise, the chemical potential appears in identical ways in eqs. (7.16) and eq. (7.35), so eq. (4.55), $\sigma_{\mathrm{f}}(T)=2 \sigma_{\mathrm{b}}\left(\frac{T}{2}\right)-\sigma_{\mathrm{b}}(T)$, continues to apply. Employing it with eq. (7.18) we get

$$
\begin{aligned}
f(T, \mu)=-2 \int_{\mathbf{p}}\left\{\epsilon_{p}+T\right. & {\left[\ln \left(1-e^{-2 \beta\left(\epsilon_{p}-\mu\right)}\right)+\ln \left(1-e^{-2 \beta\left(\epsilon_{p}+\mu\right)}\right)\right.} \\
& \left.\left.-\ln \left(1-e^{-\beta\left(\epsilon_{p}-\mu\right)}\right)-\ln \left(1-e^{-\beta\left(\epsilon_{p}+\mu\right)}\right)\right]\right\} \\
=-2 \int_{\mathbf{p}}\left\{\epsilon_{p}+T\right. & {\left.\left[\ln \left(1+e^{-\beta\left(\epsilon_{p}-\mu\right)}\right)+\ln \left(1+e^{-\beta\left(\epsilon_{p}+\mu\right)}\right)\right]\right\} . }
\end{aligned}
$$

The thermal part of this integral is well-defined for any $\mu$; thus fermions do not suffer from infrared problems with $\mu \neq 0$, and do not undergo condensation (in the absence of interactions). 


\section{How about chemical potentials for gauge symmetries?}

It was mentioned after eq. (7.2) that a chemical potential has some relation to a gauge field $A_{0}$. However, in cases like QCD, a chemical potential has no colour structure (i.e. it is an identity matrix in colour space), whereas $A_{0}$ is a traceless matrix in colour space (cf. eq. (7.30)). On the other hand, in QED, $A_{0}$ is not traceless. In fact, in QED, the gauge symmetry is nothing but a local version of that in eq. (7.31). We may therefore ask whether we can associate a chemical potential to the electric charge of QED, and what the precise relation of $A_{0}$ and $\mu$ is in this case.

Let us first recall what happens in such a situation physically. A non-zero chemical potential in QED corresponds to a system which is charged. Moreover, if we want to describe it perturbatively with the QED Lagrangian, we had better choose a system where the charge carriers (particles) are essentially free; such a system could be a metal or a plasma. In this situation, the free charge carriers interact repulsively with a long-range force, and hence all the net charge resides on the surface. In other words, the homogeneous "bulk" of the medium is neutral (i.e. has no free charge). The charged body as a whole has a non-zero electric potential, $V_{0}$, with respect to the ground.

Let us try to understand how to reproduce this behaviour directly from the partition function, eq. (7.34), adapted to QED:

$$
\mathcal{Z}(T, \mu)=\int_{\text {b.c. }} \mathcal{D} A_{\mu} \mathcal{D} \bar{\psi} \mathcal{D} \psi \exp \left\{-\int_{0}^{\beta} \mathrm{d} \tau \int_{\mathbf{x}}\left[\frac{1}{4} F_{\mu \nu}^{2}+\bar{\psi}\left(\gamma_{0}\left(\partial_{\tau}-i e A_{0}-\mu\right)+\gamma_{i} D_{i}+m\right) \psi\right]\right\} .
$$

The usual boundary conditions ("b.c.") over the time direction are assumed. The basic claim is that, according to the physical picture above, if we assume the system to be homogeneous, i.e. consider the "bulk" situation, then the partition function should not depend on $\mu$. Indeed this would ensure the neutrality that we expect:

$$
\rho=-\frac{\partial f}{\partial \mu}=0
$$

How does this arise?

The key observation is that we should again think of the system in terms of an effective potential, like in eq. (7.20). The role of the condensate is now given to the field $A_{0}$; let us denote it by $\bar{A}_{0}$. The last integral to be carried out is

$$
\mathcal{Z}(T, \mu)=\int_{-\infty}^{\infty} \mathrm{d} \bar{A}_{0} \exp \left\{-\frac{V}{T} V_{\text {eff }}\left(\bar{A}_{0}\right)\right\}
$$

Now, we can deduce from eq. (7.37) that $\mu$ can only appear in the combination $-i e \bar{A}_{0}-\mu$, so that $V_{\text {eff }}\left(\bar{A}_{0}\right)=f\left(\bar{A}_{0}-i \mu / e\right)$. Moreover, we know from eq. (6.36) that in a large volume and high temperature,

$$
V_{\mathrm{eff}}\left(\bar{A}_{0}\right) \approx \frac{1}{2} m_{\mathrm{E}}^{2}\left(\bar{A}_{0}-i \mu / e\right)^{2}+\mathcal{O}\left(\bar{A}_{0}-i \mu / e\right)^{4},
$$

where $m_{\mathrm{E}}^{2} \sim e^{2} T^{2}$. (The complete 1-loop $V_{\text {eff }}$ could be deduced from eq. (7.42) below, simply by substituting $\mu \rightarrow \mu+i e \bar{A}_{0}$ there.) In the infinite-volume limit, the integral in eq. (7.39) can be carried out by making use of the saddle point approximation, like with Bose-Einstein condensation in eq. (7.28). The saddle point is located in the complex plane at the position where $V_{\text {eff }}^{\prime}\left(\bar{A}_{0}\right)=0$, i.e. at $\bar{A}_{0}=i \mu / e$. The value of the potential at the saddle point, as well as the second derivative and so also the Gaussian integral around it, are independent of $\mu$. This leads to eq. (7.38). 
It is interesting to note that the saddle point lies at a purely imaginary $\bar{A}_{0}$. Recalling the relation of Minkowskian and Euclidean $A_{0}$ from page 66, this corresponds to a real Minkowskian $A_{0}$. Thus there indeed is a real electric potential $V_{0} \propto \mu$, just as we anticipated on physical grounds.

Finally, we note that in more complicated systems, like the Standard Model of particle physics, the proper procedure involves introducing chemical potentials for all global charges, and background values $\bar{A}_{0}^{a}$ for all gauge fields. Subsequently, we need to search for the saddle point, by minimizing the effective potential as a function of the background fields [7.6,7.7].

\section{Appendix A: Exact results in the free massless limit}

The free energy density of a single Dirac fermion,

$$
f(T, \mu)=-2 \Varangle_{\{P\}}\left\{\ln \left[\left(\omega_{n}+i \mu\right)^{2}+\epsilon_{p}^{2}\right]-\text { const. }\right\},
$$

can be computed explicitly for the case $m=0$ (i.e. $\epsilon_{p}=p$ ). We show that, subtracting the vacuum part, the result is

$$
f(T, \mu)=-\left(\frac{7 \pi^{2} T^{4}}{180}+\frac{\mu^{2} T^{2}}{6}+\frac{\mu^{4}}{12 \pi^{2}}\right) .
$$

We start from eq. (7.36), subtracting the vacuum term and setting $m=0, d=3$ :

$$
\begin{aligned}
f(T, \mu) & =-2 T \int \frac{\mathrm{d}^{3} \mathbf{p}}{(2 \pi)^{3}}\left\{\ln \left[1+\exp \left(-\frac{p-\mu}{T}\right)\right]+\ln \left[1+\exp \left(-\frac{p+\mu}{T}\right)\right]\right\} \\
& =-\frac{T^{4}}{\pi^{2}} \int_{0}^{\infty} \mathrm{d} x x^{2}\left\{\ln \left(1+e^{-x+y}\right)+\ln \left(1+e^{-x-y}\right)\right\}
\end{aligned}
$$

where we set $x \equiv p / T$ and $y \equiv \mu / T$, and carried out the angular integration.

A possible trick now is to expand the logarithms in Taylor series,

$$
\ln (1+z)=\sum_{n=1}^{\infty}(-1)^{n+1} \frac{z^{n}}{n}, \quad|z|<1 .
$$

Assuming $y>0$, this is indeed possible with the second term of eq. (7.43), whereas in the first term a direct application is not possible, because the series does not converge for all $x$. However, if $e^{-x+y}>1$, we can write $1+e^{-x+y}=e^{-x+y}\left(1+e^{x-y}\right)$, where $e^{x-y}<1$. Thereby the Taylor expansion can be written as

$$
\ln \left(1+e^{-x+y}\right)=\theta(x-y) \sum_{n=1}^{\infty} \frac{(-1)^{n+1}}{n} e^{-x n} e^{y n}+\theta(y-x)\left[y-x+\sum_{n=1}^{\infty} \frac{(-1)^{n+1}}{n} e^{x n} e^{-y n}\right] .
$$

Inserting this into eq. (7.43), we get

$$
\begin{aligned}
f(T, \mu)= & -\frac{T^{4}}{\pi^{2}}\left\{\int_{0}^{y} \mathrm{~d} x\left[y x^{2}-x^{3}+\sum_{n=1}^{\infty} \frac{(-1)^{n+1}}{n} x^{2}\left(e^{x n} e^{-y n}+e^{-x n} e^{-y n}\right)\right]\right. \\
& \left.+\int_{y}^{\infty} \mathrm{d} x\left[\sum_{n=1}^{\infty} \frac{(-1)^{n+1}}{n} x^{2}\left(e^{-x n} e^{y n}+e^{-x n} e^{-y n}\right)\right]\right\} \\
= & -\frac{T^{4}}{\pi^{2}}\left\{\int_{0}^{y} \mathrm{~d} x\left[y x^{2}-x^{3}+\sum_{n=1}^{\infty} \frac{(-1)^{n+1}}{n} x^{2}\left(e^{x n} e^{-y n}-e^{-x n} e^{y n}\right)\right]\right. \\
& \left.+\int_{0}^{\infty} \mathrm{d} x\left[\sum_{n=1}^{\infty} \frac{(-1)^{n+1}}{n} x^{2}\left(e^{-x n} e^{y n}+e^{-x n} e^{-y n}\right)\right]\right\} .
\end{aligned}
$$


All the $x$-integrals can be carried out:

$$
\begin{aligned}
\int_{0}^{y} \mathrm{~d} x\left(y x^{2}-x^{3}\right) & =\left(\frac{1}{3}-\frac{1}{4}\right) y^{4}=\frac{1}{12} y^{4}, \\
\int_{0}^{y} \mathrm{~d} x x^{2} e^{\alpha x} & =-\frac{2}{\alpha^{3}}+e^{\alpha y}\left(\frac{2}{\alpha^{3}}-\frac{2 y}{\alpha^{2}}+\frac{y^{2}}{\alpha}\right), \\
\int_{0}^{\infty} \mathrm{d} x x^{2} e^{-x n} & =\frac{2}{n^{3}} .
\end{aligned}
$$

Inserting these into eq. (7.46) we get

$$
\begin{aligned}
f(T, \mu)= & -\frac{T^{4}}{\pi^{2}}\left\{\frac{y^{4}}{12}+\sum_{n=1}^{\infty} \frac{(-1)^{n+1}}{n}\left[e^{-y n}\left(-\frac{2}{n^{3}}+e^{y n}\left(\frac{2}{n^{3}}-\frac{2 y}{n^{2}}+\frac{y^{2}}{n}\right)\right)\right.\right. \\
& \left.\left.-e^{y n}\left(\frac{2}{n^{3}}+e^{-y n}\left(-\frac{2}{n^{3}}-\frac{2 y}{n^{2}}-\frac{y^{2}}{n}\right)\right)+e^{y n} \frac{2}{n^{3}}+e^{-y n} \frac{2}{n^{3}}\right]\right\} \\
= & -\frac{T^{4}}{\pi^{2}}\left\{\frac{y^{4}}{12}+\sum_{n=1}^{\infty} \frac{(-1)^{n+1}}{n}\left[\frac{4}{n^{3}}+\frac{2 y^{2}}{n}\right]\right\},
\end{aligned}
$$

where a remarkable cancellation took place. The sums can be carried out:

$$
\begin{aligned}
& \eta(2) \equiv \sum_{n=1}^{\infty} \frac{(-1)^{n+1}}{n^{2}}=\frac{1}{1^{2}}-\frac{1}{2^{2}}+\frac{1}{3^{2}}-\frac{1}{4^{2}}+\cdots=\zeta(2)-\frac{2}{2^{2}} \zeta(2)=\frac{1}{2} \zeta(2)=\frac{\pi^{2}}{12}, \\
& \eta(4) \equiv \sum_{n=1}^{\infty} \frac{(-1)^{n+1}}{n^{4}}=\frac{1}{1^{4}}-\frac{1}{2^{4}}+\frac{1}{3^{4}}-\frac{1}{4^{4}}+\cdots=\zeta(4)-\frac{2}{2^{4}} \zeta(4)=\frac{7}{8} \zeta(4)=\frac{7}{8} \frac{\pi^{4}}{90} .
\end{aligned}
$$

Inserting into eq. (7.50), we end up with

$$
f(T, \mu)=-\frac{T^{4}}{\pi^{2}}\left\{\frac{y^{4}}{12}+\frac{\pi^{2} y^{2}}{6}+\frac{7 \pi^{4}}{180}\right\},
$$

which after the substitution $y=\mu / T$ reproduces eq. (7.42).

\section{Appendix B: Free susceptibilities}

Important characteristics of dense systems are offered by susceptibilities, which define fluctuations of the particle number in a grand canonical ensemble. For a Dirac fermion,

$$
\begin{aligned}
\chi_{\mathrm{f}} & \equiv \lim _{V \rightarrow \infty} \frac{\left\langle\hat{N}^{2}\right\rangle-\langle\hat{N}\rangle^{2}}{V}=\lim _{V \rightarrow \infty} T \partial_{\mu}\left(\frac{\langle\hat{N}\rangle}{V}\right)=\lim _{V \rightarrow \infty} \frac{T^{2} \partial_{\mu}^{2} \ln \mathcal{Z}}{V}=-T \partial_{\mu}^{2} f(T, \mu) \\
\stackrel{(7.36)}{=} & 2 T \int_{\mathbf{p}} \partial_{\mu}^{2}\left\{\epsilon_{p}+T\left[\ln \left(1+e^{-\beta\left(\epsilon_{p}-\mu\right)}\right)+\ln \left(1+e^{-\beta\left(\epsilon_{p}+\mu\right)}\right)\right]\right\} \\
& =2 T \int_{\mathbf{p}} \partial_{\mu}\left\{\frac{1}{e^{\beta\left(\epsilon_{p}-\mu\right)}+1}-\frac{1}{e^{\beta\left(\epsilon_{p}+\mu\right)}+1}\right\} \\
& =2 \int_{\mathbf{p}}\left\{\frac{e^{\beta\left(\epsilon_{p}-\mu\right)}}{\left[e^{\beta\left(\epsilon_{p}-\mu\right)}+1\right]^{2}}+\frac{e^{\beta\left(\epsilon_{p}+\mu\right)}}{\left[e^{\beta\left(\epsilon_{p}+\mu\right)}+1\right]^{2}}\right\} \\
& =\frac{1}{\pi^{2}} \int_{0}^{\infty} \mathrm{d} p p^{2}\left\{n_{\mathrm{F}}\left(\epsilon_{p}-\mu\right)\left[1-n_{\mathrm{F}}\left(\epsilon_{p}-\mu\right)\right]+n_{\mathrm{F}}\left(\epsilon_{p}+\mu\right)\left[1-n_{\mathrm{F}}\left(\epsilon_{p}+\mu\right)\right]\right\} .
\end{aligned}
$$

In the massless limit, eq. (7.42) directly gives $\chi_{\mathrm{f}}=\frac{T^{3}}{3}+\frac{\mu^{2} T}{\pi^{2}}$. On the other hand, for $m \neq 0$, $\mu=0$, one gets $\chi_{\mathrm{f}}=\frac{2 m^{2} T}{\pi^{2}} \sum_{n=1}^{\infty}(-1)^{n+1} K_{2}\left(\frac{n m}{T}\right)$, where $K_{2}$ is a modified Bessel function. In the 
bosonic case of a complex scalar field, eq. (7.18) similarly leads to

$$
\chi_{\mathrm{b}}=\frac{1}{2 \pi^{2}} \int_{0}^{\infty} \mathrm{d} p p^{2}\left\{n_{\mathrm{B}}\left(\epsilon_{p}-\mu\right)\left[1+n_{\mathrm{B}}\left(\epsilon_{p}-\mu\right)\right]+n_{\mathrm{B}}\left(\epsilon_{p}+\mu\right)\left[1+n_{\mathrm{B}}\left(\epsilon_{p}+\mu\right)\right]\right\} .
$$

In this case the massless limit is only relevant at $\mu=0$ (otherwise the integrand is singular at $p=|\mu|)$, where we obtain $\chi_{\mathrm{b}}=T^{3} / 3$. For $m \neq 0$, the susceptibility at $\mu=0$ can again be expressed in terms of modified Bessel functions, $\chi_{\mathrm{b}}=\frac{m^{2} T}{\pi^{2}} \sum_{n=1}^{\infty} K_{2}\left(\frac{n m}{T}\right)$.

\section{Appendix C: Finite density QCD at next-to-leading order}

Extending the description of finite density systems to higher perturbative orders has become an actively studied topic, cf. e.g. refs. [7.7,7.8]. An example is the susceptibility defined in eq. (7.54), evaluated at $\mu=0$ within $\mathrm{QCD}$, and the generalization thereof to the case of several quark flavours. These quantities probe finite density, but can nevertheless be compared with lattice QCD simulations that are well under control only at vanishing chemical potentials. The basic strategy in their evaluation follows the above leading-order computation in the sense that it is technically easier to first compute the entire free energy density at finite $\mu$, and only afterwards to take derivatives with respect to $\mu[7.8]$. As a by-product of evaluating susceptibilities at $\mu=0$, we therefore obtain the behaviour of the pressure at finite density.

At 2-loop order, the $\mu$-dependent part of the QCD free energy density gets contributions from one single diagram, namely the same as in eq. (5.121). Like in eq. (5.121) it is easy to see that in the limit of massless quarks (an approximation that significantly simplifies higher-order computations) this diagram can be written in the form

$$
\text { Wnin }=d_{A} g^{2} \frac{d-1}{2} \oint_{\{P\} Q}\left[\frac{1}{\tilde{P}^{2}(\tilde{P}-Q)^{2}}-\frac{2}{\tilde{P}^{2} Q^{2}}\right],
$$

where $d_{A} \equiv N_{\mathrm{c}}^{2}-1$, we set $N_{\mathrm{f}}=1$ and, in accordance with eq. (7.35), the fermionic Matsubara frequencies have been shifted by $\omega_{n} \rightarrow \omega_{n}+i \mu \equiv \tilde{p}_{n}$. Both terms in this result clearly factorize into products of 1-loop sum-integrals that (up to the shift of the fermionic Matsubara frequencies) can be identified as the functions $I(0, T)=I_{T}(0)$ and $\tilde{I}(0, T)=\tilde{I}_{T}(0)$ studied in secs. 2.3 and 4.2, respectively.

For completeness, let us next inspect the more general fermionic sum-integral

$$
\tilde{I}(m=0, T, \mu, \alpha) \equiv \oiint_{\{P\}} \frac{1}{\left(\tilde{P}^{2}\right)^{\alpha}},
$$

following a strategy similar to that in eq. (2.90). In other words we first perform the $3-2 \epsilon-$ dimensional integral over the spatial momentum $\mathbf{p}$, and afterwards take care of the Matsubara sum. Applying the familiar result of eq. (2.64), we obtain

$$
\begin{aligned}
\tilde{I}(m=0, T, \mu, \alpha)= & \frac{1}{(4 \pi)^{3 / 2-\epsilon}} \frac{\Gamma(\alpha-3 / 2+\epsilon)}{\Gamma(\alpha)} T \sum_{k=-\infty}^{\infty} \frac{1}{\left[((2 k+1) \pi T+i \mu)^{2}\right]^{\alpha-3 / 2+\epsilon}} \\
= & 2^{-2 \alpha} \pi^{-2 \alpha+3 / 2-\epsilon} T^{-2 \alpha+4-2 \epsilon} \frac{\Gamma(\alpha-3 / 2+\epsilon)}{\Gamma(\alpha)} \\
& \times\left[\zeta\left(2 \alpha-3+2 \epsilon, \frac{1}{2}-i \bar{\mu}\right)+\zeta\left(2 \alpha-3+2 \epsilon, \frac{1}{2}+i \bar{\mu}\right)\right],
\end{aligned}
$$


where $\bar{\mu} \equiv \mu /(2 \pi T)$ and we have expressed the infinite sums in terms of the generalized (Hurwitz) zeta-function

$$
\zeta(z, q) \equiv \sum_{n=0}^{\infty} \frac{1}{(q+n)^{z}}
$$

Specializing now to $\alpha=1$ and dropping terms of $\mathcal{O}(\epsilon)$, we easily get

$$
\tilde{I}(m=0, T, \mu, 1)=-\frac{T^{2}}{24}-\frac{\mu^{2}}{8 \pi^{2}}+\mathcal{O}(\epsilon) .
$$

Plugging this and $I_{T}(0)=T^{2} / 12$ into eq. (7.57) produces

$$
=\frac{d_{A} g^{2} T^{4}}{576}\left[5+\frac{18 \mu^{2}}{(\pi T)^{2}}+\frac{9 \mu^{4}}{(\pi T)^{4}}\right]+\mathcal{O}(\epsilon) \text {. }
$$

From here the next-to-leading order contribution to the quark number susceptibility can be extracted according to eq. (7.54),

$$
\left.\chi_{\mathrm{f}}\right|_{\mu=0}=T^{3}\left(\frac{N_{\mathrm{c}}}{3}-\frac{d_{A} g^{2}}{16 \pi^{2}}\right),
$$

where we have added the appropriate colour factor to the leading-order term.

\section{Appendix D: Cold and dense limit}

As discussed in secs. 3.4, 5.4 and 6.2, at higher orders of perturbation theory one encounters uncancelled IR divergences that necessitate the use of either diagrammatic resummations, or an effective theory framework for obtaining well-defined weak-coupling results. At high temperature, these IR problems can typically be traced back to the static Matsubara zero mode sector of bosonic fields, but the situation is qualitatively different in the limit of zero (or very small) temperature, where the discrete Matsubara modes $k_{n}$ merge into a continuous (Euclidean) frequency $k_{0}$. This is an active topic of research for physical systems characterized by sizable chemical potentials for fermionic fields. An example is dense quark matter possibly found inside neutron stars, where densities greatly exceed the so-called saturation density of nuclear matter, $n_{s} \approx 0.16$ baryons per $\mathrm{fm}^{3}$. In this appendix, we review tools used in higher-order computations of thermodynamic quantities in dense $T=0$ quark matter, giving special emphasis to how IR problems are treated in the evaluation of its pressure. Our treatment largely follows that of ref. [7.9].

The strict zero-temperature limit can be approached either by starting from $T \neq 0$ and then gradually lowering the temperature, following steps similar to those taken in sec. 2.2 , or by setting $T=0$ from the outset. Choosing the latter option, we end up considering the same vacuum, or bubble, diagrams as at non-zero temperature, but with two crucial differences. First, in order to yield a non-zero contribution, each Feynman graph needs to contain at least one closed fermion loop, as purely bosonic integrals vanish in the $T=0$ limit. And second, bosonic and fermionic sum-integrals get replaced by continuous integrals in $D \equiv d+1=4-2 \epsilon$ dimensions, according to

$$
\begin{aligned}
& \mathcal{F}_{K} \rightarrow \int_{-\infty}^{\infty} \frac{\mathrm{d} k_{0}}{2 \pi} \int \frac{\mathrm{d}^{d} \mathbf{k}}{(2 \pi)^{d}}=\int_{K}=\Lambda^{-2 \epsilon}\left[\Lambda^{2 \epsilon} \int_{K}\right] \text { (bosons) } \\
& \mathcal{F}_{\{K\}} \rightarrow \int_{-\infty+i \mu}^{\infty+i \mu} \frac{\mathrm{d} k_{0}}{2 \pi} \int \frac{\mathrm{d}^{d} \mathbf{k}}{(2 \pi)^{d}}=\int_{\widetilde{K}}=\Lambda^{-2 \epsilon}\left[\Lambda^{2 \epsilon} \int_{\widetilde{K}}\right] \text { (fermions), }
\end{aligned}
$$


where $\Lambda$ is a scale parameter. For QCD, the chemical potentials $\mu$ corresponding to different quark flavors are all independent if one altogether neglects the weak interactions. In a neutron-star setting, weak interactions, however, play an important role in enforcing the so-called $\beta$ equilibrium, which together with local charge neutrality only leaves one independent chemical potential. This is normally taken as that of the down quark and denoted by $\mu_{q} \equiv \mu_{d}=\mu_{B} / 3$, where $\mu_{B}$ is the baryon number chemical potential. The set of quark chemical potentials is denoted by $\left\{\mu_{f}\right\}$.

For the first two orders of perturbation theory, few subtleties arise in the determination of the pressure. In practice, one first takes care of the temporal momentum integrations using the residue theorem, which for the case of massless QCD yields the non-interacting-limit result (cf. eq. (7.42))

$$
p_{\mathrm{QCD}}^{\mathrm{LO}}\left(\left\{\mu_{f}\right\}\right)=2 N_{\mathrm{c}} \sum_{f} \int_{\mathbf{k}}\left(\mu_{f}-k\right) \theta\left(\mu_{f}-k\right)=\frac{N_{\mathrm{c}}}{12 \pi^{2}} \sum_{f} \mu_{f}^{4} .
$$

Here the $\theta$-function originates from taking the limit $n_{\mathrm{F}}(\epsilon-\mu) \stackrel{T \rightarrow 0}{\longrightarrow} \theta(\mu-\epsilon)$. The next order of the weak-coupling expansion (NLO) is similarly straightforward and automatically leads to an UV and IR finite result (cf. eq. (7.62) for $f=-p$ ).

At Next-to-Next-to-Leading Order (NNLO), or three loops in naive perturbation theory, one finally encounters uncancelled IR divergences, closely analogous to those discussed in sec. 3.4. This time they come from one diagram, namely the 3-loop vacuum graph containing two closed fermion loops, which is built by joining together two 1-loop self-energy diagrams from eq. (5.96). Technically, the divergence occurs due to the non-vanishing low-momentum limit of this self-energy, which will be thoroughly discussed in section 8.4. That discussion culminates in the derivation of eqs. (8.160) and (8.162), the Euclidean versions of the so-called Hard Thermal Loop (HTL) self-energies. These results can immediately be adopted to our $T=0$ discussion, with the only modifications being the replacement $k_{n} \rightarrow k_{0}$ and the use of the zero-temperature Debye mass from eq. (8.149), $m_{\mathrm{E}}^{2}=g^{2} /\left(2 \pi^{2}\right) \sum_{f} \mu_{f}^{2}$. Importantly, the broader HTL effective theory, also to be discussed in section 8.4, represents the correct IR effective theory of QCD at zero temperature, replacing the dimensionally reduced EQCD valid at high temperatures.

Studying the HTL self-energies, we can make an important observation: while both components reduce to mere numbers in the static $k_{0}=0$ limit, relevant for the IR physics of high temperatures, they remain non-trivial functions of the dimensionless variable $k_{0} / k$ if $k_{0} \sim k \sim m_{\mathrm{E}}$. Given that the latter represents the relevant IR limit at zero temperature, we realize that the IR physics of cold and dense QCD is qualitatively different from hot quark-gluon plasma. As a result, the leading non-analytic behavior of the weak-coupling expansion of the pressure in $\alpha_{s}$ is this time not proportional to $T^{4} \alpha_{s}^{3 / 2}$, like at high temperatures, but to $\mu^{4} \alpha_{s}^{2} \ln \alpha_{s}$. In what follows, we derive this contribution and the corresponding prefactor using relatively simple effective theory arguments.

To obtain the leading pressure contribution from the soft momentum scale $m_{\mathrm{E}}$, one can follow two different but in principle equivalent routes. The first, closely analogous to the corresponding discussion in section 3.4, involves an explicit resummation of the IR sensitive ring diagrams of the full theory and analyzing it in the limit of soft gluonic momentum. The second approach involves the use of effective theories for soft physics. In both cases, one ends up inspecting the leading-order pressure of the HTL theory, which takes the form

$$
\begin{aligned}
p_{\mathrm{HTL}}^{\mathrm{LO}} & =-\frac{d_{A}}{2} \int_{K}\left\{(d-1) \ln \left[K^{2}+\Pi_{\mathrm{T}}(K)\right]+\ln \left[K^{2}+\Pi_{\mathrm{E}}(K)\right]\right\} \\
& =-\frac{d_{A} m_{\mathrm{E}}^{D}}{2} \int_{\hat{K}}\left\{(d-1) \ln \left[\hat{K}^{2}+\hat{\Pi}_{\mathrm{T}}(\phi)\right]+\ln \left[\hat{K}^{2}+\hat{\Pi}_{\mathrm{E}}(\phi)\right]\right\},
\end{aligned}
$$


where the coefficients $d-1$ and 1 correspond to the traces of the projectors in eqs. (8.150) and (8.151), respectively. Otherwise the structure is like in eq. (5.57), omitting the longitudinal part which has no HTL self-energy. The two self-energies can be taken from eqs. (8.160) and (8.162). In eq. (7.68) we have scaled out the parameter $m_{\mathrm{E}}$ from the integral, by defining $K \equiv m_{\mathrm{E}} \hat{K}, \Pi_{i}(K) \equiv$ $m_{\mathrm{E}}^{2} \hat{\Pi}_{i}(\phi)$, with $\tan \phi \equiv k / k_{0} \cdot{ }^{20}$ From the latter form of the result, recalling $m_{\mathrm{E}}^{2} \propto \alpha_{s} \mu^{2}$, it becomes clear that the integral yields a contribution proportional to $\alpha_{s}^{2}$.

To evaluate the integral in eq. (7.68), we may take advantage of eq. (2.64), specifically

$$
\int \frac{\mathrm{d}^{D} K}{(2 \pi)^{D}} \ln \left(K^{2}+m^{2}\right)=-\lim _{A \rightarrow 0} \frac{\Phi(m, D, A)}{A}=-\frac{m^{D} \Gamma(-D / 2)}{(4 \pi)^{\frac{D}{2}}}
$$

which allows us to perform the radial integration in eq. (7.68), leaving over the angular one. Utilizing the integration measure given in eq. (8.205), with $d \rightarrow D$ and $z \rightarrow \cos \phi$, and recalling $\Gamma(1 / 2)=\sqrt{\pi}$, this leads to

$$
p_{\mathrm{HTL}}^{\mathrm{LO}}=\frac{d_{A} m_{\mathrm{E}}^{D} \Gamma(D / 2) \Gamma(-D / 2)}{(4 \pi)^{\frac{D+1}{2}} \Gamma((D-1) / 2)} \int_{0}^{\pi} \mathrm{d} \phi \sin ^{2-2 \epsilon} \phi\left[(d-1) \hat{\Pi}_{\mathrm{T}}^{D / 2}(\phi)+\hat{\Pi}_{\mathrm{E}}^{D / 2}(\phi)\right] .
$$

The prefactor here evaluates to

$$
\frac{d_{A} m_{\mathrm{E}}^{D} \Gamma(D / 2) \Gamma(-D / 2)}{(4 \pi)^{\frac{D+1}{2}} \Gamma((D-1) / 2)}=\frac{d_{A}}{32 \pi^{3}} \frac{m_{\mathrm{E}}^{4-2 \epsilon}}{\epsilon}+\mathcal{O}\left(\epsilon^{0}\right),
$$

which produces the desired logarithm of $m_{\mathrm{E}}$ (and thus of $\alpha_{s}$ ). This allows us to set $\epsilon=0$ in the angular integral, which thereby becomes analytically calculable, giving

$$
\int_{0}^{\pi} \mathrm{d} \phi \sin ^{2-2 \epsilon} \phi\left[(d-1) \hat{\Pi}_{\mathrm{T}}^{D / 2}(\phi)+\hat{\Pi}_{\mathrm{E}}^{D / 2}(\phi)\right]=\frac{\pi}{4}+\mathcal{O}(\epsilon) .
$$

Collecting all the results and explicitly reinstating the scale parameter $\Lambda$ in the integration measure, we obtain

$$
p_{\mathrm{HTL}}^{\mathrm{LO}}=\frac{d_{A} m_{\mathrm{E}}^{4} \Lambda^{-2 \epsilon}}{8(4 \pi)^{2}}\left(\frac{\Lambda^{2}}{m_{\mathrm{E}}^{2}}\right)^{\epsilon} \frac{1}{\epsilon}+\mathcal{O}\left(\epsilon^{0}\right)
$$

which upon an expansion in powers of $\epsilon$ and the insertion of $m_{\mathrm{E}}^{2} \propto \alpha_{s} \mu^{2}$ leads us to the final result for the non-analytic part,

$$
p_{\mathrm{HTL}}^{\mathrm{LO}} \quad \supset-\frac{d_{A} m_{\mathrm{E}}^{4}}{8(4 \pi)^{2}} \ln \alpha_{s}+\mathcal{O}(\epsilon)
$$

The coefficient of the $\alpha_{s}^{2} \ln \alpha_{s}$ term was originally derived back in 1977 through a tedious bruteforce calculation in full QCD [7.10]. More than forty years later, a series of systematic calculations in the HTL theory have generalized this result to include all soft contributions up to and including the full order $\alpha_{s}^{3}[7.9]$, leaving only the so-called hard and mixed contributions missing from the 4-loop pressure.

\footnotetext{
${ }^{20}$ To obtain $\hat{\Pi}_{i}(\phi)$ from $\Pi_{i}(K)$, we first remove the overall factor $m_{\mathrm{E}}^{2}$ and then write $|K|=\sqrt{k_{0}^{2}+k^{2}}$ everywhere. This allows us to write $\hat{\Pi}_{i}$ as a function of the dimensionless variable $k / k_{0}$ that can be replaced by $\tan \phi$. The resulting functions $\hat{\Pi}_{i}(\phi)$ can be read off from eq. (B.28) of ref. [7.9].
} 


\section{Literature}

[7.1] G. 't Hooft, Symmetry Breaking through Bell-Jackiw Anomalies, Phys. Rev. Lett. 37 (1976) 8.

[7.2] M. D'Onofrio, K. Rummukainen and A. Tranberg, Sphaleron Rate in the Minimal Standard Model, Phys. Rev. Lett. 113 (2014) 141602 [1404.3565].

[7.3] J.I. Kapusta, Bose-Einstein condensation, spontaneous symmetry breaking, and gauge theories, Phys. Rev. D 24 (1981) 426.

[7.4] H.E. Haber and H.A. Weldon, Finite-temperature symmetry breaking as Bose-Einstein condensation, Phys. Rev. D 25 (1982) 502.

[7.5] K.M. Benson, J. Bernstein and S. Dodelson, Phase structure and the effective potential at fixed charge, Phys. Rev. D 44 (1991) 2480.

[7.6] S.Y. Khlebnikov and M.E. Shaposhnikov, Melting of the Higgs vacuum: Conserved numbers at high temperature, Phys. Lett. B 387 (1996) 817 [hep-ph/9607386].

[7.7] D. Bödeker and M. Sangel, Order $g^{2}$ susceptibilities in the symmetric phase of the Standard Model, JCAP 04 (2015) 040 [1501.03151].

[7.8] A. Vuorinen, Pressure of QCD at finite temperatures and chemical potentials, Phys. Rev. D 68 (2003) 054017 [hep-ph/0305183].

[7.9] T. Gorda, A. Kurkela, R. Paatelainen, S. Säppi and A. Vuorinen, Cold quark matter at N3LO: Soft contributions, Phys. Rev. D 104 (2021) 074015 [2103.07427].

[7.10] B.A. Freedman and L.D. McLerran, Fermions and gauge vector mesons at finite temperature and density. III. The ground-state energy of a relativistic quark gas, Phys. Rev. D 16 (1977) 1169. 


\section{Real-time observables}

Abstract: Various real-time correlation functions are defined (Wightman, retarded, advanced, time-ordered, spectral). Their analytic properties are discussed, and general relations between them are worked out for the case of a system in thermal equilibrium. Examples are given for free scalar and fermion fields. A physically relevant spectral function related to a composite operator is analyzed in detail. The so-called real-time formalism is introduced, and it is shown how it can be used to compute the same spectral function that was previously determined with the imaginarytime formalism. The need for resummations in order to systematically determine spectral functions in weakly coupled systems is stated. The concept of Hard Thermal Loops (HTLs), which implement a particular resummation, is introduced. HTL-resummed gauge field and fermion propagators are derived. The main plasma physics phenomena that the HTL resummation captures are pointed out. A warning is issued that although necessary, HTL resummation is in general not sufficient for obtaining a systematic weak-coupling expansion.

Keywords: Wick rotation, time ordering, Heisenberg operator, Wightman function, retarded and advanced correlators, Kubo-Martin-Schwinger relation, spectral representation, sum rule, analytic continuation, density matrix, Schwinger-Keldysh formalism, Hard Thermal Loops, Landau damping, plasmon, plasmino, dispersion relation.

\subsection{Different Green's functions}

We now move to a new class of observables including both a Minkowskian time $t$ and a temperature $T$. Examples are production rates of weakly interacting particles from a thermal plasma; oscillation and damping rates of long-wavelength fields in a plasma; as well as transport coefficients of a plasma such as its electric and thermal conductivities and bulk and shear viscosities. We start by developing some aspects of the general formalism, and return to specific applications later on. Let us stress that we do remain in thermal equilibrium in the following, even though some of the results also apply to an off-equilibrium ensemble.

Many observables of interest can be reduced to 2-point correlation functions of elementary or composite operators. Let us therefore list some common definitions and relations that apply to such correlation functions [8.1]-[8.4].

We denote Minkowskian spacetime coordinates by $\mathcal{X}=\left(t, x^{i}\right)$ and momenta by $\mathcal{K}=\left(k^{0}, k^{i}\right)$, whereas their Euclidean counterparts are denoted by $X=\left(\tau, x^{i}\right), K=\left(k_{n}, k_{i}\right)$. Wick rotation is carried out by $\tau \leftrightarrow i t, k_{n} \leftrightarrow-i k^{0}$. Scalar products are defined as $\mathcal{K} \cdot \mathcal{X}=k_{0} t+k_{i} x^{i}=k^{0} t-\mathbf{k} \cdot \mathbf{x}$, $K \cdot X=k_{n} \tau+k_{i} x^{i}=k_{n} \tau-\mathbf{k} \cdot \mathbf{x}$. Arguments of operators denote implicitly whether we are in Minkowskian or Euclidean spacetime. In particular, Heisenberg-operators are defined as

$$
\hat{O}(t, \mathbf{x}) \equiv e^{i \hat{H} t} \hat{O}(0, \mathbf{x}) e^{-i \hat{H} t}, \quad \hat{O}(\tau, \mathbf{x}) \equiv e^{\hat{H} \tau} \hat{O}(0, \mathbf{x}) e^{-\hat{H} \tau} .
$$

The thermal ensemble is normally defined by the density matrix $\hat{\rho}=\mathcal{Z}^{-1} \exp (-\beta \hat{H})$, even though it is also possible to include a chemical potential, as will be done in eq. (8.38). Expectation values of (products of) operators are defined through $\langle\cdots\rangle \equiv \operatorname{Tr}[\hat{\rho}(\cdots)]$. 


\section{Bosonic case}

We start by considering operators that are bosonic in nature, i.e. commuting (modulo possible contact terms). We denote the operators by $\hat{\phi}_{\alpha}, \hat{\phi}_{\beta}^{\dagger}$. These may be either elementary fields or composite operators built from them. In order to simplify the notation, functions and their Fourier transforms are to be recognized through the argument, $\mathcal{X}$ vs. $\mathcal{K}$.

We can define various classes of correlation functions. "Physical" correlators are defined as

$$
\begin{aligned}
\Pi_{\alpha \beta}^{>}(\mathcal{K}) & \equiv \int_{\mathcal{X}} e^{i \mathcal{K} \cdot \mathcal{X}}\left\langle\hat{\phi}_{\alpha}(\mathcal{X}) \hat{\phi}_{\beta}^{\dagger}(0)\right\rangle, \\
\Pi_{\alpha \beta}^{<}(\mathcal{K}) & \equiv \int_{\mathcal{X}} e^{i \mathcal{K} \cdot \mathcal{X}}\left\langle\hat{\phi}_{\beta}^{\dagger}(0) \hat{\phi}_{\alpha}(\mathcal{X})\right\rangle, \\
\rho_{\alpha \beta}(\mathcal{K}) & \equiv \int_{\mathcal{X}} e^{i \mathcal{K} \cdot \mathcal{X}}\left\langle\frac{1}{2}\left[\hat{\phi}_{\alpha}(\mathcal{X}), \hat{\phi}_{\beta}^{\dagger}(0)\right]\right\rangle, \\
\Delta_{\alpha \beta}(\mathcal{K}) & \equiv \int_{\mathcal{X}} e^{i \mathcal{K} \cdot \mathcal{X}}\left\langle\frac{1}{2}\left\{\hat{\phi}_{\alpha}(\mathcal{X}), \hat{\phi}_{\beta}^{\dagger}(0)\right\}\right\rangle,
\end{aligned}
$$

where $\Pi^{>}$and $\Pi^{<}$are called Wightman functions and $\rho$ the spectral function, whereas $\Delta$ is sometimes referred to as the statistical correlator. We are implicitly assuming the presence of an UV regulator so that there are no short-distance singularities in the Fourier transforms.

The "retarded" / "advanced" correlators can be defined as

$$
\begin{aligned}
\Pi_{\alpha \beta}^{R}(\mathcal{K}) & \equiv i \int_{\mathcal{X}} e^{i \mathcal{K} \cdot \mathcal{X}}\left\langle\left[\hat{\phi}_{\alpha}(\mathcal{X}), \hat{\phi}_{\beta}^{\dagger}(0)\right] \theta(t)\right\rangle, \\
\Pi_{\alpha \beta}^{A}(\mathcal{K}) & \equiv i \int_{\mathcal{X}} e^{i \mathcal{K} \cdot \mathcal{X}}\left\langle-\left[\hat{\phi}_{\alpha}(\mathcal{X}), \hat{\phi}_{\beta}^{\dagger}(0)\right] \theta(-t)\right\rangle .
\end{aligned}
$$

Note that since $\Pi^{R}$ involves positive times only, $e^{i k^{0} t}=e^{i\left[\operatorname{Re} k^{0}+i \operatorname{Im} k^{0}\right] t}=e^{i \operatorname{Re} k^{0} t} e^{-\operatorname{Im} k^{0} t}$ is exponentially suppressed for $\operatorname{Im} k^{0}>0$. Therefore $\Pi^{R}$ can be considered an analytic function of $k^{0}$ in the upper half of the complex $k^{0}$-plane (it can develop distribution-like singularities at the physical boundary $\left.\operatorname{Im} k^{0} \rightarrow 0^{+}\right)$. Similarly, $\Pi^{A}$ is an analytic function in the lower half of the complex $k^{0}$-plane. These turn out to be strong and useful properties, and do not apply to general correlation functions.

On the other hand, from the computational point of view one is often faced with "time-ordered" correlators,

$$
\Pi_{\alpha \beta}^{T}(\mathcal{K}) \equiv \int_{\mathcal{X}} e^{i \mathcal{K} \cdot \mathcal{X}}\left\langle\hat{\phi}_{\alpha}(\mathcal{X}) \hat{\phi}_{\beta}^{\dagger}(0) \theta(t)+\hat{\phi}_{\beta}^{\dagger}(0) \hat{\phi}_{\alpha}(\mathcal{X}) \theta(-t)\right\rangle
$$

which appear in time-dependent perturbation theory at zero temperature, or with the "Euclidean" correlator

$$
\Pi_{\alpha \beta}^{E}(K) \equiv \int_{X} e^{i K \cdot X}\left\langle\hat{\phi}_{\alpha}(X) \hat{\phi}_{\beta}^{\dagger}(0)\right\rangle,
$$

which appears in non-perturbative formulations. Restricting to $0 \leq \tau \leq \beta$, the Euclidean correlator is also time-ordered, and can be computed with standard imaginary-time functional integrals. If the correlator is periodic (cf. text below eq. (8.10)), then $k_{n}$ is a bosonic Matsubara frequency.

It follows from eq. (8.1), by using the cyclicity of the trace, that

$$
\left\langle\hat{\phi}_{\alpha}(t-i \beta, \mathbf{x}) \hat{\phi}_{\beta}^{\dagger}(0, \mathbf{0})\right\rangle=\frac{1}{\mathcal{Z}} \operatorname{Tr}\left[e^{-\beta \hat{H}} e^{\beta \hat{H}} \hat{\phi}_{\alpha}(t, \mathbf{x}) e^{-\beta \hat{H}} \hat{\phi}_{\beta}^{\dagger}(0, \mathbf{0})\right]=\left\langle\hat{\phi}_{\beta}^{\dagger}(0, \mathbf{0}) \hat{\phi}_{\alpha}(t, \mathbf{x})\right\rangle .
$$


This is a configuration-space version of the so-called Kubo-Martin-Schwinger (KMS) relation, which relates $\Pi_{\alpha \beta}^{>}$and $\Pi_{\alpha \beta}^{<}$to each other, provided that we are in thermal equilibrium. If we set $t \rightarrow 0$ and keep $\mathbf{x} \neq \mathbf{0}$, then $\hat{\phi}_{\alpha}(0, \mathbf{x})$ and $\hat{\phi}_{\beta}^{\dagger}(0, \mathbf{0})$ commute with each other. In this case, the KMS relation implies that the integrand in eq. (8.9) is a periodic function of $\tau$, with periodicity defined in the same sense as around eq. (1.41).

It turns out that all of the correlation functions defined can be related to each other in thermal equilibrium. In particular, all correlators can be expressed in terms of the spectral function, which in turn can be determined as a certain analytic continuation of the Euclidean correlator. In order to show this, we may first insert sets of energy eigenstates into the definitions of $\Pi_{\alpha \beta}^{>}$and $\Pi_{\alpha \beta}^{<}$:

$$
\begin{aligned}
\Pi_{\alpha \beta}^{>}(\mathcal{K}) & =\frac{1}{\mathcal{Z}} \int_{\mathcal{X}} e^{i \mathcal{K} \cdot \mathcal{X}} \operatorname{Tr}[e^{-\beta \hat{H}+i \hat{H} t} \underbrace{\mathbb{1}}_{\sum_{m}|m\rangle\langle m|} \hat{\phi}_{\alpha}(0, \mathbf{x}) e^{-i \hat{H} t} \underbrace{\mathbb{1}}_{\sum_{n}|n\rangle\langle n|} \hat{\phi}_{\beta}^{\dagger}(0, \mathbf{0})] \\
& =\frac{1}{\mathcal{Z}} \sum_{m, n} \int_{\mathcal{X}} e^{i \mathcal{K} \cdot \mathcal{X}} e^{(-\beta+i t) \epsilon_{m}} e^{-i t \epsilon_{n}}\left\langle m\left|\hat{\phi}_{\alpha}(0, \mathbf{x})\right| n\right\rangle\left\langle n\left|\hat{\phi}_{\beta}^{\dagger}(0, \mathbf{0})\right| m\right\rangle \\
& =\frac{1}{\mathcal{Z}} \int_{\mathbf{x}} e^{-i \mathbf{k} \cdot \mathbf{x}} \sum_{m, n} e^{-\beta \epsilon_{m}} 2 \pi \delta\left(k^{0}+\epsilon_{m}-\epsilon_{n}\right)\left\langle m\left|\hat{\phi}_{\alpha}(0, \mathbf{x})\right| n\right\rangle\left\langle n\left|\hat{\phi}_{\beta}^{\dagger}(0, \mathbf{0})\right| m\right\rangle, \\
\Pi_{\alpha \beta}^{<}(\mathcal{K}) & =\frac{1}{\mathcal{Z}} \int_{\mathcal{X}} e^{i \mathcal{K} \cdot \mathcal{X}} \operatorname{Tr}[e^{-\beta \hat{H}} \underbrace{\mathbb{1}}_{\sum_{n}|n\rangle\langle n|} \hat{\phi}_{\beta}^{\dagger}(0, \mathbf{0}) e^{i \hat{H} t} \underbrace{\mathbb{1}}_{\sum_{m}|m\rangle\langle m|} \hat{\phi}_{\alpha}(0, \mathbf{x}) e^{-i \hat{H} t}] \\
& =\frac{1}{\mathcal{Z}} \sum_{m, n} \int_{\mathcal{X}} e^{i \mathcal{K} \cdot \mathcal{X}} e^{(-\beta-i t) \epsilon_{n}} e^{i t \epsilon_{m}}\left\langle n\left|\hat{\phi}_{\beta}^{\dagger}(0, \mathbf{0})\right| m\right\rangle\left\langle m\left|\hat{\phi}_{\alpha}(0, \mathbf{x})\right| n\right\rangle \\
& =\frac{1}{\mathcal{Z}} \int_{\mathbf{x}} e^{-i \mathbf{k} \cdot \mathbf{x}} \sum_{m, n} e^{-\beta \epsilon_{n}} 2 \pi \underbrace{\delta\left(k^{0}+\epsilon_{m}-\epsilon_{n}\right)}_{\epsilon_{n}=\epsilon_{m}+k^{0}}\left\langle m\left|\hat{\phi}_{\alpha}(0, \mathbf{x})\right| n\right\rangle\left\langle n\left|\hat{\phi}_{\beta}^{\dagger}(0, \mathbf{0})\right| m\right\rangle \\
& =e^{-\beta k^{0}} \Pi_{\alpha \beta}^{>}(\mathcal{K}) .
\end{aligned}
$$

This is a Fourier-space version of the KMS relation. Consequently

$$
\rho_{\alpha \beta}(\mathcal{K})=\frac{1}{2}\left[\Pi_{\alpha \beta}^{>}(\mathcal{K})-\Pi_{\alpha \beta}^{<}(\mathcal{K})\right]=\frac{1}{2}\left(e^{\beta k^{0}}-1\right) \Pi_{\alpha \beta}^{<}(\mathcal{K})
$$

and, conversely,

$$
\begin{aligned}
\Pi_{\alpha \beta}^{<}(\mathcal{K}) & =2 n_{\mathrm{B}}\left(k^{0}\right) \rho_{\alpha \beta}(\mathcal{K}), \\
\Pi_{\alpha \beta}^{>}(\mathcal{K}) & =2 \frac{e^{\beta k^{0}}}{e^{\beta k^{0}}-1} \rho_{\alpha \beta}(\mathcal{K})=2\left[1+n_{\mathrm{B}}\left(k^{0}\right)\right] \rho_{\alpha \beta}(\mathcal{K}),
\end{aligned}
$$

where $n_{\mathrm{B}}\left(k^{0}\right) \equiv 1 /\left[\exp \left(\beta k^{0}\right)-1\right]$ is the Bose distribution. Moreover,

$$
\Delta_{\alpha \beta}(\mathcal{K})=\frac{1}{2}\left[\Pi_{\alpha \beta}^{>}(\mathcal{K})+\Pi_{\alpha \beta}^{<}(\mathcal{K})\right]=\left[1+2 n_{\mathrm{B}}\left(k^{0}\right)\right] \rho_{\alpha \beta}(\mathcal{K}) .
$$

Note that $1+2 n_{\mathrm{B}}\left(-k^{0}\right)=-\left[1+2 n_{\mathrm{B}}\left(k^{0}\right)\right]$, so that if $\rho$ is odd in $\mathcal{K} \rightarrow-\mathcal{K}$, then $\Delta$ is even.

Inserting the representation

$$
\theta(t)=i \int_{-\infty}^{\infty} \frac{\mathrm{d} \omega}{2 \pi} \frac{e^{-i \omega t}}{\omega+i 0^{+}}
$$

into the definitions of $\Pi^{R}, \Pi^{A}$, in which the commutator is represented as an inverse transformation of eq. (8.4), we obtain

$$
\Pi_{\alpha \beta}^{R}(\mathcal{K})=i \int_{\mathcal{X}} e^{i \mathcal{K} \cdot \mathcal{X}} 2 \theta(t) \int_{\mathcal{P}} e^{-i \mathcal{P} \cdot \mathcal{X}} \rho_{\alpha \beta}(\mathcal{P})
$$




$$
\begin{aligned}
& =-2 \int \mathrm{d} t \int \frac{\mathrm{d} \omega}{2 \pi} \int \frac{\mathrm{d} p^{0}}{2 \pi} \frac{e^{i\left(k^{0}-p^{0}-\omega\right) t}}{\omega+i 0^{+}} \rho_{\alpha \beta}\left(p^{0}, \mathbf{k}\right) \\
& =-2 \int \frac{\mathrm{d} \omega}{2 \pi} \int \frac{\mathrm{d} p^{0}}{2 \pi} \frac{2 \pi \delta\left(k^{0}-p^{0}-\omega\right)}{\omega+i 0^{+}} \rho_{\alpha \beta}\left(p^{0}, \mathbf{k}\right) \\
& =\int_{-\infty}^{\infty} \frac{\mathrm{d} p^{0}}{\pi} \frac{\rho_{\alpha \beta}\left(p^{0}, \mathbf{k}\right)}{p^{0}-k^{0}-i 0^{+}},
\end{aligned}
$$

and similarly

$$
\Pi_{\alpha \beta}^{A}(\mathcal{K})=\int_{-\infty}^{\infty} \frac{\mathrm{d} p^{0}}{\pi} \frac{\rho_{\alpha \beta}\left(p^{0}, \mathbf{k}\right)}{p^{0}-k^{0}+i 0^{+}} .
$$

Note that these can be considered to be limiting values from the upper half-plane for $\Pi^{R}$ (since it is the combination $k^{0}+i 0^{+}$that appears in the kernel) and from the lower half-plane for $\Pi^{A}$ (since it is the combination $k^{0}-i 0^{+}$that appears).

Making use of

$$
\frac{1}{\Delta \pm i 0^{+}}=\mathbb{P}\left(\frac{1}{\Delta}\right) \mp i \pi \delta(\Delta)
$$

and assuming that $\rho_{\alpha \beta}$ is real, we find

$$
\operatorname{Im} \Pi_{\alpha \beta}^{R}(\mathcal{K})=\rho_{\alpha \beta}(\mathcal{K}), \quad \operatorname{Im} \Pi_{\alpha \beta}^{A}(\mathcal{K})=-\rho_{\alpha \beta}(\mathcal{K}) .
$$

Furthermore, the real parts of $\Pi^{R}$ and $\Pi^{A}$ agree, so that $-i\left[\Pi_{\alpha \beta}^{R}-\Pi_{\alpha \beta}^{A}\right]=2 \rho_{\alpha \beta}$.

We note in passing that eqs. (8.16) and (8.21) can be combined into

$$
\Delta_{\alpha \beta}(\mathcal{K})=\left[1+2 n_{\mathrm{B}}\left(k^{0}\right)\right] \operatorname{Im} \Pi_{\alpha \beta}^{R}(\mathcal{K}) .
$$

This important equality is sometimes referred to as the fluctuation-dissipation theorem; the physical reason for this nomenclature will be discussed in sec. 9.6.

Moving on to $\Pi_{\alpha \beta}^{T}$ and making use of eqs. (8.14) and (8.15) as well as of eq. (8.17), we find

$$
\begin{aligned}
\Pi_{\alpha \beta}^{T}(\mathcal{K}) & =\int_{\mathcal{X}} e^{i \mathcal{K} \cdot \mathcal{X}} \int_{\mathcal{P}} e^{-i \mathcal{P} \cdot \mathcal{X}}\left[\theta(t) 2 e^{\beta p^{0}} n_{\mathrm{B}}\left(p^{0}\right)+\theta(-t) 2 n_{\mathrm{B}}\left(p^{0}\right)\right] \rho_{\alpha \beta}(\mathcal{P}) \\
& =2 i \int \mathrm{d} t \int \frac{\mathrm{d} \omega}{2 \pi} \int \frac{\mathrm{d} p^{0}}{2 \pi}\left[\frac{e^{i\left(k^{0}-p^{0}-\omega\right) t}}{\omega+i 0^{+}} e^{\beta p^{0}}+\frac{e^{i\left(k^{0}-p^{0}+\omega\right) t}}{\omega+i 0^{+}}\right] n_{\mathrm{B}}\left(p^{0}\right) \rho_{\alpha \beta}\left(p^{0}, \mathbf{k}\right) \\
& =2 i \int \frac{\mathrm{d} \omega}{2 \pi} \int \frac{\mathrm{d} p^{0}}{2 \pi}\left[\frac{2 \pi \delta\left(k^{0}-p^{0}-\omega\right)}{\omega+i 0^{+}} e^{\beta p^{0}}+\frac{2 \pi \delta\left(k^{0}-p^{0}+\omega\right)}{\omega+i 0^{+}}\right] n_{\mathrm{B}}\left(p^{0}\right) \rho_{\alpha \beta}\left(p^{0}, \mathbf{k}\right) \\
& =i \int \frac{\mathrm{d} p^{0}}{\pi}\left[\frac{e^{\beta p^{0}}}{k^{0}-p^{0}+i 0^{+}}-\frac{1}{k^{0}-p^{0}-i 0^{+}}\right] n_{\mathrm{B}}\left(p^{0}\right) \rho_{\alpha \beta}\left(p^{0}, \mathbf{k}\right) \\
& =\int_{-\infty}^{\infty} \frac{\mathrm{d} p^{0}}{\pi} \frac{i \rho_{\alpha \beta}\left(p^{0}, \mathbf{k}\right)}{k^{0}-p^{0}+i 0^{+}}+2 \rho_{\alpha \beta}\left(k^{0}, \mathbf{k}\right) n_{\mathrm{B}}\left(k^{0}\right) \\
& =-i \Pi_{\alpha \beta}^{R}(\mathcal{K})+\Pi_{\alpha \beta}^{<}(\mathcal{K}),
\end{aligned}
$$

where in the penultimate step we inserted the identity $n_{\mathrm{B}}\left(p^{0}\right) e^{\beta p^{0}}=1+n_{\mathrm{B}}\left(p^{0}\right)$ as well as eq. (8.20). Note that eq. (8.23) can be obtained also directly from the definitions in eqs. (8.3), (8.6) and (8.8), by inserting $1=\theta(t)+\theta(-t)$ into eq. (8.3). It can similarly be seen that $\Pi_{\alpha \beta}^{T}=-i \Pi_{\alpha \beta}^{A}+\Pi_{\alpha \beta}^{>}$.

We note that both sums on the second row of eq. (8.11) are exponentially convergent for $0<$ $i t<\beta$. Therefore we can formally relate the two functions

$$
\left\langle\hat{\phi}_{\alpha}(\mathcal{X}) \hat{\phi}_{\beta}^{\dagger}(0)\right\rangle \text { and }\left\langle\hat{\phi}_{\alpha}(X) \hat{\phi}_{\beta}^{\dagger}(0)\right\rangle
$$


by a direct analytic continuation $t \rightarrow-i \tau$, or $i t \rightarrow \tau$, with $0<\tau<\beta$. Thereby

$$
\begin{aligned}
\Pi_{\alpha \beta}^{E}(K) & =\int_{X} e^{i K \cdot X}\left[\int_{\mathcal{P}} e^{-i \mathcal{P} \cdot \mathcal{X}} \Pi_{\alpha \beta}^{>}(\mathcal{P})\right]_{i t \rightarrow \tau} \\
& =\int_{0}^{\beta} \mathrm{d} \tau e^{i k_{n} \tau} \int_{-\infty}^{\infty} \frac{\mathrm{d} p^{0}}{2 \pi} e^{-p^{0} \tau} \Pi_{\alpha \beta}^{>}\left(p^{0}, \mathbf{k}\right) \\
& =\int_{0}^{\beta} \mathrm{d} \tau e^{i k_{n} \tau} \int_{-\infty}^{\infty} \frac{\mathrm{d} p^{0}}{2 \pi} e^{-p^{0} \tau} \frac{2 e^{\beta p^{0}}}{e^{\beta p^{0}}-1} \rho_{\alpha \beta}\left(p^{0}, \mathbf{k}\right) \\
& =\int_{-\infty}^{\infty} \frac{\mathrm{d} p^{0}}{\pi} \frac{\rho_{\alpha \beta}\left(p^{0}, \mathbf{k}\right)}{1-e^{-\beta p^{0}}}\left[\frac{e^{\left(i k_{n}-p^{0}\right) \tau}}{i k_{n}-p^{0}}\right]_{0}^{\beta} \\
& =\int_{-\infty}^{\infty} \frac{\mathrm{d} p^{0}}{\pi} \frac{\rho_{\alpha \beta}\left(p^{0}, \mathbf{k}\right)}{1-e^{-\beta p^{0}}} \frac{e^{-\beta p^{0}}-1}{i k_{n}-p^{0}} \\
p^{0} \rightarrow k^{0} & \int_{-\infty}^{\infty} \frac{\mathrm{d} k^{0}}{\pi} \frac{\rho_{\alpha \beta}\left(k^{0}, \mathbf{k}\right)}{k^{0}-i k_{n}}
\end{aligned}
$$

where we inserted eq. (8.15) for $\Pi^{>}(\mathcal{K})$, and changed orders of integration. This relation is called the spectral representation of the Euclidean correlator. ${ }^{21}$

It is useful to note that eq. (8.25) implies the existence of a simple "sum rule":

$$
\int_{-\infty}^{\infty} \frac{\mathrm{d} k^{0}}{\pi} \frac{\rho_{\alpha \beta}\left(k^{0}, \mathbf{k}\right)}{k^{0}}=\int_{0}^{\beta} \mathrm{d} \tau \Pi_{\alpha \beta}^{E}(\tau, \mathbf{k}) .
$$

Here we set $k_{n}=0$ and used the definition in eq. (8.9) on the left-hand side of eq. (8.25). The usefulness of the sum rule is that it relates integrals over Minkowskian and Euclidean correlators to each other. (Of course, we have implicitly assumed that both sides are integrable which, as already alluded to, necessitates a suitable ultraviolet regularization in the spatial directions.)

Finally, the spectral representation in eq. (8.25) can be inverted by making use of eq. (8.20),

$$
\begin{aligned}
\rho_{\alpha \beta}(\mathcal{K}) & =\frac{1}{2 i} \operatorname{Disc} \Pi_{\alpha \beta}^{E}\left(k_{n} \rightarrow-i k^{0}, \mathbf{k}\right) \\
& \equiv \frac{1}{2 i}\left[\Pi_{\alpha \beta}^{E}\left(-i\left[k^{0}+i 0^{+}\right], \mathbf{k}\right)-\Pi_{\alpha \beta}^{E}\left(-i\left[k^{0}-i 0^{+}\right], \mathbf{k}\right)\right]
\end{aligned}
$$

Furthermore, a comparison of eqs. (8.18) and (8.25) shows that

$$
\Pi_{\alpha \beta}^{R}(\mathcal{K})=\Pi_{\alpha \beta}^{E}\left(k_{n} \rightarrow-i\left[k^{0}+i 0^{+}\right], \mathbf{k}\right) .
$$

This last relation, which can be justified also through a more rigorous mathematical analysis [8.7], captures the essence of the analytic continuation from the imaginary-time (Matsubara) formalism to physical Minkowskian spacetime. ${ }^{22}$

In the context of the spectral representation, eq. (8.25), it will often be useful to note from eq. (1.70), viz.

$$
T \sum_{\omega_{n}} \frac{e^{i \omega_{n} \tau}}{\omega_{n}^{2}+\omega^{2}}=\frac{n_{\mathrm{B}}(\omega)}{2 \omega}\left[e^{(\beta-\tau) \omega}+e^{\tau \omega}\right]
$$

\footnotetext{
${ }^{21}$ It is more difficult but not impossible to find spectral representations for higher-point functions, cf. appendix A of ref. [8.5] for the 3-point function, and ref. [8.6] for a general discussion.

${ }^{22}$ The more general function $\Pi_{\alpha \beta}^{E}\left(k_{n} \rightarrow-i z, \mathbf{k}\right)=\int_{-\infty}^{\infty} \frac{\mathrm{d} k^{0}}{\pi} \frac{\rho_{\alpha \beta}\left(k^{0}, \mathbf{k}\right)}{k^{0}-z}, z \in \mathbb{C}$, is often referred to as the "resolvent".
} 
that, for $0<\tau<\beta$,

$$
\begin{aligned}
T \sum_{\omega_{n}} \frac{1}{k^{0}-i \omega_{n}} e^{i \omega_{n} \tau} & =T \sum_{\omega_{n}} \frac{i \omega_{n}+k^{0}}{\omega_{n}^{2}+\left(k^{0}\right)^{2}} e^{i \omega_{n} \tau} \\
& =\left(\partial_{\tau}+k^{0}\right) T \sum_{\omega_{n}} \frac{e^{i \omega_{n} \tau}}{\omega_{n}^{2}+\left(k^{0}\right)^{2}} \\
& =\frac{n_{\mathrm{B}}\left(k^{0}\right)}{2 k^{0}}\left[\left(-k^{0}+k^{0}\right) e^{(\beta-\tau) k^{0}}+\left(k^{0}+k^{0}\right) e^{\tau k^{0}}\right] \\
& =n_{\mathrm{B}}\left(k^{0}\right) e^{\tau k^{0}}
\end{aligned}
$$

This relation turns out to be valid both for $k^{0}<0$ and $k^{0}>0$ (to show this, substitute $\omega_{n} \rightarrow-\omega_{n}$ and use eq. (8.32)). We also note that, again for $0<\tau<\beta$,

$$
T \sum_{\omega_{n}} \frac{1}{k^{0}-i \omega_{n}} e^{-i \omega_{n} \tau}=T \sum_{\omega_{n}} \frac{1}{k^{0}-i \omega_{n}} e^{i \omega_{n}(\beta-\tau)}=n_{\mathrm{B}}\left(k^{0}\right) e^{(\beta-\tau) k^{0}} .
$$

In particular, taking the inverse Fourier transform $\left(T \sum_{k_{n}} e^{-i k_{n} \tau}\right)$ from the left-hand side of eq. (8.25), and employing eq. (8.32), we get the relation

$$
\begin{aligned}
\int_{\mathbf{x}} e^{-i \mathbf{k} \cdot \mathbf{x}}\left\langle\hat{\phi}_{\alpha}(\tau, \mathbf{x}) \hat{\phi}_{\beta}^{\dagger}(0, \mathbf{0})\right\rangle & \\
= & \int_{-\infty}^{\infty} \frac{\mathrm{d} k^{0}}{\pi} \rho_{\alpha \beta}(\mathcal{K}) n_{\mathrm{B}}\left(k^{0}\right) e^{(\beta-\tau) k^{0}} \\
= & \int_{0}^{\infty} \frac{\mathrm{d} k^{0}}{\pi}\left\{\frac{\rho_{\alpha \beta}\left(k^{0}, \mathbf{k}\right)+\rho_{\alpha \beta}\left(-k^{0}, \mathbf{k}\right)}{2} \frac{\sinh \left[\left(\frac{\beta}{2}-\tau\right) k^{0}\right]}{\sinh \left(\frac{\beta}{2} k^{0}\right)}\right. \\
& \left.+\frac{\rho_{\alpha \beta}\left(k^{0}, \mathbf{k}\right)-\rho_{\alpha \beta}\left(-k^{0}, \mathbf{k}\right)}{2} \frac{\cosh \left[\left(\frac{\beta}{2}-\tau\right) k^{0}\right]}{\sinh \left(\frac{\beta}{2} k^{0}\right)}\right\},
\end{aligned}
$$

where we symmetrized and anti-symmetrized the "kernel" $n_{\mathrm{B}}\left(k^{0}\right) e^{(\beta-\tau) k^{0}}$ with respect to $k^{0}$. Normally (when $\hat{\phi}_{\alpha}$ and $\hat{\phi}_{\beta}^{\dagger}$ are identical) the spectral function is antisymmetric in $k^{0} \rightarrow-k^{0}$, and only the second term on the last line of eq. (8.33) contributes. Thereby we obtain a useful identity: if the left-hand side of eq. (8.33) can be measured non-perturbatively on a Euclidean lattice with Monte Carlo simulations as a function of $\tau$, then an "inversion" of eq. (8.33) could lead to a non-perturbative estimate of the Minkowskian spectral function. Issues related to this inversion are discussed in ref. [8.8].

\section{Example: free boson}

Let us illustrate the relations obtained with the example of a free propagator in scalar field theory:

$$
\Pi^{E}(K)=\frac{1}{k_{n}^{2}+\epsilon_{k}^{2}}=\frac{1}{2 \epsilon_{k}}\left(\frac{1}{i k_{n}+\epsilon_{k}}+\frac{1}{-i k_{n}+\epsilon_{k}}\right)
$$

where $\epsilon_{k}=\sqrt{k^{2}+m^{2}}$. According to eq. (8.29),

$$
\Pi^{R}(\mathcal{K})=\frac{1}{-\left(k^{0}+i 0^{+}\right)^{2}+\epsilon_{k}^{2}}
$$




$$
\begin{aligned}
& =-\frac{1}{\mathcal{K}^{2}-m^{2}+i \operatorname{sign}\left(k^{0}\right) 0^{+}} \\
& =-\mathbb{P}\left(\frac{1}{\left(k^{0}\right)^{2}-\epsilon_{k}^{2}}\right)+\frac{i \pi}{2 \epsilon_{k}}\left[\delta\left(k^{0}-\epsilon_{k}\right)-\delta\left(k^{0}+\epsilon_{k}\right)\right],
\end{aligned}
$$

and according to eq. (8.21),

$$
\rho(\mathcal{K})=\frac{\pi}{2 \epsilon_{k}}\left[\delta\left(k^{0}-\epsilon_{k}\right)-\delta\left(k^{0}+\epsilon_{k}\right)\right] .
$$

Finally, according to eqs. (8.14) and (8.23),

$$
\begin{aligned}
\Pi^{T}(\mathcal{K}) & =\mathbb{P}\left(\frac{i}{\left(k^{0}\right)^{2}-\epsilon_{k}^{2}}\right)+\frac{\pi}{2 \epsilon_{k}}\left\{\delta\left(k^{0}-\epsilon_{k}\right)\left[1+2 n_{\mathrm{B}}\left(k^{0}\right)\right]-\delta\left(k^{0}+\epsilon_{k}\right)\left[1+2 n_{\mathrm{B}}\left(k^{0}\right)\right]\right\} \\
& =\mathbb{P}\left(\frac{i}{\left(k^{0}\right)^{2}-\epsilon_{k}^{2}}\right)+\frac{\pi}{2 \epsilon_{k}}\left[\delta\left(k^{0}-\epsilon_{k}\right)+\delta\left(k^{0}+\epsilon_{k}\right)\right]\left[1+2 n_{\mathrm{B}}\left(\left|k^{0}\right|\right)\right] \\
& =\mathbb{P}\left(\frac{i}{\left(k^{0}\right)^{2}-\epsilon_{k}^{2}}\right)+\pi \delta\left(\left(k^{0}\right)^{2}-\epsilon_{k}^{2}\right)\left[1+2 n_{\mathrm{B}}\left(\left|k^{0}\right|\right)\right] \\
& =\frac{i}{\left(k^{0}\right)^{2}-\epsilon_{k}^{2}+i 0^{+}}+2 \pi \delta\left(\left(k^{0}\right)^{2}-\epsilon_{k}^{2}\right) n_{\mathrm{B}}\left(\left|k^{0}\right|\right) \\
& =\frac{i}{\mathcal{K}^{2}-m^{2}+i 0^{+}}+2 \pi \delta\left(\mathcal{K}^{2}-m^{2}\right) n_{\mathrm{B}}\left(\left|k^{0}\right|\right),
\end{aligned}
$$

where in the second step we made use of the identity $1+2 n_{\mathrm{B}}\left(-\epsilon_{k}\right)=-\left[1+2 n_{\mathrm{B}}\left(\epsilon_{k}\right)\right]$.

It is useful to note that eq. (8.37) is closely related to eq. (2.34). However, eq. (2.34) is true in general, whereas eq. (8.37) was derived for the special case of a free propagator; thus it is not always true that thermal effects can be obtained by simply replacing the zero-temperature timeordered propagator by eq. (8.37), even if surprisingly often such a simple recipe does function. We return to a discussion of this point in sec. 8.3.

\section{Fermionic case}

Let us next consider 2-point correlation functions built out of fermionic operators [8.1]-[8.4]. In contrast to the bosonic case, we take for generality the density matrix to be of the form

$$
\hat{\rho}=\frac{1}{\mathcal{Z}} \exp [-\beta(\hat{H}-\mu \hat{Q})],
$$

where $\hat{Q}$ is an operator commuting with $\hat{H}$ and $\mu$ is the associated chemical potential.

We denote the operators appearing in the 2-point functions by $\hat{j}_{\alpha}, \hat{\bar{j}}_{\beta}$. They could be elementary field operators, in which case the indices $\alpha, \beta$ label Dirac and/or flavour components, but they could also be composite operators consisting of a product of elementary field operators. Nevertheless, we assume the validity of the relation

$$
\left[\hat{j}_{\alpha}(t, \mathbf{x}), \hat{Q}\right]=\hat{j}_{\alpha}(t, \mathbf{x}) .
$$

To motivate this, note that for $\hat{j}_{\alpha} \equiv \hat{\psi}_{\alpha}, \hat{\bar{j}}_{\beta}=\hat{\bar{\psi}}_{\beta}$, the canonical commutation relation of eq. (4.33),

$$
\left\{\hat{\psi}_{\alpha}\left(x^{0}, \mathbf{x}\right), \hat{\psi}_{\beta}^{\dagger}\left(x^{0}, \mathbf{y}\right)\right\}=\delta^{(d)}(\mathbf{x}-\mathbf{y}) \delta_{\alpha \beta},
$$

and the expression for the conserved charge in eq. (7.33),

$$
\hat{Q}=\int_{\mathbf{x}} \hat{\bar{\psi}} \gamma_{0} \hat{\psi}=\int_{\mathbf{x}} \hat{\psi}_{\alpha}^{\dagger} \hat{\psi}_{\alpha},
$$


as well as the identity $[\hat{A}, \hat{B} \hat{C}]=\hat{A} \hat{B} \hat{C}-\hat{B} \hat{C} \hat{A}=\hat{A} \hat{B} \hat{C}+\hat{B} \hat{A} \hat{C}-\hat{B} \hat{A} \hat{C}-\hat{B} \hat{C} \hat{A}=\{\hat{A}, \hat{B}\} \hat{C}-\hat{B}\{\hat{A}, \hat{C}\}$, indicate that eq. (8.39) is indeed satisfied for $\hat{\psi}_{\alpha}$. Eq. (8.39) implies that

$$
e^{\beta \mu} \hat{Q}_{j_{\alpha}}(t, \mathbf{x})=\sum_{n=0}^{\infty} \frac{1}{n !}(\beta \mu)^{n}(\hat{Q})^{n} \hat{j}_{\alpha}(t, \mathbf{x})=\sum_{n=0}^{\infty} \frac{1}{n !}(\beta \mu)^{n} \hat{j}_{\alpha}(t, \mathbf{x})(\hat{Q}-\hat{\mathbb{1}})^{n}=\hat{j}_{\alpha}(t, \mathbf{x}) e^{\beta \mu \hat{Q}} e^{-\beta \mu}
$$

and consequently that

$$
\begin{aligned}
\left\langle\hat{j}_{\alpha}(t-i \beta, \mathbf{x}) \hat{\bar{j}}_{\beta}(0, \mathbf{0})\right\rangle & =\frac{1}{\mathcal{Z}} \operatorname{Tr}\left[e^{-\beta(\hat{H}-\mu \hat{Q})} e^{\beta \hat{H}} \hat{j}_{\alpha}(t, \mathbf{x}) e^{-\beta \hat{H}} \hat{\bar{j}}_{\beta}(0, \mathbf{0})\right] \\
& =\frac{1}{\mathcal{Z}} \operatorname{Tr}\left[\hat{j}_{\alpha}(t, \mathbf{x}) e^{-\beta \mu} e^{-\beta(\hat{H}-\mu \hat{Q})} \hat{\bar{j}}_{\beta}(0, \mathbf{0})\right] \\
& =\frac{1}{\mathcal{Z}} e^{-\mu \beta} \operatorname{Tr}\left[\hat{j}_{\alpha}(t, \mathbf{x}) e^{-\beta(\hat{H}-\mu \hat{Q})} \hat{\bar{j}}_{\beta}(0, \mathbf{0})\right] \\
& =e^{-\mu \beta}\left\langle\hat{\bar{j}}_{\beta}(0, \mathbf{0}) \hat{j}_{\alpha}(t, \mathbf{x})\right\rangle .
\end{aligned}
$$

This is a fermionic version of the KMS relation.

With this setting, we can again define various classes of correlation functions. The "physical" correlators are now set up as

$$
\begin{aligned}
\Pi_{\alpha \beta}^{>}(\mathcal{K}) & \equiv \int_{\mathcal{X}} e^{i \mathcal{K} \cdot \mathcal{X}}\left\langle\hat{j}_{\alpha}(\mathcal{X}) \hat{\bar{j}}_{\beta}(0)\right\rangle, \\
\Pi_{\alpha \beta}^{<}(\mathcal{K}) & \equiv \int_{\mathcal{X}} e^{i \mathcal{K} \cdot \mathcal{X}}\left\langle-\hat{\bar{j}}_{\beta}(0) \hat{j}_{\alpha}(\mathcal{X})\right\rangle, \\
\rho_{\alpha \beta}(\mathcal{K}) & \equiv \int_{\mathcal{X}} e^{i \mathcal{K} \cdot \mathcal{X}}\left\langle\frac{1}{2}\left\{\hat{j}_{\alpha}(\mathcal{X}), \hat{\bar{j}}_{\beta}(0)\right\}\right\rangle, \\
\Delta_{\alpha \beta}(\mathcal{K}) & \equiv \int_{\mathcal{X}} e^{i \mathcal{K} \cdot \mathcal{X}}\left\langle\frac{1}{2}\left[\hat{j}_{\alpha}(\mathcal{X}), \hat{\bar{j}}_{\beta}(0)\right]\right\rangle,
\end{aligned}
$$

where $\rho_{\alpha \beta}$ is the spectral function. The retarded and advanced correlators can be defined as

$$
\begin{aligned}
\Pi_{\alpha \beta}^{R}(\mathcal{K}) & \equiv i \int_{\mathcal{X}} e^{i \mathcal{K} \cdot \mathcal{X}}\left\langle\left\{\hat{j}_{\alpha}(\mathcal{X}), \hat{\bar{j}}_{\beta}(0)\right\} \theta(t)\right\rangle, \\
\Pi_{\alpha \beta}^{A}(\mathcal{K}) & \equiv i \int_{\mathcal{X}} e^{i \mathcal{K} \cdot \mathcal{X}}\left\langle-\left\{\hat{j}_{\alpha}(\mathcal{X}), \hat{\bar{j}}_{\beta}(0)\right\} \theta(-t)\right\rangle .
\end{aligned}
$$

On the other hand, the time-ordered correlation function reads

$$
\Pi_{\alpha \beta}^{T}(\mathcal{K}) \equiv \int_{\mathcal{X}} e^{i \mathcal{K} \cdot \mathcal{X}}\left\langle\hat{j}_{\alpha}(\mathcal{X}) \hat{\bar{j}}_{\beta}(0) \theta(t)-\hat{\bar{j}}_{\beta}(0) \hat{j}_{\alpha}(\mathcal{X}) \theta(-t)\right\rangle
$$

whereas the Euclidean correlator is

$$
\Pi_{\alpha \beta}^{E}(K) \equiv \int_{0}^{\beta} \mathrm{d} \tau \int_{\mathbf{x}} e^{\left(i k_{n}+\mu\right) \tau-i \mathbf{k} \cdot \mathbf{x}}\left\langle\hat{j}_{\alpha}(X) \hat{\bar{j}}_{\beta}(0)\right\rangle .
$$

Note that the Euclidean correlator is time-ordered by definition $(0 \leq \tau \leq \beta)$, and can be computed with standard imaginary-time functional integrals.

If the two operators in the integrand of eq. (8.51) anticommute with each other at $t=0$, then the KMS relation in eq. (8.43) asserts that $\left\langle\hat{j}_{\alpha}(-i \beta, \mathbf{x}) \hat{\bar{j}}_{\beta}(0, \mathbf{0})\right\rangle=e^{-\mu \beta}\left\langle\hat{\bar{j}}_{\beta}(0, \mathbf{0}) \hat{j}_{\alpha}(0, \mathbf{x})\right\rangle=$ $-e^{-\mu \beta}\left\langle\hat{j}_{\alpha}(0, \mathbf{x}) \hat{\bar{j}}_{\beta}(0, \mathbf{0})\right\rangle$. The additional term in the Fourier transform with respect to $\tau$ in eq. (8.51) cancels the multiplicative factor $e^{-\mu \beta}$ at $\tau=\beta$, so that the $\tau$-integrand is antiperiodic. Therefore the Matsubara frequencies $k_{n}$ are fermionic. 
We can establish relations between the different Green's functions just like in the bosonic case:

$$
\begin{aligned}
& \Pi_{\alpha \beta}^{>}(\mathcal{K})=\frac{1}{\mathcal{Z}} \int_{\mathcal{X}} e^{i \mathcal{K} \cdot \mathcal{X}} \operatorname{Tr}[e^{-\beta \hat{H}+i \hat{H} t} \underbrace{\mathbb{1}}_{\sum_{m}|m\rangle\langle m|} e^{\beta \mu \hat{Q}} \hat{j}_{\alpha}(0, \mathbf{x}) e^{-i \hat{H} t} \underbrace{\mathbb{1}}_{\sum_{n}|n\rangle\langle n|} \hat{\bar{j}}_{\beta}(0, \mathbf{0})] \\
& =\frac{1}{\mathcal{Z}} \sum_{m, n} \int_{\mathcal{X}} e^{i \mathcal{K} \cdot \mathcal{X}} e^{(-\beta+i t) \epsilon_{m}} e^{-i t \epsilon_{n}} e^{-\beta \mu}\left\langle m\left|\hat{j}_{\alpha}(0, \mathbf{x}) e^{\beta \mu \hat{Q}}\right| n\right\rangle\left\langle n\left|\hat{\bar{j}}_{\beta}(0, \mathbf{0})\right| m\right\rangle \\
& =\frac{1}{\mathcal{Z}} \int_{\mathbf{x}} e^{-i \mathbf{k} \cdot \mathbf{x}} \sum_{m, n} e^{-\beta\left(\epsilon_{m}+\mu\right)} 2 \pi \delta\left(k^{0}+\epsilon_{m}-\epsilon_{n}\right)\left\langle m\left|\hat{j}_{\alpha}(0, \mathbf{x}) e^{\beta \mu \hat{Q}}\right| n\right\rangle\left\langle n\left|\hat{\bar{j}}_{\beta}(0, \mathbf{0})\right| m\right\rangle, \\
& \Pi_{\alpha \beta}^{<}(\mathcal{K})=-\frac{1}{\mathcal{Z}} \int_{\mathcal{X}} e^{i \mathcal{K} \cdot \mathcal{X}} \operatorname{Tr}[e^{-\beta \hat{H}} e^{\beta \mu \hat{Q}} \underbrace{\mathbb{1}}_{\sum_{n}|n\rangle\langle n|} \hat{\bar{j}}_{\beta}(0, \mathbf{0}) e^{i \hat{H} t} \underbrace{\mathbb{1}}_{\sum_{m}|m\rangle\langle m|} \hat{j}_{\alpha}(0, \mathbf{x}) e^{-i \hat{H} t}] \\
& =-\frac{1}{\mathcal{Z}} \sum_{m, n} \int_{\mathcal{X}} e^{i \mathcal{K} \cdot \mathcal{X}} e^{(-\beta-i t) \epsilon_{n}} e^{i t \epsilon_{m}}\left\langle n\left|\hat{\bar{j}}_{\beta}(0, \mathbf{0})\right| m\right\rangle\left\langle m\left|\hat{j}_{\alpha}(0, \mathbf{x}) e^{\beta \mu \hat{Q}}\right| n\right\rangle \\
& =-\frac{1}{\mathcal{Z}} \int_{\mathbf{x}} e^{-i \mathbf{k} \cdot \mathbf{x}} \sum_{m, n} e^{-\beta \epsilon_{n}} 2 \pi \underbrace{\delta\left(k^{0}+\epsilon_{m}-\epsilon_{n}\right.}_{\epsilon_{n}=\epsilon_{m}+k^{0}})\left\langle m\left|\hat{j}_{\alpha}(0, \mathbf{x}) e^{\beta \mu \hat{Q}}\right| n\right\rangle\left\langle n\left|\hat{\bar{j}}_{\beta}(0, \mathbf{0})\right| m\right\rangle \\
& =-e^{-\beta\left(k^{0}-\mu\right)} \Pi_{\alpha \beta}^{>}(\mathcal{K}) .
\end{aligned}
$$

Using the fact that $\rho_{\alpha \beta}(\mathcal{K})=\left[\Pi_{\alpha \beta}^{>}(\mathcal{K})-\Pi_{\alpha \beta}^{<}(\mathcal{K})\right] / 2$, we subsequently obtain

$$
\Pi_{\alpha \beta}^{>}(\mathcal{K})=2\left[1-n_{\mathrm{F}}\left(k^{0}-\mu\right)\right] \rho_{\alpha \beta}(\mathcal{K}), \quad \Pi_{\alpha \beta}^{<}(\mathcal{K})=-2 n_{\mathrm{F}}\left(k^{0}-\mu\right) \rho_{\alpha \beta}(\mathcal{K}),
$$

where $n_{\mathrm{F}}\left(k^{0}\right) \equiv 1 /\left[\exp \left(\beta k^{0}\right)+1\right]$ is the Fermi distribution. Moreover, the statistical correlator can be expressed as $\Delta_{\alpha \beta}(\mathcal{K})=\left[1-2 n_{\mathrm{F}}\left(k^{0}-\mu\right)\right] \rho_{\alpha \beta}(\mathcal{K})$.

The relation of $\Pi^{R}, \Pi^{A}$ and $\Pi^{T}$ to the spectral function can be derived in complete analogy with eqs. (8.17)-(8.23). For brevity we only cite the final results:

$$
\begin{aligned}
\Pi_{\alpha \beta}^{R}(\mathcal{K}) & =\int_{-\infty}^{\infty} \frac{\mathrm{d} \omega}{\pi} \frac{\rho_{\alpha \beta}(\omega, \mathbf{k})}{\omega-k^{0}-i 0^{+}}, \quad \Pi_{\alpha \beta}^{A}(\mathcal{K})=\int_{-\infty}^{\infty} \frac{\mathrm{d} \omega}{\pi} \frac{\rho_{\alpha \beta}(\omega, \mathbf{k})}{\omega-k^{0}+i 0^{+}}, \\
\Pi_{\alpha \beta}^{T}(\mathcal{K}) & =\int_{-\infty}^{\infty} \frac{\mathrm{d} \omega}{\pi} \frac{i \rho_{\alpha \beta}(\omega, \mathbf{k})}{k^{0}-\omega+i 0^{+}}-2 n_{\mathrm{F}}\left(k^{0}-\mu\right) \rho_{\alpha \beta}\left(k^{0}, \mathbf{k}\right) \\
& =-i \Pi_{\alpha \beta}^{R}(\mathcal{K})+\Pi_{\alpha \beta}^{<}(\mathcal{K}) .
\end{aligned}
$$

Note that when written in a "generic form", where no distribution functions are visible, the end results are identical to the bosonic ones. In addition, eq. (8.56) can again be crosschecked using the right-hand sides of eqs. (8.45), (8.48) and (8.50), and the alternative representation $\Pi_{\alpha \beta}^{T}=$ $-i \Pi_{\alpha \beta}^{A}+\Pi_{\alpha \beta}^{>}$also applies. The latter derivation implies that these "operator relations" apply even in a non-thermal situation, described by a generic density matrix (cf. sec. 8.3).

Finally, writing the argument inside the $\tau$-integration in eq. (8.51) as a Wick rotation of the inverse Fourier transform of eq. (8.44), inserting eq. (8.54), and changing orders of integration, we get a spectral representation analogous to eq. (8.25),

$$
\begin{aligned}
\Pi_{\alpha \beta}^{E}(K) & =\int_{0}^{\beta} \mathrm{d} \tau e^{\left(i k_{n}+\mu\right) \tau} \int_{-\infty}^{\infty} \frac{\mathrm{d} p^{0}}{2 \pi} e^{-p^{0} \tau} \Pi_{\alpha \beta}^{>}\left(p^{0}, \mathbf{k}\right) \\
& =\int_{0}^{\beta} \mathrm{d} \tau e^{\left(i k_{n}+\mu\right) \tau} \int_{-\infty}^{\infty} \frac{\mathrm{d} p^{0}}{2 \pi} e^{-p^{0} \tau} \frac{2 e^{\beta\left(p^{0}-\mu\right)}}{e^{\beta\left(p^{0}-\mu\right)}+1} \rho_{\alpha \beta}\left(p^{0}, \mathbf{k}\right)
\end{aligned}
$$




$$
\begin{array}{ll}
= & \int_{-\infty}^{\infty} \frac{\mathrm{d} p^{0}}{\pi} \frac{e^{\beta\left(p^{0}-\mu\right)}}{e^{\beta\left(p^{0}-\mu\right)}+1} \rho_{\alpha \beta}\left(p^{0}, \mathbf{k}\right) \int_{0}^{\beta} \mathrm{d} \tau e^{\left(i k_{n}+\mu-p^{0}\right) \tau} \\
= & \int_{-\infty}^{\infty} \frac{\mathrm{d} p^{0}}{\pi} \frac{e^{\beta\left(p^{0}-\mu\right)}}{e^{\beta\left(p^{0}-\mu\right)}+1} \rho_{\alpha \beta}\left(p^{0}, \mathbf{k}\right)\left[\frac{e^{\left(i k_{n}+\mu-p^{0}\right) \tau}}{i k_{n}+\mu-p^{0}}\right]_{0}^{\beta} \\
= & \int_{-\infty}^{\infty} \frac{\mathrm{d} p^{0}}{\pi} \frac{e^{\beta\left(p^{0}-\mu\right)}}{e^{\beta\left(p^{0}-\mu\right)}+1} \rho_{\alpha \beta}\left(p^{0}, \mathbf{k}\right) \frac{-e^{-\beta\left(p^{0}-\mu\right)}-1}{i k_{n}+\mu-p^{0}} \\
\stackrel{p^{0} \rightarrow k^{0}}{=} & \int_{-\infty}^{\infty} \frac{\mathrm{d} k^{0}}{\pi} \frac{\rho_{\alpha \beta}\left(k^{0}, \mathbf{k}\right)}{k^{0}-i\left[k_{n}-i \mu\right]} .
\end{array}
$$

Like in the bosonic case, this relation can be inverted by making use of eq. (8.20),

$$
\rho_{\alpha \beta}(\mathcal{K})=\frac{1}{2 i} \operatorname{Disc} \Pi_{\alpha \beta}^{E}\left(k_{n}-i \mu \rightarrow-i k^{0}, \mathbf{k}\right),
$$

where the discontinuity is defined like in eq. (8.28).

The fermionic Matsubara sum over the structure in eq. (8.57) can be carried out explicitly. This could be verified by making use of eq. (4.77), in analogy with the bosonic analysis in eqs. (8.31) and (8.32), but let us proceed in another way for a change. We may recall (cf. footnote on p. 11) that

$$
T \sum_{\omega_{n}} e^{i \omega_{n} \tau}=\delta(\tau \bmod \beta) .
$$

According to eq. (4.55), viz. $\sigma_{\mathrm{f}}(T)=2 \sigma_{\mathrm{b}}\left(\frac{T}{2}\right)-\sigma_{\mathrm{b}}(T)$, we can thus write

$$
T \sum_{\left\{\omega_{n}\right\}} e^{i \omega_{n} \tau}=2 \delta(\tau \bmod 2 \beta)-\delta(\tau \bmod \beta) .
$$

Let us assume for a moment that $k^{0}-\mu>0$. Employing the representation

$$
\frac{1}{\alpha+i \beta}=\int_{0}^{\infty} \mathrm{d} s e^{-(\alpha+i \beta) s}, \quad \alpha>0,
$$

and inserting subsequently eq. (8.60), we get

$$
\begin{aligned}
& T \sum_{\left\{\omega_{n}\right\}} \frac{1}{k^{0}-\mu-i \omega_{n}} e^{i \omega_{n} \tau}=\int_{0}^{\infty} \mathrm{d} s T \sum_{\left\{\omega_{n}\right\}} e^{i \omega_{n} \tau-k^{0} s+\mu s+i \omega_{n} s} \\
& =\int_{0}^{\infty} \mathrm{d} s e^{-\left(k^{0}-\mu\right) s}[2 \delta(\tau+s \bmod 2 \beta)-\delta(\tau+s \bmod \beta)] \\
& =2 \sum_{n=1}^{\infty} e^{-\left(k^{0}-\mu\right)(-\tau+2 \beta n)}-\sum_{n=1}^{\infty} e^{-\left(k^{0}-\mu\right)(-\tau+\beta n)}
\end{aligned}
$$

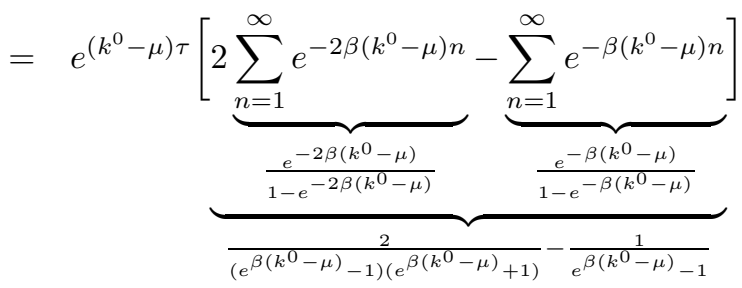

$$
\begin{aligned}
& =-e^{\left(k^{0}-\mu\right) \tau} n_{\mathrm{F}}\left(k^{0}-\mu\right),
\end{aligned}
$$

where we assumed $0<\tau<\beta$. As an immediate consequence,

$$
T \sum_{\left\{\omega_{n}\right\}} \frac{1}{k^{0}-\mu-i \omega_{n}} e^{-i \omega_{n} \tau}=-T \sum_{\left\{\omega_{n}\right\}} \frac{1}{k^{0}-\mu-i \omega_{n}} e^{i \omega_{n}(\beta-\tau)}=e^{(\beta-\tau)\left(k^{0}-\mu\right)} n_{\mathrm{F}}\left(k^{0}-\mu\right) .
$$


Furthermore, it is not difficult to show (by substituting $\omega_{n} \rightarrow-\omega_{n}$ ) that these relations continue to hold also for $k^{0}-\mu<0$.

As a consequence of eq. (8.62), we note that

$$
\begin{aligned}
T \sum_{\left\{\omega_{n}\right\}} \frac{e^{i\left(\omega_{n}+i \mu\right) \tau}}{\left(\omega_{n}+i \mu\right)^{2}+\omega^{2}} & =e^{-\mu \tau} T \sum_{\left\{\omega_{n}\right\}} e^{i \omega_{n} \tau} \frac{1}{\left(\omega-i \omega_{n}+\mu\right)\left(\omega+i \omega_{n}-\mu\right)} \\
& =e^{-\mu \tau} T \sum_{\left\{\omega_{n}\right\}} e^{i \omega_{n} \tau} \frac{1}{2 \omega}\left[\frac{1}{\omega-\mu+i \omega_{n}}+\frac{1}{\omega+\mu-i \omega_{n}}\right] \\
& =\frac{e^{-\mu \tau}}{2 \omega}\left[e^{-(\omega-\mu) \tau} n_{\mathrm{F}}(-\omega+\mu)-e^{(\omega+\mu) \tau} n_{\mathrm{F}}(\omega+\mu)\right] \\
& =\frac{e^{-\mu \tau}}{2 \omega}\left[e^{(\beta-\tau)(\omega-\mu)} n_{\mathrm{F}}(\omega-\mu)-e^{\tau(\omega+\mu)} n_{\mathrm{F}}(\omega+\mu)\right] \\
& =\frac{1}{2 \omega}\left[n_{\mathrm{F}}(\omega-\mu) e^{(\beta-\tau) \omega-\beta \mu}-n_{\mathrm{F}}(\omega+\mu) e^{\tau \omega}\right] .
\end{aligned}
$$

This constitutes a generalization of eq. (4.77) to the case of a finite chemical potential.

\section{Example: free fermion}

We illustrate the relations obtained by considering the structure of the free fermion propagator in the presence of a chemical potential. With fermions, one has to be quite careful with definitions. Suppressing spatial coordinates and indices, eq. (5.47) and the presence of a chemical potential $\grave{a}$ la eq. (7.35) imply that the free propagator can be written in the schematic form (here $A$ and $B$ carry dependence on the spatial momentum and the Dirac matrices)

$$
\langle\hat{\psi}(\tau) \hat{\bar{\psi}}(0)\rangle=T \sum_{\left\{p_{n}\right\}} e^{i\left(p_{n}+i \mu\right) \tau} \frac{-i A\left(p_{n}+i \mu\right)+B}{\left(p_{n}+i \mu\right)^{2}+\epsilon_{k}^{2}},
$$

where an additional exponential has been inserted into the Fourier transform, in order to respect the KMS property in eq. (8.43). The correlator in eq. (8.51) then becomes

$$
\begin{aligned}
\Pi^{E}\left(k_{n}\right) & =\int_{0}^{\beta} \mathrm{d} \tau e^{\left(i k_{n}+\mu\right) \tau} T \sum_{\left\{p_{n}\right\}} e^{i\left(p_{n}+i \mu\right) \tau} \frac{-i A\left(p_{n}+i \mu\right)+B}{\left(p_{n}+i \mu\right)^{2}+\epsilon_{k}^{2}} \\
& =\frac{i A\left(k_{n}-i \mu\right)+B}{\left(k_{n}-i \mu\right)^{2}+\epsilon_{k}^{2}} .
\end{aligned}
$$

The analytic continuation in eq. (8.58) yields the retarded correlator

$$
\Pi^{R}\left(k^{0}\right)=\frac{A\left(k^{0}+i 0^{+}\right)+B}{-\left(k^{0}+i 0^{+}\right)^{2}+\epsilon_{k}^{2}}=-\frac{A k^{0}+B}{\left(k^{0}\right)^{2}-\epsilon_{k}^{2}+i \operatorname{sign}\left(k^{0}\right) 0^{+}},
$$

and its discontinuity gives

$$
\begin{aligned}
\rho\left(k^{0}\right) & =\pi\left(A k^{0}+B\right) \operatorname{sign}\left(k^{0}\right) \delta\left(\left(k^{0}-\epsilon_{k}\right)\left(k^{0}+\epsilon_{k}\right)\right) \\
& =\pi\left(A k^{0}+B\right) \frac{\operatorname{sign}\left(k^{0}\right)}{2 \epsilon_{k}}\left[\delta\left(k^{0}-\epsilon_{k}\right)+\delta\left(k^{0}+\epsilon_{k}\right)\right] \\
& =\frac{\pi}{2 \epsilon_{k}}\left(A k^{0}+B\right)\left[\delta\left(k^{0}-\epsilon_{k}\right)-\delta\left(k^{0}+\epsilon_{k}\right)\right] .
\end{aligned}
$$


Any dependence on temperature and chemical potential has disappeared here. Note that (if $B$ is odd in $\mathbf{k}) \rho$ is even in $\mathcal{K} \rightarrow-\mathcal{K}$. From eqs. (8.54) and (8.56), the time-ordered propagator can be determined after a few steps:

$$
\begin{aligned}
\Pi^{T}\left(k^{0}\right)= & \left(A k^{0}+B\right)\left\{\frac{-i}{2 \epsilon_{k}}\left(\frac{1}{\epsilon_{k}-k^{0}-i 0^{+}}+\frac{1}{\epsilon_{k}+k^{0}+i 0^{+}}\right)\right. \\
& \left.-\frac{2 \pi}{2 \epsilon_{k}} n_{\mathrm{F}}\left(k^{0}-\mu\right)\left[\delta\left(k^{0}-\epsilon_{k}\right)-\delta\left(k^{0}+\epsilon_{k}\right)\right]\right\} \\
= & \frac{A k^{0}+B}{2 \epsilon_{k}}\left\{-i \mathbb{P}\left(\frac{1}{\epsilon_{k}-k^{0}}\right)-i \mathbb{P}\left(\frac{1}{\epsilon_{k}+k^{0}}\right)\right. \\
& \left.+\pi \delta\left(k^{0}-\epsilon_{k}\right)\left[1-2 n_{\mathrm{F}}\left(k^{0}-\mu\right)\right]-\pi \delta\left(k^{0}+\epsilon_{k}\right)\left[1-2 n_{\mathrm{F}}\left(k^{0}-\mu\right)\right]\right\} \\
= & \frac{A k^{0}+B}{2 \epsilon_{k}}\left\{-i \mathbb{P}\left(\frac{2 \epsilon_{k}}{\epsilon_{k}^{2}-\left(k^{0}\right)^{2}}\right)\right. \\
& \left.+\pi \delta\left(k^{0}-\epsilon_{k}\right)\left[1-2 n_{\mathrm{F}}\left(k^{0}-\mu\right)\right]+\pi \delta\left(k^{0}+\epsilon_{k}\right)\left[1-2 n_{\mathrm{F}}\left(-k^{0}+\mu\right)\right]\right\} \\
= & \frac{A k^{0}+B}{2 \epsilon_{k}}\left\{-i \mathbb{P}\left(\frac{2 \epsilon_{k}}{\epsilon_{k}^{2}-\left(k^{0}\right)^{2}}\right)+2 \epsilon_{k} \pi \delta\left(\left(k^{0}\right)^{2}-\epsilon_{k}^{2}\right)\right. \\
& \left.-2 \pi\left[\delta\left(k^{0}-\epsilon_{k}\right) n_{\mathrm{F}}\left(k^{0}-\mu\right)+\delta\left(k^{0}+\epsilon_{k}\right) n_{\mathrm{F}}\left(-k^{0}+\mu\right)\right]\right\} \\
= & \left(A k^{0}+B\right)\left\{\frac{i}{\mathcal{K}^{2}-m^{2}+i 0^{+}}-2 \pi \delta\left(\mathcal{K}^{2}-m^{2}\right) n_{\mathrm{F}}\left(\left|k^{0}\right|-\operatorname{sign}\left(k^{0}\right) \mu\right)\right\} .
\end{aligned}
$$

Medium effects are seen to reside in the on-shell part and, to some extent, one could hope to account for them simply by replacing free zero-temperature Feynman propagators by eq. (8.69). The proper procedure, however, is to carry out the analytic continuation for the complete observable considered, and this may not always amount to the simple replacement of vacuum time-ordered propagators through eq. (8.69), cf. sec. 8.3. 


\subsection{From a Euclidean correlator to a spectral function}

As an application of the relations derived in sec. 8.1, let us carry out an explicit 1-loop computation illustrating the steps. ${ }^{23}$ The computation performed here will turn out to be directly relevant in the context of particle production, discussed in more detail in sec. 9.3.

Our goal is to work out the leading non-trivial contribution to the spectral function of a righthanded lepton $(N)$ that originates from its Yukawa interaction with Standard Model particles,

$$
\delta \mathcal{L}_{M} \equiv-h \bar{L} \tilde{\phi} a_{\mathrm{R}} N-h^{*} \bar{N} \tilde{\phi}^{\dagger} a_{\mathrm{L}} L
$$

Here $\tilde{\phi} \equiv i \tau_{2} \phi^{*}$ is a conjugated Higgs doublet, $L$ is a lepton doublet, $a_{\mathrm{L}} \equiv\left(1-\gamma_{5}\right) / 2$ and $a_{\mathrm{R}} \equiv$ $\left(1+\gamma_{5}\right) / 2$ are chiral projectors, and $h$ is a Yukawa coupling constant. The Higgs and lepton doublets have the forms

$$
\tilde{\phi}=\frac{1}{\sqrt{2}}\left(\begin{array}{c}
\phi_{0}+i \phi_{3} \\
-\phi_{2}+i \phi_{1}
\end{array}\right), \quad L=\left(\begin{array}{c}
\nu \\
e
\end{array}\right) \equiv\left(\begin{array}{c}
\ell_{1} \\
\ell_{2}
\end{array}\right),
$$

where $\phi_{\mu}, \mu \in\{0,1,2,3\}$, are real scalar fields. The neutral component $\phi_{0}$ is the physical Higgs field, whereas the $\phi_{i}$ represent Goldstone modes after electroweak symmetry breaking.

Anticipating the results of sec. 9.3, we consider the Euclidean correlator of the operators coupling to the right-handed lepton through the interaction in eq. (8.70),

$$
\Pi^{E}(K) \equiv \int_{X} e^{i K \cdot X} a_{\mathrm{L}}\left\langle\left(\tilde{\phi}^{\dagger} L\right)(X)(\bar{L} \tilde{\phi})(0)\right\rangle a_{\mathrm{R}} .
$$

This has the form of eq. (8.51); the coupling constant $|h|^{2}$ has been omitted for simplicity. The four-momentum $K$ is fermionic. The operators in eq. (8.72) are of a mixed "boson-fermion" type; similar computations will be carried out for "fermion-fermion" and "boson-boson" cases below, cf. eqs. (8.135) and (8.179), respectively. The "boson-fermion" analysis is furthermore generalized to include a chemical potential around eq. (8.181).

Inserting eq. (8.71) and carrying out the contractions, we can rewrite eq. (8.72) in the form

$$
\begin{aligned}
\Pi^{E}(K) & =\frac{1}{2} \int_{X} e^{i K \cdot X} a_{\mathrm{L}}\langle\ell(X) \bar{\ell}(0)\rangle_{0}\langle\phi(X) \phi(0)\rangle_{0} a_{\mathrm{R}} \\
& =\frac{1}{2} \int_{X} \sum_{\{P\} R} e^{i(K+P+R) \cdot X} a_{\mathrm{L}} \frac{-i \not P+m_{\ell}}{P^{2}+m_{\ell}^{2}} \frac{1}{R^{2}+m_{\phi}^{2}} a_{\mathrm{R}} \\
& =\frac{1}{2} \int_{\mathbf{p}} T \sum_{\left\{p_{n}\right\}} \frac{-i \not P a_{\mathrm{R}}}{p_{n}^{2}+\epsilon_{1}^{2}} \frac{1}{\left(p_{n}+k_{n}\right)^{2}+\epsilon_{2}^{2}},
\end{aligned}
$$

where we inserted the free scalar and fermion propagators, and denoted

$$
\epsilon_{1} \equiv \sqrt{\mathbf{p}^{2}+m_{\ell}^{2}}, \quad \epsilon_{2} \equiv \sqrt{(\mathbf{p}+\mathbf{k})^{2}+m_{\phi}^{2}} .
$$

Moreover the left and right projectors removed the mass term from the numerator. We have been implicit about the assignment of the masses $m_{\ell}, m_{\phi}$ to the corresponding fields, as well as about the summation over the different field components, as details of this kind are unnecessary for now.

\footnotetext{
${ }^{23} \mathrm{~A}$ classic example of this kind of a computation can be found in ref. [8.9]. It is straightforward to generalize the techniques to the 2-loop level, cf. e.g. ref. [8.10]; at that order the novelty arises that there are infrared divergences in "real" and "virtual" parts of the result, which only cancel in the sum.
} 
The essential issue in handling eq. (8.73) is the treatment of the Matsubara sum. More generally, let us inspect the structure

$$
\mathcal{F} \equiv T \sum_{\left\{p_{n}\right\}} \frac{f\left(i p_{n}, i k_{n}, \mathbf{v}\right)}{\left[p_{n}^{2}+\epsilon_{1}^{2}\right]\left[\left(p_{n}+k_{n}\right)^{2}+\epsilon_{2}^{2}\right]},
$$

where we assume that the book-keeping function $f$ depends linearly on its arguments (this assumption will become crucial below), and $\mathbf{v}$ is a dummy variable representing spatial momenta. We can write

$$
\begin{aligned}
\mathcal{F} & =T \sum_{\left\{p_{n}\right\}} T \sum_{r_{n}} \beta \delta\left(r_{n}-p_{n}-k_{n}\right) \frac{f\left(i p_{n}, i k_{n}, \mathbf{v}\right)}{\left[p_{n}^{2}+\epsilon_{1}^{2}\right]\left[r_{n}^{2}+\epsilon_{2}^{2}\right]} \\
& =\int_{0}^{\beta} \mathrm{d} \tau e^{-i k_{n} \tau}\left\{T \sum_{\left\{p_{n}\right\}} e^{-i p_{n} \tau} \frac{f\left(i p_{n}, i k_{n}, \mathbf{v}\right)}{p_{n}^{2}+\epsilon_{1}^{2}}\right\}\left\{T \sum_{r_{n}} \frac{e^{i r_{n} \tau}}{r_{n}^{2}+\epsilon_{2}^{2}}\right\},
\end{aligned}
$$

where we have used the relation

$$
\beta \delta\left(r_{n}-p_{n}-k_{n}\right)=\int_{0}^{\beta} \mathrm{d} \tau e^{i\left(r_{n}-p_{n}-k_{n}\right) \tau} .
$$

This way of handling the Matsubara sums is sometimes called the "Saclay method", cf. e.g. refs. $[8.11,8.12]$. Now we can make use of eqs. (8.30) and (8.64) and time derivatives thereof:

$$
\begin{aligned}
T \sum_{r_{n}} \frac{e^{i r_{n} \tau}}{r_{n}^{2}+\epsilon_{2}^{2}} & =\frac{n_{\mathrm{B}}\left(\epsilon_{2}\right)}{2 \epsilon_{2}}\left[e^{(\beta-\tau) \epsilon_{2}}+e^{\tau \epsilon_{2}}\right], \\
T \sum_{\left\{p_{n}\right\}} \frac{e^{ \pm i p_{n} \tau}}{p_{n}^{2}+\epsilon_{1}^{2}} & =\frac{n_{\mathrm{F}}\left(\epsilon_{1}\right)}{2 \epsilon_{1}}\left[e^{(\beta-\tau) \epsilon_{1}}-e^{\tau \epsilon_{1}}\right], \\
T \sum_{\left\{p_{n}\right\}} \frac{i p_{n} e^{-i p_{n} \tau}}{p_{n}^{2}+\epsilon_{1}^{2}} & =\frac{n_{\mathrm{F}}\left(\epsilon_{1}\right)}{2 \epsilon_{1}}\left[\epsilon_{1} e^{(\beta-\tau) \epsilon_{1}}+\epsilon_{1} e^{\tau \epsilon_{1}}\right] .
\end{aligned}
$$

Accounting for the minus sign in eq. (8.79) within the arguments of the linear function, we then get

$$
\begin{aligned}
& \mathcal{F}=\int_{0}^{\beta} \mathrm{d} \tau e^{-i k_{n} \tau} \frac{n_{\mathrm{F}}\left(\epsilon_{1}\right) n_{\mathrm{B}}\left(\epsilon_{2}\right)}{4 \epsilon_{1} \epsilon_{2}} \\
& \times\left\{\quad e^{(\beta-\tau)\left(\epsilon_{1}+\epsilon_{2}\right)} f\left(\epsilon_{1}, i k_{n}, \mathbf{v}\right)\right. \\
& +e^{(\beta-\tau) \epsilon_{2}+\tau \epsilon_{1}} f\left(\epsilon_{1},-i k_{n},-\mathbf{v}\right) \\
& +e^{(\beta-\tau) \epsilon_{1}+\tau \epsilon_{2}} f\left(\epsilon_{1}, i k_{n}, \mathbf{v}\right) \\
& \left.+e^{\tau\left(\epsilon_{1}+\epsilon_{2}\right)} f\left(\epsilon_{1},-i k_{n},-\mathbf{v}\right)\right\} \text {. }
\end{aligned}
$$

As an example, let us focus on the third structure in eq. (8.81); the other three follow in an analogous way. The $\tau$-integral can be carried out, noting that $k_{n}$ is fermionic:

$$
\begin{aligned}
\int_{0}^{\beta} \mathrm{d} \tau e^{\beta \epsilon_{1}} e^{\tau\left(-i k_{n}-\epsilon_{1}+\epsilon_{2}\right)} & =\frac{e^{\beta \epsilon_{1}}}{-i k_{n}-\epsilon_{1}+\epsilon_{2}}\left[-e^{\beta\left(\epsilon_{2}-\epsilon_{1}\right)}-1\right] \\
& =\frac{e^{\beta \epsilon_{2}}+e^{\beta \epsilon_{1}}}{i k_{n}+\epsilon_{1}-\epsilon_{2}} \\
& =\frac{1}{i k_{n}+\epsilon_{1}-\epsilon_{2}}\left[n_{\mathrm{B}}^{-1}\left(\epsilon_{2}\right)+n_{\mathrm{F}}^{-1}\left(\epsilon_{1}\right)\right] .
\end{aligned}
$$


Thus

$$
\left.\mathcal{F}\right|_{3 \mathrm{rd}}=\frac{1}{4 \epsilon_{1} \epsilon_{2}}\left[n_{\mathrm{F}}\left(\epsilon_{1}\right)+n_{\mathrm{B}}\left(\epsilon_{2}\right)\right] \frac{f\left(\epsilon_{1}, i k_{n}, \mathbf{v}\right)}{i k_{n}+\epsilon_{1}-\epsilon_{2}} .
$$

Finally we set $k_{n} \rightarrow-i\left(k^{0}+i 0^{+}\right)$and take the imaginary part according to eq. (8.58). Making use of eq. (8.20), we note that

$$
\frac{1}{2 i}\left[\frac{1}{k^{0}+\Delta+i 0^{+}}-\frac{1}{k^{0}+\Delta-i 0^{+}}\right]=-\pi \delta\left(k^{0}+\Delta\right) .
$$

Thereby $1 /\left(i k_{n}+\epsilon_{1}-\epsilon_{2}\right)$ in eq. (8.83) gets replaced with $-\pi \delta\left(k^{0}+\epsilon_{1}-\epsilon_{2}\right)$. Special attention needs to be paid to the possibility that $k_{n}$ could also appear in the numerator in eq. (8.83); however, we can then write

$$
i k_{n}=\underbrace{i k_{n}+\epsilon_{1}-\epsilon_{2}}_{\text {no discontinuity }}+\epsilon_{2}-\epsilon_{1}
$$

so that in total

$$
\begin{aligned}
\operatorname{Im} & \left.\left\{\mathcal{F}\left(i k_{n} \rightarrow k^{0}+i 0^{+}\right)\right\}\right|_{3 \mathrm{rd}}=-\frac{\pi}{4 \epsilon_{1} \epsilon_{2}}\left[n_{\mathrm{F}}\left(\epsilon_{1}\right)+n_{\mathrm{B}}\left(\epsilon_{2}\right)\right] \delta\left(k^{0}+\epsilon_{1}-\epsilon_{2}\right) f(\epsilon_{1}, \underbrace{\epsilon_{2}-\epsilon_{1}}_{k^{0}}, \mathbf{v}) \\
& =-\frac{2 \pi \delta\left(k^{0}+\epsilon_{1}-\epsilon_{2}\right)}{8 \epsilon_{1} \epsilon_{2}} f\left(\epsilon_{1}, k^{0}, \mathbf{v}\right) n_{\mathrm{F}}^{-1}\left(k^{0}\right) \frac{e^{\beta \epsilon_{1}}+e^{\beta \epsilon_{2}}}{\left[e^{\beta\left(\epsilon_{2}-\epsilon_{1}\right)}+1\right]\left(e^{\beta \epsilon_{1}}+1\right)\left(e^{\beta \epsilon_{2}}-1\right)} \\
& =-\frac{2 \pi \delta\left(k^{0}+\epsilon_{1}-\epsilon_{2}\right)}{8 \epsilon_{1} \epsilon_{2}} f\left(\epsilon_{1}, k^{0}, \mathbf{v}\right) n_{\mathrm{F}}^{-1}\left(k^{0}\right) \frac{e^{\beta \epsilon_{1}}\left[1+e^{\beta\left(\epsilon_{2}-\epsilon_{1}\right)}\right]}{\left[e^{\beta\left(\epsilon_{2}-\epsilon_{1}\right)}+1\right]\left(e^{\beta \epsilon_{1}}+1\right)\left(e^{\beta \epsilon_{2}}-1\right)} \\
& =-\frac{2 \pi \delta\left(k^{0}+\epsilon_{1}-\epsilon_{2}\right)}{8 \epsilon_{1} \epsilon_{2}} f\left(\epsilon_{1}, k^{0}, \mathbf{v}\right) n_{\mathrm{F}}^{-1}\left(k^{0}\right) n_{\mathrm{B}}\left(\epsilon_{2}\right)\left[1-n_{\mathrm{F}}\left(\epsilon_{1}\right)\right] .
\end{aligned}
$$

We have chosen to factor out $n_{\mathrm{F}}^{-1}\left(k^{0}\right)$ because in typical applications it gets cancelled against $n_{\mathrm{F}}\left(k^{0}\right)$, cf. eq. (9.158). Moreover, we remember that $\epsilon_{2}=\sqrt{m_{\phi}^{2}+(\mathbf{p}+\mathbf{k})^{2}}$, and can therefore use the trivial identity

$$
g(\mathbf{p}+\mathbf{k})=\int_{\mathbf{p}_{2}}(2 \pi)^{d} \delta^{(d)}\left(\mathbf{k}+\mathbf{p}-\mathbf{p}_{2}\right) g\left(\mathbf{p}_{2}\right)
$$

to write the result in a somewhat more symmetric form (see below).

Let us now return to eq. (8.73). We had there the object $i \not P$, which plays the role of the function $f$, and according to eq. (8.86) becomes

$$
i \not P=i p_{n} \gamma_{0}+i p_{j} \gamma_{j} \rightarrow \epsilon_{1} \gamma^{0}+i p_{j}\left(-i \gamma^{j}\right) \equiv \not P,
$$

where we made use of the definition of the Euclidean Dirac-matrices in eq. (4.36) (eq. (8.86) shows that any possible $i \not K$ can also be replaced by $\not K)$. Furthermore, two factors of $-1 / 2$ in eq. (8.73) and (8.86) combine into $1 / 4$. Renaming also $\mathcal{P} \rightarrow \mathcal{P}_{1}$ and inserting eq. (8.87), the spectral function finally becomes

$$
\begin{aligned}
\rho(\mathcal{K})=\frac{n_{\mathrm{F}}^{-1}\left(k^{0}\right)}{4} & \int_{\mathbf{p}_{1}, \mathbf{p}_{2}} \frac{\mathcal{P}_{1} a_{\mathrm{R}}}{4 \epsilon_{1} \epsilon_{2}} \\
& \times\left\{(2 \pi)^{D} \delta^{(D)}\left(\mathcal{P}_{1}+\mathcal{P}_{2}-\mathcal{K}\right) n_{\mathrm{F} 1} n_{\mathrm{B} 2}\right. \\
& +(2 \pi)^{D} \delta^{(D)}\left(\mathcal{P}_{1}-\mathcal{P}_{2}-\mathcal{K}\right) n_{\mathrm{F} 1}\left(1+n_{\mathrm{B} 2}\right) \\
& +(2 \pi)^{D} \delta^{(D)}\left(\mathcal{P}_{2}-\mathcal{P}_{1}-\mathcal{K}\right) n_{\mathrm{B} 2}\left(1-n_{\mathrm{F} 1}\right) \\
& \left.+(2 \pi)^{D} \delta^{(D)}\left(\mathcal{P}_{1}+\mathcal{P}_{2}+\mathcal{K}\right)\left(1-n_{\mathrm{F} 1}\right)\left(1+n_{\mathrm{B} 2}\right)\right\}, \mathbb{K}_{K}^{1}
\end{aligned}
$$


where the results of the other channels were added; $D \equiv d+1$; and we denoted $n_{\mathrm{Fi}} \equiv n_{\mathrm{F}}\left(\epsilon_{i}\right)$, $n_{\mathrm{Bi}} \equiv n_{\mathrm{B}}\left(\epsilon_{i}\right)$. The graphs in eq. (8.89) illustrate the various processes that the energy-momentum constraints correspond to, with a dashed line for $\phi$, a solid for $L$, and a dotted for $N$. One immediate implication of these constraints is that for a positive $k^{0}$, the last of the four structures in eq. (8.89) does not contribute at all. In general, depending on the particle masses, some of the other channels are also kinematically forbidden.

The physics lesson to draw from eq. (8.89) is that the spectral function, as extracted here from an analytic continuation and cut of a Euclidean correlator, represents real scatterings of on-shell particles, whose distribution functions are given by the Bose and Fermi distributions. The Bose and Fermi distributions appear in a form reminiscent of a Boltzmann equation, save for the "external" line carrying the momentum $\mathcal{K}$ which appears differently (this is discussed in more detail in sec. 9.3). If we went to the 2-loop level, then there would also be virtual corrections, with the closed loops experiencing thermal modifications weighted by $n_{\mathrm{B}}$ or $-n_{\mathrm{F}}$.

As a final remark we note that the spectral function $\rho$ has the important property that, in a $\mathrm{CP}$-symmetric situation, it is even in $\mathcal{K}$ :

$$
\rho(-\mathcal{K})=\rho(\mathcal{K})
$$

(In contrast, bosonic spectral functions are odd in $\mathcal{K}$.) Let us demonstrate this explicitly with the 2nd channel in eq. (8.89). Its energy-dependent part satisfies

$$
\begin{aligned}
n_{\mathrm{F}}^{-1}\left(k^{0}\right) \delta\left(\epsilon_{1}-\epsilon_{2}-k^{0}\right) n_{\mathrm{F} 1}\left(1+n_{\mathrm{B} 2}\right) \stackrel{\mathcal{K} \rightarrow-\mathcal{K}}{\longrightarrow} & n_{\mathrm{F}}^{-1}\left(-k^{0}\right) \delta\left(\epsilon_{1}-\epsilon_{2}+k^{0}\right) n_{\mathrm{F} 1}\left(1+n_{\mathrm{B} 2}\right) \\
& =\delta\left(\epsilon_{1}-\epsilon_{2}+k^{0}\right) \frac{\left(e^{-\beta k^{0}}+1\right) e^{\beta \epsilon_{2}}}{\left(e^{\beta \epsilon_{1}}+1\right)\left(e^{\beta \epsilon_{2}}-1\right)} \\
& =\delta\left(\epsilon_{1}-\epsilon_{2}+k^{0}\right)\left(e^{\beta k^{0}}+1\right) \frac{e^{\beta\left(\epsilon_{2}-k^{0}\right)}}{\left(e^{\beta \epsilon_{1}}+1\right)\left(e^{\beta \epsilon_{2}}-1\right)} \\
& =\delta\left(\epsilon_{1}-\epsilon_{2}+k^{0}\right) n_{\mathrm{F}}^{-1}\left(k^{0}\right) \frac{e^{\beta \epsilon_{1}}}{\left(e^{\beta \epsilon_{1}}+1\right)\left(e^{\beta \epsilon_{2}}-1\right)} \\
& =n_{\mathrm{F}}^{-1}\left(k^{0}\right) \delta\left(\epsilon_{2}-\epsilon_{1}-k^{0}\right) n_{\mathrm{B} 2}\left(1-n_{\mathrm{F} 1}\right)
\end{aligned}
$$

which is exactly the structure of the 3 rd channel. The spatial change $\mathbf{k} \rightarrow-\mathbf{k}$ only has an effect on the three-dimensional $\delta$-function, turning it into that on the 3 rd row of eq. (8.89). Similarly, it can be checked that the 4 th term goes over into the 1 st term, and vice versa.

There are a number of general remarks to make about the determination of spectral functions of the type that we have considered here; these have been deferred to the end of appendix A.

\section{Appendix A: What if the internal lines are treated non-perturbatively?}

Above we made use of tree-level propagators, but in general the propagators need to be resummed (cf. sec. 8.4), and have a more complicated appearance. It is then useful to express them as in the spectral representation of eq. (8.25). In particular, the scalar propagator can be written as

$$
\langle\tilde{\phi}(K) \tilde{\phi}(Q)\rangle_{0}=\frac{\delta(K+Q)}{k_{n}^{2}+\mathbf{k}^{2}+\Pi_{\mathrm{S}}\left(k_{n}, \mathbf{k}\right)}=\delta(K+Q) \int_{-\infty}^{\infty} \frac{\mathrm{d} k^{0}}{\pi} \frac{\rho_{\mathrm{S}}\left(k^{0}, \mathbf{k}\right)}{k^{0}-i k_{n}},
$$


whereas the fermion propagator contains two possible structures in the chirally symmetric case of a vanishing mass (more general cases have been considered in ref. [8.13]):

$$
\begin{aligned}
\langle\tilde{\psi}(K) \overline{\tilde{\psi}}(Q)\rangle_{0} & =\delta(K-Q)\left[\frac{-i k_{n} \gamma_{0}}{k_{n}^{2}+\mathbf{k}^{2}+\Pi_{\mathrm{w}}\left(k_{n}, \mathbf{k}\right)}+\frac{-i k_{j} \gamma_{j}}{k_{n}^{2}+\mathbf{k}^{2}+\Pi_{\mathrm{P}}\left(k_{n}, \mathbf{k}\right)}\right] \\
& =\delta(K-Q)\left[i k_{n} \gamma_{0} \int_{-\infty}^{\infty} \frac{\mathrm{d} k^{0}}{\pi} \frac{\rho_{\mathrm{w}}\left(k^{0}, \mathbf{k}\right)}{k^{0}-i k_{n}}+i k_{j} \gamma_{j} \int_{-\infty}^{\infty} \frac{\mathrm{d} k^{0}}{\pi} \frac{\rho_{\mathrm{P}}\left(k^{0}, \mathbf{k}\right)}{k^{0}-i k_{n}}\right] .
\end{aligned}
$$

Here minus signs have been incorporated into the definitions of the spectral functions $\rho_{\mathrm{W}}$ and $\rho_{\mathrm{P}}$ for later convenience. Let us carry out the steps from eq. (8.73) to (8.89) in this situation.

The structure in eq. (8.75) now has the form

$$
\mathcal{F}=T \sum_{\left\{p_{n}\right\}} \sum_{\mathrm{F}=\mathrm{W}, \mathrm{P}} \int_{-\infty}^{\infty} \frac{\mathrm{d} \omega_{1}}{\pi} \int_{-\infty}^{\infty} \frac{\mathrm{d} \omega_{2}}{\pi} \frac{f_{\mathrm{F}}\left(i p_{n}, i k_{n}, \mathbf{v}\right) \rho_{\mathrm{F}}\left(\omega_{1}, \mathbf{p}\right) \rho_{\mathrm{S}}\left(\omega_{2}, \mathbf{p}+\mathbf{k}\right)}{\left[\omega_{1}-i p_{n}\right]\left[\omega_{2}-i\left(p_{n}+k_{n}\right)\right]}
$$

where the book-keeping function $f_{\mathrm{F}}$ is again assumed to depend linearly on its arguments. We can write

$$
\begin{aligned}
\mathcal{F}=\sum_{\mathrm{F}=\mathrm{W}, \mathrm{P}} \int_{-\infty}^{\infty} \frac{\mathrm{d} \omega_{1} \mathrm{~d} \omega_{2}}{\pi^{2}} \rho_{\mathrm{F}}\left(\omega_{1}, \mathbf{p}\right) \rho_{\mathrm{S}}\left(\omega_{2}, \mathbf{p}+\mathbf{k}\right) \\
\quad \times T \sum_{\left\{p_{n}\right\}} T \sum_{r_{n}} \beta \delta\left(r_{n}-p_{n}-k_{n}\right) \frac{f_{\mathrm{F}}\left(i p_{n}, i k_{n}, \mathbf{v}\right)}{\left[\omega_{1}-i p_{n}\right]\left[\omega_{2}-i r_{n}\right]}
\end{aligned}
$$

Employing eqs. (8.77), (8.31) and (8.63), as well as the time derivative of the last one, ${ }^{24}$

$$
T \sum_{\left\{p_{n}\right\}} \frac{i p_{n}}{\omega_{1}-i p_{n}} e^{-i p_{n} \tau}=-\frac{\mathrm{d}}{\mathrm{d} \tau}\left[n_{\mathrm{F}}\left(\omega_{1}\right) e^{(\beta-\tau) \omega_{1}}\right]=n_{\mathrm{F}}\left(\omega_{1}\right) \omega_{1} e^{(\beta-\tau) \omega_{1}}, \quad 0<\tau<\beta,
$$

we get

$$
\begin{aligned}
\mathcal{F}= & \sum_{\mathrm{F}=\mathrm{W}, \mathrm{P}} \int_{-\infty}^{\infty} \frac{\mathrm{d} \omega_{1} \mathrm{~d} \omega_{2}}{\pi^{2}} \rho_{\mathrm{F}}\left(\omega_{1}, \mathbf{p}\right) \rho_{\mathrm{S}}\left(\omega_{2}, \mathbf{p}+\mathbf{k}\right) \\
& \times \int_{0}^{\beta} \mathrm{d} \tau e^{-i k_{n} \tau} n_{\mathrm{F}}\left(\omega_{1}\right) n_{\mathrm{B}}\left(\omega_{2}\right) f_{\mathrm{F}}\left(\omega_{1}, i k_{n}, \mathbf{v}\right) e^{(\beta-\tau) \omega_{1}+\tau \omega_{2}}
\end{aligned}
$$

The $\tau$-integral can now be carried out, noting that $k_{n}$ is fermionic:

$$
\begin{aligned}
\int_{0}^{\beta} \mathrm{d} \tau n_{\mathrm{F}}\left(\omega_{1}\right) n_{\mathrm{B}}\left(\omega_{2}\right) e^{\beta \omega_{1}} e^{\tau\left(-i k_{n}-\omega_{1}+\omega_{2}\right)} & =\frac{n_{\mathrm{F}}\left(\omega_{1}\right) n_{\mathrm{B}}\left(\omega_{2}\right) e^{\beta \omega_{1}}}{-i k_{n}-\omega_{1}+\omega_{2}}\left[-e^{\beta\left(\omega_{2}-\omega_{1}\right)}-1\right] \\
& =\frac{n_{\mathrm{F}}\left(\omega_{1}\right) n_{\mathrm{B}}\left(\omega_{2}\right)}{i k_{n}+\omega_{1}-\omega_{2}}\left[e^{\beta \omega_{2}}+e^{\beta \omega_{1}}\right] \\
& =\frac{n_{\mathrm{F}}\left(\omega_{1}\right) n_{\mathrm{B}}\left(\omega_{2}\right)}{i k_{n}+\omega_{1}-\omega_{2}}\left[n_{\mathrm{F}}^{-1}\left(\omega_{1}\right)+n_{\mathrm{B}}^{-1}\left(\omega_{2}\right)\right] \\
& =\frac{1}{i k_{n}+\omega_{1}-\omega_{2}}\left[n_{\mathrm{F}}\left(\omega_{1}\right)+n_{\mathrm{B}}\left(\omega_{2}\right)\right] .
\end{aligned}
$$

Finally we set $k_{n} \rightarrow-i\left(k^{0}+i 0^{+}\right)$and take the discontinuity. The appearance of $k_{n}$ inside $f_{\mathrm{F}}$ can be handled like in eq. (8.85). Making use of eq. (8.84), the denominator in eq. (8.98) simply

\footnotetext{
${ }^{24}$ We are somewhat sloppy here: a part of the sums leads to Dirac- $\delta$ 's (cf. eq. (8.60)), which can give a contribution to $\mathcal{F}$. That term is, however, independent of $k_{n}$ and thus drops out when taking the discontinuity.
} 
gets replaced with $(-\pi)$ times a Dirac $\delta$-function, so that in total

$$
\begin{aligned}
& \operatorname{Im}\left\{\mathcal{F}\left(i k_{n} \rightarrow k^{0}+i 0^{+}\right)\right\} \\
&=- \pi \sum_{\mathrm{F}=\mathrm{W}, \mathrm{P}} \int_{-\infty}^{\infty} \frac{\mathrm{d} \omega_{1} \mathrm{~d} \omega_{2}}{\pi^{2}} \rho_{\mathrm{F}}\left(\omega_{1}, \mathbf{p}\right) \rho_{\mathrm{S}}\left(\omega_{2}, \mathbf{p}+\mathbf{k}\right) \\
& \times\left[n_{\mathrm{F}}\left(\omega_{1}\right)+n_{\mathrm{B}}\left(\omega_{2}\right)\right] \delta\left(k^{0}+\omega_{1}-\omega_{2}\right) f_{\mathrm{F}}\left(\omega_{1}, \omega_{2}-\omega_{1}, \mathbf{v}\right) \\
&=- \frac{1}{2} \sum_{\mathrm{F}=\mathrm{W}, \mathrm{P}} \int_{-\infty}^{\infty} \frac{\mathrm{d} \omega_{1} \mathrm{~d} \omega_{2}}{\pi^{2}} \rho_{\mathrm{F}}\left(\omega_{1}, \mathbf{p}\right) \rho_{\mathrm{S}}\left(\omega_{2}, \mathbf{p}+\mathbf{k}\right) \\
& \times 2 \pi \delta\left(k^{0}+\omega_{1}-\omega_{2}\right) f_{\mathrm{F}}\left(\omega_{1}, k^{0}, \mathbf{v}\right) n_{\mathrm{F}}^{-1}\left(k^{0}\right) n_{\mathrm{B}}\left(\omega_{2}\right)\left[1-n_{\mathrm{F}}\left(\omega_{1}\right)\right],
\end{aligned}
$$

where we parallelled the steps in eq. (8.86). Finally, making use of eq. (8.87) and defining $\mathcal{P}_{1} \equiv$ $\left(\omega_{1}, \mathbf{p}\right) \equiv\left(\omega_{1}, \mathbf{p}_{1}\right), \mathcal{P}_{2} \equiv\left(\omega_{2}, \mathbf{p}_{2}\right)$, the spectral function corresponding to eq. (8.89) becomes

$$
\begin{aligned}
\rho(\mathcal{K}) & =-n_{\mathrm{F}}^{-1}\left(k^{0}\right) \int_{\mathcal{P}_{1}} \int_{\mathcal{P}_{2}}\left[\omega_{1} \gamma^{0} \rho_{\mathrm{W}}\left(\mathcal{P}_{1}\right)+\not p_{1} \rho_{\mathrm{P}}\left(\mathcal{P}_{1}\right)\right] a_{\mathrm{R}} \rho_{\mathrm{S}}\left(\mathcal{P}_{2}\right) \\
& \times\left\{(2 \pi)^{D} \delta^{(D)}\left(\mathcal{P}_{2}-\mathcal{P}_{1}-\mathcal{K}\right) n_{\mathrm{B} 2}\left(1-n_{\mathrm{F} 1}\right) 2-\mathcal{K}_{K}^{1}\right\},
\end{aligned}
$$

where $\not p_{1} \equiv p_{1 j} \gamma^{j}, n_{\mathrm{Fi}} \equiv n_{\mathrm{F}}\left(\omega_{i}\right)$ and $n_{\mathrm{Bi}} \equiv n_{\mathrm{B}}\left(\omega_{i}\right)$. If we insert here the free spectral shape from eq. (8.36), recalling the extra minus sign that was incorporated into $\rho_{\mathrm{W}}$ and $\rho_{\mathrm{P}}$ in eq. (8.93), then it can be shown that this result goes over into eq. (8.89), with the four channels originating from the on-shell points $\omega_{i}= \pm \epsilon_{i}, i=1,2$.

A few concluding remarks are in order:

- Expressions such as eq. (8.100) are useful particularly if the scalar and fermion propagators are Hard Thermal Loop (HTL) resummed, cf. sec. 8.4. In that case $\rho_{\mathrm{w}}$ and $\rho_{\mathrm{P}}$ are given by eq. (8.201).

- HTL resummed spectral functions contain in general two types of contributions. First of all, there are "pole contributions", represented by Dirac $\delta$-functions. In these contributions the pole locations are shifted from the free vacuum spectral functions by thermal mass corrections. Consequently, kinematic channels which would be forbidden in vacuum (such as a $1 \rightarrow 2$ decay between three massless particles) may open up.

- The second type of HTL corrections originates from a "cut contribution". An HTL resummed fermion or gauge field spectral function $\rho(\omega, k)$ has a non-zero continuous part in the spacelike domain $k>|\omega|$. Physically, this originates from real $2 \leftrightarrow 1$ scatterings experienced by such off-shell fields. Inserted into eq. (8.100) this turns the full process into a real $2 \rightarrow 2$ scattering, which tends to play an important role for the physics of nearly massless particles, because $2 \rightarrow 2$ processes are not kinematically suppressed even in the massless limit.

A classic example of an HTL computation in which both "pole" and "cut" contributions play a role can be found in ref. [8.14]. Further processes, contributing at the same order even though not accounted for just by using HTL spectral functions, have been discussed in ref. [8.15]. A complete leading-order computation of the observable considered in the present section, related to right-handed fermions interacting with the Standard Model particles through Yukawa interactions, is presented in refs. [8.16,8.17], and a similar analysis for the production rate of photons from a QCD plasma can be found in refs. [8.18, 8.19]. We return to some of these issues in sec. 9.3. 


\subsection{Real-time formalism}

In the previous section, we considered a particular spectral function, obtained from the Euclidean correlator in eq. (8.72) through the basic relation in eq. (8.58). The question may be posed, however, whether it really is necessary to go through Euclidean considerations at all. It turns out that, within perturbation theory, the answer is negative: in the so-called real-time formalism, realtime observables can be directly expressed as Feynman diagrams containing real-time propagators. The price to pay for this simplification is that the field content of the theory gets effectively "doubled" and, in a general situation, every propagator turns into a $2 \times 2$ matrix, and every vertex splits into multiple vertices.

A full-fledged formulation of the real-time formalism proceeds through the Schwinger-Keldysh or closed time-path framework; reviews can be found in refs. [8.20,8.21]. A frequently appearing concept is that of Kadanoff-Baym equations, which are analogues of Schwinger-Dyson equations within this formalism. In the following, we only provide a short motivation for the field doubling, and then demonstrate how the result of eq. (8.89) can be obtained directly within the real-time formalism.

\section{Basic definitions}

One advantage of the real-time formalism is that it also applies to systems out of equilibrium. In quantum statistical mechanics a general out-of-equilibrium situation is described by a density matrix, denoted by $\hat{\rho}(t)$. The density matrix is assumed normalized such that $\operatorname{Tr}(\hat{\rho})=1$, and statistical expectation values are defined as

$$
\left\langle\hat{O}\left(t_{1}, \mathbf{x}_{1}\right) \hat{O}\left(t_{2}, \mathbf{x}_{2}\right) \ldots\right\rangle \equiv \operatorname{Tr}\left[\hat{\rho}(t) \hat{O}\left(t_{1}, \mathbf{x}_{1}\right) \hat{O}\left(t_{2}, \mathbf{x}_{2}\right) \ldots\right]
$$

where $\hat{O}$ is a Heisenberg operator defined like in eq. (8.1). The same 2-point functions as in sec. 8.1 can be considered in this general ensemble, and some of the operator relations also continue to hold, such as $\Pi^{T}=-i \Pi^{R}+\Pi^{<}=-i \Pi^{A}+\Pi^{>}$.

An important difference between the out-of-equilibrium and equilibrium cases is that in the former situation the considerations leading to the KMS relation, cf. eqs. (8.11) and (8.12) for the bosonic case, no longer go through. However, we can still work out the trace in eq. (8.101) in a given basis and learn something from the outcome.

Consider the same Wightman function $\Pi^{>}$as in eq. (8.11). With a view of obtaining a perturbative expansion, we now choose as the basis not energy eigenstates, but rather eigenstates of elementary field operators; for the moment we denote these by $\left|\alpha_{i}\right\rangle$. Simplifying also the operator notation somewhat from that in sec. 8.1, we can write

$$
\begin{aligned}
\Pi^{>}(t) & \equiv \operatorname{Tr}\left[\hat{\rho}(t) e^{i \hat{H} t} \hat{O}(0) e^{-i \hat{H} t} \hat{O}(0)\right] \\
& =\int \Pi_{i=1}^{5} \mathrm{~d} \alpha_{i}\left\langle\alpha_{1}|\hat{\rho}(t)| \alpha_{2}\right\rangle\left\langle\alpha_{2}\left|e^{i \hat{H} t}\right| \alpha_{3}\right\rangle\left\langle\alpha_{3}|\hat{O}(0)| \alpha_{4}\right\rangle\left\langle\alpha_{4}\left|e^{-i \hat{H} t}\right| \alpha_{5}\right\rangle\left\langle\alpha_{5}|\hat{O}(0)| \alpha_{1}\right\rangle
\end{aligned}
$$

If the operators $\hat{O}$ contain only the field operators $\hat{\alpha}$ and no conjugate momenta, then we can

directly write $\left\langle\alpha_{i}|\hat{O}[\hat{\alpha}]| \alpha_{j}\right\rangle=O\left[\alpha_{j}\right] \delta_{\alpha_{i}, \alpha_{j}}$. For the time evolution, we insert the usual Feynman 
path integral,

$$
\left\langle\alpha_{4}\left|e^{-i \hat{H} t}\right| \alpha_{5}\right\rangle=\int_{\alpha(0)=\alpha_{5}}^{\alpha(t)=\alpha_{4}} \mathcal{D} \alpha e^{i \mathcal{S}_{M}},
$$

while the "backward" time evolution $\left\langle\alpha_{2}\left|e^{i \hat{H} t}\right| \alpha_{3}\right\rangle$ is obtained from the Hermitian (complex) conjugate of this relation. Denoting the "forward-propagating" field interpolating between $\alpha_{5}$ and $\alpha_{4}$ now by $\phi_{1}$, and that interpolating between $\alpha_{3}$ and $\alpha_{2}$ by $\phi_{2}$, we thereby get

$$
\Pi^{>}(t)=\int \mathcal{D} \phi_{1} \mathcal{D} \phi_{2} O\left[\phi_{2}(t)\right] O\left[\phi_{1}(0)\right] e^{i \mathcal{S}_{M}\left[\phi_{1}\right]-i \mathcal{S}_{M}\left[\phi_{2}\right]}\left\langle\phi_{1}(0)|\hat{\rho}(t)| \phi_{2}(0)\right\rangle .
$$

Note that $\phi_{1}(t)=\phi_{2}(t)=\alpha_{3}=\alpha_{4}$ in this example because $t$ is the largest time value appearing; however $\phi_{2}(0) \neq \phi_{1}(0)$ and both are integrated over. It is helpful to use $\phi_{2}(t)$ rather than $\phi_{1}(t)$ inside $O\left[\phi_{2}(t)\right]$ in eq. (8.104), because this makes it explicit that $O\left[\phi_{2}(t)\right]$ stands to the left of the operator $O\left[\phi_{1}(0)\right]$, as is indeed implied by the definition of the Wightman function $\Pi^{>}(t)$. One should think of the field $\phi_{1}$ as corresponding to the operators positioned on the right and with time arguments increasing to the left, followed by $\phi_{2}$ for the operators positioned on the left.

A similar computation for the other Wightman function yields

$$
\Pi<(t)=\int \mathcal{D} \phi_{1} \mathcal{D} \phi_{2} O\left[\phi_{2}(0)\right] O\left[\phi_{1}(t)\right] e^{i \mathcal{S}_{M}\left[\phi_{1}\right]-i \mathcal{S}_{M}\left[\phi_{2}\right]}\left\langle\phi_{1}(0)|\hat{\rho}(t)| \phi_{2}(0)\right\rangle .
$$

This time we have indicated the field with the largest time argument by $\phi_{1}(t)$ rather than $\phi_{2}(t)$, because the corresponding operator stands to the utmost right, i.e. closest to the origin of time flow. Note that within eq. (8.105), $O\left[\phi_{2}(0)\right]$ and $O\left[\phi_{1}(t)\right]$ are just complex numbers and ordering plays no role (in the bosonic case), so we could also write $\Pi^{<}(t)=\left\langle O\left[\phi_{1}(t)\right] O\left[\phi_{2}(0)\right]\right\rangle$. Here $\langle\ldots\rangle$ refers to an expectation value in the sense of the Schwinger-Keldysh functional integral,

$$
\langle\ldots\rangle \equiv \int \mathcal{D} \phi_{1} \mathcal{D} \phi_{2}(\ldots) e^{i \mathcal{S}_{M}\left[\phi_{1}\right]-i \mathcal{S}_{M}\left[\phi_{2}\right]}\left\langle\phi_{1}(0)|\hat{\rho}(t)| \phi_{2}(0)\right\rangle .
$$

If $\hat{\rho}$ happens to be a time-independent thermal density matrix, $\hat{\rho}=e^{-\beta \hat{H}} / \mathcal{Z}$, then the remaining expectation value $\left\langle\phi_{1}(0)|\hat{\rho}(t)| \phi_{2}(0)\right\rangle$ can be represented as an imaginary-time path integral as was discussed for a scalar field in sec. 2.1. For many formal considerations it is however not necessary to write down this part explicitly.

The lesson to be drawn from eqs. (8.104) and (8.105) is that the two Wightman functions $\Pi^{>}$ and $\Pi^{<}$are independent objects if $\hat{\rho}$ is non-thermal, and that representing them as path integrals necessitates a doubling of the field content of the theory $\left(\phi \rightarrow\left\{\phi_{1}, \phi_{2}\right\}\right)$.

If we specialize to the case in which the operators in eqs. (8.104) and (8.105) are directly elementary fields, rather than composite operators, then it is conventional to assemble these propagators into a $2 \times 2$ matrix. If we add a time-ordered structure,

$$
\begin{aligned}
& \theta\left(t_{2}-t_{1}\right) \hat{\phi}\left(t_{2}\right) \hat{\phi}\left(t_{1}\right)+\theta\left(t_{1}-t_{2}\right) \hat{\phi}\left(t_{1}\right) \hat{\phi}\left(t_{2}\right) \\
& =\theta\left(t_{2}-t_{1}\right) e^{i \hat{H} t_{2}} \hat{\phi}(0) e^{-i \hat{H}\left(t_{2}-t_{1}\right)} \hat{\phi}(0) e^{-i \hat{H} t_{1}} \\
& +\theta\left(t_{1}-t_{2}\right) e^{i \hat{H} t_{1}} \hat{\phi}(0) e^{-i \hat{H}\left(t_{1}-t_{2}\right)} \hat{\phi}(0) e^{-i \hat{H} t_{2}}
\end{aligned}
$$

it corresponds to time evolution along the forward-propagating branch, denoted above by the field $\phi_{1}$. Similarly, an anti-time-ordered propagator can be represented in terms of the $\phi_{2}$-field. The general propagator is then

$$
\left(\begin{array}{cc}
\left\langle\phi_{1}(t) \phi_{1}(0)\right\rangle & \left\langle\phi_{1}(t) \phi_{2}(0)\right\rangle \\
\left\langle\phi_{2}(t) \phi_{1}(0)\right\rangle & \left\langle\phi_{2}(t) \phi_{2}(0)\right\rangle
\end{array}\right)=\left(\begin{array}{cc}
\Pi_{\phi}^{T}(t) & \Pi_{\phi}^{<}(t) \\
\Pi_{\phi}^{>}(t) & \Pi_{\phi}^{T}(t)
\end{array}\right),
$$


where $\bar{T}$ denotes anti-time-ordering. The action $\mathcal{S}_{M}\left[\phi_{1}\right]-\mathcal{S}_{M}\left[\phi_{2}\right]$ contains vertices for both types of fields, and the non-diagonal matrix structure of eq. (8.108) implies that when interactions are included, both types of vertices contribute to a given observable.

In the literature, the field basis introduced above is referred to as the $1 / 2$-basis. There is another possible choice, referred to as the $r / a$-basis, which is beneficial for many practical computations. It is obtained by the linear transformation

$$
\phi_{r} \equiv \frac{1}{2}\left(\phi_{1}+\phi_{2}\right), \quad \phi_{a} \equiv \phi_{1}-\phi_{2} .
$$

Consequently, inserting the $1 / 2$ propagators from eq. (8.108), we get

$$
\begin{aligned}
& \left\langle\phi_{r}(t) \phi_{r}(0)\right\rangle=\frac{1}{4}\left(\Pi_{\phi}^{T}+\Pi_{\phi}^{T}+\Pi_{\phi}^{>}+\Pi_{\phi}^{<}\right)=\frac{1}{2}\left(\Pi_{\phi}^{>}+\Pi_{\phi}^{<}\right)=\Delta_{\phi}(t), \\
& \left\langle\phi_{r}(t) \phi_{a}(0)\right\rangle=\frac{1}{2}\left(\Pi_{\phi}^{T}-\Pi_{\phi}^{T}+\Pi_{\phi}^{>}-\Pi_{\phi}^{<}\right)=\theta(t)\left(\Pi_{\phi}^{>}-\Pi_{\phi}^{<}\right)=-i \Pi_{\phi}^{R}(t),
\end{aligned}
$$

and similarly $\left\langle\phi_{a}(t) \phi_{r}(0)\right\rangle=-i \Pi_{\phi}^{A}(t)$ and $\left\langle\phi_{a}(t) \phi_{a}(0)\right\rangle=0$.

Among the advantages of the $r / a$-basis are that the $a a$ element vanishes, and that closed loops containing only the advanced $\left\langle\phi_{a}(t) \phi_{r}(0)\right\rangle$ or the retarded $\left\langle\phi_{r}(t) \phi_{a}(0)\right\rangle$ also vanish. In addition, the statistical function $\Delta_{\phi}$, containing the Bose distribution in the bosonic case (cf. eq. (8.16)), is the only element surviving in the classical limit (because it is not proportional to a commutator), and may thus dominate the dynamics if we consider a soft regime $\epsilon \ll T$ such as in the situation described in sec. 6.1 (cf. ref. [8.22] for a detailed discussion).

Let us conclude by remarking that at higher orders of perturbation theory, the real-time formalism quickly becomes technically rather complicated, and for a long time only leading-order results existed. The past few years have, however, witnessed significant progress in the field, which is related in particular to the handling of soft contributions in the computations, as alluded to above. Examples of next-to-leading order computations can be found in refs. [8.23]-[8.25].

\section{Practical illustration}

In order to illustrate how the real-time formalism works, let us return to the 1-loop spectral function of the operator coupling to a right-handed fermion in the Standard Model, discussed in sec. 8.2. Concretely, we wish to obtain the spectral function corresponding to eq. (8.73). In order to do this in the $r / a$-basis, let us first go back to the Lagrangian in eq. (8.70), expressed with the field components of eq. (8.71). We need to write the interaction in the form $\mathcal{S}_{M}\left[\phi_{1}\right]-\mathcal{S}_{M}\left[\phi_{2}\right]$ that appears in the exponential in eq. (8.106), so we insert $\phi_{1}=\phi_{r}+\phi_{a} / 2, \phi_{2}=\phi_{r}-\phi_{a} / 2$ from eq. (8.109), and similarly for the fermions. For the two structures in eq. (8.70) this yields

$$
\begin{aligned}
& \bar{\ell}_{1} \phi_{1} a_{\mathrm{R}} N_{1}-\bar{\ell}_{2} \phi_{2} a_{\mathrm{R}} N_{2}=\left(\bar{\ell}_{r} \phi_{a}+\bar{\ell}_{a} \phi_{r}\right) a_{\mathrm{R}} N_{r}+\left(\bar{\ell}_{r} \phi_{r}+\frac{\bar{\ell}_{a} \phi_{a}}{4}\right) a_{\mathrm{R}} N_{a}, \\
& \bar{N}_{1} \phi_{1} a_{\mathrm{L}} \ell_{1}-\bar{N}_{2} \phi_{2} a_{\mathrm{L}} \ell_{2}=\bar{N}_{r} a_{\mathrm{L}}\left(\phi_{a} \ell_{r}+\phi_{r} \ell_{a}\right)+\bar{N}_{a} a_{\mathrm{L}}\left(\phi_{r} \ell_{r}+\frac{\phi_{a} \ell_{a}}{4}\right) .
\end{aligned}
$$

The retarded correlator is obtained by considering the operators coupling to $\bar{N}_{a}$ and $N_{r}$. We observe that the part $\phi_{a} \ell_{a} / 4$ plays no role, because the propagators $\left\langle\phi_{a} \phi_{a}\right\rangle$ and $\left\langle\ell_{a} \bar{\ell}_{a}\right\rangle$ vanish. The part $\phi_{r} \ell_{r}$ can be contracted with both operators coupling to $a_{\mathrm{R}} N_{r}$ in eq. (8.112), so eq. (8.73) is 
replaced by

$$
-i \Pi^{R}(\mathcal{K})=\frac{1}{2} \int_{\mathcal{X}} e^{i \mathcal{K} \cdot \mathcal{X}} a_{\mathrm{L}}\left\{\left\langle\ell_{r}(\mathcal{X}) \bar{\ell}_{r}(0)\right\rangle\left\langle\phi_{r}(\mathcal{X}) \phi_{a}(0)\right\rangle+\left\langle\ell_{r}(\mathcal{X}) \bar{\ell}_{a}(0)\right\rangle\left\langle\phi_{r}(\mathcal{X}) \phi_{r}(0)\right\rangle\right\} a_{\mathrm{R}} .
$$

Going to momentum space; inserting propagators from eqs. (8.110) and (8.111); noting that $\Delta$ is real; and taking the imaginary part, we obtain

$$
\begin{aligned}
\operatorname{Im} \Pi^{R}(\mathcal{K}) & =\frac{1}{2} \int_{\mathcal{K}}\left\{\Delta_{\ell}(\mathcal{P}) \operatorname{Im} \Pi_{\phi}^{R}(\mathcal{K}-\mathcal{P})+\operatorname{Im} \Pi_{\ell}^{R}(\mathcal{P}) \Delta_{\phi}(\mathcal{K}-\mathcal{P})\right\} \\
& =\frac{1}{2} \int_{\mathcal{P}_{1}, \mathcal{P}_{2}}(2 \pi)^{D} \delta^{(D)}\left(\mathcal{P}_{1}+\mathcal{P}_{2}-K\right)\left\{\Delta_{\ell}\left(\mathcal{P}_{1}\right) \rho_{\phi}\left(\mathcal{P}_{2}\right)+\rho_{\ell}\left(\mathcal{P}_{1}\right) \Delta_{\phi}\left(\mathcal{P}_{2}\right)\right\}
\end{aligned}
$$

Here we have introduced a second momentum variable by inserting the relation

$$
1=\int_{\mathcal{P}_{2}}(2 \pi)^{D} \delta^{(D)}\left(\mathcal{P}_{1}+\mathcal{P}_{2}-\mathcal{K}\right)
$$

into the integral, and identified $\operatorname{Im} \Pi^{R}=\rho$. For simplicity we have also hid the chiral projectors into the definition of the lepton propagator.

According to eq. (8.15) and the line below eq. (8.54) we can write (with $\left.\mathcal{P}_{i} \equiv\left(\omega_{i}, \mathbf{p}_{i}\right)\right)$

$$
\Delta_{\phi}\left(\mathcal{P}_{2}\right)=\left[1+2 n_{\mathrm{B}}\left(\omega_{1}\right)\right] \rho_{\phi}\left(\mathcal{P}_{2}\right), \quad \Delta_{\ell}\left(\mathcal{P}_{1}\right)=\left[1-2 n_{\mathrm{F}}\left(\omega_{1}\right)\right] \rho_{\ell}\left(\mathcal{P}_{1}\right) .
$$

Combining the two terms, eq. (8.115) thereby becomes

$$
\rho(\mathcal{K})=\int_{\mathcal{P}_{1}, \mathcal{P}_{2}}(2 \pi)^{D} \delta^{(D)}\left(\mathcal{P}_{1}+\mathcal{P}_{2}-\mathcal{K}\right)\left[1-n_{\mathrm{F}}\left(\omega_{1}\right)+n_{\mathrm{B}}\left(\omega_{2}\right)\right] \rho_{\ell}\left(\mathcal{P}_{1}\right) \rho_{\phi}\left(\mathcal{P}_{2}\right) .
$$

In order to make eq. (8.118) more explicit, we insert the free spectral functions (cf. eqs. (8.36) and (8.68)),

$$
\begin{aligned}
\rho_{\phi}\left(\omega_{2}, \mathbf{p}_{2}\right) & \equiv \frac{\pi}{2 \epsilon_{2}}\left[\delta\left(\omega_{2}-\epsilon_{2}\right)-\delta\left(\omega_{2}+\epsilon_{2}\right)\right], \\
\rho_{\ell}\left(\omega_{1}, \mathbf{p}_{1}\right) & \equiv \frac{\pi}{2 \epsilon_{1}} a_{\mathrm{L}} \not_{1} a_{\mathrm{R}}\left[\delta\left(\omega_{1}-\epsilon_{1}\right)-\delta\left(\omega_{1}+\epsilon_{1}\right)\right],
\end{aligned}
$$

where $\epsilon_{1}$ and $\epsilon_{2}$ are defined in accordance with eq. (8.74) (but with spatial momenta adjusted as appropriate). Further re-organizing the phase space distributions in analogy with eq. (8.86),

$$
\delta\left(\omega_{1}+\omega_{2}-k^{0}\right)\left[1-n_{\mathrm{F}}\left(\omega_{1}\right)+n_{\mathrm{B}}\left(\omega_{2}\right)\right]=\delta\left(\omega_{1}+\omega_{2}-k^{0}\right) n_{\mathrm{F}}^{-1}\left(k^{0}\right) n_{\mathrm{F}}\left(\omega_{1}\right) n_{\mathrm{B}}\left(\omega_{2}\right),
$$

we arrive at the result

$$
\begin{aligned}
\rho(\mathcal{K}) & =n_{\mathrm{F}}^{-1}\left(k^{0}\right) \int_{-\infty}^{\infty} \frac{\mathrm{d} \omega_{1}}{2 \pi} \int_{-\infty}^{\infty} \frac{\mathrm{d} \omega_{2}}{2 \pi} \int_{\mathbf{p}_{1}, \mathbf{p}_{2}}(2 \pi)^{D} \delta^{(D)}\left(\mathcal{P}_{1}+\mathcal{P}_{2}-\mathcal{K}\right) n_{\mathrm{F}}\left(\omega_{1}\right) n_{\mathrm{B}}\left(\omega_{2}\right) \\
& \times \frac{\pi^{2}}{4 \epsilon_{1} \epsilon_{2}} \mathbb{P}_{1} a_{\mathrm{R}}\left[\delta\left(\omega_{1}-\epsilon_{1}\right)-\delta\left(\omega_{1}+\epsilon_{1}\right)\right]\left[\delta\left(\omega_{2}-\epsilon_{2}\right)-\delta\left(\omega_{2}+\epsilon_{2}\right)\right] .
\end{aligned}
$$

If we now integrate over $\omega_{1}$ and $\omega_{2}$, re-adjust the notation so that $\mathcal{P}_{i} \equiv\left(\epsilon_{i}, \mathbf{p}_{i}\right)$, and in addition make the substitution $\mathbf{p}_{i} \rightarrow-\mathbf{p}_{i}$ where necessary, we obtain

$$
\rho(\mathcal{K})=\frac{n_{\mathrm{F}}^{-1}\left(k^{0}\right)}{4} \int_{\mathbf{p}_{1}, \mathbf{p}_{\mathbf{2}}} \frac{\not{P}_{1} a_{\mathrm{R}}}{4 \epsilon_{1} \epsilon_{2}}
$$




$$
\begin{aligned}
& \times\left\{(2 \pi)^{D} \delta^{(D)}\left(\mathcal{P}_{1}+\mathcal{P}_{2}-\mathcal{K}\right) n_{\mathrm{F}}\left(\epsilon_{1}\right) n_{\mathrm{B}}\left(\epsilon_{2}\right)\right. \\
& -(2 \pi)^{D} \delta^{(D)}\left(\mathcal{P}_{1}-\mathcal{P}_{2}-\mathcal{K}\right) n_{\mathrm{F}}\left(\epsilon_{1}\right) n_{\mathrm{B}}\left(-\epsilon_{2}\right) \\
& +(2 \pi)^{D} \delta^{(D)}\left(\mathcal{P}_{1}-\mathcal{P}_{2}+\mathcal{K}\right) n_{\mathrm{F}}\left(-\epsilon_{1}\right) n_{\mathrm{B}}\left(\epsilon_{2}\right) \\
& \left.-(2 \pi)^{D} \delta^{(D)}\left(\mathcal{P}_{1}+\mathcal{P}_{2}+\mathcal{K}\right) n_{\mathrm{F}}\left(-\epsilon_{1}\right) n_{\mathrm{B}}\left(-\epsilon_{2}\right)\right\} .
\end{aligned}
$$

This becomes identical with eq. (8.89) upon using the relations

$$
n_{\mathrm{F}}\left(-\epsilon_{1}\right)=1-n_{\mathrm{F}}\left(\epsilon_{1}\right), \quad n_{\mathrm{B}}\left(-\epsilon_{2}\right)=-1-n_{\mathrm{B}}\left(\epsilon_{2}\right) .
$$

The above example confirms our expectation that real-time quantities may indeed be determined through the real-time formalism. The imaginary-time formalism is, however, equally valid for problems in thermal equilibrium, and applicable on the non-perturbative level as well. Within perturbation theory, the main difference between the two formalisms is that in the imaginary-time case Matsubara sums need to be carried out before taking the discontinuity, but there is only one expression under evaluation (cf. eq. (8.73)), whereas in the real-time case only integrations appear like in vacuum computations, with the price that there are more terms (cf. eq. (8.114)). 


\subsection{Hard Thermal Loops}

For "static" observables, we realized in sec. 3.2 that the perturbative series suffers from infrared divergences. However, as discussed in sec. 6.1, in weakly coupled theories these divergences can only be associated with bosonic Matsubara zero modes. They can therefore be isolated by constructing an effective field theory for the bosonic Matsubara zero modes, as we did in sec. 6.2.

The situation is more complicated in the case of real-time observables discussed in the present chapter. Indeed, as eq. (8.27) shows, the dependence on all Matsubara modes is needed in order to carry out the analytic continuation leading to the spectral function, even if we were only interested in its behaviour at small frequencies $\left|k^{0}\right| \ll \pi T$. (The same holds also in the opposite direction: as the sum rule in eq. (8.26) shows, the information contained in the Matsubara zero mode is spread out to all $k^{0}$ 's in the Minkowskian formulation.) Therefore, it is non-trivial to isolate the soft/light degrees of freedom for which to write down the most general effective Lagrangian. ${ }^{25}$

Nevertheless, it turns out that the dimensionally reduced effective field theory of sec. 6.2 can to some extent be generalized to real-time observables as well. In the case of QCD, the generalization is known as the Hard Thermal Loop effective theory. The effective theory dictates what kind of resummed propagators should be used for instance in the computation of sec. 8.2 , in order to alleviate infrared problems appearing in perturbative computations. An example of a computation showing that (logarithmic) infrared divergences get cancelled this way can be found in ref. [8.26].

More precisely, Hard Thermal Loops (HTL) can operationally be defined via the following steps that refer to the computation of 2 or higher-point functions [8.27]-[8.30]:

- Consider "soft" external frequencies and momenta: $\left|k^{0}\right|,|\mathbf{k}| \sim g T$.

- Inside the loops, sum over all Matsubara frequencies $p_{n}$.

- Subsequently, integrate over "hard" spatial loop momenta, $|\mathbf{p}| \gtrsim \pi T$, Taylor-expanding the result to leading non-trivial order in $\left|k^{0}\right| /|\mathbf{p}|,|\mathbf{k}| /|\mathbf{p}|$.

The soft momenta $\left|k^{0}\right|,|\mathbf{k}|$ are the analogues of the small mass $m$ considered in sec. 6.1 , and the scale $\sim \pi T$ plays the role of the heavy mass $M$. According to eq. (6.25), the parametric error made through a given truncation might be expected to be $\sim(g / \pi)^{k}$ with some $k>0$, however as will be discussed below this is unfortunately difficult to establish in general.

In order to illustrate the procedure, let us compute the gauge field self-energy in this situation. The computation is much like that in sec. 5.3, except that now we keep the external momentum $(K)$ non-zero while carrying out the Matsubara sum, because the full dependence on $k_{n}$ is needed for the analytic continuation. It is crucial to take $k^{0}, \mathbf{k}$ soft only after the analytic continuation.

As a starting point, we take the self-energy in Feynman gauge, $\Pi_{\mu \nu}(K)$, as defined in eq. (5.69). This will be interpreted as being a part of an "effective action",

$$
S_{\text {eff }}=\oiint_{K} \frac{1}{2} \tilde{A}_{\mu}^{a}(K)\left[K^{2} \delta_{\mu \nu}-K_{\mu} K_{\nu}+\frac{1}{\xi} K_{\mu} K_{\nu}+\Pi_{\mu \nu}(K)\right] \tilde{A}_{\nu}^{a}(-K)+\ldots
$$

Summing together results from eqs. (5.74), (5.79), (5.82), (5.94) and (5.101), setting the fermion mass to zero for simplicity, and expressing the spacetime dimensionality as $D \equiv d+1$, the 1-loop

\footnotetext{
${ }^{25}$ The corresponding discussion in the real-time formalism, introduced in sec. 8.3, can be found in ref. [8.22].
} 
self-energy reads

$$
\begin{aligned}
\Pi_{\mu \nu}(K) & =\frac{g^{2} N_{\mathrm{c}}}{2} \oiint_{P} \frac{\delta_{\mu \nu}\left[-4 K^{2}+2(D-2) P^{2}\right]+(D+2) K_{\mu} K_{\nu}-4(D-2) P_{\mu} P_{\nu}}{P^{2}(K-P)^{2}} \\
& -g^{2} N_{\mathrm{f}} \oiint_{\{P\}} \frac{\delta_{\mu \nu}\left[-K^{2}+2 P^{2}\right]+2 K_{\mu} K_{\nu}-4 P_{\mu} P_{\nu}}{P^{2}(K-P)^{2}} .
\end{aligned}
$$

The bosonic part is discussed in appendix A; here we focus on the fermionic part.

Consider first the spatial components, $\Pi_{i j}$. Shifting $P \rightarrow K-P$ in one term, we can write

$$
\Pi_{i j}^{(\mathrm{f})}(K)=-g^{2} N_{\mathrm{f}} \int_{\mathbf{p}} T \sum_{\left\{p_{n}\right\}}\left[\frac{2 \delta_{i j}}{P^{2}}+\frac{-K^{2} \delta_{i j}+2 k_{i} k_{j}-4 p_{i} p_{j}}{P^{2}(K-P)^{2}}\right] .
$$

For generality we assume that, like in eq. (8.65), the Matsubara frequency is of the form ${ }^{26}$

$$
p_{n} \rightarrow \tilde{p}_{n} \equiv \omega_{n}+i \mu, \quad \omega_{n}=2 \pi T\left(n+\frac{1}{2}\right) .
$$

The Matsubara sum can now be carried out, in analogy with the procedure described in sec. 8.2. Denoting

$$
\epsilon_{1} \equiv|\mathbf{p}|, \quad \epsilon_{2} \equiv|\mathbf{p}-\mathbf{k}|
$$

we can read from eq. (8.64) that

$$
\begin{aligned}
T \sum_{\left\{\omega_{n}\right\}} \frac{1}{\left(\omega_{n}+i \mu\right)^{2}+\epsilon_{1}^{2}} & =\frac{1}{2 \epsilon_{1}}\left[n_{\mathrm{F}}\left(\epsilon_{1}-\mu\right) e^{\beta\left(\epsilon_{1}-\mu\right)}-n_{\mathrm{F}}\left(\epsilon_{1}+\mu\right)\right] \\
& =\frac{1}{2 \epsilon_{1}}\left[1-n_{\mathrm{F}}\left(\epsilon_{1}-\mu\right)-n_{\mathrm{F}}\left(\epsilon_{1}+\mu\right)\right] .
\end{aligned}
$$

It is somewhat more tedious to carry out the other sum. Proceeding in analogy with the analysis following eq. (8.75) and denoting the result by $\mathcal{G}$, we get

$$
\begin{aligned}
\mathcal{G} & =T \sum_{\left\{p_{n}\right\}} \frac{1}{\left[\tilde{p}_{n}^{2}+\epsilon_{1}^{2}\right]\left[\left(k_{n}-\tilde{p}_{n}\right)^{2}+\epsilon_{2}^{2}\right]} \\
& =T \sum_{\left\{p_{n}\right\}} T \sum_{\left\{r_{n}\right\}} \beta \delta\left(\tilde{r}_{n}+k_{n}-\tilde{p}_{n}\right) \frac{1}{\left[\tilde{p}_{n}^{2}+\epsilon_{1}^{2}\right]\left[\tilde{r}_{n}^{2}+\epsilon_{2}^{2}\right]} \\
& =\int_{0}^{\beta} \mathrm{d} \tau e^{i k_{n} \tau}\left\{T \sum_{\left\{p_{n}\right\}} \frac{e^{-i \tilde{p}_{n} \tau}}{\tilde{p}_{n}^{2}+\epsilon_{1}^{2}}\right\}\left\{T \sum_{\left\{r_{n}\right\}} \frac{e^{i \tilde{r}_{n} \tau}}{\tilde{r}_{n}^{2}+\epsilon_{2}^{2}}\right\},
\end{aligned}
$$

where we used the trick in eq. (8.77). The sums can be carried out by making use of eq. (8.64),

$$
\begin{aligned}
T \sum_{\left\{r_{n}\right\}} \frac{e^{i \tilde{r}_{n} \tau}}{\tilde{r}_{n}^{2}+\epsilon_{2}^{2}} & =\frac{1}{2 \epsilon_{2}}\left[n_{\mathrm{F}}\left(\epsilon_{2}-\mu\right) e^{(\beta-\tau) \epsilon_{2}-\beta \mu}-n_{\mathrm{F}}\left(\epsilon_{2}+\mu\right) e^{\tau \epsilon_{2}}\right], \\
T \sum_{\left\{p_{n}\right\}} \frac{e^{-i \tilde{p}_{n} \tau}}{\tilde{p}_{n}^{2}+\epsilon_{1}^{2}} & =-e^{\mu \beta} T \sum_{\left\{p_{n}\right\}} \frac{e^{i \tilde{p}_{n}(\beta-\tau)}}{\tilde{p}_{n}^{2}+\epsilon_{1}^{2}} \\
& =\frac{1}{2 \epsilon_{1}}\left[n_{\mathrm{F}}\left(\epsilon_{1}+\mu\right) e^{(\beta-\tau) \epsilon_{1}+\beta \mu}-n_{\mathrm{F}}\left(\epsilon_{1}-\mu\right) e^{\tau \epsilon_{1}}\right],
\end{aligned}
$$

\footnotetext{
${ }^{26}$ As mentioned between eqs. (5.100) and (5.101), shifts like $P \rightarrow K-P$ are delicate in the presence of chemical potentials, given that we have to keep track of the relative signs of $\omega_{n}$ and $i \mu$ in the Matsubara frequency. It turns out that there is no problem with the present example, since the final result is an even function of $\mu$. To be careful, one could employ the unshifted form, given in eq. (8.226), and verify that the results do remain the same.
} 
where in the latter equation attention needed to be paid to the fact that eq. (8.64) only applies for $0 \leq \tau \leq \beta$ and that there is a shift due to the chemical potential in $\tilde{p}_{n}$.

Inserting these expressions into eq. (8.132) and carrying out the integral over $\tau$, we get

$$
\begin{gathered}
\mathcal{G}=\int_{0}^{\beta} \mathrm{d} \tau e^{i k_{n} \tau} \frac{1}{4 \epsilon_{1} \epsilon_{2}}\left\{n_{\mathrm{F}}\left(\epsilon_{1}+\mu\right) n_{\mathrm{F}}\left(\epsilon_{2}-\mu\right) e^{(\beta-\tau)\left(\epsilon_{1}+\epsilon_{2}\right)}\right. \\
-n_{\mathrm{F}}\left(\epsilon_{1}+\mu\right) n_{\mathrm{F}}\left(\epsilon_{2}+\mu\right) e^{\tau\left(\epsilon_{2}-\epsilon_{1}\right)+\beta\left(\epsilon_{1}+\mu\right)} \\
-n_{\mathrm{F}}\left(\epsilon_{1}-\mu\right) n_{\mathrm{F}}\left(\epsilon_{2}-\mu\right) e^{\tau\left(\epsilon_{1}-\epsilon_{2}\right)+\beta\left(\epsilon_{2}-\mu\right)} \\
\left.+n_{\mathrm{F}}\left(\epsilon_{1}-\mu\right) n_{\mathrm{F}}\left(\epsilon_{2}+\mu\right) e^{\tau\left(\epsilon_{1}+\epsilon_{2}\right)}\right\} \\
=\frac{1}{4 \epsilon_{1} \epsilon_{2}}\left\{n_{\mathrm{F}}\left(\epsilon_{1}+\mu\right) n_{\mathrm{F}}\left(\epsilon_{2}-\mu\right) \frac{1}{i k_{n}-\epsilon_{1}-\epsilon_{2}}\left[1-e^{\beta\left(\epsilon_{1}+\epsilon_{2}\right)}\right]\right. \\
-n_{\mathrm{F}}\left(\epsilon_{1}+\mu\right) n_{\mathrm{F}}\left(\epsilon_{2}+\mu\right) \frac{1}{i k_{n}+\epsilon_{2}-\epsilon_{1}}\left[e^{\beta\left(\epsilon_{2}+\mu\right)}-e^{\beta\left(\epsilon_{1}+\mu\right)}\right] \\
-n_{\mathrm{F}}\left(\epsilon_{1}-\mu\right) n_{\mathrm{F}}\left(\epsilon_{2}-\mu\right) \frac{1}{i k_{n}+\epsilon_{1}-\epsilon_{2}}\left[e^{\beta\left(\epsilon_{1}-\mu\right)}-e^{\beta\left(\epsilon_{2}-\mu\right)}\right] \\
\left.+n_{\mathrm{F}}\left(\epsilon_{1}-\mu\right) n_{\mathrm{F}}\left(\epsilon_{2}+\mu\right) \frac{1}{i k_{n}+\epsilon_{1}+\epsilon_{2}}\left[e^{\beta\left(\epsilon_{1}+\epsilon_{2}\right)}-1\right]\right\} \\
\frac{1}{4 \epsilon_{1} \epsilon_{2}}\left\{\frac{1}{i k_{n}-\epsilon_{1}-\epsilon_{2}}\left[n_{\mathrm{F}}\left(\epsilon_{1}+\mu\right)+n_{\mathrm{F}}\left(\epsilon_{2}-\mu\right)-1\right]\right. \\
+\frac{1}{i k_{n}+\epsilon_{2}-\epsilon_{1}}\left[n_{\mathrm{F}}\left(\epsilon_{2}+\mu\right)-n_{\mathrm{F}}\left(\epsilon_{1}+\mu\right)\right] \\
+\frac{1}{i k_{n}+\epsilon_{1}-\epsilon_{2}}\left[n_{\mathrm{F}}\left(\epsilon_{1}-\mu\right)-n_{\mathrm{F}}\left(\epsilon_{2}-\mu\right)\right] \\
\left.+\frac{1}{i k_{n}+\epsilon_{1}+\epsilon_{2}}\left[1-n_{\mathrm{F}}\left(\epsilon_{1}-\mu\right)-n_{\mathrm{F}}\left(\epsilon_{2}+\mu\right)\right]\right\} .
\end{gathered}
$$

At this point we could carry out the analytic continuation $i k_{n} \rightarrow k^{0}+i 0^{+}$, but it will be convenient to postpone it for a moment; we just need to keep in mind that after the analytic continuation, $i k_{n}$ becomes a soft quantity.

The next step is to Taylor-expand to leading order in $k^{0}, \mathbf{k}$. To this end we can write

$$
\epsilon_{1}=p \equiv|\mathbf{p}|, \quad \epsilon_{2}=|\mathbf{p}-\mathbf{k}| \approx p-k_{i} \frac{\partial}{\partial p_{i}}|\mathbf{p}|=p-k_{i} v_{i},
$$

where

$$
v_{i} \equiv \frac{p_{i}}{p}, \quad i \in\{1,2,3\},
$$

are referred to as the velocities of the hard particles.

It has to be realized that a Taylor expansion is sensible only in terms in which there is a thermal distribution function providing an external scale $T$ and thereby guaranteeing that the integral obtains its dominant contributions from hard momenta, $p \sim \pi T$. We cannot Taylor-expand in the vacuum part, which has no scale with respect to which to expand. It can, however, be separately verified that the vacuum part vanishes as a power of $k^{0}, \mathbf{k}$, which is consistent with the fact that there is no gluon mass in vacuum. Here we simply omit the temperature-independent part. 
With these approximations, the function $\mathcal{G}$ reads

$$
\begin{aligned}
\mathcal{G} \approx \frac{1}{4 p^{2}} & \left\{\frac{1}{2 p}\left[-n_{\mathrm{F}}(p+\mu)-n_{\mathrm{F}}(p-\mu)\right]\right. \\
& +\frac{1}{i k_{n}-\mathbf{k} \cdot \mathbf{v}}(-\mathbf{k} \cdot \mathbf{v}) n_{\mathrm{F}}^{\prime}(p+\mu) \\
& +\frac{1}{i k_{n}+\mathbf{k} \cdot \mathbf{v}}(+\mathbf{k} \cdot \mathbf{v}) n_{\mathrm{F}}^{\prime}(p-\mu) \\
& \left.+\frac{1}{2 p}\left[-n_{\mathrm{F}}(p-\mu)-n_{\mathrm{F}}(p+\mu)\right]\right\}+\mathcal{O}\left(k^{0}, \mathbf{k}\right) .
\end{aligned}
$$

Now we insert eqs. (8.130) and (8.138) into eq. (8.127). Through the substitution $\mathbf{p} \rightarrow-\mathbf{p}$ (whereby $\mathbf{v} \rightarrow-\mathbf{v}$ ), the 3rd row in eq. (8.138) can be put in the same form as the 2nd row. Furthermore, terms containing $k_{n}$ or $\mathbf{k}$ in the numerator in eq. (8.127) are seen to be of higher order. Thereby

$$
\begin{aligned}
\Pi_{i j}^{(\mathrm{f})}(K) \approx-g^{2} N_{\mathrm{f}} \int_{\mathbf{p}} & \left\{\frac{\delta_{i j}}{p}\left[-n_{\mathrm{F}}(p+\mu)-n_{\mathrm{F}}(p-\mu)\right]\right. \\
& -\frac{p_{i} p_{j}}{p^{2}} \frac{1}{p}\left[-n_{\mathrm{F}}(p+\mu)-n_{\mathrm{F}}(p-\mu)\right] \\
& \left.-\frac{p_{i} p_{j}}{p^{2}} \frac{i k_{n}-\mathbf{k} \cdot \mathbf{v}-i k_{n}}{i k_{n}-\mathbf{k} \cdot \mathbf{v}}\left[n_{\mathrm{F}}^{\prime}(p+\mu)+n_{\mathrm{F}}^{\prime}(p-\mu)\right]\right\} \\
=-g^{2} N_{\mathrm{f}} \int_{\mathbf{p}} & \left\{\frac{-\delta_{i j}}{p}\left[n_{\mathrm{F}}(p+\mu)+n_{\mathrm{F}}(p-\mu)\right]\right. \\
& +\frac{v_{i} v_{j}}{p}\left[n_{\mathrm{F}}(p+\mu)+n_{\mathrm{F}}(p-\mu)\right] \\
& -v_{i} v_{j}\left[n_{\mathrm{F}}^{\prime}(p+\mu)+n_{\mathrm{F}}^{\prime}(p-\mu)\right] \\
& \left.+\frac{v_{i} v_{j} i k_{n}}{i k_{n}-\mathbf{k} \cdot \mathbf{v}}\left[n_{\mathrm{F}}^{\prime}(p+\mu)+n_{\mathrm{F}}^{\prime}(p-\mu)\right]\right\} .
\end{aligned}
$$

The remaining integration can be factorized into a radial and an angular part,

$$
\int_{\mathbf{p}}=\int_{p} \int \mathrm{d} \Omega_{v}
$$

where the angular integration goes over the directions of $\mathbf{v}=\mathbf{p} / p$, and is normalized to unity:

$$
\int \mathrm{d} \Omega_{v} \equiv 1
$$

Then, the following identities can be verified (for eqs. (8.142) and (8.144) details are given in appendix C; eq. (8.143) is a trivial consequence of rotational symmetry and $\mathbf{v}^{2}=1$ ):

$$
\begin{aligned}
\int_{p}\left[n_{\mathrm{F}}^{\prime}(p+\mu)+n_{\mathrm{F}}^{\prime}(p-\mu)\right] & =-(d-1) \int_{p} \frac{1}{p}\left[n_{\mathrm{F}}(p+\mu)+n_{\mathrm{F}}(p-\mu)\right], \\
\int \mathrm{d} \Omega_{v} v_{i} v_{j} & =\frac{\delta_{i j}}{d},
\end{aligned}
$$

and, for $d=3$,

$$
\int_{p} \frac{1}{p}\left[n_{\mathrm{F}}(p+\mu)+n_{\mathrm{F}}(p-\mu)\right] \stackrel{d=3}{=} \frac{1}{4}\left(\frac{T^{2}}{3}+\frac{\mu^{2}}{\pi^{2}}\right)
$$

The integration

$$
\int \mathrm{d} \Omega_{v} \frac{v_{i} v_{j}}{i k_{n}-\mathbf{k} \cdot \mathbf{v}}
$$


can also be carried out (cf. appendix C) but we do not need its value for the moment.

With these ingredients, eq. (8.139) becomes

$$
\begin{aligned}
\Pi_{i j}^{(\mathrm{f})}(K) & =-g^{2} N_{\mathrm{f}} \int_{p} \frac{1}{p}\left[n_{\mathrm{F}}(p+\mu)+n_{\mathrm{F}}(p-\mu)\right] \\
& \times\left\{\delta_{i j}\left(-1+\frac{1}{d}+\frac{d-1}{d}\right)-(d-1) \int \mathrm{d} \Omega_{v} \frac{v_{i} v_{j} i k_{n}}{i k_{n}-\mathbf{k} \cdot \mathbf{v}}\right\} \\
& =g^{2} N_{\mathrm{f}}(d-1) \int_{p} \frac{1}{p}\left[n_{\mathrm{F}}(p+\mu)+n_{\mathrm{F}}(p-\mu)\right] \int \mathrm{d} \Omega_{v} \frac{v_{i} v_{j} i k_{n}}{i k_{n}-\mathbf{k} \cdot \mathbf{v}} .
\end{aligned}
$$

Including also gauge fields and ghosts, the complete result reads

$$
\Pi_{i j}(K)=m_{\mathrm{E}}^{2} \int \mathrm{d} \Omega_{v} \frac{v_{i} v_{j} i k_{n}}{i k_{n}-\mathbf{k} \cdot \mathbf{v}}+\mathcal{O}\left(i k_{n}, \mathbf{k}\right),
$$

where $m_{\mathrm{E}}$ is the generalization of the Debye mass in eq. (5.107) to the case of a fermionic chemical potential,

$$
\begin{aligned}
& m_{\mathrm{E}}^{2} \equiv g^{2}(d-1) \int_{p} \frac{1}{p}\left\{N_{\mathrm{f}}\left[n_{\mathrm{F}}(p+\mu)+n_{\mathrm{F}}(p-\mu)\right]+(d-1) N_{\mathrm{c}} n_{\mathrm{B}}(p)\right\} \\
& \stackrel{d \equiv 3}{=} g^{2}\left[N_{\mathrm{f}}\left(\frac{T^{2}}{6}+\frac{\mu^{2}}{2 \pi^{2}}\right)+\frac{N_{\mathrm{c}} T^{2}}{3}\right] .
\end{aligned}
$$

Eq. (8.147), known for QED since a long time [8.31]-[8.33], is a remarkable expression. ${ }^{27}$ Even though it is of $\mathcal{O}(1)$ is we count $i k_{n}$ and $\mathbf{k}$ as quantities of the same order, it depends non-trivially on the ratio $i k_{n} /|\mathbf{k}|$. In particular, for $k^{0}=i k_{n} \rightarrow 0$, i.e. in the static limit, $\Pi_{i j}$ vanishes. This corresponds to the result in eq. (5.105), i.e. that spatial gauge field components do not develop a thermal mass at 1-loop order. On the other hand, for $0<\left|k^{0}\right|<|\mathbf{k}|$, it contains both a real and an imaginary part, cf. eqs. (8.221) and (8.225). The imaginary part is related to the physics of Landau damping: it means that spacelike gauge fields can lose energy to hard particles in the plasma through real $2 \leftrightarrow 1$ scatterings.

So far, we were only concerned with the spatial part $\Pi_{i j}$. An interesting question is to generalize the computation to the full self-energy $\Pi_{\mu \nu}$. Fortunately, it turns out that all the information needed can be extracted from eq. (8.147), as we now show.

Indeed, the self-energy $\Pi_{\mu \nu}$, obtained by integrating out the hard modes, must produce a structure which is gauge-invariant in "soft" gauge transformations, and therefore it must obey a SlavnovTaylor identity and be transverse with respect to the external four-momentum. However, the meaning of transversality changes from the case of zero temperature, because the heat bath introduces a preferred frame, and thus breaks Lorentz invariance. More precisely, we can now introduce two different projection operators,

$$
\begin{aligned}
& \mathbb{P}_{\mu \nu}^{\mathrm{T}}(K) \equiv \delta_{\mu i} \delta_{\nu j}\left(\delta_{i j}-\frac{k_{i} k_{j}}{k^{2}}\right), \\
& \mathbb{P}_{\mu \nu}^{\mathrm{E}}(K) \equiv \delta_{\mu \nu}-\frac{K_{\mu} K_{\nu}}{K^{2}}-\mathbb{P}_{\mu \nu}^{\mathrm{T}}(K),
\end{aligned}
$$

which both are four-dimensionally transverse,

$$
\mathbb{P}_{\mu \nu}^{\mathrm{T}}(K) K_{\nu}=\mathbb{P}_{\mu \nu}^{\mathrm{E}}(K) K_{\nu}=0,
$$

\footnotetext{
${ }^{27}$ Recently the derivation of $m_{\mathrm{E}}^{2}$ has been extended to NLO in QED [8.34], confirming that the result agrees with that obtained in the dimensionally reduced effective theories.
} 
and of which $\mathbb{P}_{\mu \nu}^{\mathrm{T}}(K)$ is in addition three-dimensionally transverse,

$$
\mathbb{P}_{\mu i}^{\mathrm{T}}(K) k_{i}=0 .
$$

The two projectors are also orthogonal to each other, $\mathbb{P}_{\mu \alpha}^{\mathrm{E}} \mathbb{P}_{\alpha \nu}^{\mathrm{T}}=0$.

With the above projectors, we can write

$$
\Pi_{i j}(K)=m_{\mathrm{E}}^{2} \int \mathrm{d} \Omega_{v} \frac{v_{i} v_{j} i k_{n}}{i k_{n}-\mathbf{k} \cdot \mathbf{v}} \equiv \mathbb{P}_{i j}^{\mathrm{T}}(K) \Pi_{\mathrm{T}}(K)+\mathbb{P}_{i j}^{\mathrm{E}}(K) \Pi_{\mathrm{E}}(K) .
$$

Note that this decomposition applies for $(\ldots)_{i j} \rightarrow(\ldots)_{\mu \nu}$ as well. Contracting eq. (8.154) with $\delta_{i j}$ and with $k_{i} k_{j}$ leads to the equations

$$
\begin{aligned}
m_{\mathrm{E}}^{2} i k_{n} L & =(d-1) \Pi_{\mathrm{T}}+\left(1-\frac{k^{2}}{k_{n}^{2}+k^{2}}\right) \Pi_{\mathrm{E}}, \\
m_{\mathrm{E}}^{2} \int \mathrm{d} \Omega_{v} \frac{(\mathbf{k} \cdot \mathbf{v})^{2} i k_{n}}{i k_{n}-\mathbf{k} \cdot \mathbf{v}} & =0 \Pi_{\mathrm{T}}+\left(k^{2}-\frac{\left(k^{2}\right)^{2}}{k_{n}^{2}+k^{2}}\right) \Pi_{\mathrm{E}},
\end{aligned}
$$

where

$$
L \equiv \int \mathrm{d} \Omega_{v} \frac{1}{i k_{n}-\mathbf{k} \cdot \mathbf{v}} .
$$

The integral on the left-hand side of eq. (8.156) can furthermore be written as

$$
\begin{aligned}
\int \mathrm{d} \Omega_{v} \frac{(\mathbf{k} \cdot \mathbf{v})^{2} i k_{n}}{i k_{n}-\mathbf{k} \cdot \mathbf{v}} & =\int \mathrm{d} \Omega_{v} \frac{\left(-\mathbf{k} \cdot \mathbf{v}+i k_{n}-i k_{n}\right)(-\mathbf{k} \cdot \mathbf{v}) i k_{n}}{i k_{n}-\mathbf{k} \cdot \mathbf{v}} \\
& =\left(i k_{n}\right)^{2} \int \mathrm{d} \Omega_{v} \frac{\mathbf{k} \cdot \mathbf{v}}{i k_{n}-\mathbf{k} \cdot \mathbf{v}} \\
& =\left(i k_{n}\right)^{2}\left(-1+i k_{n} L\right)
\end{aligned}
$$

where we have in the second step dropped a term that vanishes upon angular integration. Solving for $\Pi_{\mathrm{T}}, \Pi_{\mathrm{E}}$ and subsequently inserting the expression for $L$ from eq. (8.213), we thus get

$$
\begin{aligned}
\Pi_{\mathrm{T}}(K) & =\frac{m_{\mathrm{E}}^{2}}{d-1}\left\{-\frac{k_{n}^{2}}{k^{2}}+\frac{K^{2}}{k^{2}} i k_{n} L\right\} \\
& \stackrel{d=3}{=} \frac{m_{\mathrm{E}}^{2}}{2}\left\{\frac{\left(i k_{n}\right)^{2}}{k^{2}}+\frac{i k_{n}}{2 k}\left[1-\frac{\left(i k_{n}\right)^{2}}{k^{2}}\right] \ln \frac{i k_{n}+k}{i k_{n}-k}\right\}, \\
\Pi_{\mathrm{E}}(K) & =\frac{m_{\mathrm{E}}^{2} K^{2}}{k^{2}}\left(1-i k_{n} L\right) \\
& \stackrel{d=3}{=} m_{\mathrm{E}}^{2}\left[1-\frac{\left(i k_{n}\right)^{2}}{k^{2}}\right]\left[1-\frac{i k_{n}}{2 k} \ln \frac{i k_{n}+k}{i k_{n}-k}\right] .
\end{aligned}
$$

Eqs. (8.160) and (8.162) have a number of interesting limiting values. For $i k_{n} \rightarrow 0$ but with $k \neq 0, \Pi_{\mathrm{T}} \rightarrow 0, \Pi_{\mathrm{E}} \rightarrow m_{\mathrm{E}}^{2}$. This corresponds to the physics of Debye screening, familiar to us from eq. (5.106). On the contrary, if we consider homogeneous but time-dependent waves, i.e. take $k \rightarrow 0$ with $i k_{n} \neq 0$, it can be seen that $\Pi_{\mathrm{T}}, \Pi_{\mathrm{E}} \rightarrow m_{\mathrm{E}}^{2} / 3$. This genuinely Minkowskian structure in the resummed self-energy corresponds to plasma oscillations, or plasmons.

We can also write down a resummed propagator: in a general covariant gauge, where the treelevel propagator has the form in eq. (5.45) and the static Feynman gauge propagator the form in eq. (5.106), we get

$$
\left\langle A_{\mu}^{a}(X) A_{\nu}^{b}(Y)\right\rangle_{0}=\delta^{a b} \oint_{K} e^{i K \cdot(X-Y)}\left[\frac{\mathbb{P}_{\mu \nu}^{\mathrm{T}}(K)}{K^{2}+\Pi_{\mathrm{T}}(K)}+\frac{\mathbb{P}_{\mu \nu}^{\mathrm{E}}(K)}{K^{2}+\Pi_{\mathrm{E}}(K)}+\frac{\xi K_{\mu} K_{\nu}}{\left(K^{2}\right)^{2}}\right],
$$


where $\xi$ is the gauge parameter.

If the propagator of eq. (8.163) is used in practical applications, it is often useful to express it in terms of the spectral representation, cf. eq. (8.25). The spectral function appearing in the spectral representation can be obtained from eq. (8.28), where now $1 /\left[K^{2}+\Pi_{\mathrm{T}(\mathrm{E})}(K)\right]$ plays the role of $\Pi_{\alpha \beta}^{E}$. After analytic continuation, $i k_{n} \rightarrow k^{0}+i 0^{+}$,

$$
\frac{1}{K^{2}+\Pi_{\mathrm{T}(\mathrm{E})}\left(k_{n}, \mathbf{k}\right)} \rightarrow \frac{1}{-\left(k^{0}+i 0^{+}\right)^{2}+\mathbf{k}^{2}+\Pi_{\mathrm{T}(\mathrm{E})}\left(-i\left(k^{0}+i 0^{+}\right), \mathbf{k}\right)},
$$

where

$$
\begin{aligned}
& \Pi_{\mathrm{T}}\left(-i\left(k^{0}+i 0^{+}\right), \mathbf{k}\right)=\frac{m_{\mathrm{E}}^{2}}{2}\left\{\frac{\left(k^{0}\right)^{2}}{k^{2}}+\frac{k^{0}}{2 k}\left[1-\frac{\left(k^{0}\right)^{2}}{k^{2}}\right] \ln \frac{k^{0}+k+i 0^{+}}{k^{0}-k+i 0^{+}}\right\}, \\
& \Pi_{\mathrm{E}}\left(-i\left(k^{0}+i 0^{+}\right), \mathbf{k}\right)=m_{\mathrm{E}}^{2}\left[1-\frac{\left(k^{0}\right)^{2}}{k^{2}}\right]\left[1-\frac{k^{0}}{2 k} \ln \frac{k^{0}+k+i 0^{+}}{k^{0}-k+i 0^{+}}\right] .
\end{aligned}
$$

For $\left|k^{0}\right|>k, \Pi_{\mathrm{T}}, \Pi_{\mathrm{E}}$ are real, whereas for $\left|k^{0}\right|<k$, they have an imaginary part. Denoting $\eta \equiv \frac{k^{0}}{k}$, a straightforward computation (utilizing the fact that $\ln z$ has a branch cut on the negative real axis) leads to the spectral functions $\rho_{\mathrm{T}(\mathrm{E})} \equiv \operatorname{Im}\left(\frac{1}{K^{2}+\Pi_{\mathrm{T}(\mathrm{E})}}\right)_{i k_{n} \rightarrow k^{0}+i 0^{+}}$, where

$$
\begin{aligned}
\rho_{\mathrm{T}}(\mathcal{K}) & = \begin{cases}\frac{\Gamma_{\mathrm{T}}(\eta)}{\Sigma_{\mathrm{T}}^{2}(\mathcal{K})+\Gamma_{\mathrm{T}}^{2}(\eta)}, & |\eta|<1, \\
\pi \operatorname{sign}(\eta) \delta\left(\Sigma_{\mathrm{T}}(\mathcal{K})\right), & |\eta|>1,\end{cases} \\
\left(\eta^{2}-1\right) \rho_{\mathrm{E}}(\mathcal{K}) & = \begin{cases}\frac{\Gamma_{\mathrm{E}}(\eta)}{\Sigma_{\mathrm{E}}^{2}(\mathcal{K})+\Gamma_{\mathrm{E}}^{2}(\eta)}, & |\eta|<1, \\
\pi \operatorname{sign}(\eta) \delta\left(\Sigma_{\mathrm{E}}(\mathcal{K})\right), & |\eta|>1 .\end{cases}
\end{aligned}
$$

Here we have introduced the well-known functions [8.31]-[8.33]

$$
\begin{aligned}
\Sigma_{\mathrm{T}}(\mathcal{K}) & \equiv-\mathcal{K}^{2}+\frac{m_{\mathrm{E}}^{2}}{2}\left[\eta^{2}+\frac{\eta\left(1-\eta^{2}\right)}{2} \ln \left|\frac{1+\eta}{1-\eta}\right|\right], \\
\Gamma_{\mathrm{T}}(\eta) & \equiv \frac{\pi m_{\mathrm{E}}^{2} \eta\left(1-\eta^{2}\right)}{4}, \\
\Sigma_{\mathrm{E}}(\mathcal{K}) & \equiv k^{2}+m_{\mathrm{E}}^{2}\left[1-\frac{\eta}{2} \ln \left|\frac{1+\eta}{1-\eta}\right|\right], \\
\Gamma_{\mathrm{E}}(\eta) & \equiv \frac{\pi m_{\mathrm{E}}^{2} \eta}{2} .
\end{aligned}
$$

The essential structure is that in each case there is a "plasmon" pole, i.e. a $\delta$-function analogous to the $\delta$-functions in the free propagator of eq. (8.36) but displaced by an amount $\propto m_{\mathrm{E}}^{2}$, as well as a cut at $\left|k^{0}\right|<k$, representing Landau damping.

So far, we have only computed the resummed propagator. A very interesting question is whether also an effective action can be written down, which would then not only contain the inverse propagator like eq. (8.125), but also new vertices, in analogy with the dimensionally reduced effective theory of eq. (6.36). Such effective vertices are needed for properly describing how the soft modes interact with each other. Note that since our observables are now non-static, the effective action should be gauge-invariant also in time-dependent gauge transformations.

Most remarkably, such an effective action can indeed be found $[8.35,8.36]$. We simply cite here the result for the gluonic case. Expressing everything in Minkowskian notation (i.e. after setting 
$i k_{n} \rightarrow k^{0}$ and using the Minkowskian $\left.A_{0}^{a}\right)$, the effective Lagrangian reads

$$
\mathcal{L}_{M}=-\frac{1}{2} \operatorname{Tr}\left[F_{\mu \nu} F^{\mu \nu}\right]+\frac{m_{\mathrm{E}}^{2}}{2} \int \mathrm{d} \Omega_{v} \operatorname{Tr}\left[\left(\frac{1}{\mathcal{V} \cdot \mathcal{D}} \mathcal{V}^{\alpha} F_{\alpha \mu}\right)\left(\frac{1}{\mathcal{V} \cdot \mathcal{D}} \mathcal{V}^{\beta} F_{\beta}{ }^{\mu}\right)\right] .
$$

Here $\mathcal{V} \equiv(1, \mathbf{v})$ is a light-like four-velocity, and $\mathcal{D}$ represents the covariant derivative in the adjoint representation.

Several remarks on eq. (8.173) are in order:

- A somewhat tedious analysis, making use of the velocity integrals listed in eqs. (8.216)-(8.224) below, shows that in the static limit the second term in eq. (8.173) reduces to the mass term in eq. (6.36) (modulo Wick rotation and the Minkowskian vs. Euclidean convention for $A_{0}^{a}$ ).

- In the static limit, we found quarks to always be infrared-safe, but this situation changes after the analytic continuation. Therefore a "dynamical" quark part should be added to eq. (8.173) $[8.35,8.36]$; some details are given in appendix B.

- In the presence of chemical potentials, additional operators, which break charge conjugation invariance, should be added to eq. (8.173) [8.37].

- Eq. (8.173) has the unpleasant feature that it is non-local: derivatives appear in the denominator. This we do not usually expect from effective theories. Indeed, if non-local structures appear, it is difficult to analyze what kind of higher-order operators have been omitted and, hence, what the relative accuracy of the effective description is.

In some sense, the appearance of non-local terms is a manifestation of the fact that the proper infrared degrees of freedom have not been identified. It turns out that the HTL theory can be reformulated by introducing additional degrees of freedom, which gives the theory a local appearance [8.22], [8.38]-[8.40] (for a pedagogic introduction, see ref. [8.41]). However the reformulation contains classical on-shell particles rather than quantum fields, whereby it continues to be difficult to analyze the accuracy of the effective description.

- We arrived at eq. (8.173) by integrating out the hard modes, with momenta $p \sim \pi T$. However, like in the static limit, the theory still has multiple dynamical momentum scales, $k \sim g T$ and $k \sim g^{2} T / \pi$. It can be asked what happens if the momenta $k \sim g T$ are also integrated out. This question has been analyzed in the literature, and leads indeed to a simplified (local) effective description [8.42]-[8.46], which can be used for non-perturbatively studying observables only sensitive to "ultrasoft" momenta, $k \sim g^{2} T / \pi$.

- Remarkably, for certain light-cone observables, "sum rules" can be established which allow to reduce gluonic HTL structures to the dimensionally reduced theory $[8.17,8.24,8.47] .^{28}$ This is an important development, because the dimensionally reduced theory can be studied with standard non-perturbative techniques [8.48].

\footnotetext{
${ }^{28}$ Picking out one spatial component and denoting it by $k_{\|}$, so that $\mathbf{k} \equiv\left(k_{\|}, \mathbf{k}_{\perp}\right)$, the sum rules can be expressed as

$$
\begin{aligned}
\int_{-\infty}^{\infty} \frac{\mathrm{d} k_{\|}}{2 \pi}\left\{\frac{\rho_{\mathrm{T}}\left(k_{\|}, \mathbf{k}\right)}{k_{\|}}-\frac{\rho_{\mathrm{E}}\left(k_{\|}, \mathbf{k}\right)}{k_{\|}}\right\} \frac{k_{\perp}^{4}}{k_{\perp}^{2}+k_{\|}^{2}} & =\frac{1}{2} \frac{m_{\mathrm{E}}^{2}}{k_{\perp}^{2}+m_{\mathrm{E}}^{2}} \\
\int_{-\infty}^{\infty} \frac{\mathrm{d} k_{\|}}{2 \pi} k_{\|}\left\{\rho_{\mathrm{P}}\left(k_{\|}, \mathbf{k}\right)-\rho_{\mathrm{W}}\left(k_{\|}, \mathbf{k}\right)\right\} & =\frac{1}{4} \frac{m_{\ell}^{2}}{k_{\perp}^{2}+m_{\ell}^{2}}
\end{aligned}
$$

where $\rho_{\mathrm{T}}, \rho_{\mathrm{E}}, \rho_{\mathrm{W}}$ and $\rho_{\mathrm{P}}$ are the spectral functions from eqs. (8.167), (8.168), and (8.201) for the last two. 


\section{Appendix A: Hard gauge boson loop}

Here a few details are given concerning the handling of the bosonic part of eq. (8.126). We follow the steps from eq. (8.127) onwards. The spatial part of the self-energy can be written as

$$
\Pi_{i j}^{(\mathrm{b})}(K)=\frac{g^{2} N_{\mathrm{c}}}{2} \mathcal{F}_{P}\left\{(D-2)\left[\frac{2 \delta_{i j}}{P^{2}}+\frac{k_{i} k_{j}-4 p_{i} p_{j}}{P^{2}(K-P)^{2}}\right]-4 \frac{k^{2} \delta_{i j}-k_{i} k_{j}}{P^{2}(K-P)^{2}}\right\},
$$

where all terms containing $k_{i}$ in the numerator are subleading. The bosonic counterpart of eq. (8.130) (cf. eq. (8.30)) reads

$$
T \sum_{p_{n}} \frac{1}{p_{n}^{2}+\epsilon_{1}^{2}}=\frac{1}{2 \epsilon_{1}}\left[1+2 n_{\mathrm{B}}\left(\epsilon_{1}\right)\right]
$$

whereas eqs. (8.131)-(8.135) get replaced with

$$
\begin{aligned}
\mathcal{G}^{\prime} \equiv T \sum_{p_{n}} & \frac{1}{\left[p_{n}^{2}+\epsilon_{1}^{2}\right]\left[\left(k_{n}-p_{n}\right)^{2}+\epsilon_{2}^{2}\right]} \\
=\frac{1}{4 \epsilon_{1} \epsilon_{2}} & \left\{\frac{1}{i k_{n}-\epsilon_{1}-\epsilon_{2}}\left[-n_{\mathrm{B}}\left(\epsilon_{1}\right)-n_{\mathrm{B}}\left(\epsilon_{2}\right)-1\right]\right. \\
& +\frac{1}{i k_{n}+\epsilon_{2}-\epsilon_{1}}\left[n_{\mathrm{B}}\left(\epsilon_{1}\right)-n_{\mathrm{B}}\left(\epsilon_{2}\right)\right] \\
& +\frac{1}{i k_{n}+\epsilon_{1}-\epsilon_{2}}\left[n_{\mathrm{B}}\left(\epsilon_{2}\right)-n_{\mathrm{B}}\left(\epsilon_{1}\right)\right] \\
& \left.+\frac{1}{i k_{n}+\epsilon_{1}+\epsilon_{2}}\left[1+n_{\mathrm{B}}\left(\epsilon_{1}\right)+n_{\mathrm{B}}\left(\epsilon_{2}\right)\right]\right\} .
\end{aligned}
$$

We observe that the bosonic results can be obtained from the fermionic ones simply by setting $n_{\mathrm{F}} \rightarrow-n_{\mathrm{B}}$. The expansions of eqs. (8.136)-(8.138) proceed as before, although one must be careful in making sure that the IR behaviour of the Bose distribution still permits for a Taylor expansion in powers of the external momentum. The partial integration identity in eq. (8.142) can in addition be seen to retain its form, so that, effectively,

$$
\mathcal{G}^{\prime} \rightarrow \frac{n_{\mathrm{B}}(p)}{2 p^{3}}\left[1-(D-2) \frac{\mathbf{k} \cdot \mathbf{v}}{i k_{n}-\mathbf{k} \cdot \mathbf{v}}\right]=\frac{n_{\mathrm{B}}(p)}{2 p^{3}}\left[D-1-(D-2) \frac{i k_{n}}{i k_{n}-\mathbf{k} \cdot \mathbf{v}}\right] .
$$

The final steps are like in eq. (8.146) and lead to eq. (8.147), with $m_{\mathrm{E}}^{2}$ as given in eq. (8.148).

\section{Appendix B: Fermion self-energy}

Next, we consider a Dirac fermion at a finite temperature $T$ and a finite chemical potential $\mu$, interacting with an Abelian gauge field (this is no restriction at the current order: for a nonAbelian case simply replace $e^{2} \rightarrow g^{2} C_{\mathrm{F}}$, where $\left.C_{\mathrm{F}} \equiv\left(N_{\mathrm{c}}^{2}-1\right) /\left(2 N_{\mathrm{c}}\right)\right)$. The action is of the form in eq. (7.34) with $D_{\mu}=\partial_{\mu}-i e A_{\mu}$. To second order in $e$, the "effective action", or generating functional, takes the form $S_{\text {eff }}=S_{0}+\left\langle S_{I}-\frac{1}{2} S_{I}^{2}+\mathcal{O}\left(e^{3}\right)\right\rangle_{1 \mathrm{PI}}$, where $S_{0}$ is the quadratic part of the Euclidean action and $S_{I}$ contains the interactions. Carrying out the Wick contractions, this yields

$$
S_{\text {eff }}=\oiint_{\{K\}} \tilde{\bar{\psi}}(\tilde{K})\left[i \tilde{K}+m+e^{2} \mathcal{F}_{\{P\}} \frac{\gamma_{\mu}(-i \tilde{P}+m) \gamma_{\mu}}{\left(\tilde{P}^{2}+m^{2}\right)(\tilde{P}-\tilde{K})^{2}}+\mathcal{O}\left(e A_{\mu}\right)\right] \tilde{\psi}(\tilde{K}),
$$


where we have for simplicity employed the Feynman gauge, and $\tilde{P}, \tilde{K}$ are fermionic Matsubara momenta where the zero component contains the chemical potential as indicated in eq. (8.128): $\tilde{k}_{n} \equiv k_{n}+i \mu$. In the momentum $\tilde{P}-\tilde{K}$, carried by $A_{\mu}$, the chemical potential drops out.

The Dirac structures appearing in eq. (8.181) can be simplified: $\gamma_{\mu} \gamma_{\mu}=D \mathbb{1}_{4 \times 4}, \gamma_{\mu} \tilde{P} \gamma_{\mu}=$ $(2-D) \tilde{P}$. Denoting

$$
f\left(i \tilde{p}_{n}, \mathbf{v}\right) \equiv i(D-2) \tilde{P}+D m \mathbb{1}_{4 \times 4}
$$

where $\mathbf{v}$ is a dummy variable for both $\mathbf{p}$ and $m$; as well as

$$
\epsilon_{1} \equiv \sqrt{p^{2}+m^{2}}, \quad \epsilon_{2} \equiv \sqrt{(\mathbf{p}-\mathbf{k})^{2}},
$$

we are led to consider the sum (a generalization of eq. (8.75))

$$
\mathcal{F} \equiv T \sum_{\left\{p_{n}\right\}} \frac{f\left(i \tilde{p}_{n}, \mathbf{v}\right)}{\left[\tilde{p}_{n}^{2}+\epsilon_{1}^{2}\right]\left[\left(\tilde{p}_{n}-\tilde{k}_{n}\right)^{2}+\epsilon_{2}^{2}\right]} .
$$

We can now write

$$
\begin{aligned}
\mathcal{F} & =T \sum_{\left\{p_{n}\right\}} T \sum_{r_{n}} \beta \delta\left(\tilde{p}_{n}-\tilde{k}_{n}-r_{n}\right) \frac{f\left(i \tilde{p}_{n}, \mathbf{v}\right)}{\left[\tilde{p}_{n}^{2}+\epsilon_{1}^{2}\right]\left[r_{n}^{2}+\epsilon_{2}^{2}\right]} \\
& =\int_{0}^{\beta} \mathrm{d} \tau e^{-i \tilde{k}_{n} \tau}\left\{T \sum_{\left\{p_{n}\right\}} e^{i \tilde{p}_{n} \tau} \frac{f\left(i \tilde{p}_{n}, \mathbf{v}\right)}{\tilde{p}_{n}^{2}+\epsilon_{1}^{2}}\right\}\left\{T \sum_{r_{n}} \frac{e^{-i r_{n} \tau}}{r_{n}^{2}+\epsilon_{2}^{2}}\right\},
\end{aligned}
$$

where we used a similar representation as before,

$$
\beta \delta\left(\tilde{p}_{n}-\tilde{k}_{n}-r_{n}\right)=\int_{0}^{\beta} \mathrm{d} \tau e^{i\left(\tilde{p}_{n}-\tilde{k}_{n}-r_{n}\right) \tau} .
$$

Subsequently eqs. (8.30) and (8.64) and their time derivatives can be inserted:

$$
\begin{aligned}
& T \sum_{r_{n}} \frac{e^{-i r_{n} \tau}}{r_{n}^{2}+\epsilon_{2}^{2}}=\frac{n_{\mathrm{B}}\left(\epsilon_{2}\right)}{2 \epsilon_{2}}\left[e^{(\beta-\tau) \epsilon_{2}}+e^{\tau \epsilon_{2}}\right], \\
& T \sum_{\left\{p_{n}\right\}} \frac{e^{i \tilde{p}_{n} \tau}}{\tilde{p}_{n}^{2}+\epsilon_{1}^{2}}=\frac{1}{2 \epsilon_{1}}\left[n_{\mathrm{F}}\left(\epsilon_{1}-\mu\right) e^{(\beta-\tau) \epsilon_{1}-\beta \mu}-n_{\mathrm{F}}\left(\epsilon_{1}+\mu\right) e^{\tau \epsilon_{1}}\right], \\
& T \sum_{\left\{p_{n}\right\}} \frac{i \tilde{p}_{n} e^{i \tilde{p}_{n} \tau}}{\tilde{p}_{n}^{2}+\epsilon_{1}^{2}}=-\frac{1}{2}\left[n_{\mathrm{F}}\left(\epsilon_{1}-\mu\right) e^{(\beta-\tau) \epsilon_{1}-\beta \mu}+n_{\mathrm{F}}\left(\epsilon_{1}+\mu\right) e^{\tau \epsilon_{1}}\right] .
\end{aligned}
$$

Thereby we obtain

$$
\begin{aligned}
& \mathcal{F}=\int_{0}^{\beta} \mathrm{d} \tau e^{-i \tilde{k}_{n} \tau} \frac{n_{\mathrm{B}}\left(\epsilon_{2}\right)}{4 \epsilon_{1} \epsilon_{2}}\left\{\quad n_{\mathrm{F}}\left(\epsilon_{1}-\mu\right) e^{(\beta-\tau)\left(\epsilon_{1}+\epsilon_{2}\right)-\beta \mu} f\left(-\epsilon_{1}, \mathbf{v}\right)\right. \\
& +n_{\mathrm{F}}\left(\epsilon_{1}-\mu\right) e^{(\beta-\tau) \epsilon_{1}+\tau \epsilon_{2}-\beta \mu} f\left(-\epsilon_{1}, \mathbf{v}\right) \\
& +n_{\mathrm{F}}\left(\epsilon_{1}+\mu\right) e^{(\beta-\tau) \epsilon_{2}+\tau \epsilon_{1}} f\left(-\epsilon_{1},-\mathbf{v}\right) \\
& \left.+n_{\mathrm{F}}\left(\epsilon_{1}+\mu\right) e^{\tau\left(\epsilon_{1}+\epsilon_{2}\right)} f\left(-\epsilon_{1},-\mathbf{v}\right)\right\} \text {. }
\end{aligned}
$$

As an example, let us focus on the second structure in eq. (8.190). The $\tau$-integral can be carried out, noting that $\tilde{k}_{n}$ is fermionic:

$$
\int_{0}^{\beta} \mathrm{d} \tau e^{\beta\left(\epsilon_{1}-\mu\right)} e^{\tau\left(-i \tilde{k}_{n}-\epsilon_{1}+\epsilon_{2}\right)}=\frac{e^{\beta\left(\epsilon_{1}-\mu\right)}}{-i \tilde{k}_{n}-\epsilon_{1}+\epsilon_{2}}\left[-e^{\beta\left(\epsilon_{2}-\epsilon_{1}+\mu\right)}-1\right]
$$




$$
\begin{aligned}
& =\frac{e^{\beta \epsilon_{2}}+e^{\beta\left(\epsilon_{1}-\mu\right)}}{i \tilde{k}_{n}+\epsilon_{1}-\epsilon_{2}} \\
& =\frac{1}{i \tilde{k}_{n}+\epsilon_{1}-\epsilon_{2}}\left[n_{\mathrm{B}}^{-1}\left(\epsilon_{2}\right)+n_{\mathrm{F}}^{-1}\left(\epsilon_{1}-\mu\right)\right] .
\end{aligned}
$$

The inverse distribution functions nicely combine with those appearing explicitly in eq. (8.190):

$$
\begin{aligned}
& \mathcal{F}=\frac{1}{4 \epsilon_{1} \epsilon_{2}}\left\{\quad \frac{f\left(-\epsilon_{1}, \mathbf{v}\right)}{i \tilde{k}_{n}+\epsilon_{1}+\epsilon_{2}}\left[1+n_{\mathrm{B}}\left(\epsilon_{2}\right)-n_{\mathrm{F}}\left(\epsilon_{1}-\mu\right)\right]\right. \\
& +\frac{f\left(-\epsilon_{1}, \mathbf{v}\right)}{i \tilde{k}_{n}+\epsilon_{1}-\epsilon_{2}}\left[n_{\mathrm{F}}\left(\epsilon_{1}-\mu\right)+n_{\mathrm{B}}\left(\epsilon_{2}\right)\right] \\
& +\frac{f\left(\epsilon_{1}, \mathbf{v}\right)}{i \tilde{k}_{n}-\epsilon_{1}+\epsilon_{2}}\left[-n_{\mathrm{F}}\left(\epsilon_{1}+\mu\right)-n_{\mathrm{B}}\left(\epsilon_{2}\right)\right] \\
& \left.+\frac{f\left(\epsilon_{1}, \mathbf{v}\right)}{i \tilde{k}_{n}-\epsilon_{1}-\epsilon_{2}}\left[-1-n_{\mathrm{B}}\left(\epsilon_{2}\right)+n_{\mathrm{F}}\left(\epsilon_{1}+\mu\right)\right]\right\} \text {. }
\end{aligned}
$$

We now make the assumption, akin to that leading to eq. (8.138), that all four components of the (Minkowskian) external momentum $\mathcal{K}$ are small compared with the loop three-momentum $p=|\mathbf{p}|$, whose scale is fixed by the temperature and the chemical potential (this argument does not apply to the vacuum terms which are omitted; they amount e.g. to a radiative correction to the mass parameter $m$ ). Furthermore, in order to simplify the discussion, we assume that the (renormalized) mass parameter is small compared with $T$ and $\mu$. Thereby the "energies" of eq. (8.183) become

$$
\epsilon_{1} \approx p+\frac{m^{2}}{2 p}+\mathcal{O}\left(\frac{m^{4}}{p^{3}}\right), \quad \epsilon_{2} \approx p-\mathbf{k} \cdot \mathbf{v}+\mathcal{O}\left(\frac{k^{2}}{p}\right)
$$

where again

$$
\mathbf{v} \equiv \frac{\mathbf{p}}{p}
$$

Combining eqs. (8.182) and (8.192) with eq. (8.193), and noting that (for $m \ll p$ )

$$
f\left( \pm \epsilon_{1}, \mathbf{v}\right) \approx(D-2)\left( \pm \gamma^{0}+v_{i} \gamma^{i}\right) p
$$

where we returned to Minkowskian conventions for the Dirac matrices (cf. eq. (4.36)), it is easy to see that the dominant contribution, of order $1 / \mathcal{K}$, arises from the 2 nd and 3 rd terms in eq. (8.192) which contain the difference $\epsilon_{1}-\epsilon_{2}$ in the denominator. Writing $-\mathbf{v} \cdot \gamma \equiv v_{i} \gamma^{i}$ and substituting $\mathbf{v} \rightarrow-\mathbf{v}$ in the 3 rd term, eq. (8.181) becomes $S_{\text {eff }}^{(0)}=\oiint_{\{\tilde{K}\}} \tilde{\bar{\psi}}(\tilde{K})[i \tilde{K}+m+\mathbb{\psi}(\tilde{K})] \tilde{\psi}(\tilde{K})$, where the superscript indicates that terms of $\mathcal{O}\left(e A_{\mu}\right)$ have been omitted, and

$$
\mathbb{\psi}(\tilde{K}) \approx-m_{\mathrm{F}}^{2} \int \mathrm{d} \Omega_{v} \frac{\gamma_{0}+\mathbf{v} \cdot \boldsymbol{\gamma}}{i \tilde{k}_{n}+\mathbf{k} \cdot \mathbf{v}} .
$$

Here we have defined

$$
\begin{aligned}
& m_{\mathrm{F}}^{2} \equiv \frac{(D-2) e^{2}}{4} \int_{\mathbf{p}} \frac{1}{p}\left[2 n_{\mathrm{B}}(p)+n_{\mathrm{F}}(p+\mu)+n_{\mathrm{F}}(p-\mu)\right] \\
& \stackrel{D \equiv 4}{=} e^{2}\left(\frac{T^{2}}{8}+\frac{\mu^{2}}{8 \pi^{2}}\right),
\end{aligned}
$$

and carried out the integrals for $D=4$ (the bosonic part gives $2 \int_{\mathbf{p}} n_{\mathrm{B}}(p) / p=T^{2} / 6$; the fermionic part is worked out in appendix C). The angular integrations can also be carried out, cf. eqs. (8.219) and (8.220) below. 
Next, we want to determine the corresponding spectral representation. As discussed in connection with the example following eq. (8.65), sign conventions are tricky with fermions. Our $S_{\text {eff }}^{(0)}$ defines the inverse propagator, representing therefore a generalization of the object in eq. (8.65), with the frequency variable appearing as $\tilde{k}_{n}=k_{n}+i \mu$. Aiming for a spectral representation directly in terms of this variable, needed in eq. (8.93), we define the analytic continuation as $i \tilde{k}_{n} \rightarrow \omega$ where $\omega$ has a small positive imaginary part. Carrying out the angular integrals in eq. (8.196) as explained in appendix $\mathrm{C}$, the analytically continued inverse propagator becomes (we set $m \rightarrow 0$ )

$$
\not{C}+\not{4}(-i \omega, \mathbf{k})=\omega \gamma_{0}\left[1-\frac{m_{\mathrm{F}}^{2}}{2 k \omega} \ln \frac{\omega+k}{\omega-k}\right]-\mathbf{k} \cdot \gamma\left[1+\frac{m_{\mathrm{F}}^{2}}{k^{2}}\left(1-\frac{\omega}{2 k} \ln \frac{\omega+k}{\omega-k}\right)\right] .
$$

Introducing the concept of an "asymptotic mass" $m_{\ell}^{2} \equiv 2 m_{\mathrm{F}}^{2}$ and denoting $L \equiv \frac{1}{2 k} \ln \frac{\omega+k}{\omega-k}$, the corresponding spectral function reads

$$
\operatorname{Im}\left\{[\not \mathcal{L}+\not{Z}(-i \omega, \mathbf{k})]^{-1}\right\}=\not(\omega, \mathbf{k}), \quad \rho \equiv\left(\omega \rho_{\mathrm{W}}, \mathbf{k} \rho_{\mathrm{P}}\right) \equiv \frac{1}{2}\left(n_{+} \rho_{+}+n_{-} \rho_{-}\right),
$$

where two separate basis choices have been introduced. Here $n_{ \pm} \equiv(1, \pm \mathbf{k} / k)$, and

$$
\begin{aligned}
& \rho_{\mathrm{W}}=\operatorname{Im}\left\{\frac{1-\frac{m_{\ell}^{2} L}{2 \omega}}{\left[\omega-\frac{m_{\ell}^{2} L}{2}\right]^{2}-\left[k+\frac{m_{\ell}^{2}(1-\omega L)}{2 k}\right]^{2}}\right\}, \quad \rho_{\mathrm{P}}=\operatorname{Im}\left\{\frac{1+\frac{m_{\ell}^{2}(1-\omega L)}{2 k^{2}}}{\left[\omega-\frac{m_{\ell}^{2} L}{2}\right]^{2}-\left[k+\frac{m_{\ell}^{2}(1-\omega L)}{2 k}\right]^{2}}\right\}, \\
& \rho_{+}=\operatorname{Im}\left\{\frac{1}{(\omega-k)\left[1+\frac{m_{\ell}^{2} L}{2 k}\right]-\frac{m_{\ell}^{2}}{2 k}}\right\}, \quad \rho_{-}=\operatorname{Im}\left\{\frac{1}{(\omega+k)\left[1-\frac{m_{\ell}^{2} L}{2 k}\right]+\frac{m_{\ell}^{2}}{2 k}}\right\} .
\end{aligned}
$$

These are well-known results $[8.13,8.32]$, generalized to the presence of a finite chemical potential [8.49]; note that the chemical potential only appears "trivially", in eq. (8.198), without affecting the functional form of the momentum dependence. The corresponding "dispersion relations", relevant for computing the "pole contributions" mentioned below eq. (8.100), have been discussed in the literature [8.50], and can be shown to comprise two branches. There is a novel branch, dubbed a "plasmino" branch, with the peculiar property that

$$
\omega \approx m_{\mathrm{F}}-\frac{k}{3}+\frac{k^{2}}{3 m_{\mathrm{F}}}<m_{\mathrm{F}}, \quad k \ll m_{\mathrm{F}} .
$$

If the zero-temperature mass $m$ is larger than $m_{\mathrm{F}}$, the plasmino branch decouples [8.51]. For large momenta, the dispersion relation of the normal branch is of the form

$$
\omega \approx k+\frac{m_{\ell}^{2}}{2 k}, \quad k \gg m_{\ell},
$$

which explains why $m_{\ell}$ is called an asymptotic mass. A discussion of the dispersion relation in various limits can be found in ref. [8.52].

\section{Appendix C: Radial and angular momentum integrals}

We compute here the radial and angular integrals defined in eqs. (8.142)-(8.145).

For generality, and because this is necessary in loop computations, it is useful to keep the space dimensionality open for as long as possible. Let us recall that the dimensionally regularized integration measure can be written as

$$
\int \frac{\mathrm{d}^{d} \mathbf{p}}{(2 \pi)^{d}} \rightarrow \frac{4}{(4 \pi)^{\frac{d+1}{2}} \Gamma\left(\frac{d-1}{2}\right)} \int_{0}^{\infty} \mathrm{d} p p^{d-1} \int_{-1}^{+1} \mathrm{~d} z\left(1-z^{2}\right)^{\frac{d-3}{2}},
$$


where $d \equiv D-1$ and $z=\mathbf{k} \cdot \mathbf{p} /(k p)$ parametrizes an angle with respect to some external vector. An important use of eq. (8.205) is that it allows us to carry out partial integrations with respect to both $p$ and $z$. If the integrand is independent of $z$, the $z$-integral yields

$$
\int_{-1}^{+1} \mathrm{~d} z\left(1-z^{2}\right)^{\frac{d-3}{2}}=\frac{\Gamma\left(\frac{1}{2}\right) \Gamma\left(\frac{d-1}{2}\right)}{\Gamma\left(\frac{d}{2}\right)},
$$

and we then denote (cf. eq. $(2.61)$, now divided by $(2 \pi)^{d}$ )

$$
c(d) \equiv \frac{2}{(4 \pi)^{\frac{d}{2}} \Gamma\left(\frac{d}{2}\right)},
$$

so that $\int_{\mathbf{p}}=\int_{p} \equiv c(d) \int_{0}^{\infty} \mathrm{d} p p^{d-1}$.

Now, eq. (8.142) can be verified through partial integration as follows:

$$
\begin{aligned}
\int_{p} \frac{1}{p}\left[n_{\mathrm{F}}(p+\mu)+n_{\mathrm{F}}(p-\mu)\right]= & c(d) \int_{0}^{\infty} \mathrm{d} p \frac{\mathrm{d} p}{\mathrm{~d} p} p^{d-2}\left[n_{\mathrm{F}}(p+\mu)+n_{\mathrm{F}}(p-\mu)\right] \\
= & -(d-2) c(d) \int_{0}^{\infty} \mathrm{d} p p^{d-2}\left[n_{\mathrm{F}}(p+\mu)+n_{\mathrm{F}}(p-\mu)\right] \\
& -c(d) \int_{0}^{\infty} \mathrm{d} p p^{d-1}\left[n_{\mathrm{F}}^{\prime}(p+\mu)+n_{\mathrm{F}}^{\prime}(p-\mu)\right] .
\end{aligned}
$$

Moving the first term to the left-hand side leads directly to eq. (8.142).

In order to derive the explicit expression in eq. (8.144), we set $d=3$; then a possible starting point is a combination of eqs. (7.36) and (7.42):

$$
\begin{aligned}
-f(T, \mu) & =2 \int_{p}\left\{p+T\left[\ln \left(1+e^{-\frac{p-\mu}{T}}\right)+\ln \left(1+e^{-\frac{p+\mu}{T}}\right)\right]\right\} \\
& \stackrel{d=3}{=} \frac{7 \pi^{2} T^{4}}{180}+\frac{\mu^{2} T^{2}}{6}+\frac{\mu^{4}}{12 \pi^{2}} .
\end{aligned}
$$

Taking the second partial derivative with respect to $\mu$, we get

$$
\begin{aligned}
-\frac{\partial^{2} f(T, \mu)}{\partial \mu^{2}} & =2 T \int_{p} \frac{\partial^{2}}{\partial \mu^{2}}\left[\ln \left(1+e^{-\frac{p-\mu}{T}}\right)+\ln \left(1+e^{-\frac{p+\mu}{T}}\right)\right] \\
& =2 T \int_{p} \frac{\mathrm{d}^{2}}{\mathrm{~d} p^{2}}\left[\ln \left(1+e^{-\frac{p-\mu}{T}}\right)+\ln \left(1+e^{-\frac{p+\mu}{T}}\right)\right] \\
& \stackrel{d \equiv 3}{=}-4 T \int_{p} \frac{1}{p} \frac{\mathrm{d}}{\mathrm{d} p}\left[\ln \left(1+e^{-\frac{p-\mu}{T}}\right)+\ln \left(1+e^{-\frac{p+\mu}{T}}\right)\right] \\
& =\frac{T^{2}}{3}+\frac{\mu^{2}}{\pi^{2}},
\end{aligned}
$$

where in the penultimate step we carried out one partial integration. On the other hand, the integral in eq. (8.210) can be rewritten as

$$
\begin{aligned}
& -4 T \int_{p} \frac{1}{p} \frac{\mathrm{d}}{\mathrm{d} p}\left[\ln \left(1+e^{-\frac{p-\mu}{T}}\right)+\ln \left(1+e^{-\frac{p+\mu}{T}}\right)\right] \\
& =-4 T \int_{p} \frac{1}{p}\left[\frac{e^{-\frac{p-\mu}{T}}}{1+e^{-\frac{p-\mu}{T}}}+\frac{e^{-\frac{p+\mu}{T}}}{1+e^{-\frac{p+\mu}{T}}}\right]\left(-\frac{1}{T}\right) \\
& =4 \int_{p} \frac{1}{p}\left[n_{\mathrm{F}}(p+\mu)+n_{\mathrm{F}}(p-\mu)\right] .
\end{aligned}
$$


Eqs. (8.211) and eq. (8.212) combine into eq. (8.144).

As far as angular integrals go (such as the one in eq. (8.145)), we start with the simplest structure, defined in eq. (8.157):

$$
\begin{aligned}
L(K) \equiv \int \mathrm{d} \Omega_{v} \frac{1}{i k_{n}-\mathbf{k} \cdot \mathbf{v}} & \stackrel{d=3}{=} \frac{1}{4 \pi} 2 \pi \int_{-1}^{+1} \mathrm{~d} z \frac{1}{i k_{n}-k z} \\
& =-\frac{1}{2 k} \int_{-1}^{+1} \mathrm{~d} z \frac{\mathrm{d}}{\mathrm{d} z} \ln \left(i k_{n}-k z\right) \\
& =\frac{1}{2 k} \ln \frac{i k_{n}+k}{i k_{n}-k} .
\end{aligned}
$$

Further integrals can then be obtained by making use of rotational symmetry. For instance,

$$
\int \mathrm{d} \Omega_{v} \frac{v_{i}}{i k_{n}-\mathbf{k} \cdot \mathbf{v}}=k_{i} f\left(i k_{n}, k\right)
$$

where, contracting both sides with $\mathbf{k}$,

$$
f\left(i k_{n}, k\right)=\frac{1}{k^{2}} \int \mathrm{d} \Omega_{v} \frac{\mathbf{k} \cdot \mathbf{v}}{i k_{n}-\mathbf{k} \cdot \mathbf{v}}=\frac{1}{k^{2}}\left[-1+i k_{n} \int \mathrm{d} \Omega_{v} \frac{1}{i k_{n}-\mathbf{k} \cdot \mathbf{v}}\right] .
$$

Another trick, needed for having higher powers in the denominator, is to take derivatives of eq. (8.213) with respect to $i k_{n}$.

Without detailing further steps, we list the results for a number of velocity integrals that can be obtained this way. Let us change the notation at this point: we replace $i k_{n}$ by $k^{0}+i 0^{+}$, as is relevant for retarded Green's functions $\left(i 0^{+}\right.$is not shown explicitly), and introduce the light-like four-velocity $\mathcal{V} \equiv(1, \mathbf{v})$. Then the integrals read $(d=3 ; i, j=1,2,3)$

$$
\begin{aligned}
\int \mathrm{d} \Omega_{v} & =1, \\
\int \mathrm{d} \Omega_{v} v^{i} & =0 \\
\int \mathrm{d} \Omega_{v} v^{i} v^{j} & =\frac{1}{3} \delta^{i j}, \\
\int \mathrm{d} \Omega_{v} \frac{1}{\mathcal{V} \cdot \mathcal{K}} & =L(\mathcal{K}), \\
\int \mathrm{d} \Omega_{v} \frac{v^{i}}{\mathcal{V} \cdot \mathcal{K}} & =\frac{k^{i}}{k^{2}}\left[-1+k^{0} L(\mathcal{K})\right], \\
\int \mathrm{d} \Omega_{v} \frac{v^{i} v^{j}}{\mathcal{V} \cdot \mathcal{K}} & =\frac{L(\mathcal{K})}{2}\left(\delta^{i j}-\frac{k^{i} k^{j}}{k^{2}}\right)+\frac{k^{0}}{2 k^{2}}\left[1-k^{0} L(\mathcal{K})\right]\left(\delta^{i j}-\frac{3 k^{i} k^{j}}{k^{2}}\right), \\
\int \mathrm{d} \Omega_{v} \frac{1}{(\mathcal{V} \cdot \mathcal{K})^{2}} & =\frac{1}{\mathcal{K}^{2}}, \\
\int \mathrm{d} \Omega_{v} \frac{v^{i}}{(\mathcal{V} \cdot \mathcal{K})^{2}} & =\frac{k^{i}}{k^{2}}\left[\frac{k^{0}}{\mathcal{K}^{2}}-L(\mathcal{K})\right], \\
\int \mathrm{d} \Omega_{v} \frac{v^{i} v^{j}}{(\mathcal{V} \cdot \mathcal{K})^{2}} & =\frac{1}{2 \mathcal{K}^{2}}\left(\delta^{i j}-\frac{k^{i} k^{j}}{k^{2}}\right)-\frac{1}{2 k^{2}}\left[1-2 k^{0} L(\mathcal{K})+\frac{\left(k^{0}\right)^{2}}{\mathcal{K}^{2}}\right]\left(\delta^{i j}-\frac{3 k^{i} k^{j}}{k^{2}}\right),
\end{aligned}
$$

where $\mathcal{V} \cdot \mathcal{K}=k^{0}-\mathbf{v} \cdot \mathbf{k}$, and

$$
L(\mathcal{K})=\frac{1}{2 k} \ln \frac{k^{0}+k+i 0^{+}}{k^{0}-k+i 0^{+}} \quad \stackrel{\left|k^{0}\right| \ll k}{\approx} \quad-\frac{i \pi}{2 k}+\frac{k^{0}}{k^{2}}+\frac{\left(k^{0}\right)^{3}}{3 k^{4}}+\ldots
$$




\section{Appendix D: Photon polarization beyond the hard thermal limit}

The Hard Thermal Loop computations that we have reviewed in this section relied on two separate approximations: the plasma particles were treated as massless, and all components of the external momentum $\mathcal{K}$ were assumed small compared with the loop momentum, $p \sim \pi T$. However, these approximations are not always justified. In this appendix, we determine the massive fermion contribution to the gauge field self-energy. The starting point is the sum-integral in eq. (5.100), expressed in the same way as on the last line of eq. (8.126), which we furthermore consider in a system with a finite chemical potential (cf. eq. (8.128)), viz.

$$
\Pi_{\mu \nu}(K) \supset-g^{2} N_{\mathrm{f}} \mathcal{F}_{\{P\}} \frac{\delta_{\mu \nu}\left[(K-\tilde{P})^{2}+\tilde{P}^{2}+2 m^{2}-K^{2}\right]+2\left(\tilde{P}_{\mu} K_{\nu}+\tilde{P}_{\nu} K_{\mu}\right)-4 \tilde{P}_{\mu} \tilde{P}_{\nu}}{\left[\tilde{P}^{2}+m^{2}\right]\left[(K-\tilde{P})^{2}+m^{2}\right]} .
$$

As a first step, consider the vacuum version of eq. (8.226). This is obtained by setting $\mathbb{E}_{P} \rightarrow \int_{P}$ and $\tilde{P} \rightarrow P$. The integrals can be solved with standard techniques, and produce a wave function correction, i.e. a term $\propto K^{2} \delta_{\mu \nu}-K_{\mu} K_{\nu}$. In the following, this vacuum part is omitted.

For the medium part, the Matsubara sums need to be carried out. It is useful to focus on the spatial part, like in eq. (8.127). Incorporating masses in the energies in eq. (8.129), the results can be taken over from eqs. (8.130) and (8.135). The terms without $n_{\mathrm{F}}$ are vacuum ones, and omitted. In the terms containing $n_{\mathrm{F}}\left(\epsilon_{2} \pm \mu\right)$, we can substitute $\mathbf{p} \rightarrow \mathbf{k}-\mathbf{p}$, whereby $\epsilon_{2} \rightarrow \epsilon_{1}$. The numerator is invariant in this substitution. Collecting the terms, the Fermi distributions can be factorized into $n_{\mathrm{F}}\left(\epsilon_{p}+\mu\right)+n_{\mathrm{F}}\left(\epsilon_{p}-\mu\right)$, where we have denoted $\epsilon_{p} \equiv \epsilon_{1} \equiv \sqrt{p^{2}+m^{2}}$.

The next step is to carry out angular integration. First, tensor integrals can be reduced into scalar ones, by making use of rotational invariance. This means that if the numerator contains $p_{i}$, the result is proportional to $k_{i}$, and if it contains $p_{i} p_{j}$, the result is a linear combination of terms proportional to $\delta_{i j}$ and $k_{i} k_{j}$. Subsequently, the angular integration can be carried out in the coefficient functions.

With the result at hand, it may be projected into the basis of eqs. (8.150)-(8.154). Finally, analytic continuation, viz. $k_{n} \rightarrow-i\left[k^{0}+i 0^{+}\right]$, leads to a retarded correlator. Given that the results are a bit lengthy, we employ an implicit notation, suppressing $i 0^{+}$and using $k_{0}=k^{0}$ :

$$
\begin{aligned}
\Pi_{\mathrm{T}}(\mathcal{K}) & \supset \frac{g^{2} N_{\mathrm{f}}}{2} \int_{p} \frac{n_{\mathrm{F}}\left(\epsilon_{p}-\mu\right)+n_{\mathrm{F}}\left(\epsilon_{p}+\mu\right)}{\epsilon_{p}} \\
& \times\left[1+\frac{k_{0}^{2}}{k^{2}}-\frac{k_{0}^{4}-k^{4}+4\left(k_{0}^{2} \epsilon_{p}^{2}-k^{2} p^{2}\right)}{8 k^{3} p} \ln \left(\mathcal{R}_{+} \mathcal{R}_{-}\right)-\frac{k_{0}\left(k_{0}^{2}-k^{2}\right) \epsilon_{p}}{2 k^{3} p} \ln \left(\frac{\mathcal{R}_{+}}{\mathcal{R}_{-}}\right)\right] \\
\Pi_{\mathrm{E}}(\mathcal{K}) & \supset g^{2} N_{\mathrm{f}} \int_{p} \frac{n_{\mathrm{F}}\left(\epsilon_{p}-\mu\right)+n_{\mathrm{F}}\left(\epsilon_{p}+\mu\right)}{\epsilon_{p}} \\
& \times\left(1-\frac{k_{0}^{2}}{k^{2}}\right)\left[1-\frac{k_{0}^{2}-k^{2}+4 \epsilon_{p}^{2}}{8 k p} \ln \left(\mathcal{R}_{+} \mathcal{R}_{-}\right)-\frac{k_{0} \epsilon_{p}}{2 k p} \ln \left(\frac{\mathcal{R}_{+}}{\mathcal{R}_{-}}\right)\right]
\end{aligned}
$$

where

$$
\mathcal{R}_{+} \equiv \frac{k_{0}^{2}-k^{2}+2\left(k_{0} \epsilon_{p}+k p\right)}{k_{0}^{2}-k^{2}+2\left(k_{0} \epsilon_{p}-k p\right)}, \quad \mathcal{R}_{-} \equiv \frac{k_{0}^{2}-k^{2}-2\left(k_{0} \epsilon_{p}-k p\right)}{k_{0}^{2}-k^{2}-2\left(k_{0} \epsilon_{p}+k p\right)} .
$$

In the Hard Thermal Loop limit, viz. $\epsilon_{p} \rightarrow p, \mathcal{K}^{2} \ll k p$, the logarithms become $\ln \left(\mathcal{R}_{+} \mathcal{R}_{-}\right) \rightarrow$ $-2 k / p$ and $\ln \left(\mathcal{R}_{+} / \mathcal{R}_{-}\right) \rightarrow 4 k L(\mathcal{K})$, and eqs. (8.165) and (8.166) are reproduced. 


\section{Literature}

[8.1] L.P. Kadanoff and G.A. Baym, Quantum Statistical Mechanics (Benjamin, Menlo Park, 1962).

[8.2] A.L. Fetter and J.D. Walecka, Quantum Theory of Many-Particle Systems (McGraw-Hill, New York, 1971).

[8.3] S. Doniach and E.H. Sondheimer, Green's Functions for Solid State Physicists (Benjamin, Reading, 1974).

[8.4] J.W. Negele and H. Orland, Quantum Many Particle Systems (Addison-Wesley, Redwood City, 1988).

[8.5] D. Bödeker and M. Sangel, Lepton asymmetry rate from quantum field theory: NLO in the hierarchical limit, JCAP 06 (2017) 052 [1702.02155].

[8.6] S. Chaudhuri, C. Chowdhury and R. Loganayagam, Spectral representation of thermal OTO correlators, JHEP 02 (2019) 018 [1810.03118].

[8.7] G. Cuniberti, E. De Micheli and G.A. Viano, Reconstructing the thermal Green functions at real times from those at imaginary times, Commun. Math. Phys. 216 (2001) 59 [condmat/0109175].

[8.8] H.B. Meyer, Transport properties of the quark-gluon plasma: A lattice QCD perspective, Eur. Phys. J. A 47 (2011) 86 [1104.3708].

[8.9] H.A. Weldon, Simple rules for discontinuities in finite-temperature field theory, Phys. Rev. D 28 (1983) 2007.

[8.10] M. Laine, Thermal 2-loop master spectral function at finite momentum, JHEP 05 (2013) 083 [1304.0202].

[8.11] R.D. Pisarski, Computing finite temperature loops with ease, Nucl. Phys. B 309 (1988) 476.

[8.12] R.R. Parwani, Resummation in a hot scalar field theory, Phys. Rev. D 45 (1992) 4695; ibid. 48 (1993) 5965 (E) [hep-ph/9204216].

[8.13] H.A. Weldon, Effective fermion masses of order $g T$ in high-temperature gauge theories with exact chiral invariance, Phys. Rev. D 26 (1982) 2789.

[8.14] E. Braaten, R.D. Pisarski and T.-C. Yuan, Production of soft dileptons in the quark-gluon plasma, Phys. Rev. Lett. 64 (1990) 2242.

[8.15] G.D. Moore and J.-M. Robert, Dileptons, spectral weights, and conductivity in the quarkgluon plasma, hep-ph/0607172.

[8.16] A. Anisimov, D. Besak and D. Bödeker, Thermal production of relativistic Majorana neutrinos: strong enhancement by multiple soft scattering, JCAP 03 (2011) 042 [1012.3784].

[8.17] D. Besak and D. Bödeker, Thermal production of ultrarelativistic right-handed neutrinos: complete leading-order results, JCAP 03 (2012) 029 [1202.1288].

[8.18] P.B. Arnold, G.D. Moore and L.G. Yaffe, Photon emission from ultrarelativistic plasmas, JHEP 11 (2001) 057 [hep-ph/0109064]. 
[8.19] P.B. Arnold, G.D. Moore and L.G. Yaffe, Photon emission from quark-gluon plasma: complete leading order results, JHEP 12 (2001) 009 [hep-ph/0111107].

[8.20] K. Chou, Z. Su, B. Hao and L. Yu, Equilibrium and nonequilibrium formalisms made unified, Phys. Rept. 118 (1985) 1.

[8.21] N.P. Landsman and C.G. van Weert, Real- and imaginary-time field theory at finite temperature and density, Phys. Rept. 145 (1987) 141.

[8.22] S. Caron-Huot, Hard thermal loops in the real-time formalism, JHEP 04 (2009) 004 [0710.5726].

[8.23] S. Caron-Huot and G.D. Moore, Heavy quark diffusion in perturbative QCD at next-toleading order, Phys. Rev. Lett. 100 (2008) 052301 [0708.4232].

[8.24] S. Caron-Huot, $O(g)$ plasma effects in jet quenching, Phys. Rev. D 79 (2009) 065039 [0811.1603].

[8.25] J. Ghiglieri, J. Hong, A. Kurkela, E. Lu, G.D. Moore and D. Teaney, Next-to-leading order thermal photon production in a weakly coupled quark-gluon plasma, JHEP 05 (2013) 010 [1302.5970].

[8.26] E. Braaten and T.C. Yuan, Calculation of screening in a hot plasma, Phys. Rev. Lett. 66 (1991) 2183.

[8.27] R.D. Pisarski, Scattering amplitudes in hot gauge theories, Phys. Rev. Lett. 63 (1989) 1129.

[8.28] J. Frenkel and J.C. Taylor, High-temperature limit of thermal QCD, Nucl. Phys. B 334 (1990) 199.

[8.29] E. Braaten and R.D. Pisarski, Soft amplitudes in hot gauge theories: A general analysis, Nucl. Phys. B 337 (1990) 569.

[8.30] J.C. Taylor and S.M.H. Wong, The effective action of hard thermal loops in QCD, Nucl. Phys. B 346 (1990) 115.

[8.31] V.P. Silin, On the electromagnetic properties of a relativistic plasma, Sov. Phys. JETP 11 (1960) 1136 [Zh. Eksp. Teor. Fiz. 38 (1960) 1577].

[8.32] V.V. Klimov, Collective Excitations in a Hot Quark Gluon Plasma, Sov. Phys. JETP 55 (1982) 199 [Zh. Eksp. Teor. Fiz. 82 (1982) 336].

[8.33] H.A. Weldon, Covariant calculations at finite temperature: The relativistic plasma, Phys. Rev. D 26 (1982) 1394.

[8.34] S. Carignano, M.E. Carrington and J. Soto, The HTL Lagrangian at NLO: the photon case, Phys. Lett. B 801 (2020) 135193 [1909.10545].

[8.35] J. Frenkel and J.C. Taylor, Hard thermal QCD, forward scattering and effective actions, Nucl. Phys. B 374 (1992) 156.

[8.36] E. Braaten and R.D. Pisarski, Simple effective Lagrangian for hard thermal loops, Phys. Rev. D 45 (1992) 1827.

[8.37] D. Bödeker and M. Laine, Finite baryon density effects on gauge field dynamics, JHEP 09 (2001) 029 [hep-ph/0108034]. 
[8.38] J.P. Blaizot and E. Iancu, Kinetic equations for long-wavelength excitations of the quarkgluon plasma, Phys. Rev. Lett. 70 (1993) 3376 [hep-ph/9301236].

[8.39] P.F. Kelly, Q. Liu, C. Lucchesi and C. Manuel, Deriving the hard thermal loops of QCD from classical transport theory, Phys. Rev. Lett. 72 (1994) 3461 [hep-ph/9403403].

[8.40] F.T. Brandt, J. Frenkel and J.C. Taylor, High temperature QCD and the classical Boltzmann equation in curved space-time, Nucl. Phys. B 437 (1995) 433 [hep-th/9411130].

[8.41] R.D. Pisarski, Kinetic Theory of Hot Gauge Theories: Overview, Details 89 Extensions, NATO Sci. Ser. C 511 (1998) 195 [hep-ph/9710370].

[8.42] D. Bödeker, Effective dynamics of soft non-Abelian gauge fields at finite temperature, Phys. Lett. B 426 (1998) 351 [hep-ph/9801430].

[8.43] P. Arnold, D.T. Son and L.G. Yaffe, Effective dynamics of hot, soft non-Abelian gauge fields: Color conductivity and $\log (1 / \alpha)$ effects, Phys. Rev. D 59 (1999) 105020 [hep-ph/9810216].

[8.44] D. Bödeker, Diagrammatic approach to soft non-Abelian dynamics at high temperature, Nucl. Phys. B 566 (2000) 402 [hep-ph/9903478].

[8.45] P.B. Arnold and L.G. Yaffe, High temperature color conductivity at next-to-leading log order, Phys. Rev. D 62 (2000) 125014 [hep-ph/9912306].

[8.46] D. Bödeker, Perturbative and non-perturbative aspects of the non-Abelian BoltzmannLangevin equation, Nucl. Phys. B 647 (2002) 512 [hep-ph/0205202].

[8.47] P. Aurenche, F. Gelis and H. Zaraket, A simple sum rule for the thermal gluon spectral function and applications, JHEP 05 (2002) 043 [hep-ph/0204146].

[8.48] M. Panero, K. Rummukainen and A. Schäfer, Lattice Study of the Jet Quenching Parameter, Phys. Rev. Lett. 112 (2014) 162001 [1307.5850].

[8.49] J.-P. Blaizot and J.-Y. Ollitrault, Collective fermionic excitations in systems with a large chemical potential, Phys. Rev. D 48 (1993) 1390 [hep-th/9303070].

[8.50] H.A. Weldon, Dynamical holes in the quark-gluon plasma, Phys. Rev. D 40 (1989) 2410.

[8.51] E. Petitgirard, Massive fermion dispersion relation at finite temperature, Z. Phys. C 54 (1992) 673.

[8.52] P.M. Chesler, A. Gynther and A. Vuorinen, On the dispersion of fundamental particles in $Q C D$ and $\mathcal{N}=4$ Super Yang-Mills theory, JHEP 09 (2009) 003 [0906.3052]. 


\section{Applications}

Abstract: A number of physical applications of relativistic thermal field theory are considered. First the basic formalism for addressing the existence of a scalar field driven phase transition is developed (sec. 9.1). Then the concept of instantons is introduced with the example of a bubble nucleation rate related to a first order phase transition (sec. 9.2). This is followed by a general discussion concerning the formalism for particle production rate computations, relevant both for heavy ion collision experiments and cosmology (sec. 9.3). How a particle production rate can be embedded in an expanding cosmological background is explained in detail (sec. 9.4). Turning to so-called transport coefficients, we first consider the effective mass and friction coefficient that a scalar field evolving within a thermal environment feels (sec. 9.5). Then transport coefficients are discussed more generally, culminating in the definition of shear and bulk viscosities, diffusion coefficients, and the electric conductivity of QCD matter (sec. 9.6). Transport coefficients are closely related to the rate at which a slightly disturbed system equilibrates, and the corresponding formalism is introduced, stressing the idea of employing operator equations of motion in order to simplify the correlation function to be computed (sec. 9.7). Finally a somewhat different but physically important topic, that of the behaviour of resonances made of a heavy particle and antiparticle, is outlined, with emphasis on the roles that "virtual" and "real" corrections play at finite temperature (sec. 9.8).

Keywords: Effective potential, condensate, first order phase transition, semiclassical approximation, saddle point, instanton, fluctuation determinant, tunnelling, sphaleron, classical limit, critical bubble, latent heat, surface tension, particle production, on-shell field operator, LandauPomeranchuk-Migdal effect, decay rate, Friedmann equations, yield parameter, Boltzmann equation, friction coefficient, damping rate, thermal mass, dilaton, axion, Chern-Simons diffusion, equilibration, fluctuation-dissipation theorem, Kubo formula, transport peak, flavour diffusion, conductivity, viscosity, Brownian motion, Langevin equation, quarkonium, Debye screening, decoherence, thermal width, real and virtual processes at finite temperature.

\subsection{Thermal phase transitions}

As a first application of the general formalism developed, we consider the existence of thermal phase transitions in models of particle physics. Prime examples are the "deconfinement" transition in QCD, and the "electroweak symmetry restoring" transition in the electroweak theory, ${ }^{29}$ both of which took place in the early universe. For simplicity, though, the practical analysis will be carried out within the scalar field theory discussed in sec. 3 .

In general, a phase transition can be defined as a line in the $(T, \mu)$-plane across which the grand canonical free energy density $f(T, \mu)$ is non-analytic. In particular, if $\partial f / \partial T$ or $\partial f / \partial \mu$ is discontinuous, we speak of a first order transition. The energy density

$$
e=\frac{1}{V \mathcal{Z}} \operatorname{Tr}\left[\hat{H} e^{-\beta(\hat{H}-\mu \hat{Q})}\right]=\frac{T^{2}}{V} \frac{\partial}{\partial T}(\ln \mathcal{Z})_{\frac{\mu}{T}}=f-T\left(\frac{\partial f}{\partial T}\right)_{\frac{\mu}{T}}
$$

is then discontinuous, with the discontinuity known as the latent heat. This means that a closed

\footnotetext{
${ }^{29}$ Here the standard terminology is used, even though it is inappropriate in a strict sense, given that both transitions are known to be of a crossover type, i.e. not genuine phase transitions.
} 


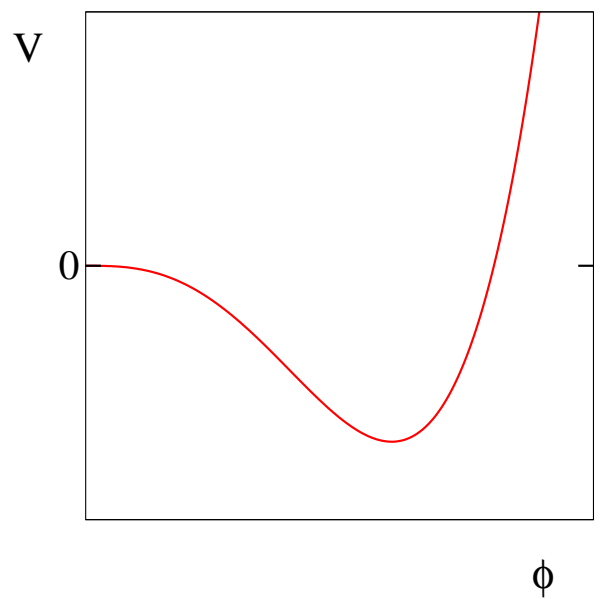

Figure 2: The potential from eq. (9.2) at zero temperature for $\phi>0$.

system can proceed through the transition only if there is some mechanism for energy transfer and dissipation; thus, first order transitions possess non-trivial dynamics.

It is often possible to associate an order parameter with a phase transition. In a strict sense, the order parameter should be an elementary or composite field, the expectation value of which vanishes in one phase and is non-zero in another. In a generalized sense, we may refer to an order parameter even if it does not vanish in either phase, provided that (in a first order transition) it jumps across the phase boundary. In a particularly simple situation this role is taken by some elementary field; in the following, we consider the case where a real scalar field, $\phi$, plays the role of an order parameter. More realistically, $\phi$ could for instance be a neutral component of the Higgs doublet (after gauge fixing).

Suppose now that the Euclidean Lagrangian of the $\phi$-field reads

$$
L_{E}=\frac{1}{2}\left(\partial_{\tau} \phi\right)^{2}+\frac{1}{2}(\nabla \phi)^{2}+V(\phi)
$$

We take the potential to be of the form $V(\phi)=-\frac{1}{2} m^{2} \phi^{2}+\frac{1}{4} \lambda \phi^{4}$, with positive real parameters $m$ and $\lambda$ and a discrete $\mathrm{Z}(2)$ symmetry. Then $\phi$ has a non-zero expectation value at zero temperature, as is suggested by a graphical illustration of the potential in fig. 2 .

Let us now evaluate the partition function of the above system with the method of the effective potential, $V_{\text {eff }}(\bar{\phi})$, introduced in sec. 7.1. In other words, we put the system in a finite volume $V$, and denote by $\bar{\phi}$ the condensate, i.e. the mode with $p_{n}=0, \mathbf{p}=0$. As our system possesses no continuous symmetry, there are furthermore no conserved charges and thus we cannot introduce a chemical potential; only $T$ appears in the result after taking the $V \rightarrow \infty$ limit. We then write

$$
\begin{aligned}
\mathcal{Z}(V, T)=\exp \left[-\frac{V}{T} f(T)\right] & =\int_{-\infty}^{\infty} \mathrm{d} \bar{\phi} \int_{P \neq 0} \mathcal{D} \phi^{\prime} \exp \left(-S_{E}\left[\phi=\bar{\phi}+\phi^{\prime}\right]\right) \\
& \equiv \int_{-\infty}^{\infty} \mathrm{d} \bar{\phi} \exp \left[-\frac{V}{T} V_{\text {eff }}(\bar{\phi})\right]
\end{aligned}
$$

We note that the thermodynamic limit $V \rightarrow \infty$ is to be taken only after the evaluation of $V_{\text {eff }}(\bar{\phi})$, and that $\int_{0}^{\beta} \mathrm{d} \tau \int_{\mathbf{x}} \phi^{\prime}=0$, given that $\phi^{\prime}$ by definition only has modes with $P \neq 0$. 


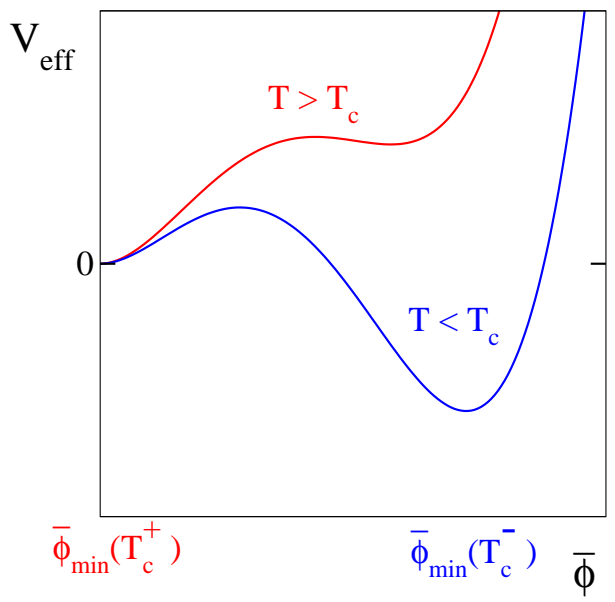

Figure 3: A thermal effective potential displaying a first order phase transition.

In order to carry out the integral in eq. (9.4), we expand $V_{\text {eff }}(\bar{\phi})$ around its absolute minimum $\bar{\phi}_{\min }$, and perform the corresponding Gaussian integral: ${ }^{30}$

$$
\begin{aligned}
V_{\text {eff }}(\bar{\phi}) & =V_{\text {eff }}\left(\bar{\phi}_{\text {min }}\right)+\frac{1}{2} V_{\text {eff }}^{\prime \prime}\left(\bar{\phi}_{\text {min }}\right)\left(\bar{\phi}-\bar{\phi}_{\text {min }}\right)^{2}+\ldots, \\
\int_{-\infty}^{\infty} \mathrm{d} \bar{\phi} \exp \left[-\frac{V}{T} V_{\text {eff }}(\bar{\phi})\right] & \approx \exp \left[-\frac{V}{T} V_{\text {eff }}\left(\bar{\phi}_{\min }\right)\right] \sqrt{\frac{2 \pi T}{V_{\text {eff }}^{\prime \prime}\left(\bar{\phi}_{\text {min }}\right) V}} .
\end{aligned}
$$

Thereby the free energy density reads

$$
f(T)=V_{\text {eff }}\left(\bar{\phi}_{\text {min }}\right)+\mathcal{O}\left(\frac{\ln V}{V}\right) .
$$

In other words, in the thermodynamic limit $V \rightarrow \infty$, the problem of computing $f(T)$ reduces to determining $V_{\text {eff }}$ and finding its minima. Note that $\bar{\phi}_{\min }$ depends on the parameters of the problem, particularly on $T$.

Let us now ask under which conditions a first order transition could emerge. We can write

$$
\begin{aligned}
\frac{\mathrm{d} f(T)}{\mathrm{d} T} & =\left[\frac{\partial V_{\mathrm{eff}}(\bar{\phi} ; T)}{\partial \bar{\phi}} \frac{\mathrm{d} \bar{\phi}_{\text {min }}}{\mathrm{d} T}+\frac{\partial V_{\text {eff }}(\bar{\phi} ; T)}{\partial T}\right]_{\bar{\phi}=\bar{\phi}_{\text {min }}} \\
& =\left.\frac{\partial V_{\text {eff }}(\bar{\phi} ; T)}{\partial T}\right|_{\bar{\phi}=\bar{\phi}_{\text {min }}},
\end{aligned}
$$

where we have written out the explicit temperature dependence of the effective potential and made use of the fact that $\bar{\phi}_{\text {min }}$ minimizes $V_{\text {eff }}$. Eq. (9.9) makes it clear (if $V_{\text {eff }}$ is an analytic function of its arguments) that $\lim _{T \rightarrow T_{c}^{+}} \frac{\mathrm{d} f}{\mathrm{~d} T} \neq \lim _{T \rightarrow T_{c}^{-}} \frac{\mathrm{d} f}{\mathrm{~d} T}$ only if $\lim _{T \rightarrow T_{c}^{+}} \bar{\phi}_{\min } \neq \lim _{T \rightarrow T_{c}^{-}} \bar{\phi}_{\min }$. In other words, a first order transition necessitates a discontinuity in $\bar{\phi}_{\text {min }}$, such as is the case in the potential illustrated in fig. 3 .

Given the above considerations, our task becomes to evaluate $V_{\text {eff }}$. Before proceeding with the computation, let us formulate the generic rules that follow from the analogy between the definition of the quantity,

$$
\exp \left[-\frac{V}{T} V_{\mathrm{eff}}(\bar{\phi})\right]=\int_{P \neq 0} \mathcal{D} \phi^{\prime} \quad \exp \left(-S_{E}\left[\phi=\bar{\phi}+\phi^{\prime}\right]\right)
$$

\footnotetext{
${ }^{30}$ To be precise an infinitesimal "source" should be added in order to pick a unique minimum.
} 
and that of the free energy density, $f(T)$, discussed in sec. 3.1:

(i) Write $\phi=\bar{\phi}+\phi^{\prime}$ in $L_{E}$.

(ii) The part only depending on $\bar{\phi}$ is the zeroth order, or tree-level, contribution to $V_{\text {eff }}$.

(iii) Any terms linear in $\phi^{\prime}$ should be omitted, because $\int_{0}^{\beta} \mathrm{d} \tau \int_{\mathbf{x}} \phi^{\prime}=0$.

(iv) The remaining contributions to $V_{\text {eff }}$ are obtained like $f(T)$ before, cf. eq. (3.12), except that the masses and couplings of $\phi^{\prime}$ now depend on the "shift" $\bar{\phi}$.

(v) However, among all possible connected diagrams, one-particle-reducible graphs (i.e. graphs where the cutting of a single $\phi^{\prime}$-propagator would split the graph into two disjoint parts) should be omitted, since such a $\phi^{\prime}$-propagator would necessarily carry zero momentum, which is excluded by the above definition.

Remarkably, as noted in ref. [9.1], these rules are identical to the rules that follow [9.2] from a totally different (but "standard") definition of the effective potential, based on a Legendre transform of the generating functional:

$$
\begin{aligned}
e^{-W[J]} & \equiv \int \mathcal{D} \phi e^{-S_{E}-\int_{X} \phi J}, \\
\Gamma[\bar{\phi}] & \equiv W[J]-\int_{X} J \bar{\phi}, \quad \bar{\phi} \equiv \frac{\delta W[J]}{\delta J}, \\
V_{\mathrm{eff}}(\bar{\phi}) & \equiv \frac{T}{V} \Gamma[\bar{\phi}] \quad \text { for } \quad \bar{\phi}=\text { constant } .
\end{aligned}
$$

However, our procedure is actually better than the Legendre transform one, because it is defined for any value of $\bar{\phi}$, whereas the existence of a Legendre transform requires certain (invertibility) properties from the functions concerned, which has led to numerous discussions about whether the effective potential necessarily needs to be a convex function.

Let us now proceed to the practical computation. Implementing steps (i) and (ii), and indicating terms dropped in step (iii) by square brackets, we get

$$
\begin{aligned}
\frac{1}{2}\left(\partial_{\mu} \phi\right)^{2} & \rightarrow \frac{1}{2}\left(\partial_{\mu} \phi^{\prime}\right)^{2} \\
-\frac{1}{2} m^{2} \phi^{2} & \rightarrow-\frac{1}{2} m^{2} \bar{\phi}^{2}-\left[m^{2} \bar{\phi} \phi^{\prime}\right]-\frac{1}{2} m^{2} \phi^{\prime 2} \\
\frac{1}{4} \lambda \phi^{4} & \rightarrow \frac{1}{4} \lambda \bar{\phi}^{4}+\left[\lambda \bar{\phi}^{3} \phi^{\prime}\right]+\frac{3}{2} \lambda \bar{\phi}^{2} \phi^{\prime 2}+\lambda \bar{\phi} \phi^{\prime 3}+\frac{1}{4} \lambda \phi^{\prime 4}, \\
\int_{0}^{\beta} \mathrm{d} \tau \int_{\mathbf{x}} & =\frac{V}{T} \\
V_{\mathrm{eff}}^{(0)}(\bar{\phi}) & =-\frac{1}{2} m^{2} \bar{\phi}^{2}+\frac{1}{4} \lambda \bar{\phi}^{4},
\end{aligned}
$$

where one should in particular note that $V_{\text {eff }}^{(0)}(\bar{\phi})$ is independent of the temperature $T$.

The dominant "thermal fluctuations" or "radiative corrections" arise at the 1-loop order, and follow from the part quadratic in $\phi^{\prime}$ as linear terms are dropped. Combining eqs. (9.15) and (9.16), the "effective" mass of $\phi^{\prime}$ reads $m_{\text {eff }}^{2} \equiv-m^{2}+3 \lambda \bar{\phi}^{2}$, and the corresponding contribution to the effective potential becomes

$$
\exp \left(-\frac{V}{T} V_{\text {eff }}^{(1)}\right)=\int \mathcal{D} \phi^{\prime} \exp \left(-\int_{0}^{\beta} \mathrm{d} \tau \int_{\mathbf{x}} \frac{1}{2} \phi^{\prime}\left[-\partial_{\mu}^{2}+m_{\text {eff }}^{2}\right] \phi^{\prime}\right)
$$



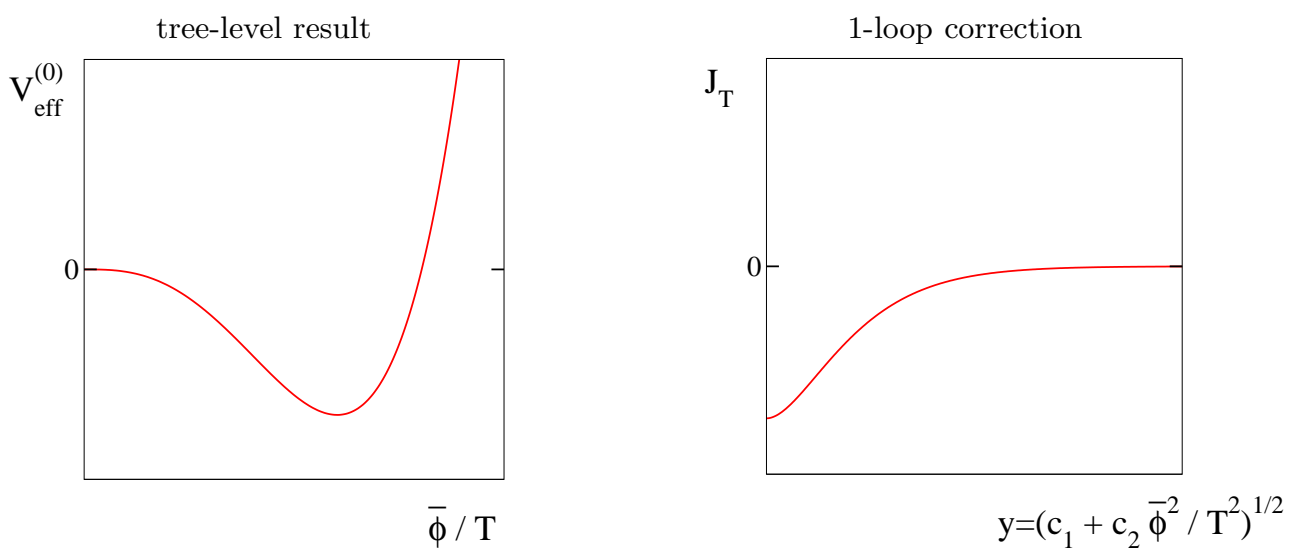

Figure 4: A comparison of the shapes of the tree-level zero-temperature potential and the 1-loop thermal correction. The function $J_{T}$ is given in eq. (9.27).

$$
\begin{aligned}
& =\int \mathcal{D} \tilde{\phi}^{\prime} \exp \left(-\frac{T}{V} \sum_{p_{n}, \mathbf{p}} \frac{1}{2} \tilde{\phi}^{\prime}\left[p_{n}^{2}+\mathbf{p}^{2}+m_{\mathrm{eff}}^{2}\right] \tilde{\phi}^{\prime}\right) \\
& =\mathcal{C}\left[\prod_{P \neq 0}\left(p_{n}^{2}+\mathbf{p}^{2}+m_{\mathrm{eff}}^{2}\right)\right]^{-\frac{1}{2}} ; \\
V_{\mathrm{eff}}^{(1)}(\bar{\phi}) & =\lim _{V \rightarrow \infty} \frac{T}{V} \sum_{P \neq 0}\left[\frac{1}{2} \ln \left(p_{n}^{2}+\mathbf{p}^{2}+m_{\mathrm{eff}}^{2}\right)-\text { const. }\right] .
\end{aligned}
$$

In the infinite-volume limit, where the omission of the zero mode is irrelevant, this goes over to the function $J\left(m_{\mathrm{eff}}, T\right)$ defined in eqs. (2.50) and (2.51). We return to the properties of this function presently, but let us first specify how higher-order corrections to this result can be obtained.

Higher-order corrections come from the remaining terms in eq. (9.16), paying attention to rules (iv) and (v):

$$
\begin{aligned}
\exp \left[-\frac{V}{T} V_{\mathrm{eff}}^{(\geq 2)}(\bar{\phi})\right] & =\left\langle\exp \left(-S_{E, I}\left[\bar{\phi}, \phi^{\prime}\right]\right)-1\right\rangle_{1 \mathrm{PI}} \\
S_{E, I}\left[\bar{\phi}, \phi^{\prime}\right] & =\int_{0}^{\beta} \mathrm{d} \tau \int_{\mathbf{x}}\left[\lambda \bar{\phi} \phi^{\prime 3}+\frac{1}{4} \lambda \phi^{\prime 4}\right]
\end{aligned}
$$

Here the propagator to be used reads

$$
\left\langle\tilde{\phi}^{\prime}(P) \tilde{\phi}^{\prime}(Q)\right\rangle=\frac{V}{T} \delta_{P,-Q} \frac{1}{p_{n}^{2}+\mathbf{p}^{2}+m_{\mathrm{eff}}^{2}} .
$$

The $V \rightarrow \infty$ limit leads to a scalar propagator, eq. (3.27), with the mass $m_{\text {eff }}$.

We now return to the evaluation of the 1-loop effective potential after taking $V \rightarrow \infty$. From eq. (2.50) we have

$$
V_{\mathrm{eff}}^{(1)}(\bar{\phi})=\int_{\mathbf{p}}\left[\frac{\epsilon_{p}}{2}+T \ln \left(1-e^{-\beta \epsilon_{p}}\right)\right]_{\epsilon_{p}=\sqrt{p^{2}+m_{\mathrm{eff}}^{2}}},
$$

the temperature-dependent part of which is given by eq. (2.58): ${ }^{31}$

$$
J_{T}\left(m_{\mathrm{eff}}\right)=\int_{\mathbf{p}} T \ln \left(1-e^{-\beta \epsilon_{p}}\right) \stackrel{d=3}{=} \frac{T^{4}}{2 \pi^{2}} \int_{0}^{\infty} \mathrm{d} x x^{2} \ln \left[1-e^{-\sqrt{x^{2}+y^{2}}}\right]_{y=\frac{m_{\mathrm{eff}}}{T}} .
$$

\footnotetext{
${ }^{31}$ Note that even though $\bar{\phi}$-dependent, the $T=0$ part "only" renormalizes the parameters $m^{2}$ and $\lambda$ that appear in $V_{\mathrm{eff}}^{(0)}$. These are important effects in any quantitative study but can be omitted for a qualitative understanding.
} 


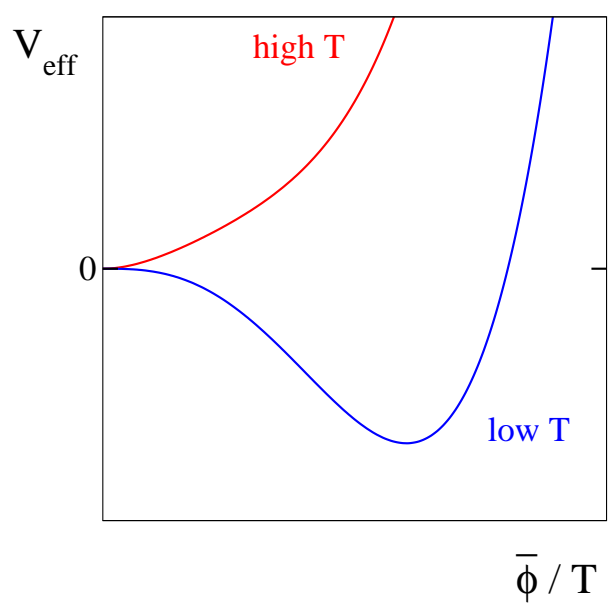

Figure 5: An illustration of the effective potential in eq. (9.29), possessing a phase transition.

This function was evaluated in fig. 1 on p. 28; its shape in comparison with the zero-temperature potential is illustrated in fig. 4. Clearly, the symmetric minimum becomes more favourable (has a smaller free energy density) at higher temperatures.

In order to be more quantitative, let us study what happens at $\pi T \gg m_{\text {eff }}$ where, from eq. (2.82),

$$
J_{T}\left(m_{\mathrm{eff}}\right)=-\frac{\pi^{2} T^{4}}{90}+\frac{m_{\mathrm{eff}}^{2} T^{2}}{24}-\frac{m_{\mathrm{eff}}^{3} T}{12 \pi}-\frac{m_{\mathrm{eff}}^{4}}{2(4 \pi)^{2}}\left[\ln \left(\frac{m_{\mathrm{eff}} e^{\gamma_{\mathrm{E}}}}{4 \pi T}\right)-\frac{3}{4}\right]+\mathcal{O}\left(\frac{m_{\mathrm{eff}}^{6}}{\pi^{4} T^{2}}\right) .
$$

Keeping just the leading mass-dependent term leads to

$$
V_{\mathrm{eff}}^{(0)}+V_{\mathrm{eff}}^{(1)}=[\bar{\phi} \text {-indep. }]+\frac{1}{2}\left(-m^{2}+\frac{\lambda T^{2}}{4}\right) \bar{\phi}^{2}+\frac{1}{4} \lambda \bar{\phi}^{4} .
$$

We already knew that for $T=0$ the symmetry is broken; from here we observe that for $T \gg 2 \mathrm{~m} / \sqrt{\lambda}$ it is restored. For the Standard Model Higgs field, this was realized in refs. [9.3]-[9.6]. Hence, there must be a phase transition of some kind; this is sketched in fig. 5 .

We may subsequently ask a refined question, namely, what is the order of the transition? In order to get a first impression, let us include the next term from eq. (9.28) in the effective potential. Proceeding for easier illustration to the $m^{2} \rightarrow 0$ limit, we thereby obtain

$$
V_{\mathrm{eff}}^{(0)}+V_{\mathrm{eff}}^{(1)}=[\bar{\phi} \text {-indep. }]+\frac{\lambda}{8} T^{2} \bar{\phi}^{2}-\frac{T}{12 \pi}(3 \lambda)^{3 / 2}|\bar{\phi}|^{3}+\frac{1}{4} \lambda \bar{\phi}^{4}
$$

This could describe a "fluctuation induced" first order transition, as is illustrated in fig. 6 .

We should not rush to conclusions, however. Indeed, it can be seen from eq. (9.30) that the broken minimum appears where the cubic and quartic terms are of similar magnitudes, i.e.,

$$
\frac{T \lambda^{\frac{3}{2}}|\bar{\phi}|^{3}}{\pi} \sim \lambda|\bar{\phi}|^{4} \Rightarrow|\bar{\phi}| \sim \frac{\lambda^{\frac{1}{2}} T}{\pi} .
$$

However, the expansion parameter related to higher-order corrections, discussed schematically in sec. 6.1 , then becomes

$$
\frac{\lambda T}{\pi m_{\mathrm{eff}}} \sim \frac{\lambda T}{\pi \sqrt{3 \lambda \bar{\phi}^{2}}} \sim \frac{\lambda^{\frac{1}{2}} T}{\pi|\bar{\phi}|} \sim \mathcal{O}(1)
$$




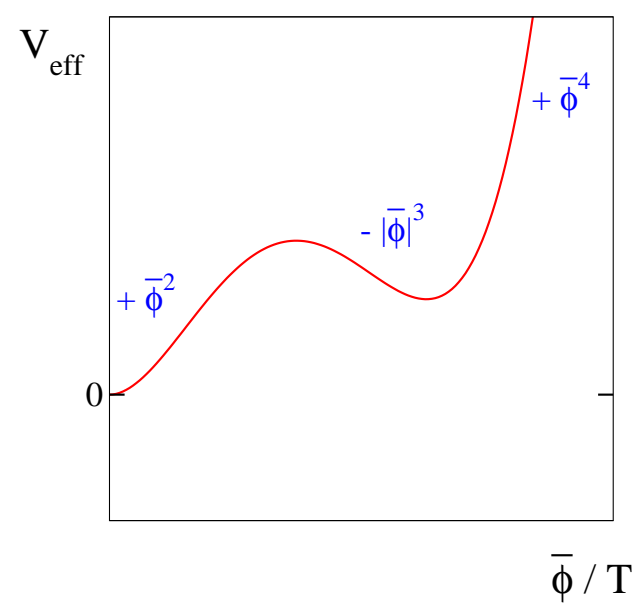

Figure 6: A sketch of the structure described by eq. (9.30).

In other words, the perturbative prediction is not reliable for the order of the transition.

On the other hand, a reliable analysis can again be carried out with effective field theory techniques, as discussed in sec. 6.2. In the case of a scalar field theory, the dimensionally reduced action takes the form

$$
S_{\mathrm{eff}}=\frac{1}{T} \int_{\mathbf{x}}\left[\frac{1}{2}\left(\partial_{i} \phi_{3}\right)^{2}+\frac{1}{2} m_{3}^{2} \phi_{3}^{2}+\frac{1}{4} \lambda_{3} \phi_{3}^{4}+\ldots\right],
$$

with the effective couplings reading

$$
\begin{aligned}
m_{3}^{2} & =-m_{\mathrm{R}}^{2}\left[1+\mathcal{O}\left(\lambda_{\mathrm{R}}\right)\right]+\frac{1}{4} \lambda_{\mathrm{R}} T^{2}\left[1+\mathcal{O}\left(\lambda_{\mathrm{R}}\right)\right] \\
\lambda_{3} & =\lambda_{\mathrm{R}}\left[1+\mathcal{O}\left(\lambda_{\mathrm{R}}\right)\right] .
\end{aligned}
$$

This system can be studied non-perturbatively (e.g. with lattice simulations) to show that there is a second order transition at $m_{3}^{2} \approx 0$. The transition belongs to the $3 \mathrm{~d}$ Ising universality class. ${ }^{32}$

Finally, we note that if the original theory is more complicated (containing more fields and coupling constants), it is often possible to arrange the couplings so that the first order signature seen in perturbation theory is physical. Examples of systems where this happens include:

- A theory with two real scalar fields can have a first order transition, if the couplings between the two fields are tuned appropriately $[9.9,9.10]$. An example is given in appendix A.

- A theory with a complex scalar field and $U(1)$ gauge symmetry, which happens to form the Ginzburg-Landau theory of superconductivity, does have a first order transition, if the quartic coupling $\lambda_{\mathrm{R}}$ is small enough compared with the electric coupling squared, $e_{\mathrm{R}}^{2}[9.11]$.

- The standard electroweak theory, with a Higgs doublet and $\mathrm{SU}(2) \times \mathrm{U}(1)$ gauge symmetry, can also have a first order transition if the scalar self-coupling $\lambda_{\mathrm{R}}$ is small enough [9.12,9.13]. However, this possibility is not realized for the physical value of the Higgs mass $m_{H} \approx$

\footnotetext{
${ }^{32}$ To be precise we should note that scalar field theories suffer from the so-called "triviality" problem (cf. e.g. refs. $[9.7,9.8])$ : the only 4-dimensional continuum theory which is defined on a non-perturbative level is the one with $\lambda_{\mathrm{R}}=0$. Therefore, our discussion implicitly concerns a scalar field theory which has a finite ultraviolet cutoff.
} 
$125 \mathrm{GeV}$ (for a review, see ref. [9.14]). On the other hand, in many extensions of the Standard Model, for instance in theories containing more than one scalar field, first order phase transitions have been found (for a review see, e.g., ref. [9.15]).

If the transition is of first order, its real-time dynamics is non-trivial. Upon lowering the temperature, such a transition normally proceeds through supercooling and a subsequent nucleation of bubbles of the low-temperature phase, which then expand rapidly and fill the volume. (If bubble nucleation does not have time to take place due to very fast cooling, it is possible to enter a regime of "spinodal decomposition" in which any "barrier" between the two phases disappears.) We turn to this problem in the next section.

\section{Appendix A: Strong phase transition with two scalar fields}

Let us generalize the theory in eq. (9.2) by including another scalar field, denoted by $\chi$ :

$$
\begin{aligned}
L_{E} & =\frac{1}{2} \partial_{\mu} \phi \partial_{\mu} \phi+\frac{1}{2} \partial_{\mu} \chi \partial_{\mu} \chi+V(\phi, \chi) \\
V(\phi, \chi) & =-\frac{m^{2} \phi^{2}}{2}+\frac{\lambda \phi^{4}}{4}-\frac{M^{2} \chi^{2}}{2}+\frac{\kappa \chi^{4}}{4}+\frac{\gamma \phi^{2} \chi^{2}}{2} .
\end{aligned}
$$

The form of the coupling between the fields has been constrained by imposing a $\mathrm{Z}(2)$ symmetry. The dimensionally reduced theory is like in eq. (9.33), with the potential having the form

$$
V_{3}\left(\phi_{3}, \chi_{3}\right)=\frac{m_{3}^{2} \phi_{3}^{2}}{2}+\frac{\lambda_{3} \phi_{3}^{4}}{4}+\frac{M_{3}^{2} \chi_{3}^{2}}{2}+\frac{\kappa_{3} \chi_{3}^{4}}{4}+\frac{\gamma_{3} \phi_{3}^{2} \chi_{3}^{2}}{2}+\ldots
$$

The most important parameters are the thermal masses,

$$
m_{3}^{2} \approx-m^{2}+\frac{(3 \lambda+\gamma) T^{2}}{12}, \quad M_{3}^{2} \approx-M^{2}+\frac{(3 \kappa+\gamma) T^{2}}{12} .
$$

Therefore, the respective symmetries tend to get restored at (we assume $\gamma>0$ here)

$$
T_{\phi}^{2} \approx \frac{12 m^{2}}{3 \lambda+\gamma}, \quad T_{\chi}^{2} \approx \frac{12 M^{2}}{3 \kappa+\gamma} .
$$

Here the parameters are renormalized even if for simplicity we do not show the subscripts \{\}$_{R}$.

The idea is now as follows. Suppose that as we cool down from very high temperatures, the symmetry gets first broken in the $\chi$-direction. According to eq. (9.40), this requires $T_{\chi}>T_{\phi}$, i.e.

$$
\frac{M^{2}}{m^{2}}>\frac{3 \kappa+\gamma}{3 \lambda+\gamma}
$$

At a lower temperature, we assume that the minimum with a non-zero expectation value of $\phi$ becomes deeper, so that we go to the "usual" vacuum. Then, as we will see, the two minima may be connected by a saddle point in between, and the transition can become strong. This scenario is known as a "two-stage" or "two-step" transition [9.10].

In order to see if this can work, let us consider the extrema of the potential at low temperatures $\left(T \ll T_{\phi}, T_{\chi}\right)$. Extrema are found at

$$
\partial_{\phi} V=\partial_{\chi} V=0 \Leftrightarrow\left\{\begin{aligned}
0 & =\phi\left(-m^{2}+\lambda \phi^{2}+\gamma \chi^{2}\right), \\
0 & =\chi\left(-M^{2}+\kappa \chi^{2}+\gamma \phi^{2}\right) .
\end{aligned}\right.
$$


There are four solutions, which we label as

$$
\begin{array}{ll}
\text { (a) : } & \phi=\chi=0, \\
\text { (b) : } & \phi=0, \quad \chi^{2}=\frac{M^{2}}{\kappa}, \\
\text { (c) : } & \chi=0, \quad \phi^{2}=\frac{m^{2}}{\lambda}, \\
\text { (d) : } & \left(\begin{array}{c}
\phi^{2} \\
\chi^{2}
\end{array}\right)=\frac{1}{\lambda \kappa-\gamma^{2}}\left(\begin{array}{c}
\kappa m^{2}-\gamma M^{2} \\
\lambda M^{2}-\gamma m^{2}
\end{array}\right) .
\end{array}
$$

Then we need to inspect which of these are minima, by considering the "mass matrix"

$$
\left(\begin{array}{cc}
\partial_{\phi}^{2} V & \partial_{\phi} \partial_{\chi} V \\
\partial_{\phi} \partial_{\chi} V & \partial_{\chi}^{2} V
\end{array}\right)=\left(\begin{array}{cc}
-m^{2}+3 \lambda \phi^{2}+\gamma \chi^{2} & 2 \gamma \phi \chi \\
2 \gamma \phi \chi & -M^{2}+3 \kappa \chi^{2}+\gamma \phi^{2}
\end{array}\right)
$$

Inserting eq. (9.43), the extremum (a) is a local maximum. Inserting eqs. (9.44) and (9.45), the extrema (b) and (c) are local minima if

$$
\begin{aligned}
& \text { (b) minimum } \Leftrightarrow \frac{M^{2}}{m^{2}}>\frac{\kappa}{\gamma}, \\
& \text { (c) minimum } \Leftrightarrow \frac{M^{2}}{m^{2}}<\frac{\gamma}{\lambda} .
\end{aligned}
$$

It follows from eqs. (9.48) and (9.49) that $\gamma^{2}>\lambda \kappa$ (recalling that we consider the case $\gamma>0$ here). By inserting eq. (9.46) into eq. (9.47) and taking the determinant, it can be verified that under these conditions the determinant is negative, i.e. the extremum (d) is a saddle point.

Finally, consider the value of the potential at the local minima. A substitution shows that

$$
V\left(0, \chi_{\min }\right)=-\frac{M^{4}}{4 \kappa}, \quad V\left(\phi_{\min }, 0\right)=-\frac{m^{4}}{4 \lambda} .
$$

The desired $\phi_{\min }$-minimum is realized at low temperatures if $\left|V\left(\phi_{\min }, 0\right)\right|>\left|V\left(0, \chi_{\min }\right)\right|$, i.e.

$$
\frac{M^{2}}{m^{2}}<\sqrt{\frac{\kappa}{\lambda}}
$$

In total, we thus have four constraints to satisfy, eqs. (9.41), (9.48), (9.49) and (9.51). Denoting $\hat{\kappa} \equiv \kappa / \gamma$ and $\hat{\lambda} \equiv \lambda / \gamma$, all of these can be respected simultaneously if

$$
\hat{\lambda} \in\left(0, \frac{1}{3}\right) \Rightarrow \hat{\kappa} \in\left(\hat{\lambda}, \frac{1}{9 \hat{\lambda}}\right), \quad \hat{\lambda} \in\left(\frac{1}{3}, 1\right) \Rightarrow \hat{\kappa} \in\left(\frac{1}{9 \hat{\lambda}}, \hat{\lambda}\right), \quad \hat{\lambda}>1 \Rightarrow \hat{\kappa} \in\left(\frac{1}{9 \hat{\lambda}}, \frac{1}{\hat{\lambda}}\right) .
$$

Given that we did not rely on loop effects but only on tree-level structures, and that the final transition can take place at a low temperature compared with vacuum mass scales, we expect that expansion parameters such as eq. (9.32) can be kept small, and that there indeed is a first order transition in this system. 


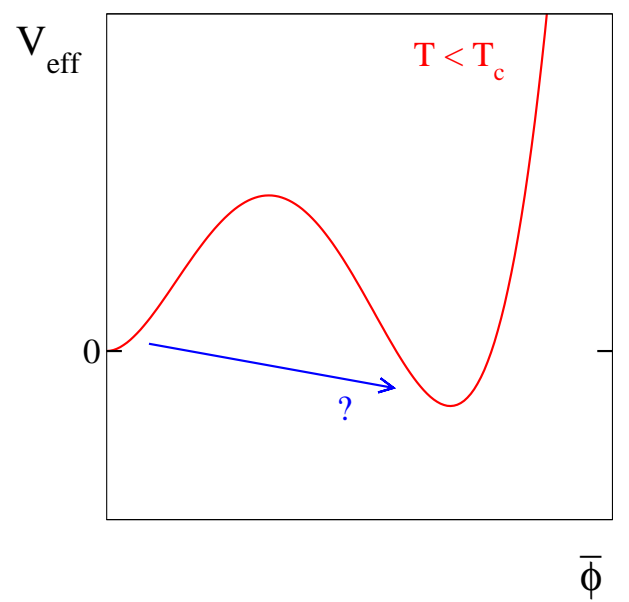

Figure 7: An illustration of the tunnelling process which a metastable high-temperature state needs to undergo in a first order phase transition.

\subsection{Bubble nucleation rate}

As was mentioned in the previous section, if a first order transition takes place its dynamics is nontrivial, because the discontinuity in energy density ("latent heat") released needs to be transported or dissipated away. The basic mechanism for this is bubble nucleation and growth: the transition does not take place exactly at the critical temperature, $T_{\mathrm{c}}$, but upon lowering the temperature the system first supercools to some nucleation temperature, $T_{\mathrm{n}}$. Around this point bubbles of the stable phase form, and start to grow; normally (in the case of a "deflagration") the latent heat is transported away in a hydrodynamic shock wave which precedes the expanding bubble.

The purpose of this section is to determine the probability of bubble nucleation, per unit time and volume, at a given temperature $T<T_{\mathrm{c}}$, having in mind the phase transitions taking place in the early universe. Combined with the cosmological evolution equation for the temperature $T$, which determines the rate $\dot{T}(t)$ with which the system passes through the transition point (cf. sec. 9.4), this would in principle allow us to estimate $T_{\mathrm{n}}$ (cf. appendix B). We will, however, not get into explicit estimates here, but rather illustrate aspects of the general formalism, given that it is analogous to several other "rate" computations in quantum field theory, such as the determination of the rate of baryon plus lepton number violation in the Standard Model.

In terms of the effective potential, the general setting can be illustrated as shown in fig. 7. For simplicity, we consider a situation in which a barrier between the minima already exists in the tree-level potential $V(\bar{\phi})$. For radiatively generated transitions, in which a barrier only appears in $V_{\text {eff }}(\bar{\phi})$, some degrees of freedom need to be integrated out for the discussion to apply.

Our starting point now is an attempt at a definition of what is meant with the nucleation rate. It turns out that this task is rather non-trivial; in fact, it is not clear whether a completely general definition can be given at all. Nevertheless, for many practical purposes, the so-called Langer formalism $[9.16,9.17]$ appears sufficient.

The general idea is the following. Consider first a system at zero temperature. Suppose we use boundary conditions at spatial infinity, $\lim _{|\mathbf{x}| \rightarrow \infty} \phi(\mathbf{x})=0$, in order to define metastable energy eigenstates. We could imagine that, as a result of the vacuum fluctuations taking place, the time 
evolution of these would-be states looks like

$$
\begin{aligned}
& |\phi(t)\rangle=e^{-i E t}|\phi(0)\rangle=e^{-i[\operatorname{Re}(E)+i \operatorname{Im}(E)] t}|\phi(0)\rangle \\
\Rightarrow \quad & \langle\phi(t) \mid \phi(t)\rangle=e^{2 \operatorname{Im}(E) t}\langle\phi(0) \mid \phi(0)\rangle .
\end{aligned}
$$

Thereby we could say that such a metastable state possesses a decay rate, $\Gamma(E)$, given by

$$
\Gamma(E) \simeq-2 \operatorname{Im}(E)
$$

Moving to a thermal ensemble, we could analogously expect that

$$
\Gamma(T) \stackrel{?}{\simeq}-2 \operatorname{Im}(F)
$$

where $F$ is the free energy of the system, defined in the usual way. It should be stressed, though, that this generalization is just a guess: it would be next to miraculous if a real-time observable, the nucleation rate, could be determined exactly from a Euclidean observable, the free energy.

To inspect the nature of our intuitive guess, we first pose the question whether $F$ could indeed develop an imaginary part. It turns out that the answer to this is positive, as can be seen via the following argument [9.18]. Consider the path integral expression for the partition function,

$$
F=-T \ln \left\{\int_{\text {b.c. }} \mathcal{D} \phi \exp \left(-S_{E}[\phi]\right)\right\},
$$

where "b.c." refers to the usual periodic boundary conditions. Let us assume that we can find (at least) two different saddle points $\hat{\phi}$, each satisfying

$$
\left.\frac{\delta S_{E}}{\delta \phi}\right|_{\phi=\hat{\phi}}=0, \quad \hat{\phi}(0, \mathbf{x})=\hat{\phi}(\beta, \mathbf{x}), \quad \lim _{|\mathbf{x}| \rightarrow \infty} \hat{\phi}(\tau, \mathbf{x})=0 .
$$

We assume that one of the solutions is the trivial one, $\hat{\phi} \equiv 0$, whereas the other is a non-trivial (i.e. $\mathbf{x}$-dependent) solution, which we henceforth denote by $\hat{\phi}(\tau, \mathbf{x})$.

Let us now consider fluctuations around the non-trivial saddle point, which we assume to have an unstable direction. Suppose for simplicity that the fluctuation operator around $\hat{\phi}$ has exactly one negative eigenmode

$$
\left.\frac{\delta^{2} S_{E}}{\delta \phi^{2}}\right|_{\phi=\hat{\phi}} f_{-}(\tau, \mathbf{x})=-\lambda_{-}^{2} f_{-}(\tau, \mathbf{x}),
$$

whereas for the non-negative modes we define the eigenvalues through

$$
\left.\frac{\delta^{2} S_{E}}{\delta \phi^{2}}\right|_{\phi=\hat{\phi}} f_{n}(\tau, \mathbf{x})=\lambda_{n}^{2} f_{n}(\tau, \mathbf{x}), \quad n \geq 0 .
$$

Writing now a generic deviation of the field $\phi$ from the saddle point solution in the form

$$
\delta \phi=\phi-\hat{\phi}=\sum_{n} \delta \phi_{n} \equiv \sum_{n} c_{n} f_{n}
$$

where $c_{n}$ are coefficients (which we assume, for simplicity, to be real), and taking the eigenfunctions to be orthonormal $\left(\int_{X} f_{m} f_{n}=\delta_{m n}\right)$, we can define the integration measure over the fluctuations as

$$
\int \mathcal{D} \phi \equiv \prod_{n} \int \frac{\mathrm{d} c_{n}}{\sqrt{2 \pi}}
$$



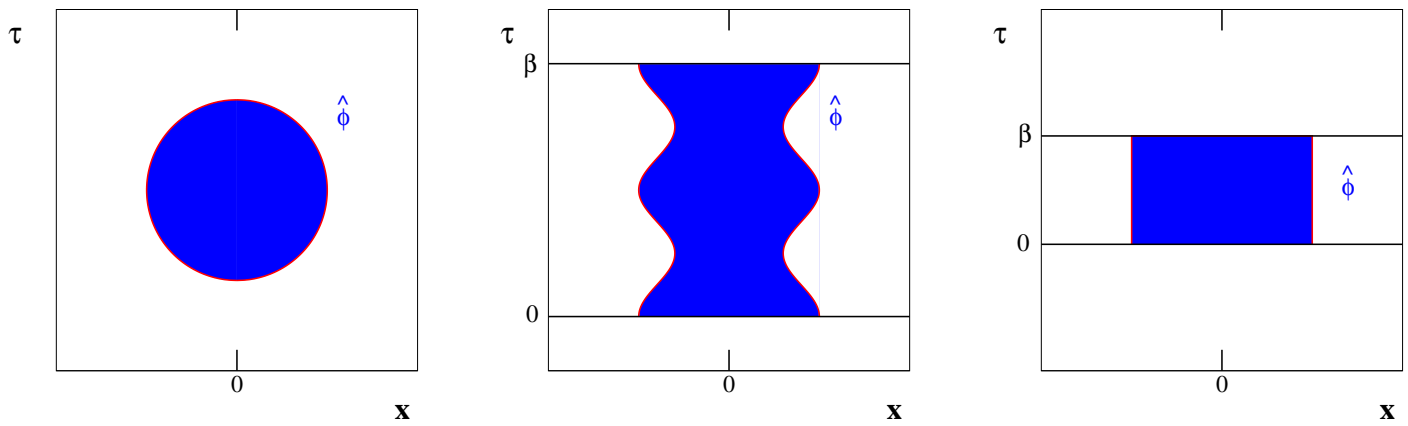

Figure 8: The form of the non-trivial saddle point solution in various regimes: at zero temperature (left); at an intermediate temperature (middle); and at a high temperature (right).

In the vicinity of the saddle point, the action can be written in terms of the eigenvalues and coefficients as

$$
S_{E}[\phi] \approx S_{E}[\hat{\phi}]+\int_{X} \frac{1}{2} \delta \phi \frac{\delta^{2} S_{E}[\hat{\phi}]}{\delta \phi^{2}} \delta \phi=S_{E}[\hat{\phi}]-\frac{1}{2} \lambda_{-}^{2} c_{-}^{2}+\sum_{n \geq 0} \frac{1}{2} \lambda_{n}^{2} c_{n}^{2} .
$$

Then, denoting $\mathcal{Z}_{0} \equiv \mathcal{Z}[\hat{\phi}=0]$, we can use the semiclassical approximation to write the free energy in a form where the contributions of both saddle points are separated,

$$
F \sim-T \ln \left\{\mathcal{Z}_{0}+e^{-S_{E}[\hat{\phi}]} \int \frac{\mathrm{d} c_{-}}{\sqrt{2 \pi}} e^{\frac{1}{2} \lambda_{-}^{2} c_{-}^{2}} \int \prod_{n \geq 0} \frac{\mathrm{d} c_{n}}{\sqrt{2 \pi}} e^{-\frac{1}{2} \lambda_{n}^{2} c_{n}^{2}}\right\} .
$$

Dealing with the negative eigenmode properly would require a careful analysis, but in the end this leads (up to a factor $1 / 2$ ) to the intuitive result

$$
\int \frac{\mathrm{d} c_{-}}{\sqrt{2 \pi}} e^{\frac{1}{2} \lambda_{-}^{2} c_{-}^{2}} \sim \frac{1}{\sqrt{2 \pi}} \sqrt{\frac{2 \pi}{-\lambda_{-}^{2}}} \sim i \sqrt{\frac{1}{\lambda_{-}^{2}}},
$$

indicating that the partition function indeed obtains an imaginary part. Assuming furthermore that the contribution from the trivial saddle point is much larger in absolute magnitude than that originating from the non-trivial one, the evaluation of eq. (9.56) leads to

$$
\Gamma \sim \frac{T}{\mathcal{Z}_{0}} \exp \left\{-S_{E}[\hat{\phi}]\right\}\left|\operatorname{det}\left(\delta^{2} S_{E}[\hat{\phi}] / \delta \phi^{2}\right)\right|^{-\frac{1}{2}}
$$

where the determinant is simply the product of all eigenvalues. Somewhat more precise versions of this formula will be given in eqs. (9.77) and (9.80) below.

The non-trivial saddle point contributing to the partition function is referred to as an instanton, or, if its shape is modified by a finite temporal extent, a caloron. By definition, an instanton is a solution of the imaginary-time classical equations of motion, but it describes the exponential factor in the rate of a real-time transition, as suggested by the intuitive considerations above.

Of course, the instanton needs to respect the boundary conditions of eq. (9.58). Depending on the geometric shape of the instanton solution within these constraints, we can give different physical interpretations to the kind of "tunnelling" that the instanton describes. In the simplest case, when the temperature is very low $(\beta=1 / T$ is very large), the Euclidean time direction is identical to the space directions, and we can expect that the solution has $4 \mathrm{~d}$ rotational symmetry, 
as illustrated in fig. 8(left). Such a solution is said to describe "quantum tunnelling". Indeed, had we kept $\hbar \neq 1$, eq. (9.66) would have had the exponential $\exp \left\{-S_{E}[\hat{\phi}] / \hbar\right\}$.

On the other hand, if the temperature increases and $\beta$ decreases, the four-volume becomes "squeezed", and this affects the form of the solution [9.19]. This caloron is depicted in fig. 8(middle). Then we can say that "quantum tunnelling" and "thermal fluctuations" both play a role.

For very large $T$, the box becomes very squeezed, and we expect that the solution only respects $3 \mathrm{~d}$ rotational symmetry, as shown in fig. 8(right). In this situation, like in dimensional reduction, we can factorize and perform the integration over the $\tau$-coordinate, and the instanton action becomes

$$
\frac{1}{\hbar} S_{E}[\hat{\phi}]=\frac{1}{\hbar} \beta \hbar \int_{\mathbf{x}} L_{E} \equiv \beta S_{3 \mathrm{~d}}[\hat{\phi}] .
$$

We say that the transition takes place through "classical thermal fluctuations".

In typical cases, the action appearing in the exponent is large, and thereby the exponential is very small. Just how small it is, is determined predominantly by the instanton action, rather than the fluctuation determinant which does not have any exponential factors, and is therefore "of order unity". Hence we can say that the instanton solution and its Euclidean action $S_{E}[\hat{\phi}]$ play the dominant role in determining the nucleation rate.

At the same time, from a theoretical point of view, it can be said that the real "art" in solving the problem is the computation of the fluctuation determinant around the saddle point solution [9.20]. In fact, the eigenmodes of the fluctuation operator can be classified into:

(1) one negative mode;

(2) a number of zero modes;

(3) infinitely many positive modes.

We have already addressed the negative mode (except for showing that there is only one), which is responsible for the imaginary part, so let us now look at the zero modes, whose normalization turns out to be somewhat non-trivial.

The existence and multiplicity of the zero modes can be deduced from the classical equations of motion and from the expression of the fluctuation operator. Indeed, assuming the action to be of the form

$$
S_{E}=\int_{0}^{\beta} \mathrm{d} \tau \int_{\mathbf{x}}\left[\frac{1}{2}\left(\partial_{\mu} \phi\right)^{2}+V(\phi)\right]
$$

the classical equations of motion read

$$
\frac{\delta S_{E}[\hat{\phi}]}{\delta \phi}=0 \quad \Leftrightarrow \quad-\partial_{\mu}^{2} \hat{\phi}+V^{\prime}(\hat{\phi})=0 .
$$

The fluctuation operator is thus given by

$$
\frac{\delta^{2} S_{E}[\hat{\phi}]}{\delta \phi^{2}}=-\partial_{\mu}^{2}+V^{\prime \prime}(\hat{\phi})
$$

Differentiating eq. (9.69) by $\partial_{\nu}$ on the other hand yields the equation

$$
\left[-\partial_{\mu}^{2}+V^{\prime \prime}(\hat{\phi})\right] \partial_{\nu} \hat{\phi}=0,
$$


implying that $\partial_{\nu} \hat{\phi}$ can be identified as one of the zero modes. Note that a zero mode exists (i.e. is non-trivial) only if the solution $\hat{\phi}$ depends on the coordinate $x^{\nu}$; the trivial saddle point $\hat{\phi}=0$ does not lead to zero modes.

Let us now turn to the normalization of the zero modes. It turns out that integrals over the zero modes are only defined in a finite volume, and are proportional to the volume, $V=L^{d}$, corresponding to translational freedom in where we place the instanton. A proper normalization amounts to

$$
\int \frac{\mathrm{d} c_{0}}{\sqrt{2 \pi}}=\left(\frac{\hat{S}_{E}}{2 \pi}\right)^{\frac{1}{2}} L
$$

for $\partial_{1} \hat{\phi}, \partial_{2} \hat{\phi}, \partial_{3} \hat{\phi}$, and $L \rightarrow \beta$ for $\partial_{0} \hat{\phi}$. This can be shown by considering (for simplicity) a onedimensional case (of extent $L$ ), where the orthonormality condition of the eigenmodes takes the form

$$
\int_{0}^{L} \mathrm{~d} x f_{m} f_{n}=\delta_{m n}
$$

We note that the classical equation of motion, eq. (9.69), implies (upon multiplying with $\partial_{x} \hat{\phi}$ and fixing the integration constant at infinity) a "virial theorem", $\frac{1}{2}\left(\partial_{x} \hat{\phi}\right)^{2}=V(\hat{\phi})$, where we assume $V(0)=0$. We then see that

$$
\int_{0}^{L} \mathrm{~d} x\left(\partial_{x} \hat{\phi}\right)^{2}=\int_{0}^{L} \mathrm{~d} x\left[\frac{1}{2}\left(\partial_{x} \hat{\phi}\right)^{2}+V(\hat{\phi})\right]=S_{E}[\hat{\phi}] \equiv \hat{S}_{E},
$$

or in other words, that the properly normalized zero mode reads

$$
f_{0}=\frac{1}{\sqrt{\hat{S}_{E}}} \partial_{x} \hat{\phi}
$$

As the last step, we note that

$$
c_{0} f_{0}(x)=\frac{c_{0}}{\sqrt{\hat{S}_{E}}} \partial_{x} \hat{\phi}(x) \approx \hat{\phi}\left(x+\frac{c_{0}}{\sqrt{\hat{S}_{E}}}\right)-\hat{\phi}(x) .
$$

This shows that the zero mode corresponds to translations of the saddle-point solution. Since the box is of size $L$ and assumed periodic, we should restrict the translations into the range $c_{0} / \sqrt{\hat{S}_{E}} \in(0, L)$, i.e. $c_{0} \in\left(0, L \sqrt{\hat{S}_{E}}\right)$. This directly leads to eq. (9.72).

We are now ready to put everything together. A more careful analysis [9.20] shows that the factor 2 in eq. (9.55) cancels against a factor $1 / 2$ which we missed in eq. (9.65). Thereby eq. (9.66) can be seen to be accurate at low $T$, except for the treatment of the zero modes. Rectifying this point according to eq. (9.72), assuming that the number of zero modes is 4 (according to the spacetime dimensionality), and expressing also $\mathcal{Z}_{0}$ in the Gaussian approximation, we arrive at

$$
\left.\frac{\Gamma}{V}\right|_{\text {low } T} \simeq\left(\frac{\hat{S}_{E}}{2 \pi}\right)^{\frac{4}{2}}\left|\frac{\operatorname{det}^{\prime}\left[-\partial^{2}+V^{\prime \prime}(\hat{\phi})\right]}{\operatorname{det}\left[-\partial^{2}+V^{\prime \prime}(0)\right]}\right|^{-\frac{1}{2}} e^{-\hat{S}_{E}},
$$

where $\operatorname{det}^{\prime}$ means that zero modes have been omitted (but the negative mode is kept).

On the other hand, in the classical high-temperature limit, we can approximate $\partial_{\tau} \hat{\phi}=0$, cf. eq. (9.67). Thereby there are only three zero modes, and

$$
-2 \operatorname{Im} F \simeq T V\left(\frac{\hat{S}_{3 \mathrm{~d}}}{2 \pi T}\right)^{\frac{3}{2}}\left|\frac{\operatorname{det}^{\prime}\left[-\nabla^{2}+V^{\prime \prime}(\hat{\phi})\right]}{\operatorname{det}\left[-\nabla^{2}+V^{\prime \prime}(0)\right]}\right|^{-\frac{1}{2}} e^{-\beta \hat{S}_{3 \mathrm{~d}}} .
$$


Furthermore, it turns out that the guess $\Gamma \simeq-2 \operatorname{Im} F$ of eq. (9.56) should in this case be corrected into [9.21]

$$
\Gamma \simeq-\frac{\beta \lambda_{-}}{\pi} \operatorname{Im} F
$$

A conjectured result for the nucleation rate is thus

$$
\left.\frac{\Gamma}{V}\right|_{\text {high } T} \simeq\left(\frac{\lambda_{-}}{2 \pi}\right)\left(\frac{\hat{S}_{3 \mathrm{~d}}}{2 \pi T}\right)^{\frac{3}{2}}\left|\frac{\operatorname{det}^{\prime}\left[-\nabla^{2}+V^{\prime \prime}(\hat{\phi})\right]}{\operatorname{det}\left[-\nabla^{2}+V^{\prime \prime}(0)\right]}\right|^{-\frac{1}{2}} e^{-\beta \hat{S}_{3 \mathrm{~d}}} .
$$

Comparing eqs. (9.56) and (9.79), we may expect the high-temperature result of eq. (9.80) to be more accurate than the low-temperature result of eq. (9.77) above the regime in which the prefactors cross each other, i.e. for

$$
T \gtrsim \frac{\lambda_{-}}{2 \pi}
$$

It should be stressed, however, that the simplistic approach based on the negative eigenmode $\lambda_{-}$ does not really give a theoretically consistent answer [9.22,9.23]; rather, we should understand the above analysis in the sense that a rate exists, and the formulae as giving its order of magnitude.

Let us end by commenting on the analogous case of the baryon plus lepton number $(B+L)$ violation rate [9.24]. In that case, the vacua (there are infinitely many of them) are actually degenerate, and the role of the field $\phi$ is played by the Chern-Simons number, which is a suitable coordinate for classifying topologically distinct vacua. However, the formalism itself is identical: in particular, at low temperatures it may be assumed that there is a saddle point solution with $4 \mathrm{~d}$ symmetry, which is a usual instanton [9.25], whereas at high temperatures (but still in the symmetry broken phase) the saddle point solution has $3 \mathrm{~d}$ symmetry, i.e. is time-independent, and is referred to as a sphaleron [9.26]. Again there are also zero modes, which have to be treated carefully [9.27]. At high temperatures a complete analysis, even at leading order in couplings, requires non-perturbative methods [9.22, 9.28, 9.29].

\section{Appendix A: Nucleation action in the classical limit}

In scalar field theory, the instanton solution (also known as the "critical bubble") and its Euclidean action can be determined in a simple form, if we assume the classical limit of high temperatures and that the minima are almost degenerate. It can be shown (cf. e.g. refs. $[9.30,9.31]$ ) that then

$$
\hat{S}_{3 \mathrm{~d}}=\frac{16 \pi}{3} \frac{\sigma^{3}}{(\Delta p)^{2}},
$$

where

$$
\sigma \equiv \int_{0}^{\bar{\phi}_{\text {broken }}} \mathrm{d} \bar{\phi} \sqrt{2 V(\bar{\phi})}
$$

is the surface tension, and

$$
\Delta p \equiv V(0)-V\left(\bar{\phi}_{\text {broken }}\right)
$$

is the pressure difference in favour of the broken phase. In this limit the configuration $\hat{\phi}$ is called a thin-wall bubble. It is important to note that eq. (9.82) implies that $\hat{S}_{3 \mathrm{~d}} \rightarrow \infty$ for $\Delta p \rightarrow 0$; this is the reason why nucleation can only take place after some supercooling, when $\Delta p>0$ and $\hat{S}_{3 \mathrm{~d}}$ becomes finite. 

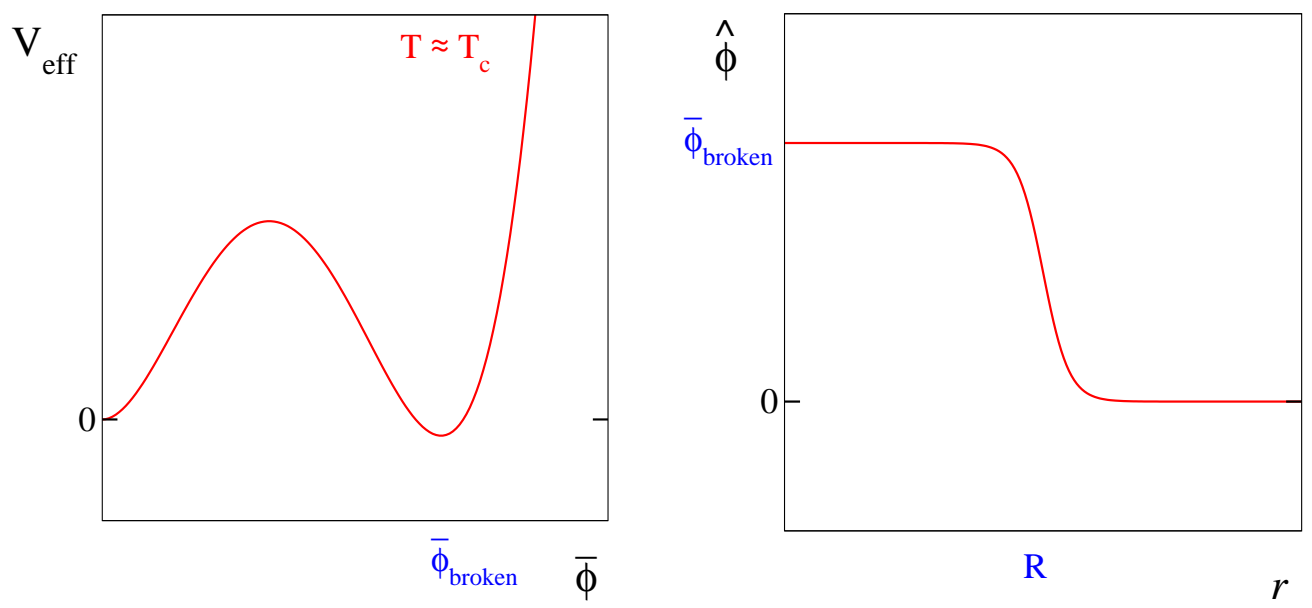

Figure 9: Left: The effective potential describing a first order phase transition at the critical temperature. Right: The profile of the critical bubble solution as a function of the radial coordinate.

The limit of almost degenerate minima is illustrated in fig. 9(left). In the classical limit, there is no dependence on $\tau$, and the equation of motion reads

$$
-\nabla^{2} \hat{\phi}+V^{\prime}(\hat{\phi})=0
$$

which, assuming spherical symmetry, can be written as

$$
\frac{\mathrm{d}^{2} \hat{\phi}}{\mathrm{d} r^{2}}+\frac{2}{r} \frac{\mathrm{d} \hat{\phi}}{\mathrm{d} r}=V^{\prime}(\hat{\phi})
$$

The boundary conditions in eq. (9.58) can furthermore be rephrased as

$$
\left\{\begin{array}{l}
\hat{\phi}(\infty)=0 \\
\left.\frac{\mathrm{d} \hat{\phi}(r)}{\mathrm{d} r}\right|_{r=0}=0
\end{array},\right.
$$

whereas the action reads

$$
\hat{S}_{3 \mathrm{~d}}=4 \pi \int_{0}^{\infty} \mathrm{d} r r^{2}\left\{\frac{1}{2}\left(\frac{\mathrm{d} \hat{\phi}}{\mathrm{d} r}\right)^{2}+V(\hat{\phi})\right\}
$$

Before proceeding, it is useful to note that eqs. (9.86) and (9.87) have a mechanical analogue. Indeed, rewriting $r \rightarrow t, V \rightarrow-U, \hat{\phi} \rightarrow x$, they correspond to a classical "particle in a valley" problem with friction. The particle starts at $t=0$ from $x>0$, near the top of the hill in the potential $U$, and rolls then towards the other top of the hill at the origin. The starting point has to be slightly higher than the end point, because the second term in eq. (9.86) acts as friction. Therefore the broken minimum has to be lower than the symmetric one in order for a non-trivial solution to exist.

Proceeding now with the solution, we introduce the following ansatz. Suppose that at $r<R$, the field is constant and has a value close to that in the broken minimum:

$$
\frac{\mathrm{d} \hat{\phi}}{\mathrm{d} r} \simeq 0, \quad V(\hat{\phi}) \simeq V\left(\bar{\phi}_{\text {broken }}\right) .
$$

This is illustrated in fig. 9(right). The contribution to the action from this region is

$$
\delta \hat{S}_{3 \mathrm{~d}} \simeq \frac{4}{3} \pi R^{3} V\left(\bar{\phi}_{\text {broken }}\right) .
$$


For $r>R$, we assume a similar situation, but now the field is close to the origin

$$
\frac{\mathrm{d} \hat{\phi}}{\mathrm{d} r} \simeq 0, \quad V(\hat{\phi}) \simeq V(0) \equiv 0,
$$

so that this region does not contribute to the action.

Finally, let us inspect the region at $r \simeq R$. If $R$ is very large, the term $2 \hat{\phi}^{\prime} / R$ in eq. (9.86) is very small, and can be neglected. Thereby

$$
\frac{\mathrm{d}^{2} \hat{\phi}}{\mathrm{d} r^{2}} \simeq V^{\prime}(\hat{\phi}),
$$

which through multiplication with $\hat{\phi}^{\prime}(r)$ can be integrated into

$$
\frac{1}{2}\left(\frac{\mathrm{d} \hat{\phi}}{\mathrm{d} r}\right)^{2} \simeq V(\hat{\phi})
$$

The contribution to the action thus becomes

$$
\begin{aligned}
\delta \hat{S}_{3 \mathrm{~d}} & \simeq 4 \pi R^{2} \int_{R-\delta}^{R+\delta} \mathrm{d} r\left(\frac{\mathrm{d} \hat{\phi}}{\mathrm{d} r}\right)^{2} \\
& \simeq 4 \pi R^{2} \int_{0}^{\bar{\phi}_{\text {broken }}} \mathrm{d} \hat{\phi} \frac{\mathrm{d} \hat{\phi}}{\mathrm{d} r} \\
& \simeq 4 \pi R^{2} \int_{0}^{\bar{\phi}_{\text {broken }}} \mathrm{d} \hat{\phi} \sqrt{2 V(\hat{\phi})} .
\end{aligned}
$$

The quantity

$$
\sigma \equiv \int_{R-\delta}^{R+\delta} \mathrm{d} r\left\{\frac{1}{2}\left(\frac{\mathrm{d} \hat{\phi}}{\mathrm{d} r}\right)^{2}+V(\hat{\phi})\right\} \simeq \int_{0}^{\bar{\phi}_{\text {broken }}} \mathrm{d} \hat{\phi} \sqrt{2 V(\hat{\phi})}
$$

represents the energy density of a planar surface, i.e. a surface tension.

Summing up the contributions, we get

$$
\hat{S}_{3 \mathrm{~d}}(R) \simeq 4 \pi R^{2} \sigma-\frac{4}{3} \pi R^{3} \Delta p,
$$

where $\Delta p>0$ was defined according to eq. (9.84). The so far undetermined parameter $R$ can be solved by extremizing the action,

$$
\delta_{R} \hat{S}_{3 \mathrm{~d}}=0
$$

leading to the radius $R=2 \sigma / \Delta p$. Substituting this back to eq. (9.96) we get

$$
\hat{S}_{3 \mathrm{~d}}=4 \pi \sigma \frac{4 \sigma^{2}}{(\Delta p)^{2}}-\frac{4}{3} \pi \frac{8 \sigma^{3}}{(\Delta p)^{2}}=\frac{16 \pi}{3} \frac{\sigma^{3}}{(\Delta p)^{2}} .
$$

Finally, if we are very close to $T_{\mathrm{c}}, \Delta p$ can be related to basic characteristics of the first order transition. Indeed, the energy density is

$$
e=T s-p,
$$

where the entropy density reads $s=\mathrm{d} p / \mathrm{d} T$. Across the transition, the pressure is continuous but the energy density has a discontinuity called the latent heat (here again $\Delta x \equiv x_{\text {broken }}-x_{\text {symmetric }}$ ):

$$
L \equiv-\Delta e=-T_{\mathrm{c}} \Delta s=-T_{\mathrm{c}} \frac{\mathrm{d} \Delta p}{\mathrm{~d} T} .
$$

Therefore

$$
\Delta p(T) \approx \Delta p\left(T_{\mathrm{c}}\right)+\frac{\mathrm{d} \Delta p}{\mathrm{~d} T}\left(T-T_{\mathrm{c}}\right)=L\left(1-\frac{T}{T_{\mathrm{c}}}\right) .
$$

At the same time, the surface tension remains finite at the transition point. Thereby the nucleation action in eq. (9.98) diverges quadratically as $T \rightarrow T_{\mathrm{c}}^{-}$. 


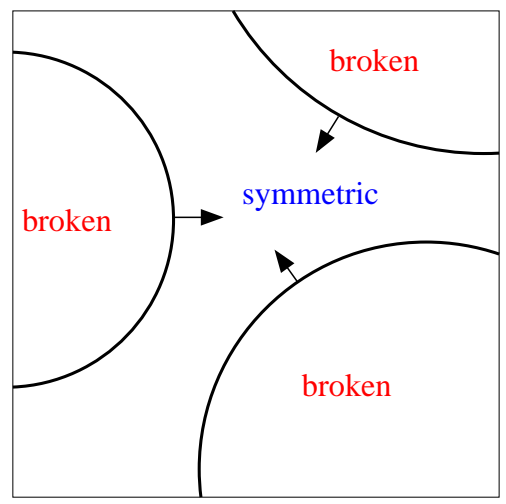

Figure 10: A situation with many bubbles having nucleated recently and growing to fill the space.

\section{Appendix B: Nucleation dynamics with many bubbles}

Above we discussed the probability of nucleating a single bubble of the low-temperature phase. In an actual transition, many bubbles nucleate with an increasing rate, and those that nucleated first have had time to grow. The transition "completes" when the bubbles have filled the space. This process is illustrated in fig. 10. Let us sketch the practical approach that is often used for estimating the "nucleation temperature", $T_{n}$, around which this process effectively takes place.

The nucleation probability per time and volume is denoted by $p=\Gamma / V=p_{0} e^{-\hat{S}_{E}}$, where in the classical limit $\hat{S}_{E}=\beta \hat{S}_{3 \mathrm{~d}}$. Let us write $\hat{S}_{E}$ as a function of time. Expanding $\hat{S}_{E}(t) \approx$ $\hat{S}_{E}\left(t_{\mathrm{n}}\right)+\hat{S}_{E}^{\prime}\left(t_{\mathrm{n}}\right)\left(t-t_{\mathrm{n}}\right)$ where $t_{\mathrm{n}}$ is the effective nucleation time, and including the volume of a bubble growing with velocity $v$, we integrate over all possible nucleation times prior to $t_{\mathrm{n}}$, and assert that the transition has completed when the probability is of order unity [9.32]-[9.34]:

$$
1 \simeq \int_{-\infty}^{t_{\mathrm{n}}} \mathrm{d} t \frac{4 \pi v^{3}\left(t_{\mathrm{n}}-t\right)^{3}}{3} p_{0} e^{-\hat{S}_{E}(t)} \approx \frac{8 \pi v^{3} p_{0}}{\left|\hat{S}_{E}^{\prime}\left(t_{\mathrm{n}}\right)\right|^{4}} e^{-\hat{S}_{E}\left(t_{\mathrm{n}}\right)}
$$

Here we noted that the nucleation action decreases with time, $\hat{S}_{E}^{\prime}\left(t_{\mathrm{n}}\right)<0$, cf. the discussion below eq. (9.101). Similarly, but this time not including the bubble growth factor, we can estimate the average inverse volume of the bubbles that did get nucleated:

$$
\frac{1}{V} \simeq \int_{-\infty}^{t_{\mathrm{n}}} \mathrm{d} t p_{0} e^{-\hat{S}_{E}(t)} \approx \frac{p_{0}}{\left|\hat{S}_{E}^{\prime}\left(t_{\mathrm{n}}\right)\right|} e^{-\hat{S}_{E}\left(t_{\mathrm{n}}\right)} \approx \frac{\left|\hat{S}_{E}^{\prime}\left(t_{\mathrm{n}}\right)\right|^{3}}{8 \pi v^{3}}
$$

In the last step here, we inserted eq. (9.102). The average distance of the bubbles is therefore

$$
\ell \equiv\left(\frac{1}{V}\right)^{-1 / 3} \simeq \frac{v}{\left|\hat{S}_{E}^{\prime}\left(t_{\mathrm{n}}\right)\right|}
$$

To summarize, the equilibrium properties of a first-order transition are characterized by the quantities $T_{\mathrm{c}}, L$, and $\sigma$, whereas the nucleation dynamics depends on $T_{\mathrm{n}}, \ell$, and the velocity $v$. We can estimate $T_{\mathrm{n}}$ from eq. (9.102) after inserting the relation between $t_{\mathrm{n}}$ and $T_{\mathrm{n}}$ from sec. 9.4. For $\hat{S}_{E}$ this is trivial because we originally expressed $\hat{S}_{E}$ in terms of $T$ (cf. appendix A), whereas for the time derivative in eq. (9.102) we can make use of an explicit relation, to be derived in eq. (9.181), in order to write

$$
\left|\hat{S}_{E}^{\prime}\left(t_{\mathrm{n}}\right)\right|=3 c_{s}^{2} T_{\mathrm{n}} H\left(T_{\mathrm{n}}\right) \hat{S}_{E}^{\prime}\left(T_{\mathrm{n}}\right)
$$


where $H(T)$ is the Hubble rate. According to the non-perturbative study in ref. [9.23], the prefactor $p_{0}$ in eq. (9.102) can be approximated as $p_{0} \simeq \alpha_{w}^{5}\left(\frac{g T}{m_{\mathrm{E}}}\right)^{2} \ln \left(\frac{1}{g}\right) T^{4}$, where $\alpha_{w} \equiv g^{2} /(4 \pi)$ and $m_{\mathrm{E}}$ is the Debye mass, provided that we make use of non-perturbative values of $L$ and $\sigma$ when evaluating eq. (9.82). The velocity $v$ appearing in eq. (9.104) is a complicated function, because it depends not only on microscopic processes [9.35] but also on global hydrodynamic aspects of the bubble dynamics which in turn depend on $T_{\mathrm{n}}$ [9.36]. All in all, it is fair to say that nucleation computations contain large uncertainties. 


\subsection{Particle production rate}

Consider a system where some particles interact strongly enough to be in thermal equilibrium, while others interact so weakly that they are out of equilibrium. We can imagine that particles of the latter type "escape" from the thermal system, either concretely (if the system is of finite size) or in an abstract sense (being still within the same volume but not interacting with the thermal particles). Familiar physical examples of such settings are the "decoupling" of weakly interacting dark matter particles in cosmology; the production of electromagnetic "hard probes", such as photons and lepton-antilepton pairs, in the QCD plasma generated in heavy ion collision experiments; as well as the neutrino "emissivity" of neutron stars, constituting the most important process by which neutron stars cool down.

The purpose of this section is to develop a general formalism for addressing this phenomenon. ${ }^{33}$ To keep the discussion concrete, we focus on a simple model: the production rate of hypothetical scalar particles coupled to a gauge-invariant operator $\mathcal{J}$ composed of Standard Model degrees of freedom. The classical Lagrangian is assumed to take the form

$$
\mathcal{L}_{M}=\partial_{\mu} \phi^{*} \partial^{\mu} \phi-m^{2} \phi^{*} \phi-h \phi^{*} \mathcal{J}-h^{*} \mathcal{J}^{*} \phi+\mathcal{L}_{\text {bath }}
$$

where $\mathcal{L}_{\text {bath }}$ describes the thermalized degrees of freedom. The first step is to derive a master equation relating the production rate of $\phi$ 's to a certain Green's function of $\mathcal{J}$ 's.

Let $\hat{\rho}$ be the density matrix of the full theory, incorporating all degrees of freedom, and $\hat{H}$ the corresponding full Hamiltonian operator. Then the equation of motion for the density matrix is ${ }^{34}$

$$
i \frac{\mathrm{d} \hat{\rho}(t)}{\mathrm{d} t}=[\hat{H}, \hat{\rho}(t)] .
$$

We now split $\hat{H}$ up as

$$
\hat{H}=\hat{H}_{\text {bath }}+\hat{H}_{\phi}+\hat{H}_{\text {int }},
$$

where $\hat{H}_{\text {bath }}$ is the Hamiltonian of the heat bath, $\hat{H}_{\phi}$ is the free Hamiltonian of the scalar fields, and $\hat{H}_{\text {int }}$, which is proportional to the coupling constant $h$, contains the interactions between the two sets:

$$
\hat{H}_{\text {int }}=\int_{\mathbf{x}}\left(h \hat{\phi}^{\dagger} \hat{\mathcal{J}}+h^{*} \hat{\mathcal{J}}^{\dagger} \hat{\phi}\right)
$$

To find the density of the scalar particles, one has to solve eq. (9.109) with some initial conditions. We assume that initially there were no $\phi$-particles, that is

$$
\hat{\rho}(0)=\hat{\rho}_{\text {bath }} \otimes|0\rangle\langle 0|,
$$

${ }^{33}$ Classic discussions of thermal particle production include refs. [9.37, 9.38], which establish the dilepton and photon production rates from a QCD plasma as

$$
\begin{aligned}
\frac{\mathrm{d} N_{\ell^{-} \ell+}}{\mathrm{d}^{4} \mathcal{X}^{4} \mathcal{K}} & =\sum_{f f^{\prime}} \frac{-2 e^{4} Q_{f} Q_{f^{\prime}} \theta\left(\mathcal{K}^{2}-4 m_{\ell}^{2}\right)}{3(2 \pi)^{5} \mathcal{K}^{2}}\left(1+\frac{2 m_{\ell}^{2}}{\mathcal{K}^{2}}\right)\left(1-\frac{4 m_{\ell}^{2}}{\mathcal{K}^{2}}\right)^{\frac{1}{2}} n_{\mathrm{B}}\left(k^{0}\right) \rho_{f f^{\prime}}(\mathcal{K}), \\
\frac{\mathrm{d} N_{\gamma}}{\mathrm{d}^{4} \mathcal{X} \mathrm{d}^{3} \mathbf{k}} & =\left.\sum_{f f^{\prime}} \frac{-e^{2} Q_{f} Q_{f^{\prime}}}{(2 \pi)^{3} k} n_{\mathrm{B}}(k) \rho_{f f^{\prime}}(\mathcal{K})\right|_{k^{0}=k},
\end{aligned}
$$

where $Q_{f}$ is the quark electric charge in units of $e$, and $\rho_{f f^{\prime}}(\mathcal{K})=\int_{\mathcal{X}} e^{i \mathcal{K} \cdot \mathcal{X}}\left\langle\frac{1}{2}\left[\hat{\mathcal{J}}_{f}^{\mu}(\mathcal{X}), \hat{\mathcal{J}}_{f^{\prime} \mu}(0)\right]\right\rangle$ is a spectral function related to flavours $f$ and $f^{\prime}$. In the present section we follow the alternative formalism of ref. [9.39].

${ }^{34}$ This is the Liouville - von Neumann equation; its derivation proceeds roughly as

$$
i \frac{\mathrm{d}}{\mathrm{d} t}|\psi\rangle=\hat{H}|\psi\rangle, \quad-i \frac{\mathrm{d}}{\mathrm{d} t}\langle\psi|=\left\langle\psi\left|\hat{H} \quad \Rightarrow \quad i \frac{\mathrm{d}}{\mathrm{d} t}\right| \psi\right\rangle\langle\psi|=[\hat{H},|\psi\rangle\langle\psi|] \quad \Rightarrow \quad i \frac{\mathrm{d}}{\mathrm{d} t} \hat{\rho}(t)=[\hat{H}, \hat{\rho}(t)] .
$$




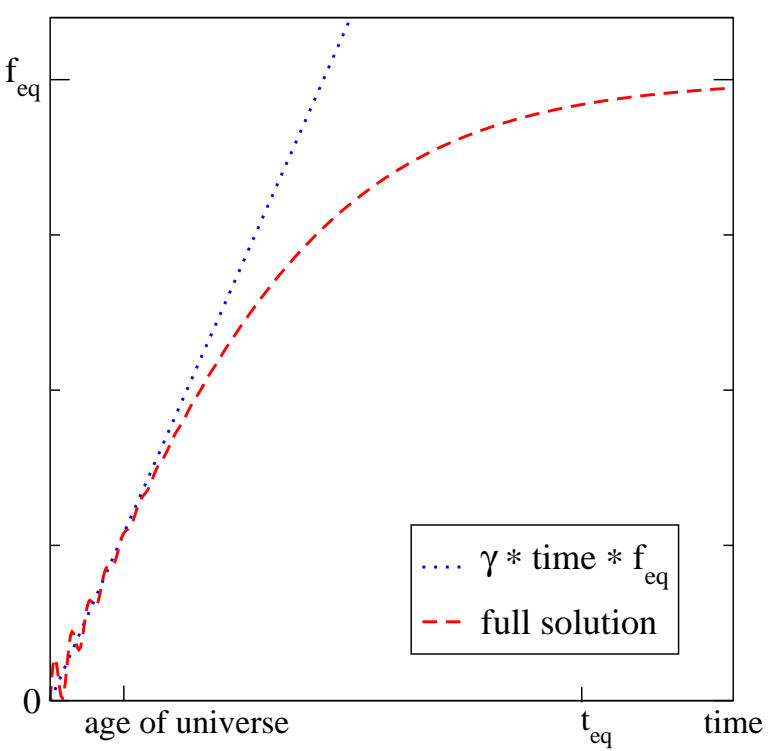

Figure 11: A sketch of how the phase space density of a weakly interacting particle species evolves from zero towards its equilibrium form. In many interesting cases, the equilibrium value is not reached within the lifetime of the system (denoted here by "age of universe"). Then it is important to know the rate $\gamma$, characterizing the linear slope at intermediate times.

where $\hat{\rho}_{\text {bath }}=\mathcal{Z}_{\text {bath }}^{-1} \exp \left(-\beta \hat{H}_{\text {bath }}\right), \beta \equiv 1 / T$, is the equilibrium density matrix of the heat bath at temperature $T$; and $|0\rangle$ is the vacuum state for the scalar particles.

Denoting by $\hat{H}_{0} \equiv \hat{H}_{\text {bath }}+\hat{H}_{\phi}$ a "free" Hamiltonian and by $\hat{H}_{\text {int }}$ an interaction term, an equation of motion can be obtained for the density matrix in the interaction picture, $\hat{\rho}_{I} \equiv$ $\exp \left(i \hat{H}_{0} t\right) \hat{\rho} \exp \left(-i \hat{H}_{0} t\right)$, in the standard way:

$$
\begin{aligned}
i \frac{\mathrm{d}}{\mathrm{d} t} \hat{\rho}_{I}(t) & =-\hat{H}_{0} \hat{\rho}_{I}+e^{i \hat{H}_{0} t}[\hat{H}, \hat{\rho}(t)] e^{-i \hat{H}_{0} t}+\hat{\rho}_{I} \hat{H}_{0} \\
& =-\hat{H}_{0} \hat{\rho}_{I}+e^{i \hat{H}_{0} t}\left[\hat{H}_{0}+\hat{H}_{\mathrm{int}}, \hat{\rho}(t)\right] e^{-i \hat{H}_{0} t}+\hat{\rho}_{I} \hat{H}_{0} \\
& =e^{i \hat{H}_{0} t}\left[\hat{H}_{\mathrm{int}}, \hat{\rho}(t)\right] e^{-i \hat{H}_{0} t} \\
& =e^{i \hat{H}_{0} t} \hat{H}_{\mathrm{int}} e^{-i \hat{H}_{0} t} e^{i \hat{H}_{0} t} \hat{\rho}(t) e^{-i \hat{H}_{0} t}-e^{i \hat{H}_{0} t} \hat{\rho}(t) e^{-i \hat{H}_{0} t} e^{i \hat{H}_{0} t} \hat{H}_{\mathrm{int}} e^{-i \hat{H}_{0} t} \\
& =\left[\hat{H}_{I}(t), \hat{\rho}_{I}(t)\right]
\end{aligned}
$$

Here, as usual, $\hat{H}_{I}=\exp \left(i \hat{H}_{0} t\right) \hat{H}_{\text {int }} \exp \left(-i \hat{H}_{0} t\right)$ is the interaction Hamiltonian in the interaction picture.

Now, perturbation theory with respect to $\hat{H}_{I}$ can be used to compute the time evolution of $\hat{\rho}_{I}$; the first two terms read

$$
\hat{\rho}_{I}(t)=\hat{\rho}_{0}-i \int_{0}^{t} \mathrm{~d} t^{\prime}\left[\hat{H}_{I}\left(t^{\prime}\right), \hat{\rho}_{0}\right]+(-i)^{2} \int_{0}^{t} \mathrm{~d} t^{\prime} \int_{0}^{t^{\prime}} \mathrm{d} t^{\prime \prime}\left[\hat{H}_{I}\left(t^{\prime}\right),\left[\hat{H}_{I}\left(t^{\prime \prime}\right), \hat{\rho}_{0}\right]\right]+\ldots,
$$

where $\hat{\rho}_{0} \equiv \hat{\rho}(0)=\hat{\rho}_{I}(0)$. We note that perturbation theory as an expansion in $\hat{H}_{I}$ may break down at a certain time $t \simeq t_{\text {eq }}$ due to so-called secular terms. Physically, the reason is that for $t \gtrsim t_{\text {eq }}$ scalar particles enter thermal equilibrium and their concentration needs to be computed by other 
means (cf. below). Here we assumed that $t \ll t_{\text {eq }}$ and thus perturbation theory should work. At the same time, $t$ is also assumed to be much larger than the microscopic time scales characterizing the dynamics of the heat bath, say $t \gg 1 /\left(\alpha^{2} T\right)$, where $\alpha$ is a generic fine structure constant. This guarantees that quantum-mechanical oscillations get damped out, and the produced particles can be considered to constitute a "classical" phase space distribution function. The situation is illustrated in fig. 11, with the slope $\gamma$ denoting the rate that we want to compute and initial quantum-mechanical oscillations illustrated with small wiggles in the full solution.

More specifically, let us consider the distribution of scalar particles "of type $a$ ", generated by the creation operator $\hat{a}_{\mathbf{k}}^{\dagger} \cdot{ }^{35}$ It is associated with the operator

$$
\frac{\mathrm{d} \hat{N}_{a}}{\mathrm{~d}^{3} \mathbf{x d}^{3} \mathbf{k}} \equiv \frac{1}{V} \hat{a}_{\mathbf{k}}^{\dagger} \hat{a}_{\mathbf{k}}
$$

where $V$ is the volume of the system, and the normalization corresponds to

$$
\left[\hat{a}_{\mathbf{p}}, \hat{a}_{\mathbf{k}}^{\dagger}\right]=\left[\hat{b}_{\mathbf{p}}, \hat{b}_{\mathbf{k}}^{\dagger}\right]=\delta^{(3)}(\mathbf{p}-\mathbf{k})
$$

or in configuration space to

$$
\left[\hat{\phi}(\mathcal{X}), \partial_{0} \hat{\phi}^{\dagger}(\mathcal{Y})\right]=i \delta^{(3)}(\mathbf{x}-\mathbf{y}) \quad \text { for } \quad x^{0}=y^{0} .
$$

Then the distribution function (in a translationally invariant system) is given by

$$
f_{a}(t, \mathbf{k}) \equiv(2 \pi)^{3} \operatorname{Tr}\left[\frac{\mathrm{d} \hat{N}_{a}}{\mathrm{~d}^{3} \mathbf{x} \mathrm{d}^{3} \mathbf{k}} \hat{\rho}_{I}(t)\right] .
$$

Inserting eq. (9.114), the first term vanishes because $\left\langle 0\left|\hat{a}_{\mathbf{k}}^{\dagger} \hat{a}_{\mathbf{k}}\right| 0\right\rangle=0$, and the second term does not contribute since $\hat{H}_{I}$ is linear in $\hat{a}_{\mathbf{k}}^{\dagger}$ and $\hat{a}_{\mathbf{k}}$ (cf. eqs. (9.111) and (9.120)), so that the corresponding trace vanishes. Thus, we get that the rate of particle production reads

$$
\dot{f}_{a}(t, \mathbf{k})=R_{a}(T, \mathbf{k}) \equiv-\frac{(2 \pi)^{3}}{V} \operatorname{Tr}\left\{\hat{a}_{\mathbf{k}}^{\dagger} \hat{a}_{\mathbf{k}} \int_{0}^{t} \mathrm{~d} t^{\prime}\left[\hat{H}_{I}(t),\left[\hat{H}_{I}\left(t^{\prime}\right), \hat{\rho}_{0}\right]\right]\right\}+\mathcal{O}\left(|h|^{4}\right) .
$$

The interaction Hamiltonian $\hat{H}_{I}$ appearing in eq. (9.119) has the form in eq. (9.111), except that we now interpret the field operators as being in the interaction picture. Since $\hat{\phi}$ evolves with the free Hamiltonian $\hat{H}_{\phi}$ in the interaction picture, it has the form of a free on-shell field operator, and can hence be written as

$$
\hat{\phi}(\mathcal{X})=\int \frac{\mathrm{d}^{3} \mathbf{p}}{\sqrt{(2 \pi)^{3} 2 \epsilon_{p}}}\left(\hat{a}_{\mathbf{p}} e^{-i \mathcal{P} \cdot \mathcal{X}}+\hat{b}_{\mathbf{p}}^{\dagger} e^{i \mathcal{P} \cdot \mathcal{X}}\right),
$$

where we assumed the normalization in eq. (9.116), and $p^{0} \equiv \epsilon_{p} \equiv \sqrt{p^{2}+M^{2}}, \mathcal{P} \equiv\left(p^{0}, \mathbf{p}\right)$. Inserting $\hat{\phi}(\mathcal{X})$ into (the interaction picture version of) eq. (9.111), we can rewrite $\hat{H}_{I}$ as

$$
\hat{H}_{I}=\int_{\mathbf{x}} \int \frac{\mathrm{d}^{3} \mathbf{p}}{\sqrt{(2 \pi)^{3} 2 \epsilon_{p}}}\left\{\left[h \hat{a}_{\mathbf{p}}^{\dagger} \hat{\mathcal{J}}+h^{*} \hat{\mathcal{J}}^{\dagger} \hat{b}_{\mathbf{p}}^{\dagger}\right](\mathcal{X}) e^{i \mathcal{P} \cdot \mathcal{X}}+\left[h^{*} \hat{\mathcal{J}}^{\dagger} \hat{a}_{\mathbf{p}}+h \hat{b}_{\mathbf{p}} \hat{\mathcal{J}}\right](\mathcal{X}) e^{-i \mathcal{P} \cdot \mathcal{X}}\right\}
$$

It remains to take the following steps:

\footnotetext{
${ }^{35}$ As our field $\phi$ is assumed to be complex-valued, the expansion of the corresponding field operator, cf. eq. (9.120), contains two independent sets of creation and annihilation operators, denoted here by $\hat{a}^{\dagger}, \hat{a}$ and $\hat{b}^{\dagger}, \hat{b}$.
} 
(i) We insert eq. (9.121) into eq. (9.119). Denoting

$$
\begin{aligned}
\hat{A} & \equiv \hat{a}_{\mathbf{k}}^{\dagger} \hat{a}_{\mathbf{k}}, \\
\hat{B}(t) & \equiv \int_{\mathbf{x}} \int \frac{\mathrm{d}^{3} \mathbf{p}}{\sqrt{(2 \pi)^{3} 2 \epsilon_{p}}}\left\{\left[h \hat{a}_{\mathbf{p}}^{\dagger} \hat{\mathcal{J}}+h^{*} \hat{\mathcal{J}}^{\dagger} \hat{b}_{\mathbf{p}}^{\dagger}\right](\mathcal{X}) e^{i \mathcal{P} \cdot \mathcal{X}}+\text { H.c. }\right\}, \\
\hat{C}\left(t^{\prime}\right) & \equiv \int_{\mathbf{y}} \int \frac{\mathrm{d}^{3} \mathbf{r}}{\sqrt{(2 \pi)^{3} 2 \epsilon_{r}}}\left\{\left[h \hat{a}_{\mathbf{r}}^{\dagger} \hat{\mathcal{J}}+h^{*} \hat{\mathcal{J}}^{\dagger} \hat{b}_{\mathbf{r}}^{\dagger}\right](\mathcal{Y}) e^{i \mathcal{R} \cdot \mathcal{Y}}+\text { H.c. }\right\},
\end{aligned}
$$

with $\mathcal{X} \equiv(t, \mathbf{x})$ and $\mathcal{Y} \equiv\left(t^{\prime}, \mathbf{y}\right)$, the trace can be re-organized as

$$
\begin{aligned}
\operatorname{Tr}\{\hat{A}[\hat{B},[\hat{C},|0\rangle\langle 0|]]\} & =\operatorname{Tr}\{\hat{A}(\hat{B} \hat{C}|0\rangle\langle 0|-\hat{B}| 0\rangle\langle 0|\hat{C}-\hat{C}| 0\rangle\langle 0|\hat{B}+| 0\rangle\langle 0| \hat{C} \hat{B})\} \\
& =\langle 0|\{\hat{A} \hat{B} \hat{C}-\hat{C} \hat{A} \hat{B}-\hat{B} \hat{A} \hat{C}+\hat{C} \hat{B} \hat{A}\}| 0\rangle \\
& =\langle 0|[[\hat{A}, \hat{B}], \hat{C}]| 0\rangle .
\end{aligned}
$$

(ii) Since $\hat{A}$ commutes with $\hat{b}_{\mathbf{p}}^{\dagger}$ in $\hat{B}$, the part of $\hat{B}$ with $\hat{b}_{\mathbf{p}}^{\dagger}$ gives no contribution; this is also true for $\hat{b}_{\mathbf{r}}^{\dagger}$ in $\hat{C}$ since an odd number of creation or annihilation operators yields nothing. A non-zero trace only arises from structures of the type $\left\langle 0\left|\hat{a} \hat{a}^{\dagger} \hat{a} \hat{a}^{\dagger}\right| 0\right\rangle$, i.e. the second and third terms in the second line of eq. (9.125), in which $\hat{A}$ is "shielded" from the vacuum state. Thus, eq. (9.119) becomes

$$
\begin{aligned}
R_{a}(T, \mathbf{k})= & \frac{|h|^{2}(2 \pi)^{3}}{V} \int_{0}^{t} \mathrm{~d} t^{\prime} \int_{\mathbf{x}} \int_{\mathbf{y}} \int \frac{\mathrm{d}^{3} \mathbf{p}}{\sqrt{(2 \pi)^{3} 2 \epsilon_{p}}} \int \frac{\mathrm{d}^{3} \mathbf{r}}{\sqrt{(2 \pi)^{3} 2 \epsilon_{r}}} \\
\times & \operatorname{Tr}\left\{\hat { \rho } _ { \mathrm { bath } } \left[\hat{\mathcal{J}}^{\dagger}(\mathcal{Y}) \hat{\mathcal{J}}(\mathcal{X}) e^{i \mathcal{P} \cdot \mathcal{X}-i \mathcal{R} \cdot \mathcal{Y}}\left\langle 0\left|\hat{a}_{\mathbf{r}} \hat{a}_{\mathbf{k}}^{\dagger} \hat{a}_{\mathbf{k}} \hat{a}_{\mathbf{p}}^{\dagger}\right| 0\right\rangle\right.\right. \\
& \left.\left.\quad+\hat{\mathcal{J}}^{\dagger}(\mathcal{X}) \hat{\mathcal{J}}(\mathcal{Y}) e^{-i \mathcal{P} \cdot \mathcal{X}+i \mathcal{R} \cdot \mathcal{Y}}\left\langle 0\left|\hat{a}_{\mathbf{p}} \hat{a}_{\mathbf{k}}^{\dagger} \hat{a}_{\mathbf{k}} \hat{a}_{\mathbf{r}}^{\dagger}\right| 0\right\rangle\right]\right\},
\end{aligned}
$$

where $\hat{\rho}_{\text {bath }}$ has appeared from eq. (9.112). Given eq. (9.116), both expectation values evaluate to

$$
\left\langle 0\left|\hat{a}_{\mathbf{r}} \hat{a}_{\mathbf{k}}^{\dagger} \hat{a}_{\mathbf{k}} \hat{a}_{\mathbf{p}}^{\dagger}\right| 0\right\rangle=\left\langle 0\left|\hat{a}_{\mathbf{p}} \hat{a}_{\mathbf{k}}^{\dagger} \hat{a}_{\mathbf{k}} \hat{a}_{\mathbf{r}}^{\dagger}\right| 0\right\rangle=\delta^{(3)}(\mathbf{r}-\mathbf{k}) \delta^{(3)}(\mathbf{p}-\mathbf{k})
$$

Thereby

$$
\begin{aligned}
R_{a}(T, \mathbf{k}) & =\frac{|h|^{2}}{V} \frac{1}{2 \epsilon_{k}} \int_{0}^{t} \mathrm{~d} t^{\prime} \int_{\mathbf{x}, \mathbf{y}} \\
& \times\left\langle\hat{\mathcal{J}}^{\dagger}(\mathcal{Y}) \hat{\mathcal{J}}(\mathcal{X}) e^{i \mathcal{K} \cdot(\mathcal{X}-\mathcal{Y})}+\hat{\mathcal{J}}^{\dagger}(\mathcal{X}) \hat{\mathcal{J}}(\mathcal{Y}) e^{i \mathcal{K} \cdot(\mathcal{Y}-\mathcal{X})}\right\rangle,
\end{aligned}
$$

where from now on the expectation value refers to that with respect to $\hat{\rho}_{\text {bath }}$.

(iii) Recalling the notation in eq. (8.3),

$$
\Pi^{<}(\mathcal{K}) \equiv \int_{\mathcal{X}} e^{i \mathcal{K} \cdot(\mathcal{X}-\mathcal{Y})}\left\langle\hat{\mathcal{J}}^{\dagger}(\mathcal{Y}) \hat{\mathcal{J}}(\mathcal{X})\right\rangle,
$$

where we made use of translational invariance, we can represent

$$
\begin{aligned}
\left\langle\hat{\mathcal{J}}^{\dagger}(\mathcal{Y}) \hat{\mathcal{J}}(\mathcal{X})\right\rangle & =\int_{\mathcal{P}} e^{-i \mathcal{P} \cdot(\mathcal{X}-\mathcal{Y})} \Pi^{<}(\mathcal{P}), \\
\left\langle\hat{\mathcal{J}}^{\dagger}(\mathcal{X}) \hat{\mathcal{J}}(\mathcal{Y})\right\rangle & =\int_{\mathcal{P}} e^{-i \mathcal{P} \cdot(\mathcal{Y}-\mathcal{X})} \Pi^{<}(\mathcal{P}) .
\end{aligned}
$$


(iv) It remains to carry out the integrals over the space and time coordinates. At this point the result can be simplified by taking the limit $t \rightarrow \infty$, which physically means that we consider time scales large compared with the interaction rate within the heat bath (cf. the figure on p. 175). Summing both terms in eq. (9.128) together and inserting eqs. (9.130) and (9.131) yields

$$
\begin{array}{cl} 
& \lim _{t \rightarrow \infty} \int \mathrm{d}^{3} \mathbf{x} \int \mathrm{d}^{3} \mathbf{y} \int_{0}^{t} \mathrm{~d} t^{\prime}\left[e^{i(\mathcal{K}-\mathcal{P}) \cdot(\mathcal{X}-\mathcal{Y})}+e^{i(\mathcal{P}-\mathcal{K}) \cdot(\mathcal{X}-\mathcal{Y})}\right] \\
= & V(2 \pi)^{3} \delta^{(3)}(\mathbf{p}-\mathbf{k}) \lim _{t \rightarrow \infty} \int_{0}^{t} \mathrm{~d} t^{\prime}\left[e^{i\left(k^{0}-p^{0}\right)\left(t-t^{\prime}\right)}+e^{i\left(p^{0}-k^{0}\right)\left(t-t^{\prime}\right)}\right] \\
\stackrel{t^{\prime \prime}=t^{\prime}-t}{=} & V(2 \pi)^{3} \delta^{(3)}(\mathbf{p}-\mathbf{k}) \lim _{t \rightarrow \infty}\left\{\int_{-t}^{0} \mathrm{~d} t^{\prime \prime}\left[e^{i\left(p^{0}-k^{0}\right) t^{\prime \prime}}+e^{-i\left(p^{0}-k^{0}\right) t^{\prime \prime}}\right]\right\} \\
t^{\prime \prime \prime} \equiv-t^{\prime \prime} & V(2 \pi)^{3} \delta^{(3)}(\mathbf{p}-\mathbf{k}) \lim _{t \rightarrow \infty}\left\{\int_{-t}^{0} \mathrm{~d} t^{\prime \prime} e^{i\left(p^{0}-k^{0}\right) t^{\prime \prime}}+\int_{0}^{t} \mathrm{~d} t^{\prime \prime \prime} e^{i\left(p^{0}-k^{0}\right) t^{\prime \prime \prime}}\right\} \\
= & V(2 \pi)^{3} \delta^{(3)}(\mathbf{p}-\mathbf{k}) \int_{-\infty}^{\infty} \mathrm{d} \tilde{t} e^{i\left(p^{0}-k^{0}\right) \tilde{t}}=V(2 \pi)^{4} \delta^{(4)}(\mathcal{P}-\mathcal{K}) .
\end{array}
$$

This allows us to cancel $1 / V$ in eq. (9.128) and remove $\int_{\mathcal{P}}$ from eqs. (9.130) and (9.131).

As a result of these steps we obtain (denoting $k^{0} \equiv \epsilon_{k}$ )

$$
R_{a}(T, \mathbf{k})=\frac{|h|^{2}}{2 \epsilon_{k}} \Pi^{<}(\mathcal{K})+\mathcal{O}\left(|h|^{4}\right) .
$$

Using eq. (8.14), viz. $\Pi^{<}(\mathcal{K})=2 n_{\mathrm{B}}\left(k^{0}\right) \rho(\mathcal{K})$, we finally arrive at the master relation

$$
R_{a}(T, \mathbf{k})=\frac{n_{\mathrm{B}}\left(\epsilon_{k}\right)}{\epsilon_{k}}|h|^{2} \rho(\mathcal{K})+\mathcal{O}\left(|h|^{4}\right) .
$$

We stress again that this relation is valid only provided that the number density of the particles created is much smaller than their equilibrium concentration.

For the production rate of $b$-particles, similar steps lead to

$$
R_{b}(T, \mathbf{k})=\frac{|h|^{2}}{2 \epsilon_{k}} \Pi^{>}(-\mathcal{K})+\mathcal{O}\left(|h|^{4}\right) .
$$

From eq. (8.15) and the identity $n_{\mathrm{B}}\left(-k^{0}\right)=-1-n_{\mathrm{B}}\left(k^{0}\right)$, we get $\Pi^{>}(-\mathcal{K})=2\left[1+n_{\mathrm{B}}\left(-k^{0}\right)\right] \rho(-\mathcal{K})=$ $-2 n_{\mathrm{B}}\left(k^{0}\right) \rho(-\mathcal{K})$, and subsequently

$$
R_{b}(T, \mathbf{k})=\frac{n_{\mathrm{B}}\left(\epsilon_{k}\right)}{\epsilon_{k}}|h|^{2}[-\rho(-\mathcal{K})]+\mathcal{O}\left(|h|^{4}\right) .
$$

In a CP-symmetric plasma (without chemical potentials), it can be shown that $\rho(-\mathcal{K})=-\rho(\mathcal{K})$ in the bosonic case, so that in fact the two production rates coincide.

In summary, we have obtained a relation connecting the particle production rate, eq. (9.119), to a finite-temperature spectral function, concerning the operator to which the produced particle couples. We return to a specific example in sec. 9.4.

Three concluding remarks are in order:

- In terms of the figure on p. 175 , the rate $\gamma$ equals $\gamma=\frac{|h|^{2} \rho(\mathcal{K})}{\epsilon_{k}}+\mathcal{O}\left(|h|^{4}\right)$ and $f_{\text {eq }}=n_{\mathrm{B}}\left(\epsilon_{k}\right)$. 
- Once sufficiently many particles have been produced, they tend to equilibrate, and the results above are no longer valid. We can expect that in this situation eq. (9.134) is modified into

$$
\dot{f}_{a}(t, \mathbf{k})=\frac{|h|^{2} \rho(\mathcal{K})}{\epsilon_{k}}\left[n_{\mathrm{B}}\left(\epsilon_{k}\right)-f_{a}(t, \mathbf{k})\right]+\mathcal{O}\left(|h|^{4}\right) .
$$

This equation is valid both for large and small deviations from equilibrium. ${ }^{36}$ It is seen how the production stops when $f_{a} \rightarrow n_{\mathrm{B}}$, as must be the case. The equilibration rate is the same $\gamma=|h|^{2} \rho(\mathcal{K}) / \epsilon_{k}$ as before.

- In this section we have related a particle production rate to a general spectral function, $\rho(\mathcal{K})$. The computation of this spectral function represents a challenge of its own. In sections 8.2 and 8.3 simple examples of such computations were given; however as alluded to below eq. (8.100), a proper computation normally requires HTL resummation, the inclusion of $2 \leftrightarrow 2$ scatterings, as well as a so-called Landau-Pomeranchuk-Migdal (LPM) resummation of almost coherent $1+n \leftrightarrow 2+n$ scatterings. Computations including these processes for the example of sections 8.2 and 8.3 have been presented in refs. [9.42,9.43], and a similar analysis for the production of photons from a QCD plasma can be found in refs. [9.44,9.45].

\section{Appendix A: Streamlined derivation of the particle production rate}

We outline here another derivation of the particle production rate, similar to the one employed in refs. $[9.37,9.38]$, which is technically simpler than the one presented above but comes with the price of being somewhat heuristic and thus implicit about the assumptions made.

Let $|\mathbf{k}\rangle \equiv \hat{a}_{\mathbf{k}}^{\dagger}|0\rangle$ be a state with one " $a$-particle" of momentum $\mathbf{k}$. Consider an initial state $|I\rangle$ and a final state $|F\rangle$, with

$$
|I\rangle \equiv|i\rangle \otimes|0\rangle, \quad|F\rangle \equiv|f\rangle \otimes|\mathbf{k}\rangle,
$$

where $|i\rangle$ and $|f\rangle$ are the initial and final states, respectively, in the Hilbert space of the degrees of freedom constituting the heat bath. The transition matrix element reads

$$
T_{F I}=\left\langle F\left|\int_{0}^{t} \mathrm{~d} t^{\prime} \hat{H}_{I}\left(t^{\prime}\right)\right| I\right\rangle
$$

where $\hat{H}_{I}$ is the interaction Hamiltonian in the interaction picture. The particle production rate can now be defined as

$$
\frac{\dot{f}_{a}(t, \mathbf{k})}{(2 \pi)^{3}} \equiv \lim _{t, V \rightarrow \infty} \sum_{f, i} \frac{e^{-\beta \epsilon_{i}}}{\mathcal{Z}_{\mathrm{bath}}} \frac{\left|T_{F I}\right|^{2}}{t V}
$$

where a thermal average is taken over all initial states, whereas for final states no constraint other than that built into the transition matrix elements is imposed. Furthermore, $\mathcal{Z}_{\text {bath }} \equiv \sum_{i} e^{-\beta \epsilon_{i}}$ is the partition function of the heat bath.

By making use of $\left\langle\mathbf{k}\left|\hat{H}_{I}\right| 0\right\rangle=\left\langle 0\left|\hat{a}_{\mathbf{k}} \hat{H}_{I}\right| 0\right\rangle=\left\langle 0\left|\left[\hat{a}_{\mathbf{k}}, \hat{H}_{I}\right]\right| 0\right\rangle$ and eq. (9.121), we immediately obtain

$$
\left\langle F\left|\int_{0}^{t} \mathrm{~d} t^{\prime} \hat{H}_{I}\left(t^{\prime}\right)\right| I\right\rangle=h \int_{\mathcal{X}^{\prime}} \frac{e^{i \mathcal{K} \cdot \mathcal{X}^{\prime}}}{\sqrt{(2 \pi)^{3} 2 \epsilon_{k}}}\left\langle f\left|\hat{\mathcal{J}}\left(\mathcal{X}^{\prime}\right)\right| i\right\rangle
$$

\footnotetext{
${ }^{36} \mathrm{~A}$ way to show this from the above formalism has been presented in ref. [9.40], and a general analysis can be found in ref. [9.41].
} 
and subsequently

$$
\left|T_{F I}\right|^{2}=\frac{|h|^{2}}{(2 \pi)^{3} 2 \epsilon_{k}} \int_{\mathcal{X}^{\prime}, \mathcal{Y}^{\prime}} e^{i \mathcal{K} \cdot\left(\mathcal{X}^{\prime}-\mathcal{Y}^{\prime}\right)}\left\langle f\left|\hat{\mathcal{J}}\left(\mathcal{X}^{\prime}\right)\right| i\right\rangle\left\langle i\left|\hat{\mathcal{J}}^{\dagger}\left(\mathcal{Y}^{\prime}\right)\right| f\right\rangle .
$$

This can be inserted into eq. (9.140). Taking the thermodynamic limit; making use of translational invariance in order to cancel the time and volume factors from the denominator; and paying attention to the ordering of the operators $\left(e^{-\beta \hat{H}}\right.$ and therefore the states $|i\rangle$ should appear at the "outer edge"), we arrive at

$$
\dot{f}_{a}(t, \mathbf{k})=\frac{|h|^{2}}{2 \epsilon_{k}} \int_{\mathcal{X}} e^{i \mathcal{K} \cdot \mathcal{X}}\left\langle\hat{\mathcal{J}}^{\dagger}(0) \hat{\mathcal{J}}(\mathcal{X})\right\rangle .
$$

If we now compare the result with eqs. (8.3) and (8.14), we can write the final expression as

$$
\dot{f}_{a}(t, \mathbf{k})=\frac{|h|^{2}}{2 \epsilon_{k}} \Pi^{<}(\mathcal{K})=\frac{n_{\mathrm{B}}\left(k^{0}\right)}{\epsilon_{k}}|h|^{2} \rho(\mathcal{K}),
$$

where $k^{0}=\epsilon_{k}$ and it is understood that the spectral function corresponds to the operator $\hat{\mathcal{J}}$.

A similar computation, making use of translational invariance and eqs. (8.2) and (8.15), yields the production rate of the " $b$-particles":

$$
\begin{aligned}
\dot{f}_{b}(t, \mathbf{k}) & =\frac{|h|^{2}}{2 \epsilon_{k}} \int_{\mathcal{X}} e^{i \mathcal{K} \cdot \mathcal{X}}\left\langle\hat{\mathcal{J}}(0) \hat{\mathcal{J}}^{\dagger}(\mathcal{X})\right\rangle \\
& =\frac{|h|^{2}}{2 \epsilon_{k}} \int_{\mathcal{X}} e^{i \mathcal{K} \cdot(\mathcal{X}-\mathcal{Y})}\left\langle\hat{\mathcal{J}}(\mathcal{Y}) \hat{\mathcal{J}}^{\dagger}(\mathcal{X})\right\rangle \\
& =\frac{|h|^{2}}{2 \epsilon_{k}} \int_{\mathcal{Y}} e^{-i \mathcal{K} \cdot \mathcal{Y}}\left\langle\hat{\mathcal{J}}(\mathcal{Y}) \hat{\mathcal{J}}^{\dagger}(0)\right\rangle \\
& =\frac{|h|^{2}}{2 \epsilon_{k}} \Pi^{>}(-\mathcal{K})=-\frac{n_{\mathrm{B}}\left(k^{0}\right)}{\epsilon_{k}}|h|^{2} \rho(-\mathcal{K}),
\end{aligned}
$$

like in eq. (9.136). Finally we note that the total number density increases as

$$
\frac{\mathrm{d}\left(N_{a}+N_{b}\right)}{\mathrm{d}^{4} \mathcal{X}}=|h|^{2} \int_{\mathbf{k}} \frac{n_{\mathrm{B}}\left(\epsilon_{k}\right)}{\epsilon_{k}}[\rho(\mathcal{K})-\rho(-\mathcal{K})] .
$$

\section{Appendix B: Particle decay rate}

The discussion above concerned the production rate of particles whose total density remains below the equilibrium value. As we now outline, one can similarly consider an "opposite" limit, in which the Hilbert space corresponding to weakly interacting particles is "full" in the initial state, and the particles are forced to decay.

Using the same notation as in appendix A, we consider an initial state $|I\rangle$ and a final state $|F\rangle$, with

$$
|I\rangle \equiv|i\rangle \otimes|\mathbf{k}\rangle, \quad|F\rangle \equiv|f\rangle \otimes|0\rangle,
$$

where $|i\rangle$ and $|f\rangle$ are the initial and final states, respectively, in the Hilbert space of the degrees of freedom constituting the heat bath. The transition matrix element can be defined and computed as before, and in the end eq. (9.142) gets replaced with

$$
\left|T_{F I}\right|^{2}=\frac{|h|^{2}}{(2 \pi)^{3} 2 \epsilon_{k}} \int_{\mathcal{X}^{\prime}, \mathcal{Y}^{\prime}} e^{i \mathcal{K} \cdot\left(\mathcal{Y}^{\prime}-\mathcal{X}^{\prime}\right)}\left\langle f\left|\hat{\mathcal{J}}^{\dagger}\left(\mathcal{X}^{\prime}\right)\right| i\right\rangle\left\langle i\left|\hat{\mathcal{J}}\left(\mathcal{Y}^{\prime}\right)\right| f\right\rangle .
$$


It follows that

$$
\dot{f}_{a}(t, \mathbf{k})=-\frac{|h|^{2}}{2 \epsilon_{k}} \Pi^{>}(\mathcal{K})=-\frac{|h|^{2}}{\epsilon_{k}}\left[1+n_{\mathrm{B}}\left(k^{0}\right)\right] \rho(\mathcal{K}) .
$$

If we take the zero-temperature limit by setting $n_{\mathrm{B}}\left(k^{0}\right) \rightarrow 0$, the result remains non-zero, and equals the text-book decay rate in vacuum (which was denoted by $\gamma$ in the figure on p. 175).

\section{Appendix C: Scattering on dense media}

We have shown that if there is an interaction of the form $\hat{H}_{\mathrm{int}} \sim \int_{\mathbf{x}}\left(h \hat{\phi}^{\dagger} \hat{\mathcal{J}}+\right.$ H.c. $)$, then an initial density matrix,

$$
\hat{\rho}(0)=\hat{\rho}_{\text {bath }} \otimes|0\rangle\langle 0|,
$$

evolves into a form whereby the number density operator of the $\phi$-particles takes a non-zero expectation value. The formal reason for this is that the amplitude $\left\langle\mathbf{k}\left|\hat{H}_{\text {int }}\right| 0\right\rangle$ is non-zero because of $\hat{\phi}$ in $\hat{H}_{\text {int }}$, cf. eq. (9.141).

Now, a variant of this situation can be envisaged, namely that of scattering of a weakly interacting particle on a thermal medium. In this case, the initial state would be something like

$$
\hat{\rho}(0)=\hat{\rho}_{\text {bath }} \otimes\left|\mathbf{k}_{\mathrm{i}}\right\rangle\left\langle\mathbf{k}_{\mathrm{i}}\right|,
$$

and the question is how fast the system evolves towards a state

$$
\hat{\rho}(t)=\hat{\rho}_{\text {bath }} \otimes\left|\mathbf{k}_{\mathrm{f}}\right\rangle\left\langle\mathbf{k}_{\mathrm{f}}\right|,
$$

with $\mathbf{k}_{\mathrm{i}} \neq \mathbf{k}_{\mathrm{f}}$. This is an interesting problem because the weakly interacting probe could effectively scatter from a collective excitation, and the relation between $\mathbf{k}_{\mathrm{i}}$ and $\mathbf{k}_{\mathrm{f}}$ could be used to determine the dispersion relation of the collective excitation. Cohen and Feynman originally proposed this method in order to determine the dispersion relation of collective excitations in liquid helium through inelastic neutron scattering [9.46], and the proposal has been successfully realized [9.47].

In the context of $\mathrm{QCD}$, it is difficult to envisage how a corresponding experiment could be realized, because QCD matter cannot be confined to a container on which a scattering experiment could be carried out. Nevertheless, on an adventurous note, we might speculate that a monochromatic $\mathrm{X}$-ray beam from a quasar scattering on a very compact neutron star could experience similar phenomenology.

In any case, the basic idea is the following. Suppose that the temperature is so low that the medium is in its ground state, without any kind of motion taking place $(T \ll m, \mu)$, and suppose that the $\phi$-particles are massless (e.g. photons). In an inelastic scattering, the momentum $\Delta p$ and the energy $\Delta E$ are transferred to the medium, with

$$
\begin{aligned}
\Delta p & =\left|\mathbf{k}_{\mathrm{i}}-\mathbf{k}_{\mathrm{f}}\right|=\sqrt{k_{\mathrm{i}}^{2}+k_{\mathrm{f}}^{2}-2 k_{\mathrm{i}} k_{\mathrm{f}} \cos \theta}, \\
\Delta E & =k_{\mathrm{i}}-k_{\mathrm{f}} .
\end{aligned}
$$

The scattering is most efficient, i.e. resonant, if the given $\Delta p$ and $\Delta E$ kick an on-shell collective excitation into motion. If the latter has the dispersion relation $\omega(k)$, resonant scattering takes place for

$$
\Delta E=\omega(\Delta p) .
$$


For a given $k_{\mathrm{i}}$ and $\theta$, this can be viewed as an equation for $k_{\mathrm{f}}$. Consequently, if the peak wave number $k_{\mathrm{f}}$ is measured as a function of the scattering angle $\theta$, one can experimentally determine the function $\omega(k)$. If $\omega(k)$ contains a scale, such as $m_{\mathrm{F}}$ (cf. eq. (8.203)), then non-trivial solutions are to be expected in the range $k_{\mathrm{i}}, k_{\mathrm{f}} \sim m_{\mathrm{F}}$.

The phenomenon just discussed might be particularly remarkable if $\Delta E<0$, i.e. more energy comes out than goes in. This can happen with the dispersion relation of eq. (8.203), and could be referred to as "Compton scattering on a plasmino". Of course, in any realistic situation, there is a background to this process from thermal free electrons with a non-trivial velocity distribution.

Formally, the amplitude for the scattering contains two appearances of $\hat{H}_{\text {int }}$, one for absorption and the other for emission. The rate will therefore be proportional to a certain 4-point function of the currents, yielding a theoretical description of scattering more complicated than for particle production, where we only encountered 2-point functions. 


\subsection{Embedding rates in cosmology}

In sec. 9.3 we considered the production rate of weakly interacting particles at a fixed temperature, $T$. In a cosmological setting, however, account needs to be taken of the expansion of the universe, which leads to an evolving temperature as well as red-shifting particle momenta. This has implications for practical computations of e.g. dark matter spectra, as will be illustrated in the current section.

Let $f(t, \mathbf{k})$ denote the phase space density of a species of particles being produced, so that their number density reads

$$
\frac{N}{V}=\int \frac{\mathrm{d}^{3} \mathbf{k}}{(2 \pi)^{3}} f(t, \mathbf{k})
$$

and the corresponding production rate (cf. eq. (9.119)) equals

$$
\dot{f}(t, \mathbf{k})=R(T, \mathbf{k}) .
$$

For the particular model considered in sec. 9.3 and particles of "type $a$ ", the production rate $R_{a}$ is given by eq. (9.134); in the following we use a slightly more realistic example, where $f$ counts

right-handed neutrinos in either polarization state. Then a computation similar to that in sec. 9.3 leads to

$$
R(T, \mathbf{k}) \equiv \sum_{a= \pm} \dot{f}_{a}(t, \mathbf{k})=\left.\frac{n_{\mathrm{F}}\left(k^{0}\right)}{k^{0}}|h|^{2} \operatorname{Tr}\left\{\not \mathcal{K} a_{\mathrm{L}}[\rho(-\mathcal{K})+\rho(\mathcal{K})] a_{\mathrm{R}}\right\}\right|_{k^{0}=\sqrt{k^{2}+M^{2}}},
$$

where the notation and the spectral function are as discussed in sec. 8.2, and the sum goes over the two polarization states of a massive Majorana fermion.

\section{Basic cosmology}

Let us begin by recalling cosmological relations between the time $t$ and the temperature $T$ and by setting up our notation. As usual, we assume that even if there were net number densities present, they are very small compared with the temperature, $\mu \ll \pi T$, so that thermodynamic quantities are determined by the temperature alone (this assumption will be relaxed in appendix B). Assuming furthermore a homogeneous and isotropic metric,

$$
\mathrm{d} s^{2}=\mathrm{d} t^{2}-a^{2}(t) \mathrm{d} \mathbf{x}_{\kappa}^{2},
$$

where $\kappa=0, \pm 1$ characterizes the spatial geometry, as well as the energy-momentum tensor of an ideal fluid,

$$
T_{\mu}{ }^{\nu}=\operatorname{diag}(e,-p,-p,-p),
$$

where $e$ denotes the energy density and $p$ the pressure, the Einstein equations, $G_{\mu}{ }^{\nu}=8 \pi G T_{\mu}{ }^{\nu}$, reduce to the Friedmann equations

$$
\begin{aligned}
\left(\frac{\dot{a}}{a}\right)^{2}+\frac{\kappa}{a^{2}} & =\frac{8 \pi G e}{3}, \\
\mathrm{~d}\left(e a^{3}\right) & =-p \mathrm{~d}\left(a^{3}\right) .
\end{aligned}
$$

We assume a flat universe, $\kappa=0$, and denote

$$
\frac{1}{m_{\mathrm{Pl}}^{2}} \equiv G
$$


where $m_{\mathrm{Pl}} \approx 1.2 \times 10^{19} \mathrm{GeV}$ is the Planck mass. We may then introduce the "Hubble parameter" $H$ via

$$
H \equiv \frac{\dot{a}(t)}{a(t)}=\sqrt{\frac{8 \pi}{3}} \frac{\sqrt{e}}{m_{\mathrm{Pl}}} .
$$

We now combine the Friedmann equations with basic thermodynamic relations. In a system with small chemical potentials, the energy and entropy densities are related by

$$
e=T s-p
$$

where $s=\mathrm{d} p / \mathrm{d} T$ is the entropy density. From here, it follows that $\mathrm{d} e=T \mathrm{~d} s$, which together with eq. (9.162) leads to the relation

$$
\begin{aligned}
0 & =\mathrm{d}\left(e a^{3}\right)+p \mathrm{~d}\left(a^{3}\right) \\
& =a^{3} \mathrm{~d} e+(p+e) \mathrm{d}\left(a^{3}\right) \\
& =a^{3} T \mathrm{~d} s+T s \mathrm{~d}\left(a^{3}\right) \\
& =T \mathrm{~d}\left(s a^{3}\right)
\end{aligned}
$$

This relation is known as the entropy conservation law, and can be re-expressed as

$$
\frac{a(t)}{a\left(t_{0}\right)}=\left[\frac{s\left(T_{0}\right)}{s(T)}\right]^{\frac{1}{3}} .
$$

We can also derive an evolution equation for the temperature. The entropy conservation law implies that

$$
\frac{\mathrm{d} s}{s}=-3 \frac{\mathrm{d} a}{a}
$$

whereas defining the "heat capacity" $c$ through

$$
\frac{\mathrm{d} e}{\mathrm{~d} T}=T \frac{\mathrm{d} s}{\mathrm{~d} T} \equiv T c,
$$

we get $\mathrm{d} s=c \mathrm{~d} T$. Inserting this into eq. (9.168) and dividing by $\mathrm{d} t$ leads to

$$
\begin{aligned}
& \frac{c}{s} \frac{\mathrm{d} T}{\mathrm{~d} t}=-\frac{3 \dot{a}}{a} \\
\stackrel{(9.164)}{\Rightarrow} & \frac{\mathrm{d} T}{\mathrm{~d} t}=-\frac{\sqrt{24 \pi}}{m_{\mathrm{Pl}}} \frac{s(T) \sqrt{e(T)}}{c(T)} .
\end{aligned}
$$

In cosmological literature, it is conventional to introduce two different ways to count the effective numbers of massless bosonic degrees of freedom, $g_{\mathrm{eff}}(T)$ and $h_{\mathrm{eff}}(T)$, defined via the relations

$$
e(T) \equiv \frac{\pi^{2} T^{4}}{30} g_{\mathrm{eff}}(T), \quad s(T) \equiv \frac{2 \pi^{2} T^{3}}{45} h_{\mathrm{eff}}(T),
$$

where the prefactors follow by applying eq. (9.165) and the line below it to the free result $p(T)=$ $\pi^{2} T^{4} / 90$ from eq. (2.81). Furthermore, for later reference, we note that the sound speed squared can be written in the forms

$$
c_{s}^{2}(T) \equiv \frac{\partial p}{\partial e}=\frac{p^{\prime}(T)}{e^{\prime}(T)}=\frac{p^{\prime}(T)}{T s^{\prime}(T)}=\frac{s(T)}{T c(T)} .
$$




\section{Production equation and its solution}

In order to generalize eq. (9.157) to an expanding background, we have to properly define our variables, the time $t$ and the momentum $\mathbf{k}$. In the following we mean by these the physical time and momentum, i.e. quantities defined in a local Minkowskian frame. However, as is well known, local Minkowskian frames at different times are inequivalent in an expanding background; in particular, the physical momenta redshift. Carrying out the derivation of the rate equation in this situation is a topic of general relativity, and we only quote the result here: the main effect of expansion is that the time derivative gets replaced as $\partial / \partial t \rightarrow \partial / \partial t-H k^{i} \partial / \partial k^{i}[9.48,9.49]$, and eq. (9.157) becomes

$$
\left(\frac{\partial}{\partial t}-H k^{i} \frac{\partial}{\partial k^{i}}\right) f(t, \mathbf{k})=R(T, \mathbf{k}),
$$

where $H$ is the Hubble parameter from eq. (9.164) and $k^{i}$ are the components of $\mathbf{k}$.

It is important to stress that the production rate $R(T, \mathbf{k})$ in eq. (9.174) can be directly taken over from the flat spacetime result in eq. (9.158). The reason is that the time scale of the equilibration of the plasma and of the scattering reactions taking place within the plasma is $\tau \lesssim 1 /\left(\alpha^{2} T\right)$, where $\alpha$ is a generic fine-structure constant. Unless $T$ is exceedingly high, this is much smaller than the time scale associated with the expansion of the universe, $H^{-1} \sim m_{\mathrm{Pl}} / T^{2}$. Therefore, local Minkowskian coordinates can be used for the duration of the plasma scatterings. Note however that the rate $R$ itself can be small; as has been discussed in sec. 9.3, the coupling $|h|^{2}$, connecting the non-equilibrium degrees of freedom to the plasma particles, is by assumption small, $|h|^{2} \ll \alpha$. In other words, the rate $R$ is determined by the physics of almost instantaneous scatterings taking place with a rate $1 / \tau \gg H$, but its numerical value could nevertheless be tiny, $R \ll H$.

Now, because of rotational symmetry, $R(T, \mathbf{k})$ and consequently also $f(t, \mathbf{k})$ are typically only functions of $k \equiv|\mathbf{k}|$. Changing the notation correspondingly, and noting that $\partial k / \partial k^{i}=k^{i} / k$, eq. (9.174) becomes

$$
\left(\frac{\partial}{\partial t}-H k \frac{\partial}{\partial k}\right) f(t, k)=R(T, k) .
$$

Furthermore, if we are only interested in the total number density, $\int_{\mathbf{k}} f(t, k)$, rather than the shape of the spectrum, we can integrate eq. (9.175) on both sides. Partially integrating $\int \mathrm{d}^{3} \mathbf{k} k \partial_{k} f(t, k)=$ $-3 \int \mathrm{d}^{3} \mathbf{k} f(t, k)$ then leads to an equation for the number density,

$$
\left(\partial_{t}+3 H\right) \int_{\mathbf{k}} f(t, k)=\int_{\mathbf{k}} R(T, k) .
$$

Eq. (9.175) can be integrated through a suitable change of variables, known as the method of characteristics. Introducing an ansatz $f(t, k)=f\left(t, k\left(t_{0}\right) \frac{a\left(t_{0}\right)}{a(t)}\right)$, and noting that

$$
\frac{\mathrm{d}}{\mathrm{d} t}\left[k\left(t_{0}\right) \frac{a\left(t_{0}\right)}{a(t)}\right]=-k\left(t_{0}\right) \frac{a\left(t_{0}\right) \dot{a}(t)}{a^{2}(t)}=-H k,
$$

eq. (9.175) can be re-expressed as

$$
\frac{\mathrm{d} f}{\mathrm{~d} t}\left(t, k\left(t_{0}\right) \frac{a\left(t_{0}\right)}{a(t)}\right)=R\left(T, k\left(t_{0}\right) \frac{a\left(t_{0}\right)}{a(t)}\right) .
$$

This can immediately be solved as

$$
f\left(t_{0}, k\left(t_{0}\right)\right)=\int_{0}^{t_{0}} \mathrm{~d} t R\left(T(t), k\left(t_{0}\right) \frac{a\left(t_{0}\right)}{a(t)}\right),
$$


where we assumed the initial condition $f(0, k)=0$, i.e. that there were no particles at $t=0$. Let us also note that the entropy conservation law of eq. (9.166) implies that $\left(\partial_{t}+3 H\right) s=0$, permitting us to re-express eq. (9.176) as

$$
\frac{\mathrm{d}}{\mathrm{d} t}\left[\frac{\int_{\mathbf{k}} f(t, k)}{s(t)}\right]=\frac{\int_{\mathbf{k}} R(T, k)}{s(t)}
$$

In cosmology, it is convenient to measure time directly in terms of the temperature. The corresponding change of variables, eq. (9.171), is often implemented in some approximate form; in its exact form, we need information concerning the pressure $p(T)$ (appearing in $e(T)=T p^{\prime}(T)-p(T)$ ), its first derivative $p^{\prime}(T)$ (appearing in $e(T)$ as well as in $s(T)=p^{\prime}(T)$ ), and its second derivative $p^{\prime \prime}(T)$ (appearing in $c(T)=s^{\prime}(T)$ ). The particular combination defining the sound speed squared, eq. (9.173), is close to $\frac{1}{3}$, so it is useful to factor it out. Inserting also eq. (9.164), eq. (9.171) then becomes

$$
\frac{\mathrm{d} T}{\mathrm{~d} t}=-\sqrt{\frac{8 \pi}{3}} \frac{T}{m_{\mathrm{Pl}}} \sqrt{e(T)}\left[3 c_{s}^{2}(T)\right]=-T H(T)\left[3 c_{s}^{2}(T)\right] .
$$

Further defining the so-called yield parameter,

$$
Y\left(t_{0}\right) \equiv \frac{\int_{\mathbf{k}} f\left(t_{0}, k\right)}{s\left(t_{0}\right)}
$$

eq. (9.180) becomes

$$
T \frac{\mathrm{d} Y}{\mathrm{~d} T}=\frac{-1}{3 c_{s}^{2}(T) s(T) H(T)} \int_{\mathbf{k}} R(T, k) .
$$

This equation implies, amongst other things, that close to a first order phase transition, where $c_{s}^{2}$ typically has a dip, the yield of produced particles is enhanced. The reason is that the system spends a long time at these temperatures, diluting the specific heat being released into the expansion of the universe, and that therefore there is a long period available for particle production.

\section{Example}

Let us write the main results derived above in an explicit form, by inserting into them the parametrizations of eq. (9.172). Denoting $k \equiv k\left(t_{0}\right)$, inserting the red-shift factor from eq. (9.167), and changing the integration variable from $t$ to $T$ according to eq. (9.181), the result of eq. (9.179) can be expressed as [9.50]

$$
f\left(t_{0}, k\right)=\sqrt{\frac{5}{4 \pi^{3}}} \int_{T_{0}}^{T_{\max }} \frac{\mathrm{d} T}{T^{3}} \frac{m_{\mathrm{Pl}}}{c_{s}^{2}(T) \sqrt{g_{\mathrm{eff}}(T)}} R\left(T, k \frac{T}{T_{0}}\left[\frac{h_{\mathrm{eff}}(T)}{h_{\mathrm{eff}}\left(T_{0}\right)}\right]^{\frac{1}{3}}\right),
$$

where $T_{\max }$ corresponds to the highest temperature of the universe. This gives the spectrum of particles produced as an integral over the history of their production. The integral over eq. (9.184), after the substitution $k=z T_{0}\left[h_{\text {eff }}\left(T_{0}\right) / h_{\text {eff }}(T)\right]^{1 / 3}$ and followed by a division by $s\left(t_{0}\right)$, or a direct integration of eq. (9.183), gives their total yield:

$$
Y\left(t_{0}\right)=\frac{45 \sqrt{5}}{(2 \pi)^{3} \pi^{5 / 2}} \int_{T_{0}}^{T_{\max }} \frac{\mathrm{d} T}{T^{3}} \frac{m_{\mathrm{Pl}}}{c_{s}^{2}(T) h_{\mathrm{eff}}(T) \sqrt{g_{\mathrm{eff}}(T)}} \int_{0}^{\infty} \mathrm{d} z z^{2} R(T, T z) .
$$

We note that if $\int_{0}^{\infty} \mathrm{d} z z^{2} R(T, T z)$ vanishes sufficiently fast at low temperatures (typically it contains a Boltzmann factor and becomes exponentially suppressed when $T$ falls below some mass scale), then the result is independent of $T_{0}$. 
To be more explicit, we need to specify the function $R(T, k)$, which for our example can be obtained from eqs. (8.89), (8.90) and (9.158). The Dirac algebra in eq. (9.158) can be trivially carried out, resulting in

$$
\operatorname{Tr}\left\{\not K a_{\mathrm{L}}\left[\not_{1}\right] a_{\mathrm{R}}\right\}=2 \mathcal{K} \cdot \mathcal{P}_{1} .
$$

Furthermore, the $\delta$-functions appearing in eq. (8.89) can be written in various ways depending on the channel (setting $D \rightarrow 4$ ),

$$
\begin{aligned}
\delta^{(4)}\left(\mathcal{P}_{1}+\mathcal{P}_{2}-\mathcal{K}\right) 2 \mathcal{K} \cdot \mathcal{P}_{1} & =\delta^{(4)}\left(\mathcal{P}_{1}+\mathcal{P}_{2}-\mathcal{K}\right)\left[\mathcal{P}_{1}^{2}+\mathcal{K}^{2}-\left(\mathcal{K}-\mathcal{P}_{1}\right)^{2}\right] \\
& =\delta^{(4)}\left(\mathcal{P}_{1}+\mathcal{P}_{2}-\mathcal{K}\right)\left[\mathcal{P}_{1}^{2}+\mathcal{K}^{2}-\mathcal{P}_{2}^{2}\right] \\
\delta^{(4)}\left(\mathcal{P}_{1}-\mathcal{P}_{2}-\mathcal{K}\right) 2 \mathcal{K} \cdot \mathcal{P}_{1} & =\delta^{(4)}\left(\mathcal{P}_{1}-\mathcal{P}_{2}-\mathcal{K}\right)\left[\mathcal{P}_{1}^{2}+\mathcal{K}^{2}-\left(\mathcal{K}-\mathcal{P}_{1}\right)^{2}\right] \\
& =\delta^{(4)}\left(\mathcal{P}_{1}-\mathcal{P}_{2}-\mathcal{K}\right)\left[\mathcal{P}_{1}^{2}+\mathcal{K}^{2}-\mathcal{P}_{2}^{2}\right] \\
\delta^{(4)}\left(\mathcal{P}_{2}-\mathcal{P}_{1}-\mathcal{K}\right) 2 \mathcal{K} \cdot \mathcal{P}_{1} & =\delta^{(4)}\left(\mathcal{P}_{2}-\mathcal{P}_{1}-\mathcal{K}\right)\left[\left(\mathcal{K}+\mathcal{P}_{1}\right)^{2}-\mathcal{P}_{1}^{2}-\mathcal{K}^{2}\right] \\
& =\delta^{(4)}\left(\mathcal{P}_{2}-\mathcal{P}_{1}-\mathcal{K}\right)\left[\mathcal{P}_{2}^{2}-\mathcal{P}_{1}^{2}-\mathcal{K}^{2}\right] \\
\delta^{(4)}\left(\mathcal{P}_{1}+\mathcal{P}_{2}+\mathcal{K}\right) 2 \mathcal{K} \cdot \mathcal{P}_{1} & =\delta^{(4)}\left(\mathcal{P}_{1}+\mathcal{P}_{2}+\mathcal{K}\right)\left[\left(\mathcal{K}+\mathcal{P}_{1}\right)^{2}-\mathcal{P}_{1}^{2}-\mathcal{K}^{2}\right] \\
& =\delta^{(4)}\left(\mathcal{P}_{1}+\mathcal{P}_{2}+\mathcal{K}\right)\left[\mathcal{P}_{2}^{2}-\mathcal{P}_{1}^{2}-\mathcal{K}^{2}\right],
\end{aligned}
$$

where the factors are all constants, independent of $\mathbf{p}_{1}, \mathbf{p}_{2}$. Thereby we arrive at

$$
\begin{aligned}
& R(T, k)=\frac{|h|^{2}}{2 \sqrt{k^{2}+M^{2}}}\left(m_{\phi}^{2}-m_{\ell}^{2}-M^{2}\right) \int \frac{\mathrm{d}^{3} \mathbf{p}_{1}}{(2 \pi)^{3} 2 \epsilon_{1}} \int \frac{\mathrm{d}^{3} \mathbf{p}_{2}}{(2 \pi)^{3} 2 \epsilon_{2}} \times
\end{aligned}
$$

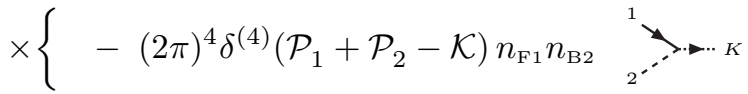

$$
\begin{aligned}
& -(2 \pi)^{4} \delta^{(4)}\left(\mathcal{P}_{1}-\mathcal{P}_{2}-\mathcal{K}\right) n_{\mathrm{F} 1}\left(1+n_{\mathrm{B} 2}\right) \\
& +(2 \pi)^{4} \delta^{(4)}\left(\mathcal{P}_{2}-\mathcal{P}_{1}-\mathcal{K}\right) n_{\mathrm{B} 2}\left(1-n_{\mathrm{F} 1}\right) \\
& \left.+(2 \pi)^{4} \delta^{(4)}\left(\mathcal{P}_{1}+\mathcal{P}_{2}+\mathcal{K}\right)\left(1-n_{\mathrm{F} 1}\right)\left(1+n_{\mathrm{B} 2}\right)\right\},{\stackrel{\mathcal{K}}{{ }^{\prime}}}_{{ }_{2}}^{1}
\end{aligned}
$$

where $\epsilon_{1} \equiv \sqrt{p_{1}^{2}+m_{\ell}^{2}}$ and $\epsilon_{2} \equiv \sqrt{p_{2}^{2}+m_{\phi}^{2}}$. In passing, we note that eq. (9.188) is equivalent to a collision term of a Boltzmann equation; the structure of the latter is recalled in appendix A.

Let us analyze eq. (9.188) in more detail, recalling that $k^{0}=\sqrt{k^{2}+M^{2}}>0$, with $M$ the mass of the produced particle. The first question is, when do the different channels get realized. Since all the particles are massive, we can go to the rest frame of the decaying one; it is then clear that the first channel gets realized for $M>m_{\ell}+m_{\phi}$; the second for $m_{\ell}>M+m_{\phi}$; the third for $m_{\phi}>M+m_{\ell}$; and the last one never. As an example, assuming that the scalar mass (the Higgs mass) is larger than those of the produced particles, $m_{\phi} \gg M, m_{\ell}$, we can focus on the third channel, where the integral to be considered reads

$$
I(k) \equiv \int \frac{\mathrm{d}^{3} \mathbf{p}_{1}}{(2 \pi)^{3} 2 \epsilon_{1}} \int \frac{\mathrm{d}^{3} \mathbf{p}_{2}}{(2 \pi)^{3} 2 \epsilon_{2}}(2 \pi)^{4} \delta^{(4)}\left(\mathcal{P}_{2}-\mathcal{P}_{1}-\mathcal{K}\right) n_{\mathrm{B}}\left(\epsilon_{2}\right)\left[1-n_{\mathrm{F}}\left(\epsilon_{1}\right)\right] .
$$

The integral in eq. (9.189) can be simplified, if we go to the high-temperature limit where the masses $M^{2}=\mathcal{K}^{2}$ and $m_{\ell}^{2}=\mathcal{P}_{1}^{2}$ of the produced particles can be neglected. ${ }^{37}$ Denoting

$$
p \equiv\left|\mathbf{p}_{1}\right|, \quad k \equiv|\mathbf{k}|,
$$

\footnotetext{
${ }^{37}$ It must be noted that, as discussed in sections 8.2 and 9.3, unresummed computations typically lose their validity in the ultra-relativistic limit when the temperature is much higher than particle masses, cf. e.g. refs. [9.42,9.44]. We assume here that $M, m_{\ell} \ll \pi T \ll m_{\phi}$.
} 
we get

$$
\begin{aligned}
I(k) & =\int \frac{\mathrm{d}^{3} \mathbf{p}_{1}}{(2 \pi)^{3} 2 p} \int \frac{\mathrm{d}^{3} \mathbf{p}_{2}}{(2 \pi)^{3} 2 \epsilon_{2}}(2 \pi)^{3} \delta^{(3)}\left(\mathbf{p}_{1}+\mathbf{k}-\mathbf{p}_{2}\right)(2 \pi) \delta\left(p+k-\epsilon_{2}\right) n_{\mathrm{B}}\left(\epsilon_{2}\right)\left[1-n_{\mathrm{F}}(p)\right] \\
& =\frac{1}{(4 \pi)^{2}} \int \frac{\mathrm{d}^{3} \mathbf{p}_{1}}{p(p+k)} \delta\left(p+k-\sqrt{m_{\phi}^{2}+\left(\mathbf{p}_{1}+\mathbf{k}\right)^{2}}\right) n_{\mathrm{B}}(p+k)\left[1-n_{\mathrm{F}}(p)\right] \\
& =\frac{1}{8 \pi} \int_{0}^{\infty} \frac{\mathrm{d} p p}{p+k} \int_{-1}^{+1} \mathrm{~d} z \delta\left(p+k-\sqrt{m_{\phi}^{2}+p^{2}+k^{2}+2 p k z}\right) n_{\mathrm{B}}(p+k)\left[1-n_{\mathrm{F}}(p)\right],
\end{aligned}
$$

where spherical coordinates were introduced in the last step. The Dirac- $\delta$ gets realized when

$$
p^{2}+k^{2}+2 p k=m_{\phi}^{2}+p^{2}+k^{2}+2 p k z
$$

i.e. $z=1-m_{\phi}^{2} /(2 p k)$. This belongs to the interval $(-1,1)$ if $p>m_{\phi}^{2} / 4 k$, so that

$$
I(k)=\frac{1}{8 \pi} \int_{\frac{m_{\phi}^{2}}{4 k}}^{\infty} \frac{\mathrm{d} p p}{p+k}\left|\frac{\mathrm{d}}{\mathrm{d} z} \sqrt{m_{\phi}^{2}+p^{2}+k^{2}+2 p k z}\right|_{\sqrt{m_{\phi}^{2}+p^{2}+k^{2}+2 p k z}=p+k}^{-1} n_{\mathrm{B}}(p+k)\left[1-n_{\mathrm{F}}(p)\right] .
$$

The derivative appearing in the above expression is taken trivially,

$$
\left.\frac{\mathrm{d}}{\mathrm{d} z} \sqrt{\cdots}\right|_{\ldots}=\frac{p k}{p+k}
$$

whereby we arrive at

$$
I(k)=\frac{1}{8 \pi k} \int_{\frac{m_{\phi}^{2}}{4 k}}^{\infty} \mathrm{d} p n_{\mathrm{B}}(p+k)\left[1-n_{\mathrm{F}}(p)\right] .
$$

This describes how a fermion of momentum $k$ is produced from a decay of a Higgs particle of energy $p+k$, with the part $p$ of the energy being carried away by the other fermionic decay product, which experiences Pauli blocking in the final state.

The integration in eq. (9.195) can be performed by decoupling the $p$-dependence via the identity

$$
n_{\mathrm{B}}(p+k)\left[1-n_{\mathrm{F}}(p)\right]=\left[n_{\mathrm{B}}(p+k)+n_{\mathrm{F}}(p)\right] n_{\mathrm{F}}(k),
$$

leading to

$$
\begin{aligned}
I(k) & =\frac{T n_{\mathrm{F}}(k)}{8 \pi k}\left[\ln \left(1-e^{-\beta(p+k)}\right)-\ln \left(1+e^{-\beta p}\right)\right]_{\frac{m_{\phi}^{2}}{4 k}}^{\infty} \\
& =\frac{T n_{\mathrm{F}}(k)}{8 \pi k} \ln \left\{\frac{1+\exp \left[-\beta\left(\frac{m_{\phi}^{2}}{4 k}\right)\right]}{1-\exp \left[-\beta\left(k+\frac{m_{\phi}^{2}}{4 k}\right)\right]}\right\} .
\end{aligned}
$$

Inserting eq. (9.197) into eq. (9.188) (with $M=m_{\ell}=0$ ) then yields

$$
R(T, k)=\frac{|h|^{2} m_{\phi}^{2}}{2 k} I(k),
$$

which in combination with eq. (9.185) produces

$$
Y\left(t_{0}\right)=\frac{45 \sqrt{5}}{\pi^{5 / 2}} \frac{|h|^{2} m_{\phi}^{2}}{16 \pi^{3}} \int_{T_{0}}^{T_{\max }} \frac{\mathrm{d} T}{T^{4}} \frac{m_{\mathrm{Pl}}}{c_{s}^{2}(T) h_{\mathrm{eff}}(T) \sqrt{g_{\mathrm{eff}}(T)}} \int_{0}^{\infty} \mathrm{d} z z I(T z) .
$$


The remaining integrals can be carried out numerically. They display the variables on which the "dark matter" abundance depends on in this model: the coupling constant $\left(|h|^{2}\right)$, the mass of the decaying particle $\left(m_{\phi}\right)$, as well as the thermal history of the universe (through the functions $c_{s}^{2}$, $h_{\text {eff }}$ and $\left.g_{\text {eff }}\right) .38$

\section{Appendix A: Relativistic Boltzmann equation}

We recall here the structure of the collision term in the relativistic Boltzmann equation, and compare the result with the quantum field theoretic formula in eq. (9.188). ${ }^{39}$

To understand the logic of the Boltzmann equation, a possible starting point is Fermi's Golden Rule for a decay rate,

$$
\Gamma_{1 \rightarrow n}(\mathcal{K})=\frac{1}{2 \epsilon_{k}} \int \mathrm{d} \Phi_{1 \rightarrow n} c \sum\left|\mathcal{M}_{1 \rightarrow n}\right|^{2},
$$

where the phase space integration measure is defined as

$$
\int \mathrm{d} \Phi_{1+m \rightarrow n} \equiv \int\left\{\prod_{i=1}^{m} \frac{\mathrm{d}^{3} \mathbf{k}_{a_{i}}}{(2 \pi)^{3} 2 \epsilon_{a_{i}}}\right\}\left\{\prod_{j=1}^{n} \frac{\mathrm{d}^{3} \mathbf{p}_{b_{j}}}{(2 \pi)^{3} 2 \epsilon_{b_{j}}}\right\}(2 \pi)^{4} \delta^{(4)}\left(\mathcal{K}+\sum_{i=1}^{m} \mathcal{K}_{a_{i}}-\sum_{j=1}^{n} \mathcal{P}_{b_{j}}\right)
$$

and $\left\{a_{i}\right\}$ and $\left\{b_{j}\right\}$ label initial- and final-state particles, respectively, with four-momenta $\mathcal{K}_{a_{i}} \equiv$ $\left(\epsilon_{a_{i}}, \mathbf{k}_{a_{i}}\right)$ and $\mathcal{P}_{b_{j}} \equiv\left(\epsilon_{b_{j}}, \mathbf{p}_{b_{j}}\right)$. Moreover, $c=\frac{1}{i_{a} ! i_{b} !}$, where $i_{a}, i_{b}$ are the numbers of identical particles in the initial and final states, whose momenta are integrated over; $\mathcal{M}_{1 \rightarrow n}$ is an invariant amplitude; and the sum in eq. (9.200) goes over unresolved polarization states.

Let now $f(\mathcal{X}, \mathbf{k})$ be a particle distribution function; we assume its normalization to be so chosen that the total number density of particles at $\mathcal{X}$ is given by (cf. eq. (9.156))

$$
n(\mathcal{X})=\int \frac{\mathrm{d}^{3} \mathbf{k}}{(2 \pi)^{3}} f(\mathcal{X}, \mathbf{k}) \text {. }
$$

In thermal equilibrium, $f(\mathcal{X}, \mathbf{k})$ is uniquely determined by the temperature and by possible chemical potentials, $f(\mathcal{X}, \mathbf{k}) \equiv n_{\mathrm{F}}\left(\epsilon_{k} \pm \mu\right)$ (or $n_{\mathrm{B}}\left(\epsilon_{k} \pm \mu\right)$ for bosons). At the same time, for a single plane wave in vacuum, regularized by a finite volume $V$, we would have

$$
f(\mathcal{X}, \mathbf{k})=\frac{(2 \pi)^{3}}{V} \delta^{(3)}\left(\mathbf{k}-\mathbf{k}_{0}\right)
$$

which would lead to $n(\mathcal{X})$ in eq. (9.202) evaluating to $1 / V$.

To convert eq. (9.200) into a Boltzmann equation, we identify the decay rate $\Gamma$ by $-\partial_{t} f / f$, and multiply that by $\epsilon_{k}$ in order to identify a Lorentz-covariant structure:

$$
-\epsilon_{k} \frac{\partial f_{1}}{\partial t} \frac{1}{f_{1}} \Rightarrow-\mathcal{K}^{\alpha} \frac{\partial f_{1}}{\partial \mathcal{X}^{\alpha}} \frac{1}{f_{1}}
$$

We also modify the right-hand side of eq. (9.200) by allowing for $1+m$ particles in the initial state, and by adding Bose enhancement and Pauli blocking factors. Thereby we obtain

$$
\begin{aligned}
& \mathcal{K}^{\alpha} \frac{\partial f_{1}}{\partial \mathcal{X}^{\alpha}}=-\frac{1}{2} \sum_{m, n} \int \mathrm{d} \Phi_{1+m \rightarrow n} c \sum|\mathcal{M}|_{1+m \rightarrow n}^{2} \\
& \quad \times\left\{f_{1} f_{a_{1}} \cdots f_{a_{m}}\left(1 \pm f_{b_{1}}\right) \cdots\left(1 \pm f_{b_{n}}\right)-f_{b_{1}} \cdots f_{b_{n}}\left(1 \pm f_{1}\right)\left(1 \pm f_{a_{1}}\right) \cdots\left(1 \pm f_{a_{m}}\right)\right\}
\end{aligned}
$$

\footnotetext{
${ }^{38} \mathrm{~A}$ phenomenologically viable dark matter scenario analogous to the one discussed here, albeit with a scalar field decaying into two right-handed neutrinos, has been suggested in refs. [9.51,9.52].

${ }^{39} \mathrm{~A}$ concise discussion of the Boltzmann equation can be found in the appendix of ref. [9.53].
} 
where + applies to bosons and - to fermions. On the last row of eq. (9.205), inverse reactions ("gain terms") have been introduced, in order to guarantee detailed balance in the case that all distribution functions have their equilibrium forms.

Let us finally compare eq. (9.205) with eq. (9.188). We observe that eq. (9.188) corresponds to the gain terms of eq. (9.205); the reason is that in the quantum field theoretic formula the produced particles were (by assumption) non-thermal, $f_{1} \equiv 0$. (This can be corrected for as discussed around eq. (9.137).) At the same time, to obtain a complete match, we should work out the scattering matrix elements, $|\mathcal{M}|^{2}$, and the factors $c$. One "strength" of the quantum field theoretic computation leading to eq. (9.188) is that these automatically come with their correct values. Another strength is that at higher orders, there are also virtual effects in the quantum field theoretic computation which lead to thermal masses, modified dispersion relations, additional quasiparticle states (cf. e.g. the discussion concerning the plasmino branch in sec. 8.4), and, last but not least, which cancel IR divergences (mass singularities) from the real processes. It is not obvious whether these can be accounted for by a simple modification of the Boltzmann equation.

\section{Appendix B: Evolution equations in the presence of a conserved charge}

Above we assumed that there were no chemical potentials affecting thermodynamic functions determining the evolution of the system; this is most likely a good assumption in cosmology, as shown e.g. by the great success of the Big Bang Nucleosynthesis computation based on this ansatz. The assumption is often quantified by the statement that the observed baryon asymmetry of the universe corresponds to a chemical potential $\mu \sim 10^{-10} T$. On the other hand, in heavy ion collision experiments and particularly in astrophysics, conserved charges and the associated chemical potentials do play an important role. Even in cosmology lepton asymmetries could in principle be much larger than the baryon asymmetry, since they cannot be directly observed, hidden as they are in a neutrino background. Let us see how the presence of a chemical potential would change the cosmological considerations presented above.

We consider a system with one chemical potential, $\mu$, and the corresponding total particle number, $N$. The energy, entropy, and number densities are defined through $e \equiv E / V, s \equiv S / V$, $n \equiv N / V$, respectively, where $V$ is the volume. The total energy of the system is then

$$
E=T S-p V+\mu N
$$

while the corresponding differential reads

$$
\mathrm{d} E=T \mathrm{~d} S-p \mathrm{~d} V+\mu \mathrm{d} N
$$

Dividing both equations by the volume, we get

$$
e+p=T s+\mu n
$$

as well as

$$
\begin{aligned}
\mathrm{de} & =\mathrm{d}\left(\frac{E}{V}\right)=\frac{\mathrm{d} E}{V}-E \frac{\mathrm{d} V}{V^{2}} \\
& =T \frac{\mathrm{d} S}{V}-p \frac{\mathrm{d} V}{V}+\mu \frac{\mathrm{d} N}{V}-T S \frac{\mathrm{d} V}{V^{2}}+p \frac{\mathrm{d} V}{V}-\mu N \frac{\mathrm{d} V}{V^{2}} \\
& =T \mathrm{~d}\left(\frac{S}{V}\right)+\mu \mathrm{d}\left(\frac{N}{V}\right)=T \mathrm{~d} s+\mu \mathrm{d} n .
\end{aligned}
$$


Taking a differential from eq. (9.208) and subtracting the result of eq. (9.209) yields the GibbsDuhem equation,

$$
\mathrm{d} p=s \mathrm{~d} T+n \mathrm{~d} \mu .
$$

As indicated by this equation, the natural variables of $p$ are $T$ and $\mu$.

The system of equations we now consider is composed of (9.161) and (9.162), complemented by the comoving conservation law for the number density,

$$
\mathrm{d}\left(n a^{3}\right)=0,
$$

as well as the thermodynamic relations just derived.

As a first step let us show that the entropy conservation law, eq. (9.166), continues to hold in the presence of the new terms. Repeating the argument leading to it with the new thermodynamic relations of eqs. (9.208) and (9.209), we obtain

$$
\begin{aligned}
0 & =\mathrm{d}\left(e a^{3}\right)+p \mathrm{~d}\left(a^{3}\right) \\
& =a^{3} \mathrm{~d} e+(p+e) \mathrm{d}\left(a^{3}\right) \\
& =a^{3}[T \mathrm{~d} s+\mu \mathrm{d} n]+[T s+\mu n] \mathrm{d}\left(a^{3}\right) \\
& =T \mathrm{~d}\left(s a^{3}\right)+\mu \mathrm{d}\left(n a^{3}\right)
\end{aligned}
$$

The relation in eq. (9.211) then directly leads to eq. (9.166).

It is considerably more difficult to find a generalization of eq. (9.171). In fact, we must simultaneously follow the time evolution of $T$ and $\mu$, solving a coupled set of non-linear differential equations. Eqs. (9.166) and (9.211) can be written as $\mathrm{d} s / s=-3 \mathrm{~d} a / a$ and $\mathrm{d} n / n=-3 \mathrm{~d} a / a$, i.e.

$$
\begin{aligned}
\frac{\partial_{T} s}{s} \dot{T}+\frac{\partial_{\mu} s}{s} \dot{\mu} & =-\frac{3 \dot{a}}{a}, \\
\frac{\partial_{T} n}{n} \dot{T}+\frac{\partial_{\mu} n}{n} \dot{\mu} & =-\frac{3 \dot{a}}{a} .
\end{aligned}
$$

Denoting (from Gibbs-Duhem, eq. (9.210))

$$
s=\partial_{T} p \equiv p_{T}, \quad n=\partial_{\mu} p \equiv p_{\mu}, \quad \partial_{T} s \equiv p_{T T}, \quad \partial_{T} n=\partial_{\mu} s \equiv p_{T \mu}, \quad \partial_{\mu} n \equiv p_{\mu \mu},
$$

and inserting the right-hand side from eq. (9.164), we obtain

$$
\begin{aligned}
\frac{\mathrm{d} T}{\mathrm{~d} t} & =\frac{p_{\mu} p_{T \mu}-p_{T} p_{\mu \mu}}{p_{T T} p_{\mu \mu}-\left(p_{T \mu}\right)^{2}} \frac{\sqrt{24 \pi e(T, \mu)}}{m_{\mathrm{Pl}}}, \\
\frac{\mathrm{d} \mu}{\mathrm{d} t} & =\frac{p_{T} p_{T \mu}-p_{\mu} p_{T T}}{p_{T T} p_{\mu \mu}-\left(p_{T \mu}\right)^{2}} \frac{\sqrt{24 \pi e(T, \mu)}}{m_{\mathrm{Pl}}},
\end{aligned}
$$

where, according to eq. (9.208),

$$
e(T, \mu)=-p+T p_{T}+\mu p_{\mu} .
$$

Therefore, in a general case, the pressure and all its first and second derivatives are needed for determining the cosmological evolution.

Finally we remark that in typical relativistic systems, mixed derivatives are small, $p_{T \mu} \sim \mu T \ll$ $p_{T T}, p_{\mu \mu} \sim T^{2}$. Setting $p_{T \mu} \rightarrow 0$, eq. (9.216) reduces to

$$
\frac{\mathrm{d} T}{\mathrm{~d} t}=-\frac{p_{T}}{p_{T T}} \frac{\sqrt{24 \pi e(T, \mu)}}{m_{\mathrm{Pl}}},
$$

which agrees with eq. (9.171). 


\subsection{Evolution of a long-wavelength field in a thermal environment}

We now move to a different class of observables: from single particles to collective "fields" that evolve within a thermal environment. In the present section we consider a field $\varphi$ that is out of equilibrium; the entire field has a non-zero expectation value, or "condensate", consisting roughly speaking of very many almost zero-momentum quanta ( $k \ll g T$ rather than $k \sim \pi T$ as is the case for typical particle states). In sec. 9.6, we then move on to cases where there is no separate field forming a condensate, but rather the degrees of freedom of a strongly interacting system contain almost conserved quantities which evolve analogously to separate weakly coupled fields.

Consider a system containing two sets of elementary fields: a scalar field $\varphi$ as well as other fields which we do not need to specify but which are contained in $\mathcal{J}_{\text {int }}$ and $\mathcal{L}_{\text {bath }}$. The setup is essentially the same as in eq. (9.108) but for simplicity now with a real scalar field, being described by the Lagrangian density

$$
\mathcal{L}_{M}=\frac{1}{2} \varphi\left(-\square-m^{2}\right) \varphi-\varphi \mathcal{J}_{\text {int }}+\mathcal{L}_{\text {bath }} .
$$

We assume that the scalar field is initially displaced from its equilibrium value $\varphi_{\text {eq }} \equiv 0$, and then evolves towards it. We also assume that this evolution is a "slow" and essentially "classical" process: the coupling between $\varphi$ and the heat bath, described by $\mathcal{J}_{\text {int }}$, is taken to be weak, implying that $\varphi$ evolves on time scales $\Delta t$ much longer than those associated with the plasma interactions $\left(\Delta t \gg 1 /\left(\alpha^{2} T\right)\right.$, where $\alpha$ is a generic fine-structure constant for plasma processes). Therefore multiple plasma collisions take place during the time interval in which $\varphi$ changes only a little, implying a smooth and decoherent evolution. Then, we may postulate a classical equation of motion for how $\varphi$ evolves towards equilibrium, which can be expanded in gradients (since the field consists of small- $k$ quanta and evolves slowly) and powers of $\varphi$ (since we assume that the initial state is already close to equilibrium):

$$
\square \varphi+V_{\mathrm{eff}}^{\prime}(\varphi)=-\Gamma \dot{\varphi}+\mathcal{O}\left(\dddot{\varphi}, \nabla^{2} \dot{\varphi}, \dot{\varphi}^{2},(\nabla \varphi)^{2}\right) .
$$

The coefficients appearing in this equation, such as $\Gamma$, are functions of the properties of the heat bath, such as its temperature $T$ and the couplings $\alpha .{ }^{40}$ We note in passing that once $\varphi$ is already close to equilibrium, the right-hand side of eq. (9.221) should be completed with a noise term representing thermal fluctuations; the general ideology for this is discussed in more detail in sec. 9.7.

There are two separate effects that the interactions of the $\varphi$-field with the heat bath lead to. The first one is that $\varphi$ obtains an "effective mass", appearing as a part of the effective potential $V_{\text {eff }}$. The second is that the interactions generate a friction coefficient $\Gamma$ as defined by eq. (9.221). The role of friction is to transmit energy from the classical field to the heat bath or, equivalently, to increase the entropy of the system. Despite different physical manifestations, the effective mass and the friction are intricately related to each other; as we will see, they are on the formal level related to the real and imaginary parts of a single analytic function (the retarded correlator of $\mathcal{J}_{\text {int }}$ ), and as such have a relation to each other, analogous to Kramers-Kronig relations. In practice, there are circumstances in which the effective mass plays a more substantial role, leading to so-called underdamped oscillations, as well as ones where the friction dominates the dynamics, referred to as overdamped oscillations. Technically, the effective mass turns out to be related to a "Euclidean susceptibility" of the operator $\mathcal{J}_{\text {int }}$, whereas $\Gamma$ is related to a "Minkowskian susceptibility", which is a genuine real-time quantity.

\footnotetext{
${ }^{40}$ For simplicity we consider a system in flat spacetime. In cosmology, the expansion of the universe causes another type of "dissipation", with the Hubble rate $H$ playing a role similar to $\Gamma$ (more precisely the Hubble friction amounts to $-3 H \dot{\varphi}$ in analogy with eq. (9.176)). The total friction is the sum of these two contributions.
} 


\section{Effective mass}

In general, an effective potential can be defined and computed as discussed around eq. (7.20). Note that for this computation $\varphi$ is treated as constant both in temporal and spatial coordinates. We integrate over all fields appearing in $\mathcal{J}_{\text {int }}$ and $\mathcal{L}_{\text {bath }}$. Denoting these by $\chi$, we obtain

$$
\exp \left(-\frac{V}{T} V_{\text {eff }}\right)=\exp \left(-\frac{V}{T} V_{0}\right) \int \mathcal{D} \chi \exp \left(-\int_{X} L_{\text {bath }}-\varphi \int_{X} \mathcal{J}_{\text {int }}\right),
$$

where $V_{0} \equiv \frac{1}{2} m^{2} \varphi^{2}$ is the tree-level potential appearing in $\mathcal{L}_{M}$; we have gone over to Euclidean spacetime as usual for static observables; and $L_{\text {bath }}$ is the Euclidean Lagrangian of the $\chi$-fields. Assuming that $\left\langle\mathcal{J}_{\text {int }}\right\rangle=0$, and defining the effective mass as

$$
V_{\text {eff }}(\varphi)=V_{\text {eff }}(0)+\frac{1}{2} m_{\text {eff }}^{2} \varphi^{2}+\mathcal{O}\left(\varphi^{3}\right)
$$

the matching of the left and right sides of eq. (9.222) leads to

$$
\delta m^{2} \equiv m_{\text {eff }}^{2}-m^{2}=-\frac{T}{V} \int_{X, Y}\left\langle\mathcal{J}_{\text {int }}(X) \mathcal{J}_{\text {int }}(Y)\right\rangle_{\mathrm{c}}=-\int_{X}\left\langle\mathcal{J}_{\text {int }}(X) \mathcal{J}_{\text {int }}(0)\right\rangle_{\mathrm{c}} .
$$

Here we made use of translational invariance, and $\langle\ldots\rangle_{\mathrm{c}}$ indicates that only the connected contraction contributes as long as $\left\langle\mathcal{J}_{\text {int }}\right\rangle=0$. The correlator in eq. (9.224) has the form of a susceptibility, cf. eq. (7.54), but with an additional integral over $\tau$ which gives it the dimension of $\mathrm{GeV}^{2}$.

In general, the 2-point correlator in eq. (9.224) has a temperature-independent divergent part, because the correlator $\left\langle\mathcal{J}_{\text {int }}(X) \mathcal{J}_{\text {int }}(0)\right\rangle_{\text {c }}$ diverges at short distances. This amounts to a renormalization of the (bare) mass parameter $m^{2}$. In addition, there can be a finite $T$-dependent correction, which can be interpreted as a thermal mass. A simple example was previously seen around eq. (3.95), and we return to a couple of further examples below.

Before proceeding let us recall that, in terms of Minkowskian quantities, the Euclidean susceptibility corresponds to a particular integral over the corresponding spectral function, cf. eq. (8.26):

$$
-\delta m^{2}=\int_{X}\left\langle\mathcal{J}_{\text {int }}(X) \mathcal{J}_{\text {int }}(0)\right\rangle_{\mathrm{c}}=\int_{0}^{\beta} \mathrm{d} \tau \Pi_{E}(\tau, \mathbf{k}=\mathbf{0})=\int_{-\infty}^{\infty} \frac{\mathrm{d} \omega}{\pi} \frac{\rho(\omega, \mathbf{0})}{\omega} .
$$

For simplicity we put $E$ in a subscript from now on (rather than in a superscript like in sec. 8.1).

\section{Friction coefficient}

Turning next to the friction coefficient, let us transform eq. (9.221) to Fourier space, writing $\varphi \propto e^{-i \omega t+i \mathbf{k} \cdot \mathbf{x}} \tilde{\varphi}:$

$$
\left[-\omega^{2}+\mathbf{k}^{2}+m_{\mathrm{eff}}^{2}-i \omega \Gamma+\mathcal{O}\left(\omega^{3}, \omega \mathbf{k}^{2}\right)\right] \tilde{\varphi}=\mathcal{O}\left(\tilde{\varphi}^{2}\right) .
$$

We compare this with the position of the "pole" appearing in the (retarded) propagator obtained after setting $\omega_{n} \rightarrow-i\left(\omega+i 0^{+}\right)$in the Euclidean propagator, cf. eq. (8.29):

$$
\frac{1}{\omega_{n}^{2}+\mathbf{k}^{2}+m^{2}-\Pi_{E}} \rightarrow \frac{1}{-\omega^{2}-i \omega 0^{+}+\mathbf{k}^{2}+m^{2}-\operatorname{Re} \Pi_{E}-i \operatorname{Im} \Pi_{E}} .
$$

The minus sign in front of $\Pi_{E}$ can be associated with that in eq. (9.224), so that $-\operatorname{Re} \Pi_{E}$ corresponds to $\delta m^{2}$ (this is an alternative interpretation for the susceptibility discussed in eq. (9.225); 
the general Kramers-Kronig relation of $\operatorname{Re} \Pi_{E}\left(-i\left[\omega+i 0^{+}\right], \mathbf{k}\right)$ and the spectral function can be deduced from eq. (8.18)). Setting $\mathbf{k} \rightarrow \mathbf{0}$ we obtain

$$
\Gamma=\lim _{\omega \rightarrow m_{\mathrm{eff}}} \frac{\operatorname{Im} \Pi_{E}\left(-i\left[\omega+i 0^{+}\right], \mathbf{0}\right)}{\omega},
$$

where

$$
\Pi_{E}\left(\omega_{n}, \mathbf{k}\right)=\int_{X} e^{i \omega_{n} \tau-i \mathbf{k} \cdot \mathbf{x}}\left\langle\mathcal{J}_{\text {int }}(\tau, \mathbf{x}) \mathcal{J}_{\text {int }}(0, \mathbf{0})\right\rangle,
$$

whose imaginary part is given by (cf. eq. (8.28))

$$
\operatorname{Im} \Pi_{E}\left(-i\left[\omega+i 0^{+}\right], \mathbf{0}\right)=\rho(\omega, \mathbf{0}) .
$$

To summarize, we have obtained

$$
\Gamma=\frac{\rho\left(m_{\mathrm{eff}}, \mathbf{0}\right)}{m_{\mathrm{eff}}},
$$

where $m_{\text {eff }}^{2}$ is the mass parameter appearing in $V_{\text {eff }}(\varphi) .{ }^{41}$ The expectation value in eq. (9.229) is taken with respect to the density matrix of the heat bath degrees of freedom. Eq. (9.231) should be contrasted with eq. (9.225): the information concerning both the mass and the friction coefficient is encoded in the same spectral function, however in different ways.

Now, if $m_{\mathrm{eff}}$ is much smaller than the thermal scales characterizing the structure of $\rho(\omega, \mathbf{0})$, in particular the width of its transport peak (this concept will be defined around eq. (9.269)), which is normally $\sim \alpha^{2} T$ (cf. sec. 9.6), then we can to a good accuracy set $m_{\text {eff }} \rightarrow 0$ in the evaluation of $\Gamma$. Then $\Gamma$ amounts to a "transport coefficient", cf. sec. 9.6.

\section{Examples}

As a first example, we let $\mathcal{J}_{\text {int }}$ be a scalar operator [9.54,9.55], in which case $\varphi$ could be called a "dilaton" field. For instance, if the medium is composed of non-Abelian gauge fields, we could have

$$
\mathcal{J}_{\text {int }}^{(\mathrm{s})}=\frac{1}{M} F^{a \mu \nu} F_{\mu \nu}^{a} .
$$

As a second example, we consider a pseudoscalar operator [9.56], whereby $\varphi$ could be an "axion" field. Then the operator appearing in the interaction term reads

$$
\mathcal{J}_{\text {int }}^{(\mathrm{p})}=\frac{q_{M}}{M}, \quad q_{M} \equiv \epsilon_{\mu \nu \rho \sigma} \frac{g^{2} F^{a \mu \nu} F^{a \rho \sigma}}{64 \pi^{2}},
$$

where $q_{M}$ is a (Minkowskian) "topological charge density". ${ }^{42}$

Now, in the case of $\mathcal{J}_{\text {int }}^{(\mathrm{s})}$, the effective mass originating from eq. (9.224) is ultraviolet divergent. Therefore a bare mass parameter needs to exist, and the susceptibility simply corrects this. There is also a finite thermal mass correction which, on dimensional grounds, is of the form $\delta m^{2}(T) \sim$ $T^{4} / M^{2}$. If $M$ is large, this thermal correction is small.

In the case of $\mathcal{J}_{\text {int }}^{(\mathrm{p})}$, in contrast, the Euclidean susceptibility is finite [9.57]. Once we go to Euclidean spacetime, the "Wick rotation" $D_{t} \rightarrow i D_{\tau}$ (cf. sec. 5.1) implies that $q_{M}$ becomes purely imaginary and thus the susceptibility in eq. (9.224) is positive. Therefore we can consider $m_{\text {eff }}^{2}$ to

\footnotetext{
${ }^{41}$ To be precise, we have here included also purely $\omega$-dependent terms such as $\omega^{3}$ into $\Gamma$; the remaining corrections are of $\mathcal{O}\left(\omega \mathbf{k}^{2}\right)$.

${ }^{42}$ In the axion literature $M$ is often denoted by $f_{a}$.
} 
be generated purely from the interaction. This is the usual scenario for axion mass generation, and corresponding measurements of the Euclidean topological susceptibility as a function of the temperature have been carried out on the lattice (cf. ref. [9.58] for a review). Consistent with the fact that the Euclidean topological susceptibility vanishes to all orders in perturbation theory and that perturbation theory works at least qualitatively at high temperatures, the measurements show a rapid decrease as the temperature increases above the confinement scale.

As far as the friction coefficients go, the operator $\mathcal{J}_{\text {int }}^{(\mathrm{s})}$ is related to the "trace anomaly" of pure Yang-Mills theory,

$$
T_{\mu}^{\mu} \approx-\frac{b_{0}}{2} F^{a \mu \nu} F_{\mu \nu}^{a}
$$

where $b_{0}$ defines the 1-loop $\beta$-function related to the running coupling, $b_{0} \equiv 11 N_{\mathrm{c}} /\left[3(4 \pi)^{2}\right]$. The trace anomaly determines a particular transport coefficient, namely the bulk viscosity $\zeta$, cf. eq. (9.286) below. This parameter has been determined in perturbation theory, with the result $[9.59]$

$$
\zeta \sim \frac{b_{0}^{2} g^{4} T^{3}}{4 \ln (1 / \alpha)}, \quad \alpha \equiv \frac{g^{2}}{4 \pi} .
$$

For $m_{\text {eff }} \ll \alpha^{2} T$, eqs. (9.231) and (9.232) now imply $\Gamma \sim 4 \zeta /\left(b_{0}^{2} M^{2}\right)$, which after the insertion of eq. (9.235) shows that $\Gamma \sim g^{4} T^{3} / M^{2}$ in the weak-coupling limit, up to logarithms.

Finally, for $\mathcal{J}_{\text {int }}^{(\mathrm{p})}$, we need the transport coefficient associated with $q_{M}$. This quantity has been studied in great detail, given its important relation to fermion number non-conservation through the axial anomaly. The quantity normally considered is the so-called "Chern-Simons diffusion rate", or (twice) the "sphaleron rate" (cf. e.g. ref. [9.60] for a discussion of these two rates). This can be defined from the time and volume average of the operator $\hat{q}_{M}$ as [9.61]

$$
\begin{aligned}
& \Gamma_{\text {diff }} \equiv \lim _{\Omega \rightarrow \infty} \frac{\left\langle\int_{\Omega} \mathrm{d}^{4} \mathcal{X} \hat{q}_{M}(\mathcal{X}) \int_{\Omega} \mathrm{d}^{4} \mathcal{Y} \hat{q}_{M}(\mathcal{Y})\right\rangle}{\Omega}=\int \mathrm{d}^{4} \mathcal{X}\left\langle\frac{1}{2}\left\{\hat{q}_{M}(\mathcal{X}), \hat{q}_{M}(0)\right\}\right\rangle \\
& \stackrel{(8.5)}{=} \quad \lim _{\omega \rightarrow 0} \Delta(\omega, \mathbf{0}) \\
& \stackrel{(8.16)}{=} \lim _{\omega \rightarrow 0} \frac{2 T \rho(\omega, \mathbf{0})}{\omega} \text {, }
\end{aligned}
$$

where $\Omega=V t$ is the spacetime volume and we made use of translational invariance. In the last equation of eq. (9.236) the integration goes over all the spacetime (positive and negative $t$ ). ${ }^{43}$ In the step leading to eq. (9.237), we furthermore exploited the fact that for $|\omega| \ll T, n_{\mathrm{B}}(\omega) \approx T / \omega$. The first equality in eq. (9.236) suggests that we call $\Gamma_{\text {diff }}$ a "Minkowskian topological susceptibility".

In order to estimate $\Gamma_{\text {diff }}$, it has been argued that at high temperatures the dominant contribution comes from the dynamics of "soft modes", which are Bose enhanced and can thus be described by classical field theory $[9.28,9.62,9.63]$. In the classical limit we can write

$$
Q(t) \equiv \int_{0}^{t} \mathrm{~d} t^{\prime} \int_{V} \mathrm{~d}^{3} \mathbf{x}^{\prime} q_{M}\left(\mathcal{X}^{\prime}\right) \equiv N_{\mathrm{CS}}(t)-N_{\mathrm{CS}}(0),
$$

where $N_{\mathrm{CS}}(t)$ is the Chern-Simons number. Therefore eq. (9.236) becomes

$$
\Gamma_{\text {diff }}=\lim _{V, t \rightarrow \infty} \frac{\left\langle\left\langle Q^{2}(t)\right\rangle\right\rangle}{V t},
$$

\footnotetext{
${ }^{43}$ To show the step in eq. (9.236) more precisely, let us denote $\int_{V} \mathrm{~d}^{3} \mathbf{x} \hat{q}_{M}(\mathcal{X}) \equiv \hat{o}_{M}(t)$. If a non-zero limit exists for the correlator after division by $t$, it means that the correlator grows linearly with $t$ at large times, so we can replace $1 / t$ through $\mathrm{d} / \mathrm{d} t$ in the prefactor. Then $\lim _{t \rightarrow \infty} \frac{\mathrm{d}}{\mathrm{d} t} \int_{0}^{t} \mathrm{~d} t^{\prime} \int_{0}^{t} \mathrm{~d} t^{\prime \prime}\left\langle\hat{o}_{M}\left(t^{\prime}\right) \hat{o}_{M}\left(t^{\prime \prime}\right)\right\rangle=\lim _{t \rightarrow \infty} \int_{0}^{t} \mathrm{~d} t^{\prime}\left\langle\left\{\hat{o}_{M}\left(t^{\prime}\right), \hat{o}_{M}(t)\right\}\right\rangle=$ $\lim _{t \rightarrow \infty} \int_{-t}^{t} \mathrm{~d} t^{\prime}\left\langle\frac{1}{2}\left\{\hat{o}_{M}\left(t^{\prime}\right), \hat{o}_{M}(0)\right\}\right\rangle$. In the last step we made use of the facts that the correlator only depends on the time difference and is symmetric in the sign of the time difference.
} 
where the expectation value refers to a classical thermal average. It is the resemblance of eqs. (9.238) and (9.239) to the usual process of particle diffusion in non-relativistic statistical mechanics (with $\left.N_{\mathrm{CS}}(t) \rightarrow x(t)\right)$ that gives rise to the above-mentioned concept of "Chern-Simons diffusion".

Practical measurements of $\Gamma_{\text {diff }}$ within classical lattice gauge theory have been carried out for pure $\mathrm{SU}(2)$ [9.64] and $\mathrm{SU}(3)$ gauge theory [9.65], and indicate that $\Gamma_{\text {diff }} \sim \alpha^{5} T^{4}$ in these cases, up to logarithms. Therefore, the axion friction coefficient scales as $\Gamma \sim \alpha^{5} T^{3} / M^{2}$. Even though smaller than the friction coefficient for $\mathcal{J}_{\text {int }}^{(\mathrm{s})}$ by $\mathcal{O}\left(\alpha^{3}\right)$, this is still parametrically larger than $m_{\text {eff }}^{2}$ which vanishes to all orders in perturbation theory in the pseudoscalar case (these arguments are relevant if $T \gg \Lambda$, where $\Lambda$ is the confinement scale). On the non-perturbative level we may write $m_{\text {eff }}^{2} \sim\left(\Lambda^{4} / M^{2}\right)(\Lambda / T)^{n}, n>0$, for $T \gg \Lambda$, leading to $\Gamma / m_{\text {eff }} \sim \alpha^{5}(T / M)(T / \Lambda)^{2+n / 2}$. Thus axion oscillations become overdamped in the regime $T \gtrsim\left(M \Lambda^{2+n / 2} / \alpha^{5}\right)^{\frac{1}{3+n / 2}} \cdot{ }^{44}$

To conclude this section, let us stress again that "Hubble friction" $H \sim T^{2} / m_{\mathrm{Pl}}$ has been omitted from the above estimates. Roughly speaking, if $M$ and $m_{\mathrm{Pl}}$ are similarly large scales, then Hubble damping dominates over $\Gamma$ below a certain temperature, because it decreases less rapidly with $T$. In particular, $H$ is generally assumed to dominate at $T \lesssim \Lambda$, in which regime $m_{\text {eff }}^{2}$ also becomes "large", $m_{\text {eff }}^{2} \sim \Lambda^{4} / M^{2}$.

\footnotetext{
${ }^{44}$ It has been proposed that such dynamics could play a role for warm inflation [9.66]-[9.69].
} 


\subsection{Linear response theory and transport coefficients}

Transport coefficients parametrize the small-frequency behaviour of long-wavelength excitations of a multiparticle system, in close analogy to the friction coefficient $\Gamma$ of the field $\varphi$ in sec. 9.5. If there is no separate field to consider, it is meaningful to speak of long-wavelength excitations only for quantities for which long-distance correlations exist. This prompts us to consider conserved (or almost conserved) currents, such as the energy-momentum tensor and various particle number currents. When the amplitude of a perturbation is so large that it requires many scatterings to change it, the dynamics of the system should be classical in nature, governed for instance by the known differential equations of hydrodynamics. The transport coefficients are then the "low-energy constants" of this infrared theory, and encode the effects of the short-wavelength modes that have been "integrated out" in order to arrive at the effective description (cf. e.g. refs. [9.70,9.71]).

Apart from a similar physical origin, transport coefficients also possess the formal property that they can be extracted from the small-frequency limit of a spectral function (cf. eq. (8.4)) as

$$
\lim _{\omega \rightarrow 0^{+}} \frac{\rho(\omega, \mathbf{0})}{\omega} .
$$

Note that the spatial momentum has been set to zero before the frequency here. Even though partly just a convention, this may be thought of as guaranteeing that the system considered is "large" and consists of very many small- $k$ quanta.

One example of a transport coefficient has already been discussed around eq. (9.231). That case was particularly simple because there were explicitly two sets of fields, one exhibiting "slow" or "soft" dynamics and another corresponding to "fast" or "hard" thermal modes. In most cases, we have just one set of fields and the task is to consistently split that set into two parts, with the transport coefficients characterizing the dynamics of the soft modes.

\section{Generic case}

We wish to illustrate generic aspects of the formalism related to transport coefficients with the example of an equilibration rate. To this end, let us assume that some external perturbation has displaced the system from equilibrium by giving it a net "charge" of some type. We assume, however, that the charge under consideration is not conserved (in the case of QCD, this is the case for instance for the spatial components of the baryon number or energy current). In this case, the system will relax back to equilibrium, i.e. the net charge will disappear, and the equilibration rate describes how fast this process takes place.

Let $\hat{N}(t)$ now be the Heisenberg operator of some almost, but not exactly conserved physical quantity. Any possible dependence on spatial coordinates has been suppressed for simplicity. According to the discussion above, we assume the equilibrium expectation value to be zero,

$$
\langle\hat{N}(t)\rangle_{\mathrm{eq}}=0 \text {. }
$$

The non-vanishing non-equilibrium expectation value, $\langle\hat{N}(t)\rangle_{\text {non-eq }}$, is assumed to evolve so slowly that all other quantities are in equilibrium. If $\langle\hat{N}(t)\rangle_{\text {non-eq }}$ is small in some sense (even though it should still be larger than typical equilibrium thermal fluctuations), we can expect the evolution to be described by an equation linear in $\langle\hat{N}(t)\rangle_{\text {non-eq }}$, and can therefore write

$$
\frac{\mathrm{d}}{\mathrm{d} t}\langle\hat{N}(t)\rangle_{\text {non-eq }}=-\Gamma\langle\hat{N}(t)\rangle_{\text {non-eq }}+\mathcal{O}\left(\langle\hat{N}(t)\rangle_{\text {non-eq }}^{2}\right)
$$


where $\mathrm{d} t>0$ is also implicitly assumed. The coefficient $\Gamma$ introduced here may be called the equilibration rate. Our goal is to obtain an expression for $\Gamma$, describing "dissipation", in terms of various equilibrium expectation values, of the type $\langle\ldots\rangle_{\mathrm{eq}}$, describing "fluctuations".

In what follows, we derive an expression for $\Gamma$ in two different ways. The first one is called matching: we consider a Green's function which is well-defined both in the classical limit as well as in the full quantum theory, compute it on both sides, and equate the results. The second method is on the other hand called linear response theory: we stay in the quantum theory all the time and try to obtain an equation of the form of eq. (9.242), from which $\Gamma$ can be identified.

As far as the matching method goes, an appropriate Green's function is a symmetric 2-point function, since it has a classical limit. Let us thus define

$$
\Delta(t) \equiv\left\langle\frac{1}{2}\{\hat{N}(t), \hat{N}(0)\}\right\rangle_{\text {eq }},
$$

as well as the corresponding Fourier transform,

$$
\tilde{\Delta}(\omega) \equiv \int_{-\infty}^{\infty} \mathrm{d} t e^{i \omega t} \Delta(t) .
$$

The value $\Delta(0)$ amounts to a "susceptibility", as defined in eq. (7.54) or in eq. (9.225),

$$
\begin{aligned}
\Delta(0)=\left\langle\hat{N}^{2}\right\rangle_{\text {eq }} & =T \partial_{\mu}\langle\hat{N}\rangle_{\text {eq }} \equiv \frac{T}{\mathcal{Z}} \partial_{\mu} \operatorname{Tr}\left\{\hat{N}\left[e^{-\beta(\hat{H}-\mu \hat{N})}\right]\right\}_{\mu=0} \\
& =\frac{T^{2}}{\mathcal{Z}} \partial_{\mu}^{2} \operatorname{Tr}\left[e^{-\beta(\hat{H}-\mu \hat{N})}\right]_{\mu=0} \\
& =\left.T^{2} \partial_{\mu}^{2} \ln \mathcal{Z}(T, \mu)\right|_{\mu=0},
\end{aligned}
$$

where in the last stage we used the fact that $\langle\hat{N}\rangle_{\text {eq }}=0$. Furthermore, by time-translational invariance it is clear that $\Delta(-t)=\Delta(t)$.

Now, on the classical side, we replace $\langle\hat{N}(t)\rangle_{\text {non-eq }}$ by $N(t)$, and instead of eq. (9.242) have

$$
\dot{N}(t) \approx-\Gamma N(t)
$$

with the trivial solution $N(t)=N(0) \exp (-\Gamma t)$. Enforcing the correct symmetry by replacing $t \rightarrow|t|$ and taking a thermal average with respect to initial conditions, denoted by $\langle\langle\ldots\rangle\rangle$, leads straightforwardly to

$$
\Delta_{\mathrm{cl}}(t) \equiv\langle\langle N(t) N(0)\rangle\rangle=\left\langle\left\langle[N(0)]^{2}\right\rangle e^{-\Gamma|t|},\right.
$$

the time integral of which yields

$$
\tilde{\Delta}_{\mathrm{cl}}(0)=\int_{-\infty}^{+\infty} \mathrm{d} t \Delta_{\mathrm{cl}}(t)=\frac{2 \Delta_{\mathrm{cl}}(0)}{\Gamma}
$$

Thus, the ratio of the susceptibility $\Delta_{\mathrm{cl}}(0)$ and the equilibration rate $\Gamma$ can be determined from the zero-frequency limit of the Fourier transform of the symmetric correlator,

$$
\frac{\Delta_{\mathrm{cl}}(0)}{\Gamma}=\frac{\tilde{\Delta}_{\mathrm{cl}}(0)}{2}=\frac{1}{2} \lim _{\omega \rightarrow 0} \int_{-\infty}^{\infty} \mathrm{d} t e^{i \omega t} \Delta_{\mathrm{cl}}(t)
$$

Let us match this equation to the quantum side. Identifying $\Delta_{\mathrm{cl}}(0) \leftrightarrow \Delta(0)$, which makes sense if the susceptibility is ultraviolet finite; inserting eq. (9.245) for the latter; and rewriting the result as in eq. (9.237), we obtain

$$
\frac{\partial_{\mu}\langle\hat{N}\rangle_{\mathrm{eq}}}{\Gamma}=\lim _{\omega \rightarrow 0} \frac{\rho(\omega)}{\omega}
$$


where $\rho(\omega)$ is the spectral function corresponding to $\hat{N}$. This is an example of a Kubo formula. However, the right-hand side does not directly give the rate of interest (as was the case in eq. (9.237)), but a susceptibility needs to be known before $\Gamma$ can be extracted. There is also the peculiar fact that $\Gamma$ appears in the denominator in eq. (9.251), to which we return in sec. 9.7.

Let us now rederive eq. (9.251) in another way, namely through a linear response analysis of $\langle\hat{N}(t)\rangle_{\text {non-eq }}$. We can assume that at time $t=-\infty$ the system was in full equilibrium, but then a source term was added to the Hamiltonian,

$$
\hat{H} \rightarrow \hat{H}(t)=\hat{H}-\mu(t) \hat{N}(t),
$$

which slowly displaced $\langle\hat{N}(t)\rangle_{\text {non-eq }}$ from zero. Solving the equation of motion for the density matrix $\hat{\rho}(t)$ (cf. eq. (9.109)),

$$
i \frac{\mathrm{d} \hat{\rho}(t)}{\mathrm{d} t}=[\hat{H}(t), \hat{\rho}(t)],
$$

to first order in the perturbation yields

$$
\hat{\rho}(t) \approx \hat{\rho}(-\infty)-i \int_{-\infty}^{t} \mathrm{~d} t^{\prime}\left[\hat{H}\left(t^{\prime}\right), \hat{\rho}(-\infty)\right]+\ldots,
$$

where we can furthermore replace $\hat{H}\left(t^{\prime}\right)$ by $-\mu\left(t^{\prime}\right) \hat{N}\left(t^{\prime}\right)$, as $\hat{H}$ and $\hat{\rho}(-\infty) \equiv \frac{1}{\mathcal{Z}} e^{-\beta \hat{H}}$ commute. Using this result in the definition of $\langle\hat{N}(t)\rangle_{\text {non-eq }}$ gives

$$
\begin{aligned}
\langle\hat{N}(t)\rangle_{\text {non-eq }} & =\operatorname{Tr}[\hat{\rho}(t) \hat{N}(t)] \\
& \approx i \int_{-\infty}^{t} \mathrm{~d} t^{\prime} \mu\left(t^{\prime}\right) \operatorname{Tr}\left\{\left[\hat{N}\left(t^{\prime}\right), \hat{\rho}(-\infty)\right] \hat{N}(t)\right\} \\
& =i \int_{-\infty}^{\infty} \mathrm{d} t^{\prime}\left\langle\left[\hat{N}(t), \hat{N}\left(t^{\prime}\right)\right]\right\rangle_{\mathrm{eq}} \theta\left(t-t^{\prime}\right) \mu\left(t^{\prime}\right),
\end{aligned}
$$

where we have denoted

$$
\langle\ldots\rangle_{\text {non-eq }} \equiv \operatorname{Tr}[\hat{\rho}(t)(\ldots)], \quad\langle\ldots\rangle_{\text {eq }} \equiv \operatorname{Tr}[\hat{\rho}(-\infty)(\ldots)] .
$$

The leading term from eq. (9.254) disappeared because of the assumption in eq. (9.241). ${ }^{45}$ In addition, we have inserted $\theta\left(t-t^{\prime}\right)$ and extended the upper end of the integration to infinity to stress the retarded nature of the correlator. The equilibrium expectation value appearing in eq. (9.255) is called a linear response function.

We define next the retarded correlator (cf. sec. 8.1),

$$
C_{R}(t) \equiv\langle i[\hat{N}(t), \hat{N}(0)] \theta(t)\rangle_{\mathrm{eq}},
$$

and its Fourier transform,

$$
\widetilde{C}_{R}(\omega) \equiv \int_{-\infty}^{\infty} \mathrm{d} t e^{i \omega t} C_{R}(t)
$$

We recall that the imaginary part of $\widetilde{C}_{R}$ yields the spectral function (cf. eqs. (8.28), (8.29)):

$$
\rho(\omega)=\operatorname{Im} \widetilde{C}_{R}\left(\omega+i 0^{+}\right) .
$$

Eq. (9.255) can now be written as

$$
\langle\hat{N}(t)\rangle_{\text {non-eq }} \approx \int_{-\infty}^{\infty} \mathrm{d} t^{\prime} C_{R}\left(t-t^{\prime}\right) \mu\left(t^{\prime}\right) .
$$

\footnotetext{
${ }^{45}$ Note that the time dependence of $\hat{N}(t)=e^{i \hat{H} t} \hat{N}(0) e^{-i \hat{H} t}$ commutes with $\frac{1}{\mathcal{Z}} e^{-\beta \hat{H}}$, so $\langle\hat{N}(t)\rangle_{\text {eq }}=\langle\hat{N}(0)\rangle_{\text {eq }}$.
} 
To proceed from here, we assume that $\mu$ varies slowly around $t^{\prime} \approx t$ and subsequently expand it in a Taylor series around this point:

$$
\mu\left(t^{\prime}\right) \approx \mu(t)+\dot{\mu}(t)\left(t^{\prime}-t\right)+\mathcal{O}\left(t^{\prime}-t\right)^{2} .
$$

Plugging this into eq. (9.260), the first term yields

$$
\begin{aligned}
\int_{-\infty}^{\infty} \mathrm{d} t^{\prime} C_{R}\left(t-t^{\prime}\right) & \stackrel{(9.258)}{=} \widetilde{C}_{R}(0) \stackrel{(8.25)}{=} \widetilde{C}_{E}(0) \\
& \stackrel{(8.9)}{=} \int_{0}^{\beta} \mathrm{d} \tau\langle\hat{N}(\tau) \hat{N}(0)\rangle \approx \beta\left\langle\hat{N}^{2}\right\rangle \stackrel{(9.245)}{=} \partial_{\mu}\langle\hat{N}\rangle_{\text {eq }},
\end{aligned}
$$

whereas the second term produces the integral

$$
\int_{-\infty}^{\infty} \mathrm{d} t^{\prime}\left(t^{\prime}-t\right) C_{R}\left(t-t^{\prime}\right) \stackrel{(9.258)}{=} i \partial_{\omega} \widetilde{C}_{R}(0) \stackrel{(9.259)}{=}-\partial_{\omega} \rho(0) .
$$

In eq. (9.262) we assumed that the equilibration rate is small (slow) compared with the temperature, $\Gamma \beta \ll 1$, so that the correlator can be assumed constant on the Euclidean time interval; in eq. (9.263) we on the other hand used the fact that the real part of $\widetilde{C}_{R}$ is even in $\omega$ (cf. eq. (8.18) together with eq. (8.20) and the antisymmetry of $\rho(\omega)$ ), such that its derivative vanishes at $\omega=0$, whereas its imaginary part gives the spectral function.

Assembling everything together, we obtain up to first order in gradients,

$$
\langle\hat{N}(t)\rangle_{\text {non-eq }} \approx \partial_{\mu}\langle\hat{N}\rangle_{\text {eq }} \mu(t)-\partial_{\omega} \rho(0) \partial_{t} \mu(t)+\mathcal{O}\left(\partial_{t}^{2} \mu\right) .
$$

The first term amounts to an equilibrium fluctuation and exists even in the absence of any time dependence, if a non-zero $\mu$ is inserted in order to displace $\langle\hat{N}\rangle$ from zero. Moreover, it implies that $\partial_{t}\langle\hat{N}(t)\rangle_{\text {non-eq }} \approx \partial_{\mu}\langle\hat{N}\rangle_{\text {eq }} \partial_{t} \mu(t)+\mathcal{O}\left(\partial_{t}^{2} \mu\right)$. If this information is inserted into the second term, we obtain

$$
\langle\hat{N}(t)\rangle_{\text {non-eq }} \approx \partial_{\mu}\langle\hat{N}\rangle_{\text {eq }} \mu(t)-\frac{\partial_{\omega} \rho(0)}{\partial_{\mu}\langle\hat{N}\rangle_{\text {eq }}} \partial_{t}\langle\hat{N}(t)\rangle_{\text {non-eq }}+\mathcal{O}\left(\partial_{t}^{2}\langle\hat{N}(t)\rangle_{\text {non-eq }}\right) .
$$

Setting $\mu(t) \rightarrow 0$, so that the first term on the right-hand side can be omitted; omitting higher derivatives; and comparing with eq. (9.242), we finally identify

$$
\frac{1}{\Gamma}=\frac{\partial_{\omega} \rho(0)}{\partial_{\mu}\langle\hat{N}\rangle_{\mathrm{eq}}}
$$

which is indeed in agreement with eq. (9.251).

\section{Transport peak}

Returning to the first method, i.e. matching with the classical limit, we note that one can extract more than just $\Gamma$ from the calculation. Indeed, the Fourier transform of eq. (9.248) implies that, for small frequencies,

$$
\begin{aligned}
\Delta_{\mathrm{cl}}(\omega) & \simeq \Delta_{\mathrm{cl}}(0)\left[\int_{-\infty}^{0} \mathrm{~d} t e^{(i \omega+\Gamma) t}+\int_{0}^{\infty} \mathrm{d} t e^{(i \omega-\Gamma) t}\right] \\
& =\Delta_{\mathrm{cl}}(0) \frac{2 \Gamma}{\omega^{2}+\Gamma^{2}} .
\end{aligned}
$$


According to eq. (8.16), the corresponding spectral function reads (for $\omega \ll T$ )

$$
\begin{aligned}
\rho(\omega) & =\Delta_{\mathrm{cl}}(0) \frac{\beta \omega \Gamma}{\omega^{2}+\Gamma^{2}} \\
& =\Delta_{\mathrm{cl}}(0) \beta \operatorname{Im} \frac{i \Gamma}{\omega+i \Gamma} .
\end{aligned}
$$

This "Lorentzian" structure is referred to as a transport peak. ${ }^{46}$

These equations contain kind of a "paradox", which becomes manifest in the free limit. In the free limit, the equilibration rate $\Gamma$ should vanish and therefore, according to eq. (9.266), the first derivative of the spectral function at zero frequency appears to diverge. In contrast, according to what we saw for the free spectral functions of single particle states (cf. eqs. (8.36) and (8.68)), we would normally expect the spectral function to be zero at small frequencies $(|\omega|<m)$.

The resolution to the paradox is to consider the free case as a limit, $\Gamma \rightarrow 0^{+}$, whereby

$$
\frac{T \rho(\omega)}{\omega}=\Delta_{\mathrm{cl}}(0) \operatorname{Im}\left(\frac{1}{\omega}-\frac{1}{\omega+i 0^{+}}\right)=\Delta_{\mathrm{cl}}(0) \pi \delta(\omega) .
$$

This indeed vanishes at $\omega>0$ but nevertheless possesses a certain structure. The distribution encountered is typical of a spectral function related to a conserved charge. Namely, plugging this into eq. (8.33), we get

$$
\langle\hat{N}(\tau) \hat{N}(0)\rangle=\int_{-\infty}^{\infty} \frac{\mathrm{d} \omega}{\pi} \rho(\omega) n_{\mathrm{B}}(\omega) e^{(\beta-\tau) \omega}=\Delta_{\mathrm{cl}}(0),
$$

where we made use of $n_{\mathrm{B}}(\omega) \approx T / \omega$ for $|\omega| \ll T$. This shows that eq. (9.270) corresponds to a Euclidean correlator which is independent of $\tau$. If the quantity is not conserved in the presence of interactions, then the transport peak gets a finite width. If, however, the quantity is exactly conserved even in the presence of interactions, the spectral function retains an infinitely narrow transport peak like in eq. (9.270), and no transport coefficient can be defined.

Let us conclude the discussion concerning the transport peak with two observations:

- As shown by eq. (9.268), the height $\left(\Delta_{\mathrm{cl}}(0) / \Gamma\right)$ and width $(\Gamma)$ of the transport peak in $T \rho(\omega) / \omega$ are two independent quantities: $\Gamma$ can be extracted from the height only if $\Delta_{\mathrm{cl}}(0)$ is known from other considerations. This, of course, was also the content of eq. (9.251).

- In order to identify $\Gamma$ from the transport peak, we need to compute the spectral function in the regime $\omega \lesssim \Gamma$. This is in general challenging because $\Gamma$ is generated by interactions, and is therefore of the type $\Gamma \sim \alpha^{2} T$, where $\alpha$ is a fine-structure constant, typically assumed small in perturbative calculations. To compute $\rho(\omega)$ correctly for soft energies $\omega \lesssim \alpha^{2} T$ requires extensive resummations [9.73] (cf. sec. 8.4 or the paragraph below eq. (9.137)).

\section{Appendix A: Transport coefficients in QCD}

Here we briefly discuss the transport coefficients most often encountered in QCD, namely flavour diffusion coefficients, the electric conductivity, as well as the shear and bulk viscosities.

The transport coefficients of QCD are all related to conserved currents. In the absence of weak interactions and non-diagonal entries in the quark mass matrix, there is a separate conserved

\footnotetext{
${ }^{46}$ Classical physics can also yield corrections to the Lorentzian shape, cf. e.g. ref. [9.72].
} 
current related to each flavour: $\partial_{\mu} \mathcal{J}_{f}^{\mu}=0, f=1, \ldots, N_{\mathrm{f}}$. The sum of all flavour currents (divided by $N_{\mathrm{c}}$ ) defines the baryon current, whereas a particular linear combination, weighted by the electric charges of each flavour, defines the electromagnetic current (denoted by $\mathcal{J}_{\mathrm{em}}^{\mu}$ ). In addition, a conserved energy-momentum tensor can be defined: $\partial_{\mu} T^{\mu \nu}=0$. Physically, diffusion coefficients describe how inhomogeneities in flavour distributions flatten out, and shear and bulk viscosities how excesses in energy or momentum flow disappear.

Now, to define the transport coefficients requires a specification of the classical description onto which to match. Let us first consider the case of a diffusion coefficient, denoted by $D_{f} .{ }^{47}$ The way it is defined is that, akin to the discussion following eq. (9.241), we assume that the system is slightly perturbed around the equilibrium state, and express $\mathcal{J}_{f}^{\mu}=\left\langle\hat{\mathcal{J}}_{f}^{\mu}\right\rangle_{\text {non-eq }}$ in terms of a gradient expansion. The equilibrium state itself is characterized by the temperature $(T)$ and chemical potentials of the conserved charges $\left(\mu_{f}\right)$, as well as a four-velocity defining the fluid rest frame $\left(u^{\mu} ; u^{\mu} u_{\mu}=1\right)$. Given $T$ and $\mu_{f}$, variables such as pressure $(p)$, energy density $(e)$, and average particle number densities $\left(n_{f}\right)$ can be defined via standard relations. The gradient expansion acts on these variables, whereas the free coefficients allowed by Lorentz symmetry are the transport coefficients (their definitions through subsequent orders of the gradient expansion are sometimes called "constitutive relations"). For instance, we can expand

$$
\mathcal{J}_{f}^{\mu}=n_{f} u^{\mu}+D_{f} \partial_{\perp}^{\mu} n_{f}+\mathcal{O}\left(\partial^{2}\right) \quad(f \text { fixed })
$$

where the transverse derivative has been defined as

$$
\partial_{\perp}^{\mu} \equiv\left(\eta^{\mu \nu}-u^{\mu} u^{\nu}\right) \partial_{\nu}, \quad \eta^{\mu \nu} \equiv \operatorname{diag}(+---) .
$$

A particular convention (called the Landau-Lifshitz convention) has been chosen whereby in the rest frame of the fluid the zeroth component of $\mathcal{J}_{f}^{\mu}$ is the number density, $u_{\mu} \mathcal{J}_{f}^{\mu} \equiv n_{f}$, to all orders in the gradient expansion. The coefficient $D_{f}$ is called the flavour diffusion coefficient.

Now, in analogy with the procedure leading to eq. (9.251), one way to determine $D_{f}$ is via matching: we need to find suitable 2-point functions on the classical side that we can equate with the corresponding quantum objects. To achieve this, we may go to the fluid rest frame and impose current conservation on eq. (9.272), producing

$$
\partial_{t} n_{f}=D_{f} \nabla^{2} n_{f}+\mathcal{O}\left(\nabla^{3}\right) .
$$

It is important to stress that even though eq. (9.274) evidently takes a non-relativistic form, the "low-energy constant" $D_{f}$ itself is defined also for relativistic flow; the corresponding covariant form of the diffusion equation follows from eq. (9.272) together with $\partial_{\mu} \mathcal{J}_{f}^{\mu}=0$.

In order to solve eq. (9.274) on the classical side, we Fourier transform in space coordinates, $\tilde{n}_{f}(t, \mathbf{k}) \equiv \int_{\mathbf{x}} e^{-i \mathbf{k} \cdot \mathbf{x}} n_{f}(t, \mathbf{x})$, to trivially obtain $\tilde{n}_{f}(t, \mathbf{k})=\tilde{n}_{f}(0, \mathbf{k}) \exp \left(-D_{f} \mathbf{k}^{2} t\right)$. If we then define a 2-point function (replacing $t \rightarrow|t|$ ), average over the initial conditions, and integrate over time like in eq. (9.267), we obtain

$$
\int_{-\infty}^{+\infty} \mathrm{d} t e^{i \omega t}\left\langle\left\langle\tilde{n}_{f}(t, \mathbf{k}) \tilde{n}_{f}(0,-\mathbf{k})\right\rangle\right\rangle=\frac{2 D_{f} \mathbf{k}^{2}}{\omega^{2}+D_{f}^{2} \mathbf{k}^{4}}\left\langle\left\langle\tilde{n}_{f}(0, \mathbf{k}) \tilde{n}_{f}(0,-\mathbf{k})\right\rangle\right\rangle
$$

\footnotetext{
${ }^{47}$ To be precise, in the case of several conserved charges the diffusion coefficients constitute a matrix, cf. e.g. ref. [9.73]. For simplicity we consider a case here where the fluctuations of the different flavours are decoupled from each other. Physically this amounts to the omission of electromagnetic effects and so-called disconnected quark contractions. In the deconfined phase of QCD both are assumed to be small effects.
} 
Let us now choose $\mathbf{k}$ along one of the coordinate axes, $\mathbf{k}=(0,0, k)$; then current conservation, $\partial_{\mu} \mathcal{J}_{f}^{\mu}=0$, allows us to re-express eq. (9.275) as

$$
\int_{-\infty}^{+\infty} \mathrm{d} t e^{i \omega t}\left\langle\left\langle\tilde{\mathcal{J}}_{f}^{3}(t, \mathbf{k}) \tilde{\mathcal{J}}_{f}^{3}(0,-\mathbf{k})\right\rangle\right\rangle=\frac{2 D_{f} \omega^{2}}{\omega^{2}+D_{f}^{2} \mathbf{k}^{4}}\left\langle\left\langle\tilde{n}_{f}(0, \mathbf{k}) \tilde{n}_{f}(0,-\mathbf{k})\right\rangle\right\rangle .
$$

Taking subsequently $\mathbf{k} \rightarrow 0$ and $\omega \rightarrow 0$, and making use of translational invariance, we obtain from here

$$
\frac{1}{3} \sum_{i} \int_{\mathcal{X}}\left\langle\left\langle\mathcal{J}_{f}^{i}(t, \mathbf{x}) \mathcal{J}_{f}^{i}(0, \mathbf{0})\right\rangle\right\rangle=2 D_{f} \int_{\mathbf{x}}\left\langle\left\langle n_{f}(0, \mathbf{x}) n_{f}(0, \mathbf{0})\right\rangle\right\rangle
$$

The left-hand side of this expression can be matched onto the zero-frequency limit of a spectral function like in eq. (9.237), whereas on the right-hand side we identify the classical limit of a Euclidean susceptibility, to be denoted by $\chi_{f},{ }^{48}$

$$
\chi_{f} \equiv \int_{0}^{\beta} \mathrm{d} \tau \int_{\mathbf{x}}\left\langle\hat{\mathcal{J}}_{f}^{0}(\tau, \mathbf{x}) \hat{\mathcal{J}}_{f}^{0}(0, \mathbf{0})\right\rangle=\beta \int_{\mathbf{x}}\left\langle\hat{\mathcal{J}}_{f}^{0}(0, \mathbf{x}) \hat{\mathcal{J}}_{f}^{0}(0, \mathbf{0})\right\rangle
$$

where we again made use of current conservation. Factors of 2 as well as of $T$ nicely cancel out at this point, and we finally obtain a Kubo relation for the flavour diffusion coefficient:

$$
D_{f}=\frac{1}{3 \chi_{f}} \lim _{\omega \rightarrow 0^{+}} \sum_{i=1}^{3} \frac{\rho_{f}^{i i}(\omega, \mathbf{0})}{\omega} .
$$

We note that, in analogy with eq. (9.251), two independent pieces of information are needed for determining $D_{f}$ : the Minkowskian spectral function of the spatial components of the current, and the Euclidean susceptibility related to the temporal component.

Let us briefly elaborate on how the structure of eq. (9.279) relates to the considerations following eq. (9.270). If we were to compute the spectral function related to the zeroth component of the current for $\mathbf{k} \rightarrow \mathbf{0}$, then we would get precisely the behaviour in eq. (9.270), because the charge $\int_{\mathbf{x}} \hat{\mathcal{J}}^{0}$ is exactly conserved even in an interacting theory. In contrast, there is no conservation law related to $\int_{\mathbf{x}} \hat{\mathcal{J}}^{i}$, and an actual transport peak exists once interactions are present. Its width, let us call it $\eta_{D_{f}}$, scales like $\Gamma$ before, i.e. $\eta_{D_{f}} \sim \alpha^{2} T$, in the massless and weakly coupled limit; at the same time $D_{f}$, which plays a role similar to $1 / \Gamma$ in eq. (9.251), diverges like $1 /\left(\alpha^{2} T\right){ }^{49}$ Physically, this is because inhomogeneities even out extremely fast in a free theory, given that there are no collisions to stop the process.

We end by summarizing the Kubo formulae for some other physically relevant transport coefficients. Let us first discuss the electric conductivity, $\sigma$, which is closely related to the flavour diffusion coefficients. It can be defined through

$$
\left\langle\hat{\mathbf{J}}_{e m}\right\rangle=\sigma \mathbf{E},
$$

where $\mathbf{E}$ is an external electric field. Recalling the (classical) Maxwell equation $\nabla \times \mathbf{B}-\partial \mathbf{E} / \partial t=$ $\left\langle\hat{\mathbf{J}}_{e m}\right\rangle$, and assuming that the external $\mathbf{B}$ has been set to zero, we obtain in analogy with eq. (9.247)

$$
\frac{\partial \mathbf{E}}{\partial t}=-\sigma \mathbf{E}
$$

\footnotetext{
${ }^{48}$ Different conventions are frequently used with regard to the trivial factor $\beta$ appearing in the second equality in eq. (9.278). If it is included in the definition of $\chi_{f}$ like here (this is natural within the imaginary-time formalism; cf. also eq. (9.224)), then $\chi_{f}$ has the dimensionality $T^{2}$. If rather the conventions of standard "canonical" statistical physics are followed, like in eq. (7.54) or (7.63), then $\chi_{f}$ has the dimensionality $T^{3}$.

${ }^{49}$ For a concise review, see ref. [9.74]. Actual expressions for $D_{f}$ in the massless limit are given in refs. [9.73, 9.75], whereas the case of a heavy flavour (with a mass $M \gg T$ ) has been discussed in ref. [9.76].
} 
Now, a Kubo formula for $\sigma$ can be derived almost trivially if we choose a convenient gauge (note that $\sigma$ as defined by eq. (9.281) is manifestly gauge independent). In particular, let us choose a gauge in which $\partial_{i} A^{0}=0$; then eq. (9.281) takes the form

$$
\partial_{t}^{2} A^{i}=-\sigma \partial_{t} A^{i},
$$

reproducing the form of eq. (9.221) in the homogeneous and massless limit. Recalling also that $A^{i}$ couples to vector currents like $\varphi$ to $\mathcal{J}_{\text {int }}$ in eq. (9.220), we can immediately write down an expression for $\sigma$ from eqs. (9.231) and (9.279),

$$
\sigma=\sigma_{d}+e^{2} \sum_{f=1}^{N_{\mathrm{f}}} Q_{f}^{2} \chi_{f} D_{f, \mathrm{c}},
$$

where $Q_{f}$ denotes the electric charge of flavour $f$ in units of the elementary charge $e$, and the subscript $(\ldots)_{c}$ refers to "connected" (or "non-singlet") quark contractions. The term $\sigma_{d}$ corresponds to a "disconnected" or "singlet" contraction, in which quark lines are contracted back to the same position $X$ from which the propagation started.

It is worth noting that, compared with eq. (9.279), no susceptibility is needed for determining $\sigma$. The formal reason for this difference is that, like with the example of sec. 9.5, there are really two sets of fields, and the electric conductivity encodes the influence of the "hard modes" (charged particles) on the dynamics of the "soft ones" (electromagnetic fields). With the diffusion coefficient, in contrast, there is only one set of degrees of freedom, but "soft modes" can be generated through fluctuations as described by the susceptibility.

The last quantities to be considered are the shear and bulk viscosities. They are defined through constitutive relations concerning the leading gradient corrections to the energy-momentum tensor: the shear viscosity coefficient $\eta$ is defined to be a function that multiplies its traceless part, while the bulk viscosity coefficient $\zeta$ multiplies the trace part. The explicit forms of the corresponding structures are most simply displayed in a non-relativistic frame, where $\left|u^{i}\right| \ll 1$; then

$$
T_{i j} \approx(p-\zeta \nabla \cdot \mathbf{v}) \delta_{i j}-\eta\left(\partial_{i} v^{j}+\partial_{j} v^{i}-\frac{2}{3} \delta_{i j} \nabla \cdot \mathbf{v}\right)+\mathcal{O}\left(\mathbf{v}^{2}, \nabla^{2}\right)
$$

where $\nabla \cdot \mathbf{v}=\partial_{i} v^{i}$ and $\delta_{i j}$ is the usual Kronecker symbol.

Once again, Kubo relations for the transport coefficients can be derived in (at least) two different ways: through a matching between quantum and classical 2-point functions, and through a linear response type computation. The former approach amounts to solving (linearized) Navier-Stokes equations for various independent hydrodynamic modes. ${ }^{50}$ The latter approach on the other hand proceeds by coupling the energy-momentum tensor to a source field which in this case is taken to be a metric perturbation, i.e. the latter part of $g^{\mu \nu}=\eta^{\mu \nu}+h^{\mu \nu}{ }^{51}$ In the following we leave out all details and simply state final expressions for the two viscosities:

$$
\begin{aligned}
\eta & =\lim _{\omega \rightarrow 0^{+}}\left\{\frac{1}{\omega} \int_{\mathcal{X}} e^{i \omega t}\left\langle\frac{1}{2}\left[\hat{T}^{12}(\mathcal{X}), \hat{T}^{12}(0)\right]\right\rangle\right\} \\
\zeta & =\frac{1}{9} \sum_{i, j=1}^{3} \lim _{\omega \rightarrow 0^{+}}\left\{\frac{1}{\omega} \int_{\mathcal{X}} e^{i \omega t}\left\langle\frac{1}{2}\left[\hat{T}^{i i}(\mathcal{X}), \hat{T}^{j j}(0)\right]\right\rangle\right\} .
\end{aligned}
$$

Note that in the case of $\zeta$, the operator could also be replaced by the full trace $\hat{T}^{i i}-\hat{T}^{00}$, given that the $\hat{T}^{00}$-part does not contribute because of energy conservation (it leads to an infinitely narrow transport peak like in eq. (9.270)).

${ }^{50} \mathrm{~A}$ review can be found in appendix $\mathrm{C}$ of ref. [9.77].

${ }^{51} \mathrm{~A}$ concise discussion can be found in ref. [9.78], while the general approach dates back to ref. [9.79]. 


\subsection{Equilibration rates / damping coefficients}

In the previous section, we already discussed an equilibration rate which we denoted by $\Gamma$, cf. eq. (9.242). However, it appears that the formalism for its determination merits further development, and this is the purpose of the present section. In short, we show that the use of operator equations of motion may simplify the structure of the 2-point correlator from which $\Gamma$ is to be extracted, thus streamlining its determination. ${ }^{52}$

\section{General analysis}

Like in the previous section, the idea is to start with an "effective" classical picture, whose free parameters are subsequently matched to reproduce quantum-mechanical correlators. Large deviations of physical quantities from their respective equilibrium values tend to decrease with time, with rates that we want to determine; however, small deviations can also be generated by the occasional inverse reactions. This is formally the same physics as that of Brownian motion, just with the momentum of the test particle replaced with the deviation of our generic "charge density" from its equilibrium value. ${ }^{53}$ In this context, it is good to note that a density, averaged over a large volume, is a continuous observable whose changes may be given a classical interpretation.

Mathematically, Brownian motion can be described via a Langevin equation,

$$
\begin{aligned}
\delta \dot{N}(t) & =-\Gamma \delta N(t)+\xi(t), \\
\left\langle\left\langle\xi(t) \xi\left(t^{\prime}\right)\right\rangle\right\rangle & =\Omega \delta\left(t-t^{\prime}\right), \quad\langle\langle\xi(t)\rangle=0,
\end{aligned}
$$

where $\delta N$ is the non-equilibrium excess in our "density" observable; $\xi$ is a Gaussian stochastic noise, whose autocorrelation function is parametrized by the coefficient $\Omega$; and $\langle\langle. .$.$\rangle denotes an$ average over the noise. This description can only be valid if the rate $\Gamma$ is much slower than that of typical reactions in the plasma, implying $\Gamma \ll \alpha^{2} T$. Then $\Gamma$ originates as a sum of very many incoherent plasma scatterings, guaranteeing the classical nature of the evolution.

Given an initial value $\delta N\left(t_{0}\right)$, eq. (9.287) admits a straightforward explicit solution,

$$
\delta N(t)=\delta N\left(t_{0}\right) e^{-\Gamma\left(t-t_{0}\right)}+\int_{t_{0}}^{t} \mathrm{~d} t^{\prime} e^{\Gamma\left(t^{\prime}-t\right)} \xi\left(t^{\prime}\right) .
$$

Making use of this expression and taking an average over the noise, we can determine the 2-point unequal time correlation function of the $\delta N$ fluctuations:

$$
\begin{aligned}
\Delta_{\mathrm{cl}}\left(t, t^{\prime}\right) & \equiv \lim _{t_{0} \rightarrow-\infty}\left\langle\left\langle\delta N(t) \delta N\left(t^{\prime}\right)\right\rangle\right\rangle \\
& =\lim _{t_{0} \rightarrow-\infty} \int_{t_{0}}^{t} \mathrm{~d} t_{1} e^{\Gamma\left(t_{1}-t\right)} \int_{t_{0}}^{t^{\prime}} \mathrm{d} t_{2} e^{\Gamma\left(t_{2}-t^{\prime}\right)}\left\langle\left\langle\xi\left(t_{1}\right) \xi\left(t_{2}\right)\right\rangle\right\rangle \\
& =\Omega \lim _{t_{0} \rightarrow-\infty} \int_{t_{0}}^{t} \mathrm{~d} t_{1} e^{\Gamma\left(t_{1}-t\right)} \int_{t_{0}}^{t^{\prime}} \mathrm{d} t_{2} e^{\Gamma\left(t_{2}-t^{\prime}\right)} \delta\left(t_{1}-t_{2}\right)
\end{aligned}
$$

\footnotetext{
${ }^{52} \mathrm{~A}$ classic example of the use of this logic comes from cosmology where, through the anomaly equation, the rate of baryon number violation can be related to the rate of Chern-Simons number diffusion [9.80] (the latter is defined around eq. (9.236)).

${ }^{53}$ The discussion here follows the description of heavy quark kinetic [9.81,9.82] or chemical [9.83] equilibration, and more generally the theory of statistical fluctuations [9.84].
} 


$$
\begin{aligned}
& =\Omega \lim _{t_{0} \rightarrow-\infty} \int_{t_{0}}^{t} \mathrm{~d} t_{1} e^{\Gamma\left(2 t_{1}-t-t^{\prime}\right)} \theta\left(t^{\prime}-t_{1}\right) \\
& =\frac{\Omega}{2 \Gamma} \lim _{t_{0} \rightarrow-\infty}\left[\theta\left(t^{\prime}-t\right)\left(e^{\Gamma\left(t-t^{\prime}\right)}-e^{\Gamma\left(2 t_{0}-t-t^{\prime}\right)}\right)+\theta\left(t-t^{\prime}\right)\left(e^{\Gamma\left(t^{\prime}-t\right)}-e^{\Gamma\left(2 t_{0}-t-t^{\prime}\right)}\right)\right] \\
& =\frac{\Omega}{2 \Gamma} e^{-\Gamma\left|t-t^{\prime}\right|}
\end{aligned}
$$

The limit $t_{0} \rightarrow-\infty$ guarantees that any initial transients have died out, making $\Delta_{\mathrm{cl}}$ an equilibrium correlation function. ${ }^{54}$ Subsequently, making use of $\partial_{t}\left|t-t^{\prime}\right|=\theta\left(t-t^{\prime}\right)-\theta\left(t^{\prime}-t\right), \partial_{t^{\prime}}\left|t-t^{\prime}\right|=$ $\theta\left(t^{\prime}-t\right)-\theta\left(t-t^{\prime}\right)$, and $\partial_{t} \partial_{t^{\prime}}\left|t-t^{\prime}\right|=-2 \delta\left(t-t^{\prime}\right)$, we obtain

$$
\partial_{t} \partial_{t^{\prime}} \Delta_{\mathrm{cl}}\left(t, t^{\prime}\right)=-\frac{\Omega \Gamma}{2} e^{-\Gamma\left|t-t^{\prime}\right|}+\Omega \delta\left(t-t^{\prime}\right) .
$$

Fourier transforming eqs. (9.290) and (9.291) leads to ${ }^{55}$

$$
\begin{aligned}
\widetilde{\Delta}_{\mathrm{cl}}(\omega) & \equiv \int_{-\infty}^{\infty} \mathrm{d} t e^{i \omega\left(t-t^{\prime}\right)} \Delta_{\mathrm{cl}}\left(t, t^{\prime}\right)=\frac{\Omega}{2 \Gamma}\left[\int_{0}^{\infty} \mathrm{d} t e^{(i \omega-\Gamma) t}+\int_{-\infty}^{0} \mathrm{~d} t e^{(i \omega+\Gamma) t}\right] \\
& =\frac{\Omega}{\omega^{2}+\Gamma^{2}}, \\
\omega^{2} \widetilde{\Delta}_{\mathrm{cl}}(\omega) & =\int_{-\infty}^{\infty} \mathrm{d} t e^{i \omega\left(t-t^{\prime}\right)} \partial_{t} \partial_{t^{\prime}} \Delta_{\mathrm{cl}}\left(t, t^{\prime}\right) \\
& =\frac{\Omega \omega^{2}}{\omega^{2}+\Gamma^{2}} .
\end{aligned}
$$

It is also useful to note that, setting the time arguments equal, we can define a susceptibility as

$$
\left\langle(\delta N)^{2}\right\rangle_{\mathrm{cl}} \equiv \lim _{t_{0} \rightarrow-\infty}\langle\langle\delta N(t) \delta N(t)\rangle\rangle=\frac{\Omega}{2 \Gamma}
$$

where we made use of eq. (9.290).

Combining eqs. (9.292)-(9.294), various strategies can be envisaged for determining the quantity that we are interested in, namely the equilibration rate $\Gamma$. One formally correct track would be to note from eq. (9.292) that $\widetilde{\Delta}_{\mathrm{cl}}(0)=\Omega / \Gamma^{2}$, and to combine this with eq. (9.294), in order to obtain

$$
\Gamma=\frac{2\left\langle(\delta N)^{2}\right\rangle_{\mathrm{cl}}}{\widetilde{\Delta}_{\mathrm{cl}}(0)} .
$$

This is equivalent to our previous approach, eq. (9.249). However, as discussed in connection with the transport peak (cf. paragraphs around eq. (9.271)), in practice it is difficult to determine $\Gamma$ from this relation, because the relevant information resides in the denominator of $\widetilde{\Delta}_{\mathrm{cl}}(\omega)$ and we would need to evaluate this function at extremely "soft" values of $\omega$.

Taking, in contrast, eqs. (9.293) and (9.294) as starting points, we obtain the alternative expressions

$$
\begin{aligned}
\Omega & =\lim _{\Gamma \ll \omega \ll \omega_{\mathrm{UV}}} \omega^{2} \widetilde{\Delta}_{\mathrm{cl}}(\omega), \\
\Gamma & =\frac{\Omega}{2\left\langle(\delta N)^{2}\right\rangle_{\mathrm{cl}}} .
\end{aligned}
$$

\footnotetext{
${ }^{54}$ In Brownian motion, when $\delta N$ corresponds to momentum, we are also interested in the correlator of particle positions, which are obtained as time integrals of the momentum. This yields $\int_{0}^{\tau} \mathrm{d} t \int_{0}^{\tau} \mathrm{d} t^{\prime} \Delta_{\mathrm{cl}}\left(t, t^{\prime}\right)=\frac{\Omega}{\Gamma^{2}}\left[\tau+\left(e^{-\Gamma \tau}-\right.\right.$ $1) / \Gamma]$. The coefficient of the linear growth is proportional to $2 D$, where $D$ is the diffusion coefficient.

${ }^{55}$ In the latter case one can literally Fourier-transform eq. (9.291), or carry out partial integrations, whereby the result can be extracted from eq. (9.292).
} 
Here $\omega_{\mathrm{UV}}$ is a frequency scale around which physics beyond the classical picture sets in, $\omega_{\mathrm{UV}} \gtrsim \alpha^{2} T$. At the same time, it has been tacitly assumed that $\Gamma$ is parametrically small compared with $\omega_{\mathrm{UV}}$. This is the case if, for instance, $\Gamma$ is inversely proportional to a heavy mass scale $M \gg T$, or proportional to a very weak coupling constant which plays no role in the dynamics of the heat bath. With these reservations, the information needed is now in the numerator of $\widetilde{\Delta}_{\mathrm{cl}}(\omega)$. Thus, if a hierarchy between $\Gamma$ and $\omega_{\mathrm{UV}}$ can be identified and an error suppressed by $\Gamma / \omega_{\mathrm{UV}}$ is tolerable, transport coefficients are most easily determined from "force-force" correlation functions (i.e. those of $\delta \dot{N}$, cf. eqs. (9.291) and (9.293)), rather than from "momentum-momentum" correlation functions (i.e. those of $\delta N$, cf. eqs. (9.290) and (9.292)).

After these preparatory steps, we can promote the determination of $\Gamma$ to the quantum level. It just remains to note that since observables commute in the classical limit, a suitable quantum version of the "momentum-momentum" correlator considered is

$$
\Delta_{\mathrm{qm}}\left(t, t^{\prime}\right) \equiv\left\langle\frac{1}{2}\left\{\delta \hat{N}(t), \delta \hat{N}\left(t^{\prime}\right)\right\}\right\rangle .
$$

With this convention, eqs. (9.296) and (9.297) can be rephrased as

$$
\begin{aligned}
\Omega & =\lim _{\Gamma \ll \omega \ll \omega_{\mathrm{UV}}} \int_{-\infty}^{\infty} \mathrm{d} t e^{i \omega\left(t-t^{\prime}\right)}\left\langle\frac{1}{2}\left\{\frac{\mathrm{d} \hat{N}(t)}{\mathrm{d} t}, \frac{\mathrm{d} \hat{N}\left(t^{\prime}\right)}{\mathrm{d} t^{\prime}}\right\}\right\rangle_{\mathrm{qm}}, \\
\Gamma & =\frac{\Omega}{2\left\langle(\delta \hat{N})^{2}\right\rangle_{\mathrm{qm}}},
\end{aligned}
$$

where the susceptibility in the denominator of the latter equation is nothing but the variance of the number density operator, $\left\langle(\delta \hat{N})^{2}\right\rangle=\left\langle\hat{N}^{2}\right\rangle-\langle\hat{N}\rangle^{2}$.

The formulae introduced above can be applied on a non-perturbative level as well, if we re-express them in the imaginary-time formalism. This means that we first define a Euclidean correlator, $\Omega(\tau)$, like in eq. (8.9); Fourier-transform it, $\Omega\left(\omega_{n}\right)=\int_{0}^{\beta} \mathrm{d} \tau e^{i \omega_{n} \tau} \Omega(\tau)$, where $\omega_{n}=2 \pi n T, n \in \mathbb{Z}$; and obtain the spectral function from its imaginary part, $\rho(\omega)=\operatorname{Im} \Omega\left(\omega_{n} \rightarrow-i\left[\omega+i 0^{+}\right]\right)$, cf. eq. (8.28). The symmetric combination needed in eq. (9.299) is subsequently given by $2 T \rho(\omega) / \omega$, where we assumed $\omega \ll T$, cf. eq. (8.16).

\section{Example}

As an example of the use of eqs. (9.299) and (9.300), let us consider the Lagrangian of eq. (9.108),

$$
\mathcal{L}_{M}=\partial_{\mu} \phi^{*} \partial^{\mu} \phi-m^{2} \phi^{*} \phi-h \phi^{*} \mathcal{J}-h^{*} \mathcal{J}^{*} \phi+\mathcal{L}_{\text {bath }}
$$

For $h=0$, the system has a conserved current (cf. eq. (7.7)),

$$
\mathcal{J}_{\mu}=-i\left(\partial_{\mu} \phi^{*} \phi-\phi^{*} \partial_{\mu} \phi\right)
$$

If the composite object $\mathcal{J}$ of eq. (9.301) does not transform under the associated symmetry, eq. (7.6), then the coupling $h$ mediates transitions through which current conservation is violated.

In eq. (9.146) of sec. 9.3, the rate at which particles and antiparticles are produced from a plasma was obtained for this model; both rates are furthermore equal if the plasma is CP-symmetric. In the present section, an initial state is considered in which the number densities of particles and antiparticles are almost in thermal equilibrium but not quite, being slightly different. Given that 
the reactions mediated by $h$ violate the conservation of the net particle minus antiparticle density, there is a rate by which the difference of the two number densities evens out, returning the system to full thermal equilibrium. This can be called a chemical equilibration rate.

Before proceeding with the calculation of the chemical equilibration rate, let us write down the canonically quantized number density operator. At this point operator ordering plays a role: if we choose the explicitly Hermitean ordering,

$$
\hat{N} \equiv-i \int_{\mathbf{x}}\left(\partial_{0} \hat{\phi}^{\dagger} \hat{\phi}-\hat{\phi}^{\dagger} \partial_{0} \hat{\phi}\right)
$$

and represent the fields in terms of the canonically normalized creation and annihilation operators like in eq. (9.120), then

$$
\hat{N}=\int \mathrm{d}^{3} \mathbf{p}\left(\hat{a}_{\mathbf{p}}^{\dagger} \hat{a}_{\mathbf{p}}-\hat{b}_{\mathbf{p}} \hat{b}_{\mathbf{p}}^{\dagger}\right)
$$

We note that this expression is not automatically "normal-ordered"; rather, it contains an infinite constant from the latter term, amounting to $\int \mathrm{d}^{3} \mathbf{p} \delta^{(3)}(\mathbf{0})=\int_{\mathbf{p}} V$, where the Dirac- $\delta$ at vanishing momentum was regulated through finite volume, $(2 \pi)^{3} \delta^{(3)}(\mathbf{p}=\mathbf{0})=\int_{V} \mathrm{~d}^{3} \mathbf{x}=V$. This infinity could be hidden by tuning the orderings in eq. (9.303). However, the infinite constant drops out in the time derivative of $\hat{N}$, so we stick to eq. (9.303) in the following.

Making next use of the operator equations of motion corresponding to eq. (9.301),

$$
\begin{aligned}
\partial_{0}^{2} \hat{\phi} & =\nabla^{2} \hat{\phi}-m^{2} \hat{\phi}-h \hat{\mathcal{J}}, \\
\partial_{0}^{2} \hat{\phi}^{\dagger} & =\nabla^{2} \hat{\phi}^{\dagger}-m^{2} \hat{\phi}^{\dagger}-h^{*} \hat{\mathcal{J}}^{\dagger},
\end{aligned}
$$

and omitting boundary terms, the time derivative of eq. (9.303) yields

$$
\partial_{0} \hat{N}=-i \int_{\mathbf{x}}\left(h \hat{\phi}^{\dagger} \hat{\mathcal{J}}-h^{*} \hat{\mathcal{J}}^{\dagger} \hat{\phi}\right)
$$

Then, according to the discussion above, we obtain to first order in $|h|^{2}$

$$
\Omega=\lim _{\Gamma \ll \omega \ll \omega_{\mathrm{UV}}} \frac{2 T \rho(\omega)}{\omega},
$$

where $\rho$ is the spectral function corresponding to the operator $\partial_{0} \hat{N}$.

Let us now define the Euclidean correlator corresponding to $\partial_{0} \hat{N}$ :

$$
\begin{aligned}
\Omega_{E}(\tau) & \equiv-\int_{\mathbf{x}, \mathbf{y}}\left\langle\left[h \phi^{*} \mathcal{J}-h^{*} \mathcal{J}^{*} \phi\right](\tau, \mathbf{x})\left[h \phi^{*} \mathcal{J}-h^{*} \mathcal{J}^{*} \phi\right](0, \mathbf{y})\right\rangle \\
& =|h|^{2} \int_{\mathbf{x}, \mathbf{y}}\left[\left\langle\phi^{*}(X) \phi(Y)\right\rangle\left\langle\mathcal{J}(X) \mathcal{J}^{*}(Y)\right\rangle+\left\langle\phi(X) \phi^{*}(Y)\right\rangle\left\langle\mathcal{J}^{*}(X) \mathcal{J}(Y)\right\rangle\right],
\end{aligned}
$$

where we defined $X \equiv(\tau, \mathbf{x}), Y \equiv(0, \mathbf{y})$ and only considered contractions allowed by the $\mathrm{U}(1)$ invariance of free $\phi$-particles. Hats have been left out from these expressions because Euclidean correlators can be evaluated with path integral techniques.

We wish to make as few assumptions about the operator $\mathcal{J}$ as possible, and simply express its 2 -point correlation function in a general spectral representation. Inverting eq. (8.9) we can write

$$
\left\langle\mathcal{J}(X) \mathcal{J}^{*}(Y)\right\rangle=\oiint_{K} e^{-i K \cdot(X-Y)} \Pi_{E}(K) .
$$


Inserting here eq. (8.25), i.e.

$$
\Pi_{E}(K)=\int_{-\infty}^{\infty} \frac{\mathrm{d} \omega}{\pi} \frac{\rho(\omega, \mathbf{k})}{\omega-i k_{n}}
$$

as well as the sum in eq. (8.32), i.e.

$$
T \sum_{k_{n}} \frac{e^{-i k_{n} \tau}}{\omega-i k_{n}}=n_{\mathrm{B}}(\omega) e^{(\beta-\tau) \omega}, \quad \text { for } 0<\tau<\beta,
$$

and substituting $\omega \rightarrow k^{0}$, we obtain

$$
\left\langle\mathcal{J}(X) \mathcal{J}^{*}(Y)\right\rangle=2 \int_{\mathcal{K}} \rho(\mathcal{K}) n_{\mathrm{B}}\left(k^{0}\right) e^{(\beta-\tau) k^{0}+i \mathbf{k} \cdot(\mathbf{x}-\mathbf{y})} .
$$

The other case is handled similarly: making use of eq. (8.31) and renaming subsequently $\omega \rightarrow-k^{0}$ and $\mathbf{k} \rightarrow-\mathbf{k}$, we obtain

$$
\begin{aligned}
\left\langle\mathcal{J}(Y) \mathcal{J}^{*}(X)\right\rangle & =\int_{\mathbf{k}} \int \frac{\mathrm{d} \omega}{\pi} \rho(\omega, \mathbf{k}) n_{\mathrm{B}}(\omega) e^{\tau \omega+i \mathbf{k} \cdot(\mathbf{y}-\mathbf{x})} \\
& =-2 \int_{\mathcal{K}} \rho(-\mathcal{K}) n_{\mathrm{B}}\left(k^{0}\right) e^{(\beta-\tau) k^{0}+i \mathbf{k} \cdot(\mathbf{x}-\mathbf{y})},
\end{aligned}
$$

where we also made use of $n_{\mathrm{B}}\left(-k^{0}\right)=-e^{\beta k^{0}} n_{\mathrm{B}}\left(k^{0}\right)$.

As far as the scalar propagators are concerned, they can be replaced with their tree-level forms, given that the fields have been assumed to be weakly interacting and we work to leading order in $|h|^{2}$. If we furthermore assume that the scalar particles are close to equilibrium, which is consistent with the linear response nature of our computation, then

$$
\begin{aligned}
\left\langle\phi(X) \phi^{*}(Y)\right\rangle=\left\langle\phi(Y) \phi^{*}(X)\right\rangle & =\sum_{P} \frac{e^{i P \cdot(X-Y)}}{p_{n}^{2}+\epsilon_{p}^{2}} \\
& =\int_{\mathbf{p}} \frac{n_{\mathrm{B}}\left(\epsilon_{p}\right)}{2 \epsilon_{p}}\left[e^{(\beta-\tau) \epsilon_{p}}+e^{\tau \epsilon_{p}}\right] e^{-i \mathbf{p} \cdot(\mathbf{x}-\mathbf{y})},
\end{aligned}
$$

where we made use of eq. (8.30).

Inserting eqs. (9.313)-(9.315) into eq. (9.309), and carrying out the integrals over $\mathbf{x}, \mathbf{y}$, and $\mathbf{p}$, yields

$$
\Omega_{E}(\tau)=|h|^{2} V \int_{\mathcal{K}} \frac{n_{\mathrm{B}}\left(\epsilon_{k}\right) n_{\mathrm{B}}\left(k^{0}\right)}{\epsilon_{k}}\left[e^{(\beta-\tau) \epsilon_{k}}+e^{\tau \epsilon_{k}}\right] e^{(\beta-\tau) k^{0}}[\rho(\mathcal{K})-\rho(-\mathcal{K})] .
$$

The Euclidean Fourier transform (cf. eq. (8.9)) turns this into

$$
\begin{aligned}
\Omega_{E}\left(\omega_{n}\right) & =\int_{0}^{\beta} \mathrm{d} \tau e^{i \omega_{n} \tau} \Omega_{E}(\tau) \\
& =|h|^{2} V \int_{\mathcal{K}} \frac{n_{\mathrm{B}}\left(\epsilon_{k}\right) n_{\mathrm{B}}\left(k^{0}\right)}{\epsilon_{k}}[\rho(\mathcal{K})-\rho(-\mathcal{K})]\left[\frac{1-e^{\beta\left(\epsilon_{k}+k^{0}\right)}}{i \omega_{n}-\epsilon_{k}-k^{0}}+\frac{e^{\beta \epsilon_{k}}-e^{\beta k^{0}}}{i \omega_{n}+\epsilon_{k}-k^{0}}\right],
\end{aligned}
$$

and the corresponding spectral function reads (cf. eq. (8.28))

$$
\begin{aligned}
\rho(\omega)= & \operatorname{Im} \Omega_{E}\left(\omega_{n} \rightarrow-i\left[\omega+i 0^{+}\right]\right) \\
=\pi|h|^{2} V \int_{\mathcal{K}} \frac{\rho(\mathcal{K})-\rho(-\mathcal{K})}{\epsilon_{k}} & \left\{\delta\left(\omega-\epsilon_{k}-k^{0}\right)\left[1+n_{\mathrm{B}}\left(\epsilon_{k}\right)+n_{\mathrm{B}}\left(k^{0}\right)\right]\right. \\
& \left.+\delta\left(\omega+\epsilon_{k}-k^{0}\right)\left[n_{\mathrm{B}}\left(\epsilon_{k}\right)-n_{\mathrm{B}}\left(k^{0}\right)\right]\right\},
\end{aligned}
$$


where we applied eq. (8.20) as well as relations satisfied by the Bose distribution.

To extract the limit needed in eq. (9.308), we note that

$$
\begin{aligned}
\delta\left(\omega-\epsilon_{k}-k^{0}\right)\left[1+n_{\mathrm{B}}\left(\epsilon_{k}\right)+n_{\mathrm{B}}\left(k^{0}\right)\right] & =\delta\left(\omega-\epsilon_{k}-k^{0}\right)\left[n_{\mathrm{B}}\left(\epsilon_{k}\right)-n_{\mathrm{B}}\left(\epsilon_{k}-\omega\right)\right] \\
& =\delta\left(\omega-\epsilon_{k}-k^{0}\right)\left[\omega n_{\mathrm{B}}^{\prime}\left(\epsilon_{k}\right)+\mathcal{O}\left(\omega^{2}\right)\right] \\
& =-\delta\left(\epsilon_{k}+k^{0}\right) \beta \omega n_{\mathrm{B}}\left(\epsilon_{k}\right)\left[1+n_{\mathrm{B}}\left(\epsilon_{k}\right)\right]+\mathcal{O}\left(\omega^{2}\right), \\
\delta\left(\omega+\epsilon_{k}-k^{0}\right)\left[n_{\mathrm{B}}\left(\epsilon_{k}\right)-n_{\mathrm{B}}\left(k^{0}\right)\right] & =\delta\left(\omega+\epsilon_{k}-k^{0}\right)\left[n_{\mathrm{B}}\left(\epsilon_{k}\right)-n_{\mathrm{B}}\left(\epsilon_{k}+\omega\right)\right] \\
& =\delta\left(\omega+\epsilon_{k}-k^{0}\right)\left[-\omega n_{\mathrm{B}}^{\prime}\left(\epsilon_{k}\right)+\mathcal{O}\left(\omega^{2}\right)\right] \\
& =\delta\left(\epsilon_{k}-k^{0}\right) \beta \omega n_{\mathrm{B}}\left(\epsilon_{k}\right)\left[1+n_{\mathrm{B}}\left(\epsilon_{k}\right)\right]+\mathcal{O}\left(\omega^{2}\right),
\end{aligned}
$$

where we made use of $n_{\mathrm{B}}(-E)=-1-n_{\mathrm{B}}(E)$ as well as $n_{\mathrm{B}}^{\prime}(E)=-\beta n_{\mathrm{B}}(E)\left[1+n_{\mathrm{B}}(E)\right]$. Therefore, for small $\omega$,

$$
\begin{aligned}
\frac{2 T \rho(\omega)}{\omega} & \approx 2 \pi|h|^{2} V \int_{\mathcal{K}} \frac{\rho(\mathcal{K})-\rho(-\mathcal{K})}{\epsilon_{k}}\left[\delta\left(\epsilon_{k}-k^{0}\right)-\delta\left(\epsilon_{k}+k^{0}\right)\right] n_{\mathrm{B}}\left(\epsilon_{k}\right)\left[1+n_{\mathrm{B}}\left(\epsilon_{k}\right)\right] \\
& =2|h|^{2} V \int_{\mathbf{k}} \frac{\rho(\mathcal{K})-\rho(-\mathcal{K})}{\epsilon_{k}} n_{\mathrm{B}}\left(\epsilon_{k}\right)\left[1+n_{\mathrm{B}}\left(\epsilon_{k}\right)\right]
\end{aligned}
$$

where in the second step we substituted $\mathbf{k} \rightarrow-\mathbf{k}$ in some of the terms, and also implicitly changed the notation: from now on $\mathcal{K}$ denotes an on-shell four-vector, $\mathcal{K} \equiv\left(\epsilon_{k}, \mathbf{k}\right)$.

In order to apply eq. (9.300), we also need the susceptibility. This can most easily be extracted from eq. (7.18), which gives the grand canonical free energy density for a complex scalar field. In the thermodynamic limit, the susceptibility is obtained from the second partial derivative of this quantity with respect to $\mu$, evaluated at $\mu=0$ (cf. eq. (7.56)):

$$
\begin{aligned}
\left\langle(\delta \hat{N})^{2}\right\rangle & =\left.T^{2} \partial_{\mu}^{2} \ln \mathcal{Z}\right|_{\mu=0}=-\left.V T \partial_{\mu}^{2} f(T, \mu)\right|_{\mu=0} \\
& =-V T \partial_{\mu}^{2} \int_{\mathbf{p}}\left\{\epsilon_{p}+T\left[\ln \left(1-e^{-\beta\left(\epsilon_{p}+\mu\right)}\right)+\ln \left(1-e^{-\beta\left(\epsilon_{p}-\mu\right)}\right)\right]\right\}_{\mu=0} \\
& =-V T \partial_{\mu} \int_{\mathbf{p}}\left\{\frac{1}{e^{\beta\left(\epsilon_{p}+\mu\right)}-1}-\frac{1}{e^{\beta\left(\epsilon_{p}-\mu\right)}-1}\right\}_{\mu=0} \\
& =2 V \int_{\mathbf{p}} n_{\mathrm{B}}\left(\epsilon_{p}\right)\left[1+n_{\mathrm{B}}\left(\epsilon_{p}\right)\right]
\end{aligned}
$$

Putting everything together, and making use of the relation $\rho(-\mathcal{K})=-\rho(\mathcal{K})$, valid for a CPsymmetric plasma, eq. (9.300) yields

$$
\Gamma=\frac{|h|^{2} \int_{\mathbf{k}} \frac{\rho(\mathcal{K})}{\epsilon_{k}} n_{\mathrm{B}}\left(\epsilon_{k}\right)\left[1+n_{\mathrm{B}}\left(\epsilon_{k}\right)\right]}{\int_{\mathbf{k}} n_{\mathrm{B}}\left(\epsilon_{k}\right)\left[1+n_{\mathrm{B}}\left(\epsilon_{k}\right)\right]} .
$$

We conclude with a discussion concerning the physical interpretation of eq. (9.323). According to eqs. (9.134) and (9.136), $|h|^{2} \frac{\rho(\mathcal{K})}{\epsilon_{k}} n_{\mathrm{B}}\left(\epsilon_{k}\right)$ gives the production rate of particles or antiparticles of momentum k; the appearance of $n_{\mathrm{B}}\left(\epsilon_{k}\right)$ indicates that the production necessitates the presence of a plasma in which collisions take place, because the energy $\epsilon_{k}$ needs to be extracted from thermal 
fluctuations. In the chemical equilibration rate, in contrast, we could think of $|h|^{2} \frac{\rho(\mathcal{K})}{\epsilon_{k}}\left[1+n_{\mathrm{B}}\left(\epsilon_{k}\right)\right]$ as the rate at which particles or antiparticles decay if they were initially in excess (cf. eq. (9.149)); only one of these processes needs to take place if there is an imbalance. Because we posed a question about the "chemical" rather than the "kinetic" equilibration of the system, eq. (9.323) contains an average over $\mathbf{k}$ of the decay rate, weighted by the kinetically equilibrated momentum distribution given by $n_{\mathrm{B}}\left(\epsilon_{k}\right)$.

\section{Appendix A: Relation of Langevin and Fokker-Planck equations}

We have seen that the dynamics following from the Langevin equation, eqs. (9.287) and (9.288), is simple enough to be exactly solvable. Nevertheless, there are circumstances where it may be advantageous to reformulate the (stochastic) Langevin equation as a deterministic (non-stochastic) equation, for the corresponding probability density. The latter is known as the Fokker-Planck equation.

Let us start by generalizing $\delta N$ to a multi-component variable, and eq. (9.289) to a corresponding solution, $\delta N_{i}(t)=\delta N_{i}\left(t_{0}\right) e^{-\Gamma\left(t-t_{0}\right)}+\int_{t_{0}}^{t} \mathrm{~d} t_{i}^{\prime} e^{\Gamma\left(t_{i}^{\prime}-t\right)} \xi_{i}\left(t_{i}^{\prime}\right)$, where $\left\langle\left\langle\xi_{i}\left(t_{i}^{\prime}\right) \xi_{j}\left(t_{j}^{\prime}\right)\right\rangle\right\rangle=\Omega \delta_{i j} \delta\left(t_{i}^{\prime}-t_{j}^{\prime}\right)$. Consider now the $n$-point function

$$
G_{i_{1} \cdots i_{n}}\left(t_{1}, \ldots, t_{n}\right) \equiv\left\langle\left\langle\delta N_{i_{1}}\left(t_{1}\right) \cdots \delta N_{i_{n}}\left(t_{n}\right)\right\rangle .\right.
$$

A time derivative yields

$$
\partial_{\tilde{t}} G_{i_{1} i_{2} \cdots i_{n}}(\tilde{t}, t, \ldots, t)=\left\langle\left\langle\left[-\Gamma \delta N_{i_{1}}(\tilde{t})+\xi_{i_{1}}(\tilde{t})\right] \delta N_{i_{2}}(t) \cdots \delta N_{i_{n}}(t)\right\rangle\right\rangle .
$$

The first term is proportional to $G_{i_{1} i_{2} \cdots i_{n}}$, whereas the second term requires knowledge about $n$ point correlators of the noise. Let us assume that the noise is "white", i.e. Gaussian. Then the noise correlator factorizes, and we get

$$
\begin{aligned}
\left\langle\left\langle\xi_{i_{1}}(\tilde{t}) \delta N_{i_{2}}(t) \cdots \delta N_{i_{n}}(t)\right\rangle\right\rangle & =\sum_{j=2}^{n}\left\langle\left\langle\xi_{i_{1}}(\tilde{t}) \delta N_{i_{j}}(t)\right\rangle \frac{\partial\left\langle\left\langle\delta N_{i_{2}}(t) \cdots \delta N_{i_{n}}(t)\right\rangle\right\rangle}{\partial \delta N_{i_{j}}(t)}\right. \\
& =\sum_{j=2}^{n}\left\langle\left\langle\xi_{i_{1}}(\tilde{t}) \int_{t_{0}}^{t} \mathrm{~d} t_{j}^{\prime} e^{\Gamma\left(t_{j}^{\prime}-t\right)} \xi_{i_{j}}\left(t_{j}^{\prime}\right)\right\rangle\right\rangle \frac{\left.\partial\left\langle\delta N_{i_{2}}(t) \cdots \delta N_{i_{n}}(t)\right\rangle\right\rangle}{\partial \delta N_{i_{j}}(t)} \\
& \stackrel{\tilde{t} \rightarrow t}{=} \theta(t-\tilde{t}) \Omega \frac{\partial\left\langle\left\langle\delta N_{i_{2}}(t) \cdots \delta N_{i_{n}}(t)\right\rangle\right\rangle}{\partial \delta N_{i_{1}}(t)} .
\end{aligned}
$$

In the third step, we inserted the noise autocorrelator. In terms of the original correlator from eq. (9.324), we see that two insertions of the fluctuation $\delta N_{i_{1}}$ have been eliminated.

Now, we return to eq. (9.325), but set $\tilde{t} \rightarrow t^{-}$, whereby the time derivative can act on any of the fluctuations. Implementing eq. (9.326) as a second derivative, then yields

$$
\partial_{t} G_{i_{1} \cdots i_{n}}(t, \ldots, t)=-n \Gamma G_{i_{1} \cdots i_{n}}(t, \ldots, t)+\frac{\Omega}{2} \sum_{i} \frac{\partial^{2} G_{i_{1} \cdots i_{n}}(t, \ldots, t)}{\partial \delta N_{i}^{2}(t)} .
$$

On the other hand, let us define a probability distribution, $\mathcal{W}$, as

$$
\mathcal{W}\left(\left\{\delta N_{i 1}\right\}, t_{1} ;\left\{\delta N_{i 0}\right\}, t_{0}\right) \equiv\left\langle\left\langle\prod_{i} \delta\left[\delta N_{i 1}-\delta N_{i}\left(t_{1}\right)\right]\right\rangle, \quad \delta N_{i}\left(t_{0}\right) \equiv \delta N_{i 0} .\right.
$$


The initial condition at time $t_{1}=t_{0}$ reads $\mathcal{W}\left(\left\{\delta N_{i 1}\right\}, t_{0} ;\left\{\delta N_{i 0}\right\}, t_{0}\right)=\prod_{i} \delta\left[\delta N_{i 1}-\delta N_{i 0}\right]$, and the normalization amounts to $\int \prod_{i} \mathrm{~d} \delta N_{i 1} \mathcal{W}\left(\left\{\delta N_{i 1}\right\}, t ;\left\{\delta N_{i 0}\right\}, t_{0}\right)=1$.

With the probability distribution, the moments appearing in eq. (9.327) are expressed as

$$
G_{i_{1} \cdots i_{n}}(t, \ldots, t)=\int\left\{\prod_{i} \mathrm{~d} \delta N_{i}\right\} \delta N_{i_{1}} \cdots \delta N_{i_{n}} \mathcal{W}\left(\left\{\delta N_{i}\right\}, t ;\left\{\delta N_{i 0}\right\}, t_{0}\right) .
$$

The time derivative then yields

$$
\partial_{t} G_{i_{1} \cdots i_{n}}(t, \ldots, t)=\int\left\{\prod_{i} \mathrm{~d} \delta N_{i}\right\} \delta N_{i_{1}} \cdots \delta N_{i_{n}} \partial_{t} \mathcal{W}\left(\left\{\delta N_{i}\right\}, t ;\left\{\delta N_{i 0}\right\}, t_{0}\right) .
$$

We envisage that the time derivative of $\mathcal{W}$ equals a differential operator, acting with respect to $\delta N_{i}$, and carry out partial integrations. In order to reproduce the structure in eq. (9.327), with the correct signs and the "counting" of insertions in the first term (i.e. the factor $n$ ), the differential operator must read

$$
\partial_{t} \mathcal{W}=\sum_{i} \frac{\partial}{\partial \delta N_{i}}\left[\left(\Gamma \delta N_{i}+\frac{\Omega}{2} \frac{\partial}{\partial \delta N_{i}}\right) \mathcal{W}\right] .
$$

This is the Fokker-Planck equation (which bears some similarity with the Schrödinger equation).

It is easy to search for a stationary solution of the Fokker-Planck equation. Physically, this corresponds to the form that $\mathcal{W}$ obtains at late times. Requiring the square brackets in eq. (9.331) to vanish, we see that

$$
\lim _{t \rightarrow \infty} \mathcal{W}\left(\left\{\delta N_{i}\right\}, t ;\left\{\delta N_{i 0}\right\}, t_{0}\right)=\mathcal{C} \exp \left(-\frac{\Gamma}{\Omega} \sum_{i} \delta N_{i}^{2}\right)
$$

The prefactor $\mathcal{C}$ can be fixed from the normalization condition as $\mathcal{C}=\prod_{i}\left(\frac{\Gamma}{\pi \Omega}\right)^{1 / 2}$. The existence of a "fixed point" shows that the system equilibrates. As a crosscheck, the width of the Gaussian distribution, as visible in eq. (9.332), agrees with what we had obtained in eq. (9.294). 


\subsection{Resonances in medium}

As a final observable, we consider the behaviour of a pair of heavy particles within a thermal medium. In the QCD context, this would be relevant for quarkonium physics [9.85]. Physically, heavy quarkonium refers to a bound state of a charm and anti-charm quark $(c \bar{c})$ or a bottom and anti-bottom quark $(b \bar{b})$. Formally, quarkonium physics refers to observables that can be extracted from 2-point correlation functions of the conserved vector current, $\hat{\mathcal{J}}^{\mu} \equiv \hat{\bar{\psi}} \gamma^{\mu} \hat{\psi}$, around the 2particle thresfold, i.e. for energies $\omega \sim 2 M$ (more precisely, $|\omega-2 M| \ll M$ ), where $M$ denotes a heavy quark "pole mass". ${ }^{56}$ In the following, we are interested in the limit $M \gg 1 \mathrm{GeV}$, so that the situation should be at least partly perturbative. The goal is to illustrate with yet another example (cf. sec. 9.5) how in a thermal medium two types of effects operate in parallel: "virtual corrections", which modify masses or effective parameters (in the present case, a potential); and "real corrections", which represent real scatterings not taking place in vacuum.

A useful starting point for our analysis is the observation that in the heavy-mass limit, the QCD Lagrangian can be simplified. Considering the extreme case in which the quarks do not move in the spatial directions at all, because the kicks they receive from medium or vacuum fluctuations are insufficient to excite them, we may keep only the temporal part of the theory, resulting in the Lagrangian

$$
\mathcal{L}_{M} \equiv \bar{\psi}\left(i \gamma^{0} D_{0}-M\right) \psi .
$$

This expression can be further split up into a form that contains explicitly a "quark" and an "antiquark", e.g. by adopting a representation for the Dirac matrices with $\gamma^{0}=\operatorname{diag}\left(\mathbb{1}_{2 \times 2},-\mathbb{1}_{2 \times 2}\right)$ and by writing

$$
\psi \equiv\left(\begin{array}{c}
\theta \\
\chi
\end{array}\right), \quad \bar{\psi} \equiv\left(\theta^{\dagger},-\chi^{\dagger}\right),
$$

or more abstractly by defining $\theta \equiv \frac{1}{2}\left(\mathbb{1}+\gamma^{0}\right) \psi, \chi \equiv \frac{1}{2}\left(\mathbb{1}-\gamma^{0}\right) \psi$. Since fermions are Grassmann fields, we must recall a minus sign when fields are commuted; in the following this concerns in particular the ordering of $\chi_{\alpha}^{*}, \chi_{\beta}$. Noting furthermore that (the Minkowskian four-vector $\mathcal{X}$ here is not to be confused with the Grassmann field $\chi$ )

$$
\int_{\mathcal{X}}-f(\mathcal{X}) \overleftarrow{D}_{\mu}^{\dagger} g(\mathcal{X})=\int_{\mathcal{X}} f(\mathcal{X}) \vec{D}_{\mu} g(\mathcal{X})
$$

where the arrow indicates the side on which the derivative operates, we obtain

$$
\int_{\mathcal{X}} \chi_{\alpha}^{*}\left[\vec{D}_{\mu}\right]_{\alpha \beta} \chi_{\beta}=-\int_{\mathcal{X}} \chi_{\beta}\left[\overleftarrow{D}_{\mu}\right]_{\alpha \beta} \chi_{\alpha}^{*}=\int_{\mathcal{X}} \chi_{\beta}\left[\vec{D}_{\mu}^{*}\right]_{\beta \alpha} \chi_{\alpha}^{*}
$$

Therefore the action corresponding to eq. (9.333) can be written as

$$
\begin{aligned}
\mathcal{S}_{M} & =\int_{\mathcal{X}}\left\{\theta^{\dagger}\left(i D_{0}-M\right) \theta+\chi^{\dagger}\left(i D_{0}+M\right) \chi\right\} \\
& =\int_{\mathcal{X}}\left\{\theta^{\dagger}\left(i D_{0}-M\right) \theta+\chi^{* \dagger}\left(i D_{0}^{*}-M\right) \chi^{*}\right\} .
\end{aligned}
$$

This shows that the (charge-conjugated) field $\chi^{*}$ represents an antiparticle to $\theta$, having the same mass but an opposite gauge charge.

\footnotetext{
${ }^{56}$ These considerations are related to the production rate of $e^{-} e^{+}$or $\mu^{-} \mu^{+}$pairs with a total energy close to the mass of a quarkonium resonance, cf. eq. (9.106). Analogous physics may also play a role in cosmology, in connection with the thermal annihilation process of non-relativistic dark matter particles into Standard Model particles, if the former interact attractively through gauge boson exchange [9.86].
} 
Next, we need a representation for the current $\mathcal{J}^{\mu}$ in terms of the spinors $\theta, \chi$. If we consider the "zero-momentum" projection $\int_{\mathbf{x}} \mathcal{J}^{\mu}$, then current conservation implies that the component $\mu=0$ must be constant in time, and hence that all interesting dynamics resides in the spatial components. It follows directly from the field redefinition in eq. (9.334) that in the standard representation ${ }^{57}$ the spatial components can be expressed as

$$
\mathcal{J}^{k}=\theta^{\dagger} \sigma_{k} \chi+\chi^{\dagger} \sigma_{k} \theta \equiv \mathcal{J}_{\mathrm{NRQCD}}^{k} .
$$

Let us note in passing that this relation does experience corrections of $\mathcal{O}\left(M^{0}\right)$ through loop effects; in fact at the next-to-leading order the relation reads $[9.87,9.88]$

$$
\mathcal{J}_{\mathrm{QCD}}^{k}=\mathcal{J}_{\mathrm{NRQCD}}^{k}\left(1-\frac{g^{2} C_{\mathrm{F}}}{2 \pi^{2}}+\ldots\right)
$$

In the following, we omit the spin structure from eq. (9.338), but simultaneously also separate the quark and antiquark from each other, connecting them with a Wilson line defined at the time slice $t$ and denoted by $W_{t}$, which is needed to keep the quantity gauge invariant. This produces the structure

$$
\mathcal{J}_{\mathrm{NRQCD}}^{k} \rightarrow \mathcal{J}_{\mathbf{r}}(t, \mathbf{x}) \equiv \theta^{\dagger}\left(t, \mathbf{x}+\frac{\mathbf{r}}{2}\right) W_{t} \chi\left(t, \mathbf{x}-\frac{\mathbf{r}}{2}\right)+\chi^{\dagger}\left(t, \mathbf{x}-\frac{\mathbf{r}}{2}\right) W_{t}^{\dagger} \theta\left(t, \mathbf{x}+\frac{\mathbf{r}}{2}\right) .
$$

A typical Green's function could be of the form $\int_{\mathbf{x}}\left\langle\hat{\mathcal{J}}_{\mathbf{r}}(t, \mathbf{x}) \hat{\mathcal{J}}_{\mathbf{r}^{\prime}}(0, \mathbf{0})\right\rangle$. Given that we have assumed the heavy quarks not to move, we can however even set $\mathbf{x}=\mathbf{0}$ and $\mathbf{r}^{\prime}=\mathbf{r}$, leaving us with

$$
C_{r}^{>}(t) \equiv\left\langle\hat{\mathcal{J}}_{\mathbf{r}}(t, \mathbf{0}) \hat{\mathcal{J}}_{\mathbf{r}}(0, \mathbf{0})\right\rangle, \quad r \equiv|\mathbf{r}|
$$

where we have used the notation of sec. 8.1 for the particular time-ordering chosen.

Now, time-translation invariance guarantees that $C_{r}^{>}(t)=C_{r}^{<}(-t)$ and $\rho_{r}(t)=\frac{1}{2}\left[C_{r}^{>}(t)-\right.$ $\left.C_{r}^{<}(t)\right]=-\rho_{r}(-t)$, which in frequency-space imply that $C_{r}^{>}(\omega)=C_{r}^{<}(-\omega)$ and $\rho_{r}(\omega)=-\rho_{r}(-\omega)$. All of these functions contain the same information but, as indicated by eqs. (8.14) and (8.15), for $\omega \gg T$ it is $C_{r}^{>}(\omega)$ that approximates $\rho_{r}(\omega)$ well, whereas for $\omega \ll-T$ the spectral information is dominantly contained in $C_{r}^{<}(\omega)$. We also note that for $|\omega| \ll T$, corresponding to the classical limit, the two orderings agree.

If we allow the Wilson lines $W_{t}$ to have an arbitrary shape, the operators $\hat{\mathcal{J}}_{\mathbf{r}}$ constitute a whole set of possible choices. Upon operating on the vacuum state, they generate a basis of gauge-invariant states in the sense of refs. [9.89]-[9.91]. These basis states are in general not eigenstates of the Hamiltonian, but the latter can be expressed as linear combinations of the basis states.

Let now $|n\rangle$ denote the gauge-invariant eigenstates of the QCD Hamiltonian in the sector of the Fock space that contains no heavy quarks or antiquarks (but does contain glueballs and their scattering states, as well as light hadrons), and $\left|n^{\prime} ; r\right\rangle$ those in the sector with one heavy quark and one antiquark, separated by a distance $r$. If $\hat{\mathcal{J}}_{\mathbf{r}}$ operates on a state of the type $|n\rangle$, the result should have a non-zero overlap with some of the $\left|n^{\prime} ; r\right\rangle$. If on the other hand the Hamiltonian operates on the states $|n\rangle$, the state does not change but gets multiplied by an $r$-independent eigenvalue $\epsilon_{n}$. And finally, if the Hamiltonian operates on the $\left|n^{\prime} ; r\right\rangle$, the result is a multiplication of the state by an $r$-dependent eigenvalue $\epsilon_{n^{\prime}}(r)$, conventionally referred to as the (singlet) static potential.

$$
{ }^{57} \text { This refers to } \gamma^{0} \equiv\left(\begin{array}{cc}
\mathbb{1} & 0 \\
0 & -\mathbb{1}
\end{array}\right), \gamma^{k} \equiv\left(\begin{array}{cc}
0 & \sigma_{k} \\
-\sigma_{k} & 0
\end{array}\right), k \in\{1,2,3\} \text {, where } \sigma_{k} \text { are the Pauli matrices. }
$$


Numerical results for several of the lowest-lying values of $\epsilon_{n^{\prime}}(r)$ in pure $\mathrm{SU}(3)$ gauge theory can be found in ref. [9.92] (to be precise, these measurements concern $\epsilon_{n^{\prime}}(r)-\epsilon_{0}$, cf. below).

We now expand the equilibrium correlator of eq. (9.341) in the energy eigenbasis. A key observation [9.93] is that in the limit $T \ll 2 M$, no states of the type $\left|n^{\prime} ; r\right\rangle$ need to be put next to the density matrix $\mathcal{Z}^{-1} \exp (-\beta \hat{H})$, because such contributions are suppressed by $\sim e^{-2 M / T}$. Thus, we obtain

$$
\begin{aligned}
C_{r}^{>}(t) & \approx \frac{1}{\mathcal{Z}} \sum_{n, n^{\prime}}\left\langle n\left|e^{-\beta \hat{H}} e^{i \hat{H} t} \hat{\mathcal{J}}_{\mathbf{r}} e^{-i \hat{H} t}\right| n^{\prime} ; r\right\rangle\left\langle n^{\prime} ; r\left|\hat{\mathcal{J}}_{\mathbf{r}}\right| n\right\rangle \\
& =\frac{1}{\mathcal{Z}} \sum_{n, n^{\prime}} e^{-\beta \epsilon_{n}} e^{i\left[\epsilon_{n}-\epsilon_{n^{\prime}}(r)\right] t}\left|\left\langle n\left|\hat{\mathcal{J}}_{\mathbf{r}}\right| n^{\prime} ; r\right\rangle\right|^{2}
\end{aligned}
$$

This function contains all relevant information about the dynamics of gauge-invariant quarkantiquark states as long as $T \ll 2 M$. Next, we discuss its basic physical features.

To start with, let us consider the case of zero temperature $(\beta \rightarrow \infty)$ and furthermore carry out a Wick rotation to Euclidean spacetime like in eq. (8.1), i.e. it $\rightarrow \tau, 0<\tau<\beta$. Then the sum over $n$, with the weight $e^{(\tau-\beta) \epsilon_{n}}$, is dominated by the ground state $n=0$, whereas the sum over $n^{\prime}$, with the weight $e^{-\tau \epsilon_{n^{\prime}}(r)}$, is dominated by $n^{\prime} \equiv 0^{\prime}$. Noting that $\mathcal{Z} \approx e^{-\beta \epsilon_{0}}$ in this limit, we observe that $\epsilon_{0^{\prime}}(r)-\epsilon_{0}$ can be extracted from the asymptotic $\tau$-dependence of $C_{r}^{>}$in the range $0 \ll \tau \ll \beta / 2$, independent of the details of the operator $\hat{\mathcal{J}}_{\mathbf{r}}$ considered. This is how the results of ref. [9.92] for $\epsilon_{n^{\prime}}(r)-\epsilon_{0}$ have been obtained.

Suppose then that we modify the setup by returning to Minkowskian signature but keeping still $T=0$. The sum over $n$ is clearly still saturated by the ground state, but in the sum over $n^{\prime}$ we now get a contribution from several states. The excited $n^{\prime} \neq 0^{\prime}$ states, however, lead to more rapid oscillations than the ground state, so that the ground state energy could be identified as the smallest oscillation frequency of the correlator. In pure $\mathrm{SU}\left(N_{\mathrm{c}}\right)$ gauge theory, $\epsilon_{n^{\prime}}(r)$ is believed to display a "string spectrum", representing vibrations of a colour "flux tube" between the quark and antiquark. The string spectrum is expected to be discrete, with level spacings $\Delta \epsilon_{n^{\prime}} \approx \pi / r$ at large $r$. Therefore $C_{r}^{>}$remains periodic, or "coherent"; this means that no information gets lost but after a certain period the time evolution of $C_{r}^{>}$repeats itself. ${ }^{58}$

Next, we switch on a finite temperature, which implies that the sum over $n$ becomes non-trivial as well. An immediate consequence of this is that, for any given $n^{\prime}$, there are contributions to eq. (9.342) which make the oscillations slower, decreasing $\epsilon_{n^{\prime}}(r)$ to $\epsilon_{n^{\prime}}(r)-\epsilon_{n}, n \geq 1$. Of course, the overlaps $\left|\left\langle n\left|\hat{\mathcal{J}}_{\mathbf{r}}\right| n^{\prime} ; r\right\rangle\right|^{2}$ also depend on $n$, so that the correlator may now be dominated by another value of $n^{\prime}$, and the "effective" magnitude of $\epsilon_{n^{\prime}}(r)-\epsilon_{n}$ is not easily deduced. Nevertheless we could refer to this phenomenon as Debye screening: in the presence of a medium the energy associated with a quark-antiquark pair separated by a distance $r$ changes from that in vacuum, because of the presence of states other than the vacuum one in the thermal average. In the language introduced at the beginning of this section, such a change in the energetics could be considered a "virtual correction".

The temperature may also lead to a more dramatic effect. Indeed, in an infinite volume the spectrum $\epsilon_{n}$ contains a continuous part, consisting e.g. of pionic states, with the pions moving with respect to the rest frame that we have chosen to represent the heat bath. If the temperature is high enough to excite this part, we may expect to find a "resonance"-type feature: the density

\footnotetext{
${ }^{58}$ Strictly speaking this is true only if the Hilbert space is finite-dimensional.
} 
of states grows with $\epsilon_{n}$, whereas $e^{-\beta \epsilon_{n}}$ decreases with $\epsilon_{n}$. To be explicit, let us model the resulting energy dependence by a Breit-Wigner shape, which implies writing

$$
\begin{aligned}
\sum_{n} e^{-\beta \epsilon_{n}+i \epsilon_{n} t}\left|\left\langle n\left|\hat{\mathcal{J}}_{\mathbf{r}}\right| n^{\prime} ; r\right\rangle\right|^{2} & \rightarrow \int \mathrm{d} \epsilon_{n} \rho\left(\epsilon_{n}\right) e^{-\beta \epsilon_{n}+i \epsilon_{n} t}\left|\left\langle n\left|\hat{\mathcal{J}}_{\mathbf{r}}\right| n^{\prime} ; r\right\rangle\right|^{2} \\
& \simeq \int \mathrm{d} \epsilon_{n} e^{i \epsilon_{n} t} \frac{\mathcal{F}\left(n^{\prime} ; r\right)}{\left[\epsilon_{n}-\mathcal{E}\left(n^{\prime} ; r\right)\right]^{2}+\Gamma^{2}\left(n^{\prime} ; r\right)} \\
& \simeq \frac{\pi \mathcal{F}\left(n^{\prime} ; r\right)}{\Gamma\left(n^{\prime} ; r\right)} \exp \left\{i \mathcal{E}\left(n^{\prime} ; r\right) t-\Gamma\left(n^{\prime} ; r\right) t\right\} .
\end{aligned}
$$

We observe that, apart from the energy shift that was referred to as Debye screening above and is now represented by $\mathcal{E}\left(n^{\prime} ; r\right)$, the absolute value of the correlator also decreases with time. This phenomenon may be referred to as decoherence: the coherent quantum-mechanical state $\left|n^{\prime} ; r\right\rangle$ loses "information" through a continuum of random scattering processes with the heat bath. According to eq. (9.343), we can also talk about a "thermal width" affecting the time evolution, or of an "imaginary part" in the effective energy shift.

Physically, imaginary parts or widths correspond to "real scatterings" ${ }^{59}$ We expect that the farther apart the quark and the antiquark are from each other, the larger should the width $\Gamma\left(n^{\prime} ; r\right)$ be. In the partonic language of quarks and gluons, this is because at a large separation the quarkantiquark pair carries a large "colour dipole", which scatters efficiently on the medium gluons. These frequent scatterings lead to a loss of coherence of the initially quantum-mechanical quarkantiquark state. (Computations of these reactions have been reviewed, e.g., in ref. [9.94].)

We can also illustrate the physical meaning of the thermal width in a gauge-invariant hadronic language. In this picture real scatterings are possible because thermal fluctuations can excite colour-neutral states, like pions, from the medium, with which the heavy quarks can interact. This mechanism can for instance dissociate the quarkonium bound state into so-called "open charm" or "open bottom" hadrons, i.e. ones in which the heavy quarks form mesons with light antiquarks, or vice versa (cf. e.g. ref. [9.95]):

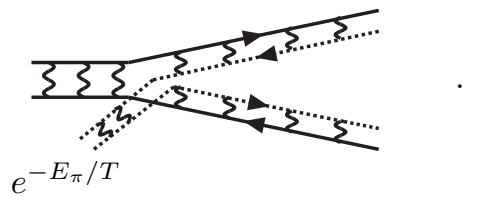

This is a purely thermal effect which would not be kinematically allowed in vacuum, because the energy of the two open states is higher than that of the single bound state. In full equilibrium, i.e. at time scales much larger than how often the process shown occurs, the opposite reaction would also take place at an equivalent rate, and the equilibrium ensemble would contain both open and bound states. The entropy of this state is maximal, i.e. all information about the coherent quantum-mechanical initial state in which the quark-antiquark pair was generated, has been lost.

\footnotetext{
${ }^{59} \mathrm{~A}$ classic example of this is the optical theorem of scattering theory.
} 


\section{Literature}

[9.1] R. Fukuda and E. Kyriakopoulos, Derivation of the effective potential, Nucl. Phys. B 85 (1975) 354.

[9.2] R. Jackiw, Functional evaluation of the effective potential, Phys. Rev. D 9 (1974) 1686.

[9.3] D.A. Kirzhnits, Weinberg model in the hot universe, JETP Lett. 15 (1972) 529 [Pisma Zh. Eksp. Teor. Fiz. 15 (1972) 745].

[9.4] D.A. Kirzhnits and A.D. Linde, Macroscopic consequences of the Weinberg model, Phys. Lett. B 42 (1972) 471.

[9.5] L. Dolan and R. Jackiw, Symmetry behavior at finite temperature, Phys. Rev. D 9 (1974) 3320.

[9.6] S. Weinberg, Gauge and global symmetries at high temperature, Phys. Rev. D 9 (1974) 3357.

[9.7] M. Lüscher and P. Weisz, Scaling laws and triviality bounds in the lattice $\varphi^{4}$ theory: (I). One-component model in the symmetric phase, Nucl. Phys. B 290 (1987) 25.

[9.8] M. Lüscher and P. Weisz, Scaling laws and triviality bounds in the lattice $\varphi^{4}$ theory: (II). One-component model in the phase with spontaneous symmetry breaking, Nucl. Phys. B 295 (1988) 65.

[9.9] J. Rudnick, First-order transition induced by cubic anisotropy, Phys. Rev. B 18 (1978) 1406.

[9.10] D. Land and E.D. Carlson, Two stage phase transition in two Higgs models, Phys. Lett. B 292 (1992) 107 [hep-ph/9208227].

[9.11] B.I. Halperin, T.C. Lubensky and S.-K. Ma, First-Order Phase Transitions in Superconductors and Smectic-A Liquid Crystals, Phys. Rev. Lett. 32 (1974) 292.

[9.12] D.A. Kirzhnits and A.D. Linde, Symmetry behavior in gauge theories, Annals Phys. 101 (1976) 195.

[9.13] P.B. Arnold and O. Espinosa, Effective potential and first-order phase transitions: Beyond leading order, Phys. Rev. D 47 (1993) 3546; ibid. 50 (1994) 6662 (E) [hep-ph/9212235].

[9.14] M. Laine and K. Rummukainen, What's new with the electroweak phase transition?, Nucl. Phys. Proc. Suppl. 73 (1999) 180 [hep-lat/9809045].

[9.15] D.E. Morrissey and M.J. Ramsey-Musolf, Electroweak baryogenesis, New J. Phys. 14 (2012) 125003 [1206.2942].

[9.16] J.S. Langer, Theory of the condensation point, Ann. Phys. 41 (1967) 108.

[9.17] J.S. Langer, Statistical theory of the decay of metastable states, Ann. Phys. 54 (1969) 258.

[9.18] S.R. Coleman, Fate of the false vacuum: Semiclassical theory, Phys. Rev. D 15 (1977) 2929; ibid. 16 (1977) 1248 (E).

[9.19] A.D. Linde, Fate of the false vacuum at finite temperature: Theory and applications, Phys. Lett. B 100 (1981) 37. 
[9.20] C.G. Callan and S.R. Coleman, Fate of the false vacuum. II. First quantum corrections, Phys. Rev. D 16 (1977) 1762.

[9.21] I. Affleck, Quantum-Statistical Metastability, Phys. Rev. Lett. 46 (1981) 388.

[9.22] P. Arnold, D. Son and L.G. Yaffe, The hot baryon violation rate is $\mathcal{O}\left(\alpha_{w}^{5} T^{4}\right)$, Phys. Rev. D 55 (1997) 6264 [hep-ph/9609481].

[9.23] G.D. Moore and K. Rummukainen, Electroweak bubble nucleation, nonperturbatively, Phys. Rev. D 63 (2001) 045002 [hep-ph/0009132].

[9.24] V.A. Rubakov and M.E. Shaposhnikov, Electroweak baryon number non-conservation in the early Universe and in high-energy collisions, Usp. Fiz. Nauk 166 (1996) 493 [Phys. Usp. 39 (1996) 461] [hep-ph/9603208].

[9.25] A.A. Belavin, A.M. Polyakov, A.S. Schwartz and Y.S. Tyupkin, Pseudoparticle solutions of the Yang-Mills equations, Phys. Lett. B 59 (1975) 85.

[9.26] F.R. Klinkhamer and N.S. Manton, A saddle-point solution in the Weinberg-Salam theory, Phys. Rev. D 30 (1984) 2212.

[9.27] P. Arnold and L.D. McLerran, Sphalerons, small fluctuations, and baryon-number violation in electroweak theory, Phys. Rev. D 36 (1987) 581.

[9.28] J. Ambjørn, T. Askgaard, H. Porter and M.E. Shaposhnikov, Sphaleron transitions and baryon asymmetry: A numerical, real-time analysis, Nucl. Phys. B 353 (1991) 346.

[9.29] M. D'Onofrio, K. Rummukainen and A. Tranberg, Sphaleron Rate in the Minimal Standard Model, Phys. Rev. Lett. 113 (2014) 141602 [1404.3565].

[9.30] L.D. Landau and E.M. Lifshitz, Statistical Physics, Part 1, §162 (Butterworth-Heinemann, Oxford).

[9.31] A.D. Linde, Decay of the false vacuum at finite temperature, Nucl. Phys. B 216 (1983) 421; ibid. 223 (1983) 544 (E).

[9.32] A.H. Guth and E.J. Weinberg, Cosmological consequences of a first-order phase transition in the SU(5) Grand Unified Model, Phys. Rev. D 23 (1981) 876.

[9.33] G.M. Fuller, G.J. Mathews and C.R. Alcock, Quark-hadron phase transition in the early Universe: Isothermal baryon number fluctuations and primordial nucleosynthesis, Phys. Rev. D 37 (1988) 1380.

[9.34] K. Enqvist, J. Ignatius, K. Kajantie and K. Rummukainen, Nucleation and bubble growth in a first order cosmological electroweak phase transition, Phys. Rev. D 45 (1992) 3415.

[9.35] D. Bödeker and G.D. Moore, Electroweak bubble wall speed limit, JCAP 05 (2017) 025 [1703.08215].

[9.36] H. Kurki-Suonio and M. Laine, On bubble growth and droplet decay in cosmological phase transitions, Phys. Rev. D 54 (1996) 7163 [hep-ph/9512202].

[9.37] L.D. McLerran and T. Toimela, Photon and dilepton emission from the quark-gluon plasma: Some general considerations, Phys. Rev. D 31 (1985) 545. 
[9.38] H.A. Weldon, Reformulation of finite-temperature dilepton production, Phys. Rev. D 42 (1990) 2384 .

[9.39] T. Asaka, M. Laine and M. Shaposhnikov, On the hadronic contribution to sterile neutrino production, JHEP 06 (2006) 053 [hep-ph/0605209].

[9.40] J. Ghiglieri and M. Laine, Improved determination of sterile neutrino dark matter spectrum, JHEP 11 (2015) 171 [1506.06752].

[9.41] D. Bödeker, M. Sangel and M. Wörmann, Equilibration, particle production, and self-energy, Phys. Rev. D 93 (2016) 045028 [1510.06742].

[9.42] A. Anisimov, D. Besak and D. Bödeker, Thermal production of relativistic Majorana neutrinos: strong enhancement by multiple soft scattering, JCAP 03 (2011) 042 [1012.3784].

[9.43] D. Besak and D. Bödeker, Thermal production of ultrarelativistic right-handed neutrinos: complete leading-order results, JCAP 03 (2012) 029 [1202.1288].

[9.44] P.B. Arnold, G.D. Moore and L.G. Yaffe, Photon emission from ultrarelativistic plasmas, JHEP 11 (2001) 057 [hep-ph/0109064].

[9.45] P.B. Arnold, G.D. Moore and L.G. Yaffe, Photon emission from quark-gluon plasma: complete leading order results, JHEP 12 (2001) 009 [hep-ph/0111107].

[9.46] M. Cohen and R.P. Feynman, Theory of Inelastic Scattering of Cold Neutrons from Liquid Helium, Phys. Rev. 107 (1957) 13.

[9.47] D.G. Henshaw and A.D.B. Woods, Modes of Atomic Motions in Liquid Helium by Inelastic Scattering of Neutrons, Phys. Rev. 121 (1961) 1266.

[9.48] J. Bernstein, Kinetic Theory in the Expanding Universe (Cambridge University Press, Cambridge, 1988).

[9.49] E.W. Kolb and M.S. Turner, The Early Universe, Front. Phys. 69 (1990) 1-547.

[9.50] T. Asaka, M. Laine and M. Shaposhnikov, Lightest sterile neutrino abundance within the

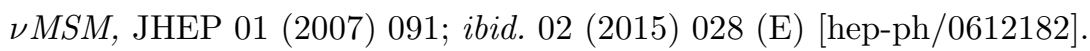

[9.51] M. Shaposhnikov and I. Tkachev, The $\nu M S M$, inflation, and dark matter, Phys. Lett. B 639 (2006) 414 [hep-ph/0604236].

[9.52] K. Petraki and A. Kusenko, Dark-matter sterile neutrinos in models with a gauge singlet in the Higgs sector, Phys. Rev. D 77 (2008) 065014 [0711.4646].

[9.53] T. Matsui, B. Svetitsky and L.D. McLerran, Strangeness production in ultrarelativistic heavyion collisions. I. Chemical kinetics in the quark-gluon plasma, Phys. Rev. D 34 (1986) 783; ibid. 37 (1988) 844 (E).

[9.54] D. Bödeker, Moduli decay in the hot early Universe, JCAP 06 (2006) 027 [hep-ph/0605030].

[9.55] M. Laine, On bulk viscosity and moduli decay, Prog. Theor. Phys. Suppl. 186 (2010) 404 [1007.2590].

[9.56] L.D. McLerran, E. Mottola and M.E. Shaposhnikov, Sphalerons and axion dynamics in high-temperature QCD, Phys. Rev. D 43 (1991) 2027. 
[9.57] M. Lüscher, Topological effects in QCD and the problem of short distance singularities, Phys. Lett. B 593 (2004) 296 [hep-th/0404034].

[9.58] M.P. Lombardo and A. Trunin, Topology and axions in QCD, Int. J. Mod. Phys. A 35 (2020) 2030010 [2005.06547].

[9.59] P.B. Arnold, C. Dogan and G.D. Moore, Bulk viscosity of high-temperature QCD, Phys. Rev. D 74 (2006) 085021 [hep-ph/0608012].

[9.60] G.D. Moore, Motion of Chern-Simons number at high temperatures under a chemical potential, Nucl. Phys. B 480 (1996) 657 [hep-ph/9603384].

[9.61] D.T. Son and A.O. Starinets, Minkowski-space correlators in AdS/CFT correspondence: recipe and applications, JHEP 09 (2002) 042 [hep-th/0205051].

[9.62] D.Y. Grigoriev and V.A. Rubakov, Soliton pair creation at finite temperatures. Numerical study in (1+1)-dimensions, Nucl. Phys. B 299 (1988) 67.

[9.63] D. Bödeker, Classical real time correlation functions and quantum corrections at finite temperature, Nucl. Phys. B 486 (1997) 500 [hep-th/9609170].

[9.64] D. Bödeker, G.D. Moore and K. Rummukainen, Chern-Simons number diffusion and hard thermal loops on the lattice, Phys. Rev. D 61 (2000) 056003 [hep-ph/9907545].

[9.65] G.D. Moore and M. Tassler, The sphaleron rate in $S U(N)$ gauge theory, JHEP 02 (2011) 105 [1011.1167].

[9.66] K.V. Berghaus, P.W. Graham and D.E. Kaplan, Minimal warm inflation, JCAP 03 (2020) 034 [1910.07525].

[9.67] S. Das, G. Goswami and C. Krishnan, Swampland, axions and minimal warm inflation, Phys. Rev. D 101 (2020) 10 [1911.00323].

[9.68] Y. Reyimuaji and X. Zhang, Warm-assisted natural inflation, JCAP 04 (2021) 077 [2012.07329].

[9.69] M. Laine and S. Procacci, Minimal warm inflation with complete medium response, JCAP 06 (2021) 031 [2102.09913].

[9.70] H.B. Meyer, Transport properties of the quark-gluon plasma: A lattice QCD perspective, Eur. Phys. J. A 47 (2011) 86 [1104.3708].

[9.71] G. Aarts, Transport and spectral functions in high-temperature QCD, PoS LAT2007 (2007) 001 [0710.0739].

[9.72] P. Kovtun, G.D. Moore and P. Romatschke, Stickiness of sound: An absolute lower limit on viscosity and the breakdown of second-order relativistic hydrodynamics, Phys. Rev. D 84 (2011) 025006 [1104.1586].

[9.73] P.B. Arnold, G.D. Moore and L.G. Yaffe, Transport coefficients in high temperature gauge theories (I): leading-log results, JHEP 11 (2000) 001 [hep-ph/0010177].

[9.74] L.G. Yaffe, Dynamics of hot gauge theories, Nucl. Phys. B (Proc. Suppl.) 106 (2002) 117 [hep-th/0111058]. 
[9.75] P.B. Arnold, G.D. Moore and L.G. Yaffe, Transport coefficients in high temperature gauge theories (II): Beyond leading log, JHEP 05 (2003) 051 [hep-ph/0302165].

[9.76] G.D. Moore and D. Teaney, How much do heavy quarks thermalize in a heavy ion collision?, Phys. Rev. C 71 (2005) 064904 [hep-ph/0412346].

[9.77] D. Teaney, Finite temperature spectral densities of momentum and $R$-charge correlators in $\mathcal{N}=4$ Yang-Mills theory, Phys. Rev. D 74 (2006) 045025 [hep-ph/0602044].

[9.78] G.D. Moore and K.A. Sohrabi, Kubo Formulas for Second-Order Hydrodynamic Coefficients, Phys. Rev. Lett. 106 (2011) 122302 [1007.5333].

[9.79] R. Kubo, Statistical-Mechanical Theory of Irreversible Processes. I. General Theory and Simple Applications in Magnetic and Conduction Problems, J. Phys. Soc. Jap. 12 (1957) 570 .

[9.80] S.Y. Khlebnikov and M.E. Shaposhnikov, The statistical theory of anomalous fermion number non-conservation, Nucl. Phys. B 308 (1988) 885.

[9.81] J. Casalderrey-Solana and D. Teaney, Heavy quark diffusion in strongly coupled $\mathcal{N}=4$ Yang Mills, Phys. Rev. D 74 (2006) 085012 [hep-ph/0605199].

[9.82] S. Caron-Huot, M. Laine and G.D. Moore, A way to estimate the heavy quark thermalization rate from the lattice, JHEP 04 (2009) 053 [0901.1195].

[9.83] D. Bödeker and M. Laine, Heavy quark chemical equilibration rate as a transport coefficient, JHEP 07 (2012) 130 [1205.4987].

[9.84] E.M. Lifshitz and L.P. Pitaevskii, Statistical Physics, Part 2, §88-89 (ButterworthHeinemann, Oxford).

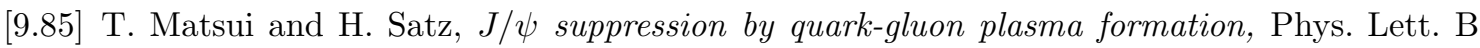
178 (1986) 416.

[9.86] S. Kim and M. Laine, On thermal corrections to near-threshold annihilation, JCAP 01 (2017) 013 [1609.00474].

[9.87] A. Czarnecki and K. Melnikov, Two-Loop QCD Corrections to the Heavy Quark Pair Production Cross Section in $e^{+} e^{-}$Annihilation near Threshold, Phys. Rev. Lett. 80 (1998) 2531 [hep-ph/9712222].

[9.88] M. Beneke, A. Signer and V.A. Smirnov, Two-Loop corrections to the Leptonic Decays of Quarkonium, Phys. Rev. Lett. 80 (1998) 2535 [hep-ph/9712302].

[9.89] C.W. Bernard, Feynman rules for gauge theories at finite temperature, Phys. Rev. D 9 (1974) 3312 .

[9.90] J.B. Kogut and L. Susskind, Hamiltonian formulation of Wilson's lattice gauge theories, Phys. Rev. D 11 (1975) 395.

[9.91] M. Lüscher, Construction of a selfadjoint, strictly positive transfer matrix for Euclidean lattice gauge theories, Commun. Math. Phys. 54 (1977) 283.

[9.92] K.J. Juge, J. Kuti and C. Morningstar, Fine Structure of the QCD String Spectrum, Phys. Rev. Lett. 90 (2003) 161601 [hep-lat/0207004]. 
[9.93] A. Rothkopf, T. Hatsuda and S. Sasaki, Proper heavy-quark potential from a spectral decomposition of the thermal Wilson loop, PoS LAT2009 (2009) 162 [0910.2321].

[9.94] J. Ghiglieri, Review of the EFT treatment of quarkonium at finite temperature, PoS ConfinementX (2012) 004 [1303.6438].

[9.95] D. Blaschke, G. Burau, Y. Kalinovsky and T. Barnes, Mott effect and $J / \psi$ dissociation at the quark hadron phase transition, Eur. Phys. J. A 18 (2003) 547 [nucl-th/0211058]. 


\section{Appendix: Extended Standard Model in Euclidean spacetime}

In eq. (5.39), the gauge-fixed imaginary-time Lagrangian of QCD was given. Here we display the corresponding structure for the Standard Model. For simplicity, terms related to gauge fixing are omitted. On the other hand, we include right-handed neutrinos as degrees of freedom, even though they are not considered to be part of the "classic" Standard Model, in which neutrinos are postulated to be massless. We include them because this does not change any of the construction principles and yet offers for a simple way to solve a number of short-comings of the classic Standard Model (for a review, see ref. [10.1]). A reader preferring not to include right-handed neutrinos may decouple them by setting the corresponding Yukawa coupling matrix $h_{\nu}$ to zero.

In QCD literature, it is common to keep the number of colours, $N_{\mathrm{c}}$, as a free parameter. When considering the Standard Model, however, its proper inclusion requires care [10.2,10.3]. For simplicity we restrict to $N_{\mathrm{c}}=3$ in the following. The gauge group is then $\mathrm{U}_{\mathrm{Y}}(1) \times \mathrm{SU}_{\mathrm{L}}(2) \times \mathrm{SU}_{\mathrm{c}}(3)$, where $\mathrm{Y}$ refers to the hypercharge degree of freedom and $\mathrm{L}$ to left-handed fermions.

The fermionic matter fields of the Standard Model carry a specific chirality. Denoting chiral projectors by $a_{\mathrm{L}} \equiv\left(1-\gamma_{5}\right) / 2$ and $a_{\mathrm{R}} \equiv\left(1+\gamma_{5}\right) / 2$, left-handed doublets are defined as

$$
Q_{a} \equiv a_{\mathrm{L}} Q_{a} \equiv\left(\begin{array}{c}
a_{\mathrm{L}} u_{a} \\
a_{\mathrm{L}} d_{a}
\end{array}\right), \quad L_{a} \equiv a_{\mathrm{L}} L_{a} \equiv\left(\begin{array}{c}
a_{\mathrm{L}} \nu_{a} \\
a_{\mathrm{L}} e_{a}
\end{array}\right),
$$

where $a \in\{1,2,3\}$ is a "family" or "generation" index. Here the fermion fields are 4-component Dirac spinors. The quark fields carry an additional colour index that has been suppressed in the notation, i.e. they are really 12-component spinors. Subsequently we redefine the notation in order to denote the right-handed components by

$$
u_{a} \equiv a_{\mathrm{R}} u_{a}, \quad d_{a} \equiv a_{\mathrm{R}} d_{a}, \quad \nu_{a} \equiv a_{\mathrm{R}} \nu_{a}, \quad e_{a} \equiv a_{\mathrm{R}} e_{a} .
$$

The scalar (Higgs) doublet is denoted by $\phi$, and $\tilde{\phi} \equiv i \sigma_{2} \phi^{*}$, where $\sigma_{2}$ is a Pauli matrix, is a conjugated version thereof, which transforms in the same way under $\mathrm{SU}_{\mathrm{L}}(2)$ but has an opposite "charge" under $\mathrm{U}_{\mathrm{Y}}(1)$. Note that the right-handed neutrino field $\nu_{a}$ was denoted by $N$ in eq. (8.70).

With this field content, the Euclidean Lagrangian can be written as $\left(Q=\left(Q_{1} Q_{2} Q_{3}\right)^{T}\right.$, etc.)

$$
\begin{aligned}
L_{E} & \equiv \frac{1}{4} F_{\mu \nu}^{a_{i}} F_{\mu \nu}^{a_{i}}+\left(D_{\mu} \phi\right)^{\dagger} D_{\mu} \phi-m^{2} \phi^{\dagger} \phi+\lambda\left(\phi^{\dagger} \phi\right)^{2} \\
& +\bar{Q} \not D Q+\bar{u} \not D u+\bar{d} \not D d+\bar{L} \not D L+\bar{\nu} \not D \nu+\bar{e} \not D e+\frac{1}{2}\left(\bar{\nu}^{c} M \nu+\bar{\nu} M^{\dagger} \nu^{c}\right) \\
& +\bar{Q} h_{u} u \tilde{\phi}+\bar{Q} h_{d} d \phi+\bar{L} h_{\nu} \nu \tilde{\phi}+\bar{L} h_{e} e \phi \\
& +\tilde{\phi}^{\dagger} \bar{u} h_{u}^{\dagger} Q+\phi^{\dagger} \bar{d} h_{d}^{\dagger} Q+\tilde{\phi}^{\dagger} \bar{\nu} h_{\nu}^{\dagger} L+\phi^{\dagger} \bar{e} h_{e}^{\dagger} L .
\end{aligned}
$$

A number of undefined symbols will be explained below. Starting from the end of the second row, $\nu^{c} \equiv C \bar{\nu}^{T}$ denotes a charge-conjugated spinor, with the charge conjugation matrix defined for instance as $C \equiv i \gamma^{2} \gamma^{0}$, where $\gamma^{\mu}$ are Dirac matrices. It is possible to verify that the "Majorana" mass matrix $M$ is symmetric, $M^{T}=M$; through the so-called Takagi factorization (a special case of singular value decomposition), it can consequently be written as $M=V \Delta V^{T}$, where $V$ is unitary and $\Delta$ is a diagonal matrix with real non-negative entries, referred to as the Majorana masses of the right-handed neutrinos.

The theory defined by eq. (10.3) contains a number of parameters: the real gauge couplings $g_{1}, g_{2}, g_{3}$; the Higgs mass parameter $m^{2}$ and self-coupling $\lambda$; the complex $3 \times 3$ Yukawa matrices 
$h_{u}, h_{d}, h_{\nu}$ and $h_{e}$; and the complex $3 \times 3$ Majorana mass matrix $M$. In the quantized theory, all of these are to be understood as bare parameters. Note that there is a fairly large redundancy in this parameter set, which could be reduced by various field redefinitions, but for simplicity we display the general expression.

Let us now define the gauge interactions. In the fermionic case, gauge interactions reside in $\not D=\gamma_{\mu} D_{\mu}$, where $D_{\mu}$ is a covariant derivative. When acting on the Higgs doublet the covariant derivative takes the form

$$
D_{\mu} \phi \equiv\left(\partial_{\mu}+\frac{i g_{1}}{2} A_{\mu}-i g_{2} T^{a_{2}} B_{\mu}^{a_{2}}\right) \phi,
$$

where $g_{1}$ and $g_{2}$ are gauge couplings related to the $\mathrm{U}_{\mathrm{Y}}(1)$ and $\mathrm{SU}_{\mathrm{L}}(2)$ gauge fields $A_{\mu}$ and $B_{\mu}^{a_{2}}$, respectively, and $T^{a_{2}}$ are Hermitean generators of $\mathrm{SU}_{\mathrm{L}}(2)$, normalized as $\operatorname{Tr}\left[T^{a_{2}} T^{b_{2}}\right]=\frac{1}{2} \delta^{a_{2} b_{2}}$. We employ a notation whereby the index $a_{2} \in\left\{1, \ldots, d_{2} \equiv 3\right\}$ implies the use of $\mathrm{SU}(2)$ generators, and repeated indices are summed over (in addition, $d_{1} \equiv 1$, and sums over $a_{1}$ are omitted whenever possible). When acting on leptons, the covariant derivative reads

$$
\begin{aligned}
D_{\mu} L_{a} & \equiv\left(\partial_{\mu}-\frac{i g_{1}}{2} A_{\mu}-i g_{2} T^{a_{2}} B_{\mu}^{a_{2}}\right) L_{a}, \\
D_{\mu} \nu_{a} & \equiv\left(\partial_{\mu}\right) \nu_{a}, \\
D_{\mu} e_{a} & \equiv\left(\partial_{\mu}-i g_{1} A_{\mu}\right) e_{a} .
\end{aligned}
$$

In the case of quarks, the $\mathrm{SU}_{\mathrm{c}}(3)$ gauge coupling $g_{3}$ and the generators $T^{a_{3}}$ and the gauge fields $C_{\mu}^{a_{3}}$ appear as well:

$$
\begin{aligned}
D_{\mu} Q_{a} & \equiv\left(\partial_{\mu}+\frac{i g_{1}}{6} A_{\mu}-i g_{2} T^{a_{2}} B_{\mu}^{a_{2}}-i g_{3} T^{a_{3}} C_{\mu}^{a_{3}}\right) Q_{a}, \\
D_{\mu} u_{a} & \equiv\left(\partial_{\mu}+\frac{2 i g_{1}}{3} A_{\mu}-i g_{3} T^{a_{3}} C_{\mu}^{a_{3}}\right) u_{a}, \\
D_{\mu} d_{a} & \equiv\left(\partial_{\mu}-\frac{i g_{1}}{3} A_{\mu}-i g_{3} T^{a_{3}} C_{\mu}^{a_{3}}\right) d_{a} .
\end{aligned}
$$

The colour index $a_{3}$ is summed over the set $a_{3} \in\left\{1, \ldots, d_{3} \equiv 8\right\}$. Finally, field strength tensors are defined in accordance with eq. (5.1),

$$
\begin{aligned}
& F_{\mu \nu}^{a_{1}} \equiv \partial_{\mu} A_{\nu}-\partial_{\nu} A_{\mu}, \\
& F_{\mu \nu}^{a_{2}} \equiv \partial_{\mu} B_{\nu}^{a_{2}}-\partial_{\nu} B_{\mu}^{a_{2}}+g_{2} \epsilon^{a_{2} b_{2} c_{2}} B_{\mu}^{b_{2}} B_{\nu}^{c_{2}}, \\
& F_{\mu \nu}^{a_{3}} \equiv \partial_{\mu} C_{\nu}^{a_{3}}-\partial_{\nu} C_{\mu}^{a_{3}}+g_{3} f^{a_{3} b_{3} c_{3}} C_{\mu}^{b_{3}} C_{\nu}^{c_{3}},
\end{aligned}
$$

where $\epsilon^{a_{2} b_{2} c_{2}}$ is the Levi-Civita symbol and $f^{a_{3} b_{3} c_{3}}$ are the structure constants of $\mathrm{SU}(3)$.

We remark that we have not included so-called $\theta$-terms in eq. (10.3), which would have the form $\delta L_{E}=i \sum_{n=1}^{3} \theta_{n} \frac{\epsilon_{\mu \nu \rho \sigma} g_{n}^{2} F_{\mu \nu}^{a_{n}} F_{\rho \sigma}^{a_{n}}}{64 \pi^{2}}$. This is because our Yukawa couplings are complex. The complex Yukawa couplings corresponding to quark masses can be tuned to be real by a chiral rotation, but this induces a QCD $\theta$-term, leading to the so-called strong $\mathrm{CP}$ problem, i.e. the unnatural-looking fact that phenomenologically $\theta_{3} \approx 0$. (It is an interesting exercise to contemplate why the $\mathrm{U}_{\mathrm{Y}}(1)$ and the $\mathrm{SU}_{\mathrm{L}}(2) \theta$-angles, $\theta_{1}$ and $\theta_{2}$, do not pose similar problems.)

Finally we recall that the quantization of chiral gauge theories is highly non-trivial. Even staying within perturbation theory, $\gamma_{5}$ has to be carefully defined in the context of dimensional regularization $[10.4,10.5]$, but this tends to break spacetime and/or gauge symmetries, leading e.g. to a complicated pattern of operator mixings $[10.6,10.7]$. 


\section{Literature}

[10.1] L. Canetti, M. Drewes, T. Frossard and M. Shaposhnikov, Dark matter, baryogenesis and neutrino oscillations from right-handed neutrinos, Phys. Rev. D 87 (2013) 093006 [1208.4607].

[10.2] A. Abbas, Anomalies and charge quantization in the Standard Model with arbitrary number of colours, Phys. Lett. B 238 (1990) 344.

[10.3] O. Bär and U.-J. Wiese, Can one see the number of colors?, Nucl. Phys. B 609 (2001) 225 [hep-ph/0105258].

[10.4] G. 't Hooft and M.J.G. Veltman, Regularization and renormalization of gauge fields, Nucl. Phys. B 44 (1972) 189.

[10.5] P. Breitenlohner and D. Maison, Dimensional renormalization and the action principle, Commun. Math. Phys. 52 (1977) 11.

[10.6] J.G. Körner, N. Nasrallah and K. Schilcher, Evaluation of the flavor-changing vertex $b \rightarrow s H$ using the Breitenlohner-Maison-'t Hooft-Veltman $\gamma_{5}$ scheme, Phys. Rev. D 41 (1990) 888.

[10.7] A.J. Buras and P.H. Weisz, QCD nonleading corrections to weak decays in dimensional regularization and 't Hooft-Veltman schemes, Nucl. Phys. B 333 (1990) 66. 


\section{Index}

Analytic continuation, 118, 123

Antiperiodic boundary conditions, 52

Asymptotic mass, 148

Axion damping coefficient, 195

Axion mass, 194

Background field gauge, 94

Blackbody radiation, 69

Boltzmann equation, 187, 189

Bose enhancement, 85, 189

Bose-Einstein condensation, 102

Brownian motion, 205

BRST symmetry, 62, 68

Bubble nucleation, 164

Caloron, 166

Canonical quantization: Dirac field, 53

Canonical quantization: fermionic oscillator, 49

Canonical quantization: gauge field, 63

Canonical quantization: harmonic oscillator, 1

Chemical equilibration rate, 207

Chemical potential: Dirac field, 105

Chemical potential: gauge field?, 106

Chemical potential: scalar field, 18, 99

Chern-Simons diffusion, 196

Classical field theory, 195

Classical limit, 92, 169, 198, 200, 205

Cold and dense limit, 110

Condensate, 102, 156

Conductivity, 203

Constrained effective potential, 103

Cosmological background, 183

Covariant derivative, 61

Critical bubble, 169

Daisy resummation, 45

Damping coefficient, 205

Damping rate, 193

Debye mass, 79, 141

Debye screening, 142, 215

Decay rate, 180
Decoherence, 216

Deflagration, 164

Density matrix, 132, 174, 199

Diffusion coefficient, 203, 206

Dilaton damping coefficient, 195

Dilaton mass, 194

Dilepton production rate, 174

Dimensional reduction, 91, 161

Dimensional regularization, 20

Dirac matrices, 53, 214

Dispersion relation, 148

Effective field theories: general, 85

Effective field theories: scalar field, 46

Effective mass, 47, 79, 193

Effective potential, 103, 106, 156, 158, 193

Einstein equations, 183

Electric conductivity, 203

Equilibration rate, 197, 205

Euclidean correlator: bosonic, 115

Euclidean correlator: fermionic, 121

Euclidean Dirac matrices, 54

Euclidean Lagrangian: Dirac field, 53, 105

Euclidean Lagrangian: gauge field, 65

Euclidean Lagrangian: harmonic oscillator, 5

Euclidean Lagrangian: QCD, 68

Euclidean Lagrangian: scalar field, 14, 30

Euclidean Lagrangian: Standard Model, 223

Euler gamma function, 25

Faddeev-Popov ghosts, 67

Fermi's Golden Rule, 189

Feynman gauge, 73

Feynman rules: Euclidean QCD, 68

Finite density, 99

First order phase transition, 160

Flavour diffusion coefficient, 202

Fluctuation determinant, 165

Fluctuation-dissipation theorem, 117, 198

Fock space, 214 
Fokker-Planck equation, 211

Fourier representation: fermion, 54

Fourier representation: harmonic oscillator, 6

Fourier representation: scalar field, 14

Free energy density: Dirac field, 57

Free energy density: QCD, 80

Free energy density: scalar field, 31

Friction coefficient, 192, 193

Friedmann equations, 183

Gauge fixing and ghosts, 67

Gauge invariance, 61

Gauss law, 63

Ghost self-energy, 83

Gibbs-Duhem equation, 191

Grassmann variables, 50

Green's functions: time orderings, 114

Gribov ambiguity, 67

Hard and soft modes: thermal QCD, 91

Hard and soft modes: vacuum example, 89

Hard Thermal Loops (HTL), 131, 137

Heisenberg-operator: bosonic, 114, 197

Heisenberg-operator: fermionic, 120

HTL: angular integrals, 150

HTL: effective action, 143

HTL: fermion propagator, 148

HTL: fermion self-energy, 148

HTL: gluon propagator, 142

HTL: gluon self-energy, 143

HTL: radial integrals, 149

HTL: spectral representation, 142

Hubble parameter, 185

Imaginary-time formalism, 5

Infrared divergence: general, 85

Infrared divergence: harmonic oscillator, 7

Infrared divergence: scalar field, 23, 38

Instanton, 166

Interaction Hamiltonian, 175

Kramers-Kronig relations, 192

Kubo formula, 199

Kubo-Martin-Schwinger: bosonic, 116

Kubo-Martin-Schwinger: fermionic, 121

Landau damping, 141, 143

Landau-Pomeranchuk-Migdal (LPM), 179

Langer formalism, 164
Langevin equation, 205

Latent heat, 155, 164, 171

Linde problem, 86

Linear response, 198

Liouville - von Neumann equation, 174

Lorentzian shape, 201

Magnetic fields, 71

Matching: equilibration rate, 198, 207

Matching: general, 90

Matching: harmonic oscillator, 7

Matching: thermal QCD, 92

Matching: transport coefficients, 203

Matsubara frequencies: bosonic, 6

Matsubara frequencies: fermionic, 54

Method of characteristics, 185

Noether's theorem, 100

Non-equilibrium ensemble, 132

Nucleation dynamics, 172

Nucleation rate, 164

On-shell field operator, 176

Order parameter, 156

Particle production rate: general, 174

Particle production rate: spectrum, 186

Partition function: complex scalar, 101

Partition function: Dirac field, 55

Partition function: fermionic oscillator, 49

Partition function: gauge field, 61

Partition function: harmonic oscillator, 2

Partition function: scalar field, 13

Path integral: complex scalar, 101

Path integral: Dirac field, 105

Path integral: fermionic oscillator, 49

Path integral: gauge field, 61

Path integral: harmonic oscillator, 3

Path integral: scalar field, 13

Pauli blocking, 188, 189

Photon production rate, 174

Plasma oscillations, 142

Plasmino, 148, 182

Plasmon, 142, 143

Propagator: Dirac fermion, 69

Propagator: gauge field, 69

Propagator: HTL-resummed, 129, 142

Propagator: scalar field, 34 
QCD, 68

QED, 71, 106, 141, 145, 151

Quantum tunnelling, 167

Quarkonium dissociation, 216

Quarkonium states, 213

Real-time formalism, 132

Real-time observables, 114

Renormalization, 40

Resolvent, 118

Resummation, 44, 86

Resummed self-energy: fermion, 145

Resummed self-energy: gluon, 141

Retarded correlator: bosonic, 115

Retarded correlator: fermionic, 121

Riemann zeta function, 26

Ring diagrams, 45

Saclay method, 127

Saddle point approximation, 104, 106, 165

Scattering on dense media, 181

Schwinger-Keldysh formalism, 133

Screening, 79, 142, 215

Self-energy: fermion, 145

Self-energy: gluon, 138, 142

Self-energy: photon, 151

Self-energy: scalar, 46

Semiclassical approximation, 166

Slavnov-Taylor identities, 78, 141

Soft and hard modes: thermal QCD, 91

Soft and hard modes: vacuum example, 89

Spectral function: bosonic, 115

Spectral function: fermionic, 121

Spectral representation, 118, 122, 129, 143

Sphaleron, 169

Sphaleron rate, 195

Spinodal decomposition, 162

Stefan-Boltzmann law, 70

Sum rule, 118, 144

Surface tension, 171

Susceptibility, 108, 193, 198, 203, 206

Susceptibility: next-to-leading order, 109

Symmetries: general effective theory, 90

Symmetries: thermal QCD, 91

Takagi factorization, 223

Thermal fluctuations, 167

Thermal mass: fermion, 147
Thermal mass: ghost?, 83

Thermal mass: gluon, 73

Thermal mass: scalar, 46

Thermal phase transitions, 155

Thermal sums: boson loop, 11, 16, 27, 35

Thermal sums: boson-boson loop, 145

Thermal sums: boson-fermion loop, 126, 146

Thermal sums: bosonic tensor, 76

Thermal sums: chemical potential, 107

Thermal sums: fermion loop, 56, 59

Thermal sums: fermion-fermion loop, 138

Thermal sums: fermionic tensor, 78

Thermal sums: high-temperature expansion, 23

Thermal sums: high-temperature fermion, 57

Thermal sums: low-temperature expansion, 19

Thermal sums: low-temperature fermion, 57

Thermal sums: non-perturbative case, 129

Thermal sums: non-relativistic boson, 119

Thermal sums: non-relativistic fermion, 123

Thermal sums: with chemical potential, 124

Thermal width, 216

Time-ordered correlator: bosonic, 115

Time-ordered correlator: fermionic, 121

Time-ordered propagator: free boson, 120

Time-ordered propagator: free fermion, 125

Transport coefficients, 197

Transport peak, 200

Triviality of scalar field theory, 161

Truncation of effective theory, 92

Two-step phase transition, 162

Viscosities, 204

Ward-Takahashi identities, 78

Weak-coupling expansion: gauge field, 67

Weak-coupling expansion: scalar field, 30

Wick contractions, 33

Wick rotation, 114

Wick's theorem, 31

Wightman function, 115, 132

Yang-Mills theory, 61, 66

Yield parameter, 186

Yukawa interaction, 126, 223

Zero mode: harmonic oscillator, 6

Zero mode: instanton, 167

Zero mode: Matsubara formalism, 85

Zero mode: scalar field, 14, 23, 44 